


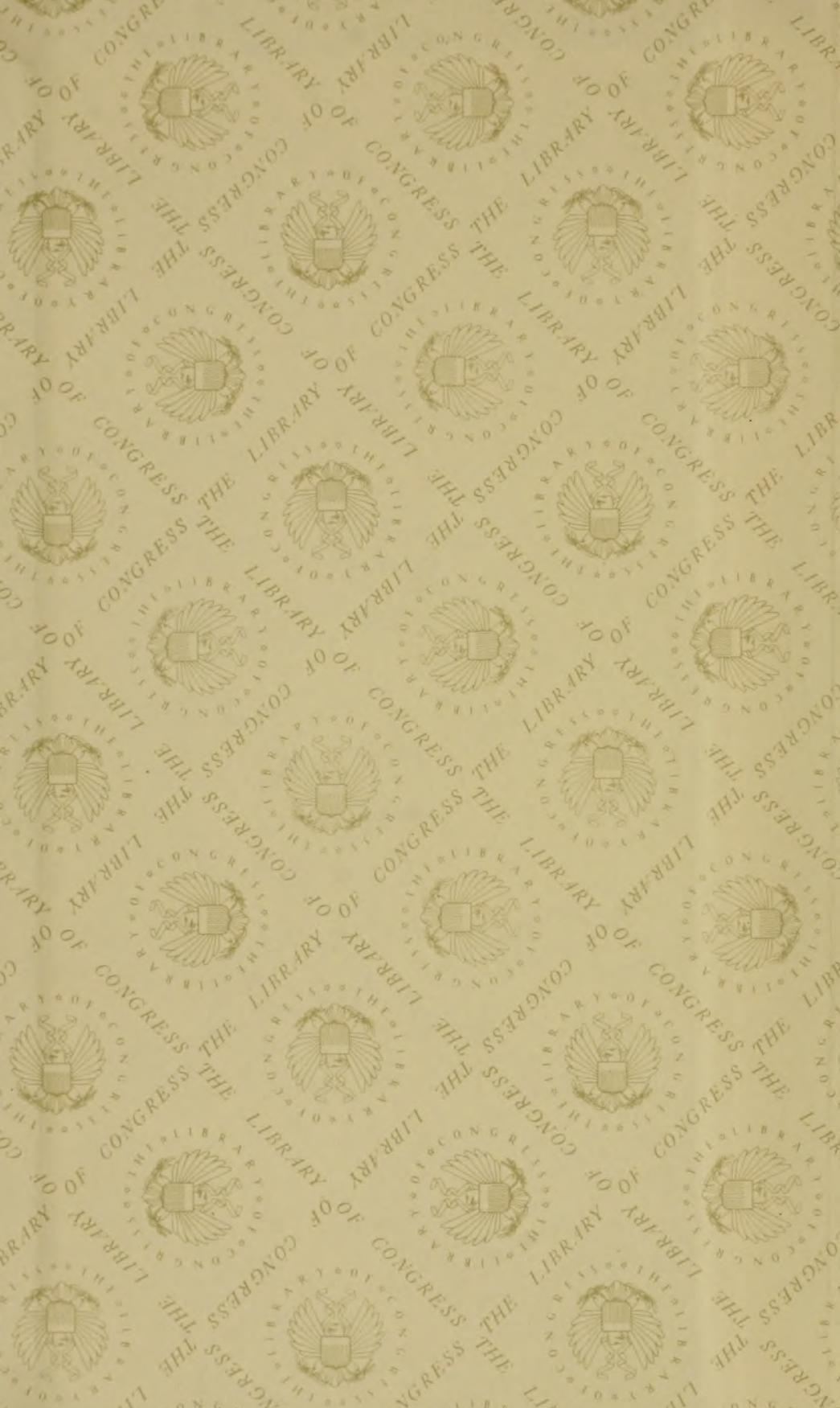





$$
\text { . }
$$



THE

\section{FRUITS AND FRUIT TREES}

- A M E R I C' A;

or,

rhB culture, propagation, aNd MaNagement, iN THE GARDEx axD ORCHARD, OF FRUIT TREES GENERALLY;

พ2т н

DESCRIPTIONS OF ALL THE FINEST VARIETIES OF FRUIT NATIVE AND FOREIGN, CULTIVATED IN THIS COUNTRY.

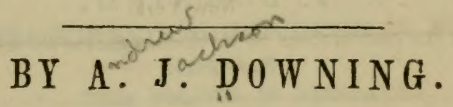

COKB FSPONDING KEMBER OF THE ROYAZ BOTANIC gOCIETY OF LONDON: AND OF TEI AORTICC:TCRAC SOCIETIES OF BERCIN; THE LOW COUNTRIKS, AABSACE-

gETTS - PENNSYLVANU ; INDIANA, CINCINNATI, ET.?.

What wondrous life is this I lead?

Ripe apples drop about my head;

The luscious clusters of the vine

Upon my month do crush their wine;

The nectarine and curious peach

Into $\mathrm{mv}$ hands themselves do -eacl.

Marvel.

FOURTEENTH EDITION.

NEW YORK :

W I L E \& H A L T E D,

No, 351 BROADWAT.

1856 . 


\section{$S B 355$ .175 1856}

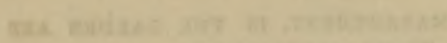

$\operatorname{con} 2 \cos 2017$

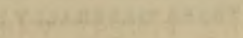

Futated wccording to Acr of Congress, in the year $1945 \mathrm{Ns}$

A. J. DOWNING,

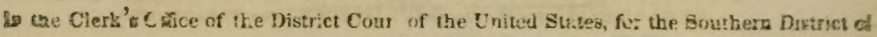
Niew-Yord.

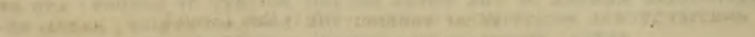

$$
\begin{aligned}
& \text { In exchange } \\
& \text { new youk state fit } \\
& \text { op. } 30,1924
\end{aligned}
$$


To

MARShall P. WiLder, Eвa.,

PRESIDENT OF THE

MAFสACHUSETTS HIRTICULTURAL SOCIETY,

THES VOLUME IS DEDICATED,

BY HIS FRIEND,

THE AUTHOR 



\section{PREFACE.}

A $n \times$ born on the banks of one of the noblest and most fruitful rive:s in America, and whose best days have been spent in gar. dens and orchards, may perhaps be pardoned for talking abous fruit trees.

Indeed the subject deserves not a few, but many words. "Fine fruit is the flower of commodities." It is the most perfect union of the useful and the beautiful that the earth knows. Trees full of soft foliage; blossoms fresh with spring beauty; and, finally,-fruit, rich, bloom-dusted, melting, and luscious-such are the treasures of the orchard and the garden, temptingly offered to every landholder in this bright and sunny, though temperate climate.

"If a ruan," says an acute essayist, "should send for me to come a hundred miles to visit him, and should set before me a bashe of fine summer fruit, 1 should think there was some proportion between the labour and the reward."

$I$ must add a counterpart to this. He who owns a rood of proper land in this country, and, in the face of all the pomonal riches of the day, only raises crabs and choke-pears, deserves to lose the respect of all sensible men. The classical antiquarian must pardon one for doubting if, amid all the wonderful beauty of the golden age, there was anything to equal our delicious modern fruits-our honied Seckels, and Beurrés, our melting Rareripes. At any rate, the science of mod ?n horticulture has restored almost everything that can be desir $d$ to give a paradisiacal richness to our fruit-gardens. Yet there are many in utter ignorance of most of these fruits, who seem to live under some ban of expulsion from all the fair and goodly productions of the garden.

Happily, the number is every day lessening. America is 3 
young orchard, but when the planting of fruit-trees in one of the newest States numbers nearly a quarter of a million in a single year; when there are more peaches exposed in the markets of New York, annually, than are raised in all France; when American apples, in large quantities, command double prices in European markets; there is little need for entering into any praises of this soil and climate generally, regarding the culture of fruit. In one part or another of the Union every man may, literally, sit under his own vine and fig tree.

It is fortunate for an author, in this practical age, when his subject requires no explanation to show its downright and direct usefulness. When I say I heartily desire that every man should cultivate an orchard, or at least a tree, of good fruit, it is not necessary that I should point out how much both himself and the public will be, in every sense, the gainers. Otherwise I might be obliged to repeat the advice of Dr. Johnson to one of his friends. "If possible," said he, "have a good orchard. I know a clergyman of small income who brought up a family very reputably, which he chiefly fed on apple dump. lings." (!)

'The first object, then, of this work is to increase the taste for the planting and cultivation of fruit-trees. The second one is to furnish a manual for those who, already more or less informed upon the subject, desire some work of reference to guide them in the operations of culture, and in the selection of varieties.

If it were only necessary for me to present for the acceptance of my readers a choice garland of fruit, comprising the few sorts shat I esteem of the most priceless value, the space and time to be occupied would be very brief.

But this would only imperfectly answer the demand that is at present made by our cultivators. The country abounds with collections of all the finest foreign varieties; our own soil has produced many native sorts of the highest merit; and from all these, kinds may be selected which are highly valuable forevery part of the country. But opinions differ much as to the mer. its of some sorts. Those which succeed perfectly in one section, are sometimes ill-adapted to another. And, finally, on 
needs solne accurate description to know when a variety coines in. to bearing, if its fruit is genuine, or even to identify an indifferent kind, in order to avoid procuring it again. Hence the number of varieties of fruit that are admitted here. Little by little I have summoned them into my pleasant and quiet court, tested them as far as possible, and endeavoured to pass the most impartial judgment upon them. The verdicts will be found in the following pages.

From this great accumulation of names, Pomology has become an embarrassing study, and those of our readers who are large collectors will best, understand the difficulty-nay, the impossibility of making a work like this perfect.

Towards settling this chaos in nomenclature, the exertions of the Horticultural Society of London have been steadily directed for the last twenty years. That greatest of experimental gardens contains, or has contained, nearly all the varieties of fruit, from all parts of the world, possessing the least celebrity. The vast confusion of names, dozens sometimes meaning the same variety, has been by careful comparison reduced to something like real order. The relative merit of the kinds has been proved anu published. In short, the horticultural world owes this Society a heavy debt of gratitude for these labours, and to the science and accuracy of Mr. Robert Thompson, the head of its fruitdepartment, horticulturists here will gladly join me in bearing the fullest testimony.

To give additional value to these results, I have adopted in nearly all cases, for fruits known abroad, the nomenclature of the London Horticultural Society. Ry this means I hope to render universal on this side of the Atlantic the same standard names, so that the difficulty and confusion which have always more or less surrounded this part of the subject may be hereafter avoided.

These foreign fruits have now been nearly all proved in this country, and remarks on their value in this climate, deduc. ed from actual experience, are here given to the public. To ou: natuve and local fruits especial care has also been devoted. Not only have most of the noted sorts been proved in the gar. 
dens here, but I have had specimens before me for comparison, the growth of no less than fourteen cf the different States. Thert are still many sorts, nominally fine, which remain to be collected, compared, and proved; some of which will undoubtedly de. serve a place in future editions. 'To the kindness of pomolo. gists in various sections of the country I must trust for the detection of errors in the present volume, and for information of really valuable new varieties.*

Of the descriptions of fruit, some explanation mav be neces. sary. First, is given the standard name in capituls, fol. lowed by the authorities - that is, the names of authors who have previously given an account of it by this title. Below this are placed, in smaller type, the various synonymes, or lo. nal names, by which the same fruit is known in various coun. tries or parts of the country. Thus, on page 386, is the follow ing :

\section{Flemish Beauty. $\oint$ Lind. Thomp.}

Belle de Flandres. Bouche Nouvelle. Bosch.

Bosc Sire.
Impératrice de France. Boschpeer.

Fondante Du Bois. $\left\{\begin{array}{c}\text { incorrectly } \\ \text { of some. }\end{array}\right.$

By this is signified, first, that Flemish Beauty is the standard name of the pear; secondly, that it has been previously described by Lindley and Thompson; thirdly, that the others-synonymes-are various local names by which the Flemish Beauty is also known in various places; and, lastly that by the two latter names-Josephine, and Fondante Du Boisit is incorrectly known in some collections; these two names really belonging to other distinct pears.

It is at once apparent that one of the chief points of value of a book like this, lies in the accuracy with which these synonymous names are given-since a person might, in looking over different

* It is well to remark that many of the so-called new varieties, especially from the West, prove to be old and well known kinds, slightly altered is appearance by new soil and different climate A new variety must pussess very superiour qualities to entitle it to regard, now that we hare so many fine fruits in our collections. 
catalogues issued here and abroad, suppose that all nine of the above are different varieties-when they are rually all different names for a single pear. In this record of synonymes, I have therefore availed myself of the valuable experience of the Lon. don Horticultural Society, and added all the additional in. formation in my own possession.

Many of the more important varieties of fruit are shown in outline. I have chosen this method as likely to give the most correct idea of the form of a fruit, and because I believe that the mere outline of a fruit, like a profile of the human face, will often be found more characteristic than a highly finished portrait in colour. The outlines have been nearly all traced directly from fruits grown here. They are from specimens mostly below the average size. It has been the custom to choose the largest and finest fruits for illustration-a practice very likely to mislead. I believe the general character is better expressed by specimens of medium size, or rather below it.

It only remains for me to present my acknowledgments to the numerous gentlemen, in various parts of the country, who have kindly furnished information necessary to the completion of the work. The names of many are given in the body of the volume. But to the following I must especially tender my thanks, for notes of their experience, or for specimens of fruits to solve existing doubts.

In Massachusetts, to Messrs. M. P. Wilder, S. G. Perkins, J. P. Cushing, B. V. French, S. Downer, and C. M. Hovey, of Boston; John C. Lee, J. M. Ives, the late Robert Manning and his son R. Manning, of Salem; and Otis Johnson, of Lynn.

In Connecticut, to Dr. E. W. Bull, of Hartford; Mr. S. Ly= man, of Manchester; and the Rev. H. S. Ramsdell, of Thompson.

In New York, to Messrs. David Thomas, of Aurora; J. J. 'Thornas, of Macedon; Luther Tucker, and Isaac Dennis'on, of Albany; Alexander Walsh, of Lansingburgh; T. II. Ilyatt, of Rochester; R. L. Pell, of Pelham; C. Downing, of New burgh; and $\mathrm{IVm}$. H. Aspinwall, of Staten Island. 
In Ohio, to Professor Kirtland, of Cleveland ; Dr. Hildreth, of Marietta; and Messrs. N. Longworth, C W. Eiliott, and A. H. Ernst, of Cincinnati.

In Indiana, to the Rev. II. W. Beecher, of Indianapolis. In New Jersey, to Messrs. Thomas Hancock, of Burlington, and J. W. Hayes, of Newark. In Pennsylvania, to Mr. Frederick Brown, and Col. Carr, of Philadelphia. In Maryland, to Lloyd N. Rogers, Esq., of Baltimore. In Georgia, to James Camak, Esq., of Athens.

A. J. D.

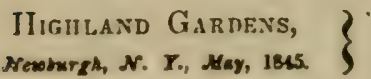




\section{ABBREVIATIONS AND BOOKS QUOTED.}

Arboretum Britannicum, or the Trees and Shrubs of Britain, pictorialls and bntanically delineated, and scientifically and pojularly described Ly J C. Loudon London, 1845, 8 vols. Svo.

Annales de la Société d'Horticulture de Paris.-Paris. In monthlv Nus. Svo. from 1827 to $1 \mathrm{S45}$.

Annales de PInstitut de Fromont. Par le Clievalier Soulange Bodin. Paris, 8vo. 1829 to 1534,6 vols.

Adium. A Mernoir on the cultivation of the Vine in America, and the best mode of making Wine. By John Allum. 1:3no. Washington, 15.29 .

Bon Jard. Le Bon Jardinier, pour l'Aunée 1914. Contenant des principes generaux de culture, etc. Par A. Poiteau and M. Vilmorin, Paris. $12 \mathrm{mo}$-yearly volume.

Busby. A Visit to the principal Vineyards of France and Spain. By Jas. Busby. New York, 12mo. 1535.

Bridgeman. The Young Gardener's Assistant. By Thomas Bridgernan. Tenth ed. New York, 1S44, 8vo.

Bumman's Cat. Catalogue des Vegretaux en tont genre dispanible dans l'Ėtablissement des Frères Baumann; à Bolwiller, 1842.

Coxe. $\wedge$ View of the Cultivation of Fruit Trees in the United States, and of the Manarement of Orchards and Cider. By Williarn Coxe..Philadelphia, 8vo, 1817.

Chaptal. Chemistry applied to Agriculture. By John Anthony Chaptal. American ed., 12mo. Boston, 153\%.

Colbett. The American Gardener. By WIn. Cobbett. London, 1921. $12 \mathrm{mo}$.

Coleman. Reports on the Agriculture of Massachusetts. By Henry Coleman. Boston, 8vo. 184U-41.

Dom. Gard. The Domestic Gardener's Manual. By John Towers. London, 1839,8 vo.

Duhamel. Traité des Arbres Fruitiers, par M. Duhamel Dumonceau. Paris, 1763,2 vols. 4 to.

Cultivator. The Cultivator, a monthly journal of Agriculture, \&c., Edited by Luther Tucker. Albany, continued to the present time, Svo.

Diel. Versuch ein Systematischen Beschreibung in Deutschland vorhandener Kernubstsorten. Von Dr. Aug. Freidr. Ad. Diel. 12no. 24 vols. 1799-1525.

De Canulle. Physiologie Végétale, ou Expositinn des Forces et des Fone tions vitales des Végétaux. Par A. P. De Candulıe. Paris, 1832, 3 rols. 8vo. 
1830,4 vols, 8 vo.

D'.Albret. Cours Thiérique et Pratique de la Taille des Arbres Fruitiers.

Par D'Albret. Paris, 1840, 8vo.

Forsyth. A Treatise on the Culture and Management of Fruit-trees. B= William Forsyth, 7th ed. London, 1824, 8 vo.

Floz Lindley's Guide to the Orchard. American ed. with addition by Michael Floy. New York, 1833, $12 \mathrm{mo.}$

Fessenden. New American Gardener, containing practical directıons fot the culture of Fruits and Vegetables. By Thos. E. Fessenden. Boston, 1528, $12 \mathrm{mo}$.

Gard. Mag. The Gardener's Magazine, conducted by J. C. Loudon, in monthly nos. 8 vo., 19 vols. to 1944 ., London.

rard. Chron. The Gardener's Chronicle, and Agricultural Gazette, edited by Professor Lindley, a weekly journal 4 to. 5 vols. 1844 to the present time.

Hoare. A Practical Treatise on the cultivation of the Grape Vine on open walls. By Clement Hoare. London, 1840, $12 \mathrm{mo.}$

Hort. Soc. Cat. See Thompson.

Hort. Trans. Transactions of the Horticultural Society of London. London, 4 to. 1815, and at intervals to the present time.

Hooker Pomona Londonensis. By William Hooker. London, 1813, 4 to.

Hayward. The Science of Horticulture. By Joseph Hayward. London, 1S24, 8vo.

Harris. A Report on the insects of Massachusetts injurious to Vegetation. By Dr. T. W. Harris. Cambridge, 1841, 8vo.

Hov. May. or $H$. $\boldsymbol{M}$. The Magazine of Horticulture, Botany and Ru. ral Affairs. Conducted by C. M. Hovey. Boston, 8vo. monthly nos. 1834 to the present time.

Jonnston. Lectures on Agricultural Chemistry and Geology. By Jas. W

F. Johnston. American ed. New York, 12mo. 2 vols. 1842.

Jard Fruit. Le Jardin Fruitier, par Louis Noisette, 2 ed Paris, 1839, 2 vols. 8 vo.

Knight Various articles in the London Horticultural Transactions. By Thomas Andrew Knight, its late President.

Knoop. Pomologie ou description des Arbres Fruitiers. Par Joh. Herm Knoop. Amsterdam, 1771, Fol.

Ken. The New American Orchardist. By William Kenrick, Boston, 1844.

Kollar. A Treatise on Insects injurious to Gardeners, Foresters and Farmers. By Vincent Kollar, Notes by Westwood. London, 1840, $12 \mathrm{mo}$.

Langley. Pomona, or the Fruit Garden Illustrated. By Batty Langley, London, 1729, Folio.

Loulon. An Encyclopedia of Gardening. By J. C. Loudon. J.ondon 1835,1 thick vol. 8 ro.

- An Encyclopedia of Plants. By the sarne. London, 1836, 1 thick vol. 8vo.

An Encyclopedia of Agriculture. By the same. London, 1931, 1 thick vol. 8vo. 
Lotudon Hurtus Britannicus. A Catalogue of all the plants in Britain, by the same. London, 8vo.

- The Suburban Horticulturist By the same. London, 1842, 870.

- The Suburban Garilener and Villa Cumpanion. By the same Zon: don, $1838,1942,8$ vo.

- Arboretum et Fruticetum Britannicum. By the same, 3 vols. London, 1838, 8vo.

Liebig. Organic Chemistry in its applications to Agriculture and Physiology. By Justus Liebig. American ed., Cambridge, 1844, 12 mo.

Lind A Guide to the Orchard and Kitchen Garden, or an account of the Fruits and Vegretables cultivated in Great Britain. By George Lindley. London, 1831, 8vo.

Lindley. An Int oduction to Botany. By John Lindley. London, 1832, 8 ro.

. An Introduction to the Natural System of Botany. By John Lindley. London, 1835, 2d ed. 8vo.

- British Fruits. See Pomological Magazine-it is the ame work.

- - The Theory of Horticulture, or an attempt to explain the Opera tion of Gardening upon Physiological Principles. By John Liriniey. London, 8vo. 1840.

. The same work with Netes by A. Gray and A. J. Downing. New York, 1941, 12 mo.

L. or Linnaus. Species Plantarum, 5th. ed. Berlin, 1810, 5 vols. 8vo.

leclieur. La Pomone Française, ou Traité le la Culture Française, et de la Tailie des Arbres Fruitiers. Par le Compte Lelieur. Paris, 1811, sro.

Man. The New England Fruit Book. By R. Manning, 2d ed. enlarged by John M. Ives, Salem, 1844, $12 \mathrm{mo}$.

Man. in H. M. Manning's articles in Hovey's Magazine.

Afill. The Gardener's and Butanist's Dictionary. By Philip Miller.revised by Professor Martyn. London, 1S19, 2 vols. 8vo.

Michaux. The North American Sylva, or Descriptions of the Forest Trees of the United States, Canada, Sc. By A. F. Michaux. Paris 1519,3 visls Svo

AF In!ush. The Circhard and Fruit Garden. By Charles McIntosh. London, 1S19, $12 \mathrm{mo}$.

N. Duh. (The New Duhamel) Traitú des f.rbres Fruitiers de Duhamel. Nouvelle edition augrmentée, etc. Par MM. Poiteau et Turpin, Paris. 5 vols. Folio, 180 et seq.

Nois. See Jardin Fruitier.

Now Eingland Farmer. A weekly periodical, devoted to Agriculture. Horticulture, \&c. Boston, 4 to. continued to the present time.

o Duh. See Duhamel.

Pom. Mag. or P. MI. The Pomological Magazine, or Figures and Descriptions of the most important varieties of Fruit cultivated in Great Britain. London, 1528, 3 vols. 8 vo.

Pom. Man. The Pomological Manual. Bg William R. Prince. New York, 1531, 2 rols. Svo.

Prince. A Treatise on the Vine. By William R. Prince, New York, 1930. 8vo. 
Prinee. A short Treatise on Horticulture. By Wiliam Prince, New York, 1828, $12 \mathrm{mo}$.

Pkillips. Pomarium Britannicum;-an Historical and Botanical Account of the Fruits known in Great Britain. By Henry Phillips, London, 1820,8 vo.

Poit. or Poiteau. Pomologie Française. Recueil des plus beaux Fruits, cultivés en France. Par Poiteau. Paris, 1833, and continued in 4to. nos.

Rivers. A Descriptive Catalogue of Pears, cultivated by T. Rivers. Sawbridgeworth, 1843-44, pamphlet, 8 vo.

Ron. ur Ronalds. Pyrus Malus Brentfordienses, or a concise description of Selected Apples, with a figure of each sort. By Hugh Ronalds, London, 1S31, 4 to.

Ray. Historia Plantarum, a John Ray, M. D., London, 3 vols. Folio, $1636-1704$.

Revue Horticole. Journal des Jardiniers et Amateurs. Audot, Editeur. Paris, 1844 , et chaque mois, $12 \mathrm{mo}$.

Switzer. The Practical Fruit Gardener. By Stephen Switzer, 172.1, 8vo

Torrey \& Gray. A Flura of North America, containing abridyed descriptions of all the known plants growing North of the Gulf of Mexico. By John Torrey, M.D , and Asa Gray, M.D., New York, vol. 1st, 8vo New York, 1840, and still in progress.

Thomp. A Catalogue of the Fruits Cultivated in the Garden of the Horticultural Society of London, 3d ed, London, 1S42. [Preprared with great care by Rubert Thompson, the head of the Fruit Department I

Thacher. The American Orchardist. By James Thacher, M.D., Bustou, 1822,8 vo.

Dan Mons. Arbres Fruitiers, ou Pomologie Belge Fxperimentale et Katsonnée, Par J. R. Van Mons. Louvain, 1535-15315, 2 vols. $12 \mathrm{mo.}$ - Catalogue des Arbres Fruitiers, Descriptif, Abrégé. Par J. B. Van Mons, Louvain, 1823.

Wilder, .MSS. Manuscript notes on Fruits, hy M. P. Wilder, Esı., Fresi dert of the Massachusetts Horticultural Society. 


\title{
FUUITS AND FRUIT TREES
}

\author{
CH A P'T E R. J,
}

THE PRODUCTION OF NEW VARIETIES OF FRUIT.

Is our survey of the culture of fruits let us begin at the be. ginning. Gradual amelioration, and the skilful practice of the cultivator, have so filled our orchards and gardens with good fruits, that it is necessary now to cast a look back at the types from which these delicious products have sprung.

In the tropical zone, amid the surprising luxuriance of vegetation of that great natural hothouse, nature offers to man, almost without care, the most refreshing, the most delicious, and the most nutritive fruits. The Plantain and Bananna, excellent either raw or cocked, bearing all the year, and producing upon a rool of ground the sustenance of a family; the refreshing Guavil and Sapodilla; the nutritious Bread-fruit; such are the natural fruit trees of those glowing climates. Indolently seated under their shade, and finding a refreshing coolness both from their ever-verdant canopy of leaves, and their juicy fruits, it is not here that we must look for the patient and skilful cultivator.

But, in the temperate climates, nature wears a harsher ard sterner aspect. Plains bounded by rocky hills, visited not only by genial warmth and sunshine, but by cold winds and seasons of ice and snow; these are accompanied by sturdy forests, whose outskirts are sprinkled with crabs and wild cherries, and festooned with the clambering branches of the wild grape. These native fruits, which at first offer so little to the eye, or the palate, are nevertheless the types of our garden varieties. [lestined in these climates to a perpetual struggle with nature, it is here that we find man ameliorating and transforming her.

Transplanted into a warmer aspect, stimulated by a richer soil, reared from selected seeds, carefully pruned, sheltered and watched, by slow degrees the sour and bitter crab expands into a Golden Pippin, the wild pear loses its thorns and becomes a Bergamotte or a Beurré, the Almond is deprived of its bitterness, and the dry and flavourless Peach is at length a tempting and delicious fruit. It is thus only in the face of obstacles, in a climate where nature is not prodigal of perfections, and in the milst of thorns and sloes, that MAN THE GARDENER arises and forces nature to yield to his art. 
These improved sorts of fruit wnich man every where causca to share his civilizaiion, bear, almost equally with himsclt, the mpress of an existence removed from the natural state. IT hen eared from seeds they always show a tendency to return to a vilder form, and it secms only chance when a new seculling is :qual to. or surpasses its parent. Removed from their natural urm, these artificially created sorts are also much more liable to tiseases and to decay. From these facts arises the fruit-garten, with its various processes of grafting, budding and other meana of continuing the sort ; with also its sheltered aspects, warm horders. deeper soils, and all its various refinements of art and sulture.

In the whole range of eares and pleasures belonging to the marden, there is nothing more truly interesting than the producinn of new varieties of fruit. It is not, indeed, by sowing the .cerls that the lover of good fruit usually undertakes to stock his parden and orchard with fine fruit trees. Raising new varietics $S$ always a slow, and, as generally understood, 2 most uncertain node of bringing about this result. The novice, plants and carefully watches his hundred scedling pippins, to find at last, perhaps, ninety-nine worthless or indifferent apples. It appears to him a lottery, in which there are too many blanks to the prizes. He, thercfore, wisely resorts to the more certain mode of grafting from well known and esteemed sorts.

Notwithstanding this, every year, under the influences of marden culture, and often without our design, we find our fruit trees reproducing themselves; and occasionally, there springs up a new and delicious sort, whose rnerits tempt us to fresh trials after perfection.

To a man who is curious in fruit, the pomologist who views with a more than common eye, the crimson cheek of a peach, the delicate bloom of a plum, or understands the epithets, rich, melting, buttery, as applied to a pear, nothing in the circle of culture, can give more lively and unmixed pleasure, than thus to pro. duce and to create-for it is a sort of creation-an entirely new sort, which he believes will prove handsomer and better than any thing that has gone before. And still more, as varieties which ori. ginate in a certain soil and climate, are found best adapted to that locality, the production of new sorts of fruit, of high merit, may be looked on as a most valuable, as well as interesting result.

Beside this, all the fine new fruits, which, of late, figure so conspicuously in the catalogues of the nurseries and fiuit gardens, have not been originated at random and by chance efforts. Some of the most distiuguished pomologists have devoted yeary io the subject of the improvement of fruit trees by seeds, and lave attained if not certain results, at least some general 
laws, which greatly assist us in this process of amelinration, Let us therefore examine the subject a little more in detail.

In the wild state, every gonus of trecs winsist of one or nore species, or strongly marked individual sints ; as, linr example. the white birch and the black birch; or, to contine nursclyes nore strictly to the matter in hanr, the different species of cherry, the will or bird cherry, the sour clierry, the mazzard cherry, de. These species, in thoir nutural state, exactly mumaluon themselves; to use a common phrase, they "exme the same" from seed. This they have done for centurics, and doubtlusg will do forever, so long as they exist under natural circumstan. ces only.

On the other hand, suppose we select one of these species of fruit-trees, and adopt it into our gardens. So long as we cultivate that individual tree, or any part of it, in the shane of sucker, graft, or bud, its nature will not be materially alteresl. It may, inlecd, through cultivation, be stimulated into a m re luxuriant growth; it will probably produce larger learis and fruit; but we shall neither alter its fruit in texture, color or taste. It will always be identically the same.

The process of amelioration begins with a new generation, an.t by sowing the seeds. Some species of tree, indeed, srem to re fuse to vield their wild nature, never producing any variation by seed; but all tivit-trees and many others, are easily domest:cated, and more readily take the impress of culture.

If we sow a quantity of secd in garden soil of the common black mazzard cherry, (Cerasus avinim,) we shall tind that, in the leaves and habit of growth, many of the soedlings rlo not entirely resemble the original species. When they come into bearing; it is probable we shall also find as great a diversity in the size, color and flavor of the fruit. Each of these individual plants. diflering from the original type, (the mazzard,) constitutes ? new varicty; though only a few, perhaps only one, may be superiour to the original species.

It is worthy of remark, that exactly in proportion as this reproduction is frequently repeated, is the change to a great ra. risty of fornis, or new sorts increased. It is likely indecd. that to gather the seeds from a wild mazzard in the woods, the in. stances of departure from the form of the original species wnuld be very fow; while if gathered from a garden tree, itself s:nmo time cultivated, or several removes from a wild state, thouchl still a mazzard, the seedlings will show great variety of character.

Once in the possession of a variety, whicin has moved out of the naturai into a more domesticated form, we have in our han ts the best material for the improving process. 'The tixed original habit of the species is broken in upon, and this variety which we have created, has always afterwaids some tendency to 
make furtier departites from the original form. It is true that all or most of its seodliner; will still retain a likeness to the pa-

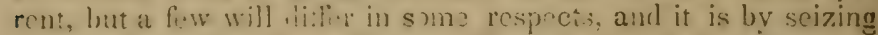
apm tho: when show svm; no of variation, that the improver of vegetable races founds his holes.

We have sail that it is a part of the character of a species to proilues the same from soed. This characteristic is retained cven where the syorl, (as garleners term it) into numbarless varicties is greatest. Thas, to returu to cherries, the Lentish or common pie chnry i.; one s.' cins, an l th" simzll black mazard another, and although a great number of varieties of each of these species have been produced, yet there is always the likeness of the species retained. From the first we may have the large and rich Mayduke, and from tho last the sweet and lu;cious B'ack-frarts; but a glanes will show us that the duke chorri.s retain the distinct dark foliacre, an l, in tlie fruit, some. thing of thr sam flavor, shape anl colne of the original species: an t the heart cherries the browl leaves and lofty growth of the mazaril. So too, the currant and (ri) neconry are ditfer. ent species of the same genus; but thourh the English gooseb rry growers have raised thousants of new varieties of this fruit, and shown them as large as hen's ecres, and of erery varicty of form and color, yet their efforts with the gooseberry have not produed any thing resembling the common currant.

Why do not varieties produce the same from seed? Why if we plant the stone of a Green Garce plum, will it not always produce a Green Gage? This is often a puzzling question to the practical gardener, while his every day experience forces him to assent to the fact.

We are not sure that the vegetable physiologists will undertake to answer this query fully. But in the mean time we can throw some light on the subject.

It will be remembered that our garden varieties of fruits are not natural forms. They are the artificial productions of our culture. They have always a tendency to inprove, but they have also another and a stronger tendency to refurn 10 a natural, or acild state. "There can be no doubt," says Dr. Lindley, "that if the arts of cultivation were abandoned for only a few years, all the annual varieties of plants in our gardens would disappear and be replaced by a few original wild forms." Be. tween these two tendencies, therefore, the one derived from nature, and the other impressed by culture, it is easily seen how little likely is the progeny of varieties always to reappear in the same form.

Again, our American farmers, who raise a rumber of kinds of Indian corn, very well know that, if they wish to licep the sorts distinct, they must grow them in different fields. Without this precaution they find on planting the seeds produced on the 
yeilow corn plants, that they have the next season a progeny, not of yellow corn alone, but composed of every color and size, ycllow, white and black, large and small, upon the farm. Now many of the varieties of fruit trees have a similar power of intermixing with each other while in blossom, by the dust or pollen of their flowers, carried through the air, by the acticn of bees and other causes. It will readily occur to the reader, in considering this fact, what an influence our custom of planting the different varieties of plum or of cherry together in a zarden or orchard, must have upon the constancy of habit in the seedlings of such fruits.

But there is still another reason for this habit, so perplexing to the novice, who, having tasted a luscious fruit, plants, watches and rears its secdling, to find it perhaps, wholly different in most respects. 'This is the infiuencs of grafting. Among the great mumber of scedling fruits produced in the United States, there is found occasionally a varicty, perhaps a plum or a peach, which. will nearly always reproduce itself from sced. From some for. unate circumstances in its origin, unknown to us, this sort, in becoming improved, still retains strongly this habit of the natu. ral oi wild form. and its seeds produce the same. We can call to mind several examples of this; fine fruit trees whose seeds have established the reputatinn in their neighborhood of fidelity to the sort. But when a graft is taken from one of these trees, and placed upon another stock, this grafted tree is found to lose its singular power of protucing the same by seed, and becumes like all other worked trees. The stock exercises some, as yet, unexplained power, in dissolving the strong natural habit of the varicty, and it becomes like its fellows, subject to the laws of its artificial life.

When we desire to raise new varicties of fruit, the enmmon practice is to collect the secds of the fincst table fruits-lhose sorts whose merits are every where acknowledered to be the highest. In procceding thus we are all pretty well aware. that the chances are grencrally a hundsed to one açainst our obtain. ine any new variety of crreat excellenes. Brfore we offer any arfvice on rearing seeflines let us examine brinfly the practice and views of two rlistinguisherl horticulturists alroal, who have paid more attention to this subject than any other persons whatever; Dr. Van Mons of Belgium, and 'Thos. Andrew Kinight, Fisq., the late President of the Horticultural Socicty of London.

\section{The Van MIons Theory.}

Dr. Tan Mnns. Professor at Louvain, dnented the ereater part of his: life to the amelioration of freits. His nurseric sontaned in 1s:3, no less than two thousand secdlings of merit. Ilis prrseverance was indefatigable, and experimenting mainly on 
Pears, he succeeded in raising an immense number of new varietic: of high excellence. The Beurre Diel, De I.ouvain, Fredorie of 17 urfombers, \&e., are a few of the many well smovn swls which are the result of his unwearied labours.

'I\%. Yau Mons theory may be briefly stated as follows:

All fine fruits are artificial products; the aim of nature, in a will state, being only a healthy, vigorous state of the tres, and perfect seerls for continuing the species. It is the object of culture, therefore, to subdue, or enfeeble this excess of vecretation; to lessen the coarseness of the tree ; to diminist. the size of the scels; and to refine the quality and increase the size of the flesh or pulp.

There is always a tendency in our varieties of fruit trees to return by their seeds towards a wild state.

This tendency is most strongly shown in the seeds borne by itd fruit-trees. And "the older the tree is of any cultivated variety of Pear," says Di. Van Mons, "the nearer will the seedlings, raised from it, approach a wild state, without however ever being able to return to that state."

On the other hand, the seeds of a young fruit tree of a good sort, heing itself in the state of amelioration, have the least ten. dency to retrograde, and are the most likely to produce improved sorts.

Again, there is a certain limit to perfection in fruits. When this point is reached, as in the finest varieties, the next generation will more probabiy produce bad fruit, than if reared from seerls of ai indifferent sort, in the course of amelioration. While, in other words, the seeds of the oldest varieties of grod fruit mostly yield inferiour sorts, seeds taken from recent viaie. tics of bad fruit, and reproduced unimterruptedly for several generations, will certainly produce good fruit.

With these premises, Dr. Van Mons begins by gathering his seeds from a young seedling tree, without paying much reyard to its quality, except that it must be in a slate of variation; that is to say, a garden variety, and not a wild sort. 'These he sows in a seedbed or nursery, where he leaves the seerlin us until they attain sufficient size to enable him to julge of ther character. He then selfets those which appear the most promising, plants them a few feet distant in the nursery, and awaits Wheir fruit. Not discouraged at finding most of them of inedinere quality, though differing from the parent, he gathes the lirst sceds of the most promising and sows them again. The next generation comes more rapidly into bearing than the first. and shows a sreater number of promising traits. Gathering inmediate?y, and sowing the seeds of this genemtion, he proluees a (limb, then a fourth, and even a fith generation, uninterupuedly, from the original sort. Eitch mencration he finds to come more quickly into bearing that the presious one, (the 5th sowiug of 
pears fruiting at 3 years,) and to produce a greater number of valuable varieties; until in the fifth generation the seedlings are nearly all of great excellence.

Dr. Van Mons found the pear to require the longest time to nltain perfection, and he carried his process with this fruil through five generations. Apples he found needed but four races, and peaches, cherries, plums, and other stone fruits, wore brought to perfection in three successive reproductions from the seed.

It will be remembered that it is a leading feature in this theory that, in order to improve the fruit, we must subdue or enfeeble the original coarse luxuriance of the tree. Keeping this in mind, Dr. Van Mons always gathers his fruit before fully ripe, and allows them to rot before planting the seeds, in order to refine or render less wild and harsh the next generation. In transplanting the young seedlings into quarters to bear, he cuts off the tap root, and he annually shortens the leading and side branches, besides planting them only a few feet apart. All this lessens the vigour of the trees, and produces an impression upon the nature of the seeds which will be produced by their first fruit; and, in order to continue in full force the progressive variation, he allows his seedlings to bear on their own roots.

Such is Dr. Van Mons' theory and method for obtaining new varieties of fruit. It has never obtained much favour in England, and from the length of time necessary to bring about its results, it is scarcely likely to come into very general use herc. At the same time it is not to be denied that in his hands it has proved a very successful mode of obtaining new varieties.

It is also undoubtedly true that it is a mode closely founclel on natural laws, and that the great bulk of our fine varieties have originated, nominally by chance, but really, by successive reproductions from the seed in our gardens.

It is not a little remarkable that the constant springing up of fine new sorts of fruit in the United States, which is every day growing more frequent, is given with much apparent force as a proof of the accuracy of the Tan Mons theory. The first colo. nists here, who brought with them many secds gathered from the lest old varieties of fruits, were surprised to find their seed. lings producing only very inferiour fruits. These seedlings hac returned by their inherent tendency almost to a wild state. $\mathrm{Bg}$ rearing from them, however, seedlings of many repeated gene. rations, we have arrived at a great number of the finest apples;

* "I have found this art to consist in regenerating in a direct line of descens, and as rapidly as possible an improving variety, taking care that there be no in. terval between the generations. To sow, to re-sow, to sow again, to sow perpetu. ally, in short to do nothing but sow, is the practice to be pursued, and which can ne: be cleparted from; and in short this is the whole secret of the art I have en Noyed."-Van MIons' Arbres Fruiliers, 1. p. 223. 
pears, peaches and plums. According to Dr. Van Mons, had this process been continued uninterruptedly, from one generation to the next, a much shorter time would have been necessary for the production of first rate varieties.

To show how the practice of chance sowing works in the other hemisphere, it is stated by one of the most celebratel of the old writers on fruits, Duhamel of France, that he had been in the habit of planting seeds of the finest table pears for fifty years without ever having produced a good variety. These seeds were from trees of old varieties of fruit.

The American gardener will easily perceive, from what wo have stated, a great advantage placed in his hands at the present time for the amelioration of fruits by this system. He will see that, as most of our American varieties of fruit are the result of repeated sowings, more or less constantly repeated, he has before him almost every day a part of the ameliorating pro. cess in progress; to which Dr. Van Mons, beginning de novo, was obliged to devote his whole life. Nearly all that it is necessary for him to do in attempting to raise a new variety of ex. cellence by this simple mode, is to gather his seeds (before they are fully ripe,) from a seedling sort of promising quality, though not yet arrived at perfection. The seedling must be quite young-must be on its own root (not grafted;) and it must be a healthy tree, in order to secure a healthy generation of seedlings. Our own experience leads us to believe that he wiil scarcely have to go beyond one or two generations to obtain fine fruit. These remarks apply to most of our table fruits common. ly cultivated. On the other hand, our native grapes, the Isabella, Catawba, \&c., which are scarcely removed from the wild state, must by this ameliorating process be carried through several successive generations before we arrive at varicties equalling the finest foreign grapes; a result, which, judging from what we see in progress, we have every reason speedily to hope for.

In order to be most successful in raising new varieties by suc. cessive reproduction, let us bear in mind that we must aroid1 st, the seeds of old fruit trees; $2 \mathrm{~d}$, those of grafted fruit trces: and $3 \mathrm{~d}$, that we have the best grounds for good results when we gather our secds from a young seedling tree, which is itself ra. ther a perfecting than a perfect fruit.

It is not to be denied that, in the face of Dr. Van Mon's theory, in this country, new varieties of rare excellence are sometimes obtained at once by planting the seeds of old grafted varieties; thus the Lawrence's Favourite, and the Columt ia plurns, were raised from seeds of the Green Gage, one of the oldest Europesn varieties.

Such are the means of originating new fruits by the lielgrian mode. Let us now examine another more direct, more interesting, and more scientific process-cross-breeding; a mode almost 
aniversally pursued now by skilful cultivators, in producing new and finer varieties of plants; and which Mr. Knigint, the most distinguished horticulturist of the age, so successfully pras. ised on fruit trees.

\section{Cross-breeding.}

In the blossoms of fruit-trees, and of most other plants, the seed is the offspring of the stamens and pistil, which may te considered the male and female parents, growing in the sume flower. Cross-breeding is, then, nothing more than removing out of the blossom of a fruit tree the stamens, or male parents, and bringing those of another, and different variety of fruit, and dusting the pistil or female parent with them,-a process sufficiently simple, but which has the most marked effect on the sceds produced. It is only within about fifty years that cross-breeding has been practised; but Lord Bacon, whose great mind scems to have had glimpses into every dark corner of human knowledge, finely foreshadowed it. "The compounding or mixture of plants is not found out, which, if it were, is more at command than that of living creatures; wherefore, it were one of the most notable discoveries touching plants to find it out, for so you mav have great varieties of fruits and flowers yet unknown."

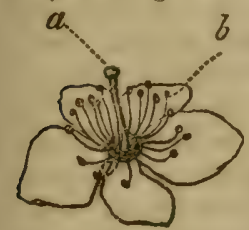

F:m. 1 .

In figure 1 , is shown the blossom of the Cherry. The central portion, $a$, connected directly with the young fruit, is the pistil. The numerous surrounding threads $b$, a re the stamens. The summit of the stamen is called the anther, and secretes the powdery substance called pollen. The pistil, has at its base, the cmbryo fruit, and at its summit, the stigma. The use of the stamens is to fortilize the young seed contained at the base of the pistil; and if we fertilize the pistil of one variety of fruit by the pollen of another, we shall obtain a new varicty partaking intermediately of the qualities of both parents. Thus, among fruits owing their origin directly to cross-breeding, Coe's Golden Drop Plum, was raised from the Grcen Gage, inpregnated by the Magnum Bonum, or Egrg plum; and the Elton cherry, from the Bigarrieu, impregnated by the White Hean.* Mr. Knight was of opinion that the habits of the new varicly would always be found to partake most strongly of the constitution, and habits of the female parent. Subsequent experience does not fully confirm this, and it would appear that the parent

* The seedlings sometimes most resembl one parent sometimes the other; but more frequently share the qualities of beth. Mr. (oxed.scribes an Apple, as rruss be-ween a Newtown Pippin and a kuset, the fruit of whic h resembled extemally at one end the Russet and at the other the Pippin, and :lie flavour at either end corresponded exactiy with the character of the exteris ur. 
whose character is most permanent, impresses its form most for cibly on the offspring.

The process of obtaining cross-bre 1 seeds of fruit trees is vervi ensily performed. It is only necessary when the tree bloom, which we intend to be the mother of the improved race, to select a blossom or blossoms growing upon it not yet fully expanded. W'ith a pair of scissors, we cut out and remove all the anthers. The next day, or as soon as the blossom is quite expanded, we collect, with a camel's hair brush, the pollen from a fully blown flower of the variety we intend for the male parent, applying the poilen and leaving it upon the stigna or point of the pistil. If your trees are much exposed to those busy little meddlers, the bees, it is well to cover the blossoms with a loose bag of thin gauze, or they will perhaps get beforehand with you in your experiments in cross-breeding. Watch the blossoms closely as they open, and bear in mind that the two essential points in the operation are; Ist, to extract the anthers carefully, before they have matured sufficiently to fertilize the pistil; and, $2 d$, to apply the pollen when it is in perfection, (dry and powdery,) and while the stigna is moist. A very little practice will enable the amateur to judge of these points.

There are certain limits to the power of crossing plants. What is strictly called a cross-bred plant or fruit is a sub-rariety raised between two varieties of the same species. There are, however, certain species, nearly allied, which are capable of fertilizing cach other. The offspring in this case is called a hybrid. or mule, and does not always produce perfect seeds. "This power of hybridising," says Dr. Lindley, "appears to be much more common in plants than in animals. It is, however, in general only between nearly allied species that this intercourse can talie place; those which are widely different in structure and constitution not being capable of any artificial union. Thus the different species of Strawberry, of the gourd or melon family, intermix with the grcatest facility, there being a great accori. ance between them in general structure, and constitution. But $\mathrm{m} n$ one has ever succecded in compelling the pear to fertilize the apple, nor the gooseberry the currant. And as species that are very dissimilar appear to have some natural impediment which prevents their reciprocal fertilization, so does this obstacle, of whatever nature it may be, present an insuperable bar to the intercourse of the different genera. All the stories that are current as to the intermixture of oranges and pomegranates, of roses and black currants, and the like, may therefore be set down to pure invention."

In practice, this power of improving varieties by crossing is very largely resorted to by gardeners at the present day. Nor only in fruit trees, but in ornamental trees, shrubs, and plants, and especially in florists' Howers, it has been carried to a greal 
extent. The great number of new and beautiful Roses, Azaleas Carnollias, Fuchsias, Dahlias, and other flowering plants so splendid in colour, and perfect in forın, owe their origin to careful cross-breeding.

In the amelioration of fruits it is by far the most certain, and satisfactory process yet discovered. Its results are more speedily obtained, and correspond much more closely to our aim, than hose procured by successive reproduction.

In order to obtain a new variety of a certain character, it is only necessary to select two parents of well known habits, and which are both varieties of the same, or nearly allied species, and cross them for a new and intermediate variety. Thus, if we have a very early, but insipid, and worthless sort of pear, and desire to raise from it a variety both early and of fine flavour, we should fertilize some of its pistils, with the pollen of the best flavoured variety of a little later maturity. Among the seed. lings produced, we should look for early pears of good auality, and at least for one or two varieties nearly, or quite as early as the female parent, and as delicious as the male. If we have a very small, but highly flavoured pear, and wish for a larger pear with a somewhat similar flavour, we must fertilize the first with the pollen of a large and handsome sort. If we desire to impart the quality of lateness to a very choice plum, we must look nut for a late variety, whether of good or bad quality: as the mother, and cross it with our best flavoured sort. If we desire to impart hardiness to a tender fruit, we must undertake a cross between it and a much hardier sort; if we seek greater beauty of colour, or vigour of growth, we must insure these qualities by selecting one parent having such quality strongly marked.

As the seeds produced by cross fertilization are not found to produce precisely the same varieties, though they will nearly all partake of the mixed character of the parents, it follows that we shall be most successful in obtaining precisely all we hope for in the new race, in proportion to the number of our cross-bred seedlings; some of which may be inferiour, as well as some superiour to the parents. It is always well, therefore, to cross several flowers at once on the same plant, when a single blossom does not produce a number of seeds.

We shculd observe here, that those who devote their time to raising new varieties, must bear in mind that it is not always by the first fruits of a seedling that it should be judged. Some of the finest varieties requ re a considerable age before their best qualities develop themselves, as it is only when the tree has arrived at some degree of maturity that its secretions, either for fower, or fruit, are perfectly elaborated. The first fruit of the Black Eagle cherry, a fine cross-bred raised by Mr. Knight, was pronounced worthless when first exhibited to the Lendon Horticultural Society; its quality now proves that the tree was not then of sufficient age to produce its fruit in perfection. 


\section{CHAPTER II.}

PROPAGATION OF VARIETIES. GRAFTING. BUDDING. CUITINGS. LAYERS AND SUCKEKS.

AfTer having obtained a new and choice kind of fruit, which in our hands is perhaps only a single tree, and which, as we have already shown, seldom produces the same from seed, the next inquiry is how to continue this variety in existence, and how to increase and extend it, so that other gardens and coun. tries may possess it as well as ourselves. 'This leads us to the subject of the propagation of fruit trees, or the continuation of varieties by grafting and budding.

Grafting and budding are the means in most common use for propagating fruit trees. They are, in fact, nothing more than inserting upon one tree, the shoot or bud of another, in such a manner that the two may unite and form a new compound. No person having any interest in a garden should be unable to perform these operations, as they are capable of effecting transformations and improvements in all trees and shrubs, no less valuable, than they are beautiful and interesting.

Grafting is a very ancient invention, having been well known and practised by the Greeks and Romans. The latter, indeed, describe a great variety of modes, quite as ingenious as any of the fanciful variations now used by gardeners. The French who are most expert in grafting, practice occasionally more than fifty modes, and within a few years have succeeded pes fectly in grafting annual plants, such as the tomato, the dahlia, and the like.

The uses of grafting, and budding, as applied to fruit trees, tnay be briefly stated as follows:

1. The rapid increase or propagation of valuable sorts of fruit not easily raised by seeds, or cuttings, as is the case with ncarly all varieties.

2. To renew or alter the heads of trees, partially or fully grown, producing in two or three years, by heading-in and grafting, a new head, bearing the finest fruit, on a formerly worthless tree.

3. To render certain foreign and delicate sorts of fruit more nardy by grafting them on robust stocks of the same species na. tive to the country, as the foreign grape on the native. And to proluce fine fruit in climates or situations not naturally farour. able by grafting on another species more hardy; as in a cool 
climate and damp strorig soil, by working the Peach on the Plum.

4. To render dwarf certain kinds of fruit, by grafting them on suitable stocks of slower growth, as in the case of the Pear on the Quince, the Apple on the paradise stock, \&c.

5. By grafting several kinds on the same tree, to be able to have a succession of fruit, from early to late, in a small garden.

6. To hasten the bearing of seedling varieties of fruit, or of such as are a long time in producing firuit, by grafting them on the branches of full grown, or mature bearing trees. Thus a seedling pear, which would not produce fruit on its own root in a dozen years, will gencrally begin to bear the third or fourtin year, if grafted on the extremity of the bearing branches of a mature tree.

The proper time for grafting fruit trees is in the spring, as soon as the sap is in motion, which commences earliest with the Cherry and Plum, and ends with the Pear and Apple. The precise time of course varies with the season and the climate, but is generally comprised from February to the middle of April. The grape vine, however, which suffers by bleeding, is not usu. ally grafted until it is in leaf. The most favourable weather for grafting is a mild atmosphere with occasional showers.

The scions are generally selected previously; as it is found in nearly all kinds of grafting by scions, that success is more complete when the stock upon which they are placed is a little more advanced-the sap in a more active state than in the scion. To secure this, we usually cut the scions very tarly in the spring, during winter, or even in the autumn, bulying their lower ends in the ground in a shaded place, or keeping them in fine soil in the cellar till wanted for use. In cutting scions, we choose straight thrifty shoots of the last year's growth, which may remain entire until we commence grafting, when they may be cut into scions of three or four buds each. In selecting scions from old trees it is always advisable to choose the most vigorous of the last year's shoots growing near the centris or top of the tree. Scions from sickly and unhealthy branches should be rejected, as they are apt to carry with them this feeble and sickly state. Scions taken from the lower bearing branches will produce fruit soonest, but they will not afford trees of so handsome a shape, or so vigorous a growth, as those taken from the thrifty upright shoots near the centre or top of the tree. Nurserymen generally take their scions from young grafted trees in the nursery-rows, these being usually in better condition than those taken from old trees not always in a healthy state.

The stock for grafting upon, is generally a tree which has been standing, at least for a year previously; on the snot where it is grafted, as success is much less certain on newly moved trees 
In the case, however, of very small trees or stocks, which are grafted below the surface of the ground, as is frequently the practice with the Apple in Amcrican nurseries, the stoclis are grafted in the house in winter, or carly spring, put away carefully in a damp cellar, and planted out in the spring; but this method is only successful when the root is small, and when the top of the stock is taken off, and the whole root is devoted to supplying the graft with nourish nent.

The theory of grafting is based on the power of union between the young tissues, or organizable matter of growing wood. When the parts are placed nicely in contact, the ascending sap of the stock passes intu and sustains life in the scion; the buds of the latter, excited by this supply of sap and the warmth of the season, begin to elaborate and send down woody matter, which, passing through the newly granulated substance of the parts in contact, unites the graft firmly with the stock. "If," says De Candolle, " the descending sap has only an incomplete analogy with the wants of the stock, the latter does not thrive, though the organic union may have taken place; and if the analogy between the albumum of stock and scion is wanting, the organic uninn does not operate; the scion cannot absorb the sap of the stock and the graft fails."

Grafting therefore is confined within certain limils. A scion finm one tree will not, from the want of affinity, succeed on every other tree, but only upon those to which it is allied. We are, in short, only successful in budding or grafting where there is a close relationship and similarity of structure between the stock and the scion. 'This is the case with varieties of the same species, which take most freely, as the different sorts of A pple; next with the differnnt species of a genus as the Apple and the Pear, which grow, but in which the union is less complete and permanent; and lastly with the genera of the same natural family, as the Cherry on the Plum-which die after a season or two. The ancients boasted of Vines and Apples grafted on Poplars and Elms; but repeated experiments, by the most skilful cultivators of modern times, have clearly proved that although we may, ....e in a thousand trials, succeed in effecting these ill assorted wrons, yet the graft invariably dies after a few months growth.*

The range in grafting or budding, for fruit trees in ordinary

The classical horticulturist will not fail to recall to mind Pliny's account of the tree in the garden of Lucullus, grafted in such a manner as to bear Olives, Almonds, Apples, Pears, Plums, Figs, and Grapes. There is little donbt, however, that this was some ingenious reception-as to this day the Italian gardenerz pretend to sell Jasmines, Honeysuciles, \&c., growing together and grafted on Oranges and Pomegranates. This is ingeniously managed, for a short lived effert, by intres. lucing she stems of these smaller plants through a hole bored up the centre of the tuck of the trees-their roots being in the same soil, and their stems, which after a litule growth fill up these boles, appearistg as if really grafted. 
sultire is as the following; Apples, on apple or crab seedlings for orcrards (standards, ) or on Paradise apple stocks, for dwarfs ; Pears, on pear seedlings for common culture, or Quince stocks for dw arfs, and sometimes un the thorn for clayey soils; Pcaches, on their own scedlings for standards or for orchards; on Almonds, for hot and dry climates; on Plums in cold or moist soils, or to secure them against the worm; Apricots, on Plum stocks, to render them hardy and productive, or on their own seedlings to render them long-lived. Nectarines are usually worked on the Peach or Plum; and Cherries on mazzard secdlings; or sometimes on the Perfumed Cherry for dwarfs.

The mamual operation of grafting is performed in a very easy and complete manner when the size of the stock, or branch to be grafted, corresponds precisely with that of the scion. In this case, which is called splice grafting, it is only necessary with a smooth sloping cut, upwards on the stock $a$, and downwards on the scion $b$, Fig. 2, to make the two fit precisely, so that the inner bark of one corresponds exactly with that of the other, to bind them firmly tngether with a strand of matting, and to cover the wound entircly with grafting clay or wax, and the whole is finished. In this, which is one of the neatest modes, the whole forms a complete union nearly at once; leaving scarcely any wounded part to heal over. But, as it is only rarely that the stock is of so small a size as to fit thus perfectly to the scion, the operation must be varied somew hat, and requires more skill. The method in most com. mon use to cover all difficulties, is called tongue

Fïg. 2. Spltce grafting. grafting.

We may remark here that grafting the shoots of Peaches, Nectarines and Apricots, owing to their large pith is more difficult than that of other fruit trees. A variation of splice-grafting, Fig. 3, has been invented to obviate this. This consists in selecting the scion $a$, so as to leave at its lower end about a fourth of an inch of two years old wood which is much firme: The bottom of the slope on the stock is cut with a dove-tail notch $b$, into which the scion is fitted.

Iomgue grafting, (or whip-grafting,) Fig. 4, resembles very nearly splice-grafting, except, instear of the simple splice, a tongue is marle to hold the two together mole firmly. In order to understand this method let us explain it a litile in detail.

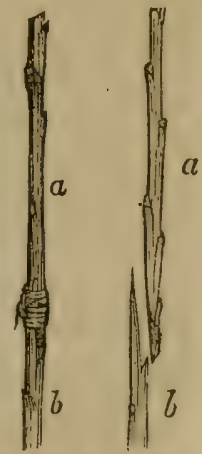

Fig. 3, Splice grafting the preach 

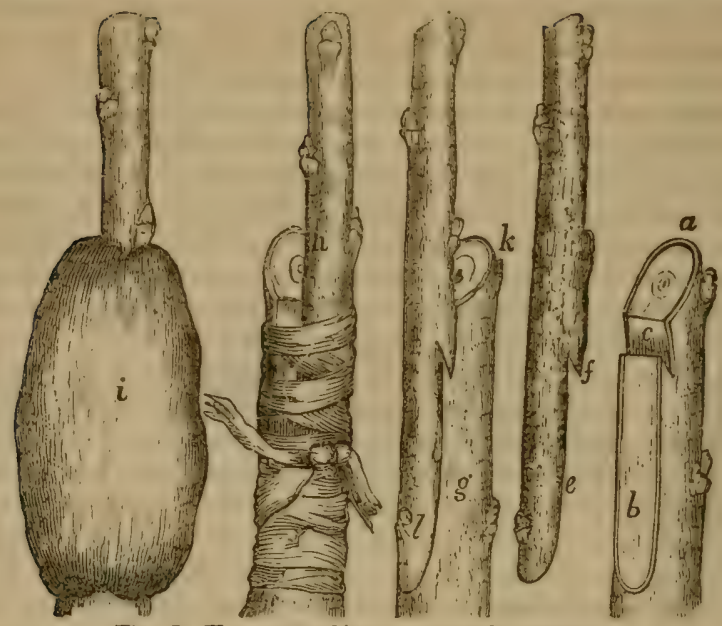

Fig. 3. Tongue-grafting, progresssive stages.

Having chosen your stock of the proper size, cut it off at the point where, $a$, it appears best to fix the graft. If the stock is quite small, it may be within three or four inches of the ground. Then, with a very sharp knife, make a smooth cut upwards $b$, about two inches in length. Next make a slit, from the top of $t^{\prime}$ is cut about one fourth of the way downwards, $c$, taking out a thin tongue of wood. Cut the scion four or five inches long, or so as to have three buds; then shape the lower end with a single smooth sloping cut, $e$, about the same length as that on the stock, and make the tongue upward $f$, to fit in the downward slit of the stock. Now apply the scion accurately to the stock making the inner bark of the scion fit exactly the inner bark of the stock, at least on one side, $g$. Without changing their position, tie them together carefully with a piece of bass-matting, or tape $h$. And finally cover the wound with well prepared grafting-clay or wax, $i$. This ball of clay should more than coves the union, by an inc: $\mathrm{ab}$ )ve and below, and should be about an inch thick. If grafting-wax is used, the covering need not be above half an inch thick.

In a month's time, if the graft has taken, it will be expanding its leaves and sending out shonts. It will then be necessary to rub or cut off all shoots between the ball and the ground, if it is a small stock, or all those which would rob it of a principal share of nourishment, if upon a large tree. If the scion or stock is very weak, it is usual to leave one or two other buds for a time, to assist in drawing up the sap. A bout the middle of July, atter a rainy day, you may remove the ball of clay, and, if the graft is 
securely united, also the bandage; and the angle left at the top of the stock, $a$, should now be cut off smoothly, in order to allow the bark of the stock and the scion to heal neatly over the whole wound.

'Though it is little attended to in common practice, the ama. teur will be glad to know that the success of a graft is always greatly insured by choosing the parts so that a bud is left near the top of the stock, $k$, and another near the bottom of the scion, $l$. These buds attract the rising sap to the portions where they are placed, form woody matter, and greatly facilitate the union of the firts near them; the upper part of the stock, and the lower part of the scion, being the portions soonest liable to perish from a want of nourishment.*

Cleft grafting is a very easy though rather clumsy mode, and is in more common use than any other in the United States. It is chicfly practised on large stocks, or trees the branches of which have been headed back, and are too large for tongue-grafting. The head of the stock is first cut over horizontally with the saw, and smoothed with a knife. A cleft about two inches deep is then made in the stock with a hammer and splitting-knife. The scion is now prepared, by sioping its lower end in the form of a wedge about an inch and half long, leaving it a little thicker on the outer edge. Opening the cleft with the splitting knife, or a small chisel for that purpose, push the scion carefully down to its place, fitting its inner bark on one side to that of one side of the stock. When the stock is large, it is

Fig. 4. usual to insert two scions, Fig. 4. On withdrawing the chisel, the cleft closes firmly on the scions, when the graft is tied and clayed in the usual manner.

Apple stoclis in many American nurseries, are grafted in greal quantities in this mortc-the stocks being previously talen out of the ground, headed down very near the root, cleft grafted with a single scion, sloping off with an oblique cut the side of tire stock upposite that where the graft is placed, and then planted at once in the rows so as to allow only a couple of buds of the scion to appear above ground. It is not usual with many, either to tic, or clay the grafts in this case, as the wound is placed below the surlace; but when this pian is adopted, the grafts must be set

* In grafting large quantities of young trees when stocks are scarce, it is not an unusual practice in some nurseries to tongue or whip-graft upon small pieces of routs of the proper sort of tree, planting the same in the earth as soon as grafted. Imleed, Dr. Van MIons considers this the most complete of all modes, with regand to the perfect condition of the grafted :ort ; $1 \mathrm{~s}$, because the smallest quantity of she stork is used; and $2 \mathrm{~d}$, because $\mathrm{tl}$ e lowerer part of the scion being thus placed in the ground, after a time it throws out fibres from that portion, and so at lest is actually growing on its own roots. 
and the trees planted at once, drawing the wcll pulverized soil with great care around the graft. Another way of grafting apple stocks, common in some westorn nurseries, consists in tongue-grafting on secdiling stocks of very small size, cut back almost to the root. This is performed in winter, lyy the fire. siue-lhe grafts carefully tied, and the roots placed in the cel. lar, in sand, till spring, when they are planted, the top of the graft just above ground.

Grafting the Vine is attended with great success in the cleft manner if treated as follows. Cut your scions during the winter or early spring, keeping them partially buried in a cool dunp cellar till wanted. As soon as the leares of the old vine or stock are tully expanded, and all danger of bleeding is past-say about the 10th of June, cut it off smoothly below the surface of the ground, and split the stock and insert one or two scions in the usual manner, binding the cleft well together if it does not close firmly. Draw the soil carefully over the whole, leaving two or three buds of the scion above the surface. If the root of the stock is a strong native grape, the graft will frequently grow ten or fifteen feet furing the first season, and yield a fair crop the second year.

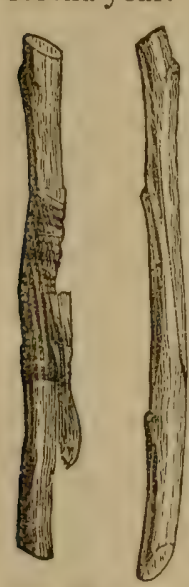

Fig. 5 .

The Vine may also be grafted with good success at the usual season if grafted below the ground, but above ground, it should not be attempted, on account of bleeding, until the leaves are nearly expanded.

Saddle grafting, Fig. 5, consists in cutting the top of the stock in the form of a wedge, splitting the scion and thinning away each half to a tongue shape, placing it astricle the stock, and fitting the two, at least on one side, as in tongue-grafting. This mode offers the largest surface for the junction of the scion and stock, and the union is very perfect. Mr. Kinight, who practised it chiefly upon Cherry trees, states that he has rarely ever seen a graft fail, even when the wood has been so succulent and immature as to preclude every hope of success by any other mode.

A varicty of this mode, for stocks larger than Sathlle grafting. the scions, is practised with much success in England atter the usual season is past, and when the bark of the stock separates readily. "The scion, which must be smaller than the stock, is split up between two or three inches from its lower end, so as to have one side stronger than the other. This strong side is then properly prepared and introduced between the bark and the wood; while the thinner division is fitted to the op. posite side of the stock." The graft, thus placed, reccives a 
large supply of the sustaining fuid from the stock, and the union is rapid; while the wound on the stock is speedily coverel by a new layer of bark from that part of the scion which slands astride it.

Grafting clay is prepared by mixing one third horsedung free from straw, and two thirds clay, or clayey loam, with a little hair, like that used in plaster, to prevent its cracking. Beat and temper it for two or three days, until it is thoroughly incorporated. When used, it should be of such a consistency as to be easily put on and shaped with the hands.

Grafting wax of excellent quality we have made by melting together three parts of bees-wax, three parts of rosin and two parts tallow. While yet warm it may be worked with the aid of a little water, like shoemaker's wax, by the hand. The
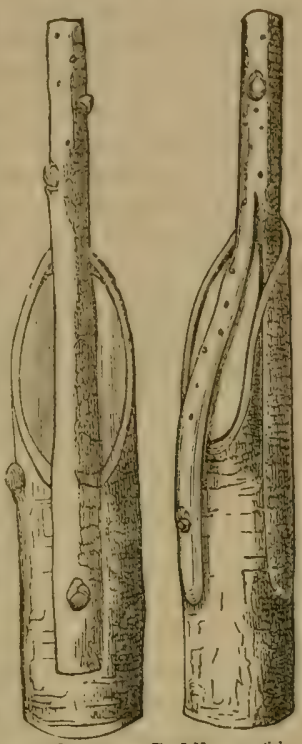

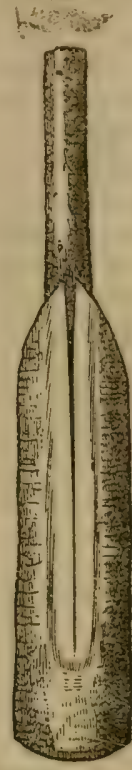

Fig. 6. Sadille grafling large sluchs. common grafting wax of the French gardeners is of two kinds. The first, is melted and laid on with a brush in a fluid state, and is made of half a pound of pitch, half a pound of bees-wax, and a pound of cow-dung boiled together. The second, which is spread while warm on strips of coarse cotton, or strong paper, and wrapped directly about the graft, answering at once to tie and to protect it, is composed of equal parts of bees-ivax, turpentine, and resin. The grafting wax most eommonly used here is made of tallow, bees-wax, and resin, in cqual parts, or, as many prefer, with a little more tallow to render it pliable.

Grafting wax is a much neater and more perfect protection than srafting clay, but the trifling cost of the latter, where a great deal of work is to be dnne, accounts for its greater use by $\mathrm{n}$ irserymen, and gardeners generally.

\section{Budding.}

Budding (inoculating, of the old authors) differs from common rratting the least in its nature or eflects. Every bud is a astinct individual, capable of becoming a tree under favourable 
circumstances. In grafting, we use a branch, composed of seve ral buds with a consilerable quantity of bark and wood; whila in budding, we employ but a single bud, with a very small quan tity of the adjoining bark and wood.

'The advantages of budding fruit trees, compared with grafting, are so considerable, that in this country it is ten times as much practised. 'These are, first, the great rapidity with which it is performed; a skilful budder, with a clever boy following him to the the buds, being able to work from a thousand to twelve hundred young nursery stocks in a day. $2 d$. The more convenient season at which it is pertormed, in all countries where a short spring crowds garden labours within a small space. 3d. Being able to perform the operation without injuring the slock in case of failure, which is always more or less the case in stocks headed down for grafting. 4th. The opportunity which it aflords, when performed in good season, of repeating the trial on the same stock. 'To these we may add that budding is universally preferred here fo. all stone fruits, such as Peaches, Apricots, and the like, as these require extra shill in grafting, but are budded with great ease.

The proper scason for budd. $n g$ fruit trees in this country is from the first of July to the mi ldle of September; the different trees coming into season as follows; Plums, Cherries, Apri. cots ou Plums, Apricots, Pears, Apples, Quinecs, Nectarines and Peaches. 'Trees of considerable size will require budding

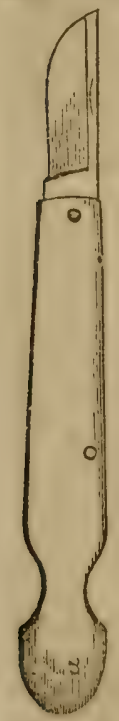
earlier than young seedling stocks. But the opera. tion is always, and only, performed when the bark of the stock parts or separates freely from the wood, and when the buds of the current year's growth are somewhat plump, and the young wood is growing firm. Young stocks in the nursery, if thrifty, are usually planted out in the rows in the spring, and buidded the same summer or autumn.

Before commencing you should provide yourself with a budiling knife, Fig. 7 , (about four and a half inches long, having a rounded blade at one end, and an ivory handle terminating in a thin rounded erige called the haft, $a$, at the other.

In choosing your buds, sclect thrifty shoots that have nearly done growing, and prepare what is called a stick of buds, Fig. 8, by cutting of a few of the imperfect huds at the lower, and such as may lee yet too soft at the upper ends, leaving only smooth well developed single buds ; dotible buds being fruitbuds. Cut off the leaves, allowing about half an incin of the foot-stalks to remain for collvenient'y inserting the bucks. Some strands of bass-matting Fig. 7. Buc about twelve or fourteen inches long, previously 
soaked in water to render them soft and pliable, (or in the absence of these some soft woollen yarn,) mist also be at hand for tying the buds

Shicld or $\mathrm{T}$ budding is the most approved mode in all countries. A new variety of this method now generally practised in this country we shall describe first as being the simplest and best mode for fruit trees.

American shicld bulding. Having your stick of buds ready, choose a smooth portion of the stock. When the latter is small, let it be near the ground, and, if equally convenient, select also the north side of the stock, as less exposed to the sun. Make an upright incision in the bark from an inch to an inch and a half long, and at the top of :uis make a cross cut, so that the whole shall form a $T$. From the stick of buds, your knife being very sharp, cut a thin, smooth slice of wood and bark containing a bud, Fig. 9, a. With the ivory haft of your budding knife, now raise the bark on each side of the inoision just wide enough to armit easily the prepared bud. T'aking hold of the footstalk of the leaf, insert the bud under the bark, pushing it gently Fig. 8. A
down to the buttom of the incision. If the upper stuck of buds.

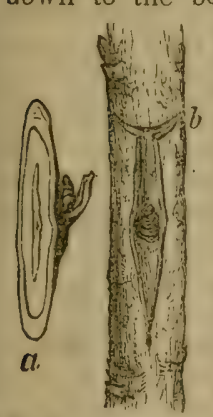

Fig. 9. American portion of the bud projects above the horizontal part of the $T$, cut it smoothly off now, so that it may completely fit, $b$. A bandage of the soft matting is now tied pretty firmly over the whole wound, Fig. 10, commencing at the bottom, and leaving the bud, and the footstalk of the leaf only exposed to the light and air.

Common shield-budding, Fig. 11, practised in all gardens in Europe, differs from the forcgoing only in one respect-the removal of the slice of wood contained in the bud. This is taken out with the point of the knife, holding the bud or shicld by the leaf stalk, with one hand, inserting shield budding. the knife under the wood at the lower extremity, and then raising and drawing out the wood by bending it upwards and downwards, with a slight jerk, until it is loosened from the bark; always taking care that a small portion of the wood remains behind to fill up the hollow at the base or heart of the bud. The bud thus prepared is inserted precisely as before described.

The American variety of shield budding is found greatly peferable to the European mode, at least for this climate. Many sorts of fruit trees, especially Plums and Cherries, nearly mature

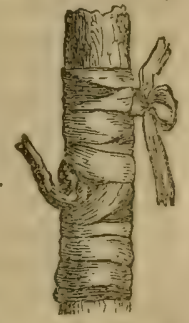

Fig. 10. 


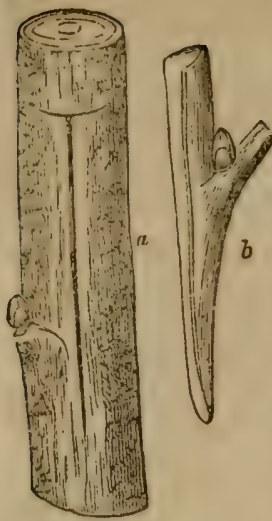

Fig. 11.

their growth, and require to ve budrled in the hottest part of our summer. In the nld method, the bud having only a shield of bark with but a particle of wood in the heart of the bud, is much more liable to be destroyed by heat, or dryness, than when the slice of wood is left behind in the American way. Taking out this wood is always an operation requiring some dexterity and practice, as few buds grow when their eye, or heart wood is damaged. The American method, therefore, requircs less skill, can be done earlier in the season with younger wood, is performed in much less time, and is uniformly more successful. It has been very fairly tested upon hundreds of thousand fiuit rrees, in our gardens, for the last twenty years, and, although practised English budrlers coming here, at first are greatly prejudiced against it, as being in direct opposition to one of the most essential features in the old mole, yet a fair trial has never failed to convince them of the superio.ity of the new.

After trentment. In two weelis after the operation you will be able to see whether the burl has taken, by its plumpness and freshmess. If it has failerl, you may, if the bark still parts rearlilv, make another trial; a clever budder will not lose more than 6 or 8 per cent. If it has succecded, after a fortnighi more has elapsed, the bandacre must he loosened, or if the stock has swelled much, it should be removed altogether. When burl. ding has been perfomed very iate, we have occasionally found it an arlvantage to leave the bandage on during the winter.

As soon as the burls commence swelling in the ensuing spring, head down the stock, with a sloping back cut, within two or three inches of the bud. The burt will then stant vigorously, and all "robbers," as the shonts of the stock near to and below the bud are termed, must be taken off from time to time. 'To secure the upright growth of the bud, and to prevent its being broken by the winds, it is tied when a fow inches loner to that portion of the stock left for the purpose, Fig. 12, $a$. About midsummer, if the shoot is stroner, this support may be removed, and the superflunus portion of the stock smonthly cut awav in the dotted line, $b$, when it will be rapilly covered with youngr bark.

IVe have found a sreat atvantage, when budling trees which do not take readily, in adopting $\mathrm{Mr}$. Knight's excellent mode of tying with two distinct bandages; ons sovering that part below the bud

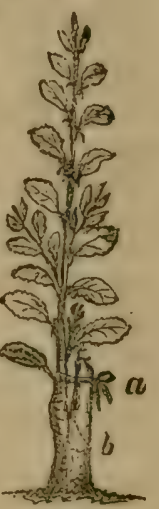

Fig. 12. Trealment of tha 
and the other the portion above it. In this case the lower bant. age is removed as soon as the bud has taken, and the upper left for twu or three wecks longer. This, by arresting the upind sap, completes the union of the upper portion of burl, (which in plums frequently dies, while the luwer part is unitcd,) and so cures success.

Reversed shield buading, which is nothing more than making the cross cut at the bottom, instead of the top of the uprigint incision in the bark, and inserting the bud from below, is a grood deal practised in the south of Europe, but we have not found that it possesses any superiour merit for fruit trees.

An ingenious application of budding, worthy the attention of amateur cultivators, consists in using a blossom-bud instead of a wood-bud; when, if the operation is carefully done, blossoms and fruit will be produced at once. This is most successful with the Pear, though we have often succecded also with the Peach. Blossom-buds are readily distinguished, as soon as well formed, by their roundness, and in some trees by their growing in pairs; while wood-buds grow singly, and are more or less pointed. We have seen a curious fruit grower borrow in this way, in September, from a neighbor ten miles distant, a single blossom-bud of a rare new pear, and produce from it a fair and beautiful fruit the next sumincr. 'The bud, in such cases, shoul,' be inserted on a favourable limb of a bearing tree.

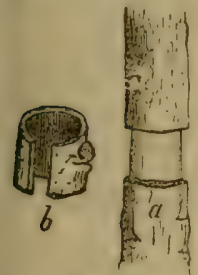

Fig. 12 . Anmular budding, Fig. 12, we have found o valuable mode for trees with hard wood, and thick bark, or those which, like the walnut, have buds so large as to render it difficult to bud them in the common way. A ring of bark, when the sap is flowing frcely, is taken from the stock, $a$. and a ring of corresponding size containing a bud, $b$, from the scion. If the latter should be too large, a picce must be taken from it to make Annular indding. it fit; or should all the scions be too small, the ring upon the stnck may extend only three fourths the way round, to suit the ring of the bud.

An application of this mode of great, value occasionally occurs in this country. In snowy winters, fruit trees in orchards are sometimes girdled at the ground by field mice, and a growth of twenty years is thus destroyed in a single day, should the girdle extend quite round the tree. 'To save such a tree, it is only necessary, as soon as the sap rises vigorously in the spring: to apply a new ring of bark in the annular mode taken from a branch of proper size; tying it firmly, covering it with grafting clay to cxclude the air, and tinally drawing up the carth so as to cover the wound completely. When the tree is too large to apply an entire ring, separate pieces, carefully fitted, will anower; and it is well to reduco the top somewhat by pruning, 
that it may not make too large a demand on the root for a sup. ply of food.

Budding may be done in the spring as well as at the latter end of summer, and is frequently so performed upon roses, and ther ornamental shrubs, by French gardeners, but is only occasional use upon fruit trees.

\section{Influence of the stock and graft.}

The well known fact that we may have a hundred different varietics of pear upon the same tree, each of which produces its fiuit of the proper form, colour, and quality; and that we may have, at least for a time, several distinct, though nearly related species upon one stock, as the Peach, Apricot, Nectarine, and Plum, prove very conclusively the power of every grafted or budded branch, however small, in prescrving its identity. To explain this, it is only necessary to recall to mind that the ascending sap, which is furnished by the root or stock, is nearly a simple fluid; that the leaves digest and modify- this sap, forming a proper juice, which re-descends in the inner bark, and that thus every bud and leaf upon a branch maintains its individuality by preparing its own proper nourishment, or orgonizing matter, out of that general aliment, the sap. Indeed, according to De Candolle, ${ }^{*}$ each separate cellule of the inner bark has this power of preparing its fool according to its nature; in proof of which, a striling experiment has been tried by grafting rings of bark, of different allied species, one above another on the sarne tree without allowing any buds to grow upon them. Con cutting down and examining this tree, it was found that under each ring of bark was deposited the proper wood of its species, thus clearly proving the power of the bark in preserving its identity, even without leaves.

On the other hand, though the stock increases in size by the woody matter received in the descending sap from the graft, yet as this desccnds through the inner bark of the stock, it is elaborated by, and receives its character from the latter; so that, atter a trce has been grafted fifty years, a shnot which springs out from its trunk below the place of union, will always be found to bear the original wild fruit, and not to have beex: in the least aflected by the graft.

But, whilst grafting never effects any alteration in the identity of the variety or species of fruit, still it is not to be deoied that the stock does exert certain influences over the habits of the graft. 'The most important of these are dwarfing, indusing fruitfulness, and adapting the graft to the soil or climate.

Thus every one knows that the slower habit of growth in .he 
Quince suck, is shared by the Pear grafted upon it, which becomes a dwarf; as does also the Apple when worked on the Paradise stock, and, in some degree, the Peach an the Plum. The want of entire similarity of structure between the stock and graft, confines the growth of the latter, and changes it, in the case of the Pear, from a lofty tree to a shrub of eight or ten feet in height. The effect of this difference of structure is very ap. parent, when the Peach is grafted on the Plum, in the greater size of the trunk above, as compared with that below the graft; a fact which seems to arise from the obstruction which the de. scending sap of the graft finds in its course through the bark of the stock.

To account for the earlier and greater fruitfulness caused by grafting on a stock of slower growth, Mr. Knight, in one of his able papers, offers the following excellent remarks.

"The disposition in young trees to produce and nourish blossom buds and fruit, is increased by this apparent obstruction of the descending sap; and the fruit, I think, ripens somewhat earlier than upon other young trees of the same age which grow upon stocks of their own species. But the growth and vigor of the tree, and its power to nourish a succession of heavy crops, sre diminished, apparently, by the stagnation in the branches sind stock of a portion of that sap which, in a tree growing on its own stem, or upon a stock of its own species, would descend 10 nourish and promote the extension of its own roots. The practice, therefore, of grafting the Pear on the Quince, and the Peach on the Plum, when extensive growth and durability are wanted is wrong; but it is eligible wherever it is wisher :o diminish the vigour and growth of the tree, and its durability is not so important."

In adapting the graft to the soil the stock has a marked influ. ence. Thus in dry chalky soils where the Peach on its own roots will scarcely grow, it is found to thrive admirably budded on the Almond. We have already mentioned that in clay soils too heavy and moist for the Peach, it succeeds very well if worked on the Plum. M. Floss, a Prussian gardener, succeeded in growing fine pears in very sandy soils, where it was nearly impossible to raise them before, by grafting them on the Mountain Ash, a nearly related tree, which thrives on the lryest and lightest soil.

A variety of fruit which is found rather tender for a certain climate, or a particular neighborhood, is frequently acclimatised by grafting it on a native stock of very hardy habits. Thus near the sea-coast where the finer flums thrive badly, we have seen them greatly improved by being worked on the beech. plum, a native stock adapted to the spot; and the foreign grape is more luxuriant when grafted on our native stocks.

1 slight effect is sometimes produced by the stock on the 
quality of the fruit. A few sorts of pear are superiour in fla. vour, but many are also inferiour, when grafted on the Quince, while they are more gritty on the thorn. The Green Gage, a Plum of great delicacy of flavour, varies considerably upon different stocks; and Apples raised on the crab, and Pears on the Mountain Ash, are said to keep longer than when grown on ibeir own roots.

In addition to the foregoing, a diseased stock should always be avoided, as it will communicate disease slowly to the graft, unless the latter is a variety of sufficient vigour to renew the health of the stock, which is but seldom the case.

The cultivator will gather from these remarks that, in a fa. vourable climate and soil, if we desire the greatest growth, duration, and devolopment in any fruit, (and this applies to orchards generally,) we should choose a stock of a closely similar nature to the graft-an apple seedling for an apple; a pear seedling for a pear. If we desire dwarf trees, that come into bearing very young, and take little space in a garden, we cm. ploy for a stock an allied species of slower growth. If our soil or climate is unfavourable, we use a stock, which is adapted to the soil, or which will, by its hardier roots, endure the cold.

The influence of the graft on the stock seems scarcely to extend beyond the power of communicating disease. A graft taken from a tree enfeebled by disease, will recover with difficulty, even if grafted on healthy stocks for a dozen times in repeated succession. And when the disease is an inherent or hereditary one, it will certainly communicate it to the stock. We have seen the yellows, from a diseased peach tree, propagated through hundreds of individuals by budding, and the stock and graft both perish together from its effects. Hence the importance, tc nurserymen especially of securing healthy grafts, and working only upon healthy stocks.

\section{Propagation by cuttings.}

Propagating by cuttinge; as applied to fruit trees, consists in causing a shoot of the previous seasons' wood to grow, by detaching it from the parent tree at a suitable season, and planting it in the ground under favourable circumstances.

In this case, instead of uniting itself by cody matter to another tree, as does the scion in grafting, the descending woody matter becomes roots at the lower end, and the cuting of which, is then a new and entire plant. Every bud being a distinct individual, capable of forming a new plant, has indeed theoretically the power, if zeparated from the parent stem, of tnrowing out mots and main. taining a separate existence; and some plants as the grape vine are frequently propagated by single buds planted in the soi? But in practice, it is found necessary, with almost all trees an. 
plants, to retain a considerable portion of the stem with the bud, to supply it with food until it has formed roots to draw nourish. i ment firorn the soil.

All fruit trees may be propagated by cuttings with prope: care and attention, but only a fow grow with sufficient fiecili!y in this way to render their propagation by cuttings a common mode. These are the Gooseberry, the Currant, the Vine, the Quince, the Fig, and the Mulberry.

Cuttings of the Currant, Gooseberry, and the hardy sorts or Vine, will root readily, in a soil not too dry, in the open garden. Currants and Gooseberries are generally taken of in the fall or winter, prepared for planting, and two thirds of their lower ends buried in the ground till the commencement of spring, when they are planted out. either where hey are to remain, or in nursery rows. If planted in autumn, they are liable ti 6 be thrown out by winter frosts. They will succeed nearly as well if talien off in the spring, but, owing to the period at which they commence growing, this must be attended to very early, if deferred till that season.

In order to raise plants of the Gooseberry and Currant, with straight, clean stems, which shall not throw up suckers, it is only nccessary, before planting the cutting, to cut out every eye or bud to bo placed below the surface of the ground, Fig. 14. The cutting should be about a foot long, eight inches of which may be inserted in the ground. To insure greater success in raising the finer sorts of gooseberry, or other shrubs, it is customary to plant the cuttings on the shaded side of a wall or fence, in deep rich loam, rather damp than dry. Cuttings of the vine are generally prepared when trimming the Fig. 14. A old plants in autumn, or winter; they may then be rooseberry cut- buried with their lower ends in the ground, or kent and planted. in earth in the cellar till spring.

Scarce sorts of foreign grapes, which it is desirable to multiply extensively, are firequently propagated by joints; that is, by buds having about two inches of wood attached to each-every bud in this way forming a plant. When this mode is adopted, it is usual to plant the joints about half an inch deep, in light soil, in a common hot bed prepared for the purpose, or each joint is planted in a pot by itself. In the first way a great number of

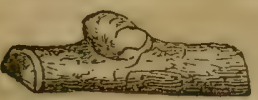

Fig. 15. plants may be grown in a srnali space. Success is more certain in propagating the vine by joints, where the joint is haved before planting, Fig. 15.

A vine joint, prezared and planted. The large English black mul. berry is propagated by cuttings 
as tollows : about the last of October, take cuttings from the thrifty shoots of a bearing tree, cut ont all the buds except two or three at the top, and piare off the bottom of the cutting just below a bud. Lay-in the cuttings in a sheltered border, biry ing them so that only the two buds at the top are exposed, ar covering them with some loose straw or litter. In the spring. make a small hot-bed with very sandy soil in which to plant the cuttings on taking them out of the ground, or place each one in a small pot in any hot-bed ready at hand, and in a few weelis they will be found to have made roots freely.

As a general rule, cuttings succeed best when they are taken off just between the young and the previous year's wood; or, in the case of young side shoots, when they are cut off close to the branch preserving the collar of the shoot. The lower end should be cut smoothily across just below a bud, the soil should in all cases be pressed firmly about the lower end of the cutting, and it should always be planted before the buds commence swelling, that the wound may in some measure heal before rrowth and the absorption of fluid commences.

\section{Propagaıton by Layers and Suckers.}

A layer may be considered as a cutting not entirely separated from the plant.

Layering is a mode of propagation resorted to in increasing some fruit tree stocks, as the Paradise stock, the Muscle Plum, and some kinds which do not grow so well from the seed. Certain varieties of native grape, as the Bland's Virginia, which do not root readily by cuttings are also raised in this way, and it may be applied to any sort of fruit tree which it is desirable to continue on its own root without grafting.

Fruit trees are generally layered in the spring, and the layers may be taken off well rooted plants in the autumn. But they may also be layered with success early in uly.

In making layers the ground around the mother plant should be made light and mellow by digging. Being provided with some hooked pegs to fasten down the layers, bend down a branch so that the end may recline upon she ground. Open a little ‘rench three or four inches deep to receive the young wood to be layered; make a cut or tongue, Fig. . $s u$, half way through the under side of the shoot, pegging down the branch wit' $b$ the hooked peg $b$, to

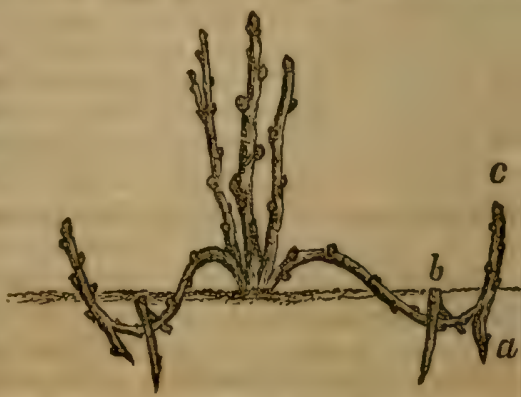

Fig. 16. Iaycring. 
keer it in its place; press the earth slightly round the tongue, and, in filling in the soil, raise nearly upright the end of the layer $c$, which remains above the surface of the ground.

'The descending sap, filled with organizable matter, is arrested by this tongue, accumulates there, and the emission of rocts speedily takes place. Ringing, wounding, or twisting the limb, answers the same purpose less perfectly, and indeed many trees root readily from the mere position of the branches as layers, and the moisture of the soil.

A tree or plant which is kept for raising layers is called a stonl, and is headed down, both to facilitate the rooting of the layers, and to afford an abundance of shoots near the eartin. Shoots of some of the fruit tree stocks in the English nurseries are pegred down to the surface before growth commences in the spring, covered about an inch deep with soil, and at the end of autumn afford hundreds of plants; almost every bud making a separate root.

Suckers are shoots sent up from the root, or from portions of the stem below the surface of the soil, which are easily separated from the parent plant.

Suckers of fruit trees aro frequently used as stocks for bud. ling or grafting upon, but they are greatly inferiour to sredliners for this purpose, as they are always more liable to produce suckers, and they have not the thrifty vigorous habit, or the same power of forming as good roots as seedlings. Beside this, strould the tree from which they are taken be diseased, they will be likely to carry the malady with them.

Propagating by suckers, is an easy and desirable way when we wish to continue a seedling fruit of value on its own ront, and some of our common fruits appear to be more healthy and permanent when growing in that way. It is also the ouly mode in use for increasing the Raspberry; as is also that of runurs, which is a kind of sucker above ground, for the Strawierry.

\section{CHAPTER III.}

\section{PRUNING.}

\section{Pruning to promote growth or modefy the form of fruit trees.}

In this country almost all fruit trees are grown as standards. In this way they develop their natural forms, attain the largest Bize, and produce the greatest quantity of fruit, with the least possible care. Our bright and powerful sun, reaching every 
part of the tree, renders the minute systems of pruning and training, which occuny so large a portion of the English works on this subject, of litile or no moment to the cultivator here. Pruning is, therefore, commonly resorted to only for the purpose of increasing the vigour of feeble trees, or to regulate and im. prove the form of healthy and luxuriant trees.

Pruning has the power of increasing the vigous of a tree in two ways. If we assume that a certain amount of nourishment is supplied by the roots to all the branches and buds of a tree, by cutting off one half of the branches, at the proper season, we direct the whole supply of nourishment to the remaining portion, which will, consequently, grow with nearly double their formes luxuriance. Again, when a tree becomes stunted or enfeebled in its growth, the thinness of its inner bark, with its consequent small sap-vessels, (which it must be remembered are the principal chan; nel for the passage of the ascending supply of food) renders the upward and downward circulation tardy, and the growth is small. By heading back or pruning judiciously, all the force of the nourishing fluid is thrown into a-smal!er number of buds, which make new and ldxuriant shoots, larger sap-vessels, and which afford a ready passage to the fluids, and the tree with these renewed energies will continue in vigour for a long time.

This treatment is especially valuable in the case of sinall trees of feeble or stunted growth, which are frequently cut back to a single bud, and a new shoot or shoots, full of vigour, gives a healthy habit to the tree. In the nurseries, this practice of heading down unthrifty trees is frequently pursued, and small orchard trees which have become enfeebled may be treated in the same manner; cutting back the head as far as the place where it is wished that new shoots should spring out. Older trees should be headed back more sparingly, unless they are greatly enfeebled; and their roots should at the same time be assisted by manure.

A judicious pruning to modify the form of our standard trees is nearly all that is required in ordinary practice. Every fruit tree, grown in the open orchard or garlen as a common standard should be allowed to tate its natural form, the whole efforts of the priner going no further than to take out all weak and crowded branches; those which are filling uselessly the interiour of the tree, where their leaves cannot be duly exposed to the licht and sun, or those which interfere with the growth of others. All pruning of large branches in healthy trees should be avoided by examining them every season and taking out superfunus shoots while small. Mr. Coxe, the best American anthor on fruit trees, remarks very truly "when orchard trees are much frumed, they are apt to throw out numerous (superfluous) sucliers trom the boughs in the following summer; these should be rubbed off when they first appear, or they may easil? 
De brokelt off while young and brittle-cutting is apt to increase their number."

Where pruning is not required to renovate the vigour of ar. enferebled tree, or to regulate its shape-in other words, in the case of a healthy tree which we wish to retain in a state of the greatest luxuriance, health, and vigour, it may be considered worse than useless. Bearing in mind that growth is always corresponding to the action of the leaves and branches, if these are in due proportion, and in perfect health, the knife will always be found rather detrimental to luxuriance and constitutional vigour than beneficial.*

The best scason for pruning to promote growth, theoretically, is in autumn soon after the fall of the leaf. Next to this, winter pruning, performed in mild weather, is best, and in orchards this is the season usually most convenient. In all parts of the coun. try where the winters are not very severe, (and always in the southern and western states,) the roots are collecting a certain stock of nourishment during the whole autumn and winter. When a tree is pruned in autumn or winter this whole supply goes to the remaining branches, while in the case of spring pruning it is partly lost. North of the $43^{\circ}$ of latitude, however, the winters are so severe that winter pruning should be deferred till the last of February.

We should especially avoid pruning at that period in spring when the buds are swelling, and the sap is in full flow, as the loss of sap by bleeding is very injurious to most trees, and, in some, brings on a serious and incurable canker in the limbs.

There are advantages and disadvantages attending all sea. sons of pruning, but our own experience has led us to believe that, practically, a fortnight before midsummer is by far the best season, on the whole, for pruning in the northern and middle states. Wounds made at this season heal over frecly and rapidly; it is the most favourable time to judge of the sliape and balance of the head, and to see at a glance which branches require removal ; and all the stock of organizable matter in the tree is directed to the branches that remain.

In pruning large limbs, some composition should always be at nand to cover the wound. 'This will not only prevent its cracking by the cold in winter pruning, but will keep out the air, and mairtain the exposed wood in a sound state, until it is covered

* Ignorant cultivators frequently weaken the energies of young trees, ant cuuse them to grow up with lean and slender stems, by injudiciously trimming off the young side shcots and leaves, in the growing season. By taking off these shootz, the stem is deprived of all the leaves which would attract and elaborate the sap, thus preparing nourishment for the growth of the stem; and the trunk of the tree does not increase in size lalf so fast as when the side branches are allowed to remain for a time, pruning them away gradually. It is better, in the case of these young trees, to slop the side branches when of moderate length by pinching out the cerminal bud. 
with a new layer of bark. Many compositions have been ir: fashion, abroad, for this purpose, which, under our summer sun and wintry frosts, are nearly worthless, as they generally crack and fall off in a single year. The following is a cheap and ad. mirable application, which we recommend to all cultivators of fruit trees.

Composition for wounds made in pruning. Take a quart of alcohol and dissolve in it as much gum shellac as will make a liquid of the consistence of paint. Apply this to the wound with a common painter's brush; always paring the wound smoothly first with the knife. The liquid becomes perfectly hard, adheres closely, excludes the air perfectly, and is affected by no changes of weather; while at the same time its thinness offers no resist. ance to the lip of new bark that gradually closes over the wound. If the composition is kept in a well corked bottle, sufficiently wide mouthed to admit the brush, it will always be ready for use and suited to the want of the moment.

\section{Pruning to induce fruitfulness.}

When a young fruit tree is too luxuriant, employing all its energies in making vigorous shoots, but forming few or no blossom buds, and producing no fruit, we have it in our power by different modes of pruning to lessen this over-luxuriance, and force it to expend its energies in fruit-bearing. The most direct and successful mode of doing this is by pruning the roots, a proceeding recently brought into very successful practice by European gardeners.

Root pruning has the effect of at once cutting off a considerable supply of the nourishment formerly afforded by the roots of a tree. The leaves, losing part of their usual food, are neither able to grow as rapidly as before, nor to use all the nutritious matter already in the branches; the branches therefore become more stunted in their growth, the organizable matter accumu. lates, and fruit buds are dircetly formed. The energies of the tree are no longer entirely carried off in growth, and the return. ing sap is employed in producing fruit buds for the next year.

Root pruning should be performed in autumn or winter, and it usually consists in laying bare the roots and cutting off smoothly at a distance of a few feet from the trunk, (in proportion to the size of the tree) the principal roots. Mr. Rivers, an English nurseryman of celebrity, who has practised this mode with great success, digs a trench early in November, eighteen inches deep, round his trees to be root pruned, cutting off the roots with a sharp spade. By following this practice every year, he not only throws his trees into early bearing, but forces Apples, Pears, and the like, grafted on their own roots, to be. come prolific dwarfs, growing only six feet apart, trained in a 
conical furm, full of fruit branches, and producing abundantly, These dwarf trees, thus annually root pruned, he supplics abun. dantly with manure at the ends of the roots, thus keeping up their health and vigour. The plan is an admirable one for small gardens, or for amateur's who wish to grow a great many sorts in a small surface. Mr. Rivers, in a pamphlet on this Eubject enumerates the following among the advaniages of systematic root pruning.

" 1 . The facility of thinning, (owing to the small size of the trees,) and, in some varieties, of setting the blossoms of shy. bearing sorts, and of thinning and gathering the fruit.

" 2 . It will make the gardener independent of the natural soil of his garden, as a few barrowsful of rich mould will support a tree for a lengthened period, thus placing bad soils nearly on a level with those the most favourable.

" 3 . The capability of removing trees of fifteen or twenty years growth, with as much facility as furniture. To tenants this will indeed be a boon, for perhaps one of the greatest annoyances a tenant is subject to, is that of being obliged to leave hehind him trees that he has nurtured with the utmost care."

In conclusion, Mr. Rivers recommends caution ; " cnough of vigour must be left in the tree to support its crop of fruit, and one, two, or three seasons cessation from root pruning, will often be found necessary."

Root pruning in this country will, we think, be most valuable in its application to common standard trees, which are thrifty, but bear little or no fruit. They will generally be found to require but a single pruning to bring them into a permanently fruitful condition; and some sorts of Pears and Plums, which do not usually give a fair crop till they are twelve or fourteen years old, may be brought into fruit by this means as soon as they are of proper size. Several nearly full grown peach, pear, and plum trees, on a very rich soil on the Hudson, which were over-luxuriant but bore no fruit, were ront pruned by nur advice two years ago, and yielded most excellent and abundant crops last season.

In the case of Apple orchards, where the permanent value depends on the size, longevity, and continued productivene'ss of the trees, it is better to wait patiently and not resort to pruning to bring them into bearing; as it cannot be denied that all exces. sive pruring shortens somewhat the lite of a tree. Mr. Coxe, indeed, recommended that the first fruit should never be allowed to ripen on a young apple orchard, as it lessens very matcrially the vigour of the trees.

Shortening-in the shoots of Peaches, Nectarines, and A pricots, is we shall hereafter point out, has a strong tendency to increase the fruitfulness of these trees, since by reducing the yourig wood, the sap accumulates in the remainder of the branch, and many 
bearing shoots are produced instead of one. And the English mactice of spurring.in, which consists in annually shortening the lateral shonts of trained Prars, Aprles, and the like, in urder (o) malie them throw out short fruit branches, or spurs, is founded on the same principle.

Bending down the limbs is an easy and simple means of throwing such branches directly into fruit. By this means the circulation is retarded, rapid growth ceases, organizable inatter accumulates, and fruit buds, as before stated, surcly follow. The limbs are bent, while fexible, in June or July, and tied down below a horizontal line until they retain of themselves their new position. When this can be easily applied, it is a never failing node of rendering such branches fruitful. It is stated in Loudon's Gardener's Magazine that " a very large crop of Pears was obtained by the Rev. Mr. Fisher, in Buckinghamshire, from trees which had not borne at all, by twisting and breaking down the young shoots, late in the autumn, when the wood had become lough; and the pendent branches afterwards continued perfectly healthy."

Disbarking and Ringing are two modes that have been recommended by some authors, but of which, except as curious experiments, we entirely disapprove. Disbarking, that is, romoving the outer bark of the trunk in February, May, or March, is and may be practised with good results on trees in very shcltcred positions, and under glass, but must always be a somewhat dangerous practice in open orchards, and in a variable climate like ours; while its good eflects may in a great measure be attained by keeping the bark in a healthy state by a wash of soft soap. Ringing, which is nothing more than stopping the descending sap in a branch and forcing it to organize blossom buds, by taking off a ring of bark, say a fourth or half an inch, near midsummer, is a mode always more or less injurious to the health of the branch, and if carried to any extent, finally destroys the tree. It is gradually falling into disuse, since root pruning, and other and better modes, are becoming known. A ligature or bandage tightly applied to the limb, will have temporarily the same offect as ringing, without so much injury to the branch.

\section{Inducing fruitfulness by other means.}

The influence of certain soils on the productiveness of frutt trees is a subject of every day observation, but the particular ingredients of the soil, which insure this abundant bearing, is not sn well known. Limestone soils are almost invariably prorluclive of all sorts of fruit; and certain strong loams in this coun. try seem to be equaliy well adapted to this end.

In a curious work called the "Rejuvenescence of Plants," etc. oy Dr. Schultz, of Berlin, the author, who has devoted consider. 
able time to the subject, states that common salt and chloride of lime confribute greatly to the flowering of most plants, to which, howerer, they can only be applied with safety, in small quantities. "Salts of lime," he continues, "appear to produce so nearly the same effect as those of potash and soda, that it is only necessary to place lime within their reach, if there is no deficiency if manure in the shape of general food. Lime will in the main promote, in an astonishing degree, the fruiting and flowering of most plants, because calcareous salts promote evaporation and the concentration of the sap."

Although we cannot coincide with many of Dr. Schultz's views as expressed in this work, yet, the remarks just quoted agree so entirely with facts that have come under our own observation, that we gladly place them before the cultivator of fruit trees. One of the most productive fruit gardens in our knowledge is on a limestone soil, and another more than usually prolific, in a neighbourhood not very fruitful, is every year treated with a top dressing of coarse salt, at the rate of two bushels to the acre. These facts are surely worth the attention of growers, and should be the subject of more extended and careful experiments.

Rendering trees more fruitful by dwarfing, and by adapting them to soils naturally unfruitful by growing them upon other and better stocks, we have already placed before the reader under the head of Grafting.

\section{CHAPTER IV.}

TRAINING.

Training fruit trees is, thanks to our favourable climate, a proceeding entirely unnecessary in the greater part of the United States. Our fine dry summers, with the great abundance of strong light... and sun, are sufficient to ripen fully the fruits of temperate climates, so that the whole art of training, at once the trial and triumph of skill with English fruit gardeners, is quite dispensed with: and in the place of long lines of brick wall and espalier rails, surrounding and dividing the fruit garden, all covered with carefully trained trees, we are proud to show the open orchard, and the borders in the fruit garden filled with thrifty and productive standards. Nothing surprises a British gardener more, knowing the cold of our winter, than the first sight of peaches, and other fine fruits, arriving at full perfection in the middle states, with so little care; and he sees at 
once that shree fourths of the great expense of a fruit garden here is rendered entirely needless.

Training fruit trees, in this country, is therefore confined in the colder districts north of the $4: 3 \circ$ of latitude, and to the gar. dens of amateurs. There can, however, scarcely be a more beautiful display of the art of the horticulturist, than a fine row of trained trees, their branches arranged with the utmost sym. metry and regularity, and covered, in the fruit season, with large and richly coloured fruit.

North of the $43^{\circ}$ latitude, (or north of the Mohawk, the peacn does not ripen well, and this, as well as some other rather tender tiees, will, in such situations, generally yield abundant crops when trained on a common upright trellis, or espalier rail, seven or eight feet high.* Still farther north, as in Maine, or Canada, a wall must be resorted to: but our own observation leads us to believe that, generally, the espalier rail will be found not only cheaper, and more easily managed in training, but really preferable to a wall, as full exposure to light is sufficient without much additional heat. With regard to walls themselves, in the middle portions of the Union, a southern aspect is almost always the worst, being too hot in midsummer; a wall running. north and south, and affording east and west aspects, is much the best. The western aspect is indeed preferable for all tender fruits, as the blossoms are not there liable to injury from early frosts. " A north wall is useful for producing a later crop.

The objects of training are, by a more complete exposure of the leaves and branches to the light and sun, to ripen fruits in a naturally unfavourable climate; to render them more fruitful,-lessening vigour and excessive growth by the lateral or horizontal arrangement of the branches; and lastly economy of space, as trees when trained on a flat surface occupy much less space in the fruit garden than standards, and leave the borders more open for cropping with vegetables.

Training conical slandarls. A very easy and simple mode of training fruit trees, which has lately come into great favour with amateurs, is the conical standard, or Quenouille, (pronounced kenool) of the French. It is applied chiefly to pears, wnich, when treated in this way, may be planted about eight feet apart, and thus a great variety of sorts may be grown in a small garden. The best example of this kind of training in this country, at present, is in the garden of Mr. Johnson of Lynn, Mass. A great number of the specimen trees in the London Horticultural Society's garden are trained in this manner; and Loudon re. marks, that in 1840 the Royal Kitchen garden of Versailles

- Ceclar or locust posts, set four or eiglit feet apart, with horizontal jars let in, and crossed by light perpendicular strips of pine from six to twelve inches apart, will form an excellent and durable trellis for espaliers. See Fig. 21. Indeed many garleners lere prefer having a light trellis a few inches from the wall, ugon which to iraist, instead of nailing direetly on the wall. 
on their own stocks, and kept within proper bounds by root pru ning, after Mr. Rivers" method, explained in a previous page.

The two best modes of training for this country, on walls or espalicers, are fan-training, and horizontal training. The first is the simplest aud easiest mode of training the Peach, the Apricot, Nectarine, and Cherry ; and the latter is best adapterl to the Pear. In training to a wall, the branches are fastened in their places by shreds of leather and nails; and, as espaliess, by tying them with slips of bass-matting to the rails of the trellis. The following account of these two modes of training is so concisely abridged from the practice of the best English gardens, in the Suburlsan Horticulturist, that we cannot do better than to place it before the reader.

Fan-training in the common English manner. A maiden plant (a tree but one year from the graft,) being planted "is to be

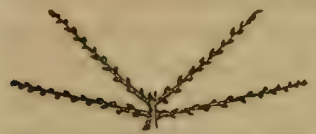

Fig. 18. Fan-training, first stage. headed down to four buds or eyes, placed in such a manner as to throw out two shoots on each side, as shown in Fig. 18. The following season the two uppermost shoots are to he headed down to three eyes, placed in such a manner as to throw out one leading shoot, and one shoot on each side; the two lowermost shoots are to be headed down to two eyes, so as to throw out one lead. ing shoot, and one shoot on the uppermost side, as shown in Fig. 19. We have now five leading shoots on each side, well placed, to form our future tree. Each of these shoots must be placed in the exact position in which it is to remain; and as it is these Fig. 19. Fan-training, second stage. shoots which are to form the future tree, none of them are to be shortened. The tree should by no means be suffered to bear any fruit this year. Each shoot must now be allowed to produce, besides the leading shoot at its extremity, two other shoots on the uppermost side, one near to the bottom and one about

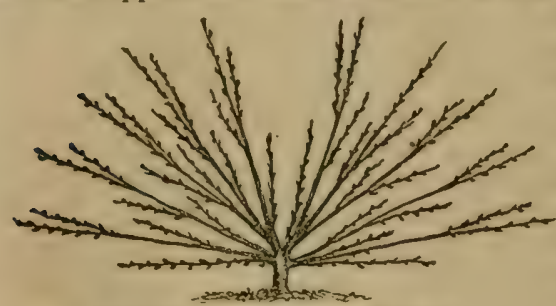

Fig. 20. Fan-training, third stage. midway up the stem; there nuust also be one shoot on the undermost side, placed about rnidway between the other two. All the other shoots must be pincked off ir their infant state. The tree will then assume, at the end of the third year, the appearance shown in Fig. 20. From this time it may be allowed to bear what crop of fruit the gar. 
dener thinks it able to carry; in determining which, he ought never to overrate the vigour of the tree. All of these shoots, exeept the leading ones, inust at the proper season be shortened. but to what length nust be left entirely to the judgment of the gardener, it of course depending upon the vigour of the tree. In shortening the shoot, care should be taken to cut back to a wond bud that will produce a shoot for the following year. Cut close to the bud, so that the wound may heal the following sea. son. The following year each shoot at the extremitics of the learling branches should produce, besides the leading shont, one on the upper and two on the under part, more or less, according to the vigour of the tree; whilst each of the sefondary branches should produce besides the leading shoot, one other placed near to the bottom; for the grand art of pruning, in all systems to which this class of trees is subjected, consists in preserving a sufficient quantity of young wood at the bnttom of the tree; and on no account must the gardener cut away clean any shoots sc placed, without well considering if they will be wanted, not only for the present but for the fuiture gosd appearance of the tree. The quantity of youing wood annualiy laid in must depend upon

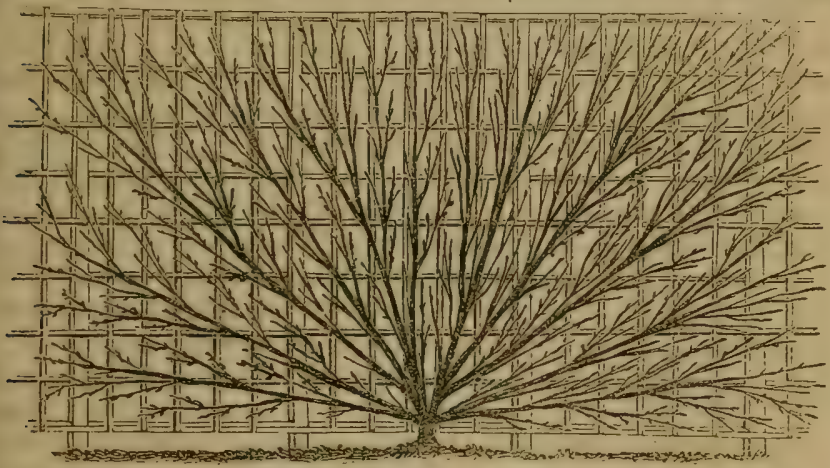

Fig. 21. Fan-training complete.

the vigour of the tree. It would be ridiculous to lay the same quantity into a weakly tree as into a tree in full vigour. The gardener here must use his own judgment. But if any of the leading shonts manifest a disposition to outstrip the others, a portion of young shoots must be laid in, and a greater quantity of fruit suffered to ripen on the over-vigorous branch. At the same time a smaller quantity of fruit than usual must be left to ripen on the weaker branch. This will tend to restore the equilitrium better than any other method. Fig. 21, presents us with the figure of a tree in a more advanced state well balanerd, and well calculated for an equal distribution of thic sap all over its surface. [We have varied this figure by representing it trained on a trellis, instead of a wall.] Whenever any of the lower shnots have advanced sefar as to incommode the others, thev 
should be cut back to a yearling shoot; this will give them room, and keep the lower part of the tree in order. In nailing to a wall, care must be taken not to bruise any part of the shoot; the wounds made by the knife heal quickly, but a bruise often proves incurable. Never let a nail gall any part of the tree; it will endanger the life of the branch. In nailing-in the young shoots, dispose them as straight and regular as possible: it will look workman-like. Whatever system of training is pursued, the leading branches should be laid-in in the exact position they are to remain; for wherever a large branch is brought down to fill the lower part of the wall, the free ascent of the sap is obstructed by the extension of the upper, and contraction of the lower parts of the branch. It is thus robbed of part of its former vigour, while it seldom fails to throw out, immediately behind the parts most bent, one or more vigorous shoots."

Horizontal training consists in preserving an upright leader, with lateral shoots trained at regular intervals. These intervals may be from a foot to eighteen inches for pears and apples, and about nine inches for cherrics and plums. "A maiden plant with three shoots having been procured, the two side shoots are laid in horizontally, and the centre one upright, as in Fig. 22; all the buds being rubbed off the latter but three, viz., one next the top for a vertical leader, and one on each side near the top, for horizontal branches. In the course of the first

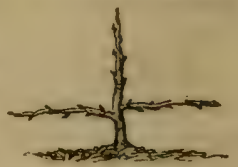
Fig. 22. Horzontal
training, first slage. summer after planting, the shoots may be allowed to grow without being stopped. In the autumn of the first year the two lat-

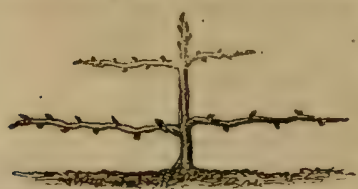

Fig. 23. Horizontal training, second stage.

stopped; which, if the plant is in proper vigour, will cause it to throw out two ho. rizontal branches, in addition to those which were thrown out 'from' those of the preceding year. The tree will now be in its second summer, and will have four horizontal branches on eacheide of the upright stcm. erals produced are nailed or tied in and also the shoots produced fror. the extremitics of the lower laterals; the centre shoot being headed down as before, as shown in Fig. 23. But in the second summer, when the main shoot has attained the length of ten or twelve inches, i: may be

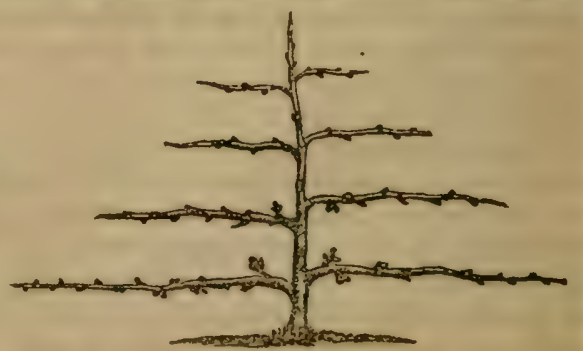

Fig. 24. Horizontal truining, third stage. 
as in Fig. 24; and by persevering in this system four horizontal branches will be produced in each year till the tree reaches the top of the wall (or espalier,) when the upright stem must terminate in two horizontal branches. In the following autumn the

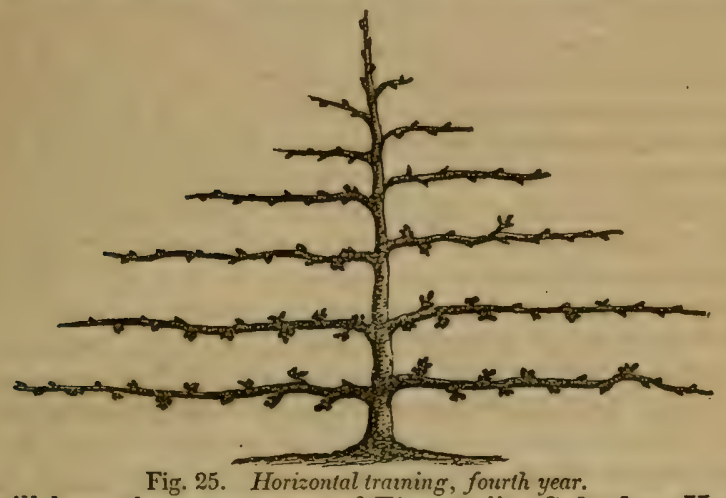

Fig. 25. Horizontal traning, fourth year.

tree will have the appearance of Fig. '25." - Suburban Horticul. turist, pp. 363 : 372.

Training fruit trees is nowhere in the United States practised to much extent except in the neighborhood of Boston; and some of the best specimens of the foregoing methods in that neighborhood are in the gardens of J. P. Cushing, Esq., Col. Perkins, and S. G. Perkins, Esq.

\section{CHAPTER V.}

\section{TRANSPLANTING.}

As nearly all fruit trees are raised first in nurseries, and then removed to their final position in the orchard or fruit garden; as upon the manner of this removal depends not only their slow or rapid growth, their feebleness or vigour afterwards, and in many cases even their life, it is evident that it is in the highest degree important, to understand and practise well this transplanting.

The season best adapted for transplanting fruit trees is a mat. ter open to much difference of opinion among horticulturists ; a difference founded mainly on experience, but without taking into account variation of climate and soils, two very importait cir. cumstances in all operations of this kind.

All phrsiologists, however, arrce that the best season for transplanting deciduous trees is in autunın, directly after the 
fall of the leaf. 'The tree is then in a completely doi mant state. Transplanted at this carly season, whatever wounds may have neen made in the roots commonce healing at once, its a deposit di. rectly takes place of granulous matter from the wound, and w'sen the spring arrives the tree is already somewhat established, aind ready to commence its growth. Autumn planting is for this reason greatly to be preferred in all mild climates, and dry soils; and even for very hardy trees as the apple, in colder latitudes; as the fixed position in the ground, which trees planted then get by the autumnal and early spring rains, gives them an advan:age, at the next season of growth, over newly moved trees.

On the other hand, in northern portions of the Union, where the winters commence early, and are severe, spring planting is greatly preferred. There, autumn and winter are not mild enough to allow this gradual process of healing and establishing the roots to go on ; for when the ground is frozen to the depth of the roots of a tree, all that slow growth and collection of nutriment by the roots is necessarily at an end. And the more tender sorts of fruit trees, the Peach and Apricot, which are less hardy when newly planted than when their roots are entire, and well fixed in the soil, are liable to injury in their branches by the inld. The proper time, in such a climate, is as early as the ground is in a fit condition in the spring.

Fiarly in autumn, and in spring before the buds expand, may as a genoral rule be considered the best seasons for transplanting. It is true that there are instances of excellent success in planting at all seasons, except midsummer; and there are nuany who, from having been once or twice successful in transplanting when trees were nearly in leaf, avow that to be the best seascn; not taking into account, that their success was probably entirely owing to a fortunately damp state of the atmosphere at the time, and abundant rains after the experiment was performed. In the niddle states, we are frequently liable to a dry period in early summer, directly following the season of removal, and if transplanting is deferred to a late period in spring, many of the trees will perish from drought, before their roots become established in the soil. Spring planting should, therefore, always be performed as soon as possible, that the roots may have the great benefit of the early and abundant rains of that season, and get well started before the heat of summer commences. For the neighborhood of NewYork, therefore, the best periods are, from the fall of the leaf, to the midlle of November, in autumn; and, from the close of 'vinter, to the middle of A pril, in the spring; though commonly, the seasons of removal are frequently extended a month beyond these limits.

Taling up the trees is an important part of the operation. A transplanter should never forget that it is by the delicate and fender points or extremities of the root that trees take up their 
lood; and that the chance of complete success is lessened, by every one of these points that is bruised or destroyed. If we could remove trees with every fibre entire, as we do a flant in a pot, they would scarcely show any sign of their char ge of position. In most cases, especially in that of trees taken from nurseries, this is, by the operation of removal, nearly impossible. But although we may not hope to get every root entire, we may, with proper care, preserve by far the larger portion of them, and more particularly the smali and delicate fibres. A fter being taken up, they should be planted directly; or, if this cannot be done, they should be liept from drying by a covering of mats, and when sent to a distance by being pasked in damp moss.*

Preparing the places. Here is the fatal stumbling block of all novices and ignorant persons in transplanting. An English gardener, when he is about to plant fruit trees, talks about preparing his borders, an American says he will dig his holes; and we cannot give a more forcible illustration of the ideas of two persons as to the wants of a fruit tree, or a better notion of the comparative provision made to supply these wants, than by contrasting the two phrases themselves. The one looks upon a tree as a living being, whose life is to be rendered long, vigorous, and fruitful by a good supply of food, and a soil mellow and easily penetrated by the smallest fibre; the other considers it very much in the light of a truncheon or a post, which he thrusts into the smallest possible hole, and supplies with the least portion of manure, trusting to what he seems to believe the inextinguishable powers of nature to make roots and branches under any circumstances. It is true that the terms differ somewhat from the nature of the culture and the greater preparation necessary in planting fruit trees in England, but this is rot by any means sufficient to justify the different modes of performing the same operation there and here.

In truth, in this country, where the sun and climate are sc favjurable, where pruning and training are comparatively so little necessary, the great requisite to success in the ordinary culture of fruit trees is the proper preparation of the soil before a tre? is planted. Whether a transplanted tree shall struggle several years to recover, or grow moderately after a short time, or at once start into a very luxuriant and vigorous growth, depends entirely upon the amount of care and labor the planter is willing to bestow on the soil for his trees. We have seen several instances where, side by side, one man planted his trees in large spaces of deeply znoved and rich soil, and another in

* We should notice an important exception to this in the case of trees packed for snipjing across the Atlantic. In this case they should be jactied only in dry moss; the molsture of the sea air being sufficient to keep the roots in good condition, whilp if facked in damp moss they will be injured by rotting or excessive growth. 
small holes in the common mode, which uniformly showed the trees of the first, larger after five years, than those of the lasi, after twelve.

No fruit tree should be planted in a hole of less size than three feet square, and eighteen inches to two feet deep. 'T'o this size and depth the soil should be removed and well pulverized, and it should if necessary be properly enrichod by the application of manure, which must be thorough mixed with the whole mass of prepared soil by repeated turnings with the spade. 'This preparation will answer, but the most skilful cultivators among us make their spaces four or five feet in diameter, or three times the size of the roots, and it is incredible how much the iuxuriance and vigour of growth, even in a poor soil, is pro moted by this. No after mending of the soil, or top dressings applied to the surface, can, in a climate of dry summers like ours, equal the effects of this carly and deep loosening and enriching he soil. Its effects on the grow'th and health of the tree are permanent, and the little expense ard carc necessary in this preparation is a source of early and constant pleasure to the planter. This preparation may be made just before the tree is planted, but, in heavy soils, it is much better to do it several months previously; and ro shallow ploughing of the soil can obviate the neccssity and advantares of the practice, where healthy, vigorous orchards or fruit gardens are desired.

The whole art of transplanting, after this, consists in placing the roots as they were before, or in the most favourable position for growth. Begin by filling the hole with the prepared soil, within as many inches of the top as will allow the tree to stand exactly as deep as it previously stood. With the spade, shape this soil for the roots in the form of a little hillock on which to place the roots-and not, as is commonly done, in the form of a hollow: the roots will then cxtend in their natural position, not being forced to turn up at the ends. Next examine the roots, and cut off all wounded parts, paring the wound smooth. Hold the tree upright on its little mound in the hole of prepared soil ; extend the roots and cover them carefully with the remaining pulverized soil. As much of the success of transplanting depends on bringing the soil in contact with every fibre, so as to leave no hollows to cause the decay of the roots, not only must this be secured by patiently filling-in all cavities among the roots, but when the trees are not quite small, it is customary to pour in a pail of water when the ronts are nearly all covered with soil. This carries the liquid mould to every hidden part. After the water has settled away, fill up the hole, pressing the earth gently about the tree with the foot, but avoiding the common practice of shaking it up and down by the stem. In windy situations it will be necessary to place a stake by the side of each tree to hold it upright, until it shall have taken f.rm root in the soil, but it is not needful in ordinary cases. 
Avoid deep planting. More than half the losses in orchard planting in Arnerica arises from this cause, and the equally common one of crowding the earth too tightly about the roots. No tree should be planted deeper than it formerly g'rew, as its roots are stifled from the want of air, or starved by the poverty of the soil at the depth where they are placed. It is much the better and more natural process in fact to plant the tree so that it shall, when the whole is complete, appear just as deep as before, but standing on a little mound two or three inches higher than the level of the ground about. This, when the mound settles, will leave it nearly on the level with the previous surface.

Mulching is an exceller.t practice with transplanted trees, and more especially for those which are removed late in the spring. Mulching is nothing more than covering the ground about the stems with coarse straw, or litter from the barn-yard, which by preventing evaporation keeps the soil from becoming dry, and maintains it in that moist and equable condition of temperature most favourable to the growth of young roots. Very many trees, in a dry season, tail at midsummer, after having made a fine start, from the parched and variable condition of the earth about the roots. Watering, frequently fails to save such trees, but mulching when they are planted will entirely obviate the necessity of watering in dry seasons, and promote growth under any circumstances. Indeed watering upon the surface, as commonly performed, is a most injurious practice, as the roots stimulated at one period of the day by water, are only rendered more susceptible to the action of the hot sun at another, and the surface of the ground becomes so hard, by repeated watering, that the beneficial access of the air is almost cut off. If trees are well watered in the holes, while transplanting is going on, they will rarely need it again, and we may say never, if they are well mulched directly after planting.

The best manure to be used in preparing the soil for transplanting trees is a compost formed of two thirds muck or black peat earth, reduced by fermenting it several months in a heap with one third fiesh barn-yard manure. Almost every farm will supply this, and it is more permanent in its effects, and less drying in its nature, than the common manure of the stable. An admirable manure, recently applied with great success, is charcoal-the small broken bits and refuse of the charcoal pits-mixed intimately with the soil. Air-slaked lime is an excellent manure for fruit trees in soils that are not naturally calcareous. Two or three handsful may be mixed with the soil when preparing each space for planting, and a top dressing may be applicd with advantage occasionally afterwards, to inerease their productivencss. But wherever large orchards or fruit gardens are to be planted, the muck compost heap should be inade ready beforchand, as it is the cheapest, most valuable, and durable of all manures for fruit trees. 
Pruting the heads of transplanted trees, at the season of re moval, we think generally an injurious practice. It is cortainly anclless and hurtful in the case of small trees, or those of such a size as will allow the roots to be taken up nearly entire; for; as the action of the branches and the roots is precisely reciproeal, and as new roots are rapidly formed just in proportion to the healthy action of the leaves, it follows that by needlessly eutting off branches we lessen the vital action of the whole tree. At the same time, where trees are transplanted of so large a size that some of the roots are lost in remoring them, it is necessary to cut back or shorten a few of the branches-as many as will restore the balance of the system-otherwise the perspiration of the leaves may be so great, as to exhaust the supply of sap faster than the roots can collect it. A little judgment only is necessary, to see at a glance, how much of the top must be pruned away before planting the tree, to equalize the loss between the branches and the roots.

When it is necessary to transplant fruit trees of large size, the best practice is to prepare them previously by digging a irench round the whole mass of roots, undermining them, and cuting off all roots projecting beyond this line. The trench should be dug at such a distance from the tree as will include all the large and sufficient ball of roots, and it should be done in the spring, or before midsummer, when it is desirable to remove the tree the next year. A fter all the ronts that extend to this circular trench are cut off, the earth is replaced, and by the season following an abundance of small fibres is sent out by the amputated roots, which, when the whole is now removed, will insure the success and speedy growth of the tree. This is more completcly the case when the tree is preparcd two years before transplanting. A variation of this mode, which has been found quite as successful and less laborious, consists in leaving the trench open, and covering it with boards only, or boards with a top layer of turf. The tree then is somewhat checlied in its growth, it throws out mn abundance of small fibres into the ball of earth containing he roots, and is the next season transplanted with rreat ease nnd safety.

The proper size for transplanting varies somewhat with the sort of tree, and the kind of culture intended. It is, however, a maxim equally well settled, both among theorists and the best practical men, that health, immediate vigour, and duration, are all greatly promoted by transplanting fruit trees of small sizefrom three to six or seven feet. We are fully aware with what impatience the beginner, or a nerson who linows little of the cul. iure of trees, looks upon trees of this size-one who is eager to plant an orchard, and stock a garden with large trees, thinking to gather a crop the next ycar. The litter may indeed be done, but the transplanting so affects the tree, that its first scanty crop 
is followed by a long sensor of rest, and fecble growth, w'ile the plantation of young trees is making wood rapidly, and soon comes into a healthy and long-continued state of productiveness-often long indeed before the large trees have fairly arrived at that condition. The small tree, transplanted with its system of roots and branches entire, suffers little or no check; the older and larger tree, losing part of its roots, requires several years to resume its former vigour. The constitution of the small tree is healthy and unimpaired; that of the large is frequently mucli enfeebled. A stout and rigorous habit-what the nurserymen call a good stocky plant-is the true criterion of merit in selecting fruit trees for transplanting.

Trees intended for orchards, being often more exposed than those in gardens, should be somewhat larger-not less than six, or more than eight feet is the best size. For gardens, all expe. rienced cultivators agree that a smaller size is preferable; we prefer plants two years old from the graft. Most gardeners abroad, when they select trees with more than usual care, take what are called maiden plants-those one year old from the graft, and there can be no doubt that, taking into account health, Juration, and the ease with which such a tree can be made to grow into any form, this is truly the preferable size for removal into a fruit garden. But we are an impatient people, and it is not till after another century of trial and experience in the culture of fruit trees, that cultivatoris generally in this country will become aware of the truth of this fact.

The facility with which the different fruit trees may be trans. planted differs considerably. Plums are generally removed with most success and after them nearly in the order as foliows: Quinces, Apples, Pears, Peaches, Nectarines, A pricots, and Cherries; the latter succeeding with some difficulty when of large size.

Laying-in by the hects is a practice adopted as a temporary kind of planting, when a larger quantity of trees is at hand than can be set out immediately. A trench is opened, and the roots are laid in and covered with soil, the tops being previously placed in a sloping position, inclining to within a few feet of the surface. In this way they are kept fresh and in goud order, until it is couvenient to plant them finally. In northern districts, where the auturnn is often too severe for planting, and the spring is frequently too late to receive trees in time from nurseries farther south, it is a eommon and successful mode to procure trees in autumn and lay them in by the heels until spring, covering over the tops of the more tender sorts if necessary with coarse littcr.

In planting an orchard, always avoid placing the trees in the same spot or near where an old tree stood before. Experience has taught us that the growth of a young tree, in such a posi. tion, is weak and.feeble; the nourishment suitable to that kind 
of tree having already been exhausted by a previous growtin, and the soil being half filled with old and decayec ronts which are detrimental to the health of the youns tree.

\section{CHAPTER VI.}

\section{THE POSITION OF ERUIT TREES. SOIL AND ASPECT.}

IN our favourable climate many fruit trees will thrive and produce some fruit in almost any soil, except dry sand, or wet swamps. But there is much to be gained in all climates by a judicious selection of soil, when this is in our power, or by that improvement which may generally be effected in inferiour soils where we are necessarily limited to such. As we shall, in treating the culture of each genus of fruit, state more in detail the soils especially adapted to its growth, our remarks here will bs confined to the subjec: of soils generally, for the orchard and fruit garden.

The soils usually selected for making plantations of fruit trees may be divided into light sandy loams, gravelly loams, strong loams, and clayey loams; the first haviny a large proportion of sand, and the last a large proportion of clay.

The soil most inviting to the eye is a light sandy loam, and, as it is also a very common soil, more than half the fruit gardens in the country are composed of this mould. The easy manner in which it is worked, owing to its loose and very friable nature, and the rapidity with which, from its warmth, crops of all kinds come into bearing, cause it to be looked upon with almost universal favour. Notwithstanding this, a pretty careful obserwa. tion, for several years, has convinced us that a light sandy soil is, on the whole, the worst soil for fruit trees. Under the bright skies of July and August, a fruit tree requires a soil which will retain and afford a moderate and continued supply of moisture, and here the sandy soil fails. In consequence of this the vigour of the tree is checked, and it becomes feeble in its growth, and is comparatively short-lived, or unproductive. As a tree in a feeble state is always most liable to the attacks of insects, those on a sandy soil are the first to fall a prey to numerous maladies.* The open loose texture of a sandy soil, joined to its warmth, affords an easy passage, and an excellent habitation for all in. sects that pass part of their lives in the ground, preparatory to

* This remark applies to the middle and southern portions of this country. North $f$ the $43^{\circ}$ a light sandy soil is perhaps preferable as warmerand earlier. 
rising out of it to attack the fruit, foliage, or branches of the tree.

Such are some of the disarlvantages of a light sandy soil ; and, in thoroughly examining many of the fruit gardens of the midule states the last fow scasons, we could not fail to be struck with the fact that in niue cases out of ten, where a variety of frunt was unusually liable to discase, to blight, or to the attacks of eytain fruit-destroying insects, as the curculio, the trees themsel res were on sandy soils; while on the other hand, and fre. quently in the same neighbourhood, the same sorts were growing luxursantly and bearing abundant crops, where the soil was a rather sirung loam.* For a few years, the growth and produc. tiveness or the trees upon sandy soil, is all that can be desired; but the trees are shorter lived and sooner fall into decay than where ine soil is stronger. If there is any exception to this rule, it is only in the case of the Peach, and judging from the superiour flavour of this fruit on stronger soils, we are inclined to doub the value of the exception even here.

Gravelly lonms are frequently much better adapted for orchards than sandy, especially where the loam is of a strong quality, and the gravel is not in cxcess; and the hardier fruits usually do well on this kind of soil.

Sirong loams, by which we mean a loam with only just a sufficient portion of sand to make it easily worked, are on the whole by far the best for fruit gardens in this country. A strong loam is usually a deep soil, and aflords during the whole heat of summer, a proper supply of moisture and nourishment to the roots of trees. Fruit trees do not come into a bearing state so soon in a strong as in a sandy loam, because the growth of wood is more vigorous, and fruit buds are not so soon formed; but they bear larger crops, are much less liable to many diseases, and their longevity is much greater. The largest and most productive orchards of the apple and pear in this country are upon soils of this kind.

Clryey loams are, when well drained, and when the clay is rot in excess, good fruit soils-they are usually strong and deep soils though rather heavy and difficult to work. T'rees that will fourish on these soils such as the Apple, Pear, Cherry, Plum, and Apricot, usually are very free from disease, or insects, and bear large crops. In a moist climate, like that of England, fruit trees on a clayey loam would die of canker, brought on by the excessive quantity of water contained in the soil, but such is

* As an instance in point, the owner of one of the most highly cultivated gar dens in the vicinity of Bustort was showing is, in despair, some trees of the Seckel pear upon which he could no longer get gool crops, or fair fruit, and lamenting the degenteracy of the sort. The next day we saw in a neighbouring garden beautiful crops of this pear growing with the least possible care. The garden in the first case was a light sandy loam; in the second, a strong loam. 
not the case under the high and warm temperature of our sum. mers. The finest, largest. and most productive Plums and Pears within our knowledge, grow in sites on the North river, when the soil is a stif? clayey loam, almost approaching a clay. Those fruits that un light sandy soils are almost worthless from their liability to disease, and the attacks of insects, are here surprisingly luxuriant and fruitful.

It is, however, well to remark, that some varieties of fruit, perhaps from the circumstances of their origin, succeed better on sandy soils than any other; thus the Newtown pippin will only arrive at perfection in a strong loam, while the Yellow Bellflower is finer when grown on a sandy soil. But these are exceptions to all rules, and what we have already stated, as to the relative quality of soils, will apply pretty generally to the whole of this country south of the Mohawk river; and it may be added that calcareous soils, of whatever texture, are better than soils of the same quality where no limestone is present.

Trenching is the most complete method of improving a soil too sandy, when the subsoil below is of a loamy or clayey nature. Deep subsoil ploughing, by bringing up a sufficient quantity of the stratum below, will answer the same purpose. When the subsoil of a sandy soil is sand or gravel, the surface can only be improved by top dressings, or the application of manures. Top-dressing with clay is the most simple means of changing the nature of such a soil, and it is surprising how moderate a quan. tity of clay will give a closer texture to light sandy soils. In manuring such soils, we may greatly improve their nature as well as condition, by using composts of peat or bog earth, swamp muck, or river mud, instead of common barn-yard or stable manure. The former are not only more permanent and hetter as manures for fruit trees, but they gradually consolidate and improve the whole texture of the soil.

Indeed no fruit garden, where the soil is not naturally deep and rich, is in perfect condition for planting trees, unless the soil has been well trenched two spades in depth. This creates a matrix for the roots, so deep and permanent, that they retain their vigour and luxuriance through the droughts of summer, and continue for a long time in a state of health and produc. tiveness.

It is difficult to give any precise rules as to aspect. We have seen fine fruit gardens here in all aspects. Perhaps the very best aspect, on the whole, is a gentle slope to the southwest, because in such positions the trees, when in blossom, are somewhat protected from the bad effects of a morning sun after spring frosts. But, to remedy this more perfectly, it is sometimes the practice to plant on the north sides of hills, and this is an effec. tual way where early frosts are fatal, and where the season is long and warm enough to ripen the fruit in any exposure. 
aue south slope, is, south of New-York, irequently found toe warm for many fruit trees, in soils that are light and dry.

Deep vallies, with small streans of water, are the worst situ. ntions for fruit trees, as the cold air settles down in thrse vallies in a calm frosty night, and buds and blossoms a re very frequently destroyed. We know a rich and fertile valley of this kind in Connecticut where the Cherry will scarcely grow, and a crop of the Apple, or the Pear, is not obtained once in ten years; while the adjacent hill tops and high country, a couple or three niles distant, yield abundant crops amnually. On the other hand the borders of large rivers, as the IIurlson, or of some of our large inland lakes, are the most farourable situations for fruit trees, as the climate is rendered milder by large borlies of water. In the garden where we write, a fourth of a mile from the Hudson, we have frequently seen ice formed during the night, of the thickness of a dellar, when the blossoms of the $\Lambda$ pricot were fully expanded, without doing the least harm to that tender fruit. This is owing to the slight fog rising from the river in the morning, which, softening the rays of the sun, and dissolving gradually the frost, prevents the injurious effects of sudden thawing. $\overline{\Lambda t}$ the same time, a couple of miles from the shores, this fruit will often be quite destroyed. In short, the season on the lower half of the Hudson, may, from the ameliorating influcnee of the river, be said to be a month longer-a fortnight earlier in spring, and later in autumn, than in the saine latitude a fow miles distant; and crops of the more tender fruits are, therefore, much more certain on the banks of large riverš or lakes, than in inland districts of the same climate.

\section{CHAPTER VII.}

\section{GENERAL, REMARKS ON INSECTS.}

'Tн insects injurious to fruit trees are numerous, and to combat them successfully requires a minute acquaintance with their character and habits. While considering the culture of each class of fruit in the succecling pages, we shall point out the habits, and suggest means of destroying the most important of these insects; but, in the meantime, we wish to call attention to some general practical hints on this sulject.

In the first place, we cannot too strongly impress upon the at. tention of the fruit grower the importance of watching carefully. and making an early attack, upon every species of insect. Ii is only necessary to look for a moment at the astonishing :apid. 
ity with which many kinds of insects increase, if allowed to get well established in a garden, to become fully aware of this. The cornmon caterpillars are the young of moths or butterflies, and that careful observer of the habits of insects, Dr. IIarris, says as each femalc lays from two to five hundred ecres, a thou. sand moths or butterflies will, on the average, produce three hundred thousand caterpillars; if one half this number, whe:n arruved at maturity, are females, they will give forty-five millions of caterpillars in the second, and six thousand seven hundred and fifty millions in the third generation.* To take another example the aphides, or plant lice, which are frequently seen in great numbers on the tender shoots of fruit trees have an almost incredibly prolific power of increase,-the investigations of Réaumur having shown that one individual, in five generations, may become the progenitor of nearly six thousand millions of descendants. With such surprising powers of propagation, were it not for the havoc caused among insects by various species preying upon each other, by birds, and other animals, and espepecially by unfavourable seasons, vegetation would soon be entirely destroyed by them. As it is, the orchards and gardens of carcless and slovenly cultivators are often overrun by them, and many of the finest crops suffer great injury, or total loss from the want of a little timely care.

In all well managed plantations of fruit, at the first appearance of any injurious insect, it will be immediately scized upon and destroyed. A few moments, in the first stage of insect lifeat the first birth of the nev colony - will do more to rid us for the season, of that species, than whole days of toil after the mat. ter has been so long neglected that the cnemy has become well established. We linow how reluctant all, but the experienced grower, are to set about cradicating what at first seems a thing of such trifling consequence. But such persons should consider that whether it is done at first, or a fortnight after, is frequently the difference between ten and ten thousand. A very little time; regularly devoted to the extirpation of noxious insects, will kecp a large place quite free from them. We know a very large garden, filled with trees, and always remarkably free from insect ravages, which, while those even in its ricinity suffer weatly, is thus preserved, by half an hour's examination of the whole premises two days in the week during the growing season. This is made early in the morning, the best time for the I urpose, as the insects are quict while the dew is yet upon the leaves, and whole races, yet only partially leveloped, may be swept off in a single moment. In lefault of other more rapid expedients, the old mode of hanrl-picling, and crushing or burring, is the safest and surest that can be adopted

* For much valuable information on the habits of inserts injurious to vegetation, see the Treatise on the. Insects of Massachusetts, by Dr. T. W. IIarris, Cambridge 
For practical purposes, the numerous insects $\mathrm{infesting}$ fruit trees may be divided into four classes; 1 st, those which for a time harbour in the ground and may be attacked in the soil ; $2 \mathrm{~d}$, winged and other species, which may be attacked among the branches; $3 \mathrm{~d}$, aphides, or plant lice which infest the young shoots ; 4th, moths, and all night-flying insects.

Insects, the larve or grubs of which harbour in the ground during a certain season, as the curculio or plum-weevil, are all more or less affected by the application of common salt as a top dressing. On a larger scale-in farm crops--the ravages of the cut-worm are frequently prevented by sowing three bushels of salt to the acre, and we have seen it applied to all kinds of fruit grounds with equal success. Salt seems to be strongly disagreeable to nearly all this class of inseets, and the grubs perish, where even a small quantity has for two ar three scasons becn applied to the soil. In a neiglibourhood where the peach worm usually destroys half the peach trees, and where whole crops of the plum are equally a victim to the plum-weevil, we have sten the former preselved in the healthiest condition by an annual application of a small handful of coarse salt about the collar of the tree at the surface of the ground; and the latter, made to hold abundant crops, by a top dressing applied every spring of packing salt, at the rate of a quart to the surface occupied by the roots of every full grown tree.

Salt, being a powerfu! agent, must be applief for this purpose with caution and judgment. In small quantities it promotes the verdure and luxuriance of fruit trees, while if applied very frequently, or too plentifully, it will certainly cause the death of any trec. "Two or three years top-dressing in moderate quantity will usually be found sufficicnt to drive away these insects, and then the application need only be repeated once in two or three seasons. Any coarse, refuse salt will answer the pur. pose; and packing sait is preferable to that of finer quality, as it dissolves slowly by the action of the atmosphere.

In the winged state, most small insects may either be driven away by powerful odours, or killed by strong decoctions of to bacco, or a wash of diluted whale-oil or other strong soap. Attention has but recently been called to the repugnance of all insects, to strong odours, and there is but little doubt that before a long time, it will lead to the discovery of the means of preventing the attacks of most inscets by means of strong smellung liquids or odourous substances. The moths that attack lurs, as every one knows, are driven away by pepper-corns or tobacco, and should future experiments prove that at certain seasons, when our trees are most likely to be attacked by insects, we may expel them by hanging bottles or rags filled with strong smelling liquids in our trees, it will certainly be a very simple and casy way of ridding ourselves of' them. "The brown scale, a trouble. 
soine enemy of the orange tree, it is stated in the Gardener's Chronicle have been destroyed by hanging plants of the common chamomile anong its branches. The odour of the coal tar of gass works is exceedingly ofiensive to some insects injurious to Iruits, and it has been found to drive away the wire worm, and other grubs that attack the roots of plants. The vapour of oil of turpentine is fatal to wasps, and that of tobacco smoke to the green fly. Little as yet is certainly known respecting the exact power of the various smells in deterring insects from at tacking trees. What we do know, however, gives us reason to believe that much may be hoped from experiments made with a variety of powerful smelling substances.

Tobacco water, and diluted whale oil soap, are the two most ellicient remedies for all the small insects which feed upon the young shoots and leaves of plants. Tobacco water is made by boimg tobacco leaves, or the refuse stems and stallss of the to bacco shops. A large pot is crowded full of them, and then filled up with water, which is boiled till a strong desoction is made. This is applied to the young shoots and leaves with a syringe, or, when the trees are growing in nursery rows, with a common white-wash brush; dipping the latter in the liquid and shaking it sharply over extremities or the infested part of each tree. This, or the whale oil soap-suds, or a mixture of both, will lill every species of plant lice, and nearly all other small insects to which young fruit trees are subject.

The wash of whale oil soap is made by mixing two pounds of this soap, which is one of the cheapest and strongest kinds, with fifteen graliuns of water. This mixture is applied to the leaves and stems of plants with a syringe, or in any other convenient mode, and there are few of the smaller insects that are not de. sroyed or driven away by it. The merit of this mixture belongs to Mr. David Haggerston, of Boston, who first applied it with great success to the rose slug, and received the premium of the Massachusetts Horticultural Society for its discovery. When this soap cannot be obtained, a good substitute may be made by turning into soap the lees of common oil casks, by the application of potash and water in the usual way:

Moths and other insects which fly at night are destroyed in large numbers by the following mode, first discovered by Victor Adouin, of France. A flat saucer or vessel is set on the ground in which is placed a light, partially covered with a common bell glass besmeared with oil. All the small moths are directly attracted by the light, fly towards it, and, in their attempts to get at the light, are either eaught by the glutinous sides of the beli glass, or fall into the basin of oil beneath, and in either case soon perish. M. Adlouin applied this to the destruction of the pyralis, a moth that is very troublesome in the French vineyards; with two hundred of these lights in a vineyard of fous 
acres, and in a single night, 30,000 moths were killed and found dead on or about the vessels. By continuing his process through the season, it was estimated that he had destroyed female moths sufficient to have produced a progeny of over a million of caterpillars. In our orchards, myriads of insects may be destroyed by lighting small bonfires of shavings, or any refuse brush ; and in districts where the apples are much worm-eaten, if repeated two or three nights at the proper season, this is a very efficient and cheap mode of getting rid of the moth which causes so much mischief. Dr. Harris, knowing how important it is to destroy the caterpillar in the moth state, has recommended flambeaux, made of tow wound round a stake and dipped in tar, to be stuck in the fruit garden at night and lighted. Thousands of moths will find a speedy death, even in the short time which these flambeaux are burning. The melon-bug may be extirpated by myriads, in the same way.

A simple and most effectual mode of ridding the fruit garden of insects of every description, which we recommend as a general extirpator, suited to all situations, is the following. Take a number of common bottles, the wider mouthed the better, and fill them about half full of a mixture of water, molasses, and vinegar. Suspend these among the branches of trees, and in various parts of the garden. In a fortnight they will be found full of dead insects, of every description not too large to enter the bottles-wasps, flies, beetles, slugs, grubs, and a great variety of others. The bottles must now be emptied, and the liquid renewed. A zealous amateur of our acquaintance, caught last season in this way, more than three busheis of insects of various kinds; and what is more satisfactory, preserved his garden almost entirely against their attacks in any shape.

The assistance of hirds in destroying insects should be duly estimated by the fruit-grower. The quantity of cggs and insects in various states, devoured annually by birds, when they are encouraged in gardens, is truly surprising. It is true that one or two species of these, as the ring-tail, annoy us by preying upon the earlier cherries, but even taking this into account, we are inclined to brlieve that we can much better spare a reasonable share of a fow fruits, than dispense with the good services of birds in riduling us of an excess of insects.

The most serviceable birds are the common sparrows, the wren, the red-breast, and, in short, most of the birds of this class. All these birds should be encouraged to build nests and inhabit the fruit garden, and this may most effectually be done by not allowing a gun to be fired within its boundaries. The introduction of hedges or live fences, greatly promotes the domestication of birds, as they afford an admirable shelter for their nests. Our own gardens are usually much more free from insects than those a mile or two distant, and we attribute this in part to our practira 
of encouraging birds, and to the thorn and arthr vitæ heages growing here, and which are greatly resorted to by those of the feathered tribe which are the greatest enemies of the insect race.

Among animals, the toad and the bat are great insect destroy. ers. The common bat lives almost entirely upon them, and in its evening sallies devours a great number of moths, beetles, veevils, etc. ; and the toad quietly makes way with numberless smaller insects.

\section{CHAPTER VIII.}

\section{THE APPLE.}

Pyrus Ma ius. L. Rosarece, of botanists.

Pommer, of the French; Apfelbaum, Germin; Apfel, Dutch; Melo pomo, Italian, and Manzana, Spanish.

TuE Apple is the world-renowned fruit of temperate climates. From the most remote periods it has been the subject of praise among writers and poets, and the old mythologies all endow its fruit with wonderful virtues. The allegrorical tree of knowledge bore apples, and the celebrated golden fruit of the orchards of Hesperus, guarded by the sleepless dragon which it was one of the triumphs of Hercules to slay, were also apples, according to the old legends. Among the heathen gods of the north, there were apples fabled to possess the power of conferring immortality, which were carefully watched over by the goddess lduna, and kept for the especial dessert of the gods whe felt themselves growing old! As the mistletoe grew chiefly on the apple and the oak, the former tree was looked upon with great respect and reverence by the ancient Druids of Britain, and even to this day, in some parts of England, the antique cus. tom of saluting the apple trees in the orchards, in the hope of obtaining a good crop the next year, still lingers among the farmers of portions of Devonshire and Herefordshire. "This odd ceremony consists of saluting the tree with a portion of the contents of a wassail bowl of cider, with a toast in it, by pouring a little of the cider about the roots, and even hanging a bit of the loast on the branches of the most barren, the farmer and his men dancing in a circle round the tree, and singing rude sougs like the following:

"Here's to thee, old apple :ree,

Whence thou mayst bud, and whence thou mayst bir w;

And whence thou mayst bear apples enow,

lfats full! eaps full-

Bushels and sacksfull!

Huvra? !" 
The species of crab from which all our sorts of Apple:s have originated, is wild in most parts of Europe. There are indeed two or three kinds of wild crab belonging to this country; as the Pyrus coronaria, or sweet scented crab, with fruit about an inch in diameter grows in many parts of the United States; and the wild crab of Oregon, $P$. rivularis, bearing a reddish yellow fruit about the size of a cherry, which the Chenook Indians use as an article of food; yet none of our cultivated varieties of apple have ween raised from these native crabs, but from seeds of the s? ines brought here by the colonists from Europe.

The Apple tree is, however, most perfectly naturalized in America, and in the northern and middle portions of the United States succeeds as well, or, as we believe, better than in any part of the world. The most celebrated apples of Germany and the north of Europe, are not superiour to many of the varieties originated here, and the American or Newtown Pippin is now pretty generally admitted to be the finest apple in the world. No better proof of the perfect adaptation of our soil and climate to this tree can be desired, than the seemingly spontaneous production of such varieties as this, the Baldwin, the Spitzenburg or the Swaar-all fruits of delicious flavour and great beauty of appearance.

The Apple is usually a very hardy and rather slow growing fruit tree, with a low spreading, rather irregular head, and bears an abundance of white blossoms tinged with red. In a wild state it is very long-lived, but the finest garden sorts usually live about fifty or eighty years; though by proper care, they may be kept healthy and productive much longer. Although the apple generally forms a tree of medium growth, there are many specimens in this country of enormous size. Among others we recollect two in the grounds of Mr. Hall of Raynham, Rhode Island, which, ten years ago, were 130 years old; the trunk of one of these trees then measured, at one foot f $f_{1}$ om the ground, thirteen feet two inches, and the other twelve feet two inches. The trees bore that season about thirty or forty bushels, but in the year 1780 they together bore one hundred and one bushels of apples. In Duxbury, Plymouth county, Mass., is a tree which in its girth measures twelve feet five inches, and which has yielded in a single season $121 \frac{1}{2}$ bushels.

UsEs of THE APPLE. No fruit is more universally liked or generally used than the apple. It is exceedingly wholesome, and, medicinally, is considered cooling, and laxative, and useful in all inflammatory discases. The finest sorts are much es. teemed for the dessert, and the little care required in its culture, renders it the most abundant of all fruits in temperate climates. As the earliest sorts ripen about the last of June, and the latest can be preserved until that season, it may be considered as a fruit in perfection the whole year. Besides its merits for the 
Jessert, the value of the apple is still greater for the kitchen. and in sauces, pies, tarts, preserves, and jellies, and roasted and joiled, this rruit is the constant and invaluable resource of the zitehen. Apple butler, made by stewing pared and sliced swee! apples in new cider until the whole is soft and pulpy, is a conlnon and excellent article of food in many farmers' families, and is frequently made by the barrel, in Connecticut. In Franee, nearly the same preparation is formed by simmering apples in $n$ ''w wine, until the whole becomes a sort of marmalade, which is called Raisine. 'The juice of the apple unfermented, is, in soine parts of the country, boiled down till it becomes molasses. When fermented it formis cider, and if this is carefully made from the best cider apples, it is nearly equal to wine; in fact many hundreds of barrels, of the cider of New-Jersey, have been manufactured in a single year, into an imitation Cham. pagne, which is scarcely distinguished by many from that made from the grape.

Dried apples are also a considerable article of commerce. Farmers usually pare and quarter then by hand, and dry them, in the sun; but those who pursue it as a matter of trade pare them by machinery, and dry them slowly in ovens. They are then packed in bags or barrels, and are used either at home, in sea stores, or are exported.

In perfumery, the pulp of this fruit, mixed intimately with lard, forms pomatum. The wood is cmployed for lasts, and for other purposes by turners; and being fine grained and compact is sometimes stained black, and used for ebony, by cabinet makers.

The quality of an apple is always judged of by the use to which it is to be applied. A table or desscrt apple of the finest quality should be of medium size, regular form and fine colour; and the flesh should be fine-grained, crisp, or tender, and of a sprightly or rich flavour, and aroma. Very large sized, or conrse apples are only adinired by persons who have little knowledge of the true critcrion of cxcellenec. Apples for kitchen use should have the property of cooking evenly into a tender pulpy consistence, and are grencrally acil in flavour; and, althouch there are many good cooking apples unfit for the table, many sorts, as the Fall Pippin and the Greening, are excellent for both purposes. To this we may add that for the common applesauce made by farmers a high flaroured sweet apple, which boils somewhat firm, is preferred, as this is generally made with cider. The very common use made of this cheap preserve at the north and west, and the recent practice of fattening hogs, horses, and other an mals upon sweet aply]es, accounts for the much greater number of varieties of swect apples hel 1 in estecm here than in any other country. In fact, so excellent has the saccharine matter of the apple been found for this purpose, that whole orchards 
of sweet applesare frequently planterl here for the purposes of fatsening swine and cattle, which are allowed to run at large in them.

Cider apples are varieties frequently useless for any other purpose. 'The best for this purpose are rather tough, piquant, and astringent ; their juice has a high specific quality, and they are usun'ly great bearers; as the Harrison, the Red Streak, and the Virginia Crab.

Propagation. The apple for propagation is usually raised from seeds obtained from the pomace of the cider mills, and a preference is always given to that from thrifty young orchards. These are sown in autumn, in broad drills, in good mellow soil, and they remain in the seed buds, attention being paid to keeping the soil loose and free from weeds, from one to three years, according to the richness of the soil. When the seedlings are a little more than a fourth of an inch in diameter, they should be taken up, in the spring or antumn, their tap roots shortened, and then planted in nursery rows, one foot apart and three to four feet between the rows. If the plants are thrifty, and the soil good, they may be budded the following autumn, within three or four inches of the ground, and this is the most speedy mode of obtaining strong, straight, thrifty plants. Grafting is generally performed when the stocks are about half an inch thick; and for several modes of performing it on the apple, see the remarks on grafting in a previous page. When young trees are feeble in the nursery, it is usual to head them back two thirds the length of the graft, when they are three or four feet high, to make them throw up a strong vigorous shoot.

Apple stocks for dwarfs are raised by layers, as pointed out in the article on Layers.

Apple trees for transplanting to orchards should be at least two years budded, and six or seven feet high, and they should have a proper balance of head or side branches.

SoIL and situation. The apple will grow on a great variety of soils, but it seldom thrives on very dry sands, or soils saturated with moisture. Its favourite soil, in all countries, is a strong loam of a calcareous or limestone nature. A deep, strong gravelly, marly, or clayey loam, or a strong sandy loam on a gravelly subsoil, produces the greatest crops, and the highest flavoured fruit, as well as the utmost longevity of the trees. Such a soil is moist rather than dry, the most favourable condition for this fruit. Too damp soils may often be rendered fit for the apple by thorough draining, and too dry ones by deep subsoil ploughing, or trenching, where the subsoil is of a heavier texture. And many apple orchards in New-England are very flourishing and productive on soils so stony and rock-covered 'though naturally fertile) as to be unfit for any other crop.*

- Blowing Eands, says Mr. Coxe, whien bottomed on a dry subetratum, ard aided 
As regards site, apple orcnards flourish best, in southern and iniddle portions of the country, on north slopes, and often even on the stcep north sides of hills, where the climate is hot and dry. Farther north a southern or southeastern aspect is preferable, to ripen the crop and the wood more perfectly.

We may here remark that almost every district of the country has one or more varieties which, having had its origin there, secms also peculiarly adapted to the sorl and climate of that Lucality. Thus the Newtown pippin, and the Spitzenburgh are the great apples of New-York; the Baldwin, and the Roxbury Rusisett, of Massachusetts; the Bellflower and the Rambo, of l'cnnsylvania and New-Jersey; and the Peck's Pleasant and the Scck-no-further, of Connecticut; and though these apples are cultivated with greater or less success in other parts of the country, yet nowhere is their flavour and productiveness so perfect as in the best soils of their native districts-excepting in such other districts where a soil containing the same elements, and a corresponding climate are also to be found.

Planting and cultivation of orchards. With the exception of a few early and very choice sorts in the fruit garden, the orchard is the place for this tree, and indeed, when we consider the rreat value and usefulness of apples to the farmer, it is easy to sce that no farm is complete wilhout a large and well selceted apple orchard.

The distance at which the trees should be planted in an orcliard, depends upon the mode in which they are to be treatcd. When it is desired finally to cover and devote the whole ground to the tress, thirty feet apart is the proper interval, but where the armer wishes to keep the land between the trees in gram and grass, fifty feet is not too great a distance in strong soils. Forty fect apart, however, is the usual distance at which the trees are planted in orchards.

Before transplanting, the ground should be well prepared for the trees, as we have insisted in a previous page, and vigo. rous healthy young trees should be selected from the nurseries. As there is a great difference in the natural growth, shape, and size of the various sorts of apple trees, those of the same kunds should be planted in the rows together, or near each other; this

by marl or meadow mud, will be found capable of producing very fine apple trees. fired cultivation, and a system of hich manuring. will always remmetate the pro prietur of an orcliard, except it be planted on a ynickennd or a colil clay; in sucen soils, ro management can prevent an early decay. One of the most thrifty orchards I possess, was planted on a hlowing sand, on which I carted three thousand loarls of mud on ten acres, at an expense of ahout iwenty-five dollars per arre, exclusive of much other mariure; on this lamd I have raised good wheai and clover. Of five rows of the Winceap apple planted ujon it eight years ago, on the summi of a sandy linoll, not one has dited out of near an hundred irces-all abundant bew ers of large and fair apjles.-View of Fruit T'rees, p. 31. 
will not only facilitate culture and gathering the fruit, but will add to the neatness and orderly appearance of the orchard.

It is an indispensable requisite, in all young orchards, to keen the ground mellow and loose by cullivation; at least for the first few years, until the trees are well established. Indeed, of twa adjoining orchards, one planted and kept in grass, and the other ploughed for the first five years, there will be an incredibie difference in favour of the latter. Not only will these trees show rich dark luxuriant foliage, and clean smooth stems, while those neglected will have a starved and sickiy look, but the size of the trees in the cultivated orchard will be treble that of the others at the end of this time, and a tree in one will be ready to bear an abundant crop, before the other has commenced yielding a peck of good fruit. Fallow crops are the best for orchards, - potatoes, vines, buckwheat, roots, Indian corn, and the like. An occasional crop of grass or grain may be taken; but clover is rather too coarse-rooted and exhausting for a young orchard. When this, or grass, is necessarily grown among young trees for a year or two, a circle of three feet diameter should be kept loose by digging every season about the stem of each tree.

When the least symptom of failure or decay in a bearing orchard is perceived, the ground should have a good top dressing of manure, and of marl, or mild lime, in alternate years. It is folly to suppose that so strong growing a tree as the apple, when planted thickly in an orchard, will not, after a few heavy crops of fruit, exhaust the soil of much of its proper food. If we desire our trees to continue in a healthy bearing state, we should, therefore, manure them as regularly as any other crop, and they will amply repay the expense. There is scarcely a farm where the waste of barn-yard manure,-the urine, etc., if properly economized by mixing this animal excrement with the muck. heap-would not be amply sufficient to keep the orchards in the highest condition. And how many moss-covered, barren orchards, formerly very productive, do we not every day see, which oniy require a plentiful new supply of food in a substantial topdressing, thorough scraping of the stems, and washing with diluted soft soap, to bring them again into the finest state of vigour and productiveness!

The bearing year of the Apple, in common culture, only takes place every alternate ycar, owing to the excessive crops which t usually produces, by which they exhaust most of the organizable matter laid up by the tree, which then requires another scason to rccover, and collcet a sufficient supply again to 'orm fruit buds. When ha.f the fruit is thinned out in a young state, leaving only a moderate crop, the apple, like other fruit trecs, will bear every year, as it will also, if the soil is kept in high condition. The hcaring ycar of an apple tree, or a whole orshard may be changed hy picking off the fruit when the trees 
first show guod crops, allowing it to remain only on the alter. nate seasons which we wish to make the bearing year.*

Prunixg. 'The apple in orchards requires very little pruning if the trees, while the orchard is young, are carefully inspected every year, a little before milsummer, and all crossing branches takien out while they are small. When the heads are onee properly adjusted and well balaneed, the less the pruning saw and knife are used the better, and the cutting out of dead limbs, and removal of such as may interfere with others, or too greatly crowd up the head of the tree, is all that an orchard will usually require. But wherever a limb is prumed away, the surface of the wound should be neatly smoothed, and if it exceeds an inch in diamcter, it should be covered with the liquid shellac previously noticed, or brushed over with common white lead, taking care with the latter, not to paint the bark also.

InsEcTs. There are three or four insects that in some parts of the country, are very destructive or injurious to this tree; a knowledge of the habits of which, is therefore, very important to

* One of the finest orchards in America is that of Pelham, farm, at Esopus, on the Hudson. It is no less remarkable for the beauty and high flavour of its fruit, than the constant productiveness of trees. The proprietor, R. L. Pell, Esq., has kindty furnished us with some notes of his experiments on fruit trees, and we sub. join the forlowing highly interesting one on the Apple.

"For several years past I have been experimenting on the apple, having an orchard of 2,000 bearing Newtown Pippin trees. I found it very unprofitable to wait for what is termed the 'bearing year,' and it has been my aim to assist nature, so as to enable the trees to bear every year. I have noticed that from the cxcessive productiveness of this tree, it requires the intermediate year to recover itself-to extract from the earth and the atmosphere the materials to enable it to produce again. This it is not able to do, unassisted by art, while it is loaded with fruit, and the intervening year is lost; if, however, the tree is supplied with proper food it will bear every year; at least such has been the result of my experiments. Three years ago, in April, I scraped all the rough bark from the stems of several thousand trees in my orchards, and washed all the trunks and limbs within reach with soft soap; trimmed out all the branches that crossed each other, early in June, and painted the wounded part with white lead, to exclude moisture and prevent decay. I then, in the latter part of the same month, slit the bark by running a sharp pointed knife from the ground to the first set of limbs, which prevents the rree from becoming bark bound, and gives the young wood an opportunity of expanding. In July 1 placed one peck of oyster shell lime under each tree, and left it piled about the trunk until November, during which time the drought was excessive. In November the lime was dug in thoroughty. The following year I collected from these trees 1700 barrels of fruit, part of which was sold in New-I urk for four, and others in London for nine dollars per barrel. The cider made from the refuse, delivered at the mill two days after its manufacture, I sold for three dollars and three quarters per harrel of 32 gallons, exclusive of the barrel. In October I manured these trees with stable manure in which the ammonin had been fixed, and covered this immediately with earth. The succeeding autumn they were literally bending to the ground ivith the finest fruit l ever saw, while the other trees in ny orchard not so treated are quite barren, the last season having been their bearing year. I am now jlacing rumd each tree one peck of charcual dust, and propose in the spring to cover it from the compost heap.

My soil is a strong, deep, sandy loam on a gravelly subsoil. I cultivate my orchard grounds, as if there were no trees on them, and raise grain of every kind except rye, when grain is so very injurious that 1 believe three surcessive crops of it would destroy any orchard youmger than iwenty years. I raised last year in an orcliard containing 20 acres, trees 18 years ald, a crop of Indian corn which averaged 140 bushels of ears to the acre." 
the orcharaist. 'These are chicfly the borer, the caterpir'ar, and the canker wrorm.

The apple Borer is, as we usually see it $\mathrm{m}$ the trunks of the apple, quince, and thom trees, a fleshy white grub, which enters the tree at the collar, just at the surface of the ground, where the bark is tender, and either girdles the tree or perforates it through every part of the stern, finally causing its death. This grub is the larva, of a brown and white striped beetle, half an inch long, (Saperda bivittata,) and it remains in this grub state two or three years, coming out of the tree in a butterfly form early in June-flying in the night only, from tree to tree after its fond, and finally depositing its eggs during this and the next month, in the collar of the tree.

The most effectual mode of destroying the borer, is that of killing it by thrusting a flexible wire as far as possible into its hole. Dr. Harris recommends placing a bit of camphor in the mouth of the aperture and plugging the hole with soft wood. But it is always better to prevent the attack of the borer, by placing about the trunk, early in the spring, a small mound of ashes or lime; and where orchards have already become greatly infested with this insect, the beetles may be destroyed by thousands, in June, by building small boufires of shavings in various parts of the orchard. The attacks of the borer on nursery trees may, in a great measure, be prevented by washing the stems in May, quite down to the ground with a solution of two pounds of potash in eight quarts of water.

The Caterpillar is a great pestilence in the apple orchard. The species which is most troublesome to our fruit trees (Clisiocampa americana,) is bred by a soint of lackey moth, different from that most troublesome in Europe, but its hatits as a caterpillar are quite as annoying to the orchardist. 'I'he moth of our common caterpillar is a reddish brown insect, whose expanded wings measure about an inch and a half. These moths appear in great abundance in midsummer, flying only at night, and often buzzing about the candles in our houses. In laying their eggs, they choose principally the apple or cherry, and they deposit thousands of small eggs about the forks and cxtremitics of the young branches. The next season, about the middle of May, these egrgs begin to hatch, and the young catcrpillars in myriads, come forth weaving their nests or tents is, the fork of the branches. If they are allowed by the careless cultivator to go on and multiply, as they soon do, incredibly fast, they will in a few seasons, - sometimes in a single ycar,-increase to such an extent as almost to cover the branches. In this caterpillar state they live six or seven wecks, feeding most voraciously upon the leaves, and often stripping whole trces of their foliage. Iheir effect upon the tree at this period of the season, when the leaves are most important to the health of the tree and 
the growth of the fruit, is most deplorable. The crop is stuntert, the health of the tree enfeebled, and, if they are allowed to remain unmolested for : everal seasons, they will often destroy its life or render it exceedingly decrepid and feeble.

To destroy the caterpillar various morles are adopted. One of the most efiectual is that practised by $M \mathrm{r}$. Pell in his orchards, which is to touch the nest with a sponge, attached to the end of a pole, and dipped in strong spirits of ammonia; the sponge should be turned slowly round in the nests, and every insect coming in contact will be instantly killed. This should be done carly in the season. Or, they may be brought down and destroyed with a round brush fixed to the end of a pole, and worked about in the nests. On small trees they may be stripped off with the hand, and crushed under the foot; and by this plain and simple mode, begun in time, with the aid of a ladder, they may in a large orchard be most effectually kept under by a few moments daily labour of a single man. As they do not leave. their nests until nine in the morning, the extirpator of caterpillars should always be abroad and busy before that time, and while they are all lying quietly in the nests. And let him never forget that he may do more in an hour when he commences early in the season, than he will in a whole day at a later period, when they are thoroughly scattered among the trees. If they are allowed to remain unmolested, they spin their cocoons about the middle of June, and in a fortnight's time comes forth from them a fresh brood of inoths-which, if they are not put an end to by bonfires, will again lay the eggs of an infinite number of caterpillars for the next spring.

The canker worm, (Anisopteryx pometaria, of Harris,) is in some parts of the country, one of the worst enemies of the apple, de. stroying also its foliage with great rapidity. It is not yet common here, but in some parts of New-England it has become a serious enemy. The male is a moth with pale, ash-coloured wings with a black dot, a little more than an inch across. 'The female is wingless, oval, dark ash-coloured above, and gray beneath.

'The canker wor:m usually rises out of the ground very early in the spring, chiefly in March, as soon as the ground is free from frost ; though a few also find their way up in the autumn. 'The females haviug no wings, climb slowly up the trunks of the trees, while the winged males hover about to pair with them. Very soon after this if we examine the trees we shall see the eggs of which every female lays some sixty or a hundred, glueri over, closely arranged in rows and placed in the forks of branches and annong the youmg twigs. About the twentieth of May, these egrgs are hatched, and the canker worms, dusky brown, or ash-coloured with a ycllow stripe, make their appearance and sommence preying upon the foliage. When they are abundaus 
they make rapid progress, and in places, where the colony is firmly established, they will sometimes strip an orchard in a few ays, making it look as if a fire had passed over it. After feedng about four weeks, they descend into the ground three or four inches, where they remain in a chrysalis form, to emerge again the next season. As the female is not provided with wings, they do not spread very rapidly from one place to another.

The attacks upon the canker worm should be chiefly made upon the female, in her way from the ground up the trunk of the tree.

The common mode of protecting apple trees is to surround the trunk with a belt or bandage of canvass, four or five inches wide, which is then thickly smeared with tar. In order to prevent the tar from soon becoming dry and hard, a tittle coarse train oil must be well mixed with it; and it should be watched and re. newed as often as it appoars necessary. This tarred belt catches and detains all the females on their upward journey. and prevents them from ascending the tree to lay their egess. And if kept in order it will very effectually deter and destroy them. When the canker worm is abundant, it is necessary to anplv the tarred bandage in October, and let it remain till the last of Mav, but. usually it will be sufficient to use it in the spring It is probable that a mixture of coal tar and common tar would be the best application; as it is more offensive and will nor so easily dry and become uscless, by exposure to the air and sun. Some persons apply the tar directly to the stems of the tree, but this has a very injurious effect upon the trunk. Old Indra rubbe $\tilde{n}_{i}$ melted in an iron vessel cver a very hot fire, forms a ve:"y adhesive fluid which is not affected by exposure to the weal'er, and is considered, by those who have made use of it, the best substance for smearing the loandages, as being a more e.f"ectuas barricr, and seldom or never requiring renewal.

Mr. Jonathan Dennis, jr. of Portsmouth, Rhode Islard, has invented and patented a circular leaden trough, which surround the trunk of the tree, and is filled with oil, and stops effectually the ascent of the canker worm. There appear, however, to bi two objections to this trough, as it is frequently used; one, the escape of the oil if not carefully used, which injures the tree; and the other, the injurious effect of nailing the troughs to the bark or trunk. They should be supported by wedges of wood driven in between the trough and the trunk, and the spaces completely filied up with liquid clay put on with a brush. 'The insects must be taken out and the oil renewed, from time to time. For districts where the canker worm greatly abounds, this leaden trough is probably the most permanent and effectual remedy yet employed.

Experiments made by the Hon. John Lowell, and Professor Peck, of Mussachusetts, lead to a belief that if the ground, under 
trees which suffer from this insect, is dug and well pulverized to the depth of five inches in October, and a good top dressing of lime applied as far as the branches extend, the canker worm will there be almost entirely destroyed. The elm, and linden trees in many places, suffer equally with the apple, from the attacks of the canker worm.

The Bark-louse, a dull white oval scale-like insect, about a tenth of an inch long, (a species of coccus, which sometimes appears in great numbers on the stems of young apple and pear trees, and stunts their growth, may be destroyed by a wash of soft soap and water, or the potash solution. "The best time to apply these is in the month of June, when the insects are young.

The Woolly aphis (aphis langinera,) or American blight* is a dreadful enemy of the apple abroad, but is fortunately, very rarely seen as yet, in the United States. It makes its appearance in the form of a minute white down, in the crotches and crevices of the branches, which is composed of a great number of very minute woolly lice, that if allowed, will increase with fearful rapidity, and produce a sickly and diseased state of the whole tree. Fortunately, this insect is too easily destroyed. "'This is tilected by washing the parts with diluted sulphuric acid; which is formed by mixing $\frac{3}{4}$ oz. by measure, of the sulphuric acid of the shops, with $7 \frac{1}{2} \mathrm{oz}$. of water. It should be rubbed into the parts affected, by means of a piece of rag tied to a stick, the operator taking care not to let it touch his clothes. After the bark of a tree has been washed with this mixture, the first shower will re-dissolve it, and convey it into the most minute crevice, so as effectually to destroy all insects that may have escaped."-(Loudon's Magazine IX. p. 336.)

The Apple worm (or Codling moth, Carpocapsa pomonana, of European writers, is the insect, introduced with the apple tree from Europe, which appears in the early worm-eaten apples and pears, in the form of a reddish white grub, and causes the fruit to fall prematurely from the tree. The perfect insect is a small moth, the fore-wings gray, with a large round brown spot on the hinder margin. These moths appear in the greatest numbers in the warm evenings af the 1st of June, and lay their egrgs in the eye or blossom-end of the young fruit, especially of the early kinds of apples and pears. In a short time, these eyrgs hatch, and the grub burrows its way till it reaches the core; the fruit then ripens prematurely, and drops to the ground. Here the worm leaves the fruit and creeps into the crevices of ahe bark and hollow of the tree; and spins its cocoon, which

* It is not a little singular that this insect which is not indigenons to this coun. try, and is never seen here except where introduced with imported trees, should be ealled in Eingland the American blight. It is the most invererate enemy of the apple in the north of France and Germany. 
asually remains there till the ensuing spring, whon the young moth again emerges from it. The readiest way of destroying them, when it can be done conveniently, is to allow swine and poultry to run at large in the orchards when the premature fruit is talling; or otherwise, the fruit may be pickel up daily and placed where the worms will be killed. It is said that if an old cloth is placed in the crotch of the tree about the time the fruit begins to drop, the apple worm will make it a retiring place, and thousands may be caught and killed from time to time. As the cocoons are deposited chiefly under the old loose bark, the thorough cultivator will take care, by keeping the trunks of his trees smooth, to afford them little harbour; and by scraping and washing the trunks early in the spring, to destroy such as may have already taken up their quarters there.

When the fruit of orchards is much liable to the attacks of this insect we cannot too much insist on the efficacy of small bonfires lighted in the evening, by which myriads of this and all other moths may be destroyed, before they have time to deposit their eggs and cause worm-eaten fruit.

The Blight which occasionally kills suddenly the ends of the limbs of the apple and the quince, appears to be caused by alp insect similar to that which produces the fire blight of the pear, and must be treated in the same way as directed for that tree.

GATHERING AND KEEPING THE FRUIT. In order to secure soundness and preservation, it is indispensably necessary that the fruit should be gathered by hand. For winter fruit the gathering is delayed as long as possible, avoiding severe frosts, and the most successful practice with our extensive orchardists is to place the good fruit directly, in a careful manner, in new, tight flour barrels as soon as gathered from the tree. These barrels should be gently shaken while filling, and the head closely pressed in ; they are then placed in a cool shady exposure under a shed open to the air, or on the north side of a building, protected by covering of boards over the top, where they remain for a fortnight, or until the cold becomes too severe, when they are carefully transferred to a cool, dry cellar, in which air can be admitted occasionally in brisk weather.

A cellar, for this purpose, should be dug in dry, gravelly, or sandy soil, with, if possible, a slope to the north; or, at any rate, with openings on the north side for the admission of air very rarely in weather not excessivcly cold. Here the barrels should be placed on tiers on their sides, and the cellar should be kept as dark as possible. In such a cellar, one of the largest apple growers in Dutchess county is able to keep the Greening apple, which, in the fruit room, usuaily decays in January, until the 1st of A pril, in the freshesi and finest condition. Some persons place a layer of clean rye straw bewween every layer of apples, when packing them in the barrels. 
App es are frequently kept by farmers in pits or ridges in the groun', covered with straw and a layer of earth, in the same manner as potatoes, but it is an infericur methorl, and the fruil very speedily decays when opened to the air. The English apple mrowers lay their fruit in heaps, in cool dry cellars, and cover them with straw.

When apples are exported, each fruit in the barrel should be wrapped in clean coarse paper, and the barrels should be placed in a dry, airy place, between decks.

Cider. To make the finest cider, apples should be chosen which are especially suited to this purpose. 'The fruit should be gathered about the first of November, and coarse cloths or straw should be laid under the tree to secure them against bruising when they are shaken from the tree. If the weather is fine the fruit is allowed to lie in heaps in the open air, or in airy sheds or lofts for some time, till it is thoroughly ripened. All immature and rotten fruit should then be rejected, and the remainder ground in the mill as nearly as possible to an uniform mass. This pulp should now remain in the vat from 21 to 49 hours, or even longer if the weather is cool, in order to heighten the colour and increase the saccharine principle. It is then put into the press (without wetting the straw,) from whence the liquor is strained through hair cloth or sieves, into perfectly clean, sweet, sound casks. The casks, with the bung out, are then placed in a cool cellar, or in a sheltered place in the open air. Here the fermentation commences, and as the pomace and froth work out of the bung-hole, the caskis must be filled up every day with some of the same pressing, liept in a cask for this purpose. In two or three weelis this rising will cease, when the first fermentation is over, and the bung should, at first, be put in loosely-then, in a day or two, driven in tight-leaving a sinall vent hole near it, which may also be stopped in a few days after. If the casks are in a cool airy cellar, the fermontation will cease in a day or two, and this state may be known by the liquor becoming clear and bright, by the cessation of the discharge of fixed air, and by the thick crust which has collecterl on the surface. The clear çider should now be drawn off and placed in a clean cask. If the cirder, which must be carefully watched in this state to prevent the fermentation going too far, remains quiet, it may be allowed to stand till spring, and the addition at first of about a gill of finely powdered charcoal to a barrel will secure this end; but if a scum collects on the sur face, and the fermentation seems inclined to proceed further, it must be immediately racked again. The vent-spile may now be driven tight but examined occasionally. In the becrinning of March a final racking should take place, when, should the eider not be perfectly fime, about three fourths of an ounce of Isikm glass should be dissolved in the cider and noured in each barreh 
which will render it perfectiy clea:. It may be bottled now, or any perior before the blossoming of the apple or afterwards, late in May. When bottling, fill the bottles within an inch of the bottom of the cork, and allow the bottles to stand an hour before the corks are driven in. They should then be sealerl, and kept in a cool cellar, with clean dry sand up to their necks; or laid on their silles in boxes or bins, with the same between each layer.

VARIETIES. The varieties of the apple, at the present time, are very numerous. The garden of the Horticultural Society, of London, which contains the most complete collection of fruit in the world, enumerates now about 900 varieties, and nearly 1500 have been tested there. Of these, the larger proportion are of course inferiour, but it is only by comparison in such an experimental garden that the value of the different varieties, for a certain climate, can be fully ascertained.

The European apples generally, are in this climate, inferiour to our first rate native sorts, though many of them are of high merit also with us. The great natural centre of the apple culture in America, is between Massachusetts bay and the Delaware river, where the Newtown pippin, the Spitzemberg, the Swaar, the Baldwin, and the yellow Belle Fleur, have originated, and are grown in the greatest perfection. The apples raised on the very fertile bottoms of the western states are very large and beautiful, but as yet, owing to the excessive luxuriance of growth, are far inferiour in flavour to those of the same quality, raised on the strong, gravelly or candy loams of this section of the country. New varieties of apples are constantly springing up in this country from the seed, in favourable soils; and these, when of superiour quality, may, as a general rule, be considered much more valuable for orchard cuiture than foreign sorts, on account of their greater productiveness and longevity. Indeed, every state has some fine apples, peculiar to it, and it is, therefore, impossible in the present state of pomology in this country, to give any thing like a complete list of the finest apples of the United States. To do this, will require time, and an extended and careful examination of their relative merits collected in one garden. The following descriptions comprise all the finest American and foreign varieties yet known in our gardens.

In the ensuing pages, apples are described as regards form as follows; round, or roundish, when the height and the diameter are nearly equal; flat, or oblate, when the height is much less; , blong, when the height is considerably more than the diameter; ovate, (egg-shaped,) when the blossom-end is narrowed and rounded; conical, when the fruit is oblong and somewhat coni cal on its sides. Pearmain-shaped is a short or flattened cone ; and Calville-shaped signifies a ribbed or furrowed surface. 


\section{Apples.*}

\section{Class 1. Summer Apples.}

\section{American Sunner Pearniain. $§$ Thomp.}

Early Summer Pearmain. Coxe.

A rich, high-flavoured fruit, much esteemed in New-Jersey, where it is most known. It appears to be quite different from the Sumrner Pearmain, (of the English,) and is probably a seed. ling rajsed from it. It ripens gradually from the tenth of August to the last of September.

Fruit of medium size, oblong, widest at the crown, and taperins slightly to the eye. Skin, red spotted with yellow in the shade, but streaked with livelier red and yellow on the sunny side. Stalk three fourths of an inch long, and pretty deeply inserted. Eye deeply sunk. Flesh yellow, remarkably tender, with a rich and pieasant flavour, and often bursts in falling from the tree. This is a valuable apple for all purposes, and it thrives admirably on sandy soils. In the nursery the tree grows slowly.

\section{Borovitsky. Thomp. Lind.}

A good early Russian apple of the middle size, which ripens here the last of July. Form roundish, a little angular. Stalk, an inch long, planted rather deeply. Skin, pale green, with a semi-transparent appearance, faintly striped on the sunny side with light and dark red. Calyx in a large basin. Flesh, white, pretty firm, and juicy, with an agreeable sub-acid flavour.

\section{Benont. Man. Ken.}

This excellent early apple is a native of Dedham, Mass. The fruit is of medium size, nearly round. Skin, deep red. Flesh, yellow, tender, and of an agreeable rich, sub-acid flavour. Ripens during the whole month of August, and is a good and regular bearer.

* In descriling apples, we shall designate the size by comparison, as follows: small, as the English Golden pippin; medium size, as the Newtown pippin, large, as the Fellow Bellfower and Fall pippin-as we consider this reference to a standard, generally known. better than an exaet deseription by measure vwing to the variation in different soils and seasons.

The blossom-end, apex or crown of the frivit. is called the cye; but we shall, for the sake of precision, call the remains of the blossom still found there the salyx and the hollow in which it is placed the basin.

This mark denotes varieties particularly recommended by the author. 
4. Cote. Thomp. Lind. Ron.

Scarlet Perfume.

$\Lambda$ variety from England of seconl quality, but admired for its beauty of appearance.

Fruit large, roundish, somewhat flattened and slightly anguar. Skin nearly covered with deep crimson on a yellowish ground, or sometimes entirely red, with a little russet. Stalk long, woolly, planted in a cavity which is sometimes nearly closed up. Calyx large, in a broad basin. Flesh white, rather firm, juicy, with a somewhat rich and agreeable flavour. August.

5. Devonshire Quarrenden. Thom. P. Mag. Fors.

Red Quarrenden. 'Lind.

Sack Apple.

A handsome English dessert fruit. The editor of the Pomological Magazine says, "there is no better autumn dessert apple ;" but after giving it a trial for soveral years by the side of the Williams' Favourite, we consider the latter greatly superiour in flavour, and equally beautiful. Fruit scarcely of medium size, roundish, flattened, and slightly narrowed at the eye. Stalk short, deeply planted. Calyx with long segments, searcely sunk in a very shallow plaited basin. Skin rich deep crimson, with lighter crimson, and occasionally a spot of green on the shadel side, sprinkled with numerous green dots. Flesh nearly white, crisp, juicy, with a pleasant sub-acid flavour. Ripe during all Augusi and September.

6. DraP D'OR. $\S$ Coxe. Thomp. Ron.

Vrai Drap d'Or. O. Dult.

Early Summer pippin, of some New-York gardens.

Bay Apple ac. to

Bonne de Mai Thomp.

This large, handsome, and excellent summer apple is highly deserving general cultivation. It is better (though incorrectly. known on the Hudson as the Summer Pippin, but it is very dis. tinct from the apple known by that name in New-Jersey, which is the Holland pippin. It is also a very different fruit from the Drap d Or of Lindley, and of Noisette, and most French ain thors, which is quite a small apple; but it is the Vrai Drap d'Or of the 3:3 Duhamel, pl. xii. Fig. 4.

Fruit large, roundish, sometimes a little oblong, narrowing slightly to the eye. Skin smooth, yellow or dead gold colour, with distinet small brown dots, or specks. Stalk short, mode- 
rately sunk. Calyx set in a shallowish basin, wlich is rather plaited or irregular. Flesh crisp, juicy, and of a pleasant, sprightly, mild favour, agreeable for the dessert or for cooking. [This is the Summer Pippin of the previous catalogues of the nurseries here.] August to October. The treo grows vigcreus. ly, and bears well, and the wood is smooth and dark brown.

\section{Early Harvest. $\oint$ Thomp. Man \\ Prince's Harvest, or Early French Reinette, of Care. \\ July Pippin. Floy. \\ Yellow Harvest. \\ Large White Juneating. \\ Tart Bough. \\ Early French Reinnette.}

An American apple; and taking into account its beauty, its excellent qualities for the dessert and for cooking, and its productiveness, we think it the finest early apple yet known. It begins to ripen about the first of July, and continues in use all that month. The smallest collection of apples should comprise

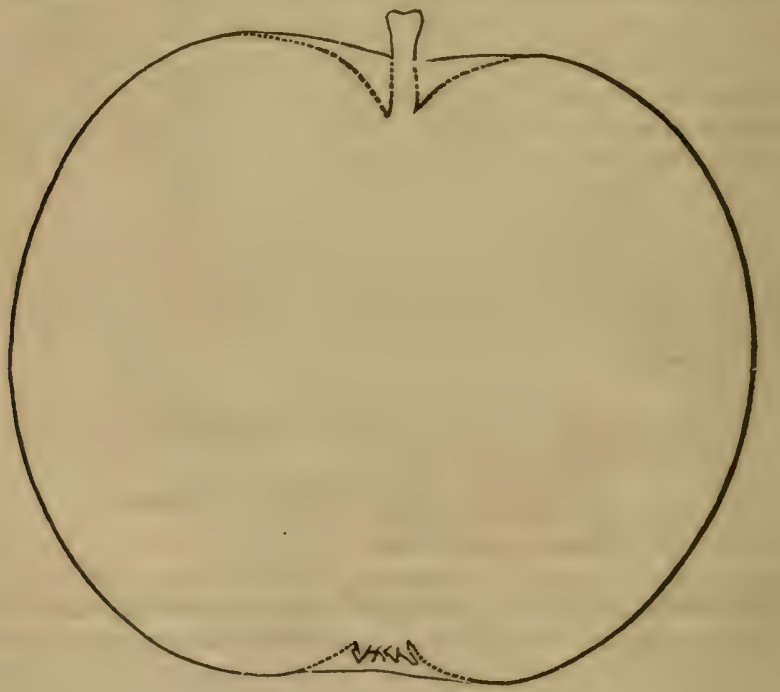

Fig. 26. Early Harvest.

this and the Red Astrachan. Form round, above medium size, rarely a little flattened. Skin very smooth, with a few is in: white dots, bright straw colour when fully ripe. Stalk half to three fourths of an inch long, rather slender, inserted in a hollow of moderate depth. Calyx set in a shallow basin. Flesh very white, tender and juicy, crisp, with a rich, sprightly, sub-acid 
flavour. The young trees of moderate vigour, with scarcely diverging shoots. Manning errs by following Coxe in calling this a flat apple.

\title{
8. Early Red Makgaret. $§$ Thomp. Lind.
}

\author{
Red Juneating. \\ Niargaret, or Striped Juneating. Ronalds. \\ Early Red Juneating. \\ Eve Apple of the Irish. \\ Striped Juneating. \\ Margaretha Apfel, of the Germans.
}

An excellent early apple, ripening about the middle of July, or directly after the Early Harvest. The tree while young is ruther slender with upright woolly shoots. It is a moderate bearer.

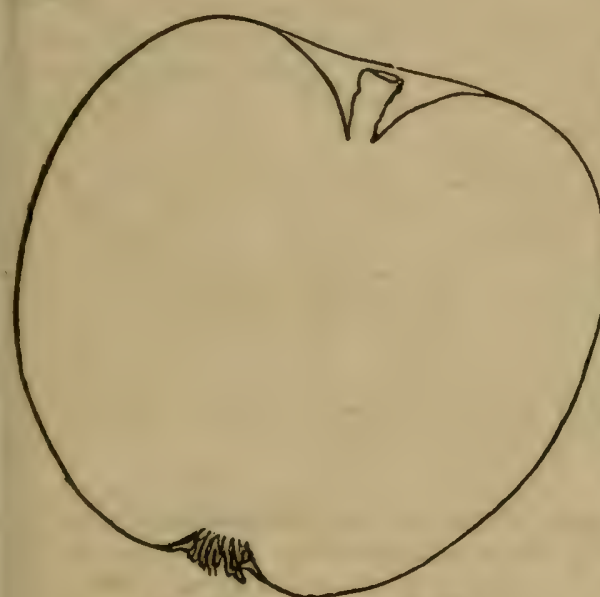

Fig. 27. Early Red Margaret.

Fruit below medium size, roundish-ovate, tapering towards the eye. Skin greenish yel. low, pretty wel! of dark red. Stalk short and thick. Calyx closed, and placed in a very shallow plaited basin. Flesh white, sub-acid, and when freshly gathered from the tree, of a rich agreeable fla. vour.

This is distinct from the Margaret Apple, of Miller, the Red Juneating of some of our gardens, which resembles it, but is round, with a short slender stalk, and dull yellow skin striped with orange red on one side, the fruit fragrant and the leaves very downy.

\section{Early Stratwberry Apple. ( ()}

Ameriran Red Juneating ?

Red Juneating, erroneorisly, of some American gardens.

A beautiful variety which is said to have originated in the neighlourhood of New-York, and appears in the markets there from July till September. Its sprightly flavour, agreeable perfume, and fine appearance, place it among the very finest sum- 
mer apples. It is quite distinct from the Early Red Margaret. which has no fragrance, and a short stem.

Fruit roundish, narrowing towards the eye. Skin smooth and fair, finely striped and stained with bright and dark red, on a yellowish white ground. Stalk an inch and a half long, rather slender and uneven, inserted in a deep cavity. Calyx rather small, in a shallow, narrow basin. Flesh white, slightly tinged with red next the skin, tender, sub-acid, and very sprightly and brisk in fla. vour, with an a. greeable aroma.

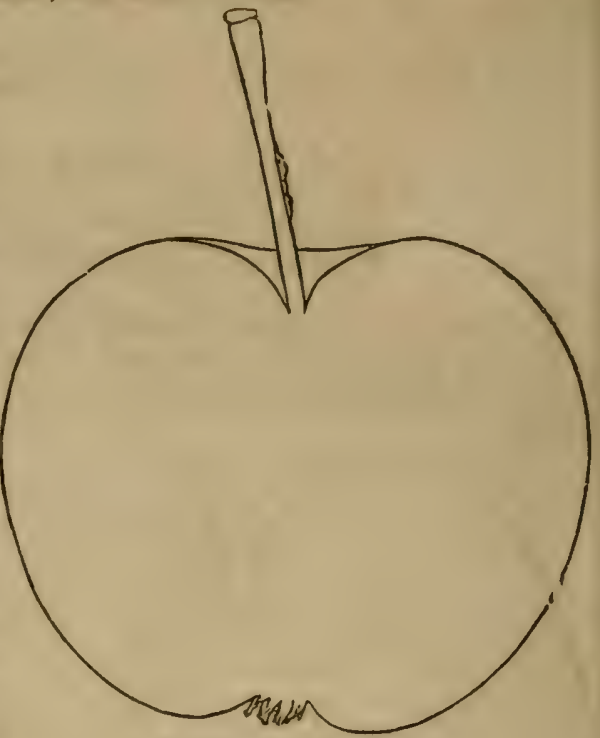

Fig. 28. Early Strawberry.

10 Irish Peach Apple. Thomp. Lind. P. Mag.

Early Crofton. Ronalds.

Fruit of medium size, round or a little flattened, and obtusely anguiar. Calyx pretty large and spreading, in a rather narrow basin. Stalk short, in a cavity of moderate depth. Skin yel. lowish green, with small dots in the shade, washed and streaked with brownish red in the sun. Flesh white, tender, juicy, and pretty well flavoured. A pleasing fruit, but does not rank so high here as in England.

\section{Large Yellow Bougri. $\S$ Thomp. \\ Early Sweet Bough. Kenrick. \\ Sweet Harvest. \\ Bough. Coxe. Floy.}

A native apple, ripening in harvest time, and one of the first quality, only second as a dessert fruit to the Early Harvest. It is not so much esteemed for the kitchen as the latter, as it is too sweet for pies and sauce, but it is generally much admired for the table, and is worthy of a place in every collection. 
Fruit above the midd'e size, an oblong-ovate in form. Skit, month, pale, greenish yellow. Stalk rather long, and the eye narrow and deep. Flesh white, very tender and crisp when fully ripe, and with a rich sweet sprightly flavour. Ripens from the middle of July to the tenth of August. Tree modo rately vigorous, bears abundantly, and forms a round head.

\section{Luman's Large Summer.}

Large Yellow Summer, Ken.

A large and handsome American fruit, introduced to notice by Mr. S. Lyman, of Manchester; Conn. The bearing trees are easily recognized by their long and drooping branches, which are almost wholly without fruit spurs, but bear in clusters at their extremities. They bear poorly until the tree attains consider. able size, when it yields excellent crops. Fruit quite large, roundish, flattened at the ends: skin smooth, pale yellow. F!esh yellow, tender, sub-acid, rich, and high flavoured, and excellent either for the table or for cooking. Last of August.

\section{OsLrn. Thomp. Lind.}

Arbroath Pippin. Forsyth.

An excellent Scotch apple, ripening early in August. Form roundish, below medium size, a little flattened. Skin rather tough, clear lemon yeliow when quite ripe, mingled with a little bright green, and sprinkled with a few grayish green dots. Stalk short and thick, set in a rather shallow depression. Calyx in a shallow basin, a little plaited, with prominent segments. Flesh yellowish, firm, crisp, juicy, with a spicy aromatic flavour. The wood is strong, and grows pretty freely from cut tings.

\section{Red Astrachan. $§$ Thomp. Lind.}

A fruit of extraordinary beauty, first imported into England with the White Astrachan, from Sweden, in 1816. It bears abundantly with us, and its singular richness of colour is heightened by an exquisite bloom on the surface of the fruit, like that of a plum. It is one of the handsomest dessert fruits, and its quality is good, but if not taken from the tree as soon as ripe, it is liable to become mealy. Ripens from the last of July to the middle of August.

Fruit pretty large, rather above the middle size, and very smooth and fair, roundish, a little narrowed towards the eye. Skin almost entirely covered with deep crimson, with sometimes a little greenish yellow in the shade, and occasionally a little 
rusaet near the stalk, and covered with a pale white bloom. Stalk rather short and deeply inserted. Calyx set in a slight

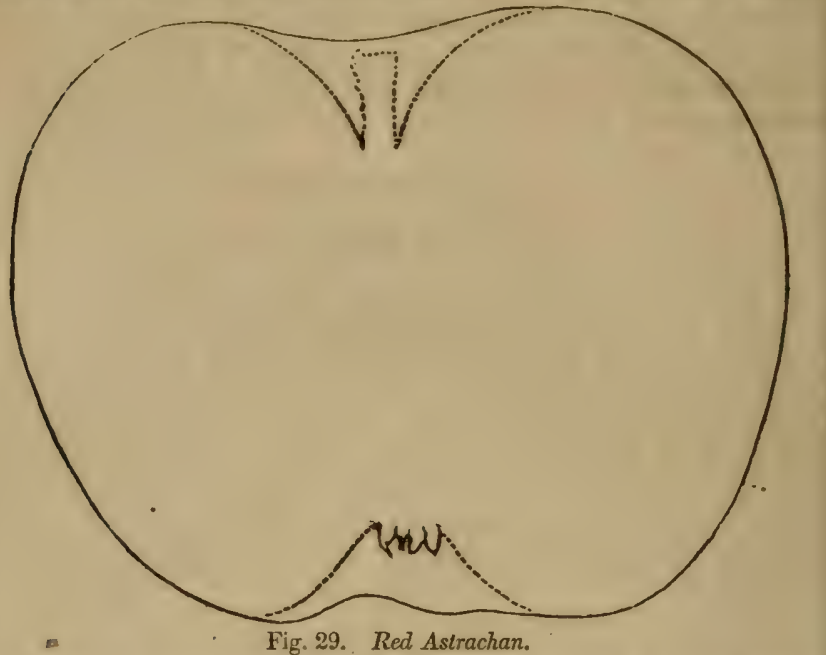

basin, which is sometimes a little irregular. Flesh quite white. crisp, moderately juicy, with an agreeable, rich, acid flavour.

\section{SinE-QUA-NoN.}

A native of Long Island, named by the late Wm. Prince. Fruit roundish - ovate, about medium size. Skin smooth, pale greenish yellow. Stalk slender. Flesh white, very tender, juicy, and of a delicate and very sprightly flavour. The young trees are rather slow and crooked in growth. August.

\section{Sugar Loaf Pipprn. Thomp. Lind. P. Mag.}

\section{Hutching's Seedling.}

A foreign sort, which is ranked among first rate sorts by Thompson, but from spccimens of two seasons produced here, it does not seem likely to take this rank with us. Fruit of medium size, oblong or conical, smooth, clear pale ycllow, be. coming nearly white on one side when fully ripe. Stalk nearly an inch long, deeply set in a regular cavity. Calyx sunk in a pretty deep basin. Flesh white, firm, very slightly acid, and moderately juicy. According to Lindley it has in England "a most agreeable lively flavour." Ripens here the latter part of July, and is very showy on the tree. 


\section{Sunmer Rose. Thomp. Coxe.}

\section{Woulman's Harvest.}

A very pretty and very excellent apple, highly esteemed as a lessert fruit.

Fruit scarcely of medium size, roundish. Skin smooth, $r$ ch waxen yellow, streaked and blotched with a little red on the sunny side. Stalk rather short, and slender. Calyx closed, set in an even basin. Flesh tender, abounding with sprightly juice. Ripens early in August.

\section{Summer Queen. Coxe.}

A popular midsummer apple for the dessert and kitchen. The fruit is large and broad at the crown, tapering towards the cye. The stalk is rather long, and is planted in a pretty deep cavity, sometimes partially closed. Calyx but little sunk in a narrow plaited basin. Skin fine deep yellow in its ground, though well striped and clouded with red. Flesh aromatic, yellow, rich and of good flavour. This variety forms a large tree with somewhat pendant boughs, and the fruit is in perfection by the tenth of August.

\section{Suniner Golden Pippin. Thomp. Lind. P. Mag.}

A nice little English dessert apple, but inferiour to many of our own. Fruit small, ovate, flattened at the eye. Stalk short and calyx set in a wide shallow basin. Skin shining bright yel. low, with a little orange next the sun. Flesh yellow, firm, crisp and rich. Ripens in August.

\section{Sops of Wine. $\S$ Lind. Ron.}

Sops in Wine. Ray, (1688.)

Rode Wyn Appel. Knoop.

Sapson. Kenirick.

A charming little apple for the dessert, which the amateur's garden should always contain. Its flavour is spr...ty, though not first rate, and its colour is very handsome. Its name pro. bably comes from the red stain in its flesh. Its branches have a spreading habit, and bear plentifully; and the fruit, in our garden, ripens gradually from the first of August to October. Fruit. small, fiom an inch and a half to two and a half in diameter, globular, narrowing to the eye. Skin smooth, crimson in the shade, stained and striped with purplish crimson in the sun, and covered with a delicate white bloom. Stalk slender, three fourths of an inch long. Calyx spreading, in a shallow basin Flesh white, with stains of a pinkish hue, firm, crisp, juicy, and of a pleasant sub-acid flavour. 


\section{Tetofsky. Themp.}

The Tetnfsky is a Russian summer apple newly introduced. which promises well. Fruit of medium size, loundish oblong, soinctimes nearly round. Skin snooth, with a yellow ground handsomely striped with red, and, like most apples of that coun. try, co:ered with a whitish bloom, under which is a shining skin. The flesh is white and juicy with a sprightly and agreealoln flavour. August.

\section{White Juneating. Ray. Thomp. Lind.}

Owen's Golden Beauty, ac. Thomp.

Juneating. Care.

This is an old variety mentioned by Evelyn in 1660, and de scribed by Ray in 1688, and is a very tolerable little apple, ripening among the very earliest, during the last of June and the first of July, and deserves a place in a large collection chiefly on that account and its excellent bearing quality. It is very distinct from the Early Harvest, sometimes called by this name. Fruit small, round, a little flattened. Calyx closed in a wrinkled basin, moderately sunk. Stalk rather long and slender, three fourths to an inch in length, slightly inserted in a shallow

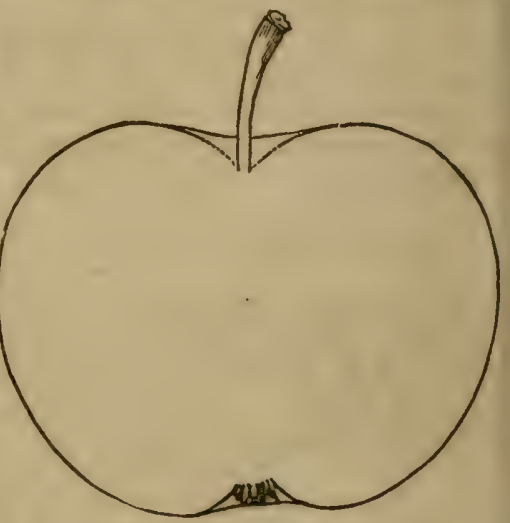

Fig. 30, White Juneating. depression. Skin smooth, pale green, at first light yellow, with sometimes a faint blush on the sunny side. Flesh crisp, and of pleasant flavour, but soon becomcs dry. Tree straight and forms an uprigit head.

\section{White Astrachan. Thomp. Lind. P. Mag.} $\left.\begin{array}{l}\text { Pyrus Astracanica. De Candolle, } \\ \text { Transparent de Moscovie, } \\ \text { Glace de Zélande, }\end{array}\right\}$ of the French gardens.

A nearly white, semi-transparent, Russian apple, which bears freely and ripens about the tenth of August ; but in this country is of little or no value, as it nearly always grows mealy and water cored as soon as ripe. Fruit of medium size, roundish, 
inclining to conical, and a little ribbed at the eye. Skın very smooth, nearly white, with a few faint streaks of red on one side, and covered with a white bloom. Stalk thick and short; calyx set in a small basin. Flesh quite white, partially transparent, tender and of delicate flavour, but rather dry.

\section{Williams's Favourite. $§$ Man. Ken.}

A large and handsome dessert apple, worthy of a place in every garien. It originated at Roxbury, near Boston, bears abundantly, and ripens from the last of July to the first of Septemler.

Fruit oi medium size, oblong, and a little one-sided. Stalk an inch long, slender, slightly sunk. Calyx closed, in a narrow angular basin. Skin very smooth of a light red ground, but nearly covered with a fine dark red. Flesh yellowish-white, and of a very mild and agreeable flavour.

\section{Class II. Autumn Apples.}

\section{Alexander. $\oint$ Thomp. \\ Emperor Alexander. Lind. Ron. \\ Russian Emperor. \\ Aporta.}

This is a very magnificent Russian sort, which thrives well in our gardens. The tree is no less striking in the spring when covered with its very large blossoms, than in autumn when loaded with its superb fruit. It is, properly, a cooking apple.

Fruit very large, frequently measuring tive inches in diame. ter, and weighing nearly a pound, regularly formed, generally conical or cordate, tapering from the base to the eye. Skiin greenish yellow, faintly streaked with red on the shaded side, but ơrange, brilliantly streaked and marked with bright red in the sun. Calyx la:gc, set in a deep basin. Stalk rather slender, three fourths of an inch long, planted in a deep cavity. Flesh yellowish white, crisp, tender and juicy, with a rather pleasant flavour. A moderate bearer. October to December. 


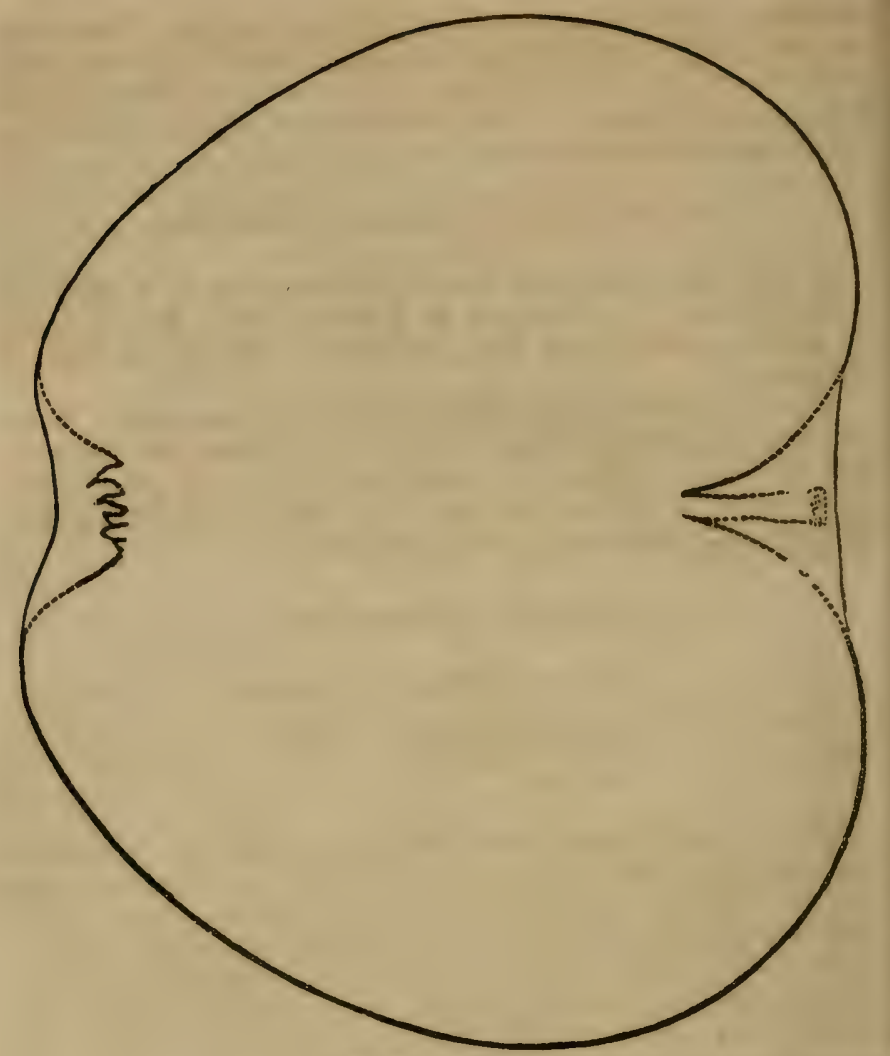

Fig. 31. Alexander.

26. Autuman Pearmaix. Thomp.

Summer Pearmain. Lind. Miller, $P$. Mag.

Winter Pearmain, of the Midlle States.

Yarmain d'Eté, Knoop.

A slow growing tree, but producing a good, high-flavoured dessert fruit. Fruit of medium size, oblong, narrowing gradu. ally towards the eye. Skin brownish yellow, mixed with green on the shaded side, but next the sun, reddish, blended with yellow, streaked with deeper red, and sprinkled with numerous small brown specks. Stalk short, obliquely planted under a fleshy lip. Calyx set in a broad shallow basin, which is some. times scarcely at all sunk, and obscurely plaited. Flesh pale yellow, crisp, firm, a little dry, but rich and high favoured. 
Branches slender. This most excellent old dessert fiuit is the "Winter Pearmain" of most old American orchards, and is a great favourite with many amateurs. October and November: and keeps till March.

\section{Blenheim Pippin. Thomp. Lind.}

\section{Blenheim Orange.}

Woodstock Pippin.

A large and showy English apple, admirable for baking, but which is not so highly esteemed for the table here, as abroad. 'The trees make strong and vigorous shoots.

Fruit very large, roundish, three inches in diameter at the base. Skin yellowish, becoming deep orange, stained on the sunny side with dull and dark red stripes. Calyx set in a large hollow basin. Flesh yellow, breaking, very sweet, and of tolerable flavour. October to December.

\section{Beauty of Kent. Thomp. Lind. Ron.}

The Beauty of Kent is, in this climate, one of the most mag. nificent of all apples, frequently measuring sixteen or eighteen inches in circumference. The flavour is of second quality, but as a kitchen fruit, it is among the finest. The tree grows very strong and upright.

Fruit very large, roundish, but flat at the base, and narrowing distinctly to the eye, where it is slightly ribbed. Skin smooth, greenish-yellow, marked with large, broken stripes of purplish red. Stalk short, slender, deeply planted in a round, shallow cavity. Calyx small, set in a narrow basin. Flesh juicy, crisp, tender, with a simple sub-acid flavour. October and November.

\section{Beauty of the West. Ken.}

A large, showy, sweet apple, of fair flavour.

Fruit large, round and regularly shaped. Skin smooth, light greenish-yellow, marked with small stripes of red. Stalk short, set in a round cavity. Flesh tender, juicy, sweet, and pleasant. A fall fruit, but may be kept for some time.

\section{Cornish' Aromatic. Thomp. Lind.}

A rich flavoured English apple. The quality is excellent, iut the fruit not very fair with us.

Fruit of medium size, roundish and angular. Skin rich red, much marked with russet yellow dots, on a pale russet ground. Stalk short, set in a deep, narrow cavity. Calyx small. in a narrow basin. Flesh yellow, with a rich aromatic suu-acid flavour. October to December. 


\title{
31. Catline. Coxe. Themp.
}

Gregson Apple.

We have not been able to procure this variety; which we be. lieve is a native of Maryland, and we therefore insert here $\mathbf{M r}$. Coxe's description. The Catline is an apple rather below ths middling size. It is a great bearer-the form is flat, the stalk short and thich, the skin smooth, and of a beautiful yellow, with a clear and brilliant red towards the sun, with numerous streaks and many dark spots scattered on the surface. The flesh is a pale yellow, tender, rich, juicy and sweet; as an eating apple in October, November and December, it is particularly fine. The tree is small, the form regular, and round in the head; the shoots straight and delicate; the foliage of a lively green-it is very productive, and in six or seven years after transplanting, it bears abundantly, when well cultivated.

\section{Dutchess of Olnenburgh. Thomp. Ron.}

A handsome Russian fruit of good quality. Fruit meäium size, regularly formed, roundish. Skin smooth, finely washed and streaked with red on a grolden or yellow ground. Calyx pretty large and nearly closed, set in a wide even hollow. There is a faint blue bloom on this fruit. The flesh is rich and juicy, with an excellent flavour. Ripens early in September.

\section{Downton Pippin. Thomp. Lind.}

\author{
Elton Pippin,
Knight's Golden Pippin, of some English garlens. \\ Downton Golden Pippin. Ken.
}

A rather early variety of the English Golden Pippin, raiserl by Mr. Knight of Downton Castle. It is a beautiful, small dessert fruit, and will ptcase those who like the rich, sharp, acid fiuvour of the Golden Pippin.

Fruit a little larger than the Golden Pippin, about two and a quarter inches in diameter, roundish, fat at the ends. Calys set in a wide, but very shallow basin. Stalk short, not deeply inserted. Skin smooth, yellow, dotted with small obscure specks.

Flesh yellowish, erisp, with a brisk, rich, tart flavour. The tree grows more vigorously and bears more abundantly than its parent, the old Golden Pippin. It is also considered a fine cider apple. October and November. 


\section{Dyer, or Pomme Royale. Ken. \\ Smithfield Spice.}

A popular New-England dessert apple, very sprightly, tende:; and excellent. It is supposed to be of French origin, and to have veen brought to Rhode Island more than a hundred years ago. It was re-named Dyer by the Mass. Hort. Society, who supposed it to be a sredling of Mr. Dyer, of R. I., but the old and familiar name of Pomme Royale should be preferred.

Fruit of medium size, roundish, pretty regularly formed. Skin smooth, pale greenish yellow, with a faint blush and a few dark specks on one side. Stalk about half an inch long, set in a smooth, round cavity. Calyx closed, basin plaited, moderately deep. Core round, hollow. Flesh white, very tender and juicy; flavour very mild and agreeable-slightly sub-acid. Sept., Oct.

\section{Dutch Codlin. Thomp. Lind. Ron.}

\section{Chalmer's Large.}

A very large kitchen apple, valued only for cooking, from August to September. Fruit of the largest size, irregularly roundish, or rather oblong, strongly marked by ribs extending from the base to the eye. Calyx set in a narrow, deep-furrowed basin. Stalk short and thick. Skin pale yellow, becoming orange yeliow on the sunny side. Flesh white, sub-acid, and moderately juicy. Inferiour to the Holland Pippin or the Drap d'Or. The tree makes very strong shoots.

\section{Flower of Kent. Thomp. Lind. Ron.}

A large and handsome English apple, chiefly valued for baking and kitchen use. Fruit quite large, roundish, a little ribbed on its sides, often considerably flattened, and rather broadest at the base. Skin tawny yellow, washed with dull red, with occasionally, a few stripes of brighter red. Calyx rather small, but set in a large basin, rather furrowed or irregular. Stalk nearly an inch long, not very deeply inserted. Flesh greenish yellow, abounding with a lively sub-acid juice. October to January.

\section{Franklin's Golden Pirpin. Thomp. Lind. Man.}

\section{Sudlow's Fall Pippin.}

This should be an American variety, named after Dr. Franklin, as it appears, by the Horticultural Transactions, to have been taken from the United States to England, in 1806; but it is, we believe, only known to nurserymen here, by importation back ugain. It is an excellent dessert fruit, larger than the common English Golden Pippin, ripening in October. Fruit of medium 
size, oval, very regular in shape, rather broadest at the base, Fye sunk in an even hcllow. Stalk short, slender, deeply planted. Skin deep yellow, freck.ed with numerous dark spots Flesh pale yellow, crisp, tender, with a fine rich aromatic fla vour. 'I'he tree grows freely, and forms an upright head.

\section{Fall Harvey. \& Man. Ken.}

A fine large Fall fruit from Essex co., Mass., very highly es. teemed in that neighbourhood. We do not think it comparable to the Fall pippin, which it a little resembles.

Fruit large, a little flattened, obscurely ribbed or irregular about the stalk, which is rather slender, an inch long, set in a wide, deep cavity. Calyx closed, small, in a rather shallow basin. Skin pale straw yellow, with a few scattered dots. Flesh white, juicy, crisp, with a rich, good flavour. October and November.

\section{Fall Pippin. § Coxe. Floy.}

The Fall Pippin is, we think, decidedly an American variety, Thompson and Lindley to the contrary, notwithstanding. It is, very probably, a seedling raised in this country, from the Whit Spanish Reinette, or the Holland pippin, both of which it so much resembles, and from which it, in fact, differs most strongly in the season of maturity. The Fall Pippin is a noble fruit, and is considered the first of Autumn apples in the middle states, where its beauty, large size, and its delicious flavour for the tablo or for cooking, render it very popular.

Fruit very large, roundish, generally a little flattened, pretty regular, sometimes with obscure ribs at the eye. Stalk rather long, three-fourths of an inch, projecting considerably beyond the fruit, (which distinguishes it from the Holland Pippin,) set in a rather small, shallow, round cavity. Calyx not very large, rather deeply sunk in a round, narrow cavity. Skin smooth, yellowish-green, becoming a fine yellow, with often a tinge of brownish blush, on one side, and with a few scattered dots. Flesh white, very tender and mellow with a rich, aromatic fla. vour. October to December.

There are several spurious sorts-the true one is always rather flattened, with a projecting stalk. (See Holland Pippin.)

\section{Gorden Sweet.}

\section{Orange Sweeting, or
Golden Sweet.}

A celebrated Connecticut fruit sent us by Mr. I.yman, of that 
state. Fruit above the medium size, roundish, scarcely fattenec. fair, and well formed. Skin, when fully ripe, pale yellow or straw colour. Stalk about an inch long, slcnder at its junction with the fruit. Calyx closed, and set in a basin of moderate dejth. Flesh tender, sweet, rich and excellent. The tree is a pretty free grower, and bears large crops. 'This we think wil] prove a valuable sort. Ripe in August ana September.

\section{Gravenstein. $\oint$ Thomp. Lind.}

\section{Grave Slije.}

A superb looking German apple, which originated at Graves.. stein, in Holstein, and is thought one of the finest apples of the north of Europe. It fully sustains its reputation here, and is, unquestionably, a fruit of first rate quality. Fruit large, rather flattened, and a little one sided or angular, broadest at the base.

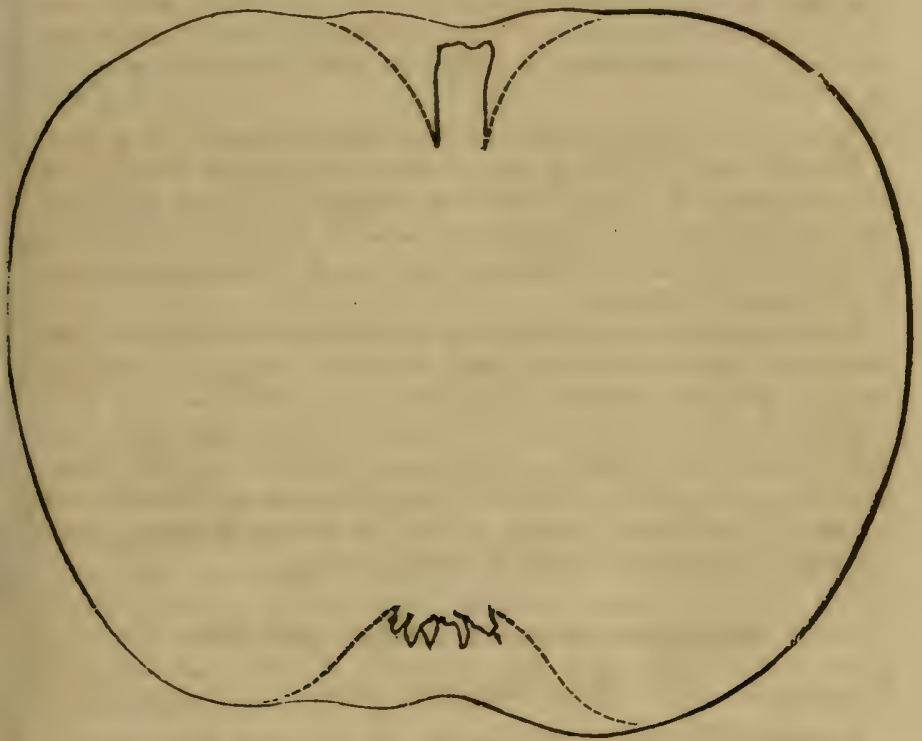

Fig. 32. Gravenstcin.

Stalk quite short and strong, deeply set. Calyx large, in a wiar deep, rather irregular basin. Skin greenish yellow at first, bus becoming bright yellow, and beautifully dashed and pencilled and marbled with light and deep red and orange. Flesh tender and crisp, with a high flavoured, somewhat aromatic taste. Ripens with us in September and Oetober, but will keepr a month longrer. The trees are very thrifty strong growers, and bear young. 


\section{Grand Sachem.}

A showy, large, dark, blond-red fruit, but rather coarse, and scarcely worth cultivation. Fruit very large, roundish, distinctly ribbed, and irregular in its outline. Stalk short and strong, and calyx set in a well marked basin. Skin smonth, deep, dingy red, over the whole surface. Flesh white, rather $\mathrm{dry}$, and without much flavour. September.

\section{Holland Pippin. Thomp. Lind. Miller.}

Reinnette d'Hollande. Norisette ?

Summer Pippin.
Pie Apple. of New-Jersey.

This and the Fall Pippin are frequently confounded together. They are indeed of the same origin, and the leaves, wood, and strong growth of both are very closely similar. One of the strongest points of difference, however, lies in their time of ripening. This being with us a late summer, the Fall Pippin a lite autumn, and the White Spanish Reinnette an early winter fruit.

The Holland Pippin, in the gardens here, begins to fall from the tree, and is fit for pies about the middle of August, and from liat time to the first of November, is one of the very best kitchen apples, making the finest tarts and pies. It is not equal to the Fall Pippin for eating.

Fruit very large, roundish, a little more square in outline than the Fall Pippin, and not so much flattened, though a good deal like it; a little narrowed next the eye. Stalk half an inch long, thick, deeply sunk. Calyx small, closed, moderately sunk in a slightly plaited basin. Skin greenish yellow or pale green, becoming pale yellow when fully ripe, washed on one side with a little dull red or pale brown, with a few scattered, large, green. ish dots. Deserves a place in every garden.

\section{Hawthorneer?. Thomp. Lind. Ron.}

White Hawthorden. Nicoll.

A celebrated Scotch apple, which originated at Hawthornden, the hirth-place of the poet Drummond. It resembles, some. what, our Maiden's Blush, but is inferiour to that fruit in flavour. Fruit rather above the medium size, (occasionally ribbed, according to Lindley, with us, pretty regularly formed, roundish, rather flattened. Skin very smooth, pale, light yellow, nearly wnite in the shade, with a fine blush where exposed to the sun. Calyx nearly closed, set in a rather shallow basin, with a few obscure plaits. Stalk half an inch long, slender. Flesh 
white, juicy, of a simple, pleasant flavour. As excellent bearer, a handsome fruit, and good for cooking or drying. The ends of the bearing branches become pendulous.

\section{Jersey Sweeting.}

A very popular apple in the middle states, where it is not only highly valued for the dessert, but, owing to its saccharine quality, it is also planted largely for the fattening of swine, which are allowed to run under the trees and gather the fruit as it falls. It is a highly valuable sort, and deserves extensive culture.

Fruit medium size, roundish-ovate, tapering to the eye. The calyx is small, closed, very slightly sunk, in a small plaited basin. Stalk half an inch long, in a rather narrow cavity. Skin thin, greenish yellow, washed and streaked, and often en. tirely covered with stripes of pale and dull red. Flesh white, fine grained, and exceedingly juicy, tender, sweet and sprightly. Young wood stout, and short jointed. This apple commences maturing about the last of August, and continues ripening till frost.

\section{Keswick Codits. Thom. Lind.}

A noted English cooking apple, which may be gathered for tarts, as early as the month of June, and continues in use till November. It is a great bearer and a vigorous tree.

Fruit a little above the middle size, rather conical, with a few obscure ribs. Stalk short and deeply set. Calyx rather large. Slrin greenish yellow, washed with a faint blush on one side. Flesh yellowish white, juicy, with a pleasant acid flavour.

\section{Kilham Hill. Man.}

A native of Essex co., Mass., raised by Daniel Kilham. Fruit Fretty large, roundish, ribbed, narrowing to the eye. Skin pale yellow, slightly splashed with red in the shade, deep red in the sun. Stalk rather long and slender, set in a wide deep hollow. Calyx in a narrow basin. Flesh of sprightly, rather high flavour, but is apt to become dry and mealy. Bears well. September.

\section{Kenrick's Autemn. Ken.}

A handsome apple of second quality. Fruit large, roundish, much flattened at the base. Stalk long, projecting beyond the fruit a good deal, set in a close cavity. Skin pale yellowish. green, striped and stained wi $h$ bright red. Flesh white, a littlo stained with red, tender, juicy, and of a sprightly acid favour. September. 
49. King of the Pippins. Thomp. Lind. Ron.

Hampshire Yellow.

An apple highly rated in England, whence it comes, but whi.fi scarcely proves first rate here. Fruit of medium siz", of a cunical or pearmain shape. Skin smooth, pale yellow, delicately streaked and washed with red next the sun. Stalk slen ler, an inch long. Calyx large, set in a deep even basin. Flesh white, very firm and of fair quality. The tree is an upright grower, and bears abundantiy. October and November.

\section{Kerry Pippin. Thomp. Lind. Ron.}

Edmonton's Aromatic Pippin. ae Thomp.

An Irish dessert anple, from the county of Kerry, as its name implies. Fruit middle size, oval, a little flattened at the eye. Skin pale yellow, mingled with a deeper yeliow, with a glossy surface, and stained and streaked with red. Stalk of medium length, sometimes short, set in a narrow cavity, with a prujection of the fruit on one side, and occasionally, a line or ridg?, running from the eye to the stalk. Calyx set in a plaited basin. Flesh yellow, tender, crisp, with a sugary flavour. Rirens in September and Octover.

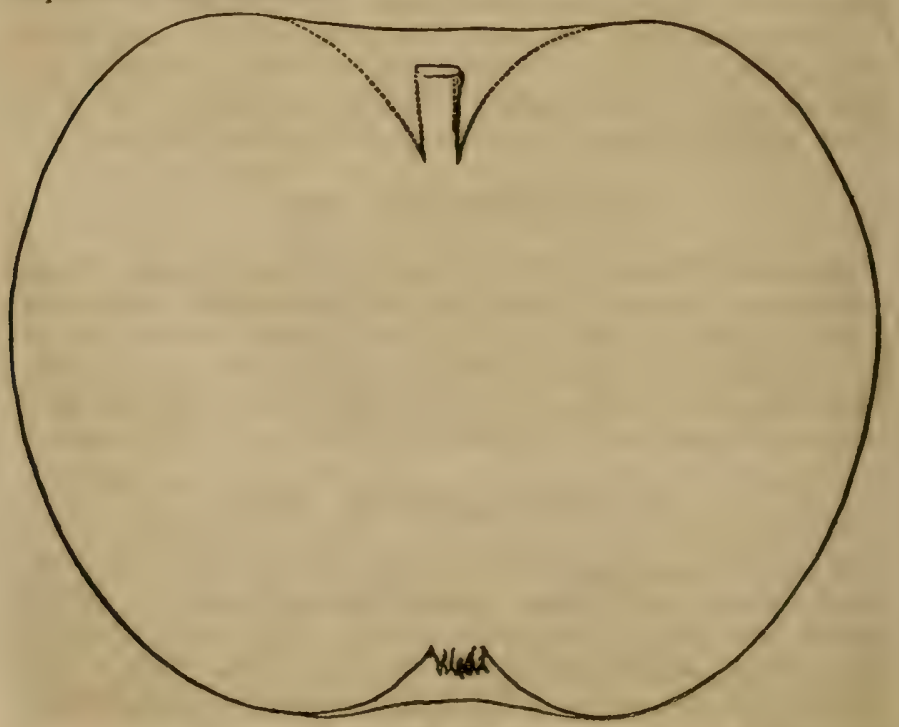

Fig. 33, Lyвcom. 


\section{Lyscom. Man. Ken.}

Osgood's Favourite.

Another Massachusetts variety of merit. Fruit large, round, skin greenish yellow, with a few broken stripes or splashes of red. Stalk short, planted in a deep, round, even cavity. Calyx small, in a very narrow, plaited basin. Flesh fine grained, and exceedingly mild and agreeable in flavour. A large, fine fruit. which is worthy of general cultivation. In use from Septembes to November.

\section{Lyman's Pumpin Sweet. Ken.}

A very large fair sweet apple which we received from $\mathrm{Mr}$. $\mathbf{S}$ T.yman, of Manchester, Conn. It is, perhaps, inferiour to the Jersey Sweet or the Summer Sweet Paradise for the table, but it is a very valuable apple for baking, and deserves a place on this account in every orchard. The original tree of this sort, is growing in Mr. Lyman's orchard.

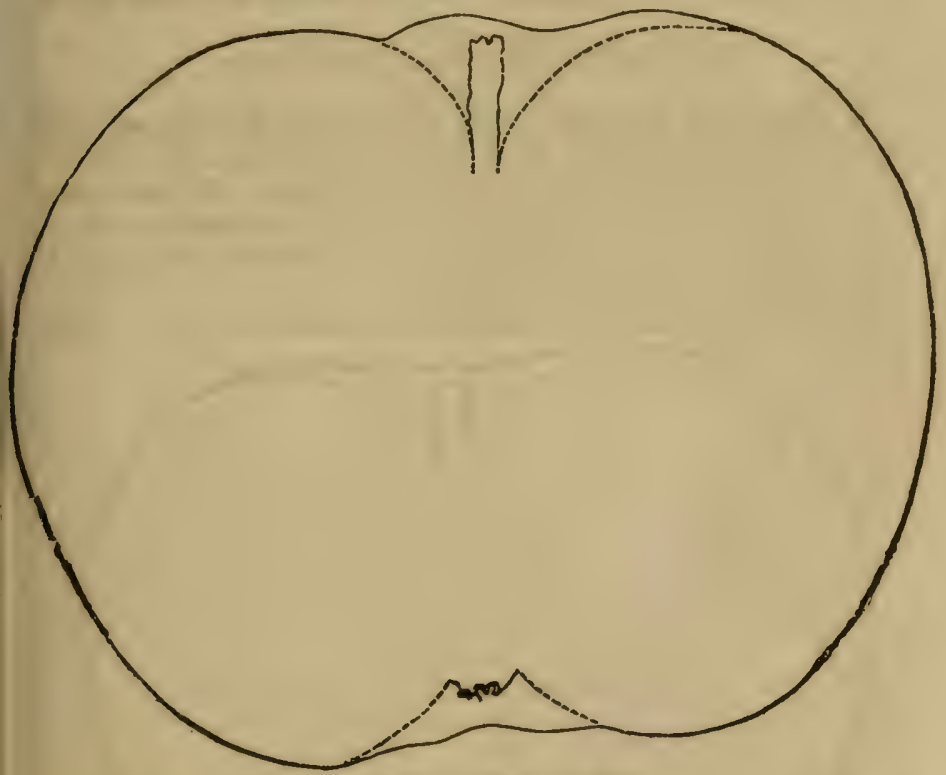

Fig. 31. Lyman's Pumpkin Sweet.

Fru:it very large, roundish, more or less furrowed or ribbed, especiai? near the stalk. Slkin smooth, pale grcen, with obscure whitish streaks near the stalk, and numerous white dots near the eye, sometimes becoming a ittle yellow next the sun. 
Stalk short, deeply sunk in a narrow cavity. Calyx rather smail, set in an abruptly sunk, rather irregular basin. Flesh white, very sweet, rich and tender, but not very juicy. Sepsember to December.

There is another Pumplin Sweeting known in this statn, which is an oblong or permain-shaped fruit, striped with yelbw and red, and ripens in $\Lambda$ ugust and September; a second rate apple.

53. Longville's Kernet. Thomp. Lind. P. Mag.

Sam's Crab.

An apple introduced into our orchards from the garden of the London Horticultural Society, but which does not compare fa. vourably with many native sorts of this season.

Fruit rather below medium size, oval, rather flattened. Stalk short, deeply inserted. Eye small, with a short erect calyx. Skin greenish yellow, streaked with pale brownish red, with a few streaks of bright red. Flesh firm, yellow, slightly perfumed, sub-acid. The tree is a great bearer. August and September.

\section{Maiden's Buush. Coxe. Thomp.}

A remarkably beautiful apple, a native of New-Jersey, and first described by Coxe. It begins to ripen about the 20th of August, and continues until the last of October. It has all the beauty of colour of the pretty little Lady Apple, and is much cultivated and admired, both for the table and for cooking. It is also very highly esteemed for drying.

Fruit medium sized, flat, and quite smooth and fair. Skir

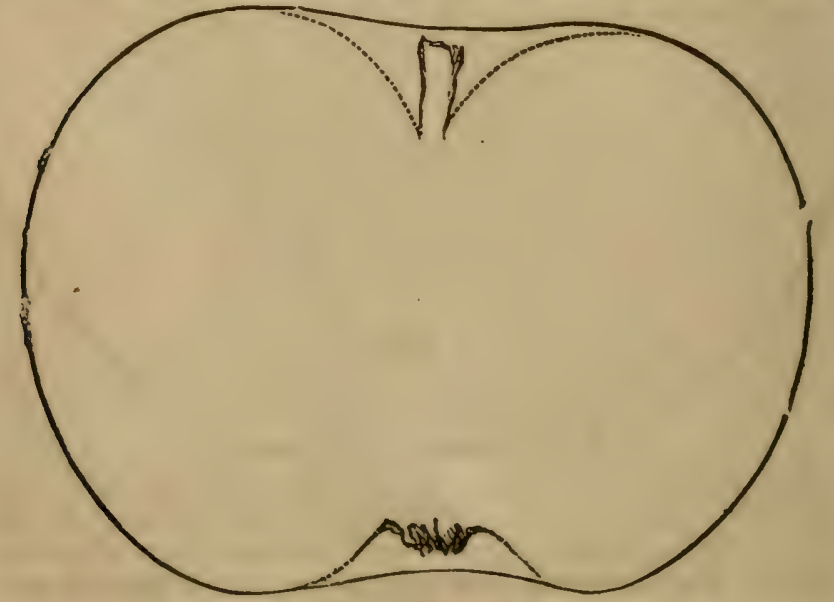

Fig. 35. Maiden's Blust. 
thin, clear lemon yellow, with a coloured cheek, sometimes ieli cately tinted like a blush, and in others with a brilliant red. Stalk short, planted in a rather wide, deep hollow. Basin mode. rately depressed, calyx closed. Flesh white, tender, sprightly with a pleasant sub-acid flavour. The fruit is very light. 'This variety forms a handsome, rapid growing tree, with a fine spreading head, and bears large crops.

\section{Nonsucr. Thomp. Lind.}

Nonsuch. Ron. Forsyth.

An old English sort, chiefiy valued for the beautiful transparent jelly which it makes.

Fruit of medium size, regular form, flat. Skin greenish yel. low, striped and spotted with dull brick red. Calyx set in a wide, regular, shallow basin. Stalk short and slender. Flesh white, soft, with a plentiful sub-acid juice. A great bearer.

\section{Ord English Codlin. Thomp.}

English Codlin. Coxe. Lind. Ray.

A large and fair cooking apple, in use from July to Novemher. Fruit generally above medium size, oblong or conical, and a iittle irregular. Skin clear lemon yellow, with a faint blish next the sun. Stalk stout and short. Flesh white, tender, and of a rather pleasant, sub-acid flavour. Much esteemed for cooking, ripens gradually upon the tree, and is free from liability to rot. In New-Jersey this fine old fruit is largely cultivated for market, as it produces handsome and abundant crops. The leaves are large, and the trees are very vigorous and fruitful.

\section{Peach-Pond Sweet.}

This is a most excellent autumn variety, from a small village of this name, in Dutchess county, N. Y., which we received from Mr. J. R. Comstock, an extensive orchardist near Poughkeepsie. It appears well worthy of a more general dissemination.

Fruit of medium size, rather flat, and a little one-sided or an. gular in its form. Skin striped light red. Stalk long and slender. Flesh tender or very mellow, moderately juicy, with a very rich,sweet, and agreeable flavour. September to Noverrber.

58. Ponme de Neige. Thomp. Lind.

Fameuse. Forsyth.

Sanguineus.

A very celebrated Canada fruit, which has its namo from the 
snow-whit? colour of its flesh, or, as some say, from the village whence it, was first taken to England. It is an excellent, pro. ductive, wutumn apple, and is especially valuable in northern latimdes.

Fruil of medium size, roundish, sonewhat flattened. Skip with a ground of pale greenish yellow, mixed with faint streaks of pale red on the shady side, but marked with blotches and short stripes of darker red, and becoming a fine deep red in the sun. Stalk quite slender, half an inch long, planted in a narrow funnel shaped cavity Calyx small and set in a shallow rather narrow basin. Flesh emarkably white, very tender, juicy and good, with a slight perfume. Ripe in October and November. A regular bearer, and a handsome dessert fruit.

\section{Porter. $§$ Man. Thomp.}

A first rate New-England fruit, raised by the Rev. S. Porter, of sherburne, Mass., and deservedly a great favourite in the Boston market. The fruit is remarkably fair, and the tree is very productive.

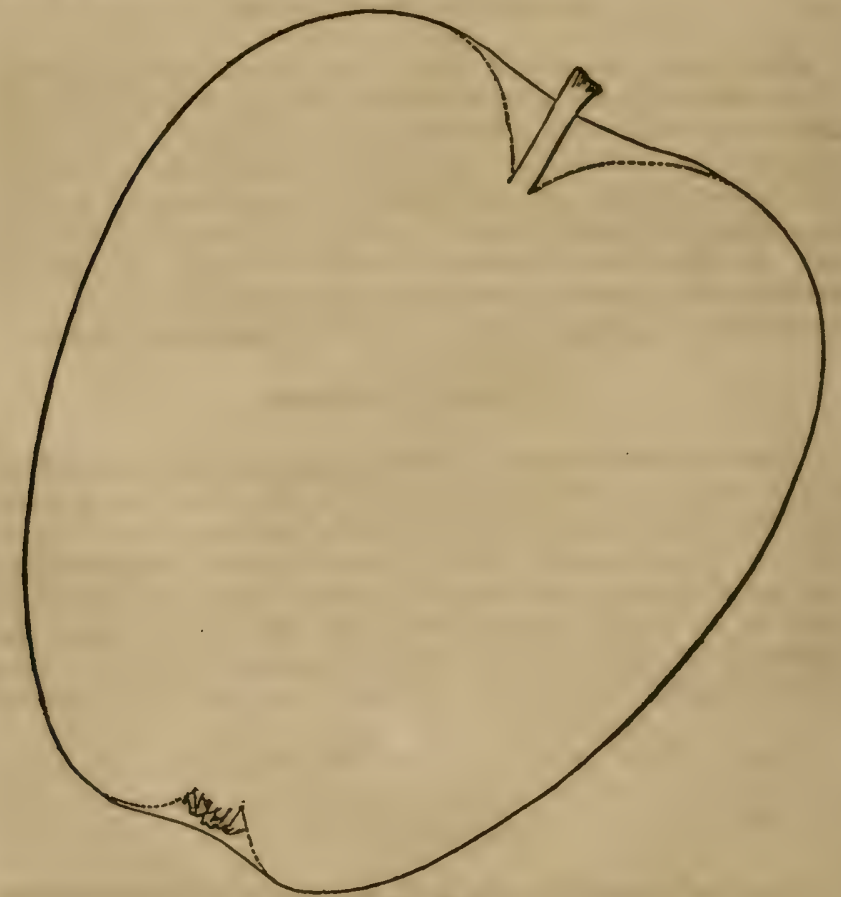

Fig. 37. Porter. 
Fruit rather large, regular, oblong, narrowing to the eye. Skin clear, glossy, bright yellow, and when exposed, with a dull blush next the sun. Calyx set in a narrow and deep basin. Stalk rather slender, not three fourths of an inch long. Flesh fine grained, and abounding with juice of a sprightly agreeable flavour. Ripens in September, and deserves general cultivation.

\section{Pine Apple Russet. Lindley.}

We have at last been able to procure this variety, and we insert Lindley's description, in order to draw attention to a sort which appears to be highly deserving of trial in this country.

"Fruit above the middle size, roundish, ovate, with broad ob tuse angles on its sides, abont two inches and three quarters in diameter, and two inches and a haif deep. Eye small, with a very short, connivent calyx, placed in a shallow depressim, surrounde: by ten rather unequal plaits. Stalk an inch long, inserted in an uneven cavity, one half of which protrudes beyond the base. Skin pale greenish yellow, almost covered with white specks on one part, and a thick scabrous, yellowish russet on the other, which extends round the stock. Flesh very pale yellow, crisp, very short, and tender. Juice more abundant than in any apple I have ever met with, as it generally runs very copiously as soon as cut open, saccharine, with that just proportion of acid which characterizes our most valuable fruits, and of a spicy aromatic flavour, with a high perfume.

A dessert apple from the end of September to the middle of October."

\section{Pumpktn Russet.}

\section{Swcet Russet. Kenrick. Pumpkin Sweet,
Flint Russet,}

This is another of the large sweet apples so popular in Now England, and is considered valuable by many. It is, however nuch inclined to rot.

Fruit large, round; fiesh, pale yellowish green, slightly covered with russet. Stalk long, set in a wide shallow cavity, Eye narrow, slightly sunk. Flesh exceedingly rich and sweet. September to Janusry. Trees large and spreading.

\section{Rムмво. $\S$ Coxe. Thomp.}

$\left.\begin{array}{l}\text { Romanite, } \\ \text { Srek-no-further, } \\ \text { Bread and Clieese Apple, }\end{array}\right\}$ of Now-Jersey.

The Rambo is one of the most popular autumn fruits to be 
found in the Philadelphia markets. It is a highly valuable apple for the table or kitchen, and the tree thrives well on light sandy soils, being a native of the banks of the Delaware.

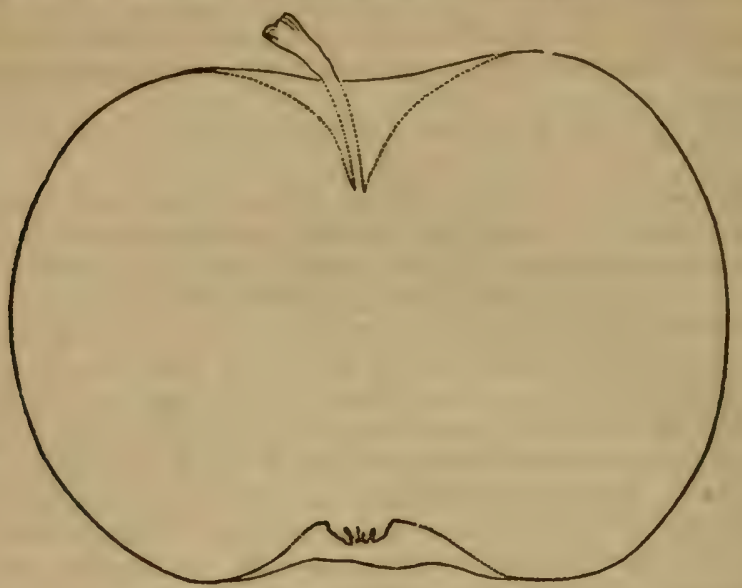

Fig. 37. Rambo.

Fruit of medium size, flat. Skin smooth, yellowish white in the shade, streaked and marbled with pale yellow and red in the sun, and speckled with large rough dots. Stalk long, rather slender, curved to one side, and deerly planted in a smooth, funnel-like cavity. Calyx closed, set in a broad basin, which is slightly plaited around it. Flesh greenish white, very tender, with a rich, sprightly, sub-acid flavour. October to December. This apple resembles externally the American Domine, which, however, is a very late-keeping winter fruit.

63. Rambour Franc. Duh. Thomp.

Frank Rambour. Lindley.

Rambour d'stri, or

Summer Rambour. Caxe.

Rambour d'Fite. Puriteau.

This is a French fruit, common in many parts of this country, and according to Coxe, was introluced from the garden of St. Cloud. It is of pretty good quality, though most esteemed for cooking.

Fruit a little above medium size, (sometimes quite large,) flat, generally evenly formed, but occasionally a little irregular. Skin pale, greenish yellow, slightly stained and streaked with red on the sunny side. Stalk short, rather firshy and deeply inserted. Eye large, the nearly closed calyx set in a deep, sliphtly furrowed basin. Flesh rather soft, of a sprightly, sub. 
acid flavour, a little bitter before maturity. Ripens eariy in September.

64 Ross Nonpareil. $\S$ Thomp. Lind. Ron.

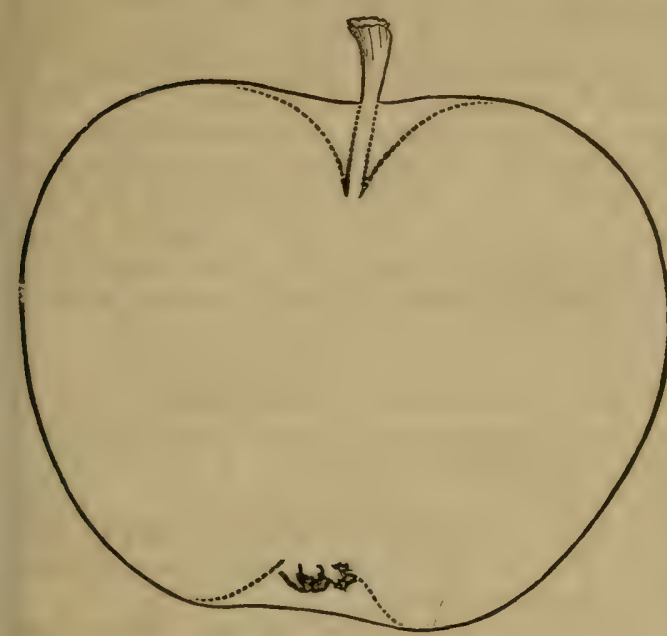

Fig. 38. Ross Nonpareil.

This is an Irish fruit, and, to our taste, one of the highest flavoured and most delicious of all apples, for the dessert, approaching in flavour some kinds of pear. In Engiand this is a winter fruit, but with us, owing io the greater warmth of our autumn, it is in perfec. tion the last of October, and will keep a month.

Fruit rather below medium size, roundish, narrowing a little to the eye. Skin covered with a thin mellow russet, and faintly stairsed with red on the sunny side. Stalk an inch or more long, slender, and rather deeply inserted. Calyx set in a shallow basin. Flesh greenish white, tender, with a rich aromatic flavour, - what is called a Fennel flavour by the English. A pro. fuse bearer, and worthy of a place in every amateur's garden.

\section{ReD Ingestrif. Thomp. Lind.}

This, and the Yellow Ingestric, are cross-bred seedlings raised by Mr. Knight, from the English Golden Pippin. 'They are greatly admired as dessert apples in England.

Fruit small, about two and a half inches in diameter, oblong or ovate, with a wide basin at the eye, and a short and slender stalk. Skin bright yellow, tinged and mottled with red on the sunny side, and speckled with obscure dots. Flesh very firm, juicy and high flavoured. Ripens in September and October.

The YELLOw Ingestrie differs from the above as follows: fruit of smaller size, of a clear, bright gold colour, without red. Eye small and shallow. Flesh tender and delicate, with a plentiful juice when freshly gathered from the tree. October. 


\section{Summer Sweet Paradise.}

A Pennsylvania fruit, sent to us by J. B. Garber, Esq., a realous fruit-grower of Columbia, in that state. It is a large, fair, sweet apple, and is certainly one of the finest of its class, for the dessert. The tree is an abundant bearer, begins to bear while young, and is highly deserving general cultivation. It has no afinity to the Paradise Apple used for stocks.

Fruit quite large, round and regular in its form, a little flattened at both ends. Skin rather thick, pale green, sometimes fainily tinged with yellow in the sun, and very distinctly marked with numerous, large, dark, gray dots. Stalk strong, and set in an even, moderately deep hollow. Flesh tender, crisp, very juicy, with a sweet, rich, aromatic flavour. Ripe in August and September.

\section{Scarlet Pearmain. Thomp. Lind.}

Bell's Scarlet Pearmain. Ronalds.

Oxford Poach of some English gardens.

A showy dessert apple, raised, according to Ronalds, by $\mathrm{Mr}$. Bell, land stewart at Sion House, the seat of the Duke of Nor. thumberland, about the year 1800 .

Fruit medium sized, pearmain or conical shaped. Skin light crimson, or yellow, in the shade, rich crimson on the sunny side; sialk nearly an inch long, deeply set. Calyx full and spreading, in a deeply sunk basin, surrounded by a few plaits. Flesh white, stained with a tinge of pink, crisp, juicy, and of good flavour. In eating from the last of August to the tenth of Octo. ber. A plentiful bearer.

\section{Seek-No-Further. Coxe.}

\section{Autumn Seek-no-further. Ken.}

This seems to be a favourite name in this country, and it is difficult to say to what variety it should be exclusively applied. The Seek-no-further of New-Jersey and Pennsylvania is the Rambo, (see Rambo;) that of some parts of New.York is the American Domine, (see the latter.) The Seek-no-further of Coxe is a large, roundish fruit, narrower at the eye. Skin smooth, pale yellowish green, or nearly white; the flesh yellow, juicy, rich and tender. The trunk straight and tall, supporting a regular well-formed head. Ripe in October, and will keep a couple of months.

The Westfield Seek-no-further is the Seek-no-further of Connecticut, and is an old and highly esteemed variety of that district. It has a Pearmain flavour, and is much superior to the 
('rreen Seek-no-further just described. Fruit large, pretty regu . larly round. Skin pale, or dull red over a pale clouded green ground-the red sprinkled with obscure russety yellow dots. Stalk very slender, three-fourths of an inch long, inserted in an even cavity. Calyx closed, or with a few reflexed segments, and set in an even basin of moderate depth. Flesh white, fine grained, tender, with a rich, pearmain flavour. A first rate fruit. October to February.

\section{Stroat. Floy. Ken.}

\section{Straat. Thomp.}

An apple in high esteem among the descendants of the Dutch settlers on the North River, the original tree of which is said to have grown in a street (stroat, Dutch) of Albany. It is well known at Kingston, N. Y.

Fruit above the middle size, regularly formed, roundish, oblong, and tapering a little to the eye. Skin smooth, yellowish green. Stem short, pretty stout, and planted in a rather shallow cavity. Flesh yellow, very tender, with an excellent, rich, brisk flavour. In eating from September to December.

70. Wormsley Piprin. $\S$ Thomp. Lind. P. Mag.

Knight's Codlin.

A well-flavoured autumnal fruit, from the English Gardens, ripening the last of August and beginning of September.

Fruit middle-sized, roundish, tapering a little towards the eye, which is deeply sunk, and the basin slightly plaited. Skin pale green, or straw colour, darker next the sun, and sprinkled with dark specks. Stalk deeply planted, nearly an inch lonis. Flesh white, crisp, firm, with a rich high flavoured juice. This is considered, abroad, one of the richest flavoured apples, but it appears to us to have been over-praised, being rather too firm and too acid.

\section{Class III. Winter Apples.}

\section{Alfriston. Thomp. Lind. Ron.}

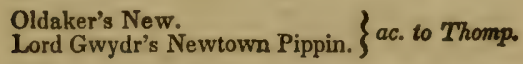

A third rate apple, valued in England as excellent for cooking. Fruit large, roundish, a little ribbed, and rather broadest at the base. Skin pale greenish-yellow, faint.y marked with 
streaks or network of russet. Stalk short, planted in a deep eavity. Calyx with open, long segments set in a deep, rather uneven basin. Flesh yellowish white, crisp, tender, with a tolerable, somewhat acid flavour. The English trace some re. scmblance between this and the Newtown pippir, but we per. ceive no similarity. October to January.

\section{American Pippin. Coxe. Thomp.}

\section{Grindstone.}

Valuable only for its late keeping and for cider, the American pippin has never been much cultivated out of New-Jersey. The Newtown pippin which is frequently called by this name abroad, is very different, and infinitely superiour to this.

Fruit of medium size, and regular form, roundish, somewhat flattened. Skin dull red in patches and stripes, or a dull green ground, marked by pretty large star-like, yellowish russet specks, which make the surface rather rough. Stalk short, somewhat fleshy and set in an irregular shallow cavity. Calyx small, set almost even with the surface of the fruit. Flesh white, firm, juicy, with a somewhat brisk, acid flavour. Keeps till June. Trees with crooked shoots.

73. BaLDwin. $\S$ Ken. Thomp. Man.

Woodpecker.

Pecker.

'The Baldwin stands at the head of all New-Fngland apples, and is unquestionably a first rate fruit in all respects. It is a native of Massachusetts, and is more largely cultivated for the Boston market than any other sort. It bears most abundantly with us, and we have had the satisfaction of raising larger, more beautiful, and highly flavoured specimens here, than we ever saw in its native region. The Baldwin, in flavour and general characteristics, evidently belongs to the same family as our Esopus Spitzenburgh, and deserves its extensive popularity.

Fruit large, roundish, and narrowing a little to the eye. Skin yellow in the shade, but nearly covered and striped with crimson, red, and orange, in the sun; dotted with a few large russet dots, and with radiating streaks of russet about the stalk. Calyx closed, set in a rather narrow, plaited basin. Stalk half to three fourths of an inch long, rather slender for so large a fruit, planted in an even, moderately deep cavity. Flesh yellowish white, crisp, with that agrecable mingling of the saccharine and acid which constitutes a rich, high flavour. The tree is a vigo. rous, upright grower, and bears most abundantly. Ripe from November to March, but with us, is in perfection in January. 


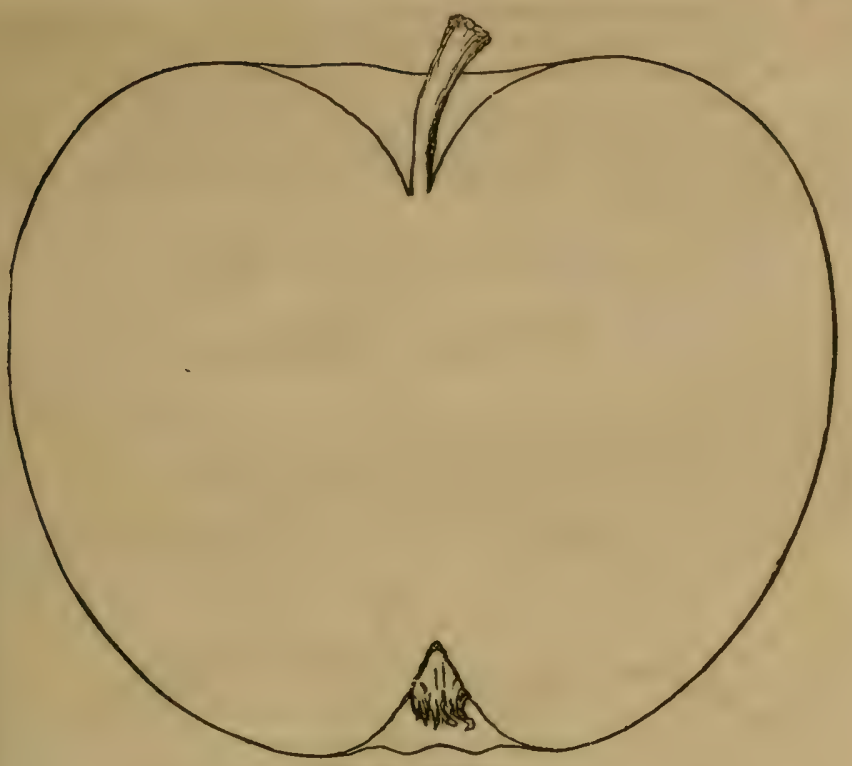

Fig. 39. Baldwin.

\section{Black Apple. Coxe.}

Black American. Thomp.?

A native fruit, of a very dark red colour, and of a mild, rather agreeable flavour.

Fruit rather below medium size, round or very slightly flattened. Skin dark red, almost black, with a mealy, whitish bloom on the surface. The stalk half to three fourths of an inch long, pretty deeply inserted. Calyx in a rather shallow basin. Flesh yellowish red, crisp, juicy, and of medium quality. The tree when fully grown has a rather drooping head. Rips from November to February.

75. Borsdorffer. Thomp. Knoop.

Borsdorff. Lind.

King George the Third. Ron.

Queen's,

Reinnette Bâtarde,

Edler Winter Borsidorffer,

Reinnette de Misnie,

Ganet Pippin,

King,

Le Grand Bohemian Borsdorffer,

A small, celebraied German apple introduced into England by Queen Charlotte. It is much admired as a dessert fruit. 
Fruit about two inches in diameter, rounàish-oval, narrowing at the eye. Skin pale yellow, with a full red cheek, sprinkled with a little russet. Calyx set in a small, even basin but little sunk. Stalk half an inch long, slender. Flesh yellowish-white, very firm and crisp, with a rich, brisk, perfumed flavour. The tree grows rather loosely, and the blossoms appear late. November to February.

76. Belle-FleUR, Yellow. $§$ Thomp.

Bell-Flower. Caxe, Floy. Ken.

Yellow Bellifower, of most nurserics.

The Yellow Belle-Fleur is a large, handsome, and excellen? winter apple, every where highly esteemed in the United States. It is most abundantly seen in the markets of Philadelphia, as if thrives well in the sandy soils of New.Jersey. Coxe first described this fruit; the original tree of which, grew in Burling.

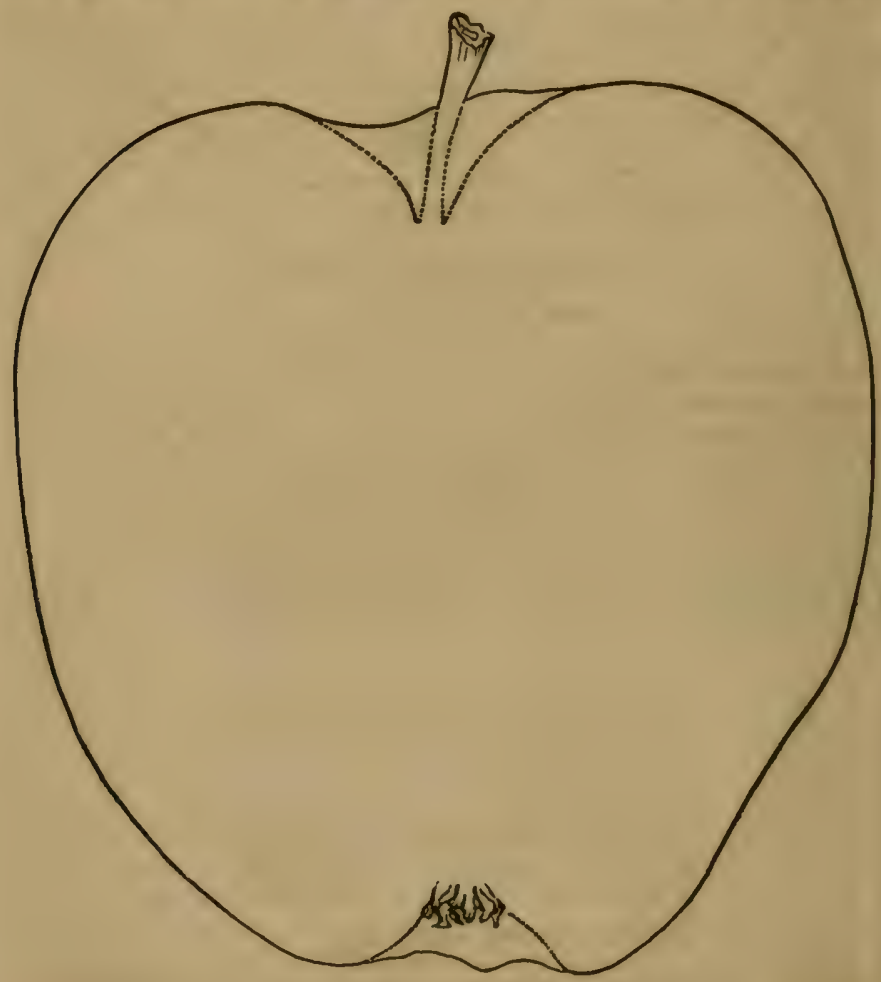

Fig. 40. Yollow belle-Ficur. 
ton, New.Jersey. We follow Thompson, in calling it Belle. Fleur, from the beauty of the blossoms, with the class of Frencli apples, to which it belongs.

Fruit very large, oblong, a little irregular, tapering to the eve. Skin smooth, pale lemon yellow, often with a blush next the sun. Stalk long and slender, in a deep cavity. Calyx closed and sel in a rather narrow, plaited basin. Seeds in a large hollow capsule or core. Flesh tender, juicy, crisp, with a sprightly, sub. acid flavour; before fully ripe, it is considerably acid. Wood yellowish, and tree vigorous, with spreading, drooping branches. A regular and excellent bearer, and worthy of a place in every orchard. November to March,

\section{Belle-FleUR, White. $\oint$}

White Bellfower. Zof Indiana, and the Green Bellitower. $\}$ North, and West.

Detroit.

White Detroit. $\}$ nati.
White Pippin.

Crivie's Pippin.

Ohio Favourite.

Hollow Cored Pippin, (of some.

The White Belle-Fleur is one of the most widely dissemina. :ed and popular apples in the Western states. It is a native, and was originally carried to the west by Mr. Brunson, a nur. seryman, who emigrated from New-York first to IIuron co., ()hio, and afterwards to Wayne co., Indiana-disseminating it

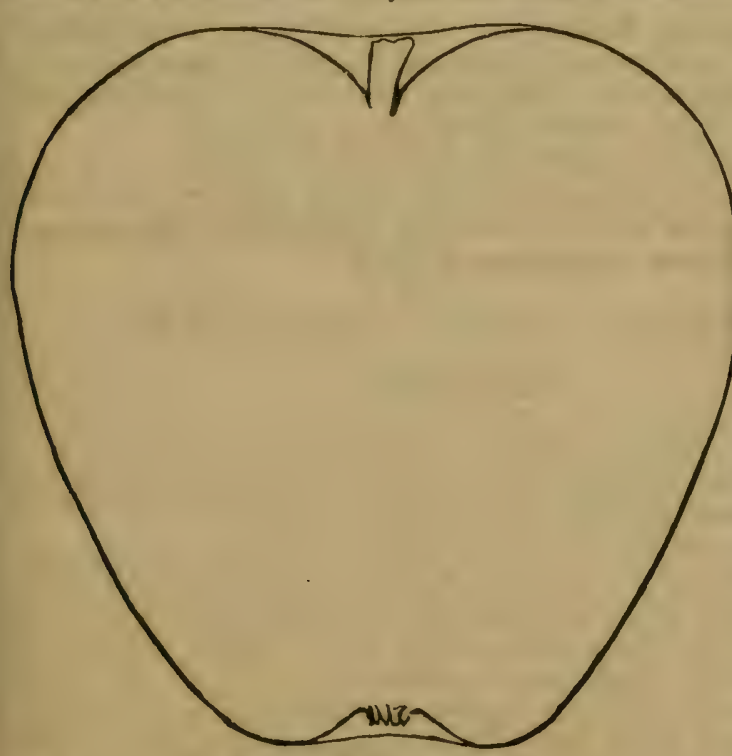

Fig. 41. White Belle-Fleur.

largely.

It grows

pretty strongly, bears ve.

ry abun. dantly, and its brittle bearing shootsare inclined to break. IIead spreading, but notdrooping.

This is a very fair and hands'me fruit, less acid than the Yellow Belle.Fleur. The Cumberland Spice, of Coxe, well 
known here, and given in previous editions as a synonym, is, we find on fartlier acquaintance with the white Belle-Fleur, an en. tirely distinct variety.

Fruit medium to large. Skin smooth, pale yellowish-white, marked with small brown dots, and rarely with a faint blush. Stalk long, slender, planted in a deep cavity. Calyx small, set in a small, narrow basin. Flesh white, tender and juicy, with a delicate, sprightly flavour. Core large and hollow. October to March.

\section{Belle-Fleur, Red.}

\section{Belle-Fleur. Poileau. Belle.Fleur Rouge? Thomp.}

f. third rate, well known, French variety, scarcely worth cultivation.

Fruit large, regular, oblong-conical. Skin pale greenishyellow, but nearly covered with red, striped with dark red, and dotted with yellow. Stalk pretty long, planted in a deep, narrow cavity. Calyx closed, sunk in a deep, narrow basin. Flesh white, tender, of tolerable, and mild flavour, apt to becorne mealy. November to January.

\section{Belle-Fleur, Brabant. Thomp. Ron.}

The Brabant Belle-Fleur is a new variety from Holland. The fruit is large and heavy, and bids fair to prove a very excellent winter apple. The habit of the tree is spreading, and it requires to be grafted high to make a good head.

Fruit large, roundish-oblong, slightly ribbed. Skin pale yellow, slightly striped with red. Calyx large, set in a pretty wide, irregular basin. Flesh firm, juicy, with a rich, pleasant, sub-acid flavour. December to April.

82. Cornisir Gilliflower. Thomp. Lind. Ron.

Cornish July-flower.

Pomme Regelans.

This is considered one of the highest flavoured apples in Eng. land, whence it comes; it is rather a shy bearer there, but we think it promises better here, in this respect.

Fruit medium size, ovate, narrowing much to the eye where it is ribbed. Skin dull green, or dark yellowish green, with a sunny side of brownish red, intermixed with a few streaks of richer red. Calyx large, set in a very narrow, furrowed or knobby basin. Stalk three fourths of an inch long. Flesh yel lowish, firm, with a rich, high flavour, and a slight perfume, re. sernbling that of the Gilliflower. November to April. 


\section{Catshead. Coxe. Lind.}

Round Catshead. Thomp.? Cathead Greening.

A very large apple, cultivated for drying in some parts of tho country, but of little other value except as a cooking apple.

Fruit of the largest size round. Stalk half an inch long, ano very deeply sunk. Calyx set in a deep, open basin. Skin quite smooth, pale green. Flesh tender, with a sub-acid juice. Oc tober and November.

\section{Calville, White Winter. Lind.}

Calville Blanche d'Hiver. Thomp. O. Duh. Noisette. White Calville. Care.

The White Winter Calville is a celebrated old French sauce and cooking apple; but like most others of its class, is not worthy of cultivation here.

Fruit large, rather flat, with the broad uneven ribs on its sides which characterize Calville apples. Skin smooth, pale greenish yellow, becoming when fully ripe, yellow, with a faint blush on one side. Calyx small, deeply set in an angular irregular basin. Stalk three fourths of an inch long, slender, deeply planted. Flesh white, large grained, tender and light, with a pleasant, third rate flavour; juice scarcely acid. A strong growing tree, and a good bearer. November to February.

85. Calville, Red Winter. Lind.

Calville Rouge d'Hiver. Thomp. Noisetle.

Calville Rouge. O. Duh.

Red Calville. Care.

The Red Winter Calville is another old French variety of the same general character as the foregoing-good for culinary use, but of very indifferent flavour.

Fruit pretty large, roundish-oblong, a little flattened at the stem, and narrowing to the cye. Stalk stout, of medium length, deeply planted. Calyx in a large deep basin. Skin on the shaded side pale red, on the sunny side dark red, covered with bloom. Flesh tender, and flavour a mild sub-acid. November to March.

86. Cos, or CaAs, Ken. Buel.

A native of Kingston, N. Y., where it is productive, and very highly esteemed.

Fruit large, one sided or angular, roundish, broad and flatten 
ed at the stalk, narrowing a good deal to the eye. Skin smooth, pale greenish yellow in the shade, but red in the sun, with splashes and specks of bright red, and a few yellow dots. Stalk very short, and rather strong, downy, deeply inserted in a wide one sided cavity. Calyx small, in a narrow, shallow basin. Flesh white, tender, with a mild, agreeable flavour. December to March.

\section{Chandler. $\oint$}

We received this fine variety, which is a great favourite in Connecticut, from the Rev. H. S. Ramsdell, of Thompson, in that state. He informs us that it originated in the town of Pomfret, Conn., (celebrated as the place of Gen. Putnam's adventure with the wolf.)

Fruit large, roundish, slightly flattened, and one-sided or angular in its form; obscurely ribbed on its sides. Skin thickly streaked and overspread with dull red, (with a few streaks of brighter red) on a greenish yellow ground; the red sprinkled with light gray dots. Stalk short, deeply sunk in a wide cavity. Calyx small and closed, set in a plaited, wide basin. Core and seeds small. Flesh greenish white, tender, juicy, with a mode. rately rich, sub-acid flavour. The tree is one of moderate vigour, and is a great bearer. November to February.

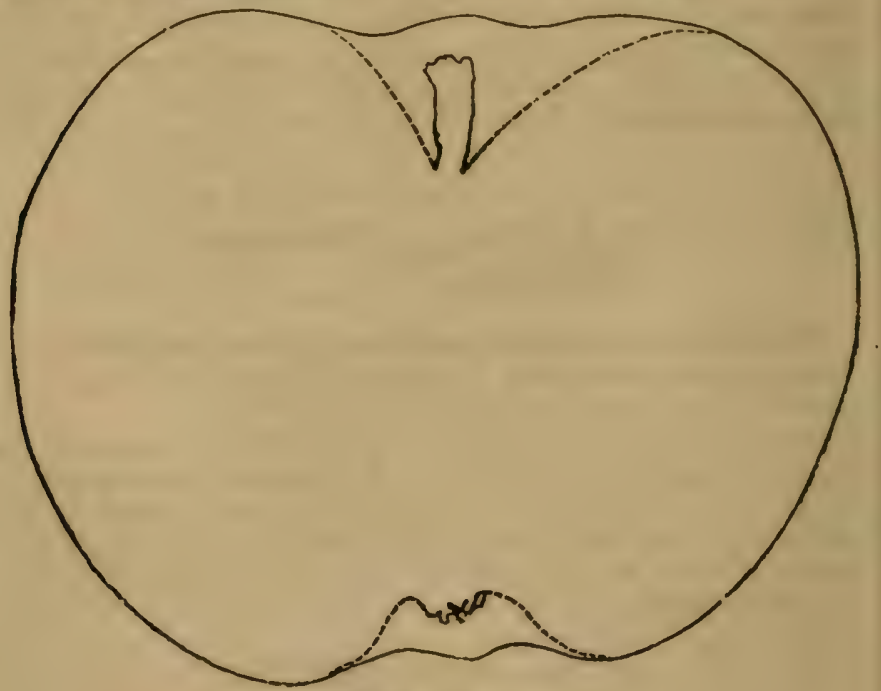

Fig 42. Chandler 
88. Court-pendu Plat. $\S$ Thomp.

Court-pendu. Lind. P. Mag. Noisette.

Court-pendu plat rougeatre. Ron.

Capendu. O. Duh.

Garnon's Apple,

Court-pendu Extra,

\begin{tabular}{l}
\multicolumn{1}{c}{$\begin{array}{l}\text { Rond Gros, } \\
\text { Rose, } \\
\text { Rouge Mus Mué, }\end{array}$} \\
$\left.\begin{array}{l}\text { Coriandra Rose, } \\
\text { Pomme de Berlin, } \\
\text { Wollaton Pippin, } \\
\begin{array}{l}\text { Russian, } \\
\text { Princisse Noble Zoete, }\end{array}\end{array}\right\} \begin{array}{c}\text { of varrous } \\
\text { European } \\
\text { collections, } \\
\text { according } \\
\text { to } \\
\text { Thompson. }\end{array}$ \\
\end{tabular}

This handsome French apple is very popular abroad, as may readily be seen by the great variety of names under which it is known in various nurseries in England, and on the continent. It thrives equally well here, and proves a beautiful acquisition to the dessert.

Fruit of medium size, regularly formed and quite flat. Skin rich, deep crimson on the sunny side, with a little pale greenish yellow in the shade. Stalk short, inserted in a very deep cavity. Calyx large, set in a wide shallow basin. Flesh yellowcrisp, with a rich, brisk, acid flavour. The tree bears young and plentifully. November to February.

This sort in England is frequently grafted on the French $\mathrm{Pa}$ radise stock, when it forms a neat little bush, not much larger than a Gooseberry, and bears an abuudance of handsome and good fruit.

89. Court of Wick. $\S$ Thomp. Ron.

Court of Wick Pippin. Lind. P. Mag.

Court de Wick. Hooker.

Rival Golden Pippin,

Fry's Pippin,

Golden Drop,

Wood's Huntingdon,

Transparent Pippin,

Phillip's Reinette,

Knighitwick Pippin,

Week's Pippin,

Yellow,

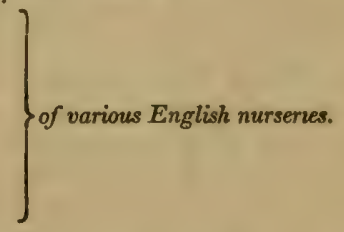

A high flavoured English dessert apple, of the Golden pippın class, which succeeds well with us.

Fruit below the middle size, regularly formed, about two and a half inches in diameter, roundish-ovate, somewhat flattened. Skin greenish yellow in the shade, but becoming a warm orange, with a little red, and dotted with small russet brown specks in the sun. Calyx with wide spread segments, and set in a wide 
even shallow basin. Stalk short, rather slender. Flesh yellow, crisp, and juicy, with a ligh, poignant flavour. October to February.

The Court of Wyck is an exceedingly hardy tree, and is therefore, well adapted for Carada or Maine.

\section{Cranberky Pippin.}

This strikingly beautiful apple we found growing on a farm near Hudson, N. Y. It is only second rate in point of flavourabout equal to the Hawthornden-but it is an excellent cooking apple, and its beautiful appearance and great productiveness, will, we think, render it a popular variety. It is not unlike in appearance a very handsome specimen of the Maiden's Blush, and it comes into use just as that sort goes out.

Fruit above medium size, very regularly formed, a little flattened. Skin very smooth, of a fine clear yellow in the shade, with a bright scarlet cheek. Stalk nearly three fourths of an inch long, slender, planted in a very even and moderately deep cavity. Calyx rather small, set in a deep, regular basin. Flesh white, moderately juicy, with a mild, sub-acid flavour. No. vember to February.

\section{Detroit.}

Red Detruit.

Black Detroit.

Black Apple.

Large Black.

Crimson Prppin.

This fruit, commonly known in Western New-York and Michigan as the Detroit, is supposed to have been brought to the neighbourhood of Detroit by early French settlers, and thence disseminated. There is little doubt that, like many other varieties grown at the west, and supposed to be indigenous there, this will yet prove to be some old variety. It is a very good fruit, of striking appearance.

There is another apple incorrectly called Detroit, or White Detroit, at Cincinnati, which is synonymous with the White Belle-Fleur. [See the latter.]

Fruit of medium or rather large size, roundish, somewhat flattened, and pretty regular. Stalk three-fourths of an inch long, planted in a deep cavity. Skin pretty thick, smooth and glossy, bright crimson at first, but becoming dark blackish pur. ple at maturity, somewhat dotted aind marbled with specks of fawn colour on the sunny side. Calyx closed, set in a rather deep, plaited basin. Flesh white, (sometimes stained with red to the core in exposed specimens,) crisp, juicy. of agreeable sprightly, sub-acid flavour. October to February. 
92. Bedfordshire Founding. Thomp. Lind.

A large green English apple, excellent for kitchen use. Fruit sarge, roundish, obscurely ribbed. Skin deep green, paler at maturity. Stalk short, deeply planted. Calyx open, rather deeply set. Flesh yellowish, tender, juicy, with a pleasant acid flavour. October to February.

92. Dutch Mignonne. § Thomp. Lind. P. Mag.

Reinette Dorée, (of the Germans.)

Pomme de Laak.

Grosser Casselar Reinette.
Paternoster Apfel.

Settin Pippin.

Copmanthorpe Crab.

This magnificent and delicious apple from Holland, proves one of the greatest acquisitions that we have received from abroad. We believe, indeed, that the Dutch Mignonne is larger and finer here than at home. At any rate we know none superior to it in superb appearance and rich flavour as an early winter fruit. The tree makes very strong upright shoots, and bears fine crops. (Hawthornden, incorrectly, of some gardens here.)

Fruit large, often very large, roundish, very regularly formed. Skin dull orange, half covered or more with rich, dull red, dotted and mottled with large yellow russet specks. Calyx open, set in a deep, round, regular basin. Stalk nearly an inch long, slender, bent, and planted in a narrow, deep cavity. Flesh at first firm, but becoming tender, with a rich, very aromatic fla. vour. November to February.

\section{Doctor. Coxe. Thomp.}

\section{Red Doctor.}

Do Witt.

A Pennsylvania apple, named in honour of a physician of Germantown, who first brought it into notice. It is not so much estepmed here at the north, as the tree is rather an indifferent prower and bearer.

Fruit mediúm sized, regularly formed and flat. Skin smooth, yellow, striped and washed with two or three shades of red, with a fow darker spots. Calyx set in a deep basin. Stalk very siort, deeply inserted. Flesh tender, juicy, and breaking in its texture, with an excellent, slightly aromatic flavour. October to January.

\section{Donine.}

This apple, extensively planted in the orchards on the Hudson, on much resembles the Rambo externally, that the two are ofter. annfounded together, and the outline of the latter fruit (see 
Rambo,) may be taken as nearly a fac-simile of this. The Domine is, however, of a livelier colour, and the flavour and season of the two fruits are very distinct,- the Rambo being rather a high flavoured early winter or autumn apple, while the Domine is a sprightly, juicy, long keeping winter fruit.

Fruit of medium size, flat. Skin lively greenish-yellow in the shade, with stripes and splashes of bright red in the sun, and pretty large russet specks. Stalk long and slender, planted in a wide cavity and inclining to one side. Calyx small, in a brrad basin moderately sunk. Flesh white, exceedingly tender and juicy, with a sprightly pleasant, though not high flavour. Young wood of a smooth, lively, light brown, and the trees are the most rapid growers and prodigious bearers that we knowthe branches being literally weighed down by the rope-like clusters of fruit.

The Domine does not appear to be described by any foreign author. Coxe says that he received it from England, but the apple he describes and figures does not appear to be ours, and we have never met with it in any collection here. It is highly probable that this is a native fruit. It is excellent from De. cember till April.

\section{Danver's Winter Sweet. Man. Ken.}

Epse's Sweet.

In Massachusetts, from a town in which this variety takes its name, it has been for a long time one of the best market applesbut we think it inferiour to the Ladies' Sweeting. It is an abundant bearer, and a very rapid tree in its growth.

Fruit of medium size, roundish-oblong. Skin smooth, dull yellow, with an orange blush. Stalk slender, inclining to one side. Calyx set in a smooth, narrow basin. Flesh yellow, firm, sweet, and rich. It bakes well, and is fit for use the whole winter, and often till April:

\section{De Saint Julien. Thomp.}

Seigneur d'Orsay.

Saint Julian. P. Mag.

This French apple of considerable reputation has not yet borne with us, and we therefore copy Mr. Thompson's descrip. tion in the Pomological Magazine, vol. iii. p. 165.

"Fruit large, roundish, slightly and obtusely angular on the sides. Eye in a moderate sized cavity, surrounded with slight plaits. Stalk slender, about an inch in length, inserted very shallow. Skin a little rough, with scars of gray russet, beneath which it is remarkably, though somewhat obscurely, striped 
with yellow and grayish green. Flesh firm, yellowish-white rich, sweet and excellent. Shoots strong, dark chestnut, mode. rately downy, with numerous distinct whitish spots. A good bearer, in perfection in December, January, and February."

\section{Easter Piprin. Thomp. Lind.}

Young's Long Keoping.

Claremont Pippin

Ironstone Pippin.

French Crab. Forsyth, (not of Caxe.)

Remarkable for keeping sound and firm two years. It is an English variety, rare with us. Fruit of medium size, skin deep green, with a pale brown blush. Stalk short, slender, deeply inserted. Calyx small, in a plaited basin. Flesh very firm. and though not juicy, of a good, sub-acid flavour.

\section{Fallawater. Thomp.}

This is a native of Pennsylvania, and was first brought irsto notice by Mr. Garber, of Columbia, Pa. It is a very good aud productive apple, with a rich flavour. Fruit rather large, regularly formed, ovate or slightly conical. Skin smooth, green, with a brown blush, dotted with large, gray spots. Stalk slender, set in a narrow, round cavity. Calyx small, closed, and placed in a smooth, narrow basin. Flesh greenish, juicy, with a rich, agreeable, sub-acid flavour. November to February.

99. Fennoullete Jaune. Thomp. Poit. Coxe.

Embroidered Pipnin. Lind.

Drap d'Or. O. Duh. No. 12. Knnoop.

Pomme de Caractére.

A beautiful, little, French dessert fruit, of that class of highly aromatic apples, which are called Fenouillets-(fennel flavour,) in France.

Fruit small, about two and a half inches in diameter, regularly formed, a little broadest at the base. Skin fine bright yellow, marked with a gray russet network, slightly resembling letiers or characters. Stalk short, deeply inserted. Calyx quite small, set in a rather small basin. Flesh white, quite firm, with a high, and peculiarly aromatic flavour. The tree rather low October to March.

100. Fenoulletet Rouge. Thomp. Poit. Lind. O. Duh.

Bardin.

Court-pendu Gris.

Fruit under medium size, between two and three inches in 
diameter, regularly formed, roundish, a little flattened. Skin grayish in the ground, but nearly overspread with dark brown. ish-red and rather rough. Stalk quite short, and sunk in a small cavity. Eye rather narrow and shallow. Flesh firm, withering a little when fully ripe, with a sugary and somewhat musklike, perfumed flavour. October to January.

\section{Fenoulliet Gris. Thomp. Poit. Nois.}

Pomme d'Anis.

A neat little Anise flavoured apple, but the tree is of too weakly and feeble a growth to be worth cultivation. Its leaves are very small and narrow, and the branches slender. The fruit is small, roundish, slightly flattened. Skin fawn-coloured russet on a yellowish ground, and rather rough. Eve quite small, in a small basin. Stalk three fourths of an inch long. Flesh firm, with a saccharine, perfumed favour. December to February.

\section{Gloria Mundi. Thomp.}

Monstrous Pippin. Caxe. Floy. Ken.

Baltimore.

Glazenwood Gloria Mundi.

New-York Gloria Mundi.

Amencan Mammoth.

Ox Apple.

This magnificently large apple is a native fruit, and we have frequently seen it weighing nearly a pound and a half, and measuring 14 inches in circumference. It is an excellent cooking apple, and, when in perfection, of a fair quality for eating; but, owing to its great weight, it blows from the tree, and is rather unproductive.

Fruit very large, roundish, rather angular, and slightly flattened at the ends. Skin smooth, greenish-white before fully ripe, when it is pale lemon yellow, becoming a little darker on one side, with very rarely a faint blush, and sprinkled with dull whitish spots imbedded under the surface. Stalk strong, deeply inserted in a large cavity. Calyx large, set in a very deep, wide basin, a little irregular, or obscurely furrowed. Core small. Flesh white, tender, with a pleasant, acid flavour. Oc. tober to January.

After a careful comparison of the fruit and wood, we do not hesitate to pronounce this synonymous with the Baltimore apple. (The Alfriston is sometimes erroneously called Baltimore.)

It is not a little curious that the origin of this apple, is claimed for Red Hook (on the Hudson,) for Long Island, and Baltimore. 


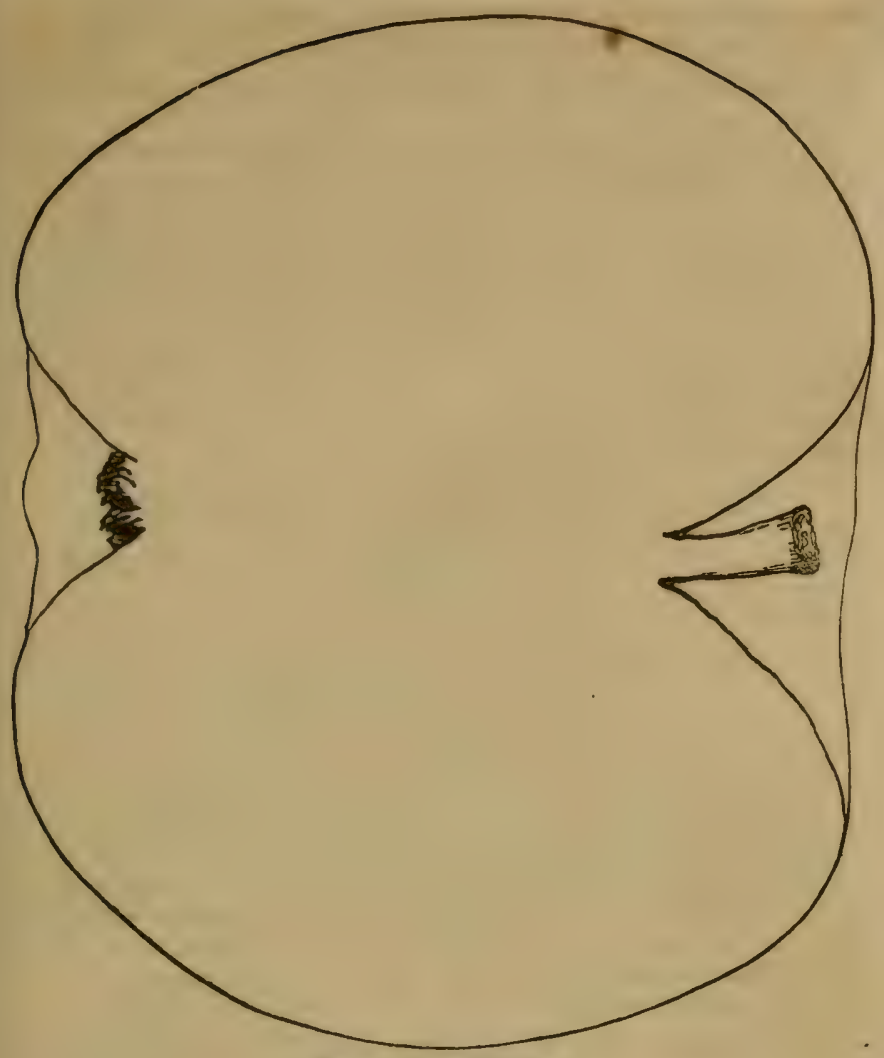

Fig. 13. Gloria Mund.

103. Golden Ball. Ren.

'This is a favourite apple in the state of Maine, and a vigorons, hardy variety. Fruit large, roundish, narrowing a little to the 'ye, about three inches deep-and a good deal ribbed at the sides and towards the crown. Skin smooth, golden yellow, with a few dots. Stalk set in a broad, shallow cavity. Eye rather narrow. Flesh crisp, tender, with a rich, aromatic flavour. Jec. to March. A native of Connecticut. Moderate bearer.

104. Golden Harvey. Thomp. Lind. Ron.

Brandy Apple. Forsyth.

An excellent, high flavoured little dessert apple from Eng 
land, which bears well, and retains its character with us. It is rather adapted for the fruit garden than the orchard-as the treo is of slender growth, and it would not be a popular market fruit here.

Fruit small, irregularly round, and about two inches in di. aneter. Skin rather rough, dull russet over a yellow ground, with a russety red cheek. Calyx small, open, with stiff seg. ments, and set in a very shallow basin. Stalk half an inch long, and rather slender. Flesh yellow, of remarkably fine texture, with a spicy, rich, sub-acid flavour. The fruit should be kept in a cellar, or it is apt to shrivel. December to April.

\section{Goj.den Pippin. Ray. Thomp. Lind.}

English Golden Pippin,
Old Golden Pippin,
Balgone Pippin,
Milton Golden Pippin,
Russet Golden Pippin,
Herefordshire Golden Pippin,
London Golden Pippin,
Warter's Golden Pippin,
Bayfordhury Golden Pippin,
Pepin d'Or. Knoop,
Pomme d'Or. Noisette o. Duh.
Koening's Pippelin.
Reinette d'Angleterre.

The Golden Pippin of the English, is the queen of all dessert apples, in the estimation of the English connoisseurs, as it unites the qualities of small size, fine form, and colour, with high flavour and durability. It is a very old variety, being mentioned by Evelyn, in 1660, but it thrives well in many parts of England still. The frolden Pippin has never become popular in this country, either because the taste here, does not run in favour of small apples, with the high, sub-acid flavour of the Golden

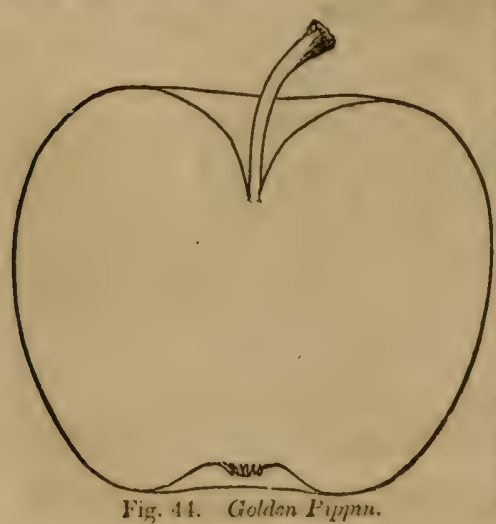

Pippin, and other favourite English sorts, or because our Newtown pippins, Swaars, and Spitzenburghs, etc., are still higher flavoured, and of a size more admired in this country. The Golden Pippin is not a very strong grower, and is rather suited to the garden than the orchard, with us.

Fruit small, round, and regularly formed. Skin gold colour, dotted with gray, russety dots, with alsa obscure white specks im 
bedded under the skin. Stalk nearly an inch long, slender. Calyx small, and set in a regular, shallow basin. Flesh yel lowish, crisp, rather acid, but with a rich, brisk, high flavour. A great bearer, but requires a strong, deep, sandy loam. No. vember to March.

There are many varieties of the English Golden Pippin, differing but little in general appearance and size, and very little in fiavour, from the old sort, but of rather more thrifty growth; the best of these are Hughes', and Kirke's new Cluster, Golden Pippins.

There are half a dozen sorts of apples which are improperly called American Golden Pippin, but we have never yet been able to find a distinct and new variety of this name. What are so termed are, usually, the Fall, or the Yellow Newtown Pippin.

106. Hoary Morning. Thomp. Lind. Ron.

Dainty Apple.

Downy.

Sam Rawlings.

A large and handsome English fruit, of good flavour, and esteemed for culinary purposes.

Fruit large, roundish, a little flattened. Skin broadly and irregularly striped with red, on a yellowish ground, and covered with a downy bloom, which gives it a somewhat hoary appearance. Calyx quite small, in a narrow, and shallow, plaited basin. Stalk of medium length, inserted in a wide depression. Flesh firm, sometimes a little pinkish next the skin, with a brisk, subacid flavour. October to December.

\section{Hubbardston Nonsuch. $\S$ Man. Ken.}

A fine, large, early winter fruit, which originated in the town of Hubbardston, Mass., and is of first rate quality. 'The tree is a vigorous grower, forming a handsome branching head, and bears very large crops. It is worthy of extensive orchard culture

Fruit large, roundish-oblong, much narrower near the eye. Skin smooth, striped with splashes, and irregular broken stripes of pale and bright red, which nearly cover a yellowish ground. The calyx open, and the stalk short, in a russetted hollow. Flesh yellow, juicy, and tender, with an agreeable mingling of sweetness and acidity in its flavour. October to January.

108. Jonathan. $\oint$ Buel. Ken.

Philip Rick.

King Philip.

The Jonathan is a very beautiful dessert apple, and its $10^{*}$ 
great beauty, good flavour, and productiveness in all soils unite to recommend it to orchard planters. The original tree of this new sort is growing on the farm of Mr. Philip Rick, of Kingston, New-York, a neighbourhood unsurpassel in the world for its great natural congeniality to the apple. It was first described by the late Jurge Buel, and named by him, in compliment to Jonathan Hasbrouck, Esq., of the same place, who made known the fruit to him. The colour of the young wond is a lively light brown, and the buds at the ends of the shoots are large. Growth rather slender, slightly pendulous.

Fruit of medium size, regularly formed, roundish-ovate, or tapering to the eye. Skin thin and smooth, the ground clear light yellow, nearly covered by lively red stripes, and deepening into brilliant or dark red in the sun. Stalk three fourths of an inch long, rather slender, inserted in a deep, regular cavity. Calyx set in a deep, rather broad basin. Flesh white, rarely a little pinkish, very tender and juicy, with a mild sprightly flavour. 'This fruit, evidently, belongs to the Spitzenburgh class. November to March.

109. Kirke's Lord Nelson. Thomp. Lind. Ron.

A large and beautiful English, early winter sort; of good quality. Fruit, about three and a half inches in diameter, roundish, and regularly formed. Skin straw colour, nearly covered with red, and washed and stained with very bright red in the sun. Calyx open, set in a pretty large and regular basin, with a few small plaits at the bottom. Stalk rather slender and short. Flesh yellowish, juicy, firm, with an agreeable, though not very high flavour.

110. Kentish Frld-Basket. Thomp. Lind. Ron.

Potter's Large Seedling Ron.

Lady de Grey's.

An immense English fruit, properly named, and much adinired by those who like great size, and beauty of appearance. The flavour is tolerable, and it is an excellent cooking apple. The tree grows strongly, and bears well.

Fruit very large-frequently four and a half inches in diameter, roundish, slightly ribbed or irregular. Skin smooth, yellowish green, in the shade, but pale yellow in the sun, with a hrownish red blush on the sumny side; slightly streaked or spot. ted with darker red. Calyx large, set in a pretty large, slightly irregular basin. Flesh tender, juicy, with a sub-acid, sprightly flavour. October to January. 


\title{
111. Lady Apple. $\S$ Coxe
}

\author{
Api. O. Duh. \\ Api Petit. Thomp. Ron. \\ Pomme Rose. \\ Pomme d'Api Rouge. Poit. \\ $\left.\begin{array}{l}\text { Petit Api Ruuge, } \\ \text { Gros Api Rouge, }\end{array}\right\}$ Nois.
}

An exquisite little dessert fruit, the pretty size and beautiful colour of which, render it an universal favourite; as it is a great. beare ${ }^{*}$ it is also a profitable sort for the orchardist, bringing the highest price of any fancy apple in market. It is an old French variety, and is nearly always known abroad by the name of Api; but the name of Lady Apple has become too uni. versal here, to change it now. No amateur's collection should be without it.

Fruit quite small, but regularly formed and flat. Skin smooth and glossy, with a brilliant deep red cheek, contrast. ing with a lively lemon yellow ground. Stalk of medium length, and deeply inserted. Calyx small, sunk in a basin with small plaits. . Flesh white, crisp, tender and juicy, with a pleasant flavour. The tree has

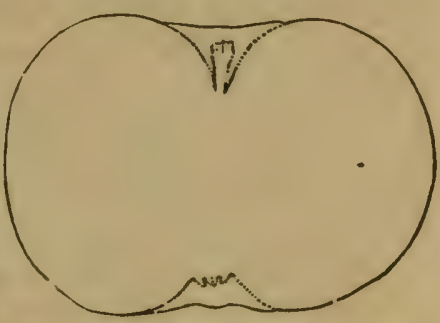

Fig. 45. Lady Apple. straight, almost black shoots, with small leaves; forms a very upright, small head, and bears its fruit in bunches. The latter is very hardy, and may be left on the tree till severe frosts. The Lady Apple is in use from December to May.

The API NoIr, or Black Lady Apple, differs from the foregoing sort only in the colour, which is nearly black. In shape, size, season, and flavour, it is nearly the same. It is, from its un. usually dark hue, a singular, and interesting fruit.

The true Apt Étollé, or Star Lady Apple, figured and described by Poiteau, in the Pomologie Française, is another very distinct variety; the fruit, which is of the same general character, but having five prominent angles, which give it the form of a star. This variety is rather scarce, the common Lady Apple being frequently sent out for it, by French nurserymen. It keeps until quite late in the spring, when its flavour becomes excellent, though in winter it is rather dry. The growth of the tree resembles that of the other Apis.

112. Lemon Pippin. Thomp. Fersyth.

Kirke's Lemon Pippin.

This is an old variety, which has been for a long time in higb 
estimation. It is, properly, an autumn sort, though it w ill kecp till January.

Fruit of medium size, and of a regular oval shape. Calyx shor and slender, set in a small, evenly formed basin. The stalk is short, fleshy, and curled round, and it grows from a small fleshy protuberance, giving the apple the form of a lemon. Skin pale green, becoming nearly lemon yellow when ripe. Flesh firm, with a brisk, and pleasant, sub-acid flavour. The tree grows prect, and produces good crops. October to January.

\section{Minister. Man. Ken.}

A very excellent New-England variety, introduced to notice by the late $\mathrm{R}$. Manning. It originated on the farm of $\mathrm{Mr}$. Saunders, Rowley, Mass. ; but was first exhibited to Mr. M. by a minister-the Rev. Dr. Spring, of Newburyport, whence its name. Mr. Manning recommended it very strongly for orchard culture.

Fruit large, oblong, tapering to the eye, around which, are a few furrows-and resembling the Yellow Belle-Fleur in outline. Skin striped and splashed near the stalk, with bright red on a greenish yellow ground. Stalk an inch long, slender, curved to one side, and pretty deeply inserted. Caly $\mathrm{x}$ small, closed, in a very narrow, plaited or furrower basin. Flesh yellowish white, very tender, with a somewhat acid, but very agreeable flavour. October to January.

\section{Male Carle. Thomp. Lind.}

Mela di Carlo.
Mela Carla.
Pomme de Charles.
Pomme Finale.
Charles Apple.

'The Male Carle is the most celebrated of all apples in Italy and the south of Europe, whence it comes. It is raised in great quantities about Genoa, and its great beauty, and delicacy of flavour, render it quite an article of commerce in the Italian and Spanish seaports. Here or in New.England, it does not always attain perfection, but south of New-York it becomes beautiful and fine, as it needs a warm and dry soil.

Fruit of medium size, very regularly shaped, and a little narrower towards the eye. Skin sinooth, with a delicate, waxen appearance, pale lemon yellow in the shade, with a brilliant crimson cheek next the sun, the two colours often joining in strong contrast. Stalk an inch long, slender, planted in a nar. row, regular cavity. Calyx set in an even, rather narrow and deep basin. Flesh white, not very juicy, but tender, and with a delicate, slightly rose-perfumed flavour. September to January. 


\section{Maclean's Favourite. Thomp.}

This is a new variety, lately received from England, which has not yet borne fruit. Mr. Thompson describes it as follows : "Middle size, roundish, yellow, crisp, rich, with the flavour of the Newtown pippin. November to February. Tree modp rately vigorous, a good bearer, of the highest excellence."

\section{Mouse Apple. $\S$}

\section{Moose Apple.}

This is an excellent, native fruit, which originated in Ulster county, on the west bank of the Hudson. It is there, one of the most popular winter fruits, being considered, by some, superiour to the Rhode Island Greening, and it deserves extensive trial elsewhere.

Fruit in weight, light; in size, large, roundish-oblong, or slightly conical. Skin, when first gathered, dull green, but when ripe, it becomes pale greenish yellow, with a brownish blush on one side, and a few scattered, russety gray dots. Stalk three fourths of an inch long, rather slender, not deeply inserted. Calyx closed, and set in a narrow basin, slightly plaited at the bottom. Flesh very white and fine grained, and moderately juicy, with a sprightly, delicate, and faintly perfumed flavour

\section{MargIL. Thomp. Lind. Ron.}

\section{Neverfail.}

Munche's Pippin.

A weil flavoured, old English dessert apple, but rather a slow grower. It is of too small size to be popular here, without greater beauty of appearance. Fruit small, a little angular, ovate, about an inch and a half in diameter. Skin orange in the sun, dull yellow in the shade, streaked and mottled with red. Calyx set in a small irregular basir. Stalk short. Flesh yellow, firm, with a high flavoured, aromatic juice. November to January.

\section{Menagère. Thomp. Man.}

We received this fruit from Mr. Manning, who, we believe. had it from Germany. It is an immense, flat, turnip-shaped apple, but, so far as we have yet tested it, with but little flavour, and only fit for cooling. Fruit very large, regularly formed, but very much flattened. Stalk short. Skin pale yellow, with sometimes a little red in the sun. Flesh tolerably juicy. Sep. tember to January. 


\section{Murphy. Man. Ken.}

This is an agreeable, Pearmain flavoured apple, strongly re. sembling, indeed, the Blue Pearmain. It is a seedling, raised by Mr. D. Murphy, of Salem, Mass. Fruit pretty large, roundish, ohlong. Skin pale red, streaked with darker red, and marked with blotches of the same colour. Calyx set in a narrow basin. Flesh white, tender, with an agreeable, rather rich flavour. November to February.

\section{Michael Henry Pippin. Coxe. Thomp.}

A New.Jersey fruit, a native of Monmouth county, first described by Coxe, and highly esteemed in many parts of the Middle States. Fruit of medium size, roundish, oblong or ovate, narrowing to the eye, smooth, and when first picked, of a dull green, resembling slightly the Newtown Pippin. Skin when ripe, of a lively yellowish green. Stalk short and rather thick. Caly $x$ set in a narrow basin. Flesh yellow, very tender, juicy, and high flavoured. The tree forms a very upright head, with pretty strong shoots. November to March.

\section{Newtown Pippin. $\S$ Coxe. Thomp.}

Green Newtown Pippin.

Green Winter Pippin.

American Newtown Pippin.

Petersbargh Pippin.

The Newtown Pippin stands at the head of all apples, and is, when in perfection, acknowledged to be unrivalled in all the qualities which constitute a high flavoured dessert apple, to which it combines the quality of long keeping without the least shrivelling, retaining its high flavour to the last. It is very largely raised in New-York and New-Jersey for exportation, and commands the highest price in Covent Garden Market, London. This variety is a native of Newtown, Long Island, and it requires a pretty strong, deep, warm soil, to attain its full perfection, and in the orchard it should bo well manured every two or three years. For this reason, while it is planted by acres in orchards in New-York and the Middle States, it is rarely raised in large quantities or with much success in New-England. On the Hudson, thousands of barrels of the fairest and richest Newtown pippins are constantly produced. The tree is of rather slender and slow growth, and even while young, is always remarlsable for its rough bark.

Fruit of medium size, roundish, a little irregular in its outline, caused by two or three obscure ribs on the sides-and 
broadest at the base, next the stalk; about three inches in di. ameter, and two and a half decp. Skin dull green, becoming oive green when ripe, with a faint, dull brownish blush on one side, dotted with small gray specks, and with delicate russet rays around the stalk. Calyx quite small and closed, set in a narrow and shallow basin. Stalk half an inch long, rather slender, deeply sunk in a wide, funnel-shaped cavity. Flesh gieenish-white, very juicy, crisp, with a fine aroma, and an exceedingly high and delicious flavour. When the fruit is not grown on healthy trees, it is liable to be spotted with black spots. This is one of the finest keeping apples, and is in eating from December to May-but is in the finest perfection in March.

122. Newtown Pippin, Yellow. $§$ Coxe. Thomp.

The Yellow Newtown Pippin strongly resembles the forego ing, and it is difficult to say which is the superiour fruit. The Yellow is handsomer, and has a higher perfume than the Green, and its flesh is rather firmer, and equally high flavoured; while the Green is more juicy, crisp, and tender. 'The Yellow Newtown Pippin is rather flatter, measuring only about two inches

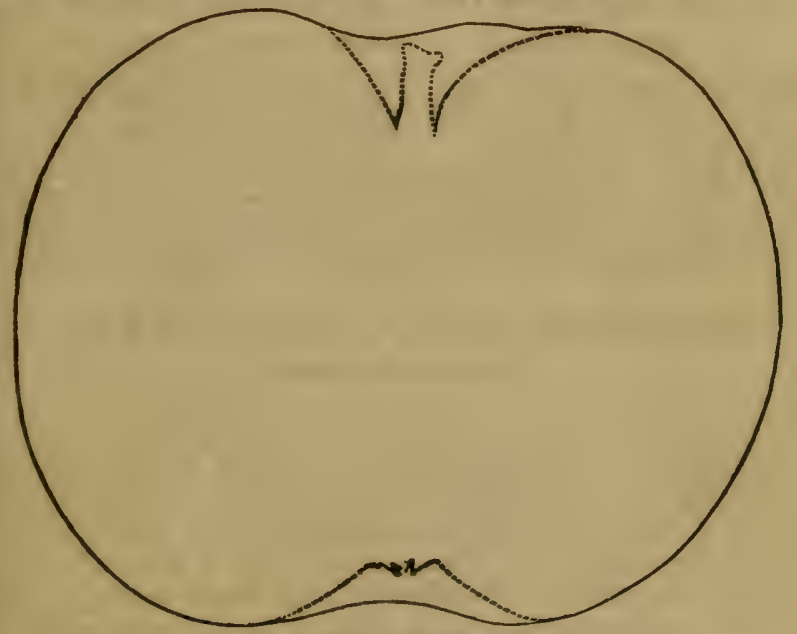

Fig. 46. Yellow Newtoun Pipjin.

deep, and it is always quite angular-projecting more on one side of the stalk than the other. When fully ripe, it is yellow, with a rather lively red cheek, and a smooth skin, few or none of the spots on the Green variety, but with the same russet marks at the stalk. It is also more highly fragrant before, and after, it is cut than the Green. The flesh is firm, crisp, juicy, and with a very rich and high flavour. Both the Newtown pippins grow 
alike, and they are both excellent bearers. This variety is rather hardier and succeeds best in the eastern states. We have kept the fruit until the 4 th of July.

\section{Northern Spy.}

This beautiful new American fruit is one of the most delicious, fragrant, and sprightly of all late dessert apples. It ripens in January, keeps till June, and always commands the highest market price. The tree is of rapid, upright growth, and bears moderate crops. It originated on the farm of Oliver Chapin, of Bloomfield, near Rochester, N. Y.

Fruit large, conical-flattened. Skin thin, smooth, in the shado greenish or pale yellow, in the sun covered with light and dark stripes of purplish-red, marked with a few pale dots, and a thin, white bloom. Stalk three-fourths of an inch long, rather slender, planted in a very wide, deep cavity, marked with russet. Calyx small, closed; basin narrow, abrupt, furrowed. Flesh white, fine-grained, tender, slightly sub-acid, with a peculiarly fresh and delicious flavor.

\section{Nonpareil, Old. Lang. Lind. Thomp.}

\section{English Nonpareil. Non Pareille. O. Duh.}

The Old Nonpareil is a favourite apple in England, but it is little esteemed in this country. November to January.

Fruit below medium size, roundish, a little ovate, and flattened. Skin greenish-yellow, thinly coated with pale russet. Stalk slender, an inch long. Calyx small, set in a narrow, round basin. Flesh firm, crisp, with a rich, acid, poignant' flavour.

124. Nonpareil, Scarlet. Thomp. Lind. Ron.

New Scarlet Nonpareil.

A handsomer and larger variety of the foregoing. Fruit of medium size, roundish, two and a half inches in diameter, and half an inch less in depth-regularly formed. Skin, in the sun deep red, sprinkled with brownish gray dots on a ground of yellowish green, slightly streaked. Calyx set in a regularly formed, shallow basin, with a few small plaits. Stalk nearly an inch long, and rather stout. Flesh firm, yellowish-white, with a rich, acid juice. The tree is a much stronger grower than the old sort. November to February.

125. Norfolk Beaufin. Thomp. Lind.

Read's Baker.

Catshead Beaufin.

Chiefly valued for drying In Norfolk, England, quite a 
trate is carried on in the dried fiuit of this apple-which is also in high esteen? for preserves, and all kitchen uses.

Fruit large, flat, a little irregular in outline. Skin dark dingy red, or copper colour, on a greenish ground. Stalk ialf an inch lone, fleshy, deeply sunk. Calyx set in an irregular, plaited basin. Flesh firm, of poor flavour, with a sub-acid juice. November to May. A great bearer.

\section{Newark King. Coxe. 'Thomp.}

Hinckman.

A new-Jersey fruit, of medium size, conical or Yearmam. sinaped, and of handsome appearance. Skin smooth, red, with a few yellow streaks and dots, on a greenish yellow ground. Calyx set in a narrow basin. Flesh tender, with a rather rich, pleasant flavour. The tree is spreading, and bears well. November to February.

\section{Newark Pippin. Coxe.}

\section{French Pippin.
Yellow Pippin. of some Amercan gardens.}

A handsome and very excellent early winter variety, easily known by the crooked, irregular growth of the tree, and the drooping habit of the branches.

Fruit rather large, roundish-oblong, regularly formed. Skin greenish yellow, becoming a fine yellow when fully ripe, with clusters of smill black dots, and rarely a very faint blusin. Calyx in a regular and rather deep basin. Stalk moderately long, and deeply inserted. Flesh yellow, tender, very rich, juicy, and high flavoured. A very desirable fruit for the ama. teur's garden. November to February.

128. Pearimain, Herefordshire. § Thomp.

Winter Pearmain. Care.

Roy, 1 Pearmain. Lind. Ron.

Parmin Royal. Kroop.

Old Pearmain.

Royale d'Angleterre.

This delicious old variety, generally known here as the English or Royal Pearmain, is one of the fincst of all winter dessert firuits, and its mild and agreeable flavour renders it here, as abroad, an universal favourite, both as a dessert apple, and for cooking.

Fruit of medium size, oblong, and of a pretty regular Pearmain-shape. Skin stained, and mottled with soft, brownish red on a dull, russety green ground, dotted with grayish specks. The red thickly mottled near the eye, with yellowish russet spots. 
Stalk slender, half an inch long. Calyx with wide-spread, re. flexed segments, and set in a shallow, narrow, slightily plaited basin. Flesh pale yellow, very mollow and temter, with a nleasant, aromatic flavour. A moderate bearer, but often pro.

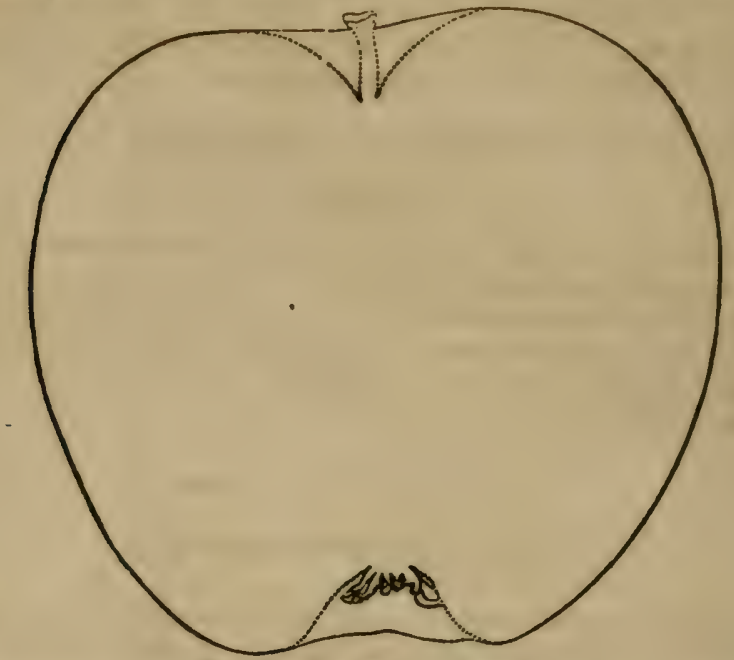

Fig. 47. Herefordshire Pearmain.

duces large crops on light soils, which are well ndapted to this sort. November to February. A strong grower.

The Winter Pearmain of most American orchards, is the Au. tumn Pearmain of this, and most English works.

129. Pearmain, Blue. $§$ Man. Ken. Thomp.

'The Blue Pearmain is a large and very showy fruit, and is therefore popular in the New-England markets. The nume. rous large russetty yellow dots which are sprinkled over the skin, and the bloom which overspreads it, mark this apple.

Fruit of the largest size, roundish, regularly formed, very slightly conical. Skin covered with stripes and blotches of dark purplish-red, over a dull ground-and appearing bluish from the white bloom. Stalk short, slender, sunk in a deep hollow, rather uneven. Calyx small, pretty deeply sunk in an even basin. Flesh yellowish, mild, rather rich and good. The tree grows strongly, and bears moderate crops. October to February.

130. Pearmaix, Claygate. Thomp. Lind.

This is a new kind of Pearmain, lately received from Eng. land, which has not yet fruites witb us, but bears the highes charartar abrond. 
Fruit of meduum size, and Pearmain shape. Skin \{rreenish. yellow, nearly covered with brownish red. Flesh yellow, tender, with a very rich, aromatic "Rihston pippin llavour." 'The tron is very laardy. November to March.

\section{Pearifain, Adans. Thomp. Lind.}

\section{Norfolk Pippin.}

'The Adams' Pearmain is a handsome variety, which stands high in England, but, as yet, does not hold its charact:r with us.

Fruit above medium size, of a roundish, Peaserain-shape. Skin pale yellow, with a few stripes and patches or salmon red and yellow, on the sunny side, and dotted with white specks near the stalk-and slightly touched with russet. Stalk three fourtlis of an inch long, rather slender. Calyx "losed, and set in a narrow basin, slightly plaited. Flesh yellow ish, quite firm and crisp, with a brisk, sub-acid, and rather rich flavour. November to February.

\section{Pearmain, Sweet. $\S$}

A handsome, dark red, sweet apple, of the Pearmain class, of very saccharine flavour, and much esteemed in some plarts of

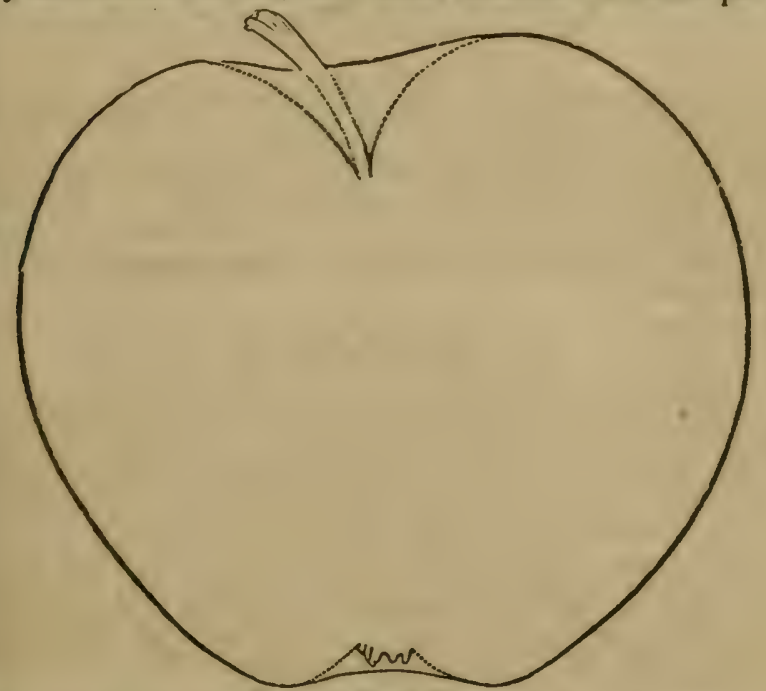

Fig. 48. Siuce Pexurmuin.

the eastern states for baking and eating. It has long ben cul tivated near Ilartford, and also in Rhode Island, and was intro duced from England be.ore the revolution. 
Fruit of redium size, and roundish Pearmain shape. Skin fine lask red, with rough russet dots, and envered with a bluish

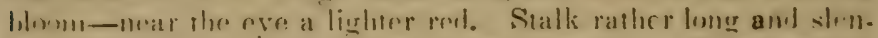
d.r. herply sulk in a wide limmel-shaped carils. (aly wooliy, su' in a very shallow and narmw basin. Flish tender, mode rately juicy, and very sweet and rich. December.

\section{3:3. Piradise, Wixter Sweet.}

The Winter Sweet Parailise is a very proluctive and excel. Ierit orchard fruit, always fair, and of fine appearance. $\mathrm{We}$ received it snme years agn, alonge with the Summer Swret Parallise, from Mr. Ciarber, of Columbia, I'a., and consider it a native lruit.

Fruit rather larere, recrularly formed, roundish. Skin fair and smonth, dull green when picked, wish a bownish blush, becoming a linte paler at maturity. Stalk short, set in a round eaviry. Calyx small, hasin shallow and narrow. Flesh white, fine grained, juicy, sweet, sprightly, and very good. November to March.

\section{Ponine Grise.}

Grise. Thomp.

Gray Apple.

A small qray applo, from Canarla, and undoubtedly one of the fincsi dessrit apples for a morihern elinute. It is not a strong grower, but is a grond hearer, and has an excellent flayeur.

Fruit below medium size, roundish, somewhat flattened Skin greenish gray or russet, with a little rod towards the cye. Calyx small, set in a round basin. Flesh tender, rich, and high flavoured.

\section{Pound Royal. $\S$}

A charming winter apple, as yet only known in Connecticut, but deserving extensive cultivation. We have this sort from the Kov. Mr. Ransdell, of that state, who informs us that the oldest known trees are growing on the Putnam estate, in Pomfret, Comn. It is not unlikely from the name by which it is generally known, that it may be of French origin,-cither introduced as a young tree, or raised from seeds given Gen. Putnam by the French officers of his acquaintaluce, during the war. The treed nie vigorvus growers, and abundant bearers. 
Fruit large, roundish-oblong, with a slightly uneven surface-ani sometimes an obscure furrow on one side. Slin pale yel. lnwish-white, rarely with a faint blush, and marlied when ripe with a f: w large rucidy or dark epcclis. Stalk an inch and a quarter long, slender, rather recply inserted. Calyx set in a furrowed, irregular basin. Flesh very tender, breaking, fine graincel, with a mild, agrceable, sprightly favour. Seeds enlosed in a hollow chamber. In use from December to April. 'This is listinct from the Pomme Royale (p. 83).

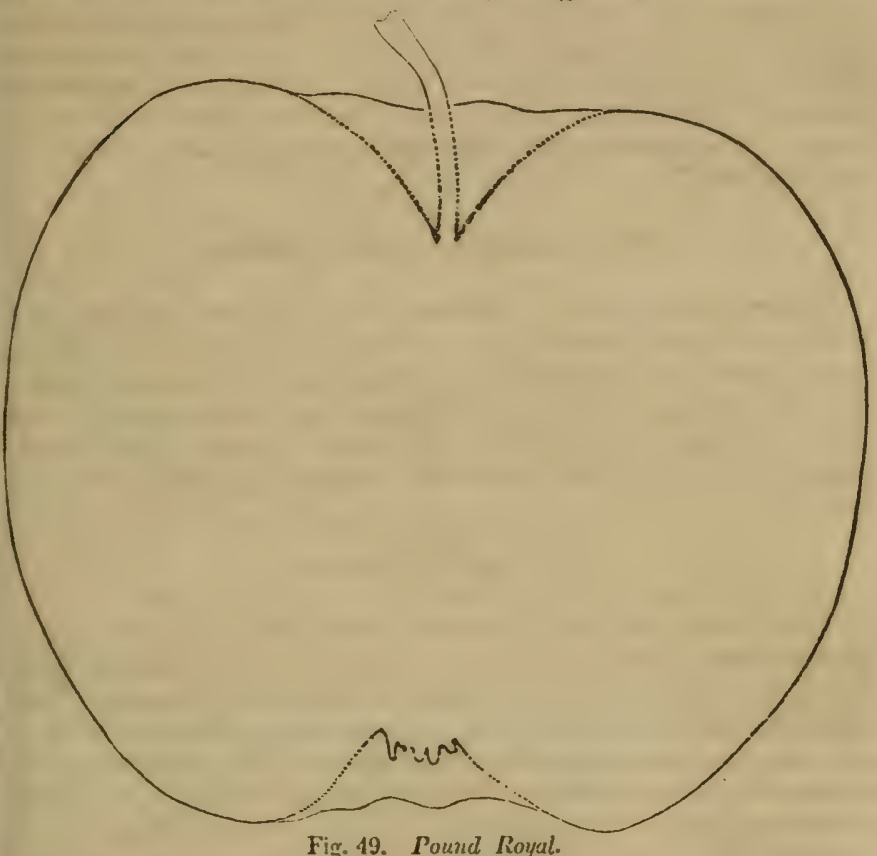

135. Pexyock's Red Winter. 'Thomp.

I'cunock. C'oxe.

This is a Ponnsylvania fruit of enos quality for the table, and an rxcellent lakiner apple. Cnformuatrly is is, of late, so liable to the bitter-rot, that it is scarcely worth cultivation.

Fruit quite large, angular or nue-sided, generalls fat,

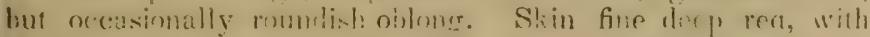
faint, indistinct strals of ycllow, and a fitw black spreks. Stalk short. Flesh yollow, tonder and juicy, with a pleasint, sweet flavour. The tree is lare, makes a firm, spreading bead, and is a regular bearer. November to March. 


\section{Priestry. Coxc. Thomp.}

I'riestley's Imerican.

Inother native of the same state as the foregoing varicty, anc named, like it, afte: the cultivator who first brought it into no. tiee. This sort has a pleasant, spicy flavour, and is much es. teemed for eating and cooking.

Fruit large, roundish-oblong. Skin smooth, dull red, with small streaks of yellowish green, dotterl with greenish specks. Siall of molium length, and inserted in a round, pretty deep cavity. Flesh white, moderately juicy, with a spicy, agreeable navour. The foliage is large, and the tree, which is a hand. some upright grower, bears well on light salidy soils. Decem. ber to March.

\section{Pearson's Plate. Thomp.}

A new variety, lately received from England, and not yet well tested here, but which has a very high reputation. Fruit small, about two and a half inches in diameter, regularly form. ed, that. Skin greenish-yellow, becoming yellow, with a little red in the sun. Flavour first rate in all respects. Mr. Thom. son says this is a good bearer, and a remarkably handsome ${ }^{\text {les. }}$ sert fruit.

\section{Peck's Pleasant.}

A first rate fruit in all respects, belonging to the Newtown pippin class. It has long been cultivated in Rlode Lsland, where we think it originated, and in the northern part of $C_{\text {inn. }}$ necticut, but as yet is little known out of that district of country, hut deserves extensive dissemination. It considerably resembles the Yellow Ne.rtown pippin, though a larger fruit, with more tender flesh, and is scarcely inferiour to it in flavour.

Fruit above merlium size, roundish, a little angular, and slightily flatened, with an indistinct furrow on one side. Slin smooth, a- when first gathered, green, with a little dark red; but when ripe, a beautiful clear yellow, with bright blush on the sunny side and near the stalk, marked with scattered gray dots. The stalk is peculiarly fleshy and fattened, short, and sunk in a wide, rather wavy cavity. Calyx woolly, sunk in a narrow, abruply, and prelty deeply sunk basin. Flesh yeilnwish, fine grained, juicy, crisp and tender, with a delicious, high aromatic flavour. The tree is only a moderate grower, but bears regu. larly and well, and the fruit conımands a high price in market. Mr. S. Lyrnan, who raises this fruit in great perfection, informs us that with him the apples on the lower branches of old trees 
are flat, while those on the upper branches are nearly conical. November to March.

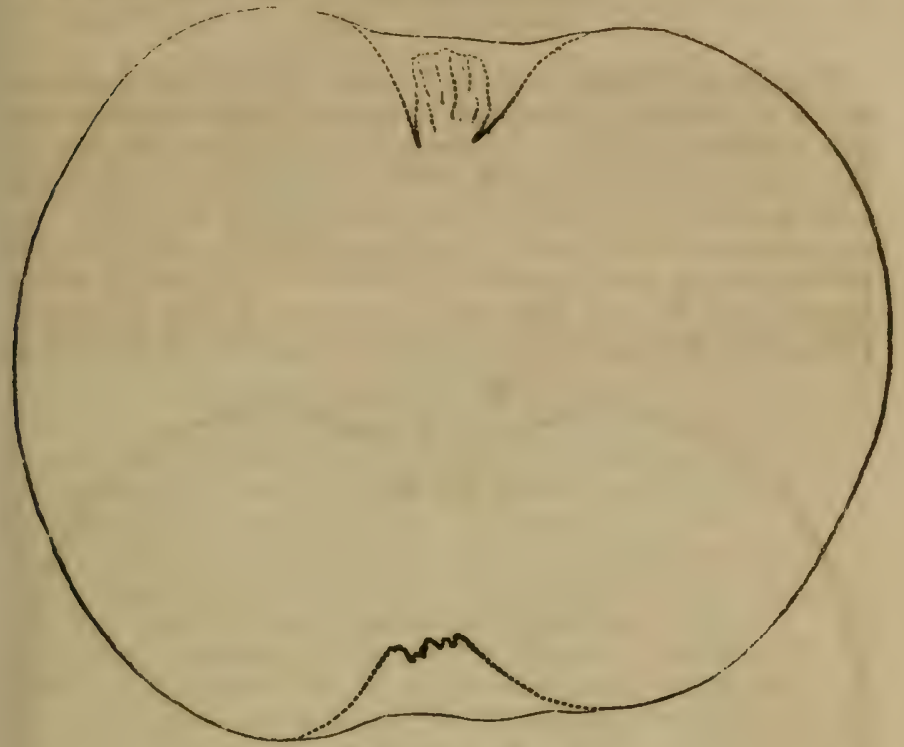

Fig. 50. Peck's Pleasant.

139. Pennington's Seeding. Thomp. Lii d.

This is a new russet variety from England, which, we think, will prove a valuable one.

Fruit of medium size, nearly flat, a little angular, and broariest at the base. Skin mostly corered with rough yellow russet, with a little pale brown in the sum. Stalk three fourths of an inch lıng, pretty stout, planted in a wide, irregular cavity. Calyx with long segments, set in a rather shallow, wavy basin. Flesh yellowish, firm, crisp, with a brisk, high flavoured, acid juice. November to March.

\section{Pound. Coxe. Thomp.}

$\Lambda$ very large and showy fruit, but of very indifferent quality, and not worth cultivation where better sorts are to be had. The fruit is roundish-chiong, striped with red, on a dull greenish ycllow grourd. The stalk short, and deeply inserted. The fesh yellowish green, and without much tlavour. October to January. 
141. Rhode Island Greening. Coxe. Thomp. Man.

\section{Burlington Greening. \\ Jersey Greening? Care.}

The Rhode Island Greening is such an universal favourite; and is so generally known, that it seems almost superfluous to give a description of it. It succeeds well in almost all parts of the country, and on a great variety of soils, and is, perhaps, more generally esteened than any other early winter fruit. In the eastern states where the Newtown pippin does not attain full perfection, this apple takes its place-and in England, it is fre. quently sold for that fruit, which, however, it does not equal. [The Green Newtown Pippin described by Lindley is this fruit.]

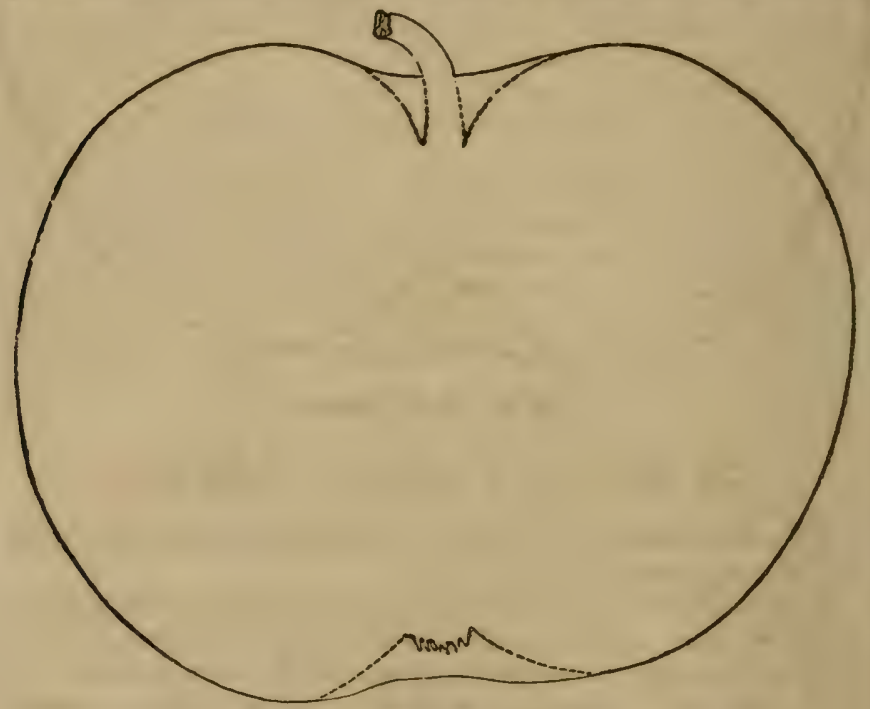

Fig. 51. Rhode Island Greening.

Fruit large, roundish, a little flattened, pretty regular, bul riften obscurely ribbed. Skin oily smooth, dark green, becom. ing pale green when ripe, when it sometimes shows a dull blush rear the stalk. Calyx small, woolly, closed, in a slightly sunk, ccarcely plaited basin. Stalk three fourths of an inch long, r.urved, thickest at the bottom. Flesh yellow, fine graineri, tenler, crisp, with an abundance of rich, slightly aromatic, lively, icid juice. The tree grows very strongly, and resembles the Yall pippin in its wood and leaves, and bears most abundant rrops. The fruit is as excellent for cooking, as for the dessert. November to February-Or, in the north, to March. 


\section{Reinette, Canada. Thomp. Nois.}

Canadian Reinette. Lind.

Grosse Reinette d'Angleterre. O. Duh.

Pomme du Caen.

Reinette du Canada Blanche. of various

Reinette Grosse du Canada. Eiropeun

Reinette du Canada à Cortes. collections

De Bretagne.

Portugal.

Januarea.

Wahr Reine:te.

It is ensy to see that the Canada Reinette is a popular and highly esteemed variety in Europe, by the great number of syn. onyms under which it is known. It is doubtful, notwithstanding its name, whether it is truly of Canadian origin, as Merlet, a French writer, describes the same fruit in the 17th century; and some authors think it was first brought to this continent from Normandy, and carried back under its new name. At any rate, it s a very large and handsome fruit, a good bearer, and of excellent quality in all respects. It is yet little known in the United States, but deserves extensive orchard culture.

Fruit of the largest size, conical, flattened; rather irregular, with projecting ribs ; broad at the base, narrowing towards the eye, four inches in diameter, and three decp. Skin greenishyellow, slightly washed with brown on the sunny side. Stalls chort, inserted in a wide hollow. Calyx short and larre, set in a rather deep, irregular basin. Flesh nearly white, rather firm, juicy, with a rich, lively, sub-acid flavour. Ripe in Decembes. and, if picked early in autumn, it will keep tili A pril.

143. Reinetre, Golden. 'Thomp. Ron. Lind.

\begin{tabular}{|c|c|}
\hline $\begin{array}{l}\text { Anrore. } \\
\text { Kirke's Golden Reinette. } \\
\text { Fellow German Reinette. } \\
\text { Reinette d'Aix. } \\
\text { English Pippin. } \\
\text { Court-pendu Doré. } \\
\text { Wyker Pippin. } \\
\text { Elizabet. } \\
\text { Wygers. } \\
\text { Niegginch Favourite. } \\
\text { Dundee. }\end{array}$ & $\begin{array}{c}\text { of various } \\
\text { Europeran } \\
\text { collections, } \\
\text { ac: to } \\
\text { Thomp. }\end{array}$ \\
\hline
\end{tabular}

The Golden Reinctte is a very popular dessert fruit in Eng. lund and oll the continent, combining beauty and high flavour It is yet but little known here.

Fruit below medium size, very regularly formed, roundish, a liule flattened. Skin smnoth, greenis's__breoming golden yel. low in the shade, washed and striped with fine soft red, on the 
sunny side, mingled with scattered, russet dots. Stalk long, anr! inserted moderately deep. Calyx large, set in a broad, but shallow basin. Flesh yel'nw, crisp, with a rich, sugary, or scarcely acid juice. October to January.

This is ditlerent and superiour to the Reinette Dorce, or Jaune Iative of the French, which is more yellow, and somewhat re. sembles it.

\section{Reinette Blanche d'Espagne. Thomp. Nois.}

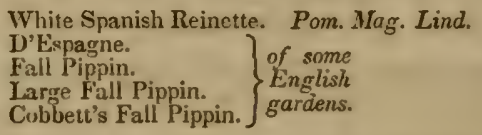

A very celebrated old Spanish variety, which is said to be the national apple of Spain, where it is called Cameusar. Notwithstanding that Thompson and other English authorities consider this apple the same as our Fall Pippin, we are yet strongly of opinion that it is different. The true Fall Pippin is only an autumn variety, while this is a winter sort, keeping till midwinter here, and in Enrrland till March. It is quite probable that the Whire Spanisin Reinette is the parent of both the Fall and Holland Pippins. 'The fruit of the present varicty is rather more oblong than that of the Fall Pippin.

Fruit very large, roumlish-ob?ong, somewhat angular, with broad rits on its sirles, terminating in an uneven crown, where it is nearly as lumed as at the base. Calyx large, open, very docply sunk in a broal-angled, oblique, irreguiar basin. Stalk ha!f an inch lonk, set in a rather small, even cavity. Skin smorth, yellowish-green on the sharled side, orange, tinged with brownish-red rext the sun, and sprinkled with l.ackish dots. Flesh yellowish-white, crisp, tender, with a sugary juice. Noisette, (Jardin Fruitier) adds, "the skin is covered with a bloom, like that on a plum, which distinguishes this variety from all those most resenbling it." The tree has the same woorl, foliage, and vimnrous habit, as our Fall Pippin, and the fruif lieeps from November to February, or March.

\section{Reinette Trtomphante. M. Christ.}

Victorious Reinette.

A German early winter apple, which we have recently re. crived, and which has only borne once in this country.

Fruit large, oblong, regularly formed. Skin paie yellow, thickly dotted with white specks, and rough, projecting warts. Flesh ycllow, firm, juicy, with a pleasant aromatic flavour. The tree is of thrifty growth, and is said to bear well. 
146. Ribston Pippin. Thomp. Lind. Ron.

Glory of York.

Travers'.

Formosa Pippis.

The Ribston Pippin, a Yorkshire apple, stands as high is Cr. eat Britain as the Bank of England, and to say that an apple ha i a Ribston flavour is, there, the highest praise that can be bestowed. But it is scarcely so much esteemed here, and must be content to give place, with us, to the Néwtown Pippin, the Swaar, the Spitzemberg, or the Baldwin. In Maine, and parts of Canadn, it is very fine and productive.

Fruit of medium size, roundish. Skin greenish-yellow, mix. ed with a little russet near the stalk, and clouded with dull red on the sunny side. Stalk short, slender, planted in a rather wide cavity. Calyx small, closed, and set in an angular basin. Flesh deep yellow, firm, crisp, with a sharp, rich, aromatic fla. vour. The tree forms a spreading top. November to February.

\section{Roman Stem. Coxe.}

The Roman Stem is not generally known out of New-Jersey. It originated at Burlington, in that state, and is much esteemed in that neighbourhood. In flavour, it belongs to the class of sprightly, pleasant apples, and somewhat resembles the Yellow Belle Fleur. Tree very productive.

Fruit scarcely of medium size, roundish-oblong-or often ovate. Skin whitish-yellow, with a faint brownish blush, sprinkled with patches of small black dots, and, when ripe, having a few reddish specks, unless the fruit is very fair. Stalk three-fourths of an inch long, inserted in a shallow cavity, under a fleshy protuberance, which the farmers have likened to a Roman nose, whence the name. Calyx set in a rather narrow basin, with a few plaits. Core hollow. Flesh tender, juicy, with a sprightly, agreeable flavour-not first rate. November to March.

\section{Russet, American Golden. \&}

Golden Russet. Man. KEn.

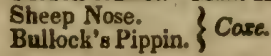

The American Golden Russet is one of the most delicious and tender apples, its flesh resembling more in texture that of a but. tery pear, than that of an ordinary apple. It is widely cultivated at the west, and in New.England as the Golden Russet, and though neither handsome, nor large, is still an universal favourite from its great productiveness and admirable lavour. The 
uncouth name of Coxe, Sheep-nose, is nearly obsolete, except in New.Jersey, and we therefore adopt the present one, to which it is well entitled. The tree is thrifty, with upright drad coloured shoots.

Fruit below medium size, roundish-ovate. Skin dull yellow, sprinkled with a very thin russet. Stalk rather long and slen der. Calyx closed, and set in a rather narrow basin. Flesh yellowish, very tender, (almost melting, juicy, with a mild, rich, spicy flavour. October to January.

The ENGlish Golden Russet is a sub-acid sort, much inferiour to the above. Fruit middle sized, ovate. Skin rough and thick, of a dingy, yellow russet, rarely with a red blush. Stalk very short, deeply planted in a narrow cavity. Flesh pale yellow, very firm and crisp, with a brisk, rather aromatic flavour. Trees with many slender, weeping branches. November to March.

\section{Russet, Putnaly. $\$$}

For a knowledge of this celebrated western apple, we are in. debted to that zealous pomologist, our friend, Professor Kirtland, of Cleveland. It is considered decidedly the most valu. able late keeping apple in the West, not inferiour to the Newtown Pippin, and the growth of the tree very luxuriant. It originated at Marietta, Ohio, and is largely grown for the NewOrlcans and West India markets. Fruit medium, or large, form rather flat. Skin yellow, blotched with russet, and at times tinged with a dull red cheek. Flesh firm, yet tender, deep yellow, juicy, sub-acid, rich, and very high flavoured March and April.*

\section{Russet, English.}

The English Russet is a valuable, long keeping variety, ex tensively cultivated, and well known by this name on the Hud. son, but which we have not been able to identify with any linglish sort. It is not fit for use until February, and may be kept till July, which, together with its great productiveness and groou Havour, renders it a very valuable marker fruit. It is acknow ledyed one of the most profitable orchard apples.

Fruit of medium size, ovate, or sometimes conical, and very regularly formed. Skin pale greenish yellow, about two-thirds covered with russet, which is thickest near the stalk. Colyx sinall, closed, and set in an even, round basin, of moderate depth. Stalk rather small, projecting even with the base, and pretty deeply inserted, in a narrow, smooth cavity. Flesh yellowishwhite, firm, crisp, with a pleasant, mild, slightly sub-acid flavour.

" This is since ascertained to bo identical with the Roxbury RusGes [7th Fid.? 
The trees grow very straight, and form spright heads, ana the wood is smooth and of a lively brown.

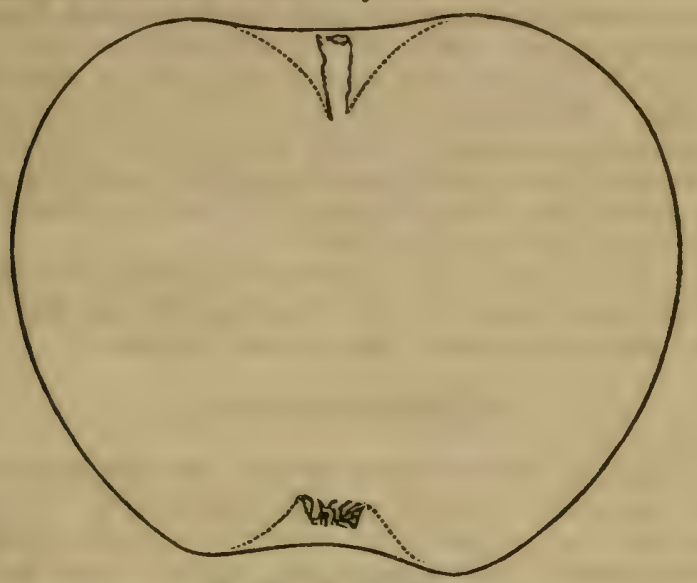

Fig. 53. English Russet.

150. Russet, Boston or Roxbury. Man. Thomp.

Roxbury Russeting. Ken.

This Russet, a native of Massachusetts, is one of the most nopular market fruits in the country, as it is excellent, a pro.

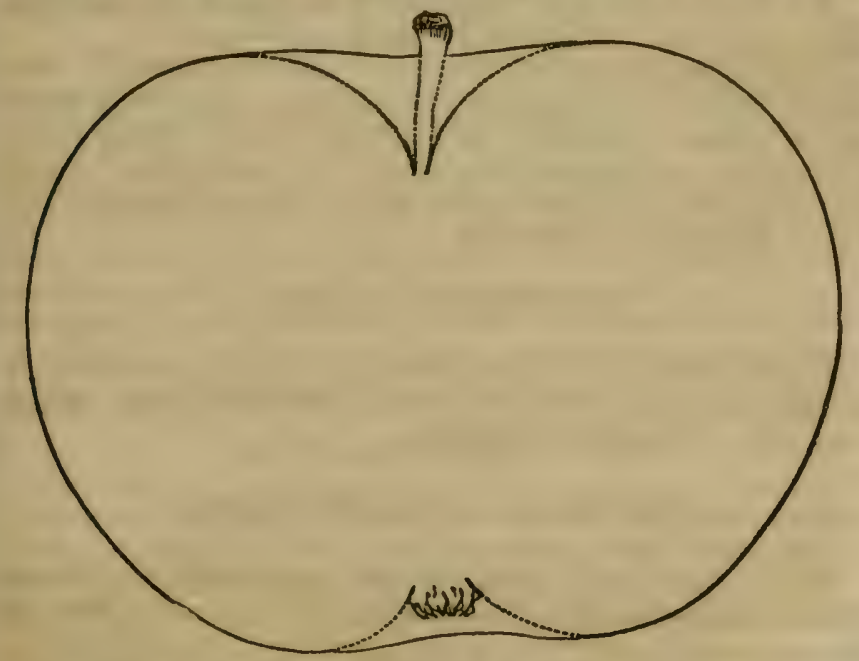

Fig. 54. Boston Russct.

12 
digious bearer, and keeps till late in the spring. It is in everv ray, highly deserving extensive cultivation.

Fruit of medium size, often large roundis's, a little flattened, and slightly angular. Skin at first dull green, covered with brownish-yellow russet when ripe, with, rately, a faint blush on one side. Stalk nearly three fourths of an inch long, rather slender, not deeply inserted. Calyx closed, set in a round basin, of moderate depth. Flesh greenish-white, moderately juicy, with a rather rich, sub-acid flavour. Ripens in January, and may be brought to market in June.

There are several native varieties of Russet or "Leather Coats," of larger size than the foregoing, but they are much inferior, being apt to shrivel and become tasteless.

\section{Red Gilliflower.}

This appears to be a native variety, and, although second rate, is esteemed in some parts of the country. Fruit of medium size, oblong, narrowing rapidly to the eye, where it is somewhat ribbed. The skin is smooth, and of a fine dark red. The calyx is set in a narrow, rather shallow, furrowed basin. Flesh white, of a mild flavour. November to January.

152. SAM Young. Thomp. Lind. P. Mag.

Irish Russet.

An exceedingly high flavoured, little dessert Russet from Killienny, in Ireland, and fit for use in early winter.

Fruit small, slightly flattened, and regularly formed. Skin bright yellow, a good deal covered with gray russet, and dotted on the yellow portion with small brown specks. Stalk short. Calyx large and expanded, placed in a broad basin. Flesh grecnish, quite juicy and tender, with a rich and excellent fla. vous. November to January.

\section{Surprise. Thomp.}

A small, round, whitish-yellow apple, of little or no value, but adinired by some, for its singularity, - the flesh being stained witu red. November to January.

154. Swaar, Coxe. Floy. Thomp.

This is a truly noble American fruit, produced by the Dutch settlers on the IJudson, near Esopus, and so termed, from its unusual weight, this word, in the Low Dutch, meaning heavy, It requires a deep, rich, sandy loam, to bring it to perfection. 
and, in its native soils, we have seen it twelve inches in circum. ference, and of a deep golden yellow colour. It is one of the finest flavoured apples in America, and deserves extensive cul. tivation, in all favourable positions, though it does not succeed well in damp or cold soils.

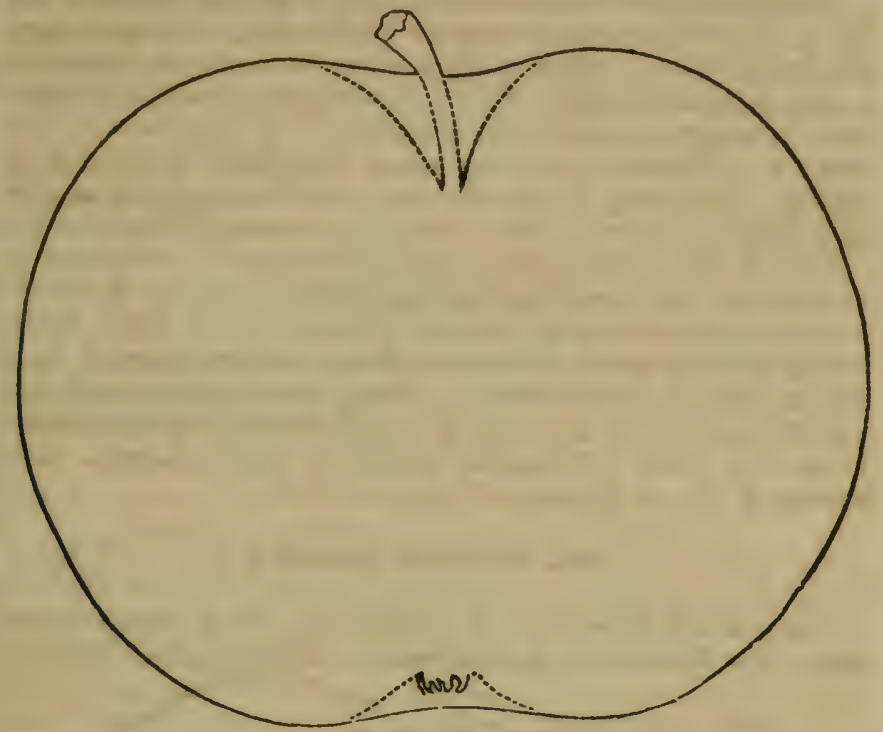

Fig. 55. Swaar.

Fruit large, regularly formed, roundish. Skin greenish-vel. low when first gathered, but when entirely ripe, of a fine, dead gold colour, dotted with numerous distinct brown specks. and sometimes faintly marbled with gray russet on the side, and round the stalk. Stalk slender, three four.hs of an inch long, inserted in a very round cavity. [Sometimes this cavity is partially closed.] Calvx small, greenish, set in a shallow basinscarcely plaited. Flesh yellowish, fine grained, tencler, witt. an exceedingly rich, aromatic flavour, and a spicy smell. Core small. The trees bear fair crops, and the fruit is in season from December to March.

\section{Sturmer Pipein. Thomp.}

This is a new English variety, of the very highest reputation. We have just received trees, but we have, for the following de. scription, the high authority of Mr. Thompson. Fruit of midlle size, short, conical. Skin yellowish.grenn, and brownish rerl; flesh firm, with a brisk, rich flavour. The tree is healthy, and a good bearer, and the fruit retains its flavour and brisliness till midsummer. 


\section{Sweetivg, Hartford.}

Spencer Sweeting.

$\Lambda$ very excellent winter sweet apple, introduced to notice by Dr. F. IV. Bull, a zealous amateur of Hartford. It may be kept till June, and this, added to its great productiveness, renders it a most profitable market fruit. The original tree of the Hartford Swecting is growing on the farm of Mr. Spencer, a few miles from Hartford, and has borne over forty bushels in a season. 'The wood is rather strong, but of slow growth, and is very hardy; (branches not pendulous, as stated by Kenrick.)

Fruit rather large, roundish, slightly flattened. Skin smooth, and fair, almost covered and striped with fine redover a yellowish-green ground,-and sprinkled with small gray dots. Stalk nearly three quarters of an inch long, slender, inserted in a rather shallow, round cavity. Calyx broad, closed, with few segments, set in a slightly uneven basin which is but little sunk. Flesh very juicy, tender, with a rich, agreeable flavousr. De. cember to May or June.

\section{Sweeting, Ladies'. $\S$}

The Ladies' Sweeting we consider the finest winter sweet Epple, for the dessert, yet known or cultivated in this country.

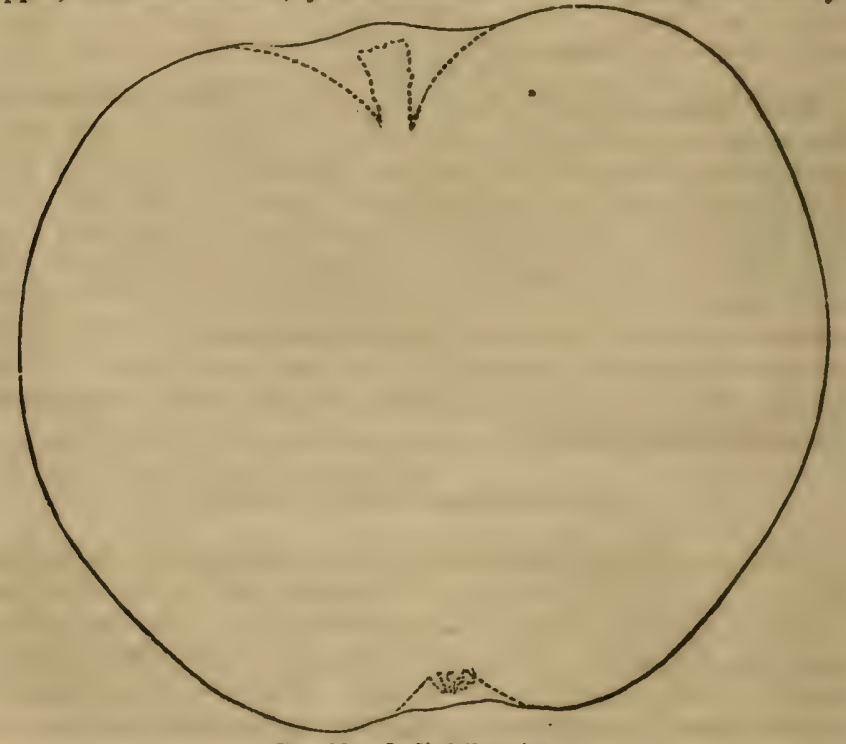

Fig. 56. Lndies' Surectinz. 
its handsome appearance, delightful perfume, sprightly flaveur, and the long time which it remains in perfection, render it universally admired wherever it is known, and no garden should be without it. It is a native of this neighbourbood, and thou. samis of trees of this variety, have been sent from this garden, to various parts of the union. The wood is not very strong, but it grows thriftily, and bears very abundantly.

Fruit large, roundish-ovate, narrowing pretty rapidly to the eye. Skin very smooth, nearly covered with red in the sun, but pale yellowish-green in the shade, with broken stripes of pale red. The red is sprinkled with well marked, yellowish-gray dnts and covered, when first gathered, with a thin white blonn. 'There is also generally a faint marbling of cloudy white over the red, on the shady side of the fruit, and rays of the same around the stalk. Calyx quite small, set in a narrow, shallow, plaited basin. Stalk half an inch long, in a shalluw cavity. Flesh greenish-white, exceedingly tender, juicy and crisp, with a delicious, sprightly, agreeably perfumed flavour. Keens without shrivelling, or losing its flavour, till May.

\section{Sweering, Tolman's.}

The Tolman's Sweeting is scarcely second rate as a table fruit, but it is one of the most popular orchard sorts, from its great productiveness, its value as food for swine and cattle, as well as for baking. Form nearly globular. Skin, when fully ripe, whitish-yellow, with a suft blush on one side. Stalk rather long and slender, inclining to one side, and inserted in a rather wide, shallow, but regular cavity. Calyx set in a small basin, slightly depressed. Flesh quite white, rather firm, fine grained, with a rich, sweet favour. November to April. This fruit, a native of Rhode Islaud, considerably resembles the Danver's Winter Sweet, of Massachusetts.

\section{Sweeting, Ramsdelis's. $\oint$}

\section{Ramsdel's Red Pumpkin Sweet. Ken. Kamidell's Swet. \\ Ked Punpkin Sweet.}

Ramsdell's Siveeting we have lately received from Connec. ticut, where it is greatly esteemed for the very large crops it bears, as well os for its remarkably rich saccharitıe navour. IV'e believe It is a native of Connecticut ; and it derives its name froin the Rev. H. S. Ramsdell, of Thompson, in that 
138

APPLES.

state, who has introd aced it to public attention. The tree is very vigorous, grows remarkably straight, and upright, comes carly into bearing, and yields every year enormously.

Fruit rather above medium size, oblongs, regularly shaped. and tapering slightly towards the eye. Skin rich, dark red dotted with fawn-coloured specks, and covered with a blue bloom. Stalk quite short, deeply sunk in a rather narrow cav. it. Calyx set in a pretty deep even basin. Flesh yellowish, very tender and mellow, unusually sweet and rich. In weight the apple is light. October to February.

161. Spitzenburgh, Esopus. Coxa.

Æsopus Spitzemberg. Thorp. Lind.

Esopus Spitzenburg. Ken.

True Spitzenburgh.

The Esopus Spitzenburgh is a handsome, truly delicious apple, and is generally considered, by all rood judges, equal to the

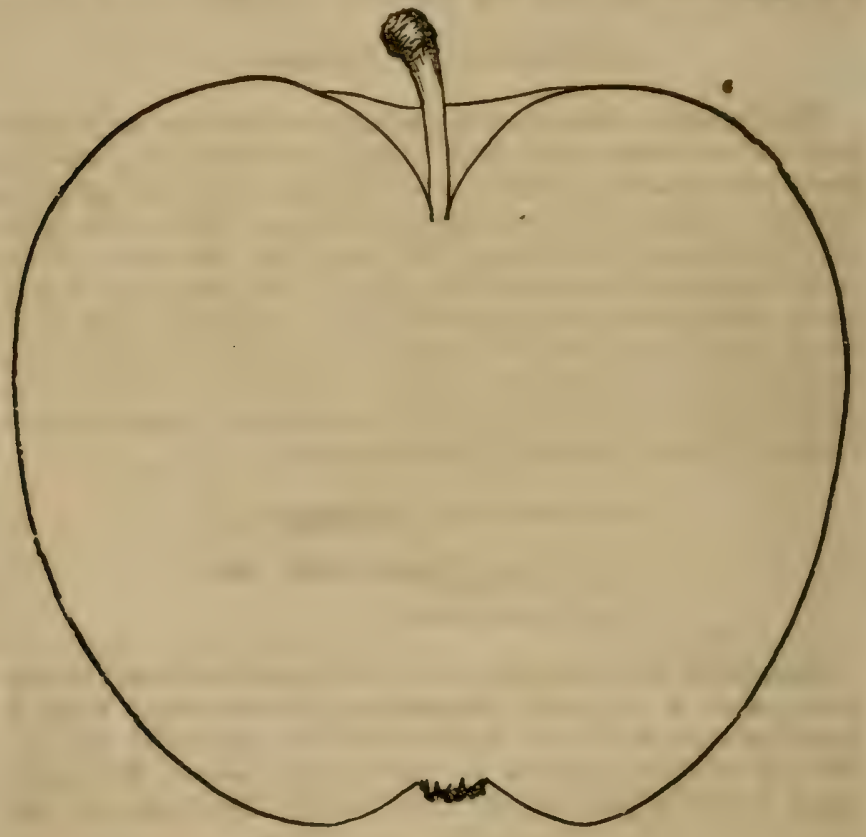

Fire. 5\%. Fsozus Spitzenturgh. 
Newtown Pippin, and unsurpassed as a dessert frutt, by any other variety. It originated at Esopus, a famois apple district, originally settled by the Low Dutch, on the Hudson, where it is still raised in its highest perfection. But throughout the whole of New-York, it is considered the first of apples, and its beauty and productiveness render it highly profitable for orehard cul. ture. The fruit of this varicty brought from IVestern New. York, seems deficient in flavour, which is, perhaps, owing to the excessive richness of the soil there. The tree has rather sien der shonts, and when in bearing, has long and hanging limbs.

Fruit larce, oblong; tapering roundly to the eye. Skin smooth, nearly covered with rich, lively red, dotted with distinct yellowish russet dots. On the shaded side, is a yellowish g:ound, with streaks and broken stripes of red. Stalk rather long,- - three fourths of an inch-and slender, projecting beyond the base, and inserted in a wide cavity. Calyx small, and closed, set in a shallow basin, which is slightly furrowed. Flesh yellow, rather firm, crisp, juicy, with a delicious rich, brisk flavour. Seeds in a hollow core. December to February

\section{Sitzenburgh, Flushing.}

This variety has been confounded by Coxe, and more recently by Thompson, with the foregoing, but is really quite distinct. The tree inakes strong, brown shoots, different from the slender yelinwish ones of the Esopus Spitzenburgh.

The fruit is roundish conical, stalk set in a narrow cavity, projecting beyond the fruit. Skin nearly covered with red, on a greenish yellow ground, dotted with large fawn spots, and cuated with a slight lloom. Calyx small, in an even basin. Flesh white, juicy, crisp, nearly sweet. and of pleasant flavour, but without the brisk richness, or yellow colour of the Esopus Spitzenlurerh. October to February.

Kaigirx's Sfitzenbergh is an inferior variety, of a conical form, and pale red colour. It originated in New. Jersey and is only of third rate quality. The tree is also an ugly, rambling grower: The fruit keeps till A pril.

163. Spitzenberg, Newtown. Coxe. Thomp. Lind.

Matchless.

Burlingturi Spitzenberg.

The Newtown Spitzenberg comes from Newtown, on I,ong Island. It is a mundish, handsome fruit, of good navour, but inferiour to the Esopus variety.

Fruit of medium size and rrgular form, roundish, slightly flattened. Skin smooth, beautiful yellow, with a fine red cheek, 
n little streaked with brighter red, and marked with nurneron dots. Calyx set in a rather wide, even basin. Stalk short, deeply inserterl. Flesh rather yollowish, firm, with a mild and agrecable fluvour. November to February.

\section{Siveeting, Wells'. $\oint$}

Wells' Swecting is one of the most sprightly and agreeable, for the dessert, of all the early winter sweet apples. The only oll tree in our knowledge, grows in the orchard of Mr. John Wells, near Newburgh, N. Y. IVe have not been able to trace it farther than this neighbourhood, though it may not have cri. ginated here. It makes stout, stiff, uprigltt shonts, and bears well.

Fruit of medium size, roundish, broadest in the middle, and lessening each way. Skin smooth, pale, dull green, (like a Rhode Island (Greening in colour, but paler,) with a dull red, or bruwn ish cheek. Stalk rather slender and short. Calyx short, set in quice a shallow basin. Flesh very white, ani very terider, abounding with a rich, agreeable, sprightly juice. November to January.

\section{Tiventy Ounce. H. Mag.}

\section{Tiventy Ounce Apile. of Cayuga}

Eighteen Uunce Apple. $\}$ co., N. Y. Cayura Red Streak ?

A very large and showy apple, well known in Cayuga $\mathrm{cn}$., N. Y., and probably a ilative there. It is a goorl, sprightly fruit, though not very hish flivoured, but its remarkably handsome appearance, and large size, render it onr of the most pi)pular fruits in market. The tree is thrifty and makes a compract, near hearl, bears regular crops, and the fruit is always fair and handsome.

Fruit very litrege, roundish. Skin s'ighttly uneven, grentish. yollow, boldly splashed and marbled with stripes of pmplish.red. Stalk short, set in a wide derp carity. Calyx small, basin mole. rately deep. Flesh coarse-grained, with a sprightily, brisk sub. acil flavor. Oet. to Jan. This is quite distinct from the TwEXTY ouxce PIPPis, a large, smooth, dull-coloured cooking anple.

\section{Temksbury Winter Buusir. Coxe.}

Mr. Coxe says, this apple was hroutht fron Tewkshury, IIuterdon county, N. J. It is a hantinne, fair fruit, with more flavour and juicines than is usual in long lieeping apples.

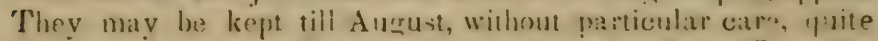
plump and sound. The size is small, rather llu. The skin smoth, vellow, with a rest chesk. Fiesh yellow, with more juice and fiavour than any other long-kerping variety. The tree srows rapidly and straight-and the fruit hangs till late in the autumn. January to july. 


\section{Victuats aNd Utint.}

\section{Big Sweet. \\ l'uimpey}

Thes is a large and delicious sweet apple, highly estecnnd in the neighbourhoo! of Newark, New-Jersey, where it origrinated, abrut 175:1. It was first introduced to notice by Mr. I. IV. Jayes, of Newark, from whom we first reccived trees and slecimens of the fruit. The fruit is very light.

Fruit large, oblong, rather irregular, and varies a good deal in size. Skin thin, but rough, dull yellow, marbled with russet, with a iaint russet blush on the sunny side. Stalk moderately long and slender, deeply inserted in an irregular eavity. Caly $\mathrm{x}$ small, set in a rather shallow basin. Flesh yellowish, tender, breaking, with a rich, sprightly, sweet flavour. In perfection from (Oetober to January, but will keep till April. The tree is a moderate bearer.

\section{VAxpervere. Coxe. Thomp. Floy.}

\section{Stalcubs.}

The Vandervere, when in perfection, is one of the most beautiful and finest apples. But it requires a rich, light, sandy soil, as in a damp heavy s il, it is almost always liable to be spoted, anfair, and destitute of flavour. It is a native of IVilmington, Delaware, and took its name finm a family there. It is a fine old variety, and is high!y worthy of extensive cultivation, whese

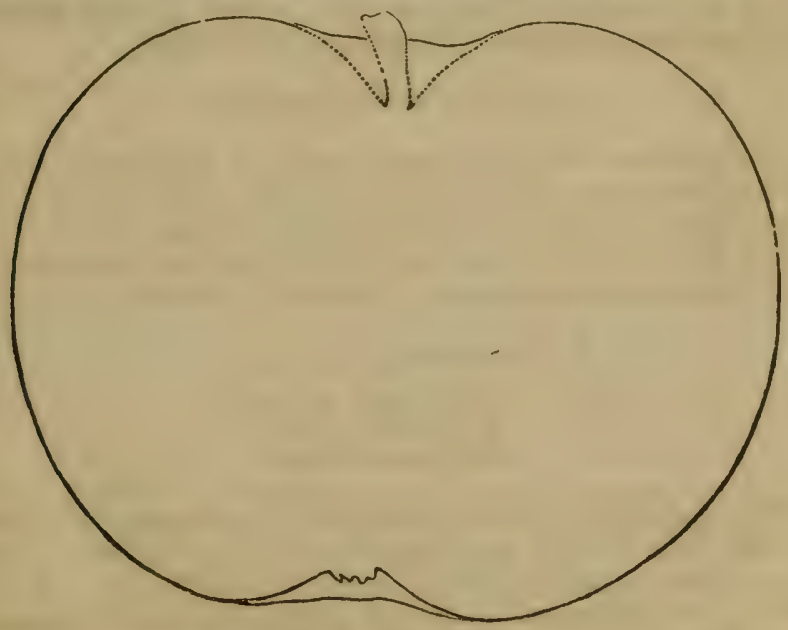

Fig 53. Finderare 
the soil is favourablo. We have before us some apples of this sort, which are exceedingly beautiful and excellent.

Protit of medium size, flat. Skin, in its ground colnur, yel. inw, stresker! and stained with elourled red, bun on the sumny silde derperening into rich rel. dotted with light gray specks Stalk slurt, inserted in a smooth, rather wide, cavity. Calys small, chesed, set in a regular, well-formed basin, of moderate depul. Flesh yellow, crisp and tender, with a rich and sprighty juice. October to January.

\section{W Axen Apple. Coxe.}

\section{Fate Apple. \& of vanous parts White Apple. $\}$ of Uhio. \\ Belsnont. Ken.}

The IVaxen Apple, for whose correct history we are indebted to that careful ponologrist, Professor Kirtland, of Clevelanil, is estecmed in Ohio, where it is now most largely cultivater!, nne of the very finest of all early winter varieties. It was carried from eastern to western Virginia, by Neisley, a nurseryman on the bauks of the Ohio, aljout the commencement of the present century. Thence it was introduced into Belınont cn., anil other parts of Ohio. From Rockport it was carried by C. Olunstead, Esr., to Boston in 1834, incorrectly under the name of [3.lmont.

Fruir of iniclle size, globular, a little flattened and nurrower towarts the eye-sometimes oblong; when of the latter liorm, the rye is knolshy. Stalk short. Skin pale yollow, rarely tinered with a bright vermillion blush, wasy, or nily smonil. Flesh white, crisp, tender, sometimes almost melting, and of a mild, agreeable flavour. November to February.

\section{Watson's Dumpling.}

A very large, English litchen apple, of fair quality. Fruit about four inches in diameter, of regular form, nearly round. Skin smooth, yellowish-green, faintly striped with dull rerl. Stalk short. Flesh juicy, rather tender, with a pleasant, sub. acill flavour, and stews wcll. October to January.

\section{Woolman's Long.}

Ortley Apple. Lind. Ortley Pippin. Mnn. Van Dyne, (of some.)

This high flavoured and cxcellent fruit, was sent to England . hy Mr. Floy, in is:5, who named it after Michael (;rtley, Esq., irom 1 hose orchard, in South Jersey, it was obtained. But we observe that Thompson, in the last edition, makes it synonymous with Woolman's Long, which is, perhaps, an English variety. 
The Ortley lias, hitherto, always been thought an Amorican variefy, and we ingret that it is so little cultivated here.

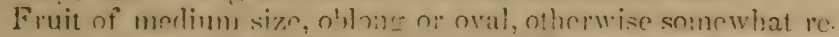
armbles the Yoliow Nowtown Piprin. Skin lively rellow, in the sharle, with a scorlet hlush, sprinkl, wihh white speceks amb gray russet patches in the sun. Stalk slenter, ins rted in an even, smooth depression. Calys large, set in a plisited, rather shallow basin. Flesh nearly white, crisp, and rather firm, breaking, with an excellent, sprightly, perfumed flavour. An abundant bearer, and will, no doubt, prove a most valuable sort. November to A pril.

\section{WiNe A PPLE. $\oint$ CoxG.}

Ilay's Winter.

The Wine Apple is a very handsome, and an admirable winter fruit, a most abundant bearer, and a hardy tree; all of which qualities render it a very popular orchar.l and market fruit. It is a native of Delaware, but is now very largely cultivated, also in IVestern New-York. The tree has small leaves, grows thriftily, and makes a fine, spreading head.

Fruit rather above medium size-in rich soils large; form regular, nearly round, a little flattened at the endis. Siriu smooth, of a lively deep red, ovf $\mathbf{r}$ a yellow ground, or, more frequently, with a few indistinct strip ss of vellow. Stalk short, inserted in a round, smooth cavity, with a little russet arouml it. Flesh yellowish-white, juicy and crisp, with a rather vinnus, rich, and pleasant flavour. This apple is not only fine for the table, but is also excellent for cooking and cider. October to March.

\section{Winesap. $\oint$ Coxe.}

\section{Wine Sop? Thomp.}

This is not only a good apple for the table, but it is also one of the very finest cider fruits, and its fruitfulness renders it a great favourite with orchardists. The tree grows ra:her irremularly, and does not form a handsome hearl, but it bears early, and the apples have the good quality of hanging late upon the trees, without injury, while the tree thrives well on sandy light soils.

Fruit of medium size, ratheroblong. Skin smooth, of a fine dark red, with a few streaks, and a little yellow ground, appearing on the shady side. Stalk nearly an inch long, slencer. set in an irregular cavity. Calyx small, placed in a regilar tasin. with fine plaits. Flesh yellow, firm, crisp, with a rich, lis? tavour. November to May. 


\section{WINTER QueEN. Coxe.}

\section{Winter Quoening. Thomp.}

A iruit of medium quali:y, much cultivated in the lower part of New.Jersey. Fruit conical, considerably broadest at the base. Skin fine deen crimson in the sun, dotted with yellow; of a paler and livelier red, in the shade. Stalk slender, threc fourths of an inch long, planted in a wide cavity. Calyx small, morlerately sunk. Flesh yellowish, of a rnild and rather plea. sant, sub-acid flavour. The tree is an abundant bearer. No vember to February.

Class IV. Cider Apples.

\section{Cooper's Russeting. Coxe.}

This native apple is especially suited to light sandy soils, where some other sorts fail. It makes an exceedingly strong cider, of delicious flavour.

Fruit small, oblong or ovate, pale ycllow, partially covered with russet. Stalk slendrr, and very long. Flosh dry, rich and swret. The fruit is fit for ciller in November, keeps well through the winter, and is estecmed by many for cooking. 'Tree small, with numerous little branches.

\section{Campfield. Coxe.}

\section{Newark Sweeting.}

Another capital New-Jersey, cider apple, ranking next to the Harrison. It forms a fine large tree, with straight, spreading limbs, and is very productive.

Fruit of medium size, roundish, rather flattened. Skin smooth, washed and striped with red, over a greenish-yollow zround. Flesh white, rather dry, firm, rich and sweet.

\section{Gilrin. Coxe. Thomp.}

Carthouse.

A handsome cider fruit, from Virginia, which is also a very good table fruit from February to May. A very hardy, vigorous and fruitful tree.

Fruit of medium size, roundish-oblong. Skin very smooth and handsome, richly streaked with deep red and yellow. Stalk 
short, deeply inserted. Calyx in a round, rather deep basin. Flesh yellow, firm, juicy and rich, becoming tender and sprightly in the spring.

\section{Harrison. Coxe.}

New-Jersey is the most celebrated cider making district in America, and this apple, which originated in Essex county, of that state, has long enjoyed the highest reputation as a cider fruit. Ten busneis of the apples make a barrel of cider. The tree grows thriftily, and bears very large crops.

Fruit medium size, ovate or roundish-oblong. Skin yellow, with roughish, distinct black specks. Stem one inch, or more, long. Flesh yellow, rather dry and tough, but with a rich fiavour, producing a high coloured cider, of great body. The fruit is very free from rot, falls easily from the tree about the first of November, and keeps well. The best cider of this variety, is worth from six to ten dollars a barrel, in New-York.

\section{Hewe's Virginia Crab. Coxe.}

The Virginia Crab makes a very high flavoured dry cider, which, by connoisseurs, is thought unsurpassed in flavour by any other, and retains its soundness a long time. It is a prodigious bearer, and the tree is very hardy, though of small size.

Fruit quite small, about an inch and a half in diameter, nearly round. Skin dull red, dotted with white specks, and obscurely streaked with greenish-yellow. Stalk long and slender. Flesh fibrous, with an acid, rough, and astringent flavour, and when ground, runs clear and limpid from the press, and ferments very slowly. The Virginia Crab is often mixed with rich pulpy apples, to which it imparts a good deal of its fine quality.

The RoANE's White CRAB is a sub-variety of the foregoing, about the same size, with a yellow skin. It makes a rich, strong, bright liquor, and keeps throughout the summer, in a well-bunged cask, perfectly sweet.

\section{Hagloe Crab. Lind.}

This is a celebrated old English cider fruit, scarcely known in this country. Lindley says, when planted on a dry soil, with a calcareous bottom, it produces a most excellent cider. The specific gravity of its juice is 1081 .

"Fruit small, ill-shaped, something between an apple and a crab, more long than broad, wide at the base and narrow at the crown, which is a little sunk, and the eye flat. Skin pale yellow, a little marbled in different directions with a russet-gray, and having a few red specks or streaks on the sunny side. Eye tlat, with a spreading calyx. Stalk short." 
This is totally distinct from the SuMnen IIAgLoe of Amer n nurseries [Hagloe Crab, of Coxe], a large, handsome, round in, purplish-red apple, covered with bloom and ripe in August-hesh soft and woolly, of pleasant sub-acid flavor-the tree a slow grower, with thick, blunt shoots.

\section{Red Streak. Coxe. \\ Herefordshire Red Streal,
Scudamore's Crab, of English gardens.}

A capital English cider apple, which thrives admirably in this country, and is very highly esteemed, as it makes a rich, high flavoured, strong liquor. It is a handsome grower, and a great bearer.

Fruit of medium sizc, roundish. Calyx small, set in a rather deep basin. Stalk rather slender and short. Skin richly streaked with red, with a few yellow streaks and spots. Flesh yellow, rich, firm and dry.

\section{Styre. Thomp.}

Forest Styre. Lind.

Styre. Caxe.

'The Stire is a famous old English cider fruit, and Lindley remarks that Styre cider may be found in the neighbourhood of Chepstow, thirty or forty years old. Fruit middle sized, round, pale yellow, with a orange cheek. Stalk short. Flesh firm, of high flavour and makes a high coloured liquor. The tree thrives well here, and forms a very upright, broom-like head. October to January.

In addition to the foregoing, several of the table apples al. ready described are esteemed for cider, as the Newtown Pippin, Wine Apple, Winesap, etc., and some of the high flavoured Einglish varieties in the preceding pages are very highly valued for cider in Britain,- the Golden Pippin, Golden Harvey, Downton, \&c. The Fox Whelp is a very celebrated apple of thiz class, used to flavour and give strength to nearly all the choice cider of Herefordshire, which is not yet introduced here, to our knowledge. It is middle sized, ovate, dark red, with a rich, heavy juice, of the specific gravity 1078. The Siberian Bit. TER SwEET is a variety of crab raised by Mr. Knight, and aboui twice the size of the Siberian Crab, small roundish, ovate, yel. low; an immense bearer, and held in very high esteem in Eng. land, for mixing with other cider apples, to impart richness. 
Class V. Apples for Ornament or Preserving.

\section{Siberian Crab. Arb. Brit.}

MIalus haccata. Lind.

Pyrus baccata. Arb. Brt.

'The common Siberian Crab is a beautiful little fruit, which is produced in rich clusters on the branches, and, at a distance, resernbles large and handsome cherries. It is highly esteemed for preserving, and almost every large garden in the middle states, contains a tree of this variety. It forms a vigorous, neat tree, of rather small size, and its blossoms, which are white, are produced in beautiful profusion in spring, and a large crop of fruit regularly follows.

Fruit about three fourths of an inch in diameter, very regu. larly formed, and rather flat. Slin smooth, of a lively scarlet. over a clear yellow ground, and when the bloom is rubbed off, is highly polished. Stalk nearly two inches long, and very slender. Calyx small, slightly sunk. Fit for preserving in September and October.

\section{Large Red Stbertan Crab.}

Pyrus Pruifolia. Arb. Brit.

This variety is about twice the size of the foregoing, round. ish.ovate, with a large and prominent calyx, and a pale red and yellow skin. It forms a larger tree, with rather coarser foliage than the common variety, and is esteemed for the same purposes. September and October.

\section{Yellow Siberian Crab.}

Amber Crab.

This scarcely differs from the common Siberian Crab, excert in its fruit, which is rather larger, and of a fine amber or golden yellow. Both this and the red are beautiful ornaments to the fruit garden in summer and autumn, and are equally esteemed for preserves and jellies. September.

Quite a number of seedlings have been raised from the Silbe. rian Crab in this country, mostly of larger size-some by Mr. Manning, of Salem, and several by Mr. Thompson, of Catskill, but scarcely deserving of especial notice here. 


\title{
186. Double Plotverixg Chinese Crab.
}

\author{
Pyrus Spectatilis, Arh. Bril. \\ Malus Spectabilis. N. Duh. \\ Double flowering Apple.
}

This very beautiful crab tree from China, which produces a small green fruit, of no value, is highly admired for its showy blossoins. These are large, tipperl with deep red in the bud, but when open, are of a pale rose colour, semi-double, large: and produced in fine clusters. It is an exceedingly ormamental, sulall tree, growing from ten to twenty feet in height.

Select list of apples for a small garden, to ripen in succession.

\begin{tabular}{|c|c|}
\hline $\begin{array}{l}\text { Summer. } \\
\text { Early Harvest. } \\
\text { Red Astrachan. } \\
\text { Early Strawberry. } \\
\text { Drap d'Or. } \\
\text { Early Red Margaret. } \\
\text { William's Favourite. } \\
\quad \text { Aulumn. } \\
\text { Porter. } \\
\text { Fall Pippin. } \\
\text { Ross Nrnpareil. } \\
\text { Maiden's Blusl. } \\
\text { Jerse Sweet. } \\
\text { Fall Harvey. } \\
\text { Gravenstein. } \\
\text { Summer Sweet Paradise. } \\
\text { Golden Sweet. }\end{array}$ & $\begin{array}{l}\text { Rambo. } \\
\text { Ninter. } \\
\text { Newtown Pippin. } \\
\text { Dutch Mignonne. } \\
\text { Esopus Spitzenburgh. } \\
\text { Baldwin. } \\
\text { Ladies' Sweeting. } \\
\text { Northern Spy. } \\
\text { Swaar. } \\
\text { Buston Russet. } \\
\text { Rhode Island Greening. } \\
\text { Yellow Belle-Fleur. } \\
\text { American Golden Russet. } \\
\text { Iady Apple. } \\
\text { Peck's Pleasant. } \\
\text { Herefurdshire Pearmain. }\end{array}$ \\
\hline
\end{tabular}

.dpples of fine quality, suited to a cold northern climate. Fa. meuse, Canada Reinctte, Poinme de Neire, Rhode Island Grrecning, Boston Russet, Porter, Baldwin, Swaar, Red As. trachan, Ladies' Sweeting, Northern Spy, Golden Ball.

Apples of fine quality, suited to a southern climate. Farly Red Margaret, Large Yellow Bough, English Golden Pippin, Sheep.Nose, Lady Apple, Maiden's Blush, Gravenstein, Golden Reinette, Green Newtown Pippin, English Russet, Mal Carle, Iellow Belle-Fleur, Wine Apple, Roman Stem.

A number of native varicties, which have originated at the extreme south, are found to succed better there than most of our nothern apples. Among these are the Horse Apple, Moun. tain l'ippin, Father tbram, ctc. These are not fine at the north, but are well adajted to the climate of Georgia, Alabama, sce. 


\section{* chapter ix.}

\section{THE ALMOND.}

\section{Amygdalus communis, Dec. Rosaceœ, of hotanists. \\ Amandier, of the French; Mandelhuum, German; Mandorlo, Italian ; Almendra Spanish.}

Tur. Almond tree, which is a native of the north of Africa, and the mountains of Asia, has long been cultivated, and is mentioned in seripture as one of the charms of the fertile land of Canaan. It so strongly resemibles the peach tree that it is diflicult to distinguish it by the laves and wood only; inteed, several hotanists are of opinion, from experiments made in raisine the almond from seed, that this tree and the peach are originally the same species, and that the rireh and luscious peach is the effect of accidental variation, produced by culture on the almond. The chicf distinction between the two in our gardens lies in the fruit, which, in the almond, consists of little more than a stone covered with a thick, dry, wonlly skin, while the Pcach has in addition a rich and luscious flesh. The blossoms of the almond resemble those of the peach, but are larger; they are produced in great profusion, carly in the season, before the leaves, and are very ornamental.

Uses. The kernel of the sweet almond is highly esteemed as an article of food, and is larocly used as an ingredient in confectionary, cookery, and perfuncry. It is raised in great quantities in the south of Europe, esprecially in Portugal, and is an important article of commerce. The bitter almond is used in conkery and confectionary, and in medicine, it furnishes the prussic acid of the shops, one of the most powerful of poisons. From both species an oil is also obtained.

In France the almond is preferrerl as a stock on which to bud and graft the peach, which, in a very dry climate or challiy scil, it is found, renders the latter more healthy and fruitful than its own bottom. The sweet hard-shelled variety (Douce à coque dure,) is preferred for stocks by French nurserymen.

Cultuation. 'The almond thrives best in a warm dry soil, and its general cultivation in this country is precisely like that of the P'each. 'The sweet almond is the only variety considered of value here, and it is usnally propagater by butding $t \mathrm{nn}$ Plum stock, or on the biter almont seedlings. It is rather more hardy at the north whon budided on the former, and as the buds of the sweet almond are rather slender and small, the pium stocks to be budded should be thrifty seedlings not more 
than a fourth of an inch in diameter at the place where the bud is inserted.

'The Common Almond, the IIard-Shell Sweet Almond, and the bitter Almoml, are harly in the latitude of New.York, and will bear tulerable crops without care. 'The Soft.Shel! Sivect Alinond, or Ladies' Almond, will not thrive well in the open farlen as a standard, north of Philadelphia; but they succeed well trained to a wall or on espalier rails in a warm situation; the branches being slightly protected in winter.

T'here is no apparent reason why the culture of the Almond should not be pursued to a profitable extent in the warm and favourable climate of some of the southern states. Especially in the valley of the Ohio and Tennessee it would be likely to succeed admirably.

1. Comnon Almond. $\S$ Thomp. Lind.

A. c. dulcis. Dec.

Amandier à Petit Fruit, commun, $\}$ O. Duh.

Amande commune.

Common siweet.

This is the common Sweet Almond of France and the south of Furope, and is one of the most hardy and productive sorts here. Nuts hard, smooth, about an inch and a quarter long, compressed and pointed, of an agreeable flavour, but inferior to the following. Flowers expand before the leaves. Ripens last cf September.

\section{The Long Mard-Shell Almond. $\oint$}

Amandier à gros fruit. $O, D u h$.

Amandier à gros.

A variety with handsome large, pale rose coloured fowers, opening before the leaves, and large and long fruit a third longer than other varieties. The stone is about as large as the snftshell variety, but the kernel is larger and plumper. This is a good hardy sort and it is very ornamental when in blossom. Ripens about the last of September.

3. Soft-Sitell Sweet Alimond. § Lind.

Doux à coque tendre,
Sultan à coupue tendre, T\% omp.

Amandier à coque tendre. $O$. Duh.

- des Dames. N. Duh. Poit.

$\left.\begin{array}{l}\text { Amandier des Dames, } \\ \text { Ou Amande Princesse. }\end{array}\right\}$ Noisetle.

Ladies' thin Shell.

The Sof.-Shell or Ladies' Almond, is the finest of all the $\mathrm{Al}$. 
monds. F: is the variety very common in the shops of the con. fectioners, with a shell so thin as to be easily crushed betweer. the fingers, and the kernel of which is so highly esteemed at the dessert. It ripens early in the season, and is also highly es. teemed in a young or fresh state, being served on the table for this purpose about the middle of July in Paris. The blossoms of this variety expand at the same time with the leaves, and are more deeply tinged with red than the foregoing. Several varieties are made of this in France, but they are (as quoted above) all essentially the same.

Fruit two inches long, oval, compressed. The nut is more than an inch long, oval pointed, one-sided, with a light coloured, porous, very tender shell. The kernel sweet and rich.

On the plum stock, in a favourable aspect, this almond suc. ceeds, with a little care, in the middle states.

\section{Sultaina Sweet Almond. Lind.}

Amande Sultane. O. Duh. Nois.

Amandier Sultane.

Sultan. Thomp.*

A tender shelled almond of excellent quality, with smaller fruit and narrower kernel than the Soft-Shell Almond, but of equally excellent flavour, and which is preferred by many. It is thought, by Poiteau, to be scarcely different from the SoftShiell or Ladies' Almond.

\section{Pistachia Siveet Almond. Lind.}

Amande Pistache. O. Duh. Nois.

Amandier Pistaclie.

A variety of almond with a very small pointed fruit, about the size and shape of that of a Pistachia, eliclosing a kernel of a delicate sweet fiavour. The shell not quite so soft as the SoftSheil Alınoml. 'This is scarcely known yet in this country, but is worth further trial at the south.

\section{Peach Almond.}

Pêcher, Peach Almond, $\{$ Thomp.

Amandier-Pêcher. N. Duh. Nois. Poil.

A rather indifferent variety, nearly sweet, lut often slightly gitter. It is a true cross between the peach and the almond, and

* We cannot follow Mr. Thompson in his nomenclature of Almonds, as he (or fis printer, mistalies the meaning of the French terms; Amande Sultane of all the Freuch autiors stoould be translated Sultana, not Sultan. 
in its leares, flowers, and stone strongly resembles the peach. the fruit is also pulpy and of tolerable flavour, like an indifferen. neach. The nut scarcely ever ripens well as far north as this.

\section{Bitter Almond. Thomp. Lind.}

The Bitter Almond has large pale blossoms, differing little from the common almond, except in the kernel, which is bitter. There are two varieties one with a hard, and the other with a brittle shell. The fruit, which is produced abundantly, ripens in September. The leaves are longer and of a darker green than those of most of the sweet fruited varieties.

Ornanental Varieties. The Dwarf Double Flowering Almond, (Amygdalus pumila, Lin. Prunus sinensis, of some,) is a beautiful, well known, low shrub, extremely ornamental in spring, being covered with a profusion of small pink blossoms, very double.

The Large Double Flowering Almond ( $A$. à grand fleur, $N$. Duh.) (A communis pleno,) is a beautiful French variety, with large, nearly white flowers, two inches in diameter. It also hears a good, small, hard-shell Almond.

\section{CHAPTER X.}

\section{THE APRICOT.}

Armeninca vulgaris, Dec. Rasacee, of botanists.

Abricotier, of the French; Avrikosenbaum, German; Alburcoco, Italian; Abraricoque, Spanisl.

Tire Apricot is one of the most beautiful of stone fruit trees, easily known by its glossy heart-shaped foliage, large white blossoms, and smooth-skinned, golden or ruddy fruit. In the fruit crarden it is a highly attractive object in early spring, as its charming flowers are the first to expand. It forms a fine spreading tree of about twenty fect in height, and is hardy enough to bear as an open standard south of the 420 of latitude in this country.

The native countries of this tree are Armenia, Arabia, and the higher regions of central Asia. It is largely cultivated in China and Japan; and, indeed, according to the accounts of Grosier the mountains west of Pelin are covered with a natural growth of apricots. The names by which it is know $n$ in vari. 
sus Europear. countries all seem to be corruptions of the original A rabic term Berkoche.

UsEs. A very handsome and delicious dessert fruit, only in ferior to the peach, ripening about midsummer, after cherrivs, and before plums, at a season when it is peculiarly acceptahle. For preserving in sugar or brandy, for jellies, or pastries, it is highly esteemed, and, where it is abundant, an admirable liqqueur is made from the fruit; and it is also dried for winter use. In some parts of Germany, the free bearing sorts-the 'Turkey, Orange, and Breda-are largely cultivated for this purpose.

Cultivation. This tree is almost always budded on the plum stock (on which in July it takes readily,) as it is found more hardy and durable than upon its own root. Many Ainerican nurserymen bud the apricot on the peach, but the trees, so produced, are of a very inferior quality-short lived, more liable to diseases, and the fruit of a second rate flavour. I3udded on the plum they are well adapted to strong soils, in which they always hold their fruit better than in light sandy soils.

Apricots generally grow very thriftily, and soon make fine heads, and produce an abundance of blossoms and young fruit; but the crop of the latter frequently falls of when half grown, from being stung by the Plum-weevil or curculio, to which the smooth slin of this fruit seems highly attractive. To remedy. this, the same course must be pursued as is directed for the plum. Seelling apricots are usually more hardy and prolluctive here, than the finer grafted sorts.

This is a favourite tree for training on walls or espaliers, and, in town gardens especially, we often see it trained against the sides of brick houses, and yielding most abundantly. As it bears its fruit in the same way as the peach, and requires the same management, we must refer our readers to the latter head for direction as to pruning and training. As the apricot, however, expands its blossoms very carly, it should not be placed on an east wall, or in a situation where it is too much exposed to the full morning sun.

Diseases. When budded on the Plum, this tree is but little liable to diseases, and may be considered a hardy fruit trce. In order to render it fruitful, and keep it for a long time in a productive state, we cannot too strnngly urge the advantages of the shortening.in system of pruning recommended for the peach.

\section{Albergier. Thomp. N. Duh. Nois,}

Alberge. O. Duh. Bon. Jard.

This is a variety very common in the interinur of France, where it is constantly reproduced with but little variation froin the seed-Alberge being the nane of the apricot in some of the 
provinces. It is a free grower, and bears well, but is neither se large nor fine as many other varieties. The leaves are small, anil often have little wing-like ears at the base. The Alber. giers are much used for stocks in France.

Fruit small, roundish, deep yellow. Flesh reddish, firm, with a brisk, vinous flavnur. Stone compressed; kernel bitter. Estremed for preserving. There are several varieties of this not yet introluced into the United States, the finest of which are the Albergier de Tours, and $A$. de Montgamet. Ripe middle of nugust.

\section{Breda. $\S$ Thomp. Lind. P. Mag.}

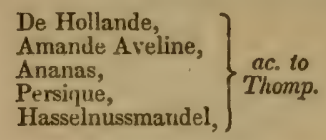

This is a very excellent small $A$ pricot, said to be originally from Africa, which bears well with common culture, and deserves a place in all gardens, as it is not only a high flavoured dessert sort, but it makes one of the richest preserves. The blossom buds are tinged with deep red before they expand.

Fruit rather small, about an inch and a half in diameter, roundish, sometimes rather four sided. Suture well marked. Skin orange, becoming dark orange in the sun. Flesh deep orange, rich, high flavoured and rather juicy - separating freely from the stone. The kernel, which is sweet, is eaten in France, whence the name Amande Aveline. First of August.

3. BLACK. Thomp. Fors.

Amygdalus dasycarpa. Dec.

Purple Apricot. Lind.

Angoumois? O. Duh.?

Noir.

Violet.

Du Pape.

This remarkable little A pricot so strongly resembles a dar mund Plum, that, at a little distance, it might easily be mistaken for one. (It was indeed calle 1 Prunus dasyearpa by the ola botanists.) It is pretty grood, and very hardy, and its unique appearance renders it sought after by amateurs. The tree has a rough, somewhat croolied trunk, and small, oval foliage.

Fruit about an inch and a half in diameter, round. Skin pale red in the shade, but dull reddish purple in the sun, covered with a slight down. Flesh pale red next the skin, yellow near the stone, adhering somewhat to the stone, juicy, with a pleasant, slightly astringent flavour. Kernel sweet. August. 


\section{Brussezs. Thomp. Lind. Miller.}

The Brussels Apricot is not a fine fruit in this country, but is is a good bearer in light soils. Fruit of medium size, rathe? oval, and flattened on its sides. Skin pale yellow, dotted with white in the shade, but often marked with a little russety brown in the sun. Suture deep next the stalk. Flesh yellow, rather firm, with a lively but not rich flavour. Kernel bitter. Middle of August. The Brussels of some collections is the Breda.

5. Hensmirke. § Thomp. Lind. P. Mag.

A large and beautiful English variety, of the finest quality, yet little disseminated in the United States, but which highly deserves extensive planting. It strongly resembles the Moor. park, from which it is known by its stone not being perforated like that variety. It also ripens a little earlier.

Fruit large, roundish, but considerably compressed or flatten. ed on its sides. Skin orange, with a red cheek. Flesh bright orange, tender, rather more juicy and sprightly than the Moorpark, with a rich and luscious plum-like flavour. Stone rather small, and kernel bitter. End of July.

6. Large EARLy. \& Thomp. Lind. P. Mag.

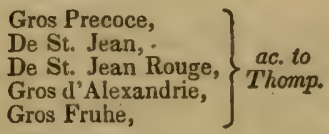

This variety which we have just received from abroad, nas the reputation of being the finest large early Apricot known. It ripens in France on midsummer day (the fete de St. Jean,) which will be about its season here.

Fruit of medium size, rather oblong, and compressed. Suture deep. Skin slightly downy, pale orange in the shade, fine bright orange with a few ruddy spots in the sun. Flesh separating readily from the stone, orange coloured, rich and juicy. Keruel bitter.

7. Moorpark. \& Thomp. Lind.

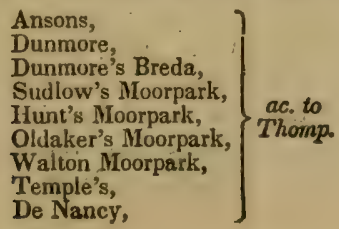

This fine old variety is the most popular and widely dissem. 
inated in this country, except the Red Masculine. It has its name from Moorpark, the seat of Sir William Temple, in Eingland, where it was cultivated more than one hundred and forty years ago. It is only a moderate bearer here, and especially requires the shortening-in mode of pruning as recommended for the Peach.

Truit large, roundish, about two inches and a quarter in di amete: each way, on a standard iree; rather larger on one side of the suiure than the other. Skin orange in the shade, but deep orange or brownish red in the sun, marked with numerous lark specks and dots. Flesh quite firm, bright orange, parting free from the stone, quite juicy, with a rich and luscious flavour. Stone peculiarly perforated along the back, where a pin may be pushed through, nearly from one end to the other. Kernel bitter. Ripe early in August.

\section{Musch-Musch. Thomp. Nois.}

D'Alexandrie.

'This delicious little Apricot takes its name from the city of Musch on the frontiers of Turkey, in Asia ; but it is also common about Alexandria, and in northern Egypt it is said to be raised in such abundance that the dried fruit is an article of commerce. The tree is rather delicate, and requires a sheltered position.

Fruit rather small, about an inch and a half in diameter, round. Skin deep yellow, with a little orange red on the sunny side. Flesh yellow, with a transparent pulp, tender, melting, and very sweet. Kernel sweet.

9. Orange. Thomp. Lind. Mill.

\section{Early Orange. \\ Royal Orange. \\ Royal George. \\ Persian. \\ Royal Persian.}

An Apricot of only tolerable quality for the dessert, but it 18 much esteemed by many for preserving; and it makes delicious tarts, even before the fruit begins to acquire colour.

Fruit of medium size, roundish, with a well marked suture, deeply hollowed near the stalk. Skin firm, orange, sometimes tinged with a ruddy tint in the sun. Flesh dark orange, moderately juicy, but often rather dry and insipid, (unless ripener in the house, not separating entirely from the flesh. Stone small, roundish. Kerne! sweet. Middle of July. 
10. PEACH. $\{$ Thomp. Fors. Lind.

\author{
Anson's Imperial. \\ Royal Peach. \\ Pêche. \\ Abricot Pêche. N. Duh. Poit. \\ De Nancy. O. Du/. \\ Du Luxembourg. \\ Pèche Grosse. \\ Wurtemburg. \\ Pfirsiche.
}

The Peach Apricot, originally from Piedmont, has long been considered the finest variety; and it is with us the largest and most excellent sort cultivated-being often as large as a Peach of medium size, handsome, and of delicious flavour. It very strongly resembles the Moorpark, but the two are readily dis. tinguished by the eye when standing near each other, and the fruit of the Peach is rather larger and finer, and a few days earlier.

Fruit of the largest size, about two and a half inches in di. ameter, roundish, rather flattened, and somewhat compressed on its sides, with a well marked suture. Skin yellow in the shade, but deep orange, mottled with dark brown, on the sunny side. Flesh of a fine yellow saffron colour, juicy, rich, and high flavoured. Stone with the same pervious passage as the Moorpark, and with a bitter kernel.

\title{
11. Ronan. Thomp. Lind. \\ Abricot Commun. O. Duh. \\ Germine. \\ Grosse Germine. \\ Transparent.
}

This is with us one of the largest growing and hardiest Apr1cot trees, and produces good crops every year in cold or unfavourable situations, where none of the other solts, except the Masculine, succeed. It is, therefore, though inferior in flavour, a valuable sort for northern situations. The blossoms will bear quite a severe frost without injury.

Fruit middle sized, oblong, with the sides slightly compressed, with but little or no suture. Skin entirely pale yellow; or very rarely dotted with a few red spots on one side. Flesh dull yel. low, soft, rather dry. When ripened by keeping a few days in the house, the flavour is tolerably good. Stone oblong, with a bitter kernel. Ripe the last of July and first of August.

There is a Blotched LEAVED Ronas, (commun à feuilles panaches, of the French,) preciscly like the foregoing in all respects, except the white or ycllow stain in the leaf-but it is quite distinct from the Blotehed leaved Turkey, cultivated here. 


\section{Royai. § Thomp. Nois. P. Mag.}

A fine large French variety, raised a few years since at the Royal T,uxembourg gardens. It is nearly as large as the Moorpark, bui with larger leaves borne on long footstalks, and witicut the pervious stone of that sort. It is quite as high flavoured and ripens a week or ten days earlier.

Fruit roundish, large, oval, slightly compressed. Skin dull yellow, with an orange cheek, very faintly tinged with red, and a shallow suture. Flesh pale orange, firm and juicy, with a rich vinous flavour. Ripe the latter end of July.

\section{Red Masculine. Thomp. Lind.}

Early Masculine.

Brown Masculine.

$\left.\begin{array}{l}\text { Abricot precoce, } \\ \text { Abricot hâtif musqueé, }\end{array}\right\}$ O. Düh.

Abricotier hatif. N. Duh.

Abricotier

Frülıne Muscateller.

The Red Masculine is a good deal cultivated with us. It is very hardy, ripens the earliest, and bears very regularly and well. On the other hand the fruit is quite small, and only of second rate flavour. It is likely, therefore, to give place to the Large Early, which ripens only a few days later, and is much superiour.

Fruit small and nearly round, scarcely an inch and a half in diameter, with a well marked suture on one side. Skin bright yellow, tinged with deep orange and spotted with dark red on the sunny side. Flesh yellow, juicy, with a slightly musky, pleasant flavour. Stone thick, obtuse at the ends. Flowers smaller than in most other sorts. Kernel bitter. Ripe abrut the 12th of July.

\section{Shipleys. Thomp.}

Blenheim.

Shipley's Large.

This is a new variety which we have lately received from England, and which is not yet fully proved in this country. It has the reputation of being nearly equal to the Moorpark, and more productive, while it is next in point of earliness to the Large Early.

Fruit large, oval, orange, with a deep yellow, juicy, and tolerably rich flesh. Stone roundish, impervious, with a bitter kernel. Ripens here about the 25th of July. 


\title{
15. TÜвеY. $\S$ Thomp. P. Mag. Lind.
}

\section{Large Turkey.}

De Nancy, (if some.)

'I'he T'urkey Apricot is a fine old variety, which is seldom seen in our gardens, the sort generally sold under this name being the Roman. It is quite a late sort, ripening after the Moor. oark, from which it is easily known by its impervious stone, and sweet kernel.

Fruit of middle size, nearly round, not compressed. Skin fine deep yellow in the shade, mottled with brownish orange in the sun. Flesh pale yellow, firm, quite juicy, with a flavour in which there is an excellent mingling of sweet and acid. Kernel nearly as sweet as that of an almond, which, as well as the form and colour, distinguishes this sort from the Roman. Ripe tle middle of August.

The Blotched Leaved Turiey, or Gold Blotched, (Abricot macule, $)$ is a sub-variety, very well lnown here, resembling the common Turkey in all respects, except that it has in the centre of each leaf a large yellowish spot. It is a thrifty tree and bears delicious fruit. Ours is not identical with the Turkey, as the last edition of the L. H. S.'s Catalngue arranges it, but is a globular fruit, and a true variation of the Turkey.

16. White Masculine. Thomp. Lind. Fors.

\author{
White Apricot. \\ Abricot Blanc. O. Duh. Nois. \\ Abricotier Blanc. N. Duhl. \\ Early White Masculine. \\ Blanc. $a c$. to \\ White Algeirs? T'homp.
}

This scarcely differs from the Red Masculine before described, except in colour. It is four or five days later.

Fruit small and roundish. Skin near!y white, rarcly with a little reddish brown on one side. Flesh white, delicate, a litlle fibrous, adheres a little to the stone, and has a delicate, pleasarit 'un':. Kernel bitter.

Cerrious, or ornamental varieties. The Briancon APRICot, (A. brigantiaca, Dec.) a very distinct species so much resenbling a plum as to be called the Briancon Plum by many au. thors, (Prune de Briancon, Poit.) is a small, irregular tree or shrub, ten or twelve feet high, a native of the Alps. It bears a great abundance of small round yellow plum-like fruit in clus. ters, which are scarcely eatable; but in Fuance and Piedmont 
the kerncls of this varicty make the "huile de marmotte," which is worth double the price of the olive vil.

The Double Flowering Apkicot is a pretty ornamental tree, yet rare with us.

Selection of Apricots for a small garden. Large Early, Breda, Peach, Moorpark.

Selection for a cold, or norlhern climate. Red Masculıne, Roman, Breda.

\section{CHAPTER XI.}

\section{THE BERBERRY.}

Berberis vulgans.* L. Berberacea, of botanists.

tipine-vinelle, of the French ; Berberilzen, German; Berbero, Italian; Berberis, Spanish.

The Berberry (or barberry) is a common prickly shrub, from cight to ten feet high, which grows wild in both hemispheres, and is particularly abundant in many parts of New-England. The flowers, the roots, and the inner wood are of the brightest yellow colour, and the small crimson fruit is borne in clusters. It is a popular but fallacious notion, entertained both here and in England, that the vicinity of this plant, in any quantity, to grain fields, causes the rust.

The barberry is too acid to cat, but it makes an agrecable preserve and jelly, and an ornamental pickle for garnishing some dishes. From the seedless sort is made in Roven a celebrated sweetmeat, confiture d'epine-vinctte. The inner bark is used in France for drying silk and cotton bright yellow.

Cucturs. The culture is of the easiest description. A ruch light soil, gives the largest fruit. It is easily propagated by seed, layers, or suckers. When fine fruit of the barberry is desired it should be kept trained to a single stem-as the suck. crs which it is liable to produce, frequently render it barren or make the fruit small.

\section{Cominon ReD.}

This is too well known to need description. In good soils it grows twelve or fifteen feet high, and its numerous clusters of bright, oval berrics, are very omamental in autumn. There is a Large Red variety of this, which is only a variation produced berry.

* Or B. Canadensis-they are scarcely distinet-ours las rather the most fleshy 
by cultivation in rich soil. There are also varietics of this in Lurope with pale yellow, white, and purple fruit, which are not yet introduced into this country, and which scarcely differ in any other respect than the colour. Finally, there is a so-called sweet variety of the common Berberry from Austria, (ji. v. clulcis,) but it is scarcely less acid than the common.

\section{Stoneless.}

\section{B. v. Asperma.}

Seedless.

Vinetier saus noyeau.

The fruit of this, which is only a variety of our common barberry, is without seeds. But it does not appear to be a permanent varicty, as the plants frequently do produce berries with seeds; and it is stated in the New Duhamel that in order to guard against this, the sort must be propagated by layers or cuttings, as the suckers always give the common sort. It is considered the best for preserving.

\section{Black Sweet Maget.lan. Loudon.}

Berberis dulcis, D. Don.

B. ratundifolia.

A new evergreen sort from the Straits of Magellan, South America. It is very rare, and has not yet fruited in this country, but it is likely to prove hardy. Loudon, in the Suburban Gardener, says it bears round black berries, about the size of those of the black currant, which are used in its native country for pies and tarts, both green and ripe. It has ripened fruit in Edinburgh, in the nursery of Mr. Cunningham, who describes it as large and excellent.

\section{NEPAL.}

Berberis aristata.

This is a new variety from Nepal, India. We have cuitivated it three or four years, and find it tolerably hardy, but, though it has produced flowers, it has yet given no fruit. It is said to yield "purple fruit, covered with a fine bloom, which in India are dried in the sun like raisins, and used like them at the dessert."

The Mahonias, or Holly Teaved Berberries, from Oregon aro handsome low evergreen ornamental sbrubs, with large deep green prickly leaves, and yellow flowers, but the fruit is of no value. 


\section{CHAPTER XII.}

\section{THE CHERRY.}

Cerasus sylestris, anil C. vulgars, Arb. Brit. Rosaceos, of butanirts. Cerister, of the French; Ḱirschenbaum, German; Ciriego, Italian; Cerezo, Spen ish.

Tue cherry is a fine, luxuriant fruit tree, with smouth, light coloured bark, and, generally of rapid growth. The varisties of the black and heart-shaped cherries are always vigorous, and form fine larre spreading heark, forty or fifty feet in height; but those of the acid or red cherry are of lower, more bushy and tardy growth. In the spring the cherry tree is profusely covered with elusters of snow white blossoms, and earlier in summer than upon any other tree, these are followed by abun. dant crops of juicy, sweet, or acid fruit hanging upon long stalks, and enclosing a smooth stone.

The cherry comes originally from Asia, and the Roman gene. ral, Lucuilus, after a victorious expedition into Pontus, has the reputation of having brought it in Italy, from Cerasus, a town in that province, in the year $69, \mathrm{~B}$. C. According to Pliny, the Romans, 100 year's after this, had eight varieties in cultivation, and they were soon afterwards carried to all parts of Europe. The seeds of the cultivated cherry were brought to this country very early after its settlement, both from England and Holland.

UsEs. As a pleasant and refreshing dessert fruit, the cherry is every where highly esteemed. The early season at which it ripens, its juiciness, delicacy, and richness, render it always acceptable. While the large and fleshy varieties are exceed. ingly sweet and luscious, others which are more tender, and more or less acid, are very valuable for pies, tarts, and various kinds of cookery. The fruit of the Kentish or Early Richinond is excellent when stoned and dried, and the Mazzard, and our wild Virginia cherries, are used to give a flavour to brandy.

The celebrated German Kir schwasser is made by distilling the liquor of the common black mazzard or gean, (in which the stones are ground and broken, and fermented with the pulp,) and the delicions Ratifu cordial of Grenoble, is also rrade from this fruit. Mtaraschino, the most celebrated liqueur of Italy, is distilled from a small gean or mazzard, with which, in formenting, honey, and the leaves and kernels of the fruit are mixed.

The gum of the cherry is near!y identical with gum aralsic, and there are some marvellous stories told of its nutritive properties. The wood of the cherry is hard and durable, and is, therefore, valuable for many purposes, but the best wood is 
afforded by our common wild or Virginia cherry, which is a very grool substitute for mahogany, taking a fire polish.

The laren growing sorts of black cherry are the finest of all fruit trees for shade, and are, therefore, generally chosen by farmers, who are always desirous of combining the us ful and the ornamental. Indeed, the cherry, from its symmotrical form, its rapid growth, its fine shade, and beautifuil blossoms, is exceedingly well suited for a road side tree in agricultural dis. tricts. We wish we could induce the planting of avenues of this and other fine growing fruit trees in our country neighbour. lıoods, as is the beautiful custom in Germany, affording orna. mont and a grateful shade and refreshment to the traveller, at the same moment. Mr. Loudon in his Arboretum, gives the following account of the cherry avenues in Germany, which we gladly lay before our readers.

"On the continent, and more especially in Germany and Sivitzerland, the cherry is much used as a road side tree; particularly in the northern parts of Germany, where the apple and the pear will not thrive. In some countries the road passes for many miles together through an avenue of cherry trees. In Moravia, the road from Brunn to Olmutz, passes through such an avenue, extending upwards of sixty miles in length; and, in the autumn of 1828 , we travelled for several days through almost one continuous avenue of cherry trees, from Strasluurg by a circuitous route to Munich. 'These avenues, in Germany, are planted by the desire of the respective governinents, not only for shading the traveller, but in order that the poor pedestrian may obtain refreshment on his journey. All persons are allowed to partake of the cherries, on condition of not injuring the trees; but the main crop of the cherries when ripe, is gathered by the respective proprietors of the land on which it grows; and when these are anxious to preserve the fruit of any particular tree, it is, as it were, tabooed; that is, a wisp of straw is tied in a conspicuous part to one of the branches, as vines by the road sides in France, when the grape are ripe, are protected by sprinkling a plant, here and there, with a mixture of lime and water, which marks the leaves with conspicuous white blotches. Every one who has travelled on the Continent in the fruit sea. son, must have observed the respect that is paid to these appropriating marlis; and there is something highly gratifying in this, and in the humane feeling displayed by the princes of the diflerent countries, in causing the trees to be planted. It would indeed be lamentable if kind treatment did not produce a corresponding return."

Soli, and situation. A $d r y$ soil for the cherry is the uni. rersal maxim, and although it is so hardy a tree that it will thrive in a creat variety of soils, yet a good, sandy, or gravelly loam is its favourite place. It will indeed grow in much thin. 
ner and drver soils than most other fruit trees, but to ohtain the firest fruit a deep and mellow soil, of crood quality, is desirable. When it is forced to grow in wet places, or where the reots are constantly daunp, it soon decays, and is very short lived. Aud we have seen this tree when fored into too juxuriant a growth in our over-rich western soils, become so gross in its wood as to bear little or no fruit, and split open in its trunk, and soon perish. It is a very hardy trec, and will bear a great variety of exposures without injury. In deep warm valleys, liable to spring frosts, it is, however, well to plant it on the north sides of hills, in order to retard it in the spring.

Propagation. The fince sorts are nearly always propagated by budding on seedlings of the common black mazzard, which is a very common kind, producing a great abundance of fruit, and very bealthy, free growing stocks. To raise these stouks, the cherries should be gathered when fully ripe, and allowerl to lie two or three days together, so that they may be partially or wholly freed from the pulp by washing them in water. They should then be planted immediately in drills in the seed plot covering them about an inch leep. They will then vegetate in the following spring, and in good soil will be fit for planting out in the nursery rows in the autumn or following spring, at a distance of ten or twelve inches apart in the rows. Many per. sons preserve their cherry stones in sand, either in the cellar or in the open air until spring, but we have found this a more precarious mode; the cherry being one of the most delicate of sceds when it commences to vegetate, and its vitality is fre. quently destroyed by leaving it in the sand twenty-four hours too long, or after it has commenced sprouting.

After planting in the nursery rows, the seedlings are gone. rally fit for budding in the month of August following. And in order not to have weak stueks overpowered by virgorous ones, they should always be assorted before they are planted, placing those of the same size ir rows together. Nearly all the cherries are grown with us a: standards. The English nurserymen usually bud their standard cherries as hich as they wish them to form heads, but we always prefer to bud them on quite young stincks, as near the ground as possible, as they then shoot up clean, straight, smooth stems, showing no clumsy joint when the bud and the stock are united. In good soils, the buds will frequently make shoots, six or eight feet high, the first season after the stock is headed back.

When divarf trees are required, the MLorello seedlings are used as stocks; or when very dwarf trees are wished the P'erfumed Cherry, (Cerasus Malhileb.) is mmplnyed; but as standarls are almost universally prefered, these are schlom secn nere. Dwarfs in the nursery must be hearled back the second year, in order to form lateral shoots near the ground. 
Curtivation. The cherry, as a standard tree, may be saia o require little or no cultivation in the middle states, further than occasionally supplying old trees with a little manure to keep up their vigour, pruning out a deut or erossing branch, and washing the stem with soft soap should it become hard and bark bound. Pruning, the eherry very little needs, and as it is always likely to produce gum (and this decay,) it should be avoided, except when really required. It should then be done in midsummer, as that is the only season when the gun is not more or less exuded. 'The cherry is not a very long lived tree, but in favourable soil the finest varieties generally endure about thirty or forty years. 'Twenty feet apart for the strong, and cighteen feet for the slow growing kinds is the proper distance for this tree.

Training the Cherry is very licle practised in the United States. The Heart and Bigarreau cherries are usually trained in the horizontal manner, explained in page 40 . When the wall or espalier is once filled, as there directed, with lateral branches, it is only necessary to cut off, twice every season-in the month of May and July - all alditional shoots to within an inch or so of the branch from which they grew. As the trees grow older, these fruit spurs will advance in length, but by cutting them out whenever they exceed four or tive inches, new ones will be produced, and the tree will continue to keep its proper shape and yield excellent fruit. The Morello cherries, being weaker growing sorts, are trained in the fan-manner, (page 38.)

Gathering the fruit. This tender and juicy fruit is best when freshly gathered from the tree, and it should always be picked with the stalks attached. For the dessert, the flavour of many sorts in our climate, is rendered more delicious by placing the fruit, for an hour or two previous, in an ice-house or refrigerator, and bringing them upon the table cool, with dew drops standing upon them.

VARIETIES. For the sake of enabling the amateur the more readily to identify varieties, we shall divide cherries into four classes, viz.

I. Heart cherries. The Common Mazzard and the Black Heart may be taken as types of this division. 'The trees are rapid growing, with ample and lofty heads, and broad, light green, waved leaves. The fruit is more or less heart-shaped, with rich, tender, sweet flesh. (This includes the MTtrisiers and Guiniers of the French, which scem to us, practically, not distinct.) This section comprises exccllent cherries, universally admired. Colour mostly black.

II. Bigurreuu cherries. 'This term comes originally from the Erench bigarrée-speckled or variegated skin-but it is now in general use by all pomologists, to signify hard, or firm.fleshecl, 
sweet cherries-those which are firm anil crackling, as com. pared with the melting, tender flesh of section I. The Common Bigarreau o: (rraftion, may be taken as the type of this cluss, which is mainly composed of the largest and most beautiful of cherries, admirable for the dessert, and whose firmness renders them werl suited for carriage to rnarlet. The trees like the Heart cnerries, are lofty and spreading, with similar foliage. (B'garreaux, and Bigarreantiers, of the French.)

III. Duke cherrics. This class is characterised by the round. ish form of its fruit, thin skin, and juicy, melting flesh; the flavour being generally sub-acid before fully ripe, when it is rich, and nearly or quite sweet. The Mayduke is the type of this class. The trees are upright in their growth while young, and finally form lower heads than those of the two previous sections, with narrower leaves, which are flat and darker coloured. The young wood is also darke;, and a iittle less strong than that of the Heart and Biggarreau classes. These are excellent varicties, succeeding well in almost all soils and climates, and invaluable both for the dessert and for cooking. (Cerisiers, of the French.)

IV. Morello cherrics. The common Kentish or pie cherry, and the Morello, are well known types of this class. The fruit is mostly round, with thin skin, juicy, tender, and quite acid, being chiefly valued for cooking, preserving, and various cu. linary purposes. The trees are of rather low and spreading growth, with small wiry branches, and narrow dark green foliage. (Griottiers, and Cerisiers, of the French.)

In describing cherries we shall designate their size by com. parison, as follows: Targe, as the Tartarian, and Bigarreau; middle sizod, as the Mayduke and Black Heart; small, as the Transparent Guigne and Honey, (see the outlines of these sorts.) As regards form, heart-shaped, as the Black Heart; obluse heart-shaped, as the Bigarreau; and round, as the Kentish. As regards texture, tender, as the Mayduke; half tender, as the 'Tartarian, and firm, as the $\mathrm{B}$ gas reau. 


\section{Class I. Heart Cherries.}

Fruil sweet, with tenizer or half tender flesh, heart-shaped, or oval; trens with broad, somewhat pendant foliage.)

\section{American Amber.}

Bloodgood's Amber.

Bloodgood's Honey.

Bluodgood's New Honey.

This exceedingly bright and pretty cherry, was raised soine years ago by Mr. Daniel Bloulgood, of Flushing, Long Island. It is a most abundant bearer, the fruit hanging in the richest bunches from the branches, giving the tree a fine apfearance when in fruit, but it is only second rate in point of flavour. At a little distance it resembles the Amsrican Heart,

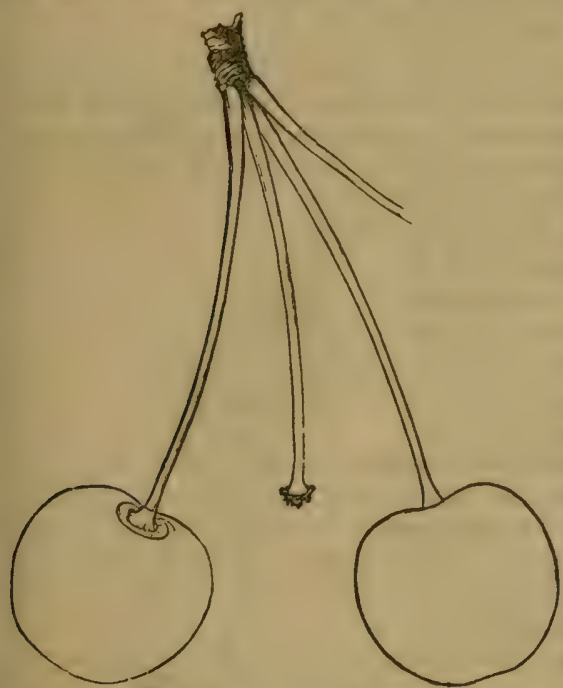

Fig. 59. American Amber. from which, however, it differs in being a tender fleshed fruit, of very regular outline, while the latter is partially firm, (belonging to the Bigarreau class, ) and of an irregular figure.

Fruit of medium size, (borne three or four in a cluster, ) and very regular form, roundish heartshaped, often nearly round, with a slightly indented point at the apex, (like a Mayduke.) Skin very thin, smooth, eveil, and glossy, clear light amber at first, but, when ripe, delicately mottled and oversprcad with clear bright red. Stalk long, slender, and inserted in a very slight and narrow cavity. Flesh amber colour, tender, abnunding with a sprightly, though not high flavoured juice. Ripe about the 25th of June. 'This is nearly related, but is inferior in quality, to Downer's Late, ard Sparhawk's Honey, which ripen at the same time. 


\section{Amber Gein. Thomp.}

Gean Amber.

A protty little Gean (or Mazzard,) with a very hin ana mansparent skir, and sweet flavour. It is exceedingly produc. tive, mpens late, and hangs till the middle of July.

Fruit small, oval or obtuse heart-shape, quite regular in form, generally borne in threes. Skin very thin and pellucid, showing the texture of the flesh beneath, colour pale yellow, partially overspread with a very faint red. Stalk long and slender, very slightly inserted. Flesh white, juicy, melting, of a sweet and pleasant flavour.

'This considerably resembles the Transparent Guigne, but it is rather smaller and less handsome. Jt is also more pellucid, mnre yellow, less distinctly spolted with red, and is borne in clusters, which the latter is not.

\section{Baumann's May. \\ Bigarreau de Mai. Ken. \\ Wilder's Bigarreau de Mai. \\ Bigarreau de Mai. Thomp.?}

This cherry, which, under the name of Bigarreau de Mai, has already obtained quite a reputation as the earliesi cherry, was received several years ago by our friend M. P. Wilder, Esq., President of the Massachusetts Horticultural Society, from the Messrs. Baumann, of Bollwyller, in France. The label was lost on the passage, and the "Bigarreau de Mai," being in the invoice it was supposed that such might be the name of this variety. As, however, it is not a Bigarreau, but a tender fleshed cherry, we think it best for the present to call it Baumann's May. The young branches are literally covered with the abundance of the fruit, it being a most prolific bearer. Branches strong, leaves large.

Fruit rather small, oval heartshaped, and rather angular in outline. Skin deep rich red, becom. ing rather dark when fully rip?. Stalk an inch and three fourths

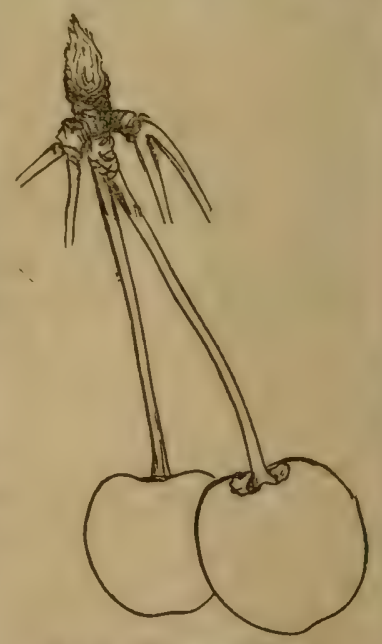

Fig. 60. Baumann's May. long, pretty stout at either end, and set in a very narrow, and rather irregular cavity. Flesh purplish, tender, juicy, and when fully ripe, tolerably sweet and good. Ripens here the 20th of May. 
4. Beack Heart. § Thomp. Mill. Lind.

Early Black.

Ansell's Fine Black.

Spanish Black Heart.

Black Russian, (of American gardens.

Black Caroon, (crroneously, of some.)

Guinier à fruit noir. O. Duh̆.

Guigne grosme noir.

Grosse Schwarze Hertz Kirsche.

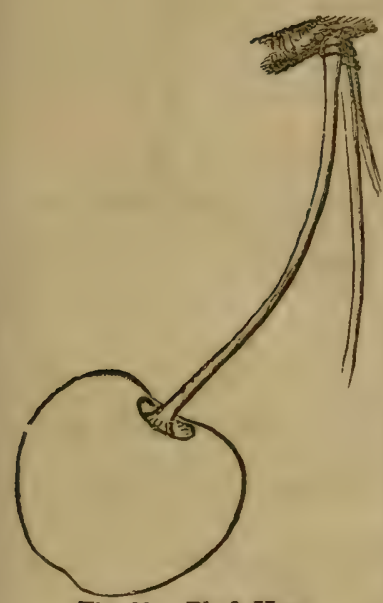

Fig. 61. Black Heart.

The Black Heart, an old variety, is better known than almost any other cherry in this country, and its great fruitfulness and good flavour, together with the hardiness and the large size to which the tree grows, render it every where esteemed.

Fruit above medium size, heartshaped, a little irregular. Skin glossy, dark purple, becoming deep black when fully ripe. Stalk an inch and a half long, slender, set in a monerate hollow. Flesh, before fully ripe, half tender, but finally becoming tender and juicy, with a rich, sweet flavour. Ripens the last of June, about ten days after the Mayduke.

5. Black Heart, Manning's Eariy. Man.

This is a seedling raised by the late Robert Manning, of Salem, Mass., from the common Black Heart. In size, form and colour, it scarcely differs from the original variety, but it has the merit of ripening ten days earlier-about the same time, or even a little before the Mayduke.

\section{Black Heart, Werder's Early. Thomp.}

Werder's Early Black.

Werdersche Fruilse Schwarze Herz IKirsche.

A new variety, recently introduced from England, and which p:omises to be very valuable on account of its ripening among the very earliest cherries. Fruit of medium size, heart-shaped; skin black ; flesh purplish, tender, sweet and excellent. Ripens the last of May, or very early in June. 


\section{Blacr Eagre. $§$ Thomp. Lind.}

A very excellent English variety, raised by the daughter of Mir. Knight, at Downton Castle, in 1806, from the seed of the Bigarreau fertilized by the Mayduke. It ripens at the beginning of July or a few days later than the Black Tarta rian.

Fruit rather above medium size, borne in pains and threes; obtuse heart-shaped. Skin deen purple, or nearly black. Stalk of mrdium length, and rather slender. Flesh deep purple, tender, with a rich, high flavoured juice, superiour to the Black Heart. Branches strong, with large leaves.

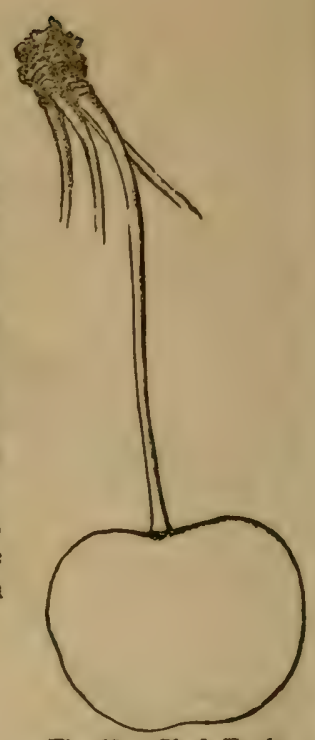

Fig. 62. Black Eagle.

8. Black Tartarian. $\S$ Thomp. Lind. P. Mag.

'Tartarian.

Fraser's Black Tartarian, $\{$ Fors.

Black Circassian. Hooker.

Superb Circassian,

Ronald's large Black Heart, lionald's Heart,

Fraser's Black Heart,

Fraser's Black,

Fraser's T'artarisclie,

Schwarze Herz Kirsche,

Black Russian, of the English, but not of American gardens.

This superb fruit has already become a general favourite in all our gardens; and in size, flavour, and productiveness, it has no superiour among black cherries. It is a Russian, and West Asian variety, introduced into England about 1796 , and brought thence to this country about twenty years ago. It is remark. able for its rapid, vigorous growth, large leaves, and the erect habit of its head. The fruit ripens about the middle of June, a few days after the Mayduke.

Fruit of the largest size, heart-ohaped, 
(snnetimes rather obiusc,) irregular and uncven on the surface, Skin glossy, bright purplish black. Flesh purplish, thick, (the stone being quite small, half-tender, and juicy. Flesh very rich and delicious.

\section{Bowyer's Early Heart. Thomp.}

A new Finglish variety, as yet little known with us. It is one of the earliest of light coloured cherries, and a good bearer, being in eating very early in June.

F'ruit rather below medium size, obtuse heart-shaped. Skin amber, mottled with red. Flesh white, soft, or very tender, juicy, with a pleasant, sweet fiavour.

10. Brack Mazzard. Thomp. Lind.

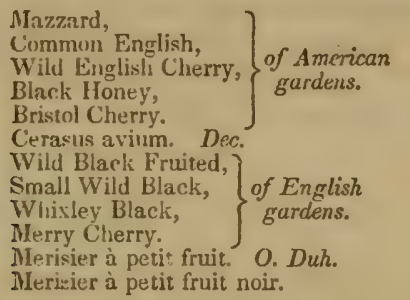

This is ine wild species of Europe, being common in the for ests of France and some parts of Kingland; and it has now be. come naturalized, and grows spontaneously on the borders of woods in many parts of the Atlantic states. It is the original species from which nearly all the fine Heart and other sweet cherries, have sprung. It is small, and of little value for cating, retaining, unless very ripe, a certain bitterness; but it ripens and hangs on the tree until the middle or last of July, sc that it then becomes somewhat acceptable. It is, however, chicfly valued for the manufacture of cherry brandy, and in districts where this is carried on, from the large size and great fruitfulness of the trees it is quite a profitable sort. It afiorile the most valuable seedling stocks on which to bud and grinlt finer varieties.

Fruit small, roundish or oval heart-shaped, flattened a little on both sides. Stalk long and very slender, inserted in a small depression. Skin thin, and when fully ripe, jet black. Flesh soft and melting, purple, with an abundant, somewhat bitter juice.

The White Mazzard, of Mr. Manning, is a seedling raised by that pomologist, which differs little except in its colour. 


\title{
11. Conove. Thomr: Fors.
}

\author{
Couronne. Iind. \\ Corrulu. Sang. \\ Hlerefordshire Black. \\ Black Orleans. \\ Large Wild Black.
}

The Corone is a natural cherry in many paris of England, reprolucing itself from seed, growing with great vigour, and bearing most abundantly. It is only of second quality being merely an improved Mazzard, and does not deserve a place in a smali collection, but as it is very hardy and ripens late, it is of some value at the north on that account.

Fruit below middle size, roundish heart-shaped. Skin dingy black when fully ripe. Stalk two inches long, slender, and inserter in a deep and narrow cavity. Flesh when ripe, tender, abounding in a deep purple juice, of tolerably good flavour. Middle of July. The Black Heart is often incorrectly called by this name in the middle states.

12. Downton. § Thomp. Lind.

A very beautiful and excellent large variety raised by T. A. Knight, Esq., of Downton Castle, from the seed, it is believed, of the Elton. It ripens a little later than the majority of sorts, and is a very desirable cherry.

Fruit large, very blunt heart-shaped, nearly roundish. Stalk one and a half to two inches long, slender, set in a pretty deep, broad hollow. Skin pale cream colour, semi-transparent, deli. cately stained on one side with red, and marbled with red dots. Flesh yellowish, without any red, tender, adhering slightly to the stone, with a delicious, rich flavour. Early in July.

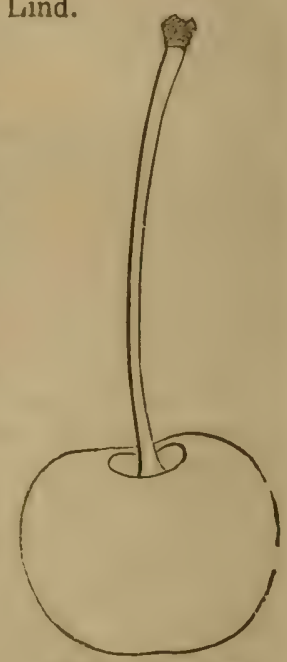

Fig. 64. Dounton.

13. Davenport's Early.

Davenport.

Davenpurt's Early Black. Ken.

Svarcely different from the Black Heart-indeed, we fino it 
mpnssible to distinguish any difference in the fruit-except that it ripens a few days earlier. The leaves, however, are larger and of a lighter crreen slin, and waved on the margin, and the tree eomes early into bearing. The thin, light brown bark, on the young trees, resembles that of the Birch. This native va. riety has been called New MIayduke by some, but it has no resemblance to a Duke Cherry.

\section{Downer's Late. $\S$}

Downer. Min.

Duwner's late Red.

This valuable late cherry was raised by Samuel Downer, Esq., an ardent cultivator of Dorchester, near Boston. It is a very regular and great bearer, ripens about a rveek after the cherry season, and hangs for a considerable time on the tree. It is a delicious, melting fruit, and deserves a place in every garden.

Fruit of medium size, roundish, heart-shaped, inclining to oval. Skin very smooth, of a soft but lively red. mottled with a little amber in the shade. Stalk inserted with a very slight depression. Fruit bornc t!ıckly, in clusters. Flesh tender, melting: with a sweet and luscious flavour. Ripens from the 4 th to the 10th of July.

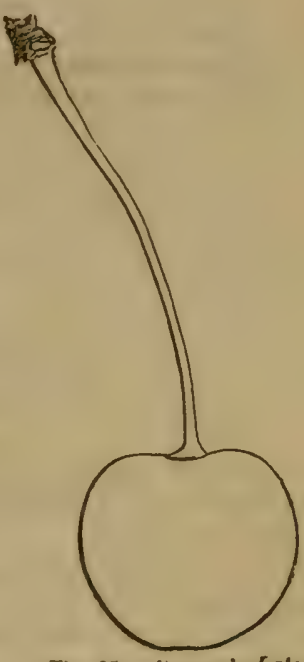

Fig. 65. Duwner's Late.

\section{Early Wiitte Heart.}

Arden's Early White Heart.

White Hpart. Coxe. Prince's Pom. Man.

White Heart,

Tredge's Early White Ieart,

White Transparent,

Ainber Heart.

An old varicty, long cultivated in this country, and one of the earliest, ripening before the Mayduke. At Ardenia, the seat of R. Arden, Esq., opposite West Point, on the 11 c.dson, there are many large trees of this variety, received by him originally from France, which are most abundant and regular bearers-and we do not perceive that in this part of the coun. try this cherry is open to Coxe's accusation of being a bad 


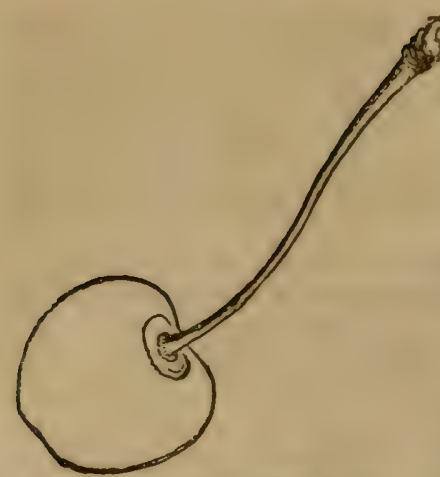

Fig. 66 Early White Heart.

bcarer. 'Though a very gnod carly fruit, this will no dnubt be supplanted by Bowyer's Early Heart, and other newer and finer sorts.

The White Ileart of Thompson and Lindley, may perhaps prove the same variety as this, though they describe it as a late ripening sort.

Fruit below medium size, rather oblong healt-shaperloften a little one-sirnd. Suture quite distinct. Stalk an inch and three fourths long; rather slender, inserted in a wide shallow cavity. Skin dull whitish yellow, tinged and speckled with pale red in the sun. Flesh half tender, unless fully ripe, when it is melting, with a sweet and pleasant flavour. Tree grows rather erect, with a distaff. like head when young. In the nursery the young trees are easily kn wn by their long and slender shoots, with few branches First of June.

\section{Early Purple Gutgne. \$ Thomp.}

Early Purple Griotte.

An erceedingly early variety ripening the last of May, newly introduced from England, and which promises to be a most valuable acquisition.

Fruit of medium size, and very handsome appearance. Skin smooth, dark red, becoming purple. Flesh purple, tender, juicy, with a rich and sweet flavour. The leaves have longer petioles than those of most other sorts.

\section{Gascolgne's Heart. Thomp. \\ Bleeding Heart. Lind. \\ Redl Ileart, (of some, ) ac. to \\ $\left.\begin{array}{l}\text { Ilerefurdshire lleart, } \\ \text { Guigne Rougre Ilative, }\end{array}\right\} \begin{aligned} & \text { ac. to } \\ & \text { Thomp. }\end{aligned}$}

An old Fnglish varicty, very seldom seen in our gardens. Fruit of medium size, long heart.shaped, and remarkable for the smal! drop or tear, with which the end is terninated. Skin rlark red. Flesh reddish, lalf tender, with only a solerab'e favour. Ripe the last of June. Unfortunately, this variety has the reputation of being a bad bearer. 


\section{Honey. Thomp.}

Large Honey.

Yellow Honey.

Late Honey.

Merisier à fruit blanc. $N .11 u h$.

The Honey cherry is a variety of the Mazzard but little larger than the common black variety, and its chief merits are great productiveness and lateness of ripening. It is exceedingly sweet when fully ripe, and will hang for a long time upon the tree, which is one of the hardiest and thriftiest in its growth; but Downer's Late, which ripens at the same time is, every way, so much superior, that when that variety can be had, the Honey cherry will scarcely find a place in the garden.

Fruit small, roundish or oval. Skin smooth, yellowish, marbled with red at first, but becoming deep amber-red. Stalk long and slender, very slightly inserted. Flesh tender, melting, with a honied sweetness. Middle of July.

\section{Hyde's Ren Heart. Man.}

A new variety which we received from Mr. Manning, not yet proved here. The fruit is said to be heart-shaped, medium size; skin, at first, pale, but becoming a rather livoly red. Flesh tender, with a pleasant, sprightly flavour. The young trees make strong shoots, the bark of which is light gra:r, dotted with clusters of small white specks.

20. KNight's Early Black. § Thomp. Lind. P. Mag.

A most admirable early cherry, resembling the Black Tartarian, though much more obtuse in form, but ripening nearly a week earlier, or about the tenth of June. It is one of Mr. Knight's seedlings, a crossbreed between the Bigarreau or Graffion and the Mayduke, originated about 1810, and is universally admired.

Fruit large, a little irregular in outline, obtuse heart-shaped. Stalk of moderate length, rather stout, (much more so than in the Black Eagle,) and inserted in a deep, open cavity. Skin dark purple, becoming black. Flesh purple, tender, juicy, with a rich and sweet juice of high Gavour. Tree spreading:

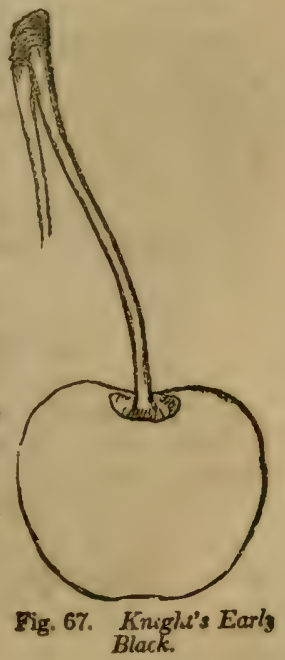




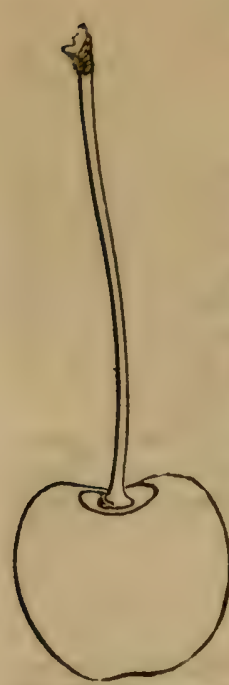

Fig. 68. Manning's Mottled.

\section{Manning's Mottued. $\S$}

Mottled Bigarreau, Man.

A beautiful cherry, raised by Mr. Manning, from the seed of the Bigarreau. It is a very tender fleshed, heart cherry, and, therefore, should not be called a Bigarreau. It is a most abundant bearer, and will soon become a favourite variety. Bark on the young tree, dark, with a few large, scattered dots.

Fruit rather large, roundish heart-shaped, flattened on one side, with distinct suture lines. Skin amber colour, finely mottled and overspread with red, with a semi-transparent, glossy appearance. Stalk slender, inserted in a shallow hollow. Flesh when fully ripe, yellow, tender, with a sweet and delicious juice. Stone pretty large. Ripens the last of June.

\section{Ox Heart. Thomp.}

\section{Iion's Heart.}

Bullock's Heart.

Very Large Heart.

Ochsen Herz Kirsche.

This has been made synonymous, by Manning, with Gas. coigne's Heart; but it is a larger and later fruit, obtuse, instead of pointed in its form. It is very scarce in collections here, the White Bigarreau being generally known by the name of $\mathrm{Ox}$ Heart in New-York. Fruit large, obtuse heart-shaped. Skin dark red. Flesh red, half tender, with a pleasant juice, of second quality in point of flavour. Ripens about the eighth of July.

\section{Roberts' Red Heart. Man.}

A new variety, which originated in the garden of David Roberts, Fisq., of Salem, Mass. Fruit large, obtuse heartshaped. Skin a bright, lively red. Stalk set in a rather wide hollow. Flesh red, juicy, tender, with an excellent, sweet fla vour. Slioots on young trees strong, dotted with large white dots. Rather late, ripening the last of June. 


\section{Rivers' Early Heart.}

A variety, raised by Mr. Rivers, a noted Engli:sh uursery man, which has not yet borne fruit with us. It is ctescriberi ass a mediurn sized heart-shaped cherry, ripening about the midule of June, and a very hardy and productive tree.

\section{Rivers' Early Amber. $\oint$}

Another seedling from the same source. A large and beau. tiful amber coloured cherry, tinged with a soft red on the sunny sille, heart-shaped, a hardy and very prolific tree. It is alsa one of the earliest in maturing its fruit, which will be in perfec. tion here about the tenth of June.

\section{Sparhatwk's Honey. § Man. Ken. \\ Sparrowhawk's Honey. Thomp.}

A delicious, melting, sweet cherry, introduced to the notico of fruit growers by Edwarl Sparhawk, Esq., of Brighton, near Boston. It ripens a little later than most varieties, is a profuse bearer, and a truly valuable sort.

Fruit of medium size, roundish heart-shaped-very regular in form. Stalk of moderate length, rather slender, set in a round, even depression. Skin thin, of a beautiful glossy pale amber-red, becoming a lively red when fully ripe, partially transparent. Flesh melting, juicy, with a very sweet and de icate flavour. Ripe the last of June.

27. Transparent Guigne. § Forsyth. Prince. Pom. Man.

Transparent Gean. Forsyth.

Transparent.

A nice little fruit, ripening with the common Ioncy cherry, about ten days after the cherry season. The skin is thin and pellucid, so that the stone is nearly visible on holding the fruit up to the light. Some writers have stated this to be a bad learer; this is incorrect. We have uniformly found it a most productive variety, the tree growing large with spreading branches. It is a valuable and pretty variety for the dessert, hanging late on the tree, and is admired by all amateurs.

Fruit small, borne in pairs, recular, oval heartshaped. Skin glossy, thin, and nearly transparent, showing the network texture of the flesh bencath, yelllowish-white, delicately blotched with finc red; distinot suture line on both sides. Stalk

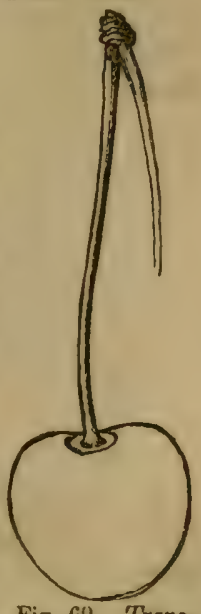

Fig. 69. Trans jorent Grutgne. 
long and slender set in a slight hollow. Flesh tender and mel\%. ing, and when fully ripe very sweet, mingled with a very slight portiun of the piquant bitter of the Mazzard class of cherries. First of July.

\section{Watertoo. \$ Thomp. Lind. P. Mag.}

A capital variety, cross-bred by $\mathrm{Mr}$. Knight, by fertilizing the Bigarreau with the pollen of the Mayduke. It retains, ir part, the habits of both parents, the flowers and the tender flesh resembling considerably those of the Mayduke, and the strong wood and leaves those of the Bigarreau. It was named from the eircumstance of its having first shown fruit about the time of the Battle of Waterloo. 'The tree is rather irregular and spreading in its head, and is, with us, only a moderate bearer.

Fruit large, obtuse heart-shaped. Skin dark purplish, becoming black at maturity. Stalk long and slender. Flesh purplish-red, juicy, tender when fully ripe, with a rich and sweet flavour. Beginning of July. A thrifty, spreading tree.

\section{White Thirtarian. Thomp.}

\section{Fraser's White Tartarian, Fraser's White Transparent, $\left\{\begin{array}{c}a c . \text { to } \\ \text { Thomp. }\end{array}\right.$}

The White Tartarian is a pretty cream coloured fruit of medium size and delicate flavour, ripening the last of June. The skin is somewhat pellucid, but not so much so as in the Trans. parent Gean.

Fruit of medium size, obtuse heart-shaped. Skin pale yellow or cream colour. Stalk of moderate length, slender. Flesh whitish yellow, half tender and of very sweet and excollent flavour. The tree is a moderate bearer.

\section{Class II. Bigarreau Cherries.}

(F'ruit sweet, heart-shaped, with flesh more or lass firm, and crisp or crackling ; trees with tall and snreading heads, and large leaves.)

\section{American Heart.}

American Heart. Thomp.

This productive and good cherry, which we have cultivated for the last eighteen years, and widely disseminated under this name, came to us from Long Island, as a native, and is really 
tne Bigarreau class. Its origin is uncertain, ana thera

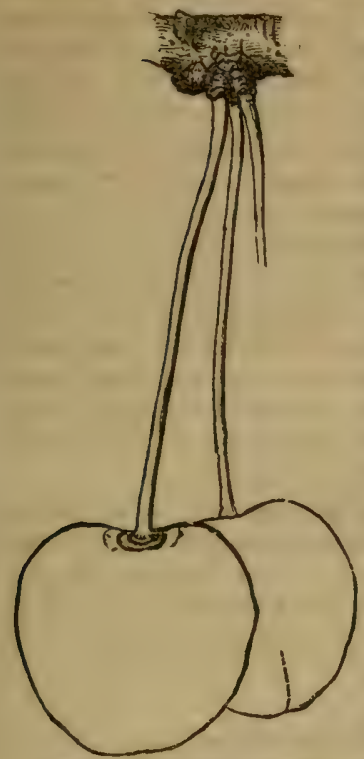
are other soris often incorrectly called by this name. The fruit is remarnable for its pink colnur, ana rather square form, often being nearly as broad at the apex as at the base near the stalk. The tree is quite luxuriant, with wide-spreading branches, a very horizon'al head, and large, rather waved leaves.

Fruit pretty large, heait-shaped, often nearly four sided, and irregular, in its outline-borne ic clusters. Skin, at first, pale, but becoming covered with light red or yink, mix. ed with very little amber. Stalk rather long and slender, iriserted in a small and shallow cavity. Flesh half tender and crackling, adhering to the skin, which is rather tough; juice abundant, and, in dry seasons, sweet and excellent, but ratner want. ing in sweetness in cool or wet sea. sons. Ripens a week before the

Fig. 70. American Heart. following - or early in June.

Mr. Manning, who confounds this with the Early White Heart, had evidently never seen it correct.

31. Bigarreat. $\oint$ Thomp. Lind.

Graffion.

Yellow Spanish, (of most American gardens.)

White Bigarreau, (of Manning and Kenrick.)

Amber, or Imperial. Coxe.

Turkey Bigarreau?

Bigarreau Koyal,

Italian Heart,

Bigarreau Gros?

West's White Heart,

Bigarreau 'Tardif,

Groote Princess,

Hollandische Grosse,

J'rinzessin Kirsche,

Cerise Ambrée. N. Duh.

This noble fruit is the Bigarreau par excellence, and is unquestionably one of the largest, most beautiful and delicious of cherries. It was intro. duced into this country about the year 1800 , by the late William

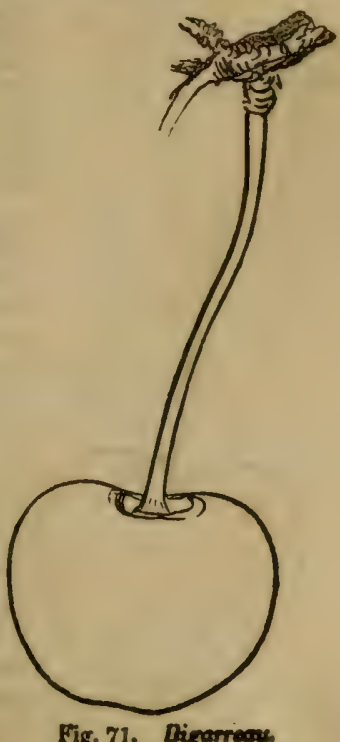


Prince, of Flushing, and has been very extensively disseminates under the names of Yellow Spanish, Graffion, ard Bigarreau. The tree is short but thrifty in growth, making strong lateral shoots, and forming a large and handsome head with spreading branches-and it commences bearing abundantly and regularly even while young. Its very large size and beautiful appear. ance, together with the firmness of its flesh renders it a very valuable variety to cultivate for market.

Fruit very large, and of a beautiful waxen appearance, regu. larly formed, obtuse heart-shaped, the base a good deal flatlen. ed. Stalk stout, nearly two inches long, inserted in a wide hollow. Skin pale whitish yellow on the shaded side, bordered with minute earmine dots and deepening into bright red finely marbled on the sunny side. Flesh pale yellow, quite firm, juicy, with a rich, sweet, and delicious flavour if allowed fully to ripen. In perfection the last of June.

This is often confounded with the following sort, from which it is easily known by its long and broad leaves. It is most commonly known in the middle states as the Yellow Spanish, an incorrect name, which has been applied to two or three sorts, and the cherry so-called by the older pomologists does not now appear to be known.

32. Bigarreau, White. Prince's Pom. Man.

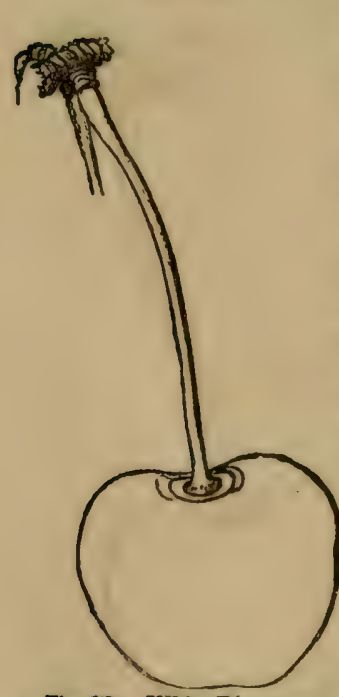

White Ox Heart, (of the middle states.)

White Bigarreau. Thomp.?

Large White Bigarreau.

Tradescant, $\}$ Caxe.

Harrison Heart?

Turkey Bigarreau?

Bigarreau blanc?

The White Bigarreau, which is more common in the neighbourhood of New. York and Philadelphia, than any other part of the country, is frequently confounded with the foregoing, from which it is materially distinct. The first trees of this cherry were, we believe, intro. duced from France, by Chancellor Livingston. It does not appear, at this time, to be known in England, though it is probably identical with the Harrison Heart of Forsyth, and the Bigarreau of Hooker. It is inferior to the Bigarreau or Graffion in hardiness, and in

Fig. 72. White Bigarreau. the circumstance that it is a vers poor bearer while the tree is young, though it bears fine crops when 
it has arriver at from twelve to fifteen years' growtin. The fruit strongly resembles that of the Bigarreau, but is not so obtuse heart-shaped, and is more irregular in it; outline. But the trees nay be readily distinguished even when very sinall, as the Bigarreau has broad flat follage, while the White Bigarreau has narrow waved leaves. Growth upright.

Fruit of the largest size, heart-shaped, with a rather irregular outline, and a pretty distinct suture line on one side. Skin yellowish white at first, but becoming quite overspread with mar. bling of red. Flesh firm, but scarcely so much so as that of the Digarreau, and when fully ripe, half tender, and more luscious than the latter cherry. It is very liable to crack after rain. Middle and last of June.

Mr. Kenrick, in his description of the White Bigarreau, has confused the characteristics of this and the former variety.

On the whole, this variety is likely to be supplanted by the Bigarreau, which joins to most of its good qualities those of greater hardiness, vigour and productiveness.

\section{Bigarreau Rouge. Thomp.}

This variety, which we have cultivated for several years, scarcely differs from the foregoing, except in the colour of the fruit, which is a little darker red.

\section{Bigarreau, Holland. $\S$}

Bigarreau d'Hollande. Noisette. Spotted Bigarreau. Armstrong's Bigarreau.

The Holland Bigarreau is certain ly one of the most beautiful of all clierries. The tree first imported into this country from France, is now growing at Dans Kamer, on the Hudson, the seat of the late Edward Armstrong, Esq.; and it appears to us identical with the Bigarreau de Hollande, of which a coloured figure and description are given by Noisette, in the second edition of his Jardin Fruitier. It is there stated to have been received from Holland in 1828.*

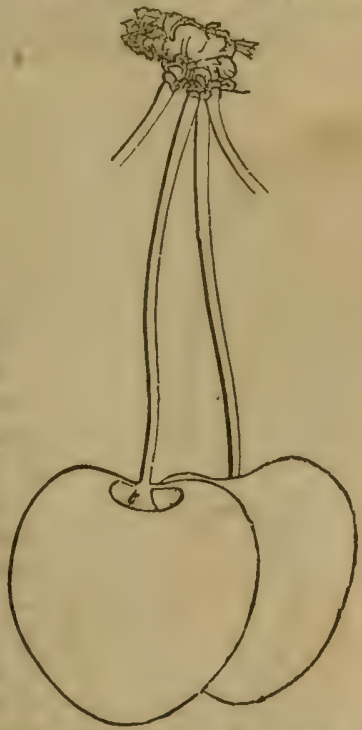

Fig. 73. Holland Bigarreck.

* Th: B. d'Hollande is made synonymons with the Bigarreau by Thompon in the 3il edition of the London Horticultural Society's Catalogue. 'This is undoubs edly an error. 
A cherry so large, fine and beautiful, and so productive ew when young, and which is of rapid and hardy growth, canmot fail scon to become a general favourite in our fruit gardens. II ripens about a week earlier than the Bigarreau, and the branch. es, which are spreading, or even drooping, are literally loaded with heavy bunches of fruit.

Fruit very large, of a regular heart-shape, rather pointerl. Slin white or very pale vellow in the shade, beattifully mottled and spotted on the sunny sicie, with bright carmine red. Stem rather slender, set in a deep hollow, and the fruit borne in thick clusters. Flesh firm, but not so much so as that of the Bigarreau; a little more juicy; sweet and excellent, perhaps scarcely so high flavoured, but this depends somewhat on the dryness of the season. Leaves very large and broad with rather light footstalks. Ripens 20 th of June.

\section{Bigarreau, Couleur de Cilirr. $\S$ Thomp.}

Flesh-coloured Bigarreau.

Gros Bigarreau, Couleur de Chair,
Gros Bigarreau Blanc.

Bigarreau ù Gros Fruit Blane.

Large Ileart-shaped Bigarreau, of Manning.

Bigarreau de Rucmont.

Coenr de Pigeon.

Belle de Rocmunt?

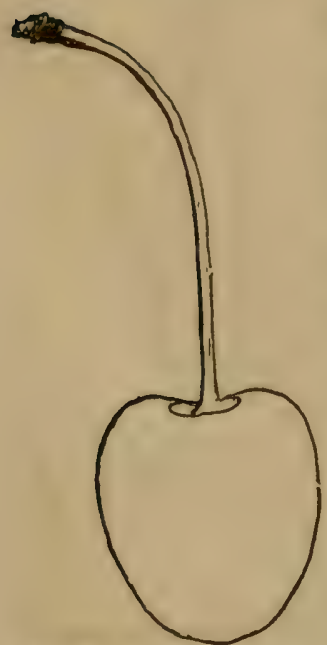

Fig. 74. Flesh-coloured Big. arreau.
The Flesh-coloured Bigarreau is a beautiful and excellent variety, much more tender in its flesh than most of its class, and which attains, under our warm skies, a higher flavour than it does in England. The leaves are not large, dark green, flat, with purplish footstalks. Tree moderately vigorous.

Fruit very large, of a very oblong heart-shape, pointed at the end. Skin shining, of the palest yellow or flesh colour, with a bright red marbled cheek. Stalk moderately long and slender, set in a rather deep and narrow hollow. Flesh half tender, or lie. coming nearly tender when fully ripe, quite juicy and sweet, and in this climate high flavoured and luscious. Ripe the middle and last of June. On fruiting several of the synonymes above, we find them identical with this va. riety, which is truly first rate. 
36. Bigarreau, Napoleov. § Thomp.

$\left.\begin{array}{l}\text { Bigarreau Lauermann, } \\ \text { Lauermann's Kirsche, } \\ \text { Lauermann's Grusse Kirsche, } \\ \text { Lauermann's Herz Kirsche. }\end{array}\right\} \begin{aligned} & \text { ac. to } \\ & \text { Thomp. }\end{aligned}$

'I he Napoleon Bigarreau is one of the finest of the firm fleshed cherries-large, well flavoured, handsome, and productive. It was introduced into this country from Holland, by the late Andrew Parmentier, of Brooklyn. (The fruit cultivated and desuribed by Mr. Manning and Kenrick under this name is, we think, not the true sort.)

Fruit of the largest size, very regularly heart-shaped, a little inclining to oblong. Skin pale yellow, becoming amber in the shade, richly dotted and spotted with very deep red, and with a fine marbled dark crimson cheek. Flesh very firm, (almost too much so,) juicy, with an excellent flavour. Stalk very stout, short and set in a narrow cavity. Ripens a few days after the Bigarreau, about the first of July, and is a good and constant bearer. The fruit is not so obtuse as the Bigarreau, and is much more firm than the Holland, or the Flesh coloured varieties.

\section{Bigarreau Gros Ceruret. Thomp. Poiteau.}

Large Heart-shaped Bigarreau.

Bigarreau Gros Monstrueux.

Gros Cceuret. Bon. Jard.

This, the true Large Heart-shaped Bigarreau, is a French variety only rarely seen in the fruit gardens of this country.

Fruit large, roundish heart-shaped, with a suture line fre. quently raised, instead of being depressed. Skin at first yellowish red, marked with deeper red strealss, but becoming, when fully ripe, a dark shining red, almost black. Stalk inserted in a shallow hollow. Stone oval and rather large. Flesh firm, purplish, a little bitter at first, but of an excellent rich flasour when fully matured. Ripe from the 10th to the middle of July.

38. Bigarreau, Large Red.

Gros Bigarreau Rouge. Poiteau.

Bigarreau à Gros Fruit Rouge. Bon. Jard.

Bigarreau à Gros Fruit Rouge. Thomp.?

Belle de Rocmont, (of some.)

The Large Red Bigarreau is another handsome French va. rety, very rare in the United States, and which we hope to seв more extensively known.

Fruit very large, of an oblong heart-shape, rather irregular in its outline, a good deal swollen on one side, the shoulders 
prijecting and marked with a distinct suture line often on both sides. Stalk rather large, and planted in a very deep and large hollow. Skin shining, ycllowish, dotted and streaked with red in the shade, but dark red in the sun. Flesh yelluwish, or stained with red next the stone, firm, and of a rich and very excellent flavour. Matures early in July. Tree of very stıong growth.

\title{
39. Bigarreat; China. Prince's Pom. Man.
}

Chinese Heart. Thomp. ?

A very striking and peculiar variety, having the skin beautifully mottled and of a shining waxen colour. It was raised by the late $\mathrm{Wm}$. Prince, of Flushing, from the seed of the Bigarreau, and is worthy of a place in the amateur's garden.

Mr. Thompson incorrectly calls this, "Chinese Heart." It is a true Bigarreau, and we prefer to continue its proper name. The tree grows large, and the lateral branches are somewhat drooping, leaves broad, light green.

Fruit of medium size, roundish or oval heart-shaped, with a distinct suture line. Skin shining, at first light amber colour, mottled with red spots, but becoming red, speckled with numerous lighter spots when fully ripe. Stalk long and slender, set in a shallow hollow. Flesh firm, or half tender when fully ripe, with a sweet, rich and peculiar flavour. This variety is the more valuable as it ripens a few days later than the cherry season.

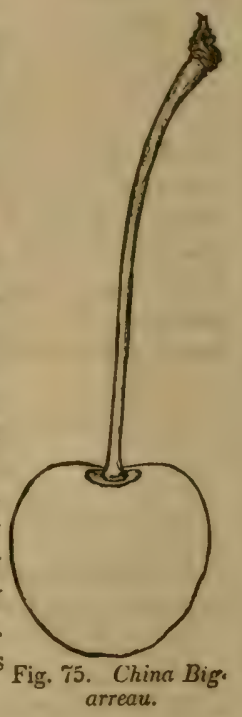

40. Bigarreau Tardif de Hildesiemin. Thomp. Sickler.

\author{
Bigarreau marbré de Hildesheim. Dict. D'Agr. \\ Bigarrean Blane 'Tardif de Hildesheim. \\ Hildesheimer ganz Späte Knorpel Kirsche. \\ Hildesheimer Späte Herz Kirsche. \\ Späte Hildesheimer Marmur Kirsche. \\ Hildesheim Bigarreau. Prince.
}

The IIildesheim Bigarreau is a rare German variety, which ripens here in August, and according to Thompson, is the latest sweet cherry known; a quality that renclers it peculiarly valu. able.

Fruit of medium size, heart-shaped. Skin yellow, mottled and marbled with red. Flesh pale yellow, firm, with a sweet and agreeable flavour. The tree is hardy, and will doubtless prove a valuable variety in this country. 


\section{Bigarreau, Buack,}

Bigarreau Noir.

The Black Bigarreau is a second rate sort, an 1 an indifferent bearer. Fruit middle sized, heart-shaped, .ooking much like a Black Heart. Skin at first dotted with red, but finally becoming quite black. Flesh firm and rather dry. First of July. Scarcely worth cultivation.

\section{Black Bigarreau of Savoy. Ken.}

A very firm, large, black cherry, very recently imported from Savoy, by George Brown, Esq., of Beverly, near Boston. It has been wather more highly rated by the cultivators of Boston, than it deserves, as, though a handsome and rich fruit, it is rather too firm and dry in its flesh to rank as first rate. Its chief merit is that of hanging late upon the tree-till the middle of July.

Fruit large, regularly heart-shaped, very slightly obtuse. Skin smooth and even on the surface, not very glossy, quite black at maturity. Stalk an inch and three fourths long, rather stout, set in a narrow even hollow. Flesh purple, quite firm and solid, with a rich but not abundant juice. Stone rather large.

\section{Bigarreau, New Large Black. Ken.}

The new large black Bigarreau, a variety recently obtained from France, appears likely to prove one of the finest. The fruit is very large quite as handsome as that of the Black Tartarian, and ripens among the late varieties.

Fruit very large, obtuse heart-shaped. Skin quite black and glossy at maturity. Flesh purple, pretty firm, but with a very rich and luscious flavour, more juicy than the other Black Bigarreaus. The tree is very thrifty in its growth, with large broad leaves. [This proves to be only the Black Tartarian. 81 h ed.]

44. Buttner's Yellow. Thomp.

Biittner's Wachs-Knorpel Kirsche.

Büttner's Gelbe-ISnorpel' Kirsche.

Raised by Bütner, of Halle, in Germany, and one of the tew cherries entirely yellow. We have just received this variety from abroad. It is said to be a good bearer, and will no doubt, be a very interesting addition to the desscrt. Mr. Thompson describes it as follows:

Fruit of medium size, roundish, a little compressed on its sides. Skin pale yellow. Flesh firm, yellowish, sweet, and gnod. It ripens at the usual cherry season. 


\section{Downing's Red Chesr.}

A very handsome and excel. lent seedling cherry, just raised at this establishment, and which promises to be a charming addition to the dessert. It somewhat resenibles the Bigarreau, but is more tender and sweet, and rather more highly coloured.

Fruit rather large, regularly ohtuse heart-shaped, with a pretty distinct suture. Skin thin, (slightly pellucid when fully ripe,) white, with a rich dark crimson cheek (somewhat mottled,) covering more than half the fruit. Stalk an inch and a half long, set in an even hollow of moderate depth. Flesh yellowish, half tender, and of a very

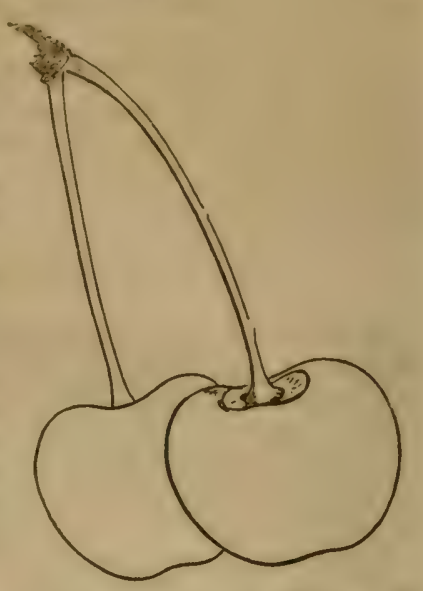

Fig. 76. Downing's Red Cheek. delicately sweet and luscious favour. Leaves coarsely serra. ted, with dark footstalks. Ripens a few days before the Bigarreau, or about the 14th of June.

\section{Elton. $\S$ Thomp. Lind. P. Mag.}

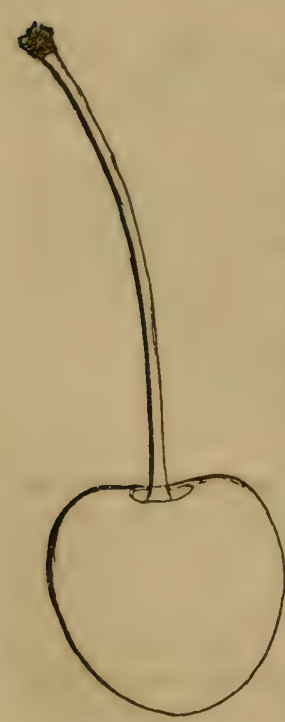

Fig. 77. Eiton.

The Elton, a seedling raised in 1806 , by the late President of the London Horticultural Society, is certainly one of the first of cherries in all respects. Its large size, early maturity, beautiful appearance, luscious flavour, and productiveness, render it universally esteemed. It is a cross-bred variety raised from the Bigar. reau or Graffion with the White Heart for its male parent. The trees grow very vigorously, and are readily known, when in foliage, by the unusually dark red co. lour of the footstalks of the leaves.

Fruit large, rather pointed, nenrt shaped. Skin thin, shining pale yellow on the shaded side, but with a cheek nex: the sun delicately mottled and streaked with bright red. Stalk long and slender. Flesh somewhat firm at first, but becoming nearly tender, juicy, with a very rich and luscious flavour, not surpassed by any large cherry known. Ripens about the middle of June, or directly after the May. duke. 


\section{Florence. $\oint$ Thomp. Lind.}

\section{Knnevett's Late Bigarreau.}

A most exerllint cherry, originally brought from Flerence, in Italy, "hich consicierably resembles the Bigarreau, but ripens a little later, and has the additional good quality of hanging a long time on the tree.

Fruit large, heart-shaped, and regularly formel. Skin amber yellow, delicately marbled with rel, with a bright rerl cheek, and when fully exposed, the whole fruit becomes of a line lively red. Stalk over two inches long, slender, set in a deep hollow. Flesh yellowish, firm, very juicy, and sweet. In perfection from the last of June till the 10th or 15 th of July.

\section{Gridley. Man. Ken. \\ Apple Cherry. Haccarty.}

A native of Roxbury, Mass., which sprung up on the farm of Mr. Samuel Gridley, of that town. An excessive bearer, and from its firmness, bears carriage well, and is a good sort to cultivate for market.

Fruit of medium size, about that of the Black Heart, round. ish. Stalk rather short, and inserted in a shallow cavity. Skin black. Flesh quite firm, purplish, moderately juicy and of quite a rich flavour. Stone small. Ripens after the Black Heart, about the 4 th of July.

49. Lady Southampton's Yellow. Thomp.

$$
\left.\begin{array}{l}
\text { Lady Southampton's Duke, } \\
\text { Iellow ur Golden, } \\
\text { Spanish Yellow. }
\end{array}\right\} \begin{aligned}
& n c, \text { to } \\
& \text { I\%omp. }
\end{aligned}
$$

A yellow cherry, very rare yet in our collections and scarcely meriting general cultivation, being more admired for its colour. 'The best flavoured yellow cherry is Buttner's Yellow.

Fruit of medium size, heart-shaped. Skin yellow. Flesh firm; not very juicy. Ripens about the middle of July.

\section{Madison Bigarreau. Man.}

The Madison Bigarrcau was recently raiserl, and named, by Mr. Manuing, froni the common Birarreau. It is a pretly fruit, and of good llavour, but only of medium size, and not, there. fore, equal to rnany of this class. 
Fruit of medium size, half as large as the Bigarreau, very regularly heart-shaped. Skin much dotted and marbled with rich red on an amber ycliow ground. Stalk rather short and slender. Flesh yollowish, half tender, with a sweet and pleasant flavour. Kipe midule to the last of June. Young trees thrifty, with spreading, rather drooping branches-slender at the ends, and light gray bark. A good bearer.

\section{Manning's Late Black. Man. \\ Manning's Late Black Heart.}

A seedling, raised by Mr. Manning, of Salem, its parent the Black Heart. Fruit large, roundish. Skin deep purple, or nearly black when fully ripe. Flesh purplish, pratty firm, mo. derately juicy and sweet. Ripens about the second week in July.

\section{Renington.}

Remington White Heart. Prince.

Rernugton Heart.

A small, firm fleshed, yellow cherry, a native of Rhode Island, which ripens very late, but is of too indillerent flavour to be worthy of cultivation.

Fruit small, heart-shaped. Skin yellow, rarely with a faint inge of red on one side. Flesh yellowish, dry, and somew.zat bitter. Middle and last of August.

53. 'Tradescant's Black Heart. § Thomp.

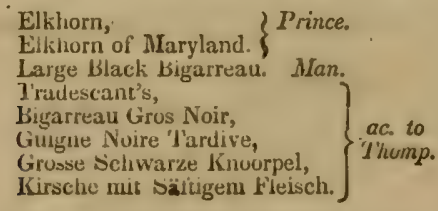

A. very remarkable, and a very good fruit. It is remarkable for the exceed. jngly solid "liver like" consistence of its flesh, and the irregular surface of its skin. Its good qualities are, handsome appearance, late ripening, rich flavour, and moderate and uniform productiveness. When the trees are young and thrifty, the fruit is often of the largest size, fully as large as that of the Black 'iartarian. It is an Hu. ropean yariety, but a tree growing about 40 years since in the garden of an inn in

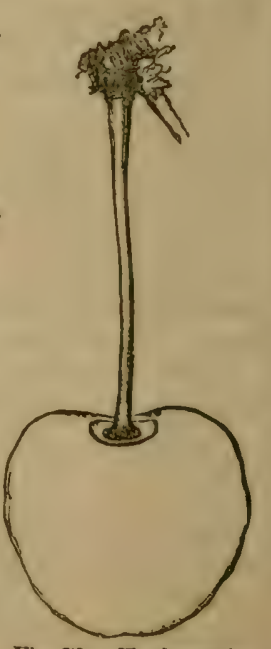

Fig. 73. Tradescant's Black Heart. 
Maryldut, attracted the notice of the late $\mathrm{Wm}$. Prince, who propagated it under the name of Ellihorn, by which it was there known. The leaves are broall, the bark of a peculiarly gray colour, and the growth quite vigrorous.

Fruit large, heart-shaped, with a very irregular or uncren surface. Skin deep black, slossy, (before fully ripe, decp purple, mottled with black.) Stalk rather short, set in a pretty doep hollow. Flesh very solid and firm, dark purple, moderately juicy, with an excellent flavour. Ripe first and second week in July.

\section{Toвacco Leaved. Thomp. Lind.}

Four to the Pound.

Cerisier de 4 a Livre.

Bigarreautier à Fevilies de Tabac.

Bigarreautier a Grandes Fenilles.

Guignier à Feuilies de 'l'abac.

Vier auf ein riund.

The tobaccolcaved cherry is an example of one of the impositions sometimes practised upon the public by dishonest. nurserymen. It has been extensively sold, both in Europe and this country, under the high sounding title of "Four (cherries) to the Pound," while in fact it only bears a very small hard fleshed yellowish cherry tingred with a little red, with a long stalk, and a large stone, and of infericr flavour. The leaves are very large and coarse.

\section{Class III. Duke Cherries.}

Fruit roundish, sub-acid at first, becoming nearly sweet; skir thin; flesh very juicy and melting. 'Trees of upright or hurizontal growth, with that, dark ro. loured leaves.)

\section{Arch Duke. Thomp. Lind. Fors.}

Griotte de Portugal. O. Duh. Nois.

Portugal Duke.

Late Arch Duke.

late Duke, (of some.)

This is a variety of the Mayduke, with considerably laiger fruit; ripening a fortnight later, but we think inferior to it in flavour. It is very scarce in this country, and even abroad more than ialf the cherries sold under this name are either the Mayduke or the Late Duke. The trees of the true sort are goud bearers, rather more vigorous than those of the Mayduke, with longer diverging branches, which become slightrly pendu. lous in bearing specimens. 
Frnit large, aisout a fourth larger than that of the Mayduko, nearly round or a little flattened. Skin, at first, red, but becom. ing a very dark red, almost black. Stalk an inch and a half long: ratlier stout at its point of insertion in the fruit. Flesh dark red, melting, juicy, slightly bitter until fully ripe, when it is of an excellent, rich, sub-acid flavour. Ripe about the first of July.

56. Belle de Choisy. $§$ Thomp. Nois. P. Mag.

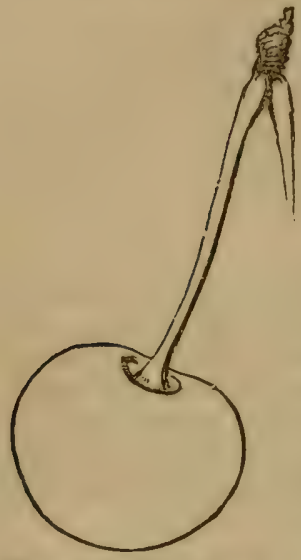

Fig. 79. Belle de Choisy.

\section{Ambré de Choisey, Arnbreè à Gros Fruit, Cerise Doucette, Cerise de la Palembre, $\}$ gurdens Cerise à Noyau 'I'endre, Schóne vun Clivisy.}

In our estimation, there is no cherry for the dessert, more delicate or delicious than the Belle de Choisy. It comes from the village of Choisy near Paris, where it was raised in 1760 . The habit of the tree is nearly that of the Mayduke, the leaves dark, and the head upright. But the fruit is rounder, of a beautiful cornelian colour, and the flavour is very sweet and delicious. It thrives well, appear's very hardy, is a regular, moderate bearer, and deserves a widely extended planting in this country.

Fruit round or slightly depressed. Shin very thin and trans:ucent, showing a net-like texture of nesh beneath; in colour, pale amber in the shade, but in the sun finely mottled with yel. lowish-red--the fruit fully exposed beconing a bright cornelian red. Flesh amber coloured, very tender and inelting, of a delicate, sweet flavour. Stalk rither short, swollen at the upper end. Middle of June, or directly after the Maydulie.

\section{JEFFREY'S DUKE. Thomp. \\ Jeffrey's Royal. Lind. \\ Jetrrey's Ruyal Caroon. \\ Royale, \\ Cherry Duke. O. Duh. \\ kuyale Ordinaire. Poiteas.}

Jeffiey's Duke, or the Cerise Royale of the French gardens, is a fine sort considerably resembling the Mayduke, and is yet very rare in this country. It is much rounder than the May. duke, and seldom or ever becomes of that dark hue which the latter fruil always assumes when fully ripe. 
Fruit of mediun size, round, or a little flattened at the aper nnd base. Skin of a fine lively red. Stalk moderately inng. Flesh yellowish amber, scarcely red. Juice abundant, and of a rich flavour. The trees are of a distinet habit of growth, being very compact, and growing quite stowly. The buids are very closely set, and the fruit is borne in thick clusters. Mid dle and last of June.

58. Late Duke. $\S$ Thomp. Lind. P. Mag.

Anglaise Tardive.

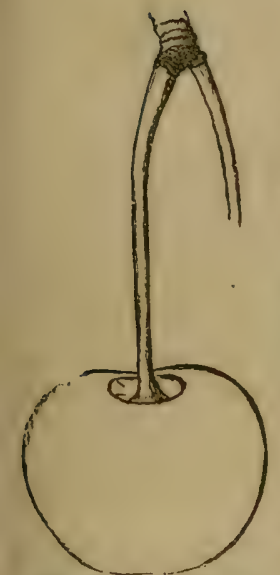

Fig. 80. Late Duke.

A very large and fine Duke cnerry, ripening a month later than the Mayduke, and therefore a very valuable surt for the dessert or for cooking. 'The tree is of vigo. rous growth, but when of bearing size, the whole branches spread almost horizontally. Leaves larger than those of the Mayduke.

Fruit large, flattened or obtuse heart. shaped, much more depressed in its figure than the Mayduke. Colour, when fully ripe, rich dark red; (but at first white, mottled with bright red.) Stalk rather slender, inserted in a shallow hollow. Flesh yellowish, tender, juicy, with a sprightly sub-acid flavour, not quite so sweet and rich as the Mayduke. Ripens grartually, and hangs on the tree from the imide"e of July till the 10th of August.

The branches of this tree are slender in their growth, and the whole habit of the tree seems to incline more to the Morellin than the Duke class.

59. Mayduke. $\S$ Mill. Thomp. Lind.

Royale Hâtive,

Cherry Duke, (of some,)

Cerise Guigne,

Coularde,

De Hollande,

D'Espagne,

Griotte Grosse Noire,

Griolle D'Espagne, (of some,

Griotte Précoce, (of some,)

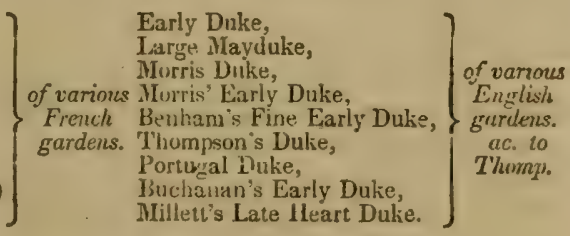

This invaluable early cherry is one of the most ponular sorts in ail countries, thriving almost cqually well in cold or warm climates. 'This, the Black Heart, and the Bigrarreau, are the most extensively diffused of all the finer varieties in the United States. And among all the naw varieties none has been found 
to supplant the Maydulie. Before it is fit for table use, it is admirably adapted for cooking; and when fully ripe, it is, perhaps, the richest of the sub-acid cherries. In the grardens here, we have noticed a peculiar habit of this tree of producing very frequently some branches which ripen much later than the others, thus protracting for a long time the period in which its fruit is in use. 'The Mayduke is remarkable for its upright, or, as it is called, fastigiate head, especially while the tree is young, in distinction to other sorts, which produce many lateral branches.

Fruit roundish or obtuse heart-shaped, growing in clusters. Skin at first of a lively red, but when fully ripe of a rich dark red. Flesh reddish, tender and melting, very juicy, and, at maturity,

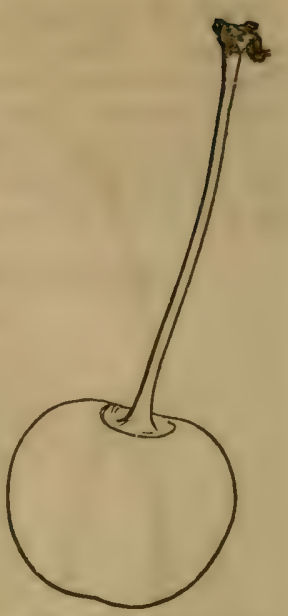

Fig. 81. Mayduke. rich and excellent in fiavour. This fruit is most frequently picked while it is yet red, and partially acid, and before it attains its proper colour or flavour. It begins to colour, about New. York, in favourable seasons, the last of May, and ripens during the first half of June.

Mayduke is said to be a corruption of Medoc, the province in France, where this variety (the type of all the class now called Dukes) is believed to have originated.

Holman's Duke, appears to be only an accidental variety of the Mayduke, ripening from a week to two weeks later. The Late Mayduke, of some gardens, is of similar character, and was obtained by grafting from the late ripening branches of the common Mayduke

\section{Royal Duke. Thomp.}

\section{Royale Anglaise Tardive.}

Fruit large, roundish, and distinctly oblate or flattened. Skin dark red. Flesh reddish, tender, juicy and rich. A good bearer. Ripens in the last of June.

The true Royal Duke is very rare in this country. The fruit is a good deal larger than the May Duke, and mure flattened at the top and bottom. It is readily known from the La:e Duke and Archduke, by its upright growth, which is similas to that of the Mayduke. 
61. Sweet Montmonency. $\S$ Man.

Allen's Sweet Mloztmorency.

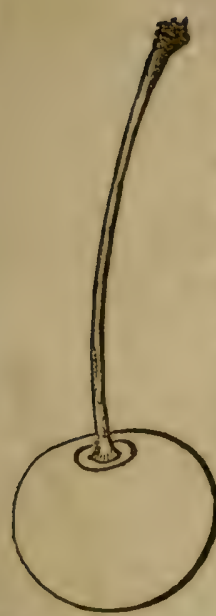

fig. 82. Suect Montmorency.

This cherry, a seedling raised by J. F. Allen, Esq., of Salem, Mass., does not properly be. long here, as, though in external appearance it resembles a Mntmorency, it is of very sweet and delicate flavour, and the whole growth and habit of the tree is rather that of a Heart cherry than a Duke, or Morello. It is no doubt an accidental hybrid between these two classes. It is a good bearer, ripens long after sweet cherries are gone, and is a valuable acquisition to all collections of this fruit.

Fruit of medium size, round, sightly flattened at the base, with a distinctly depressed point at the apex. Skin pale amber in the shade, light red, slightly mottled, in the sun. stalk an inch and three fourths long, rather slender, inserted in a small, shallow, even hollow. Flesh yellowish, tender, sweet and excellent. Ripens here the last week in July; in Boston during the early part of August.

\section{Class IV. Morello, or Acid Cherries.}

Gruit round, or flattened, acid, skin thin, flesh juicy and melting. Trees of low and spreading growth, with slender branches, which are often drooping and wiry, and small dark green foliage.)

\section{Belle Magnifique. Man.}

Belle et Magnifique. Ken.

A sort recently imported into the neighbourhood of Boston, from France, and first introduced to notice here by Gen. Dear. horn. The tree is of stronger growth than most of its class, and bears moderate crops.

Fruit large, round. Skin light red, mottled with darker spots. Stalk pretty long and inserted in a hollow of moderate depth. Flesh juicy, but quite acid. Good for preserving. Ripens about the middle or last of July.

63. Buttner's October Morello. Thomp.

Buittner's October Zucker Weichsel.

A new, Dutch, acid cherry, said to be the latest variety innown 
in Europe, and chiefly valued for ripenir, long after all others have disappeared. We have received trees, but they have rot yet borne fruit. It is described as a dark red fruit of mechum size, round, flesh juicy and tender, and quite acid, being miy fit for culinary uses. Ripens in September and hangs on the tree till October.

64. Carnation. \& Thomp. Lind.

Wax Cherry.

Crown.

Cerise Nouvelle d'Angletorre,

Cerise de Portugal,

Grosse Cerise Rouye Pále,

Griottier Ro'ıge Pále,

Griotte de Villennes.

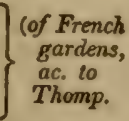

A very handsome, light red, large cherry, highly esteemed here for brandying and pre. serving.

Fruit large, round. Skin at first yellowish white, mottled with red, but becoming a lively red slightly marbled. Stalk about an inch and a half long, stout. Flesh tender, a little more firm than most of this division, but juicy, and when fully ripe, of a sprightly and good sub-acid flavour. The foliage is pretty large, and the wood strong, but the tree has a spreading, rather low habit. It is a moderate but regular bearer, and the fruit hangs a long while on the branches, without decaying.

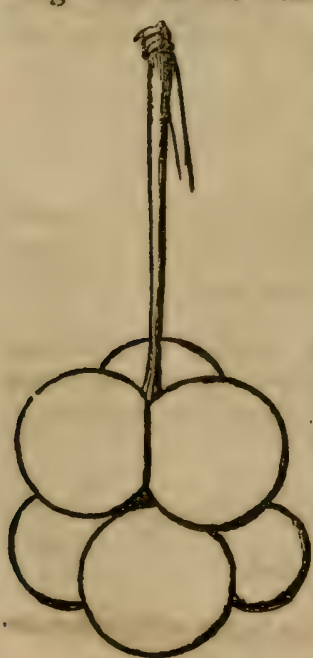

Fig. 84. Cluster.
Ripe the middle and last of July.

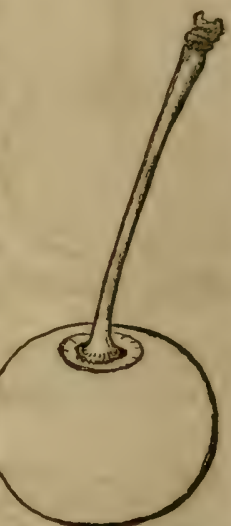

PRINCE'S DUKE is a Fig. 83. Carnation. very large variety of this cherry, raised from a seed of it, by Mr. Prince, of Long lsland. Its shy habit of bearing renders it of little value.

\section{Cluster. Thomp.}

Cerise à Bouquet. Poiteau. Duh.

Cerisier à Trochet,

Commune à Trochet, of French

Tres Fertile, gardens.

Grivtier a Bouquet.

Bouquet Amarelle,

Trauben Amarelle,

Busch Weichsel,

Flandrische Weichsel, Germans.

Büschel Kirsche.

A very curious fruit, growing closely elustered around a common stalk. Eaab 
flower varies from the normal state, by having several pistils or styles, several of which, frequently five or six, become perfect fruits forming a bouquet or cluster. The fruit is too avid to be of any value except for preserving.

Fruit of small size, borne in clusters of from two to six; round, of a lively red. Ripens the last of June. The tree is small in all its parts.

\section{Early May. Thomp. Lind.}

May Cherry. Lang.

Smali May.

Cerisier Nain à Fruit Roud.

- Précoce. O. Duh.

Griottier Nain Precoce.

Hative.

Précoce.

Nain Précoce.

Petite Curise Rouge Précoce.

Künigliche Amarelle.

Frühe k'leine Runde.

Zwerg Weichsel.

Une of the smallest, as well as the earliest of cherries. The tree very small and dwarfish, scarcely grrowing more than eight feet high. It is not worth cultivation now that we have the Early Purple Guigne, Baumann's May, and the like.

Fruit small, round, slightly flattened. Stalk an inch long, rather slender, pretty deeply set. Skin pale red, or, at matu. rity, a rather lively light red. Flesh soft, juicy, and quito 'icid

\section{Flemish. Thomp.}

Montmorency, (of Lindley.)

Kentish, (of some.)

Cerise a Cuurte Quene. Poit.

Monunorency à Gros Fruit, )

Girus Grobet, O. Duh.

Grubst a Courte Queus. .

A Cuurte Que us de Provence.

English Weichsel.?

Weichsel mit gauzkurzen stiel, \} of the

Nuuble Vulgers. Jiukh.

This is a very odd looking fruit, being much flattened, and having a very short stalk. The only bearing tree we have seen, in this country, is one in the garden of Madame Parmenties', Brooklyn, N.Y. 'The Flemish is not a good bearer, and has not, therefore, ever become a popular Guit even in France or Flanders,

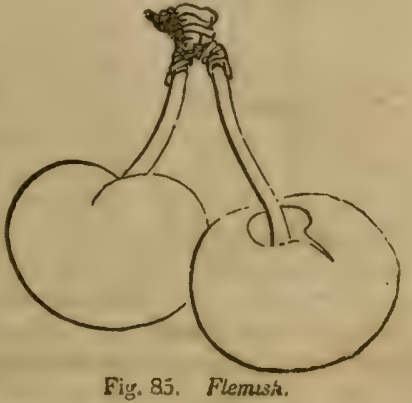

Fig. 85. Flemush. 
where it originated. It is well suited to the grounds of the curious amatcur.

Fruit rather laree, very much fattencl both at the top and base, and grencrally growing in pairs. Staili :t ut, scurcely ever an inch long. decply insert o in a hollow which has often a furroue or hollowed shope on one side. Shin shining, of a bright lively red. Flesh vellowish white, juicy, and sub-acid. Cionl for preservin-bu;, unless very ripe, scarcely rich enough for table usc. Last of July.

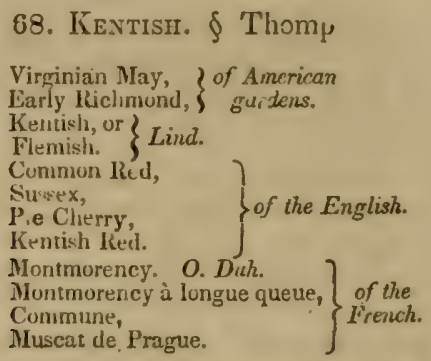

The true Kentish cherry, an old European sort, better known here as the Early Richmond is one of the most valuable of the acid cherries. It bergins to colour about the 20th of May, and may then be used for 1arts; while it will hang upon the tree, gradually growing larger, and losing its acidity, until the last of June, or, in dry seasons, even till July, when it becomes of a

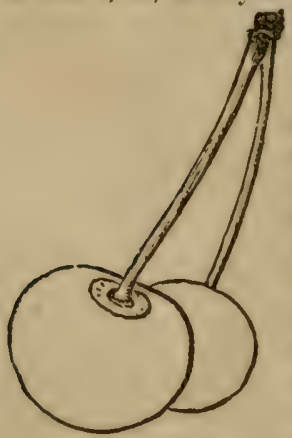
rich, sprightly, and excellent acid flavour.
The tree grows about 18 feet high, with a
roundish spreading head, is exceedingly
productive, and is from its early maturity
a very profitable market fruit, being
largely planted for this purpose in New.
Jersey. This kind is remarkable for the
tenacity with which the stone adheres to
the stalk. Advantage is taken of this to
draw out the stones. The fruit is then
exposed to the sun, and becomes one of the
most excellent of all dried fruits.

Fruit when it first reddens rather small, Fig. 86. Fentish. but, when fully ripe, of medium size, round, or a little flattened; borne in pairs, (our fig. should be one half larger.) Skin of a fine bricht red, growing somewhat dark when fully ripe. Stalk an inch and a quarter long, rather stout, set in a preity deep hollow. Flesh melting, juicy, and, at maturity, of a sprightly rather rich acid flavour.

We follow Thompson in making the true MontMorency of the French synonymous with this. But we confess that we are 
inclined to believe that it may prove distinct. The true Mont. moreney, which is now very scarce in France, (amd is rather a shy bearer,) is carefully driscribed and figured by P'itrau and others, as a larerer ermiving tree, proluches much richer fruit, wilh a longre! and thicker stall, and quite as sweet and high flavoured as that of the finest Duke cherry.

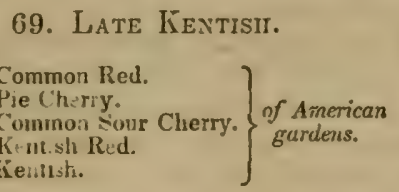

This cherry, a variety of the Kentish, is better known among us than any other acid cherry, and is especially abundant on the Hudson, and in the neighbourhood of New-York, where it is most extensively disseminated along the fences and road sides, propagating itsclf readily by seeds and suckers. It does not seem to be exactly identical with any one of this class known abroad, and is perhaps a seedling sort belonging to America. It is emphatically the Pie Cherry of this country, being more generally grown than any other sort, the pourest and most neglected garden affording so hardy a fruit in abundance. It is quite acid even when fully ripe, and the stone does not adhere to the stalk, like that of the foregoing. It ripens two or three wecks after the cherry season, or about the middle or last of July. It is two weeks later than the preceding sort, and is much more acid.

Fruit of medium size, round, slightly flattened. Stalk an inch, to one and a half long, strong, and straight. Skin deep lively red, when fully ripe. Flesh very tender and abounding with a highly acid juice.

\title{
70. Morelto. Thomp. Lind. Lang.
}

\author{
English Morello. \\ Large Norello. \\ Duteh Dlurello. \\ Iate Morello. \\ Ronalui is Large MIorello. \\ Mlilan. Lang. \\ Cerise du Nord. Nois. \\ Grionte Ordinaire du Nord. \\ September Weichsel Grusse.
}

The Morello is a fine fruit. Its name is said to be derived from the rlark purple colour of its juice, which resembles that of the Morus or Mulberry.* When grown in a shaded situation

* Or, as others say, from the French morelle, (a negress,) from the dark and thining skin. 
the fruit will hang on the free, here, til. August, and in England, whre it is trinined on north walls especially to retarct its seasin, it frequently haness till near frost, when it becomes a rich and atrecrables table fruit. 'This sort, the Large or true . Morello, is yet very scarce in this country, but we hope wil not long continue so, as it is highly valuali e for all kinds of preserves, and is an agree. able addition to a dessert.

Fruit of pretty large size, round-or slightitly obtuse heart-shaped. Skin dark red, becoming nearly b'ack when fully ripe. Flesh dark purplish rel, tender, juicy, and of a pleasant sursacid flavour when quite mature. Ripe 20th of Julv.

The Common Moratuo of this country, large. ly cuitivated in some districts, is a smaller va. ricty of the foregoing, its fruit being about two thirils the size, and a little darker in colour. It is of equally fine flavour, and is highly es. teerned for drying, for preserving in suggar or brandy, or for bottling; keeping, in the latter Fig. 86. Morello. molr, like green gronseberries without sugar or brandy, for several muntsis. The branches are stmaller and more slenier than those of the true Morello, and unfortunately are more liable to the attarks of the wereil, which catuses the knots on the Plum, than those of any other cherry; for which cutting off and burning, early in the spring, is the only remedy.

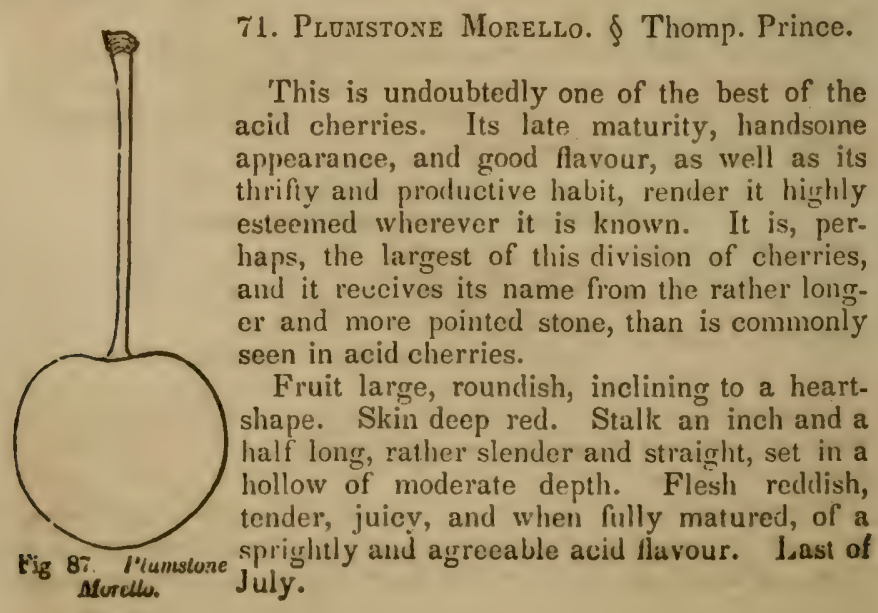


72. Rumsey's Late Morello. $\oint$

A new variety, of remarkable habit, of which the origina: tree now ten or twelve years old, was raised by our fiend, Dr. J. S. Rumsey, of Fishkill Landing, on the IIudson. It is just coming into bearing, and gives promise, from its extraordinary late. ness, large size, and handsome appearance, of becoming a very favourite acid cherry for preserving and cooking. The tree has the Morello habit, with, however, unusually light coloured wood and leaves.

A few of the fruit commence ripening about the first of August, while many on the tree are yet small and green, and they con. tinue ripening gradually until the first frosts.

Fruit frequently borne in pairs, large, roundish heart-shaped. Skin very smooth and polished; before fully ripe, of a light yel. lowish red or cornelian colour-becoming at maturity, a rich lively red, with a distinct suture line on one side. Stalk long (for a Morello, inserted in a narrow and rather deep hollow. Flesh very juicy and melting, with too much acid for the table. Stone long, resembling that of the Plumstone Morello.

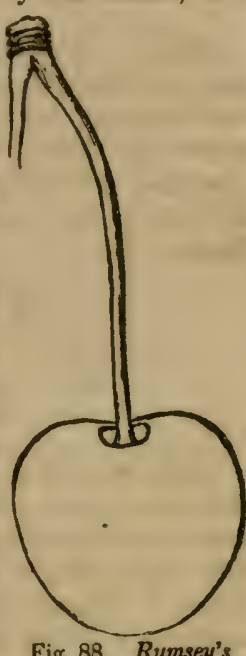

Fig. 88. Rumsey's Late Morello.

\section{Ornamental Varieties.}

\section{Large Double Fiowering.}

Double French Cherry.

Merisier à Fleurs Doubles. Thomp. Duh.

Prunus cerasus pleno.

Cerasis sylvestris, flore pleno. Arb. Bril.

The double blossomed cherry bears no fruit, but whoever ad. mires a beautiful llowering tree, cannot refuse a place in his garden to this one, so highly ornamental. Its blossoms, which appear at the usual season, are produced in the most showy profusion; they are about an inch and a half in diameter, and resemble elusters of the rnost lovely, full double, white roses. The tree has the habit and foliage of the Mazzard Cherries, and roon forms a large and lofty head. 


\section{Dwarf Double Frowerivg.}

Double Flowering Kentish. Snall Double Flowering.

Cerisier a Fleurs Doubles. Thomp. N. Theh.

This is a double flowering variety of the sour, o* Kentis? cherry, and has the more dwarfish habit and smaller leaves and branches of that tree-scarcely forming more than a larire shrub, on which account it is perhaps more suitable for small gardens. The flowers are much like those of the large double flowering, but they are not so regular and beautiful in theip form.

\section{Chinese Double Flotvering.}

Yung To.

Cerasus serrulata.
Serrulated leaved Cherry.

This is a very rare variety, recently imported from China, with the leaves cut on the edges in that manner known as serrulate by botanists. Its flowers which are borne in fuscicles are white, slightly tinged with pink, and nearly as double as those of the large double flowering. The tree considerably resembles the sour cherry tree, and appears rather dwarfish in its growth.

\section{Weeping, or Alisain'is. Thomp.}

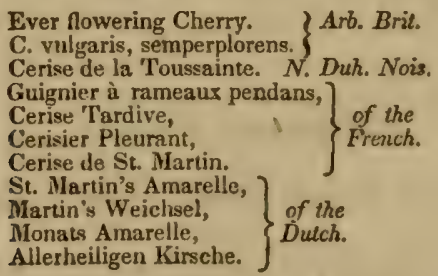

This charming little tree, with slender, weeping branches, clothed with small, almost myrtle.like foliage, is a very pleasing ornament, when introluced on a lawn. Its fruit is a small, deep red Morello, which is acid, and in moist seasons, is produced for a considerable period successively. When grafted, as it generally is, about the height of one's head, on a straight stem of the common Mazzard, it forms a beautiful parasol.like top, the ends of the branches weeping half way down to the ground 


\section{Virgtnian Wild Cherry.}

Wild Cherry, of the Inited States.

Cerasus Virginiana. Arb. Brit. Dcc.

Ceracier de Virginie. French.

Virginisch Kirsche. German.

Our na ive wild cherry is ton woll known to need minute de. seription. It forms a lare and lofiy forest tree, with glossy, dark green leaves, and bears currant-like bunches of small fruit, which are palatable, sweet, and slightly bitter when fully ripe, at milsummer. They are, however, innst esteemed for preparing cherry bounce, a favourite liqueur in many parts of the country, made by putting the fruit along with sugar in a demijohn or cask of the best old rum.

The black will cherry, (C. serotina, Torrey and Gray,) which ripens the first of September, is the best kind. 'The other specirs, (C. Virginiuna.) which is commonly known as the Choke Cherry, bears reddish coloured fruit, which is more astringent, and ripens a month earlier.

Selection of cherries for a small garden. Early Purplo Guigne, Buumann's Early, Lñight's Early Black, Mayduke, Bigarreau, Tartarian, Downer's Late, Ėton, Tradescant's Black, Belle de Choisy, swcet Montmorency, Kentish, Mordlo.

The hardiest cherries are the Kentish, (or Virginian May,) the Morello, and the Mayduke. 'These succeed well at the farthest limits, both north and south, in which the cherry can be raised, and when all other varieties fail, they may be dependec on for regular crops. Next to these, in this respect, are the. Black Heart, Downer's Late, Downton, and Elton.

\section{CHAPTER XIII.}

\section{THE CURRANT.}

Rihes rubrum, Lin. Grossulacece, of botanists.

Frossilluer commun, of the French; Die Johannicbeere, German; Aalbessctioom, Dutch; Ribes rosso, Italian; and Grossella, Spanish.

THE name currant is said to be derived from the resemblance in the fruit to the little Corinth grapes or raisins, which, under the name of currants, are sol $\mathrm{t}$ in a dried state in such quantities sy grocers; the latter word reing only a corruption of Corinth, 
and, the frut of this little grape, being familiarly known as such long before the common currants were cultivated.

The currant is a native of Britain, and the north of Europe, and is, therefore, an exceedingly hardy fruit bearing shrub, seldom growing more than three or four feet high. The fruit of the original wild species is small and very sour, but the largo garden sorts produced by cultivation, and for which we are chiefly indebted to the Dutch gardeners, are large and of a more agreeable, sub-acid flavour.

The Black Currant, (Ribes nigrum, is a distinct species, with larger leaves, and coarser growth, and which, in the whole plant, has a strong odour, disagreeable, at first, to many persons.

UsEs. The cooling acid flavour of the currant is relished by most people, in moderate quantities, and the larger varieties make also a pretty appearance on the table. Before fully ripe, currants are stewed for tarts, like green gooseberries, and are frequently employed along with cherries or other fruits in the same way ; but the chief value of this fruit is for making currant jelly, an indispensable accompaniment to many dishes. Currant shrub, made from the fruit in the same manner as lemonade, is a popular summer drink in many parts of the country, and corresponds to the well known Paris beverage, eau de grosseilles. A sweet wine, of very pleasant taste, is made from their expressel juice, which is very popular among farmers, but which we hope to see displaced by that afforded by the Isabclla and Catawba grapes, - which every one may make with less cost and trouble, and which is infinitely more wholesome, because it re. quires less additions, of any kind, to the pure juice.

The fruit of the black currant is liked by some persons in tarts, but it is chielly used for making a jam, or jelly, much valued as a domestic remedy for sore throats. The young leaves dried, very strongly resemble green tea in flavour, and have been used as a substitute for it.

The season when currants are in perfection is midsummer, but it may be prolonged until October by covering the bushes with mats, or sheltering them otherwise from the sun.

Propagation and culture. Nothing is easier of culture than the currant, as it grows and bears well in any tolerable garden soil. Never plant out a currant sucker. To propagate $\mathrm{it}$, it is only necessary to plant, in the autumn, or early in the spring, slips or cuttings, a foot long, in the open garden, where they will root with the greatest facility. The currant should never be allowed to produce suckers, and, in order to ensure against this, the superfluous eyes or buds should be taken out befure planting it, as has been directed under the head of Cuttings. When the planis are placed where they are finully to remain, they should always be kept in the form of trees-that iq co say, with single stems, and heads branching out at from onn 
funt, to three feet from the ground. The after treatment is of the simplest kind, thinning out the superfluous wood every winter, is all that is required here. Those who desire berries of an extra large size stop, or pinch out, the ends of all the strong growing shoots, about the middle of June, when the fruit is two. thirds grown. This forces the plant to expend all its strength in enlarging and maturing the fruit. And, we may add to this, that it is better not to continue the cultivation of currant trees after they have borne more than six or eight years, as finer fruit will be obtained, with less trouble, from young plants, which are so easily raised.

There are, nominally, many sorts of currants, but the following sorts comprise all at present known, worthy of cultivation. The common Red, and the common White, are totally undeserving a place in the garden, when those very superior sorts, the White, and Red Dutch, can be obtained.

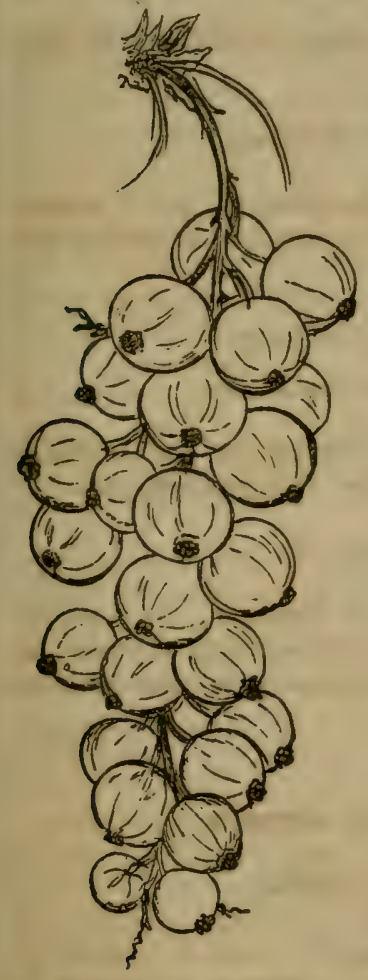

Fig. $x$. Whate Dulch.

\section{Red and White Currants, ( $R$. rubrum.)}

1. RED DUTCH. $\oint$ Thomp. Lind.

Large Red Dutch.

New Red Dutch.

Morgan's Red.

Red Grape.

Large Bunched Ked.

Long Bunched Red.

Grosillier Rouge à Gros-Fruit.

Fruit twice the size of the common currant, red, and a little less acid. Clusters two to three inches long.

2. Whrте Duтch. \& Thomp. Lind.

New White Dutch.

Reeve's White.

White Crystal.

Morgan's White.

White Leghorn.

This is precisely simuar to the foregoing in size and habit, and the fruit is equally large and of a fine yellowish white colour with a very transparent skin. It is considerably less acid than the Red Currants, and is therefore much preferred for the table. 
3. Cinampagne. § Tlomp. Lind.

Pleasant's Eye.

Grosellier a Fruit Couleur de Chair.

A large and handsome currant, of a pale pink, or flesh co. lour, exactly intermediate, in this respect, between the red and white Dutch. It is quite an acid sort, but is admired by many for its pretty appearance.

4. Knigur's Large Red. Thomp.

This seedling of Mr. Knight's is one of the largest of cur rants, being a third larger than the Red Dutch.

5. KNight's EARLY Red. Thomp.

The merit of this variety is its ripening ten days earlier than other sorts.

6. Knignt's Sweet Red. § Thomp.

This is not a sweet currant, in a literal sense, but it is con. siderably less acid than the White Dutch, and much less so than all other varieties.

\section{Striped Frutted. Thomp.}

Grosse Weiss und Rothgestreifte Johannisberre.

A very pretty new currant from Germany, the fruit of which is distinctly striped with white and red. It is yet very rare.

$$
\text { 3. MaY's Victoria. § }
$$

A new variety recently received from England. It is said to bear very large bright red fruit, in bunches 5 or 6 inches in length. The fruit is bright red and hangs on the tree a month longer than any other sort.

\section{Black Currants, (R. nigrum.)}

9. Common Black. Thomp.

Black English.

Cassis, (of the French.)

The common Black English Currant is well known. The 
berries are quite black, less than half an inch in diameter, and borne in clusters of four or five berries. It is much inferior to the following.

10. Black Naples. $\S$ Thomp. P. Mag. Lind.

The Black Naples is a beautiful fruit, the finest and largesi of all black currants, its berries often measuring nearly three fourths of an inch in diameter. Its leaves and blossorns appear carlier than those of the conmon black, but the fruit is later, and the clusters, as well as the berries, are larger and more numerous.

Ornamental Varieties. There are several very ornamental species of currant, among which we may here allude to the Missouri Currant (Ribes Aureum,) brought by Lewis and Clark from the Rocky Mountains, which is now very commor in our gardens, and generally admired for its very fragrant yellow blossoms. Its oval blue berries, which are produced in great abundance, are relished by some persons. But there is a Large Fruited Missouri Currant, a variety of this, which bears berries of the size of the Black Naples, and of more agreeable flavour.

The Red Flowering Currant ( $R$. sanguineum,) is a very beautiful shrub from the western coast of America, with foliage somewhat like that of the common black, but which bears very sharming clusters of large light crimson blossoms, in April. It is not quite hardy enough to stand the winters to the north of this. There are several varieties with white and pale pulk flowers.

\section{CHAPTER XIV.}

\section{THE CRANBERRY.}

Oxycoccus, Arb. Brit. Ericacea, of botanists.

Airelle, of th French; Die Moosebeere, German; Veen bessen, Dutch; Osstcoct, Italian.

TuF cranberry is a familiar trailing shrub growing wild in swampy, sandy meadows, and mossy bogs, in the northern por. tions of both hemispheres, and produces a round, red, acid fruit. Our native species, ( . macrocarpus,) so common in the swamps of New-England, and on the borders of our inland lakes, as tc form quite an article of commerce, is much the largest and finest species; the European cranberry, (O. palustris,) being 
much smaller in its growth, and producing fruit inferior ir. size and quality.

The value of the common cranberry for tarts, preserves and other culinary uses, is well known, and in portions of the country where it does not naturally grow, or is not abundantly pro. duced, it is quite worth while to attempt its culture. Although naturally, it grows mostly in mossy, wet land, yet it may be easily cultivated in beds of peat soil, made in any rather moist situation, and if a third of old thoroughly decayed manure is added to the peat, the berries will be much larger, and of more agreeable fiavour than the wild ones. A square of the size of twenty feet, planted in this way, will yield three or four bushels annually - quite sufficient for a family. The plants are easily procured, and are generally taken up like squares of sod or turf, and planted two or three feet apart, when they quickly cover the whole beds.

In some parts of New-England, low and coarse meadows, of no value, have been drained and turned to very profitable account, by planting them with this fruit. The average product is from eighty to 100 bushels of cranberries, worth at least one dollar a bushel, and the care they require after the land is once prepared and planted is scarcely any at all, except in gathering. Some of the farms in Massachusetts, yield large crops. partly from natural growth, and partly from cultivated plantations. The "New-England Farmer" states that Mr. Hayden, of Lincoln, Mass., gathered 400 bushels from his farm in 1830 . The cranberry grows wild in the greatest abundance, on the sandy low necks near Barnstable, and an annual cranberry festival is made of the gathering of the fruit, which is done by the mass of the population, who turn out on the day appointed by the authorities, and make a general gathering with their cranberry rakes, a certain portion of the crop belonging, and being delivered, to the town.

Capt. Hall, one of the most successful cranberry cultivators of that neighbourhood, thus turns his sandy bogs and rush. covered land to productive beds of cranberry. After draining the land well, and removing all brush, he ploughs the soil where it is possible to do so; but he usually finds it sufficient to cove $i$ the surface with a heavy top-dressing of beach sand, digging holes four feet apart into which he plants sods, or square bunches, of the cranberry roots. These soon spread on every side, overpowering the rushes, and forming a thick coating to the surface. A laborer will gather about thirty bushels of the fruit in a day, with a cranberry rake.

Cranherry culture would be a profitable business in this neighbourhond, where this fruit is scarce, and, of late years, sells for two or three dollars a bushel. 


\section{CHIAPTER XV.}

\section{THE FIG.}

Ficus Carica, L. Arb. Brit. Urticacea, of botanists. Figuicr, of the French; Feigenbuum, German ; Fico, Italian; Higuera, Spantsh

Tuis celebrated fruit tree, whose history is as ancient as that of the world, belongs properly to a warm climate, though it may be raise 1 in the open air, in the middle states, with proper care.

In its native countries, Asia and Africa, near the sea-coast it forms a low tree, twenty feet in height, with spreading branch. es, and large, deeply lobed, rough leaves. It is completely naturalized in the south of Europe, where its cultiration is one of the most important occupations of the fruit grower.

The fruit of the Fig $t 1$ ee is remarkable for making its appearance, growing, and ripening, without being preceded by any apparent blossom. The latter, however, is concealed in the interior of a fleshy receptacle which is called, and finally be. comfs, the fruit. The flavour of the fig is exceedingly sweet and luscious, so much so as not to be agreeable to many per. sons, when tasted for the first time; but, like most fruits of this kind, it becomes a great favourite with all after a short trial, and is really one of the most agreeable, wholesome, and nutritious kinds of food. It has always, indeed, been the favourite fruit of warm countries, and the ideal of earthly happiness and content, as typified in the Bible, consists in sitting under one's own fig tree.

Its cultivation was carried to great perfection arnong the Ancient Rornans, who had more than twenty varieties in their gardens. But the Athenians seem to have prided themselves most on their figs, and cven made a law forbidding any to be exported from Attica. Smuggling, however, seerns to have been carried on in those days, and a curious little piece of etymological history is connected with the fig. The informers against those who broke this law were called sukophantar, from two words in the Greek, meaning the "discoverers of figs." And as their power appears also to have been used for malicious purposes, thence arose our word sycophant. The fig was firs introduced from Italy about 1543 , by Cardinal Poole, and to this country about 1790 , by $\mathrm{Wm}$. Hamilton, Esq.*

* Dr. Pocock, the oriental traveller, first brought the fig to Oxford, and planted a tree in 1643, in Oxfurd Cullege Garden, of which tree the following anerdute is tinld. Dr. Kennirutt, the celebrated Hebrew scholar, and compiler of the Polygint Bible, was passionately fond of this fruit, and, seeing a very fine fig on this trec that he wished to preserve, wrote on a label "Dr. Kennicott's fig," which he tied to the fruit. An Oxonian wag, who had observed the transaction, watched the fruit daily, and, when ripe, gathered it, and exchanged the label for one thas worded-" a fig for Dr. Kennicott." -McInlash. 
PROFAG.LTION. This tree is very readily increased by cut tings taken off in the month of March, and planted in light soil in a hot bed, when they will make very strong plants the samz scasson. Or, they may be planted in a shady border in the open? air, quite early in April, with tolerable success. In either case the cuttings should be made eight or ten inches long, of the last ycar's shoots, with about half an inch of the old, or previous vear's wood left at the base of each.

Soil and culture. The best soil for the fig is one moln. rately deep, and neither too moist nor dry, as, in the formar case, the plant is but too apt to run to coarse wood, and, in the latter, to drop its fruit before it is fully ripe. A inellow, calcareous loam, is the best soil in this climate-and marl, or mild lime in compost, the most suitable manure.

As in the midrile states this tree is not hardy enough to be allowed to grow as a standard, it is the policy of the cultivator to keep it in a low and shrub-like form, near the ground, that it may be easily covered in winter. 'The great difficulty of this mode of training, with us, has been that the coarse and over. luxuriant growth of the branches, when kept down, is so great as to render the tree unfruitful, or to rob the frum of tis due share of nourishment. Happily the system of root-prunung, recently found so beneficial with some other trees, is in inis cli. mate, most perfectly adapted to the fig. Shor pinted wont, and only moderate vigour of growth, are well known accom. paniments of fruitfulness in this tree; and there is no means by which firm, well ripened, short-jointed wood is so easily obtain. ed as by an annual pruning of the roots-cuiting off all that project more than half the length of the branches. In this way the fig tree may be kept in that rich and somewhat strong soil necessary to enable it to hold its fruit, and ripen it of the largest size, without that coarseness of growth which usually happens in such soil, and but too frequently renders the tree barren. The mode of performing root-pruning we have already described, but we may add here that the operation should be performed on the fig early in November. When this mode is adopted but little pruning will be necessary, beyond that of keeping the plant in a somewhat low, and regular shape, shortening-in the branches occasionally, and taking out old and decaying wood.

In winter, the branches of the fig must be bent down to the ground, and fastened with hooked pegs, and covered with three or four inches of soil, as in protecting the foreign grape. This covering should be removed as soon as the spring is well set. tled. Below Philadelphia, a covering of straw, or branches of evergreens, is sufficient-and south of Virginia the fig is easy of culture as a hardy standard tree.

Two erops are usually produced in a year by this tree; the first which ripens here in midsummer, and is borne on the pre. 
vious season's shoots; and the second which is yielded by the young shnots of this summer, and which rarely ripens well in the niddle states. It is. therefore, a highly advantaureous prac. tice to rub off all the young figs of this second crop after mil. sum ner, as soon as they are formed. The consequence of this is to retain all the organizable matter in the tree; and to furm new embryo figs where these are rubbed off, which then ripen the next season as the first crop.

liIPENING THE FRUIT. In an unfavourable soil or climate, th ? ripening of the fig is undoubtedly rendered more certain anci speedy by touching the eye of the fruit with a little oil. This is very commonly practised in many districts of France. "At Argenteuil," says Loudon, "trie maturity of the latest figs is hastened by putting a singl a drop of oil into the eve of each fruit. This is done by a woman who has a phial of oil sus. pended from her waist, and a piece of hollow rie straw in her hand. This she dips into the oil, and afterwards into the eye of the fig."

We have ourselves frequently tried tho experiment of touching the end of the fig with the finger dipped in oil, ard have always found the fruits so treated to ripen much more certainly and speedily, and swell to a larger size than those left untouched.

There are forty-two varieties enumerated in the last edition of the London Horticultural Society's Catalogue. Few of these have, however, been introduced into this country, and a very. few sorts will comprise all that is most desirable and excellent in this fruit. 'The following selection includes those most suit$a b^{\prime} . c$ for our soil and climate. Fruit nearly all ripen in August.

\section{Class I. Red, Brown, or Purple.}

1. Brunswick. Thomp. Lind. P. Mag.

$\left.\begin{array}{l}\text { Madonna. } \\ \text { Hanover. } \\ \text { Brown Hamburgh. } \\ \begin{array}{l}\text { Black Naples. } \\ \text { Clementine. } \\ \text { Bayswater } \\ \text { Red. }\end{array}\end{array}\right\}$ ac. to

One of the largest and finest purple figs, well adapted to hardy culture. Fruit of the largest size, pyr:form in shape, with an oblique apex. Eye considerably sunk. Stalk short, and thick, of a fine violet brown in the sun, dotted with sinall pale brown speclis, and, on the shaded side, pale greenish yel. sow. Flesh reddish brown, slightly fink near the centre, and 
sorrewhat transparent. Flavour rich and excellel.t. The only fault of this variety for open air culture is, that it is rather too strong in its growth, not being so easily protected in winter as n:ore dwarfish sorts.

2. Brown Turkey. $\oint$ Thomp.

\author{
Brown Italian. Forsyzh. \\ Large Blue; of Lind. \\ Italian. \\ Brown Naples. \\ Murrey. Lind. \\ Lee's Perpetual.
}

This is undoubtedly one of the very best for this country, and for open air culture, as it is perhaps the very hardiest, and one of the most regular and abundant bearers. Fruit large, oblong, or pyriform. Skin dark brown, covered with a thick blue bloom. Flesh red, and of very delicious flavour.

\title{
3. Black Ischia. Thomp. Lind. \\ Early Forcing. \\ Blue Ischia.
}

One of the most fruitful sorts, and pretty hardy. Fruit of medium size, roundish, a little flattened at the apex. Skin dark violet, becoming almost black when fully ripe. Flesh deep red, and of very sweet, luscious flavour.

\section{Brown Ischia. § Thomp.}

Chestnut. Lind. Mill.

\section{Chestnut-coloured Ischia.}

A good variety, with, however, a rather thin skin, rendering it liable to crack or burst open when fully ripe. It is hardy, of good habit, and a very excellent bearer.

Fruit of medium size, roundish-obovate. Skin light or chestnut-brown; pulp purple, very sweet and excellent.

\section{Black Genoa. Lind.}

The fruit of this fig is long-obovate, that portion next the stalk being very slender. Skin dark purple, becoming nearly black, and covered with a purple bloom. Pulp bright red, fla vour excellent. Habit of the tree moderately stror.g. 


\section{Malta. $\S$ Lind.}

Small Brown.

A small, but very rich fig, which will often hang on the tree until it begins to shrivel, and becomes "a fine sweetmeat." Fruit much compressed at the apex, and very much narrowed in towards the stalk. Skin light brown. Pulp pale brown, and of a sweet, rich flavour. Ripens later than the foregoing, about the last of August.

\section{Sirall Brown Isciua. $\S$ Lind.}

$\Lambda$ very hardy sort, which, in tolerably warm places south of Philadclphia, will make a small standard tree in the open air, bearing pretty good crops, that ripen about the first of September. Fruit small, pyriform, with a very short footstalk. Skin light urown. Pulp pale purple, of high flavour. Leaves more entire than those of the common fig.

\section{VIoletTe. Lind. Duh.}

A very good sort from the neighbourhond of Paris, where it prorluces two crops ammually. Fruit small, roundish-obovate, flattened at the apex. Skin dark violet. Pulp nearly white, or a little tinged with red on the inside, and of pleasant flavour.

\section{Violette de Bordeaux. Thomp.}

Bordeaux. Lind. Duh.

A figr which is much cultivated in France, being quite productive, though of inferior flavour to many of the foregoing sorts. Fruit. large, pyriform, about three inches long, and two in diameter. Skin deep violet when fully ripe, but at first of a browr:sh red. Pulp reddish purple, sweet and good.

Class II. Fruit, While, Green, or Yellow.

10. Angelique. $\S$ Thomp. Lind. Duh.

Concourelle Blanche

Melitte.

This little fir is a very abundant bearer, and a pretty haidy bort. Fruit small, obovate. Skin pale greenish yellow, dotted 
with lighter coloured specks. Pulp white, but only tolerably sweet. It will usually bear two crops.

11. Larie IVitte Graxoa. Thomp. Lind. Fors.

Fruit large, roundish-obovate. Skin thin, pale yellow. Fulk red, and well flavoured.

12. Marseilles. Thomp. Lind.

White Mrameilles.

White Niaples.

Poconck.

Forl's Seedling.

White Standard.

Figue Blanche. Duh.

A very favourite sort for forcing and raising under glass, but which does not succeed so well as the Brown Turkey, and the Ischias, for open culture. Fruit small, roundish-oborate, slightly ribbed. Skin nearly white, with a little yellowish green remaining. Flesh white, rather dry, but sweet and rich.

13. Nern. $\oint$ Thomp. Lind.

A fruit rather smaller and longer than the Marseilles, and which, from a mingling of slight acid, is one of the most exquisite in its flavour. Fruit small, roundish-ohovate. Skin pale greenish yellow. Pulp red. Flavour at once delicate and rich. This is a very favourite variety, according to Loudon, "the richest fig known in Britain."

\section{Pregussata. $\oint$ Thomp.}

A sort lately introduced from the Ionian Isles into England. It is tolerably hardy, quite productive, and succeeds admirably under glass. Fruit of medium size, roundish, a good deal flattened. Skin purplish brown in the shade, dark brown in the sun. Pulp deep red, with a luscious, high flavour. Seeds un. usually small. Ripens gradually, in succession.

\section{White IscmiA. 'Thomp.}

Green Ischia. Lind. Fors.

A very small hg, but one of the hardiest of the light eoloured ones. Fruit about an inch in diameter. roundish-obovate. Skin pale yellowish green, very thin, and, when fully ripe, the darker coloured pulp appears through it. Pulp purplish, and higt fla voured. A moderate grower, and good bearer. 


\section{CHAPTER XVI.}

\section{THE GOOSEBFRRY.}

Rihes Grossularia, Arb. Brit. Grossulacece, of notanists.

Frossciller, of the Frencli; Stachelherstrauch, Germin; Voa Spino, Italien; Grossella, Spanish.

Tire gooseberry of our gardens is a native of the north of Europe, our native species never having been improved by gar. den culture. This low prickly shrub, which, in its wild state bears small round or oval fruit, a'uut half an inch in diameter, and weighing one fourth of an oun'-e, has bcen : o greatly im. proved by the system of successive reproduction from the seed, and high culture by British gardeners, that it now bears fruit nearly, or quite two inches in diameter, and weighing an ource and a half. Lancashire, in England, is the meridian of the gooseberry, and to the Lancashire weavers, who seem to have taken it as a hobby, we are indebted for nearly all the surprisingly large sorts of modern date. Their annual shows exhibit this fruit in its greatest perfection, and a Gooseberry Book is published at Manchester every year giving a list of all the prize sorts, etc. Indeed the climate of England seems, from its moistness and coolness, more perfectly fitted than any other to the growth of this fruit. On the continent it is considered of little uccount, and with us, south of Philadelphia, it succeeds but indifferently. In the northern, and especially in the eastern states, however, the gooseberry, on strong soils, where the best sorts are chosen, thrives admirably, and produces very fine crops.

Uses. This fruit is in the first place a very important one in its green state, being in high estimation for pies, tarts, and puddings, coming into use earlier than any other. The carliest use made of it appears to have been as a sauce with green goose, whence the name, goose-berry. In its ripe state, it is a very agreeable table fruit, and in this country, following the season of cherries, it is always most acceptable. Unripe gonseberries are bottled in water for winter use, (placing the bottles nearly filled, a few moments in boiling water, afterwards corking and sealing them, and burying them in a cool cellar, with the necks downward.) As a luxury for the poor, Mr. Lo:Idon considers this the most valuable of all fruits " since it can be grown in less space, in more unfavourable circum. stances, and brought sooner into bearing than any other." Is t) e United States the gooseberry, in humble gardens, is fre. 
quently seen in a very wretched state-the frie poor and small, and covered with mildew. This a.ises partiy fiom ignurance of a proper mole of cultivation, but chiefly from the solts grown being very inferior ones, always much liable to this disease.

Pliopagation. Gooseberry plants should only be raised from cuttings. New varieties are of course raised from seed, but no one here will attempt to do what, under more favourable cir. cumstances, the Lancashire growers can do so much better. In preparing cuttings select the strongest and straightest young shoots of the current year, at the end of October (or very early in the ensuing spring;) cut out all the buds that you intend to go below the ground (to prevent future suckers,) and plant the cuttings in a deep rich soil, on the north side of a fence, or in some shaded border. The cuttings should be inserted six inches deep, and from three to six or eight inches should remain above ground. The soil should be pressed very firmly about the cuttings, and, in the case of autumn planting, it should be examined in the spring, to render it firm again should the cutting have been raised by severe frost. After they have become well rooted-generally in a year's time-they inay be transplanted to the borders, where they are finally to remain.

Cultivation. The gooseberry in our climate is very impatient of drought, and we have uniformly found that the best soil for it is a deep strong loam, or at least whatever may be the soil, and it will grow in a great variety, it should always be deep-if not naturally so, it should be made deep by trenching and manuring. It is the most common error to plant this fruit shrub under the branches of other trees for the sake of their shade-as it always renders the fruit inferior in size and fla. vour, and more liliely to become mouldy. On the contrary, we wculd always advise planting in an open border, as if the soil is sufficiently deep, the plants will not suffer from dryness, and should it unfortunately be of a dry nature, it may be ren. dered less injurious by covering the ground under the plants with straw or litter. In any case a rich soil is necessary, and as the gooscberry is fond of manure a pretty heavy top-dressing should be dug in every year, around bearing plants. For a later crop a few bushes may be set on the north side of a fence or. wall.

For the gooseberry, regular and pretty liberal pruning is a solutely necessary. Of course no suckers should be allowed to grow. In November the winter pruning should be perform. ed. The leaves now being off it is easy to see what proportion of the new as well as old wood may be taken away; and we will bere remark that it is quite impossible to obtain fine gonseber. ries here, or any waere, without a very thorough thinning out of the branches. As a general rule, it may safely be said that one half of the head, including old and young branches (more 
especially the former, as the best fruit is borne on the young wookl, ) should now be taken out, leaving a propor distribution of shoots throughout the bush, the head being sufficiently thimerl to admit freely the light and air. An allitional proning is, in England, performed in June, which consists in stopping the growth of long shoots by pinching out the extremities, and thimning out superfluous branches; but if the annual pruning is properly performed, this will not be found recessary, except to obtain fruit of extraordinary size.

The crop should always be well thinned when the berries are about a quarter grown. The gooseberry is scarcely subject to any disease or insect in this country. The mildew, which attacks the half grown fruit, is the great pest of those who are un. acquainted with its culture. In order to prevent this, it is only necessary-1st, to root up and destroy all inferior kinds subject to mildew ; $2 d$, to procure from any of the nurseries some of the best and hardiest Lancashire varietics; $3 \mathrm{~d}$, to keep them wel? manured, and very thoroughly pruned every year.

We do not think this fruit shrub can be said to bear well for more than a half dozen years successively. After that the fruit becomes inferior and requires more care in cultivation. A succession of young plants should, therefore, be kept up by striking some cuttings every season.

VARIETIEs. The number of these is almost endless, new ones being produced by the prize growers every yea. 'The last edition of the London Horticultural ociety's Catalngue enumerates 149 sorts considered worthy of notice, and Lindlicy's Guide to the Orchard, gives a list of more than seven hundred prize sorts. It is almost needless to say that many of these very closely resemble each other, and that a small number of them will comprise all the most valuable.

The sorts bearing fruit of medium size are generally more highly flavoured than the very large ones. We have selected a sufficient number of the most valuable for all practical pur. poses.

\section{Red Gooseberries.}

1. Boardian's Brttish Crown. Fruit very large, round. ish, hairy, handsome and good. Branches spreading.

2. Cilampagine. A fine old variety, of very rich flavour. Fruit small, roundish-oblong, surface hairy, pulp clear; branch. es of very upright growth.

3. Chrprr's Top SAwYer. Fruit large, roundish, pale red, hairy; rather late, flavour very good. Branches dronping

4. Farrow's Roaring Lion. An immense berry, and hangs late. Fruit oblong, smooth; flavour excellent; branches drooping. 
5. Hartshonn's Lavcasmine Lad. Fruit large, roundish Jark red, hairy; llavour very good; branches erect.

6. Keny's Sefoling. Fruit of medium aig, nlalong, hairy. favour fir ate ; branches drooping. Liariy and protuctive.

7. Leigh's Riflenan. Fruit large, roundish, hairy; flu. vour first rate; branches erect.

8. Melifvg's Crown Boß. Fruit large, oblong, hairy; flavour first rate; branches spreading.

9. Miss Bold. Fruit of medium size, roundish, surface downy ; flavour excellent; branches spreading.

10. RED IVARRINGTON. Fruit large, roundish-oblong, hairy; flavour first rate; branches drooping.

\section{Yellow Gooseberries.}

11. Buerdsilu's Duckwrivg. Fruit large and late, obovate, smooth; Havour good; branches erect.

12. Capper's Bunker Hill. Fruit large, roundish, smooth; flavour good ; branches spreading.

13. Early Sulphur. Fruit middle size, and very early, soundish, hairy; flavour first rate ; branches erect.

14. Gorton's VIPER. Fruit large, obovate, smooth; fla. vour good; branches drooping.

15. Hill's Golden Gourd. Fruit large, oblong, hairy; flavour good; branches drooping.

16. Part's Golden Fleece. Fruit large, oval, hairy ; fla. vour first rate; branches spreading.

17. Prophet's Rockwood. Fruit large and early, roundisli, hairy ; flavour good ; branches erect.

18. Yellow Chaipagne. Fruit small, roundish, hairy; fla. vour first rate; branches erect.

19. YELLOW Ball. Fruit of middle size, roundish, smooth; flavour first rate; branches erect.

\section{Green Gooseberries.}

20. Coliters' Jolly Axgler. Fruit large and late, oblong, downy ; flavour first rate; branches erect.

21. Berry's Greenwood. Fruit large, oblong, smouth; flavour good ; branches drooping.

22. Early Green Hairi, (or Green Gascoigne.) Fruit small and early, round, hairy; flavour excellent; branches spreading.

23. Edward's Jolly Tar. Fruit large, obovate, smooth; Savour first rate: branches drooping.

24. Glenton Green. Fiuit of middle size, oblong, hairy; favour excellent; branches drooping. 
25. GreEN WALnUT. Fruit middle sized. obovate. smooth; flavour first rate; branches spreading.

26. Hepburn Green Prolific. Fruit of middle size, round. ish, hairy ; flavour first rate; branches erect.

27. Massey's Heart of OAk. Fruit large, oblong, smooth ; flavour first rate; branches drooping.

28. Parkinson's Laurel. Fruit large, obovate, downy; flavour first rate; branches erect.

29. Pituaston Green Gage. Fruit small, and hangs long, olovate, smooth ; flavour rich and excellent; branches erect.

30. Wainuax's Green Ocean. Fruit very large, oblong, smooth; flavour tolerably good; branches drooping.

\section{White Gooseberries.}

31. Cleworth's White Liov. Fruit large and hangs late, obovate, downy, flavour first rate; branches drooping.

32. Cronpton Sreba Queen. Fruit large, obovate, downy, flavour first rate; branches erect.

33. Cook's White Eagle. Fruit large, obovate, smooth; flavour first rate; branches erect.

34. Capper's Bonny Lass. Fruit large, oblong, hairy; flavour good; branches spreading.

35. Haplex's Lady of the Manor. Fruit large, roundishoblong, hairy ; flavour good; branches erect.

36. Saunder's Cheshire Lass. Fruit large and very early, oblong, downy ; flavour excellent ; branches erect.

37. Woodivard's Whitesmith. Fruit large, roundish-nblong, downy ; flavour first rate ; branches erect.

38. Wellington's GloRY. Fruit large, rather oval; very dowy; skin quite thin; flavour excellent; branches erect.

39. White Honey. Fruit of middle size, roundish-oblong, smooth ; flavour excellent; branches erect.

40. TAylor's Bright Venus. Fruit of middle size, hangs a long time, obovate, hairy; flavour first rate; branches erect.

Selection of sorts for a very small garden. RED; Red Warrington, Keen's Seedling, Crown Bob. Yellow ; Early Sulphur, Yellow Ball. WhiTe; Woodward's Whitesmith, Early White, T'aylor's Bright Venus, White Honey. Gresn; Pit. maston Green Gage, Green Walnut, Parkinson's Laurel. 


\section{CIIAPTER XVII.}

\section{THE GRAPE.}

Vilus vinifera, L. Vitacec, of botanists.

Vigne, of the French; Weintrauben, German; Vigna, Italian; Vid, or Vina, Spanash

THE history of the grape is almost as old as that of man. Growing in its highest perfection in Syria and Persia, its lus. cious fruit and the unrivalled beverage which its fermented juice affords, recommended it to the especial care of the patriarchat tillers of the soil, and vineyards were extensively planted, long before orchards or collections of other fruit trees were at all common.

The grapes of the old world are all varieties of the wine grape, (Vilis vinifera, which, though so long and so universally cultivated and naturalized in all the middle and southern por tions of Europe, is not a native of that continent, Lut came ori ginally from Persia. From the latter country, as civilization ad vanced westward, this plant accompanied it-first to Egypt, ther to Greece and Sicily, and gradually to Italy, Spain, France and Britain, to which latter country the Romans carried it about two hundred years after Christ. To America the seeds and plants of the European varieties were brought by numerous emigrants and colonists within the first fifty years after its settlement.

'The wild grapes of our own country are quite distinct species from the wine grape of Europe-are usually stronger in their growth, with larger and more entire foliage, and, in their native state, with a peculiar foxy odour or flavour, and more or less hardness of pulp. These traits, however, disappear in process of cultivation, and we have reason to hope that we shall soon obtain, from the wild type, new varieties of high quality, and of superiour hardiness and productiveness in this climate.

The grape vine is in all cases a trailing or climbing deciduous shrub, living to a great age, ${ }^{*}$ and, in its native forests, clarnber. ing over the tops of the tallest trees. In the deep rich alluvial soils of western America, it is often seen attaining a truly prodigious size, and several have been measured on the banks of the Ohio, the stems of which, were three feet in circumference, and the branches two hundred feet long, enwreathing and fes.

* Pliny gives an account of a vine six hundred ycars old, and there are said te be vines in Burgundy more than four hundred years old. 
tooning the tops of huge poplars and sycamores. In a cultivated stite, however, it is found that fine flavour, anil uniform pro. ductiveness, require the plants to be liept pruned within a sinali compass.

Uses. 'The grape in its finest varieties, as the Hamburgh and the Muscat, is in flavour hardly surpassed by any other fruit in delicacy and richness, and few or none are more beau. tiful in the dessert. Dried, it forms the raisin of commerec. the most excellent of al! dried fruits, every where esteemed, And wine, the fermented juice, has always been the first of all exhilarating liquors. Some idea of the past consumption of this product may be formed from the fact that more than $500,000,000$ imperial gallons have been made in France, in a siny?c year; and as a data to judge of its value, we may add, that, while a great proportion of the vin ordinaire, or cornmon wine, is sold at 10 or 12 cents a bottle; on the other hand, particular old and rare vintages of Marleiras or Sherries will not unfrequently command twenty or thirty dollars a gallon.

Sorl. The universal experience in all countries has establish. ed the fact that a dry and warm soil is the very best for the vine. Where vineyards are cultivated, a limestone soil, or one composed of decaying calcareous rocks, is by far the best; but where, as in most gardens, the vine is raised solely for its fruit, the soil should be highly enriched. The foreign grape will scarcely thrive well here on a heavy soil, though our native varieties yrow and bear well on any strong land, but the essence of all that can be said in crrape culture respecting soil is that it be dry and light, deep and rich. Frequent top-dressings of well rotted manure should be applied to vines in open borders, and this should every third or fourth year be alternated with a dressing of slacked lime.

Propagation. The grape vine makes roots very freely, and Is, therefore, easy of propagation. Branches of the previous or current year's wood bent down any time before midsummer, and covered with earth, as layers, root very freely, and make bearing plants in a coup: $=$ of years, or very frequently indeed bear the next season.

But the finer varieties of the vine are almost universally pro. pagated by cuttings, as that is a very simple mode, and an abundance of the cuttings being aftorded by the annual trim. ming of the vines.

When cuttings are to be planted in the open border, a somewhat moist and sharled place should be chosen for this purpose. The cuttings should then be made of the young wood of the previous years' growth, cut into lengths about a foot or eighteen inches long, and having three buds-one near the top, one at the bottom, and the third in the middle. Before planting the cutting pare off its lower end smoothly, close below the buds, 
and, finally, plant it in mellow soil, in 3 slit made by the spade, pressing the carth firmly about it with the foot.*

The rarer kinds of foreign grapes are usually grown by cut. tings of shorter length, consisting only of two buds; and the most successful mode is to plant each cutting in a small pot, and plunge the pots in a slight hotbed, or place the cuttings at once in the mould of the bed itself. In either case they will make strong plants in the same season.

But the most approved way of raising vine plants in pots is that of propagation by eyes, which we have fully explained in the first part of this work. This, as it retains the least portion of the old wood, is manifestly the nearest approach to raising a plant from the seed, that most perfect of all modes with respect to the constitution of a plant. In the case of new or rare sorts it offers to us the means of multiplying them with the greatest possible rapidity. As the grape usually receives its annual pruning in autumn or winter, the cuttings may be reduced to nearly their proper length, and liept in earth, in the cellar, until the ensuing spring. The hardier sorts may be buried in the spen ground.

The foreign and the native grapes are very different in their habits, in this climate, and, therefore, must be treated differently. The native sorts, as the Isabella and Catawba, are cultivated with scarcely any further care than training up the branches to poles or a trellis, and are, on this account, lighly valuable to the farmer, while the European varieties are of little value in this climate except with especial care, and are, therefore, confined to the garden.

\section{Culture of the Foreign Grape.}

The climate of the temperate portion of this country, so favourable to all other fruits, is unfortunately not so for the foreign grape. This results, perhaps, from its variability, the great ob. stacle being the mildew, which, seizing upon the young fruit, prevents its further growth, causer it to crack, and renders it worthless. Unwilling to believe that this was not the fault of bad culture, many intelligent cultivators, and among them inen of capital, and much practical skill, have attempted vineyard culture, with the foreign sorts, in various sections of the country, under the most favourable circumstances, and have uniformly failed. On the other hand, the very finest grapes are producea under glass, in great quantities, in our first rate gardens, espe. cially in the neighbourhood of Boston; $f$ in the small yards or

* In sandy or dry soils the cuttings may be left longer.

t The vineries at the seats of J. P. Cushing and Col. Perkins, near Boston, pro luce annually many tons of these grapes, grown in the highest porfection. 
gardtns of our cities, owing to the more uniform state of the at. mospliere, the foreign grape thrives pretty well; and, funally, in all gardens of the midlle states, the hardier kinds may, uniey eertain modes of culture, be made to bear good fruit.

iV ithout entering into any inquiries respecting the particulat way in which the mildew (which is undoubtedly a parasitical plant, ) is caused, we will endeavour to state concisely some practical truths, to which our own observation and experience have led us, respecting the hardy culture of the foreign grape.

In the first place, it is well known, to gardeners here, that young and thrifty vines generally bear one or two fair crops of fruit; sccond, that as the vine becomes older if it is pruned in the common mode, (that is to say the spurring-in mode of shortening the side branches, and getting fresh bearing shoots from main branches every year,) it soon bears only mildewed and imperfect fruit; and, finally, that the older and larger the vine: the less likely is it to produce a good crop.

This being the case, it is not difficult to see that, as the vine like all other trees is able to resist the attacks of diseaso on uniavourable climate just in proportion as it is kept in a youn? and highly vigorous state, it follows if we allow a plant to retain only young and vigorous wood, it must necessarily presurv? much of the necessary vigour of constitution. And this is only to be done, so far as regards training, by what is called the renewal system.

The renewal system of training consists in annually providing a fresh supply of young branches from which the bearing shoots are produced, cutting out all the branches that have borne the previous year. Fig. 61 represents a bearing vine treated in tnis manner, as it would appear in the spring of the year, after having been pruned. In this firure, $a$, represents the two branches of last year's growth trained up for bearing the present year; $b$, the

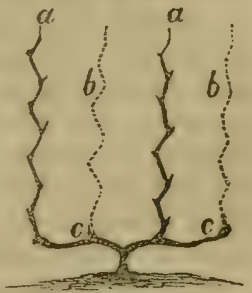

Fig. 91. Renewul Lran. ing. places occupied by the last year's wood, which, having borne, has been cut down to within an inch of the main arm, $c$. 'The present year, thereiore, the two branches $a$, will throw out sile shoots, and hear a good crop, while the young branches will be trained up in the places of $b$, to bear the next year when $a$, are in like manner cut down.

This renewal training will usually produce fair fruit, chiefly as it appears to us, because the ascent and circulation of 1. sap bring mainly carried on through young wood, is vigorous, and the plant is healthful and able to resist the mildew, while, on the contrary, the circulation of the sap is more feeble and tardy. 
through the more compact and rigid sap vessels of a vine fuli of old wood.*

The above mode of training is very easily understood, but we may add here for the benefit of the novice; 1st, that vines, in order that they may bear regularly and well, should always be kept within small bounds; $2 d$, that they should always be trained to a wall, building, or upright trellis ; $\nmid$ and, $3 \mathrm{~d}$, that the leaves should never be pulled off to promote the ripening of the fruit. The ends of the bearing shoots may be stopped, (pinched off,) when the fruit is nearly half grown, and this is usually all the summer pruning, that under our bright sun the grape vine properly treated requires.

Following out this hint, that here, the vine only bears well when it is young, or composed mainly of young wood, an intel. ligent cultivator near us secures every year abundant crops of the Chasselas, by a system of renewal by layers. Every year, from his bearing vines, he lays down two or more long and clean shoots of the previous year's growth. These root freely, are allowed to make another season's growth, and then are made to take the place of the old plants which are taken out; and by this continual system of providing young plants by layers, he al. ways succeeds in obtaining from the same piece of ground fair and excellent grapes.

Culture under glass without artificial heat. The great superiority of this fruit when raised under glass, renders a vinery an indispensable feature in every extensive garden. Even without fire-heat grapes may, under our bright sun, be grown admirably; the sudden changes of the weather being guarded against, and the warmth and uniformity of the atmosphere surrounding the vines being secured. In the neighbourhood of Boston, cheap structures of this kind are now very common, and on the North River, cven the Muscat of Alexandria and other sorts which are usually thought to require fire-heat, ripen regularly and well, with moderate attention.

A vinery of this kind may be erected so as to cost very litlle, nearly after the following manner. Its length may be thirty feet; its width sixteen feet; height at the front, two feet ; at the back twelve feet. This part of the structure may all be built of wood, taking, for the frame, cedar or locust posts, setting them three and a half feet in the ground, the portion rising above the ground being squared to four or five inches. On these posts, (which are placed six feet apart,) nail, on both silfes, matched and grooved planks, one and a quarter unches thick. The space between these planks not occupied wo the

- See Hoare on the Grape Vine

$t$ And never on an arbour, except for the purposes of shade. 
post, fill in with dry tan, which should be well rammed down The rafters should be fixed, and from three to four feet apart. The sashes forming the roof, (which are all the glass that will be necessary,) must be in two lengths, lapping in the middle, and arranged with a double groove in the rafters, so that the top and bottom ones may run free of each other. The building will, of course, front the south, and the door may be at either end.

The border for the grapes should be made partly on the inside and partly on the outside of the front wall, so that the roots of the vines may extend through to the open border. A trellis of wire should be fixed to the rafters, about sixteen inches from the glass, on which the vines are to be trained. Early in the spring, the vines, which should be two year old roots, may be planted in the inside border, about a foot from the front wallone vine below each rafter.

SoIL. The border should be thoroughly prepared and pulver. ized before planting the grapes. Two thirds of mellow sandy loam mixed with one third of a compost formed of well fermented manure, bits of broken charcoal, and a little lime rubbish, forms an excellent soil for the grape in this climate. If the soil of the garden is old, or is not of a proper quality for the basis of the border, it is best to prepare some for this purpose by rotting and reducing beforehand, a quantity of loamy turf from the road sides for this purpose. The depth of the border need not exceed two feet, but if the subsoil is not dry at all seasons, it should be well drained, and filled up half a foot below the border with small stones or brick bats.

Pruning. Decidedly the best mode of pruning for a cold house, or vinery without fire-heat, is what is called the long or renewal mode, which we have already partially explained. Supposing the house to be planted with good young plants, something like the following mode of training and pruning may be adopted. The first season one shoot only is allowed to proceed from each plant, and this, at the end of the first season, is cut down to the second or third eye or bud. The year following two leading shoots are encouraged, the strongest of which is headed or stopped when it has extended a few joints beyond tho middle of the house or rafter, and the weaker about half that lensth. In November these shoots are reduced, the strong one having four or live joints cut from its extremity, and the weaker one to the third eye from its lower end or place of origin. In the third season one leading shoot is laid in from cach of these, the stronger one throwing out side shoots on which the fruit is produced, which side shoots are allowed to mature one bunch of grapes each, and are topped at one or two joints above the fruit. No side shoots are allowed to proceed from the weaker shoot, out it is laid in, to produce fruit the ensuing season, so that by 
the third season after planting, the lower part of the house or rafters is furnished with a crop of fruit proceeding from wood of the preceding year. At nex autumn pruning, the longest of these main shoots is shortened about eighteen inches from the top of the rafter, and the next in strength to about the middle of tiue rafter, and all the spurs which had borne fruit are removed. Each vine is now furnished with two shoots of bearing wood, a part of old barren wood which has already produced fruit, and a spur near the bottom for producing a young shoot for the follow. ing year. In the fourth summer a full crop is produced, both in the upper and lower part of the house, the longer or oldest shoot producing fruit on the upper part of its length, and the shorter on its whole length; from this last, a leading shoot is laid in, and another to succeed it is produced from the spur near the bottom. At the next autumn pruning, the oldest or longest shoot, which has now reached the top of the house, is entirely cut out and removed, and replaced by that which was next in succession to it, and this in its turn is also cut out and replaced by that immediately behind it, a succession of a yearly shoot being obtained from the lower part of the old stem. (McIntosh.) This is deciledly the most successful mode for a vinery without heat, producing abundant and fair crops of fruit. Hoare, who is one of the most experienced and ingenious writers on the Grape, strongly recommends it, and suggests that " the old wood of a vine, or that which has previously produced fruit, is not only of no further use, but is a positive injury to the fertility of the plant. The truth of this remark depends on the fact that every branch of a vine which produces little or no foliage, appropriates for its own support a portion of the juices of the plant that is generated by those branches that do produce foliage."

Routine of cultere. In a vinery without heat this is com. paratively simple. As soon as the vines commence swelling their buds in the spring, they should be carefully washed with mild soap suds, to free them from any insects, soften the wood, and assist the buds to swell regularly. At least three or four times every week, they should be well syringed with water, which, when the weather is eool, should always be done in the morning. And every day the vine border should be duly sup. plied with water. During the time when the vines are in blossom, and while the fruit is setting, all sprinkling or syringing over the leaves must be suspended, and the house should be kept a little more closed and warm, than usual, and should any indications of mildew appear on any of the branches it may at once be ehecked by dusting them with flower of sulphur. Air must be given liberally every day when the temperature rises in the house, beginning by sliding down the top sashes a little in the morning, more at mid.day, and then gradually closing then 
in tne same marues. To guard against the sudden changes of temperature out uf doors, and at the same time to keep up as moist and warm a state of the atmosphere within the vinery as is consistent with pretty free admission of the air during sur -hine, is the great object of culture in a vinery of this kind.

Thinning the fruit is a very nccessary practice in all vine. ries-and on it depends greatly the flavour, as well as the fine appearance and size of the berries and bunches. The first thinning usually consists in taking off all superfluous blossom buds, leaving only one bunch in the large sorts or two in the small ones to each bearing shoot. The next thinning takes place when the berries are set and well formed, and is performed with a pair of scissors, taking care not to touch the berries that are left to grow. All this time, one third of the berries should be taken off with the point of the scissors, especially those in the centre of the cluster. 'This allows the remainder to swell to double the size, and also to form larger bunches than would otherwise be produced. Where the bunches are large, the shoulders should be suspended from the trellis by threads, in order is take off part of the weight from the stem of the vine. The last thinning, which is done chiefly to regulate the form of the bunch, is done by many gardeners, just before the fruit begins to colour-but it is scarcely needed if the previous thinning of the berries has been thoroughly done.

The regular autumnal pruning is best performed about the middle of November. The vines should then be taken down, laid down on the border, and covered for the winter with a thick layer of straw, or a slight covering of earth.

Culture under glass, With FIRE-heat. As the forcign grape is almost the only fruit of temperate climates, which cannot be raised in perfection in the open air in this climate, we shall give some concise directions for its culture in vineries with artificial heat. Those who only know this fruit as the Chassclas or Sweetwater appears, when grown in the open air, have little idea of the exceeding lusciousness, high flavour, size and beauty of such varieties as the Black Hamburgh or Mluscat of Alexandria, when well grown in a first rate vinery. By the aid of artificial heat, which, in this climate, is, after all, chiefly required in the spring and autumn, and to counteract any sudden cold changes of atmosphere, this most admirable fruit may easily be produced for the dessert, from May till December. Indeed by vineries constructed in divisions, in some of which vines are forced and in others retarded, some gentle. men near Boston, have grapes nearly every month in the year.

Construction of the vincry. The vinery with fire-heat may be built of wood, and in the same simple manner as just de. scribed, with the addition of a flue above the surface of the ground, running close along the end, two feet from the front 
wall, and about a foot from the back wall, and returning into a chimney in the back wall over the furnace.

For the sake of permanence, however, a vinery of this kind is usually built of brick; the ends and front wall eight inches shick; the back wall a foot thick-or eight inches with occa. sional abutments to increase its strength. In fig. $92(\mathrm{I})$ is shown

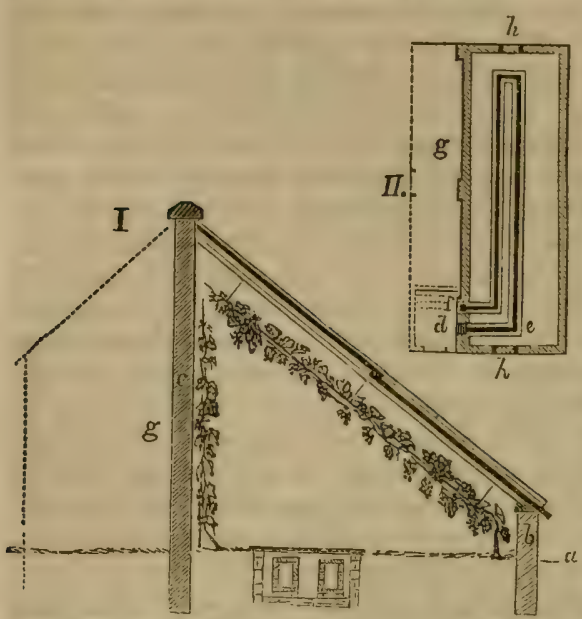

a simple plan of a vinery of this kind. In this the surface of the ground is shown at $a$, below which, the foundation walls are sunk three feet. Above the surface the front wall $b$, rises two feet, the back wall $c$, twelve feet, and the width of the house is fourteen feet. On these walls are placed the rafters, from three to four feet distant, with the sashes in

Fig. 92. Plan and section of a vinery, with fire-heat. two lengths.

in the present example the flues are kept out of the way, and the space clear, by placing them in a square walled space, directly under the walk; the walk itself being formed by an opers grating or lattice, through which the heat rises freely. The arrangement of the flue will be better understood by referring to the ground plan (II.) In this the furnace is indicated at $d$, in the back wall; ${ }^{*}$ from this the flue rises gradually to $c$, whence it continues nearly the length of the house, and returning enters the chimney at $f$. For the convenience of shelter, firing, etc., it is usual to have a back shod, $g$, behind the back wall. In this shed may be a hin for wood or coals, and a sunk area (shown in the dotted lines around $d, f$ ) with steps to descend to the furnace and ash-pit. $†$ There are two doors $h$, in the vinery at either end of the walk.

* 'This furnace should be placed two feet below the level of the fue at $\boldsymbol{e}$, in order to secure a draught. after which it may be carried quite level till it er.ters the chimney. An air chamber may be formed round it, with a register to admit heated air to the house when necessary. A furnace fourteen inches square and deep, with an ash-pit below, in which anthracite coal is burned, will be found a very easy and perfect mode of heating a house of this widih, and thirty feet long.

t The most perfect vinery that we have seen in this country is nut of two hunWred fret long at the country residence of Horace (iray, Ery. Newlown, neaz Bra:ton. It is built of wood, with a curved span roof, after a plan of Mr. Gray'z which secms to th to combine fitnoss and beauty in an unusual degree. 
The border should be thoroughly preparea previously to planting the vines, by excavating it two feet deep and filling it up with suitable compost. 'This is best formed of one half loamy turf, well rotted by having been previously laid up in heaps, (or fresh and pure loamy soil from an old pasture or common;) one third thoroughly fermented horse or cow manure, which has laid in a turf-covered heap for three months; and one third broken pieces of charcoal and old lime rubbish. The whole to be thoroughly mixed together before planting the vines.

The vines themselves should always be planted in a border prepared inside the house, and in order to give the vines that extent of soil which is necessary for them, the best cultivators make an additional border twelve or fourteen feet wide outside, in front of the vinery. By building the foundation of the front wall on piers within a couple of inches of the surface, and supporting the wall above the surface on slabs of stone reaching from pier to pier, the roots of the vines easily penetrate to the border on the outside.

The vines should be planted early in the spring. Two year old plants are preferable, and they may be set eighteen inches from the front wall一one below each rafter, or, if the latter are over three feet apart, one also in the intermediate space.

The pruning and training of the vines we have already described. The renewal system of pruning we consider the best in all cases. The spur system is, however, practised by many gardeners, with more or less success. This, as most of our readers are aware, consists in allowing a single shoot to extend from each root to the length of the rafters ; from the sides of this stem are produced the bearing shoots every year; and every autumn these spurs are shortened back, leaving only one bud at the bottom of each, which in its turn becomes the bearing shoot, and is again cut back the next season. The fruit is abundantly produced, and of good flavour, but the bunches are neither so large nor fair, nor do the vines continue so long in a productive and healthy state as when the wood is annually renewed.

"'The essential points in pruning and training the vine, whatever mode be adopted, according to Loudon, "are to shorten the wood to such an extent that no more leaves shall be produced than can be fully exposed to the light; to stop all shoots pro. duced in the summer that are not likely to be required in the winter pruning, at two or three joints, or at the first large healthy leaf from the stem where they originate; and to stop all shoots bearing bunches at one joint, or at most two, beyond the bunch. As shoots which are stopped, generally push a gecond time from the terminal bud, the secondary shoots thus $f$ oduced should be stopped at one joint. And if at that joint 
they push also, then a third stopping must take place at one joint, and so on as long as the last terminal bud continues to break Bearing these points in mind, nothing can be more simple than the pruning and training of the vine."

When early forcing of the vines is commenced, the heat should be applied very gently, for the first few days, and afterwards very gradually increased. Sixty degrees of Fahrenheit's thermometer may be the maximum, till the buds are all nearly expanded. When the leaves are expanded sixty-five may be the maximum and fifty-five the minimum temperature. When the vines are in blossom, seventy-five or eighty, in mid. day, with the solar heat should be allowed, with an abundance of air, and somewhat about this should be the average of midday temperature. But, as by far the best way of imparting in. formation as to the routine of vine culture under glass is to present a precise account of a successful practice, we give here the diary of O. Johnson, Esq., of Lynn, Mass., as reported by him in Hovey's Magazine. Mr. Johnson is a very successful amateur cultivator, and we prefer to give his diary rather than that of a professional gardener, because we consider it as likely to be more instructive to the beginner in those little points which most professional men are likely to take for granted as being commonly known. We may premise here that "the vines were planted out in the border in May, 1835; they were then one year old, in pots. In 1836 and 1837 , they were headed down. In 1838 they bore a few bunches of grapes, and made fine wood for the following year, when the date of the diary commences.

\begin{tabular}{|c|c|c|c|c|}
\hline \multirow{2}{*}{ 密 } & \multicolumn{3}{|c|}{$\begin{array}{c}\text { Feb. } 1839 . \\
\text { Temperature. }\end{array}$} & \multirow[b]{2}{*}{ DIARY OF THE VINERY. } \\
\hline & हूँ & 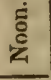 & 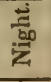 & \\
\hline 13 & & & & $\begin{array}{l}\text { Commenced fire heat in the vinery. [The } \\
\text { thermometrical observations are taken at } 6 \\
\text { o'clock in the morning, at noon, and } 10 \\
\text { o'clock at night.] }\end{array}$ \\
\hline 14 & 50 & 80 & 60 & $\begin{array}{l}\text { Placed horse manure in the house to warm the } \\
\text { border. Washed the house. 'Trok up the } \\
\text { vines, (which had been covered to protect } \\
\text { them from the frost,) and washed them } \\
\text { with warm soap suds : raised as much } \\
\text { moisture as possible. Weather moderate } \\
\text { and cloudy. }\end{array}$ \\
\hline 15 & 50 & 70 & 58 & Weather quite moderate and thawy. Sleet. \\
\hline 16 & 48 & 60 & 55 & $\begin{array}{l}\text { Covered inside border with sand for sprinkling } \\
\text { Thaw. Whitewashed the vir ery. }\end{array}$ \\
\hline
\end{tabular}



58 Earthen pans on the flues kept filled with water, but syringing suspended on account of the moisture in the atmosphere, it having been damp for three days. Cloudy.
Washed vines with soap suds. Weather mo. derate : a slight snow last night.
60 Pans kept full of water for the sake of steam and vines syringed twice a day in sunny weather. Weather changed suddenly last night ; cold, and temperature fell $10^{\circ}$ below minimum point.
A Sweetwater vine in a pot, taken from the cellar on the 18th, and pruned at that time, is now bleeding profusely. At this season of the year, in order to economize with fuel, the furnace should be managed carefully. We found it a good plan about 10 o'clock at night to close the door of the ash-pit and furnace, and push the damper in the chim- ney as far in as possible. No air is then admitted, except through the crevices of the iron work. The thermometer fell only $4^{\circ}$ during the night. Watered vines with soap suds.

61 The last seven days have been very mild for the season : to-day appears like an April day.

63 Weather became cold during the night.

Weather cloudy and thawy for the last three days. The floor of the vinery kept con. stantly damp, and the flues watered twice at night.

64 Rainy and thaw:

Muscat of Alexandria vine bleeding at the buds. Weather clear and rather cool.

64 Muscat vine continuing to bleed excessively, and finding all attempts to stop it unsuccessful, we hastily concluded to prune it down beyond the bleeding bud, and cover the wound with bladder of triple thickness (two very fast :) this, it was supposed, would stop it ; but in a few moments the sap re-appeared, forcing its way through other buds, and even through the smooth bark in many places. The buds on the Sweetwater vines in pots began to swell. Rain last night : dull weather during the day: snow nearly gone. 


\section{8}

71 Bright morning; weather

68 Quite warm and pleasant for the season.

64 Weather changed last evening suddenly; a cold snow storm set in to-day. Afternoon clear.

63 Buds of some black Hamburg vines beginning to swell. Dug up the inside border, and, notwithstanding all precautions, destroyed a few of the grape roots, which were within three inches of the surface. From this circumstance, we have determined not to disturb the border outside, but merely to loosen two inches below the surface: we are satisfied that the vines have been injured by deep digging the borders. Cold severe. last night temperature $2 \circ$ below 0 .

The cold very severe. The sudden changes render it almost impossible to keep a regular temperature in the house, which should not stand (at this stage of forcing) below $60 \circ$. The house having originally been intended for a grapery without fire heat, it is not well adapted to forcing.

68 Weather cool and pleasant.

68 Buds of the vine in pot breaking.

63 Buds of Hamburgs breaking. Snow last night. 63

Quite cold last night. Windy.

Buds of Hamburgs mostly breaking. Owing to the changeable weather, there is some fear that there has been too much heat, as a few of the shoots appear weak. Plenty of air has been given daily.

65 Morning fine; afternoon cloudy. When the fire is at a red heat, the damper and furnace door are closed to keep up the heat. 


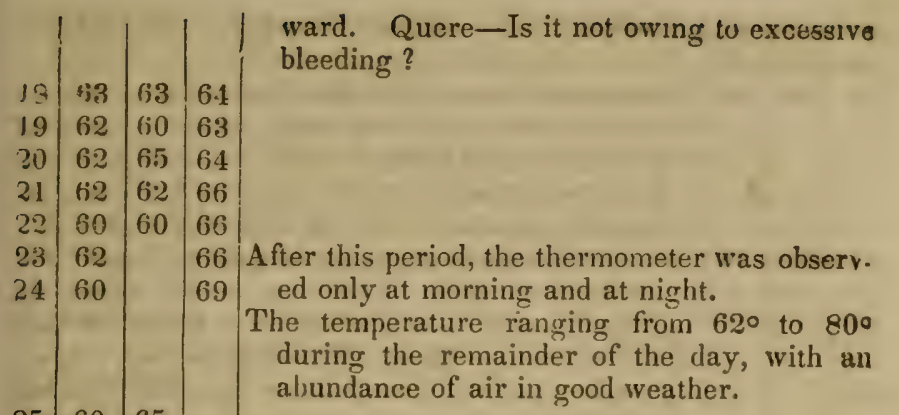

The last six days cloudy; wind east; quite cold last night for the season.

Topped the fruit-bearing shoots one joint above the fruit, and when the lower shoots appear weak, top the leading shoot of the vine.

Discontinued syringing the vines.

A few clusters of flowers began to open on two vines.

The last three days wind north-east, with much rain; to-day sleet and rain.

Grapes blooming beautifully: keep up a high temperature with moisture, when the wea. ther is cloudy during the day.

Floor sprinkled to create a fine steam.

A few clusters of flowers open on the Muscal of Alexandria. 


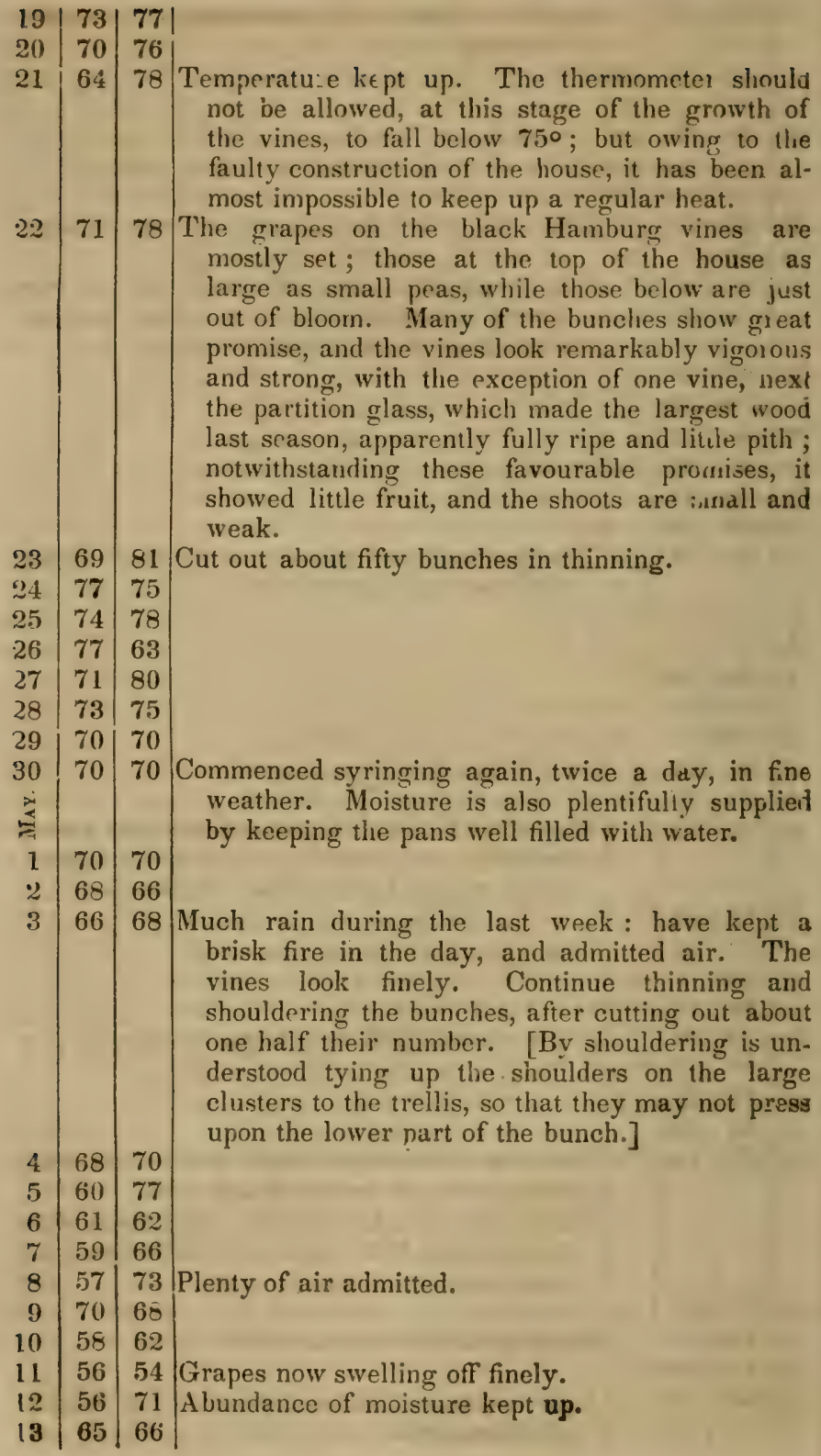


14 63 73|A fine rain to-day. The month has beer :ather cool; several nights the past week the earth has frozen slightly. The grapes are now swelling finely. Continue to thin the fruit daily.

The process of thinning the berries continued, taking out some almost every day, and always the smallest.

Abundance of air given in fine weather.

72 Next year's bearing wood carefully laid in.

The month of May has been, as a whole, unta. vourable for the grape. Much rainy and dull weather: we have been obliged to light fires every night, and occasionally in the day. The grapes have been often looked over and thinned, yet there is no doubt the scissors have been used too sparingly.

All lateral branches cut clean out.

Bunches supported by tying to the trellis.

The grapes have now completed their stoning pro. cess, and a few near the furnace swelling off. No mildew, or disease of any kind, has yet been discovered, and the vines generally have the most healthy and vigorous appearance. The weather has been dull and disagreeable, which has rendered fires necessary. 


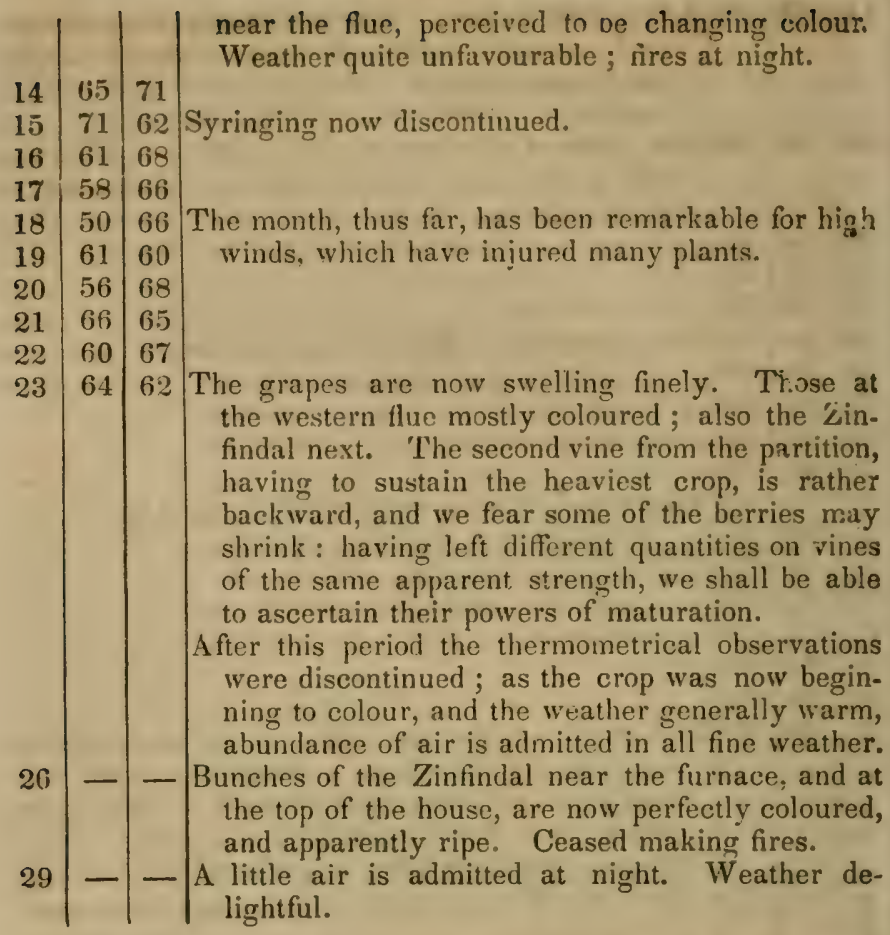

July 4.-Cut six bunches of Zinfindal grapes; the largest a pound and a half; weight of the whole, five pounds and a quarter.

6th.-Exhibited Zinfindal grapes at the Massachusetts Hor. ticultural Society.

13th.- Exhibited black Hamburg grapes at the Massachu. setts Horticultural Society's room.

15th.-A few bunches of the Muscat of Alexandria are now ripe; the flavour exceedingly fine.

20th.-Continued to cut Zinfindal grapes.

$22 d$. - The ripening of all the grapes being now completed, we have not decmed it necessary to continue the diary. In the vinery we shall eut about two hundred and thirty pounds of grapes from nine vines, [being about twenty-five pounds to each.] The Hamburgs a verage nearly one pound and a quar. er to the bunch throughout.

In the cold house, separated from the vinery by the partition, a little mildew was perceived. By dusting sulphur on the in. fected brshes, the mischief is instantly checked. Most of the 
cultivators with whom we have conversed complain grievously of mildew this season, and some have lost part of then crops by inattention on its first appearance.

Aug. 10/h.-Again exhibited some of the Hamburg grapes at the Missachusetts Horticultural Society's room. One fine ounch weighed two and a half pounds, and a beautiful cluster of Muscat of Alexandria one pound Some of the berries of the former measured three inches in circumference, and the latter three and a quarter by three and three quarter inches.

Another season we intend to use a larger quantity of soap suds on the grape border. Have not naid sufficient attention to the watering of the border, and the inside, especially, must have suffered. Another fault to be removed next year is, to tie up all the projecting grapilons as well as the shoulders, which would allow the grapes to swell without crowding.

The grapes in the cold house are swelling finely. The bunches were thinned much more severely than in the vinery, but, notwithstanding this, they are all filled un, and many are too crowded. The berries are also larger than the grapes in the vinery, though none of the clusters have attained the same size.

Much has been written upon the suijject of the shrivelling or shrinking of grapes: none of the clusters in the vinery were affected; but in the cold house, some shriveiling was perceived on a few bunches. We are inclined to believe tiat the moisture given after the grapes begin to colour, and want of sufficient air, are the causes.

To insure a good crop of grapes, we are satisfied that they must have-plenty of heat-plenty of air-plenty of moisturesevere thinning of bunches-and severe thinning of berries. The vines, also, must be pruned often, and kept free: the wood never crowded. Great attention must be paid to the airing of the house, which must be done gradually, that there may be at no time a sudden change in the temperature.

With such attention, and the prerequisite of a rich border, on a dry subsoil, good crops of fine grapes are always to be ob. tained. 'The vines require much moisture until they have com. pleted their last swell, when the moisture should be withdrawn."

INSECTS AND DISEASES. When properly grown unded glass, the grape is a very vigorous plant, liable to few diseases. The bleeding which often happens at the commencement of growth, usually ceases without doing harm, when the foliage begins to expand. If excessive, it may be stopped by a mixture of three parts cheese parings and one part lime, applied to the wound. The red-spider which sometimes infests vineries kept at a high temperature, is usually destroved by coating over the flues with a wash of quick !ime and sulphur, after which, the house must be kept closed for half a day. The smaller insects which ozea. 
sionally prey on the young shoots, are easily kept down by syringing the parts affected, with a solution of whale onl soap.

VARIETIES. There are in the catalorues a vast number of names of grapes, many of which belong to the same fruit. But there are really ouly twenty or thirty varioties which are $a$ : all worthy of cultivation in gardens. Indeed, the most experienced gardeners are satisfied with half a dozen of the best sorts for their vineries, and the sorts universally admired are the black Hamburgh, Black Prince, White Muscadine, and Musce. of Alexandria. We will describe all the fuest foreign grapes that have been introduced, and for the sake of simplifying their arrangement, shall divide them into three classes; 1st, those with dark red, purple or black berries; 2d, those with white or yellow berries; $3 d$, thase with light red, rose-coloured, gray, or striped berries.

Class I. Grapes with dark red, purple, or black berries.

\section{Black Cluster. 'Thomp.}

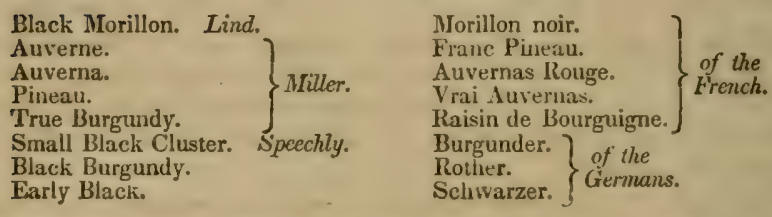

This excellent hardy grape is the true Burgundy grape so highly valued for wine in France. It is readily distinguished from Miller's Burgundy, by the absence of the down on its leaves, which peculiarly distinguishes that sort. The fruit is very sweet and excellent, and the hardiness of the vine renders it one of the best varieties for the open air in this climate.

Bunches small, compact, (i. e. berrics closely set.) Berries middle sized, roundish-oval. Skin deep black. Juice swcet and good. Ripens in the open air about the 20 th of September. Thompson gives more than 40 synonymes to this grape.

2. Black Frontignan. \& Thomp.

Muscat Noir.

Sir William Rowley's Black.

Muscat Noir Ordinaire.

Purple Frontignan.

Black Frontignac.

Purple Constantia.

Black Constantia, (of some.)

Buurdales des Hautus Pyrénées.

ac. to

Thoinp.

Muscat Noir de Jura.

An excellent grape for the vinery, originally trom the town 
of Frontignan, in France, where it and other similar sorts ere sargely cultivated for making the Muscadine or Frontignac wine.

Bunches rather long. Berries of medium size, round, quite black. Skin thin, flavour musky and rich. Ripens in Octocer. A good bearer.

The blue frontignav, (Violet Frontignan and Black Conantion, of some,) is rather inferiour to the above, having only a slightly musky flavour; the bunches are more compact, the berries not quite round, purplish, with a thick skin.

\section{Black Haiburgh. § Thomp. Lind. Speechly.}

\author{
Wamer's Black Hamburgh. \\ Purple Hamburgh. \\ Red Hamburgh. \\ Brown Hamburgh. \\ Vutch Hamburgh. \\ Victoria. \\ Salisbury Violet. \\ Hampton, Court Vine. \\ Valentine's. \\ Gibaralter. \\ Frankendale.
}

\begin{tabular}{|c|c|}
\hline $\begin{array}{l}\text { Frankenthaler. } \\
\text { Frankentlialer Gros Noir. } \\
\text { Troi.inger. } \\
\text { Elue 'Trollinger. } \\
\text { 'Troller. } \\
\text { Welscher. } \\
\text { Fleish Traube. } \\
\text { Hudler. } \\
\text { Languedoc. } \\
\text { NIolirendutte. } \\
\text { Weissholziger Trollinger. }\end{array}$ & $\begin{array}{c}\text { of various } \\
\text { European } \\
\text { gardens. } \\
\text { ac. to } \\
\text { Thomp. }\end{array}$ \\
\hline
\end{tabular}

Frankenthaler. Frankentlialer Gros Noir Troi.anger.

Bıve Trollinger.

'Troller.

Weischer.

Hudler.

Nolurendutte.

Weissholziger Trollinger.

of various gardens.

ac. to

Thomp.

The Black Hamburgh has long been considered the first of black grapes for the vinery, but it will very rarely perfect its fruit out of doors. Its very large size and most luscious flavour render it universally esteerned.

Bunches large, (about nine iriches deep,) and mostly with two shoulders, making it broad at the top. Berries very large (fig. 93,) roundish, slightly inclining to oval. Skin rather thick, deep brownish purple, becoming nearly black at full maturity. Flavour very sugary and rich. A good and regular bearer.

Wilmot's New Black Hamburgh is a recent variety which is said to bear larger and handsomer fruit.

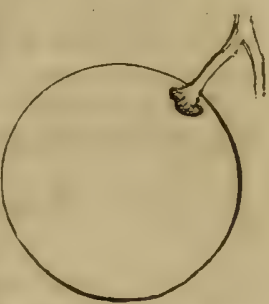

Fig. 93. Black Hamburgh.

\section{Black Prince. $\oint$ Lind. Thomp.}

Alicant.

Black Spanish.

Black Valentia.

Black Portugal.

Buston.

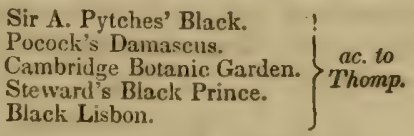

The Black Prince is very highly estermed. It is hardier than the Black Hamburgh, ripening very well here in good situations in the open air, and bearing profusely, with the easiest culture, in the vinery: 
Bunches long and not generally shouldeted, berries large, ralier thinly set, oval Skin thick, black, covered with a thicis jlue bloom. Flavour first rate-swcet and excellent.

\title{
5. Black Lombardy. § Lind. Thomp.
}

\author{
Wests' St. Peters. \\ Puonah. \\ Money's. \\ Raisin des Carmes. \\ laisin de Cuba.
}

Bunches large and long, with shoulders. Berries large, roundish-oval. Skin thin, very black at maturity. Flavour very rich and sugary. The leaves are rather small, and turn purple as the fruit ripens. 'Thompsou considers this synonymous with the Poonah grape introduced by Sir Joseph Banlis, from Bombay. It requires a pretty high temperature, and is then a great bearer.

\section{Black Morocco. Thomp.}

Le Cour. Lind.

Ansell's Large Oval Black.

Black Muscadel.

Ruisin d'Espagne.

A large and showy grape, ripening late, but requiring a gond deal of heat. The blossoms are a little imperfect, and require to be fertilized with those of the Black Hamburgh, or some other hardy sort.

Bunches large; berries very large, oval; skin thick, dark sed, flavour tolerably sweet and rich.

\section{Black Salnt Peter's. $§$ Thomp.}

Saint Peter's. Lind. Speechly.

Black Palestine.

Oldaker's West's St. Peter's.

A capital variety, ripening quite late and wnich may be kept on the vimes if it is allowed to ripen in a cool house until wiuter. This is one of the best sorts for a vinery without fire-heat.

Bunches of pretty good size, rather loose. Berries rather large, round. Skin thin and black. Flavour delicate, sweet, and excellent.

\section{Black Muscat of Alexandria. Thomp.}

Ren Nuscat of Alexandria. Lind.

Red Hi ntinac of Jerusalem.

Bunches large, and shouldered. Berries large, oval, skin 
thick, of a reddish colour, becoming black at maturity. F!esh quite firm, with a rich musky flavour. Requires a vinely wıth fire-heat.

\section{Black Tripoli. Thomp.}

\section{Black Grape from Tripoli. Lind. Speech.}

This grape, which we have not yet seen in fruit, is said to be a large and very excellent one, ripening late, and well worthy of a place in the vinery. It requires some fire-heat.

Bunches of medium size, shouldered, rather loose. Berries large, round, often slightly flattened. Stones quite small. Skin thin, purplish black, slightly covered with bloom. Flesh tender and sweet, with a very high flavoured, rich juice.

10. Black Muscadine. Lind. Thomp.

Black Chasselas

Chasselas noir?

A pretty good black grape, scarcely succeeding well, however, in the open air, and inferior to other sorts for the vinery.

Bunches of medium size, compact. Berries roundish-oval. Skin thick, black, overspread with a blue bloom. Juice sweet, and of pretty good flavour.

\section{Black Sweetwater. Thomp. Lind.}

Water Zoet Noir.

Bunches small, compact. Berries small, round. Skin thin, with a sweet and pleasant juice. A second rate, but rathe harily sort.

12. Harly Black July. § Thomp. Lind.

July Grape.

Mirdeleine.

Madeleine Noir.

Ruisin précoce. Portécu.

Morillon Hâtif. O. Duh.

De St. Jean.

Schwarzer Frihzeitiger

Burgunder.

August Traube.

Jacubs straube.

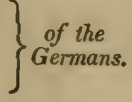

The earliest of grapes, and chiefly valued for the dessert or that account. In the open air it ripens, here, the last of July, or early in August. The leaves are rather small, and ligh: green above and beneath.

Bunches small and compact. Berries small, quite round. 
Slin thick, black, covered with a blue bloom. Flavour mode. ratelv sweet, but not rich or perfumed.

\section{Esperione. Thomp. Lind.}

Turner's Black.

Hardy Blue Wisdsor.

Cumberiand Lodge.

The Esperione is a hardy, luxuriant, and prolific grape: growing as well in the open air as the Muscadine, and even better in many situations. It is yet very rare with us, but merits more general cultivation.

Bunches large, shouldered, like the Biack Hamburgh in size. Berries round, or occasionally flattened, and often indented with a groove. Skin thick, dark purple, powdered with a thick blus bloom. Flesh adheres to the skin, of a pleasant, sprightly fla v sur, not very rich.

\section{Mrllen's Burgundy. Lind. Thomp. Speechly.}

Miller Grape.

Lo Meunier.

Morillon 'Taconné.

Fromenté.

Aleutica du Po.

Sauvignien noir.

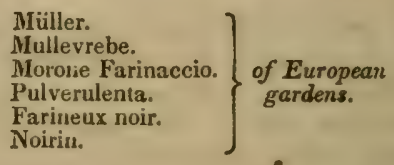

Müller.

Mullevrebe.

Morolie Farinaccio.

Pulverulenta.

Noiriu.

A favourite variety, long known and cultivated in all parts of the world as a hardy grape for wine and table use. It ripens pretty well in the open air, and is readily known by the dense cuvering of cottony down which lines both sides of the leaves, whence the name miller's grape.

Bunches short, thick, and compact. Berries roundish-oval, very closely set together. Skin thin, black, with a blue bloom. Flesh tender, abounding with a sweet high flavoured juice. Each berry contains two small seeds.

Class II. Grapes with White or Yellow berries.

15. Сiotat. Thomp. Lind. Duh.

Parsley-leaved.

White Parsley-leaved.

Parsley-leaved Muscadine.

Malmsey Muscadine.

Raisin d'Autriche.

The Parsley-leaved grape, as its name denotes, is remarkable for its very deeply divided leaves, quite unlike those of anv 
atnor sort. It succeeds very well with us in the open air, and may therefore be considered a valuable sort, but it is greatly superior in flavour when grown under glass.

Bunches of middle size, long, rather loose. Berries round Skin thin, white, with a sweet and pleasant, but not rich fla voured juice.

There is a variety of this grape with red fruit.

16. Chasselas Musqué. § Thomp. Duh.

Musk Chasselas.

Le Cour.

A very delicious grape, the highest flavoured Chassela:, naving much of the flavour of the Muscat of Alexandria.

Bunches of medium size, long and rather loose. Berries middle size, round. Skin thin, yellowish white. Flesh tender with an abundant juice, of a rich musky flavour. Leaves smaller and decper green than those of the Sweetwater or Mus cadine.

\section{Charlsworth Tokay. Thomp.}

A new variety very recently received from England, reputea to be of superior quality.

Bunches long, compact. Berries large, oval; skin thick, white. Flavour rich and excellent, with a Muscat perfume.

\section{Early White Malvasia. Thomp}

Morna Chasselas.

Early Chasselas.

Grove End Sweet Water.

White Melier.
Mornair blanc.

Le Melier.

Melier blanc.

Blanc de Bonncuil.

A nice early grape, and a good bearer, which is in fuct only an earlier variety of the Chasselas. It bears very well in the open air.

Bunches in size and form, much like those of the white Chas selas or Royal Muscadine. Berries round, yellowish white. Skin thin. Flesh sweet, juicy and agreeable in flavour. Ripens in August. The leaves ase pale green on the upper side, slightly downy below, cut into five, rather deep lobes.

\section{Pitmaston White Clustar. $\S$}

A pretty hardy grape, raised in Pitmaston, England, from the Black Cluster, ripening rather earlier than the Sweetwater, of good quality and well deserving a place where the foreign grapes are cultivated in the open air. 
Bunches of snedium size, compact and shouldered. Bermes middle sized, round. Skin thin, amber colour, occasionaı:v tiriged with a little russet when fully ripe. Flesh tender, juic:v, cweet and excellent.

\section{Royal Muscadine. § Thomp. Lind. Mill.}

Amber Muscaline

Early White Teneriffe.

rolden Chasselas.

IVhite Chasselas.

Chasselas doré.

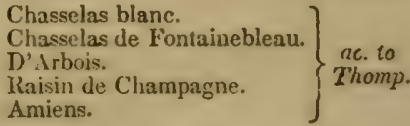

A truly excellent grape in all respects-one of the very best for harly culture in this climate, or for the vinery. It is every

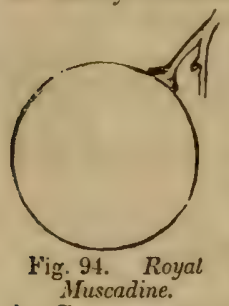

the Sweetwater. where highly esteemed, and is the Chasselas par excellence of the French.

Bunches large, and shouldered. Berries, (fig. 94,) larger than those of the Sweetwater, round. Skin thin, at first greenish white, but turning to an amber colour when fully ripe. Flesh tender, with a rich and delicious fla. vour. Ripens here about the 20th of Septem. ber. Wood and foliage stronger than those of

\section{Scotch White Cluster. $\S$ Thomp.}

Blacksmith's White Cluster.

This is a new grape, not yet fairly testea in this country, but which is likely to prove a valuable one for garden culture, as it has the reputation in England of being very hardy, very carly, and a great bearer. It was raised from the seed by a blacksmith of Edinburgh in 1812.

Bunches of middle size, compact. Berries medium sized, roundish-oval. Skin white, thin. Flesh tender, juicy, swcet, and excellent.

22. Syrian. Thomp. Lind. Speech.

Jews.

'This is believed to be the grape mentioned in the scriptures as found by the Israelites on the brook of Eschol, the bunches of which were so large as to be borne on a staff by two men. It is a very superb looking fruit, and has been grown in this country to very large size. In England, bunches of it have been produced weighing $19 \frac{1}{2}$ lbs. It is much inferiour in fla. vour to No. 24, and is, perhaps, therefore scarcely desiruble in a small collection. 
Bunches enormously large, and regularly formed, with broad shou,ucrs. Berries laree, oval. Skin thick, white at first, but becoming a tawny yellow, or amber when at full maturity. Flesh firm and solid, moderately juicy and sweet, though not rich. Will hang till Christmas in a vinery. The wood and foliage ars very large.

\title{
23. VERDELHo. Thomp. Lind. \\ Verdal. \\ Verdilhio. \\ Madeira Wine Grape.
}

A vigorous growing crrape, of grooi quality, from Madeirs. which is largely used in that island for making the best wines.

Bunches rather small, lonse. Berries small, rather uncqual in size, and often without sechls. Skin thit, semitrans, parent, yellowish-green, a little tinged with russet when ve, y ripe. Juice a little acid at first, but rich and excellent at math rity.

24. White Muscat of Alexandria. § Thomp. I. inl.

\author{
$\left.\begin{array}{l}\text { Frontnias of Alexandria. } \\ \text { Jeru:alem Muscat. }\end{array}\right\}$ Niller. \\ Malaga. \\ White Mnscat. \\ Totfenham Park Muscat. \\ White Huscat of Lunel. \\ Lunel. \\ Mn:cat d'Mlexandria. \\ Passe-longue Musqué. Duh. \\ Passe Musqué. \\ Zebibo, (of Sicily.)
}

'The most delicious of all grapes, but requires to be grown under glass in this climate. In favourable scasons it reacheg maturity well in a vincry without fire-heat, but it can scarcely be said to atiain its highest flavour cxcept with the aid of artificial heat.

Bunches very large, often 9 to 12 inches long, rather loose and irregular. Berries, (ing. 95, very large, an inch or more long, oval. Skin thick, white or pale amber when fully ripe. Flesh quite firm and crisp, with a peculiarly musky, rich, perfumed flavour, very delicious. Seeds small, and occasionally absent from the larger berries. This variety is a very strong grower, and is raised in great perfection about Boston. It will hang a long time on the vines.

Mr. Thompson considers the MaLAGA Fig. 95. White Ifuscal grape (brought to this country in jars,) as of Alexandra. synonymous. It is picked so early for importation as to have little fiavour. 
The Cannon.Halid Muscat, an English seedling, closel- re. sembles this grape, but the flest is firmer, the skin yellower, and it is not yuite so rich in favour. It also sets rather badly, re. quiring to be fertilized by hand with the pollen of some other sort.

25. White Frontignan. $\S$ Lind. Thomp.

White Constantia.

White Frontniac.

Nepean's Constantia.

Nuscat Blanc.

Kaisin de Frontignan.

Museat Blanc de Jura.
Moschata Bianca.

Muscado Bianco.

Moseatel Cummun

Muscateller.

Wiesser Mluscateller.

Weisse Muscaten 'Traube.

The White Frontignan is a very favourite grape, as the 1... xy names, quoted above, by which it is known in various pu. ts of Europe sufficiently prove. Its hardy habit, uniform plcuuctiveness in the vinery, and most luscious flavour, make it every where estecmed.

Bunches of medium size, or pretty long, and without shoulders. Berries middle sized, round, rather thickly set. Skin thin, crull white or yellow, covered with a thin bloom. Flesh tender, wih a rich, perfumed, musky flavour.

\section{White Siveetwater. Thomp. \\ Early White Muscudine. \\ White Muscadine, (of Lind.) \\ Early Sweetwater. \\ Stillward's Swetiwater. \\ Dutch Siveetwater. \\ Chasselas Precoce. \\ Chasselas Roval. \\ Water Zoete Blanc.}

This garve is better linown, and mote commonly cultivated than any viter in this country, althougn at is inferior to the Royal Muscatıne. It difiers from the latter in having weaker wood, and open, loose, bunches of a pales colour.

Bunches middle sized, loose or open, usually with many small imperfect berries, shouidered. Berries of the middle size, round. Skin thin, clear watery green, rarely becoming amber except very fully exposed to the sun. Flesh crisp, watery, sweet, but not high flavoured. Ripens in the open air from the 20th to the last of August-a fortnight earlier than the Royal Muscadine.

\section{White Toray, Thomp.}

Genuine Tokay. Lind. Specch.

Gray Tukay?

Tokai blanc.

This is the fruit from which the delicious Tokay wine of 
Hungary is made. We have ripened it very well in the open thir. Its flavour is good and its aroma peculiarly agreeable.

Bunches of medium size, compact. Berries rounded oval. closely set. Slin thin, of a dull white. Flesh very delicate, siveet and perfumed. Leaves deeply 5-lobed, and covered with a sutiny down on the lower surface.

\section{White Haniburgh. Thomp \\ White Lishon. \\ White Portugal. \\ White Raisin.}

This is the Portugal grape of cornmerce which is so largely exported to different parts of the world in jars. It is not a high flavoured though a very showy grape, and will hang a long time on the vines after maturity. It requires a vinery.

Bunches very large and loose. Berries large, oval. Slin thick, greenish-white. Flesh solid, sweet, and sometimes with a slight Muscat flavour. Bunches of this varicty weighinis over three pounds have been grown near Boston.

\section{White Nice. Thomp. Míntosh.}

A very large and showy fruit, and, in a vinery with fire-heat, a very excellont sort. M. Intosh, an English garlener of reputation, has grown bunches of this the White Nice to the enormous weight of eighteen pounds, and consider's it "one of the noblest of grapes."

Bunches very large, with lonse shoulders. Berries roundish, medium size, thinly distributed over the shoulders and sides of the bunch. Skin thin, rather tough, greenish-white, becoming, finally, a little yellowish. Flesh crisp, sweet, and of very good flavour. Leaves and wood very strong, the latter remarkably downy beneath.

\section{White Rissing. Thomp.}

Schloss Johannisberg.

Rudesheimerberg.

Reissling.

Petit Riessling.

Grosser Riessling.

Rössling.

Kleier Rissling.

The most celebrated grape of the Rhine, producing th: wele. brated hock wines. It is yet little known in this country, but from its very great hardiness and productiveness, in the cold districts of its native soil, we hope to find in it a valuathle ac. quisition for sur gardens-if not for our vineyards. 
Bunches of melium size, compact. Berries rather sinall: rnund. Skin thin. Flesh tender and juicy, with sweet and sprightly pleasant flavour.

Class III. Grapes with light rell, rose-coloured, or striped berries.

\author{
31. Alepro. Thomp. Lind. \\ Switzerland Grape. \\ Siriped Muscadine. \\ Variegated Chasselas. \\ Raisin Suisse. \\ Raisin d'Aless. \\ * Chasselas panaché. \\ Maurillan panaché. \\ Maurillan noir panaché.
}

A very singular grape, the berries being mostly striped with white and black in distinct lines; or sometimes half the bunclı will be black, and half white. It bears very well, and is de. serving a place in the vinery of the amateur. The foliagre is also prettily striped in autumn.

Bunches rather below medium size. Berries medium size, roundish. Skin thin, striped with white and dark red, or black Flesh juicy, and of a rich and excellent flavour.

32. Grizzly Frontignax. $§$ Thomp. Lind.

\author{
Red Frontignan, (of some. \\ Grizzly Frontignac. \\ Red Constantia. \\ Muscat Rouge. \\ Muscat Gris. \\ Muscalo Rosso. \\ Kiimmel Traube. \\ Grauer Muscateller.
}

This delicious grape requires to be grown in a vinery when it is, to our taste, scarcely surpassed.

Bunches rather long, with narrow shoulders. Berries round, of melium size, and growing closer upon the bunches than those of the White Frontignan. Skin thick, pale brown, blended with red and yellow. Flesh very juicy, rich, musky and high flavoured.

The Rev Frontignan 'Thompson considers the same as the foregoing only being more decply coloured in some situations. But Lindley, with whom we are inclined to agree in this case, keeps it distinct. The latter describes the Red Frontignan as having bunches without shouiders, berries perfectly round, and 
deep red, flavour excellent. These two sorts require more nareful comparison.

\section{Knight's Variegated Chasselas. 'Thomp.}

Variegated Chasselas. Lind

A hybrid seedling, raised by Mr. Knight from the White Chasselas, impregnated by the Aleppo. A curious and pretty fruit, but not first rate in flavour.

Bunches rather long, unshouldered. Berries below the mid. dle size, round, loosely set. Skin quite thin, white, shaded with bluish violet, sometimes becoming purplish in the sun. Flesh tender, sweet, and pleasant. The leaves die off in au. tumn of fine red yellow and green colours.

\section{Lombardy. Thomp. Lind. \\ Flame Coloured Tokay. \\ Rhemish Red. \\ Wantage. \\ Red Grape of Taurida.}

The Lombardy is remarkable for the very large size of the bunches, which are frequently twelve to eighteen inches long. It is a handsome fruit, the berries thickly set, (so much so as to need a good deal of thinning, ) and it requires fire-heat to bring it to full perfection.

Bunches very larre, handsomely formed, with large ', houlders. Berries large roundish. Skin thick, pale red or flame colour. Flesh firm, sweet, with a sprightly, very good flavour.

\section{Red Chasselas. Thomp. Lind. Fors.}

Red Muscadine. Mill.

Chasselas Rouge. Duh.

This grape a rood deal resembles the White Chasselas, cx. cept that the berries are slightly coloured with red. Very rare. ly, when over ripe, they become a dark red.

Bunches loose, not large; berries medium size, round. Skin thin, at first pale green, but when exposed to the sun they become red. Flesh tender, sweet, and very good. Not very hardy.

\section{Cultwvation of the Native Grapes.}

The better varieties of the native grapes, as the Isabella, Ca. tawba, etc., are among the most valuable of fruits in the middle 
Etates. Hardy, vigorous, and productive, with very trifling care they yield the farmer, and the common gardener, to whom the finer foreign sorts requiring much attention and consirlerabie expense in culture, are denied, the enjoyment of an abundance of very good fruit. In the neighbourhood of New. Tork and Philadelphia their culture is carried to a large extent for suf. plying the markets, a single grower on the Huison, (Dr. Ur. derhill,) sending thousands of baskets to New-York annually. In this part of the country no fruit is more common than the Isabella grape, and many families preserve large quantities for use during the winter months, by packing thern away, as soon as ripe, in jars, boxes, or barrels, between layers of cotton battingin which way they may be kept plump and fresh till March.

North of the $42^{\circ}$ of latitude, and east of the Hudson river, these varieties, except in favourable situations, do not alwavs succeed perfectly - the summers being frequently ton short to mature their fruit, and the winter injuring the vines; but this may be guarded against by planting them against the south side of walls and buildings. In nearly all the middle and western states they thrive perfectly. But in many localities ai the south, especially in Georgia, the fruit is very liable to rot before ripening, and this is most successfully remedied by allowing the vines to run very high-in the tops of trees, or apon a very tall trellis.

The varieties of native grapes at present grown are chicfly either the finer sorts of wild species, or, which is most grnerally the case, they are accidentally improved varieties, t'sat have sprung up in woods and fields from wild vines. 'ney are, therefore, but one remove from a wild state, and, as extensive trials are now being made by various cultivators is produce new varieties from these, there is little doub! that in a few years we shall have many new native sorts, combinirg the good qualities of the best foreign grapes, with the hardiness of the indigenous ones, and with also the necessary adaptation to the various soils ard climates of the United States.

Gandin Culture. The garden culture of these grapes is very easy. They grow with vigour in any soil not atsulute?ponr, and bear abundant crops in sandy or heavy soiis, shough being of grosser habit than many of the forcign grapes, they prefer a rather strong and rich soil. One of the first points ts be attended to in planting them is to secure a periectly summy. open exposure, as it may always be assumed that, with us, no at. mosphere can be too bright or sunny for the grape; tor although it will make the most luxuriant and vigorous shoos in the shado of trees or buildings, yet the crops will be small end uncertain, and the berries will be likely to fall a prey to mirlew.

In the second place the vines should be liept unthrn miderate bounds, and trained to all uright trellis. The ssabella and $\mathrm{Ca}$ 
Lawba are so rampant in thrir growth, when young, that the in. dulgent and gratified cultivator is but ton apt to allow them tc cover a large space. Experience, however, has convincell us that this is an error. For two or three seasons, vines of great size will produce enormous crops, ${ }^{*}$ but they soon exhaust the supply of nourishment at hand, (which, indeed, it is difficult to supply again,) the vine becomes filled with liseless, old wood, and speedily becomes unfruitful and worthless. About 6 or 8 feet apart we have found to be the best distance at which to plant the native grapes. Assuming the trellis to be 8 feet high, then each vine will extend either way 3 or 4 feet, covering a space 8 feet square. In this form, the roots and branches extending but a short distance, they may be kept in high vigour, and a state of constant productiveness, for a great length of time.

The system of pruning and training these grapes generally pursued is the upright mode, with the spur mode of training. T'he first seasons' growth of a newly planted vine is cut back to two buds the ensuing fall or spring. These two buds are allowed to form two upright shoots the next summer, which, at the end of the season, are brought down to a horizontal position, and fastened each way to the lower horizontal rail of the trellis, being shortened at the distance of three or four feet from the root-or as far each side as the plant is wished to extend. 'The next season, upright shoots are allowed to grow one foot apart, and these, as soon as they reach the top of the trellis, are also stopped. The next year, the trellis being filled with the vines, a set of lateral shoots will be produced from the upright leaders with from one to three bunches upon each, which will be the first crop. The vine is now perfect, and, in the spur mode of pruning, it is only necessary at the close of every season, that is, at the autumnal or winter pruning, to cut back these lateral shoots, or fruit spurs, to within an inch of the upright shoot from which they sprung, and a new lateral producing fruit will annually supply its place, to be again cut out at the winter pruning.

After several years bearing, if it is found that the grapes fail in size or flavour, the vines should be cut down to the main horizontal shoots at the bottom of the trellis. They will then speedily make a new set of upright shoots which will produce very abundantly, as at first.

It cannot be denied that the renewal system of training, (see page 221,) is certain of yielding always the largest and finest fruit, though not so large a crop-as half the surface of the vine is every year occupied with young wood, to take the place of that annually cut out.

What we have already stated, in page 222 , respecting pruning will apply equally well here. If the vine is fully exposed to the

*An old vine of the Isabella, still standing in these gardens, has ! roduced 3,000 alusters of grapes in a year. 
sun it will require very little suromer pruning; in fact, none, excent stopping the young shoots three joints beyond the farthesi bunch of grapes, at midsummer-for the leaves being intended by nature to claborate the sap, the more we can retain of them (without robling the fruit unduly of fluids expended in masing new growth.) the larger and higher flavoured will be the fruit; cirreful experiments having proved that there is no more successful mode of impoverishing the crop of fruit than that of pulling off the leaves.

The annual pruning of the hardy grapes is usually perform. ed during mild days in February or March-at least a month betore vegetation is likely to commence. Many cultivators prefer to prune their vines in November, and, except for cold latitudes or exposures, this is undoubtedly the better season.

Fivery third year, at least, the borders where the vines are growing should have a heavy top-dressing of manure. The vine soon exhausts the soil within its reach, and ceases bearing welı when that is the case. We have frequently seen old and impuverished vines entirely resuscitated by digging in about the roots, as far as they extend, a very heavy top-dressing of slightly fermented stable manure.

Vineyard CUlture. While many persons who have either made or witnessed the failures in raising the foreign grapes in vineyards in this country, believe it is folly for us to attempt to compete with France and Germany in wine-making, some of our western citizens, aided by skilful Swiss and German vinedressers-emigrants to this country, have placed the fact of profitable vineyard culture beyond $a$ doubt, in the valley of the Ohio. 'The vineyards on the Ohio, now eovering many acres, produce regular, and very large crops, and their wine of the diferent characters of Markiri, Hock, and Champagne, bringr very readily from 75 cents to nue dollar a gallon in Cincinnatı. 'The Swiss, at Vevav, furst comnenced wine-making in the IVest, but to the zeal and fostering care of $\mathrm{N}$. Longworth, F.q., of Cincimati, one of the most energetic of western horticulturists, that district of country owes the firm basis on which the vine culture is now placed.* The native grapes-chiefly the Catawba--are entirely used there, and as many parts of the midule

\footnotetext{
* From an interesting lefter on this subject which Mr. Longworth has kindly favoured 12 with, we glailly extract the following, knowing how much it will in. terest the practical reader.

"I can scarcely now state the present extent of the culture of the grape for wine in this comitry. We have a large German pupulation who are yearly plant. ing new vineyards, and 1 believe the Ohio river will be, in the course of the next century, as crelelorated for it: wine as lie Rhine. Af er 30 years of experience, with vues from Madeira in the south, and the monutains of Jura in the north, ant mosi of the intermedate latitniles. I an satisfied that the foreign vine can never steceed with us. Nor do I believe in its arclimation. I have cultivated the chaselas for the table for 33 years, and it dors not now succecd so well as it did the first few seasons that I hat it. I have fonnd two o- three foreign varie
} 
etates are quite as favourable as the banks of the Olio for these varieties, the much greater yield of these grapes leads us to be lieve that we may even here pursue wine-making profitably.

The vineyard culture of the native grape is very simple

Ues that may be worthy of cultivation, and one that may make a wine equal te Mideira, but it produces small wood and will not bear a large crop. It was re. coived from ['rince under the nams of the Missouri, but it is clearly a foreign grapr, and I bulieve of the Pineau family. We must rely on our native grapes, aud new varieties raised from their seed. Our beet success, with present materials, will be with the Citawha grape, as we can make from it a wine equal to the bes: Huck, anil with a firk $r$ aroma. I sent recently a sample of some Catawba wine to Niw-Oileans, and was offered the highest price of Hock wine if I would forwarl a quantity for sale.

The lsabilla rots with us more than other grapes, and is only fit to make a sweet wine by alling surar. I have made a fine, white, sweet wine from it, and have sam;les now 12 years of age. The Otho grape is, with us, quite as hardy as tho Catawha and lsabel!a. It does not bear to be crowded, hut requires the full benefit of the sun and air. I deem it better for the table then for wine, as it is free from the hard pulp common to must of our native grapes.

The cultivation of the grape at Vevay is on the wane, as they cultivate only one variety - the Cape grape-a native sort, otherwise known as the Alexander";, or Schuyllisil Muscadell. From it they may make a rough, red, acid wine. This same graps makes a wine resembling, and equal to, the Tenereiffe, when mades without being fermented on the skins, and with the addition of brandy as is usua! with the 'Tenereiffe.

I have now 1.4 vineyards, under the mangement of Germans and Swiss, aidd containing about 70 acres. The wine meets a ready sale ivith our German population, at prices varying from 75 cents to one and a half dollars per gallon, by the quantity.

The grape requires a good soil, and is benefitted by well rotted manure. For aspect I prefer the sides of our hills, but our native grapes would not succeed well in a $d y$ sandy soil, particularly the Catawba, which is a cousin german to the old fox-grape, that prefers a spot near a stream of watar. The north sides of our hills are the richest, and I believe they will, as our summers are warm, in the majority of seitsons produce the best crops. In my first attempts at vineyard culture, to gratify my Germans, I went to unnecessary expense in duep trenching. In a loose soil, like miue, it can do nn good; in a clay soil it is injurious to put the rich soil baluw and have from one to two feet of clisy on the top. 'The root seldom gets to the rich soil, and grows too near the surface, which should be guarded against, as the fruit then suffers from the drouglt. Deep ploughing is better, and is not a twentieth part of the expense. Where it hill is steep, trenching and walling-or sod terracing, is necessary.

I believe our best wine will be made in latitudes similar to ours. A location farther north ma answer well if the groland be covered with snow all the winter, to protect the vine. It is to this cause that they are indebted for their saccess in the cultivation of the grape on the Jura mountains, in France. There is little doubt that the grape will bear better with us, and (judging from samples I have had from the first grower at the south,) will make a better wine here than in Caroliıa.

There was lately published an absurd statement respecting the product of a ineyard at the sont $h_{-}$-that the product was at the rate of 3,400 gallons of wine to she acre. This arose from a false calculation, made by measuring the yield of a single vine, which grew over the top of a tree, and calculating the product of the vineyard by the space occupied by the root of this vine! One favourable vear I selected, from the best part of one of my vineyards, the fourteenth part of ar. kcre, the product of which was 105 gallons-at the rate of 1,470 gallons per acre. The best crop $I$ have ever seen, was here, at the vineyard of MIr. Hackinger, a German-about 900 gallons to the acre, from the Catuwba grape. It was a truly gratifying sight to see, in the midst of the vintage, his aged father sitting in his nrm chair, under the shade of a tree, in the centre of the vineyard, with his bot. the and glass "just as he did in Gernany."

We generally leave six feet between the rows, and use the plough, setting tho plarts 3 to 4 feet apart, and tr tining them to stakes about 6 feot high. The Gier 
Strong loamy or gravelly soils are preferable-linestone soils being usually the best-and a warm, open, sunny exposure being indispensable. The vines are planted in rows, about six feet apart, and trained to upright stakes or posts as in Europe. The ordinary culture is as simple as that of a field of Indian corn-one man and horse with the plough, and the horse-cultivator, being able to keep a pretty large surface in good order. The annual pruning is performed in winter, top-dressing the vines when it is necessary in the spring; and the summer work, stopping side shoots, thinning, tying, and gathering, being chiefly done by women and children. In the fermentation of the newly made wine lies the chief secret of the vigneron, and, much as has been said of this in books, we have satisfied ourselves that careful experiments, or, which is better, a resort to the experience of others, is the only way in which to secure success in the quality of the wine itself.

Diseases. The mildew, which is troublesome in some districts, is easily prevented by keeping the vine of small size, and by the renewal system of pruning, or, never allowing the vine to bear more than two years on spurs from the same old wood.

The beetles which sometimes infest the grape vines in summer, especially the large brownish yellow vine beetle, (Pelicinota punclata,) and the grape-vine flea-beetle (Haltica chalybea, ) are very destructive to the foliage and buds, and the most effectual remedy is hand-picking when taken in time. But we would also very strongly recommend again the use of open mouthed bottles, half filled, (and kept renewed,) with a mixture of swec:ened water and vinegar, and hung here and there among the vines. Indeed, we have seen bushels of beetles, and other in. sects, destroyed in a season, and all injury prevented, simply by the use of such bottles.

VARIETIES. There are yet but few varieties of our native grapes that are really worthy of cultivation. Adlum and Prince, in their treatises, describe quite a large number, but many of these are really quite worthless. In the following list will be found described all that we have yet been able to find of any value. Most of these as the Isabella, Catawba, Alexander's, \&c., are no doubt accidental seedlings from the wild Fox.

man women and ehildren do most of the labor, in tying the vines, trimming the lateral shoots, tupping the fruit branches, etc.

In our furst experiments we generally used to add sugar to the juice, but our Germuns, and indeed all foreigners, give the wine made without sugar the pre: ference. I have now Catawba wine made without sugar 20 years old, sourid and still improving. The Catawba will convert from 8 to $10 \mathrm{oz}$. to the gallon, but when sugar is added it does not so readily fine itself. When I add sugar I mix it with the must as it comes from the press. The wines our climate will best pra duce are the dry Hock wines; though, from our dry Catawba wine, a skilful wine. cooper can make all the varieties of sparkling Hock, etc., equal to the imported. The best Chainpagne I have ever dru'k was made by one of my Germen tenanta, from the Catawba grape." 
grape of the northern states. (Vitis Labrusca, L.) While others of a different habit in leaf, and berry, as the Elsing. burgh, Ohio, \&c., are, perhaps, the offspring of the Frost Grape, (V. cordifolia,) or the Summer Grape, (V. estivalis.) 'I he most popular American Grapes, as yet, are the Isabella and Catawba. More delicate sorts for the dessert, being free from pulp, are the Ohio, Elsingburgh and Bland. All these grapes require a warm summer to enable them to attain their flavour, which is the reason why, in the cool climate of England, they have been pronounced so "harsh and disagreeable."

Native Grapes.

1. Alexander's. Thomp. Prin.

Schuylkill Muscadell. Adlum.

Capeadine.

Cape Grape.

Clifton's Constantia.

Madeira, of York, $\boldsymbol{P}$ a.

Tasker's Grape.

Winne.

This grape, a natural seedling, was first discovered by $\mathrm{Mr}$. Alexander, gardener to Gov. Penn, before the war of the revolution. It is not unfrequently found, as a seedling, from the wild Foxgrape, on the borders of our woods. It is quite sweet when ripe, and makes a very fair wine, but is quite too pulpy and coarse for table use. The bunches are more compact, and the leaves much more downy, than those of the Isabella.

Bunches rather compact, not shouldered. Berries of medium size, oval. Skin thick, quite black. Flesh witn a very firm pulp, but juicy, and quite sweet and musky, wnen fully ripe, which it is not till the last of October.

\section{BLAND.}

Bland's Virginia.

Bland's Madeira.

Bland's Pale Red.

Powell.

Red Suppernong, (of some)

The Bland is one of the best of our native grapes, approach. mg, in flavour and appearance, the Chasselas grapes of Europe, with very little pulp, and only a slight astringency. It does not ripen well to the north of this, except in favourable situations, and should always be planted in a warm exposure. It is a genuine native sort, (doubtless a natural seedling,) and 
is said to have been found on the eastern shore of Virginia, by Col. Bland of that state, who presented scions to Mr. Bartram, the botanist, by whom it was first cultivated. The Bland is not a great bearer, but it merits a place in every good garden in this country. The fruit keeps admirably, in jars, for winter use.

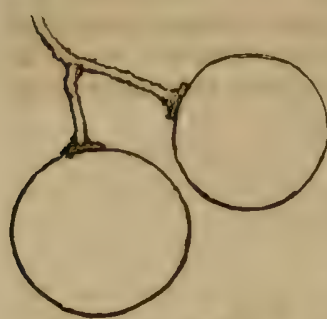

Fig. 96. Bland.

Bunches rather long, loose, and often with small, imperfect berries. Berries, (fig. 96, ) round, on long stalks-hang. ing rather thinly. Skin thin, at first, pale green, but pale red when ripe. Flesh slightly pulpy, of a pleasant, sprightly, delicate flavour, and with little or no musky scent, but a slight astringency. Ripens pretty late. Fo. liage lighter green than that of the Catawba, smoother, and more delicate. This vine is quite difficult of propagation by cuttings.

\section{Catatrba. Adlum. Ken.}

Red Muncy.

Catawba Tolkay.

This excellent native grape was first introduced to notice by Major Adlum, of Georgetown, D. C., and was found by him in Maryland. It probably has its name from the Catawba river, but it has been found growing at various points from that river to Pennsylvania. It is one of the hardiest, most productive, and excellent of our native sorts, either for wine or table use, and succeeds well in all situations not too cold for grape culture. In habit of growth, it so closely resembles the Isabella that it is difficult to distinguish the two, except in the colour and shape of the fruit. Unless it be very ripe, it is, perhaps, a little more musky in flavour, than the Isabella.

Bu::ches of medium size, somewhat loose, shouldered. Berries, (fig. 97,) round, (or sometimes slightly oval, pretty large. Skin rather thick, pale red in the shade, but pretty deep red in the sun, covered with a lilac bloom. Elesh slightly pulpy, juicy, very sweet, with an aromatic, rich, musky flavour. Ripe from the 1 st to the

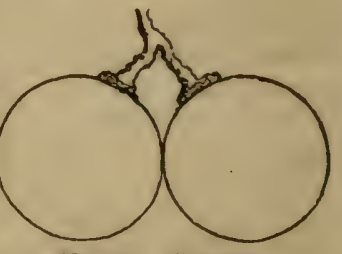

Fig. 97. Catawba. middle of October, and should be allowed to hang till fully ripe.

The To KALoN, is a seedling of this grape raised by the late Dr. Spofford, of Lansingburgh, N. Y. It has no pulp, tut it oroves, with us, too shy a bearer to be worth cultivation. 
Pond's Sreding is a sub-variety from the neighbourhood of Boston, which ripens earlier than the Catawba, but is also a shy bearer.

\section{Diana.}

A seedling of the Catawba, raised by Mrs. Diana Crehore, of Boston, and named by the Massachusetts Horticultural S ciety. It is said to be of superiour quality, and to ripen a fortnight earlier, which will make it valuable at the north.

Fruit much resembling the Catawba, but paler in colour, being a pale grayish red. Bunches loose. Berry round, almost vithout pulp, juicy, with a sweet, rich flavour.

\section{Elsinburgir. Ken. Prin. Adlum.}

Smart's Elingburg.

Elsenborough.

A very nice little grape for the dessert, perfectly sweet and melting, without pulp, originally brought from a village of this na ne in Salem co., New-Jersey. It is not a great deal larger than the common Frost grape, in the size of the berry. A moderate, but regular bearer, ripens well, and much esteemed by many for the table.

Bunches pretty large, loose, and shouldered. Berries, (fig. 98,) small, round. Skin thin, black, covered with a blue bloom. Flesh entirely with. Fig 93. Flsing. out pulp, melting, sweet, and excellent. The burgh. leaves are deeply 5-lobed, pretty dark green, and the wood rather slender, with long joints.

\section{Isabella. Prin. Ken. Adlum.}

This very popular grape, a native of South Carolina, was brought to the north and introduced to the notice of cultivators, about the year 1818, by Mrs. Isabella Gibbs, the wife of George Gibbs, Esq., in honor of whom it was named. Its great vigour, hardiness, and productiveness, with the least possible care, have caused it to be most widely disseminated. A vine growing here has borne 12 bushels of grape in a single year. It is, perhaps, a little more hardy, and ripens earlier than the $\mathrm{Ca}$ tawba, which renders it valuable at the northern part of this state, or the colder portions of New-England No farmer's garden, however small, should be without this and the $\mathrm{Ca}$. tawba.

Bunches of good size-five to seven inches long, rather loose. shouldered. Berries, (fig. 99,) oval, pretty large. Skin thicki 
dark purple, becoming at last nearly black, covered with a blue bloom. Flesh tender, with some pulp, which nearly dissolves when fully mature; juicy, sweet, and rich, with slight musky aroma.

This grape is frequently picked as soon as it is well coloured, and long before it is ripe.

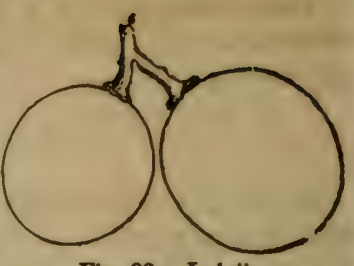

Fig. 99. Isabella.

\section{LENOIR}

\section{Sumpter?}

Clarence?

A very excellent table grape, perhaps superior in flavour for the dessert to any of the foregoing. It comes, originally, from Mr. Lenoir, of the Santee River, Carolina, and is believed to be a seedling raised by him from a seed of one of the Burgundy grapes. It has very much the habit of a foreign vine, but ap. pears to bear well, and ripen admirably here. The wood is grayish brown, strong, and long jointed, and, like the Elsingburgh and Ohio grapes should be left rather long in pruning.

Bunches very handsome, barge, compact, not much shouldered. Berries small, round. Skin thin, purple, with a slight bloom. Flesh tender, melting, (without pulpiness,) sweet, and excellent.

\section{Norton's Virginta. Prin. Ken.}

Norton's Seedling.

A native seedling, produced by a cross betweel, the Bland and Miller's Burgundy, by Dr. N. Norton, of Richnond, Virginia. It is a most productive grape in garden or vineyard, bearing very large crops (especially at the south, where many kinds rot,) is all seasons. It has been confounded by some with Ohio grape, from which it is quite distinct, more pulpy, and less agreeable for the dessert, though, probably a much better wine grape.

Bunches long, sometimes eight or nine inches, occasionally shouldered, somewhat compact. Berries small, round. Skin thin, dark purple. Flesh pulpy, with a brisk, rather rough fla. vour. The foliage is light coloured, shaped like the Fisingburgh. Shoots strong and hardy.

\section{Missouri.}

Missouri Seedling.

This grape we reccived from Cinciannti, where it is con 
little, however, is yet known of it, as it has not yet been placed in the hands of cultivators generally for trial, but we hope soon to see it disseminated.

Bunches large, often weighing a pound, shouldered. Berries nearly as large as Black Hambargh, oval, pretty thickly set. Skin thick, light purple, or lilac, (obscurely spotted,) with a grayish bloom. Flesh firm, with a rich, sweet, and very excel. lent flavour. This fruit ripens early in September, or, in favourable situations the last of August.

\title{
12. Scuppernong. Prin. Adlum.
}

\author{
Fox Grape. \\ $\left.\begin{array}{l}\text { Bull or Bullet. } \\ \text { American Muscadine. } \\ \text { Ruanoake. }\end{array}\right\}$ the south. \\ Ruanoake. \\ Vitis vulpina. Land. \\ - rotundifolia. Mrchs.
}

The Scuppernong grape is a very distinct southern species, found growing wild, from Virginia to Florida, and climbing the tops of the tallest trees. It is easily known from every other grape by the small size of its leaves, which are seldom over two or three inches in diameter, and by their being glossy and smooth on both the under and upper surfaces. These leaves are roundish, and coarsely serrated, and the young shoots are slender; the old wood is smooth, and not shaggy, like that of most vines. This species is diœcious.

We have made several trials with the Scuppernong grape, but find it quite too tender for a northern climate, being killed to the ground by our winters. At the south it is a very hardy, productive, and excellent wine grape. The White and Black Scuppernong scarcely differ, except in the colour of the fruit. The tendrils of each correspond in hue with the fruit.

Bunches small, loose, seldom composed of more than six berries. Berries round, large. Skin thick, light green in the white, dark red in the black variety. Flesh quite pulpy, except when very thoroughly ripe, juicy and sweet, but with a strong, musky scent and flavour.

\section{WARREN.}

A variety recently brought into notice by $\mathrm{Mr}$. Prince, of Flushing, which may prove synonymous with Nos. 7 or 9 . We made the following memoranda from an examination of the fruit last season.

Bunches long, loose, slimhtly shouldered. Berries round, small, of the same size, and form of those of the Elsingburgh, but rather more clnsely set. Skin thin, dark purple, with but little bloom. Flesh tender, melting, with no pulp, and a very sweet, pleasant flavour. 
OtHer wild varieties. There are many other wild varie. ties recently introdured into gardens, but whith are of little or no value for the table. Among these, the Cumningham and Wnodson Prince Edward, from Virginia, are pretty good wine grapes, though the former is a shy bearer. Gimbrecie's Hudson and Ladies' grapes, as well as the Hyde's Black, Red River, Perfumed Red, and several others, are Foxgrapes, with a strong scent, and harsh flavour, of no value for the dessert, and unworthy of cultivation. The Troy Grape, Hyde's Eliza, and some others, are varieties of the Isabella, in no way remarkable. Worthington and Luffborough are recommended by Adlum for wine; we have found them harsh and worthless for the dessert.

Selection of foreign grapes for hardy culture. Royal Musca. dine, Early Black July, Early White Malvasie, Black Prince.

Selection of foreign grapes for a vinery. Black Hamburgh, White Muscal of Alexandria, White Frontignan, Royal Musca. dine, West's St. Peters, Red Frontignan.

Selection of nalive grapes for a small garden. Calawba, Bland, Ohio, Lenoir, Isabella.

\section{CHAPTER XVIII.}

\section{THE MULBERRY.}

Mornes, Tourn. Urticacea, of botanists.

Murter, of the French; Muulbeerbaum, German; Moro, Italian; Murel, Spanish.

Tre Mulberry is a hardy, deciduous fruit tree, but little cul. tivated in this country, though it is really a very considerable acquisition to our list of summer fruits, and every garden of considerable size, ought to contain one or two trees. The fruit ripens in July, very soon after the season of cherries. It is rarely picked from the trees, as it falls as soon as ripe, and it is therefore the custom to keep the surface below it in short turf, and the fruit is picked from the clean grass. Or, if the surface is dug ground, it may be sown thickly with cress seed, six weeks previously to the ripening of the fruit, which will form a tem. porary carpet of soft verdure.

'I'he Red Mulderrey (Morus rubra, L.) is a native species, more or less cornmon in nur wools, with large, rough, heartshaped or lobed l-aves. The fruit is about an inch long, and very pleasant and palatable-though much inferiour to the fol. iowing sort. It bears transplanting well, or is casi"y raised 
from seed, and may, un loubtedly, be greatly imps , ved by re. peated reproduction in this way. As it forms a iarge orna. mental tree with a fine spreading head, 40 feet higl, it is wel' deserving a place on the lawn, or near the house, in ornamental plantations.

Johnson Mulberry. We have lately received from Profes. sor Kirtland, of Cleveland, one of the most intelligent hosticultu. rists in the country: this new variety of our native Mulberry; the fruit of which is said to be of extra large size and superior Havour.

Fruit very large, oblong, cylindric; blackish colnur, sub. acid, and of mild, agreeable flavour. Growth of the wond strong and irregular. Leaves uncommonly large.

The Black Mulberry, or Fnglish Mulberry, (Morus mgra, L.) is a very celebrated old fruit tree, originally from Asia, more or less commonly cultivated in all parts of Europe, hut yet quite rare in this country. Its growth is slow, and it seldem altains a height of more than 12 or 15 feet, forming a low, branching tree, with lobed leaves, but it is very long lived, and there is a specimen in England, at the seat of the duke of Northumberland, 300 years old. In this country it is scarcely hardy enough for the eastern states; but it thrives pretty well

- here, and we have seen very fine crops on a tree in a sheltered position, at Hyde Park, on the Hudson, 80 miles above NewYork. The fruit is incom, arably larger and fines than that of the Red Mulberry, being an inch and a half long, and nearly an inch across-black, and of delicious flavour.

There are many varieties of the White Mulberry, commonly cultivated for silk, but which produce fruit of no value.

The best soil for the Mulberry, is a rich, deep, sandy loam. The tree requires little or no pruning, and is of very easy cul. ture. It is usually propagated by cuttings, three feet long, planted in the spring, half their depth in the ground; cuttings made of pieces of the roots will also send up shoots and becoine nlants.

\section{CHAPTER XIX.}

NUTS.

The Etropean Walnut, (Jugltns regia, L.; Noyer, of the French; IValnaussbaum, German; Nocil, Italian; and Nngal, Bpanish;) better known here as the Madeira $N^{\top} u t$, is a fine lnfty 
growing tree, with a handsome spreading head, and bearing crops of large and excellent nuts, enclosed like those of our native black walnut in a simple husk. It stands the winter very wcll here, and to the south of this it would undoubtedly be a profitable fruit to plant for the market. The fruit in a green state is very highly esteemed for pickling, and the great quan. tities of the ripe nuts annually imported and sold here, prove the estimation in which they are held for the table. There are several varieties reputed to be of rather finer quality, which, however, have not displaced the original species, even in the gardens of Europe, and have not yet borne fruit here.

This tree is usually propagated by the seed, and transplanted from the nurseries when from three to six feet high. But it may also be grafted, with due care, on the common hickory nut.

The Hicliony Nut (Carya alba,) or shell-bark. The Black Walnut (Juglans nigra, $)$ and the Butternut, $(J$. cineria, $)$ are native nut-bearing trees, common in our forests, and too well known to need description here. There are occasionally found in the woods, accidental varieties of the shell-bark hickory, of much larger size and finer flavour than the common species, which are highly worthy of cultivation, as we confess, to our own taste, this nut is much superiour to the European walnut. There is indeed no doubt, that with a little care in reproduction by seed, the shell-bark may be trebled in size, and greatly improved in flavour.

The Filbert, (Noisette, of the French; Nassbaum, German ; Avellano, Spanish;) is an improved variety of the common hazel-nut of the woods of Europe, Corylus avellana, L.) The fruit is three or four times as large as that of our common hazelnut, and from its size and excellent flavour is admired for the dessert. The old Spanish filbert common in many of our gar. dens, is a worthless, nearly barren variety, but we have found the better English sorts productive and excellent in this climate, and at least a few plants of them, should have a place in all our gardens. They are generally raised from layers, made in the spring, but they may also be grafted readily on the commor. hazel-nut, or the Spanish nut. When planted out they should not be permitted to sucker, and should be kept in the form of bushes with low heads, branching out about two feet from the ground, and they should be annually pruned somewhat like the gooseberry, so as to preserve a rather thin, open headshortening back the extremities of the young shoots one half, every spring.

The following are the best filberts known.

1. Cosford. (Thomp. P. Mag.) Nut large, oblong; husk hairy ; shell remarkably thin, and kernel of excellent flavour. A gond bearer.

2. Frizzled. (Thomp. P. Mag.) Easily known by its hand 
some, deeply cut husk. Nut of medium size, oval, compressed; husk hairy; shell thick; kernel sweet and good.

3. Nortianiptonsuire Prglific. ('Thomp.) Ripens early. Nut of medium size, oblong, husk hairy ; shell thick.

4. Red Filbert. Easily known from other sorts, by the crimson skin of the kernel. Fruit of medium size, cvate. Shell thick. Kernel with a peculiar, excellent flavour.

5. White Filbert. (Thomp. Lind.) Resembles the last, but with a light yellow or white skin. The tree is also quite bushy. Nuts ovate. Husk long and tubular.

The English generally call those varieties with long husks, filberts, (full-beards,) and those with short husks, simply unis.

The Chestnut, (Castanea vesca, W.; Chatagnier, of the French ; Castainenbaum, German ; Castagno, Italian ;) is one of our loftiest forest trees, common in most parts of the United States and Europe, and bearing excellent nuts. The foreien variety best known in this country, is the Spanish Chestnut, with fruit nearly as large as that of the Horse-Chestnut, and which is excellelit when boiled or roasted. It thrives very well heie, but is not quite hardy to the north or east of this. One or two English varieties have been produced, of considerable excellence, among which, the Downton is considered the best. The French cultivate a dozen or more varieties of greater or less excellence, but though some of them have been introduced, we have not yet fairly tested them in this country.

The Chrvquapin, or Dwarf Chestnut, common in some parts of the mid lle and southern states, is a diwarf species of the chestnut, usually growing not more than six to ten feet high, and bearing fruil of half the size as the common shestnut, with the same flavour. It is worth a place in a small fruit garden, as a curiosity.

All the chestnuts are very easily cultivated in ary gnod, light soil, and may be propagated by grafting, anc' by sowing the seeds.

\section{CHAPTER XX.}

\section{THE PLUM.}

Prunus domestica, L. 1hosacece, of botanists.
Prunter, of the French; Pjhumsnbaim. Girman; Prugno, Italiar: Cirucio,
Spanish.

THE original parent of most of the cultivated plurns of out gardens is a native of Asia and the snuthern parts of Eurone 
but it has become nuturalized in this country, anci in many parts of it is produced in the greatest abundance.* 'i'hat the soil and climate of the middle states are admirably suited to this fruit is sufficiently proved by the almost spontaneous production of such varieties as the IV ashington, Jefferson, Lawrence's $\mathrm{Fa}$ vourite, etc.; sorts which equal or surpass in beauty or flavour the most celehrated plums of France or England.

Usıs. The finer kinds of plums are beautiful dessert fruits, of rich and luscious flavour. They are not, perhaps, so entirely wholesome as the peach or the pear, as, from their somewhat cloying and flatulent nature, unless when very perfectly ripe, they are more likely to disagrce with weak stomachs.

For the kitchen the plum is also very highly estecmed, being prized for tarts, pies, sweetmeats, etc. In the south of France an excellent spirit is made from this fruit fermented with honey. In the western part of this state, where they are very abundant, they are halved, stoned, and dried in the sun or ovens, in large quantities, and are then rxcellent for winter use. For eating, the plum should be allowed to hang on the tree till perfectly ripe, and the fruit will always be finer in proportion as the tree has a more sunny exposure. The size and quality of the fruit is always greatly improved by thinning the fruit when it is half grown. Indeed to prevent rotting and to have this fruit in its highest perfection, no two plums should be allowed to touch each other while growing, and those who are willing to take this pains, are amply repaid by the superior quality of the fruit.

One of the most important forms of the plum in commerce is that of prunes, as they are exported from France to every part of the world. We quote the following interesting account of the best mode of preparing prunes from the Arboretum Brit. tanicum.

The best prunes are made near Tours, of the St. Catherine

* There are tliree species of wild plum indigenous to this country-of tolerablo flavour, but seldom cultivated in our gardens. The $y$ are the following.

1. The Curckasaw Plus. (Prumus Chicasa, Micliaux.) Fruit about three fourths of an inch in diameter, round, and red or yellowish red, of a pleasant, subacid favour, ripens pretty early. Skin thin. The branches are thurny, the head rather bushy, with narrow lanceolate, serrulate leaves, louking at a litile distance, scmewhat like those of a pench tree. It usually grows abuut 12 or 14 feet high, but on the Prairies of Arkansas it is only 3 or 4 feet high. and in this form it is atso common in 'Texas. The Dwar F Texas PLum described by Kenrick is only this species. It is quite ornamental.

11. WILD RED oR Yellow P'LUM. (P. americana, Marshall.) Fruit ronndishoral, skin thick, reddisls orange, with a juicy, yellow, sub-acid pulp. The lenves ar ovate, coarsely serrate, and the old branches rourh and sumewhat thorny. Grows in hedgres, and by the banks of streams, from Canada to the Gulf of Mex1co 'Tree from 10 to 15 feet high. Fruit ripens in July and August.

III. Tlie Be.sci Plua, or Sind Pium. (P. marutima, Wang.) A low shrub,

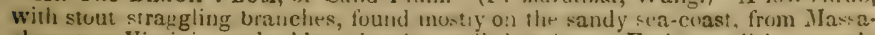
thusetts to Virginia, and seldum ripening well elsewlere. Fruit roundish, scarce!y an inch in diameter, red or purple, covered with a bloom; pleasant, but some what rstringent. I,eaves oval, finely serrate. 
plum and the prune d'Agen; and the best French plumis (so. called in England,) are made in Provence, of the Perdrigon blanc, the Brignole, and the prune d'Ast ; the Provence plums being most fleshy, and having always most blnom. Both kinds are, however, made of these and other kinds of plums, in various parts of France. The plums are gathered when just ripe enough to fall from the trees on their being slightly shaken. They are then laid, separately, on frames, or sieves, made of wicker-work or laths, and exposed for several days to the sun, till they become as soft as ripe medlars. When this is the case, they are put into a spent oven, shut quite close, and left there for twenty-four hours; they are then taken out, and the oven being slightly reheated, they are put in again when it is rather warmer than it was before. The next day they are again taken out, and turned by slightly shaking the sieves. The oven is heated again, and they are put in a third time, when the oven is one-fourth degree hotter than it was the second time. After remaining twenty four hours, they are taken out, and left to get quite cold. They are then rounded, an operation which is performed by turning the stone in the plum without breaking the skin, and pressing the two ends together between the thumb and finger. 'They are then again put upon the sieves, which are placed in an oven, from which the bread has been just drawn. The door of the oven is closed, and the crevices are stopped round it with clay or dry grass. An hour afterwards, the plums are taken out, and the oven is again shut with a cup of water in it, for about two hours. When the water is so warm as just to be able to bear the finger in it, the prunes are again placed in the oven, and left there for twenty-four hours, when the operation is finished, and they are put loosely into small, long, and rather deep boxes, for sale. The common sorts are gathered by shaking the trees; but the finer kinds, for making French plums, must be gathered in the morning, before the rising of the sun, by taking hold of the stalk, between the thumb and finger, without touching the fruit, and laid gently on a bed of vine-leaves in a basket. When the baskets are filled, without the plums touching each other, they are removed to the fruit room, where they are left for two or three days exposed to the sun and air; after which, the same process is employed as for the others; and in this way the delicate bloom is retained on the fruit, even when quite dry.

Propagation and culture. The plum is usually propagated in this country by sowing the seeds of any common free growing variety, (avoiding the damsons which are not readily worker.) and budding them when two years old, with the finer sorts. The stones should be planted as soon as gathered, in broad drills, (as in planting peas,) but about an inch and a half deep. In good soil the seedlings will reach eighteen inches or two feet 
?. reight, the next season, and in the autumn or the ensuing pring, they may be taken from the seed beds, their tap roots educed, uud all that are of suitable size, planted at once in the tursery rows, the smaller ones being thickly bedded until after inother season's growth.

The stocks planted out in the nursery will, ordinarily, be ready for working about the ensuing midsummer, and, as the plum is quite diflicult to bud in this dry climate, if the exact season is not chosen, the budder must watch the condition of the trees, and insert his buds as early as they are sufficiently firm,-say, In this neighbourhood, about the 10th of July. Insert the buds, if possible, on the north side of the stock, that being more pro. lected from the sun, and tie the bandage rather more tightly than for other trees.

The English propagate very largely by layers three varieties of the common plum-the Muscle, the Brussels and the Pear Plum, which are almost exclusively employed for stocks with them. But we have not found these stocks superiour to the seedlings raised from our common plums, (the Blue Gage, Horse-plum, \&c.,) so abundant in all our gardens. For dwarf. ing, the seedlings of the Mirabelle are chiefly employed.

Open standard culture, is the universal mode in America, as the plum is one of the hardiest of fruit trees. It requires little or no pruning, beyond that of thinning out a crowded head, or taking away decayed or broken branches, and this should be done before midsummer, to prevent the flow of gum. Old trees that have become barren, may be renovated by heading thern in pretty severely, covering the wounds with our solution of gum shellac, and giving them a good top dressing at the roots.

SorL. The plum will grow vigorously in almost every part of this country, but it only bears its finest and most abundant crops in heavy loams, or in solls in which there is a considerable mixture of clay. In sandy soils, the tree blossoms and sets plentiful crops, but they are rarely perfected, falling a prey to the curculio, an insect that harbors in the soil, and seems ic find it difficult to penetrate or live in one of a heary texture, while a warm, light, sandy soil, is exceedingly favorable to its propagation. It is also, undoubtedly true, that a heavy soil is naturally the most favourable one. The surprising facility with which superior new varieties are raised merely by ordinary re. production from sced, in certain parts of the valley of the Hudson, as at Hudson, or near Albany, where the soil is quite clayey, and also the delicious flavour and great productiveness and health of the plum tree there almost without any care, while in adjacent districts of rich sandy land it is a very uncertain bearer, are very convincing proofs of the great importance of clayey soil for this fruit.

Where the whole soil of a place is light and sandy, we would 
recommend the employment of pure yellow loam or yellow clay, in the place of manure, when preparing the border or spaces for planting the plum. Very heavy clay, burned slowly by mixing it in large heaps with brush or faggots, is at once an admirable manure and alterative for such soils. Swamp muck is also one of the best substances, and especially that from salt watos marshes.

Common salt we have found one of the best fertilizers for the plum tree. It not only greatly promotes its health and luxuri. ance, but from the dislike which most insects have to this sub. stance, it drives away or destroys most of those to which the plum is liable. The most successful plum grower in our neigh. bourhood, applies, with the best results, half a peck of coarse salt to the surface of the ground under each bearing tree, annually, about the first of April.

INSECTS AND DISEASES. There are but tiwn drawbacks to the cultivation of the plum in the United States, but they are in some districts so great as almost to destroy the value of this tree. 'These are the curculio, and the knots.

The curculio, or plum-weevil, (Rhynchcenus Nemuphar, is the uncompromising foe of all smooth stone fruits. The cultivator of the Plum, the Nectarine, and the Apricot, in many parts of the country, after a llattering profusion of snowy blos. soms and an abundant promise in the thickly set young crops of fruit, has the frequent mortification of seeing nearly all, or indeed, often the whole crop, fall from the trees when half or two-thirds grown.

If he examines these falling fruits, he will perceive on the surface of each, not far from the stalk, a small semi-circular scar. This star is the crescent-shaped insignia of that little Turk, the curculio; an insect so small, as perhaps, to have es caped his observation for years, unless particularly drawn to it but which nevertheless appropriates to himself the whole firoduct of a tree, or an orchard of a thousand trees.

The habits of this curculio, or plum-weevil, are not yet fully and entirely ascertained. But careful observation has resultce in establishing the following points in its history.

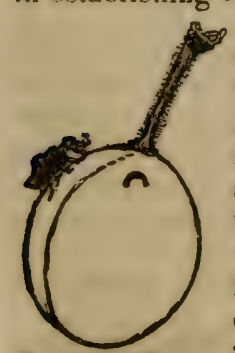

The plum-weevil is a small, dark brown beetle, with spots of white, yellow, and black. Its length is scarcely one-fifth of an inch. On its back are two black humps, and it is furnished with a pretty long, curved throat and snout, which, when it is at rest, is bent between the forelegs. It is also provided with two wings with which it flies through the air. How far this insect flies is yet a disputed point, sone cultivators affirming that it scarcely goes far. Fig. 101. The cur ther than a single tree, and others believing
outio, and its mark. that it fies over a whole neighbourhood. Our 
own olservation inclines us to the belief that this insect erm grates just in proportion as it finds in more or less abumdances the tender fruit for depositing its eggss. Very rarely do we see more than one puncture in a plum, and, if the insects are abun dant, the trees of a single spot will not atTord a sufficient number for the purpose; then there is little doubt (as we have seen them flying through the air,) that the insect flies farther in search of a larger supply. But usually, we think it rernains nearly in the same neighbourhood, or migrates but slowly.

A bout a week or two after the blossoms have fallen from the trees, if we examine the fruit of the plum in a district where this insect abounds, we shall find the small, newly formed fruit, beginning to be punctured by the proboscis of the plum-weevil. 'The insect is so small and shy, that unless we watch closely it is very likely to escape our notice. But if we strike or shake the tree suddenly, it will fall in considerable numbers on the ground, drawn up as if dead, and resembling a small raisin, or, perhaps more nearly, a ripe hemp seed. From the first of A pril until August, this insect may be found, though we think its depredations on fruit, and indeed its appearance in any quantity, is confined to the month of May in this climate. In places where it is very abundant, it also attacks to some extent the cherry, the peach, and even the apple.

Early in July the punctured plums begin to fall rapidly from the tree. The egr deposited in each, at first invisible, has become a white grub or larva, which slowly eats its way towards the stone or pit. As soon as it reaches this point, the fruit falls to the ground. Here, if left undisturbed, the grub soon finds its way into the soil.

There, according to most cultivators of fruit, and to our own observations, the grubs or larva remain till the ensuing spring, when in their perfect form they arain emerge as beetles and renew their ravages on the fruit. It is true that Harris, and some other naturalists, have proved that the insect does sometimes undergo its final transformation and emerge from the ground in twenty days, but we are inclined to the opinion that this only takes place with a small portion of the brool, which, perhaps, have penetrated but a very shor. distance below the surface of the soil. These making their appearance in mid. summer, and finding no young fruit, deposit their ergs in the young branches of trees, etc. But it is mueniable that the season of the plum-weevil is early spring, and that incst of the larvre which produce this annual swarm, remain in the soil during the whole period interven ing since the fall of the previous vear's fruit.

There are several modes of destroving this troublesome insect. Before detailing them, we will again allude to the fact, that we have never known an instance of its being troublesome 
in a heavy soil. Almost always the complaint comes from por tions of country where the soil is light and sandy. The explana. tion of this would seem to be that th: compe ct nature of a clayey soil is not favourable to the passage or life of this insect, while the warm and easily permeable surface of sandy land nurses every insect through its tender larræ state. Plum trees grow. ing in hard trodden court-yards, usually bear plentiful crops. Following these hints some persons have deterred the plumweevil by paving beneath the trees; and we have lately seen a most successful experiment which consisted in spreading be. neath the tree as far as the branches extended a mortar made of stiff clay about the thickness of two or three inches-which completely prevented the descent of the insect into the earth. This is quickly and easily applied, and may therefore be renewed every season until it is no longer found necessary.

The other modes of destroying the plum-weevil are the fol. lowing :-

1. Shaking the tree and killing the beetles. Watch the young fruit, and you will perceive when the inscet makes its appearunce, by its punctures upon them. Spread some sheets under the tree, and strike the trunk pretty sharply several times with a wooden mallet. The insects will quiclily fall, and should be killed immediately. This should be reprated daily for a week, or so long as the inscets continue to make their appearance. Repeated trials have proved, beyond question, that this lather tedious mode, is a very effectual one if persisted in.* Coops of chickens placed about under the trees at this season will assist in destroying the insects.

2. Guthering the fruit and destroying ihe larve. As the insect, in its larræ or grub form, is yet within the plums when they fall promaturely from the tree, it is a very obvious mode of extrorminating the next year's brood to gather these fallen fruits: taily, and feed them to swine, boil, or otherwise destroy them, In our own garden, where several years ago we suffered by the plum-weevil, we have found that this practice, pursued for a couple of seasons, has been pretty effectual. Others have re. ported less favisurably of it ; but this, we think, arose from their trying it too short a time, in a soil and ncighbourhood where the insect is very abundant, and where it conscquently had sought extensively other kinds of fruit besides the plum.

* Merely shating the tree is not sufficient. The following memorandum, as ad. ditional proof, we quote from the Genesee Farmer. "Under a tree in a remote part of the fruit garden, having spread the sheets, I made the following experiment. On shaking lhe tree wrill I canght five eurculios; on jarring it with the hand I caught twelve more; and on striking the tree with a stone, eight more dropped on the sheets. I was now convine: d that I had be en in error; and calling in assistance, and uving a hammer to jar the tree violently, we caught in less than an hour, more than two hundre 1 and sixty of these insects." We will arld to this, that to prevent injury to tise tree a large wooden mallet should be substituted for a hammer, and it is better if a thick layer of cloth is bound over its head. 
A more simple and easy way of covering the diffictilty, where there is a plum orchard or enclosure, is that of turning in swine and fowls during the whole season, when the stung plums are droppoing to the ground 'The fruit, and the insects contained in it, will thus be devoured torether. This is an excellent expe. dient for the farmer, who bestors his time grudgingly on the nares of the garden.

5. The use of salt. A good deal of attention has lately been drawn to the use of common salt, as a remedy for the curculio. Trials have been marle with this substance in various parts of the country, where scarcely a ripe plum was formerly obtained, with the most complete success. On the nther hand, some per. sous after testing it, have pronnunced it of no value. Our own experience is greatly in favour of its use. We believe that, properly applied, it is an effectual remedy against the curculio: while it also promotes the growth of the tree, and keeps the soil in that state most contrenial to its prorluetiveness. The fuilures that have arisen in its use, have, doubtless, grown out of an im. perfect application, either in regard to the quantity or the timn of applying it.

In the directions usually given, it seems only considered ne. cessary to apply salt, pretty plentifully, at any spason. If the soil be thoroughly saturated with salt, it is probable thit it would destroy insects therein, in any srage of their growth. But, though the plum tree seems fond of saliue matter, (and nne of the imst successful experimenters applied strong fish brine, at the rate of three or four pails full to a tree of $m$ derate size, it must be confessed this is a somewhat dangerous mole, as the roots are forced to receive a large supply of so [mwerful an agrent at once.

'Tlue best methor of applying salt against the plum-weevil is that of strewing it prenty thickly over the surface, when the punthred plums commence drophing. The surface of the ground shonid be made smooth and hard, and fine packing salt may then be evenly sprearlover it, as far as the branches extend, and about a fourth of an inch in rlepth. Should the weather be fine, this cont will last until the fruit infected has all fallen; stonuld it be dissolved or carried off by showers, it must be replaced directly. The larve or grubs of the weevil, in this most tender state, emerging from the plum to enter the ground, will fall a prey to the elfect of the salt before they are able to reach the snil. If this is carefully and generally practised, we have little cluubt of its finally ridding the cultivator of this troublesome enemy, even in the worst districts and soils.

The knols, or black gum. In some parts of the country this is a most iroublesome disease, and it has, in neighbourhoods where it. lias been suffered to take its course, even destroyed the who: race of plum trees. 
The knots is a disease attacking the bark and wook. Tia former at first becomes swollen, afterwards bursts, and, finally. assumes the apparance of lare, irmgular, black lumps, with a narl, cracked, uneven surface, quite dry within. 'The passage of the sap upwards, becomes stopped by the compression of the branch by the tumor, and, finally, the poison seems to dissemi nate itseif by the downward fow of the sap through the whole trunk, breaking out in various parts of it.

The sorts of plum most attacked by this disease, are those with purple fruit, and we have never known the green or yellow fruited varisties infected, until the other sorts had first become filled with the knots. The common horse plum, and damson, appar to be the first to fall a prey to it, and it is more difficult to eradicate it from them, than from most other sorts. 'The common Morella cherry is, also, very often injured by the same thisease in Pennsylvania.

'There is yet some donbt respecting the precise cause of these knotty excresconces, though there is every reason to think it is the work of an insect. Professor Peck and Dr. Harris believe that they are caused by the same curculio or plum-weevil that stings the fruit; the second brood of which, finding no fruit rearly, choose the branches of this tree and ne clerry. This olsservation would seem to be confirmed by the fact that the grubs or larve of the plum-weevil are frequenty ticund in these warts, and that the beetles have been seen stining the branches.

On the other hand, the following facts are wort'ny of attention. First, in some parts of the country, where the curculio has been troublesome for many years, the knots have never been known. Secondly, in many cases, the knots have been abun. dant on plum trees, when the fruit was entirely fair and uninjured by the curculio, even upon the same branches.

These ficts seem so irreconcilable with the opinion that the curculio produces both these effects, that we rather incline at present to the belief, that though the curculio deposits its engs in the fumors on the branches while they are yet soft and tender, yet it is not to the curculio, but to some other insect or cause, that we owe this unsightly disease.

Practically, however, this is of little account. The experience of many persons, besides ourselves, has proved, most satisfactorily, that it is easy to extirpate this malady, if it is taken in season, and unremittingly pursued. As early as possible in the spring, the whole of the infected trees should be cxamined, and every branch and twig that shows a tumor, should be cut off, and inmediately burned. Whatever may be the insect, we thus destroy it, and, as experience has taught us that the malarly spreads rapilly, we will thus effectually prevent its increase. If the trees are considerably attacked by it, it will probably be necessary to go over them again, about the middle of May, 
Dut, usually, once a year will be sufficient. If any of the trees are very much covered with these knots, it is better to head back the shoots severely, or dig them up and burn them outright, and it will be necessary to prevail on your neighbours, if they are near ones, to enter into the plan, or your own labors will be of little value. Pursue this simple and straightforward practice, for two or three seasons, (covering any large wounds made, with the solution of gum shellac,) and the knots will be found to disappear; the curculio to the contrary notwithstanding.

VARIETIES. 'There are now a pretty large number of fine plums, and some most important additions have been made by the secdlings raised in this country. The Green Gage still stands at the head of the list for high flavour, though several other sorts are nearly or quite equal to it. The Washington, the Jefferson and the Columbia, are among the largest and most beautiful; and Coe's Golden Drop and Roe's Autumn Gage, are very desirable for their late maturity.

In describing plums, the surface of the young wood, when just ripened, is an important character; as it is smooth, in some varieties. and downy, or covered with soft hairs, in others. In some varieties, the flesh parts from the stone, while in others, it ad. heres. And, finally, the depressed line or channel which runs down one side of the exterior surface of the plum, is called the suture, and the prominence or absence of this feature enables us to distinguish many kinds at first sight.

Class I. Green, White, or Yellow Plums.

\section{Autumn Gage.}

Roe's Autumn Gage.

A new plum, raised by $\mathrm{Wm}$. Roe, Esq., of Newburgh, of good quality, a very abundant bearer, and so late in its maturity; as to be valuable. The tree forms a spreading head, with regular, pointed, ovate leaves; the branches drooping with the weight of the fruit, which is in perfection about the middle of Sep. tember.

Branches smooth. Fruit medium size, oval, rather broadest towards the stall. Stalk three-fourths of an inch long, inserted without any de.

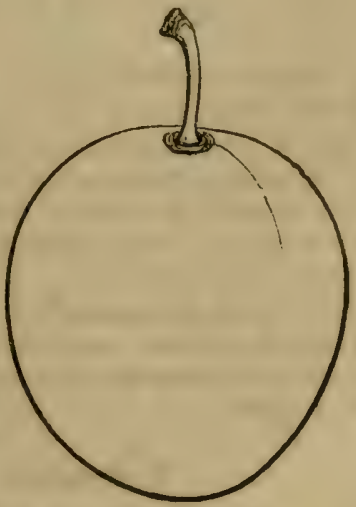

Fig. 102 Autumn Gaga 
pression. Skin pale yellow, covered with thin whitısh bloom. Flesh greenish yellow, scparating from the stone; juicy, sweet, and of delicate, pleasant flavour. Stone long, compressed, point. ed at both ends.

2. Apricor. Lind. Miller.

Apricot Plum of Tours.

Abricoteé de 'I'ours.

Abricotee.

$\grave{D}$ ih.

Yellow Apricot.

Branches quite downy, neariy white. Fruit above medium size, roundish, with a deep suture or furrow. Stalk very short, seldum half an inch long. Skin yellow, dotted and tinged with red on the sunny side, covered with a white bloom. Flesh yellow, rather firm, separates from the stone; slightly bitter, unti! fully ripe, when it is melting, juicy, and high flavoured. Ripe the middle of August.

This is the true old Apricot plum of Duhamel. The Apricot plum of Thomson is an inferiour, clingstone, oval fruit, with smooth branches, ) fit only for cooking.

\section{Byfield. Man.}

This plum, not having yet borne fruit with us, we can only give its character from the mss. of Mr. Manning.

Branches smooth. Fruit small, round; suture a mere line. Stalk half an inch iong, set in an even basin. Skin light vel. low, with red spots around the stem. Flesh yellow, of good flavour, adheres to the stone, which is thick. Middle in last $0^{\circ}$ August.

\section{BUEL'S FAVUURITE.}

An excellent new plum, raised by that successful growor Isaac Ienniston, of Albany, and named after his friend, the dis. +inguished agriculturist, Judge Buel.

Branches smooth, reddish. Fruit pretty large, ovate, broadest towards the stalk. Suture quite distinct for half the circumference. Stalk nearly three quarters of an inch long, rather stout, slightly inserted. Skin pale green, thickly sprinkled with lighter dots, and speckled with a little red next the stalk. Flesh greenish-yellow, rather firm, juicy, and quite rich and high flavourtd, acheres to the stone, which is long and pointed. Last of August.

5. Bivghan. §Man. Ken. Thomp.

A rative fruit, originally from Pennsylvania, and named afto. 
the Bingham family, but better known now near Boston, where it is very popular.

Fruit large, handsome, productive, and excellent. Branches downy. Fruit an inch and three fourths long, oval, rather widest towards the stalk. Skin deep yellow, somewhat spotted with rich red on the sunny side. Stalk slightly inserted. Flesh yellow: adhering to the stone, juicy, and of rich and delicious flavour. Last of August and first of September.

\section{(i. Blfecker's Gage. $\S$ Man.}

\section{German Gage.}

A fruit of the first quality, and the most prpular plum in the northern and western portion of this state, being not only excellent, but remarkably hardy, and a good and regular learer. It was raised by the late Mrs. Bleecker, of Albanv, about $3 \mathrm{C}$ years ago, from a prune pit given her by the Rev. Mr. Dull, of

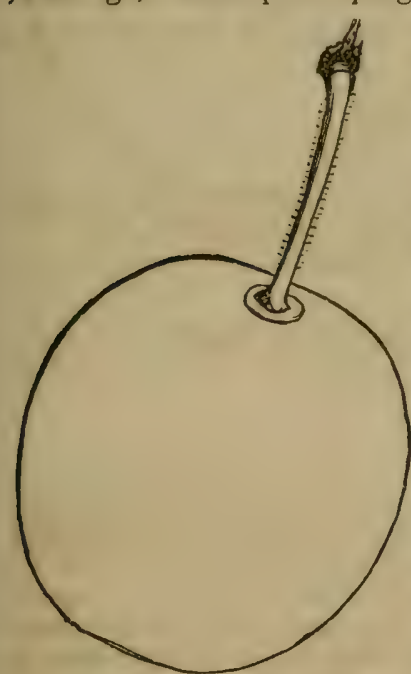

Fig. 103. Bleecker's Gage.

Kingston, N. Y., which he receiv. ed from Germany. The origina! tree still stands in her garder.

It ripens the last of August: from a week to two weeks lates than our Yellow Gage. Branches downy. Fruit of niedium size, roundish-oval, very regular. Suture scarcely perceptible. Stalk quite long, an inch or more, straight and pretty stout, downy, slightly inserted. Skin yellow, with numerous imbedded whito specks, and a thin white bloom. Flesh yellow, rich, sweet, and luscious in flavour. Separates almost entirely from the stone, which is pointed at both ends. Leaves dark green. Easily dis. tinguished from Yellow Gagre by its longer and stouter stalk.

7. Coe's Golden Drnp. Thomp. Lind. P. Mag.

Bury Seedling.

Coe's Imperial.

New Golden Drop.

Fair's Golden Drop.

Gulden Gage.

Coe's Golden Irop is worthy of its name, being the largest, most beautiful, and delicious of late plums. It succeeds admi. 
rably in the middle states, ripening from the middle to the last of September. It - bears abundantly, keeps well, and frequensly grows larger than the Magnum Bonum. No garden is complete with. out it. It is an English variety, raised by a market gardener, in Suffolk, whose name it bears.

Branches smooth. Fruit of the largest size, oval, with a well marked suture, on one side of which it is a little more swollen than the other, the outline narrowing towards the stalik. Skin light yellow, with a number of rich, dark red spots on the sunny side. Stalk nearly an inch long, rather stiff, set on the end of the fruits. Flesh yellow, rather firm, athering closely to the stone, which is quite pointed. Flavour rich, sweet, and delicious.

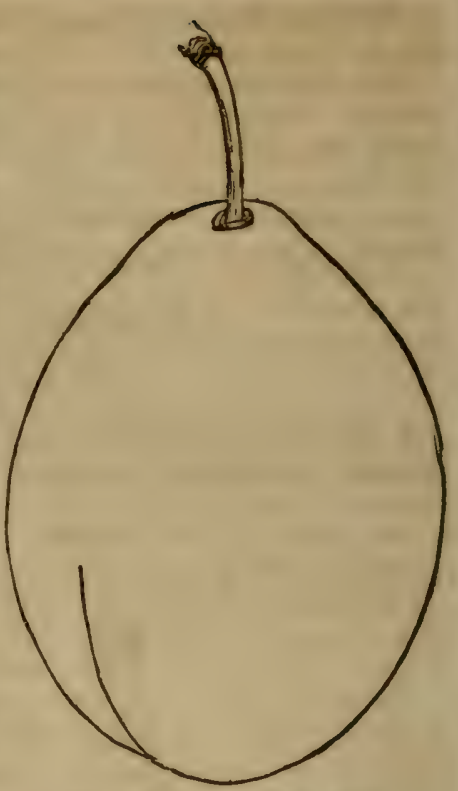

Fig. 104. Coe's Golden Drop.

8. DrAP D'OR. Thomp. Lind. Lang.

Mirabelle Double. Duh.

Mirabelle Grusse.

lellow Perdrigon.

The Mrap d'Or, or Cloth of Gold, Plum is about the size and figure of the (ircen Gage, but of a fine golden yellow and ripens a week earlier.

Branches slightly downy. Fruit below medium size, round, with an indistinct suture and a dimpled or pitted apex. Stalk slender, half an inch long. Skin rich bright yellow, with a few crimson suecks when fully exposed. Flesh yellow, sugary and rich, but sometimes a little dry; separates freely from the stone. Early in August.

\section{Downton Inperatrice. Thomp. Lind.}

A hybrid, raised by $\mathrm{Mr}$. Knight, from the White Magnum Bonum, fertilized by the Blue Imperatrice. A strong, upright growing tree, and a brisk, sprightly flavoured fruit. Ripens late, and is valuable for preserving. 
Branches long, smooth. Fruit of medium size, oval, narrow. ing a little to the stalk. Skin pale yellow, quite thin. Flesh yellow, melting and sweet when fully ripe, with a little acidity before; adhering to the stone. Ripens last of September, and hangs some time on the tree.

\section{Denniston's Albany Beauty.}

A good variety. Branches slightly downy. Fruit rather be. low medium size, roundish-oval, with an obscure suture. Skin pale whitish-green, marked with numerous small purplish dots, and covered with a thin bloom. Stalk an inch or more long, slenter, very slightly inserted. Flesh yellow, moderately juicy, rich, and sweet, separates from the stone, which is small and pointed. : Ripe 24th of August.

\section{Denniston's Superb. $\S$}

An excellent seedling, from Mr. Denniston's famous plum or. chard, near Albany, N. Y., of the Green Gage family, a third larger than the latter variety, and nearly as rich in fiavour.

Branches downy. Fruit round, a little flattened, and having a distinct suture, often extending quite round the fruit. Skin pale yellowish-green, marked with a few large purple blotches and dots, and overspread with a thin bloom. Stalk rough, three fourths of an inch long, set in a cavity of moderate size. Flesh very thick, (the stone being small,) moderately juicy, with a rich vinous flavour. Stone parts readily, and is roundish and thick. Middle and last of August.

\section{Dana's Yellow Gage. Man.}

A New-England variety, raised by the Rev. Mr. Dana, of Ipswich, Mass. It is a very haruy and healthy tree, and bears abundantly. The flavour good, and rather more sprightly than our common Yellow Gage, though not so luscious.

Fruit of medium size, oval, pale yellow, with a very thin bloom, the skin clouded like that of the Imperial Gage. Flesh adheres to the stone, juicy, sweet, with a lively, pcculiar flavour. Last of August and first of September.

\section{Emerald Drop.}

A variety produced in our own gardens, from a stone of the Washington. It is a rich juicy plum, nearly first rate, and a most abundant bearer. 


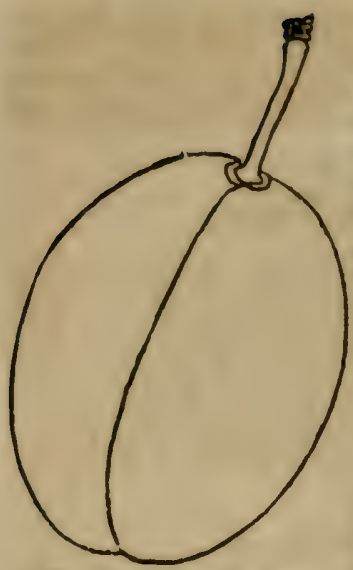

Fig. 105. Emerald Drop.

Branches long and smooth. Fruit of medium size, long-oval. Suture strongly marked, and the fruit larger on one of its sides. Skin pale yel. lowish-green, sometimes dull green only, in the shade. Stalk three. fourths of an inch long, inserted with scarcely any depression. Flesh greenish-yellow, very juicy and rich, ad. heres somewhat to the stone, which is long and pointed. Last of August.

\section{Ghiston's Early.}

A large and handsome (native ?) fruit, resembling, a good deal, the Magnum Bonum or Yellow Egg Plum, but a freestone. Branches smooth, short-jointed. Fruit large, oval, skin clear yellow, with a light bloom. Flesh yellow, separates from the stone, of pleasant flavour. Middle of August.

15. Green Gage. § Lang. Lind. Thomp.

Bruyn Gage.

Bradfori Gage,

Schuyler's Gage ?

Wilmot's Green Gage.

$\left.\begin{array}{l}\longrightarrow \text { New (ireen Gage. } \\ \begin{array}{l}\text { Isleworth Green Gage. } \\ \text { Burgnon Gage. }\end{array}\end{array}\right\} \begin{aligned} & \text { of some } \\ & \text { English } \\ & \text { gardens. }\end{aligned}$
Reine Claude.

Grosse Reine Claude.

Grosse Rrine.

Damas Vert.

Sucrin Vert.

Vert Bonne.

Abricnt Vert.

Dauphine. of varous

Fren h gardens.

The Green Gage is universally admitted to hold the first rank in flavour among all plumis, and is every where highly esteemed. In France, this variety is gerierally known as the Reine Ciaude, having, it is said, been introduced into that country by Queen Claude, wife of Francis I. During the last century, an English family by the name of Grage, obtained a number of fruit trees from the morks of Charteuse, near Paris. Among them was a tree of this plum, which, having lost its name, was called by the gardener the Green Gage. It is pronounced, by Lindley, the best flum in England, and we must ad. mit that we have no superiour to it here.

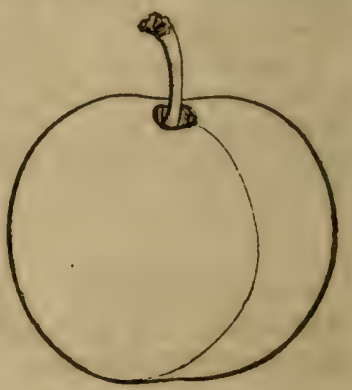

Fig. 106. Grem Gage. 
The Green Gage is a very short-jointed, slow growing tree, of spreading and rather dwarfish habit. It is an abundant and pretty regular bearer, though the fruit is a little liable to crack upon the tree in wet seasons.

Branches smooth. Buds with large shoulders. Fruit round, ratiser small, seldom of medium size. Suture faintly marked, but extending from the stalk to the apex. Skin green, or yellowish-green at full maturity, when it is often a little dottel or marbled with red. Stalk half to three-fourths of an inch long, slender, very slightly inserted. Flesh pale green, exceedingly melting and juicy, and usually separates freely from the stone. Flavour, at once, sprightly and very luscious. Ripe about the middle of August.

There are several seedling varieties of this plum in various parts of this country-but none superiour or scarcely equal to the old. That known as the Bruyn Gave, which has been disseminated from the garden of A. Bruyn, Esq., of Kingston, N.Y., is only the true Reine Claude, brought by Chancellor Livingston from France.

\section{Hudson Gage.}

A new early plum, which promises to rank among the first, of the season at which it ripens. It is one of the seedlings which Mr. Lawrence, of the city of Hudson, (see Lawrence's Favour. ite,) has had the good fortune to give to the public within a few years. It has some affinity to the Imperial Gage, but ripens three weeks earlier.

Branches slightly downy. Fruit of medium size, oval, a little enlarged on one side of the obscure suture. Skin yellow, clouded with green streaks under the skin, and covered with a thin white bloom. Stalk short, little more than half an inch long, inserted in a moderate hollow. Flesh greenish, very juicy and melting, with a rich, sprightly, excellent flavour. It separates from the stone, (adhering very slightly, which is quite small. First week in August, two weeks before the Washington.

\section{Hulings' Superb. $\oint$ Pom. Man.}

\section{Keyser's Plum.}

A noble plum, of the largest size, raised from seed by $\mathrm{Mr}$. Keyser, of Pennsylvania, but first made known to cultivators by Dr. Wm. G. Hulings, of the same state. It is as large as the Vashingtou, frequently measuring six inches in circumference, or two and a furth in diameter, the longest way. In flavour, it is more sprightly than that plum, having its siveetness relieved by a little aciclity, and is scarcely inferiour to the Green Gage. It is productive, and in every way, a fruit of great merit. 
The tree is remarkable for its vigorous growin, its stout, blunt shoots with large shouldered buds, and its fine luxuriant foliage. It is a good bearer, especially in strong soils.

Branches downy. Fruit very large, globular, a little incli. ning to ovate, with a distinct suture. Stalk three fourths of an inch long, set in a shallow depression. Skin dull, greenish. yellow. Flesh pale greenish-yellow, rather firm, with a rich, brisk, excellent flavour; partly clinging to the stone. Ridens middle of August.

\section{Inpertal Ottonan. Thomp.}

A very neat, early plum, of good flavour, and a prolific bearer. It has the reputation of having been brought from Turkey, but it is uncertain whether this is correct.

Branches slightly downy. Fruit scarcely below medium size, roundish, between Green Gage and the American Yellow Gage in appearance, and having a suture on one side, from the stalk half way down. Stalk downy, slender, curved, three-fourths of an inch long, inserted in a very slight cavity. Skin dull yellow, clouded with darker streaks, and covered with a thin bloom. It adheres considerably to the stone, which is pointed at both ends. The flesh is juicy, sweet, melting, and of very good flavour. It ripens the last of July, or four or five days before the American Yellow Gage.

\section{Imperial Gage. $§$ Pom. Man. Ken.}

Flushing Gage. Thomp. Floy.

Prince's Imperial Gage.

White Gage, of Boston.

Superiour Green Grage.

The Imperial Gage has long enjoyed the reputation of one of the most excellent and produclive of plums. It was raised at Prince's Nursery, Flushing, N. Y.; from the seed of the Green Gage, and the fact of the fruit of a single tree near Boston having produced fruit to the value of near fifty dollars, annually, has often been repeated as a proof of the profit of its cultivation for market. It should be remarked, however, as an exception to the general rule, that it is peculiarly fitted for dry, light soils, where many sorts drop their fruit, and

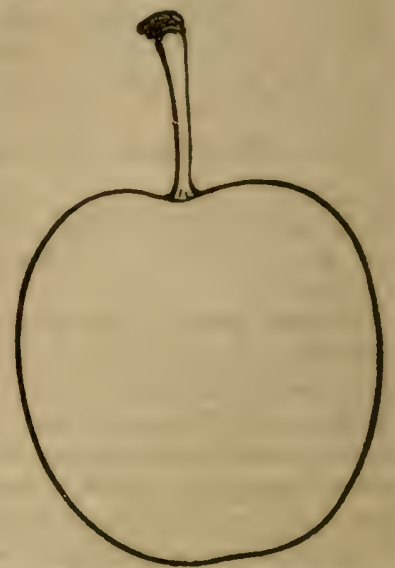

Fig. 107. Imperial Gage. 
that in rich heavy soils, like those of Albany, the fruit is ofter. insipid.

The tree grows freely and rises rapidly, and has long dark shoots and leaves, slightly downy. Fruit rather above medium size, oval, with a distinct suture. Stalk nearly an inch long, slightly hairy, and pretty stout, inserted in an even hollow. Skin pale green, until fully ripe, when it is tinged with yellow, show. ing a peculiar marbling of dull green stripes, and covered with copious white bloom. Flesh greenish, very juicy, melting, and rich, with a very sprightly, agreeable flavour. In some situ. ations it adheres to the stone, but it generally separates pretty freely. The latter is oval, and pointed at both enris. It is a great and regular bearer, and the fruit is therefore improved by thinning, when half grown. Ripens about the 1st of September, or a week later than the Washington.

\section{JaUne Hative. Thomp: Lind. O. Dul.}

Early Yellow.

Catalonian.

White Primordian.

Amber Primurdian.
Jaune de Catalogne.

Prune de St. Barnabe.

D'A voine.

The earliest of plums, which is its chief reconmendation. It is a very old variety from Catalonia, and the south of France, and has been in cultivation more than two hundred years. It is a pretty little fruit, and is worthy of a place in the garden of the amateur. The tree has lonir, slender, downy branches.

Fruit small, oval, or obovate, with a shallow suture on one side. Stalk slender, half an inch long. Skin pale yellow, thinly coated with bloom. Flesh yellow, tolerably juicy, and melting, of sweet and pleasant flavour; separates from the stone. Ripens from the 10 th to the middle of July.

\section{JEFFERSON. $\oint$}

If we were asked which we think the most desirable and beautiful of all dessert plums, we should undoubtedly give the narne of this new variety. When fully ripe, it is nearly, shall we not say quite-equal in flavour to the Green Gage, that unsurpassable standard of flavour. But when we contrast the small and rather insignificant appearance of the Green Gage, with the unusual size and beauty of the Jefferson, we must admit that it takes the very first rank. As large as the Washington, it is more richly and deeply coloured, being dark yellow, uniformly and handsomely marked with a fine ruddy cheek. It is about ten days or a fortnight later than the Washington, ripen. ing the last of August, when it has the rare quality of hanging long on the tree, gradually improving in flavour. It doss not, 
like many sorts, appear liable to the attacks of wasps, whiob destroy so many of the light coloured plums as soon as they arrive at maturity.

We received the Jefferson Plum a few years ago, from the late Judge Buel, by whom it was raised and named. The original tree is still, we believe, growing in his garden near Albany. It is a good and regular bearer, and the crop is very handsome upon the tree.

Branches slightly downy, leaves oval, flat. Fruit large, oval, slightly nar. rowed on one side, towards the stalk. Skin golden yul. low, with a beautiful purplish-red cheek, and covered with a thin white bloom. Stalk an inch long, pretty stout, very slightly inserted.

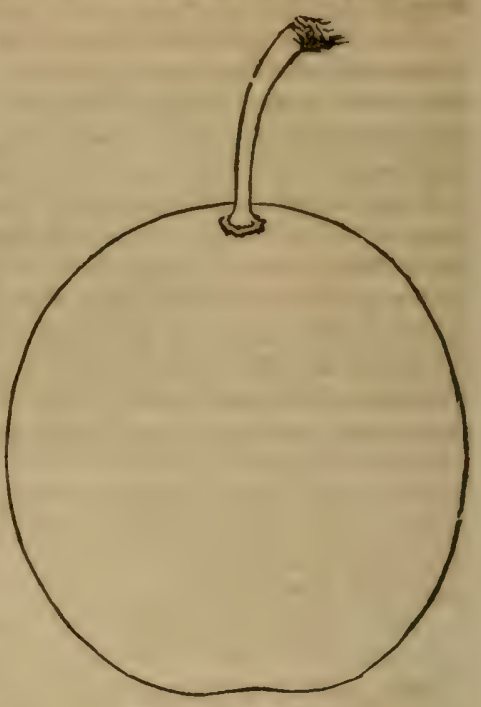

Fig. 103. Jefferson.

Suture indistinct. Flesh deep orange, (like that of an Apricot,) parts freely, and almost entirely from the stone, which is long and pointed; very rich, juicy, luscious and high flavoured. Hangs a fortnight on the tree.

\section{Lawrence's Favourite. $\S$}

\section{Lawrence's Gage.}

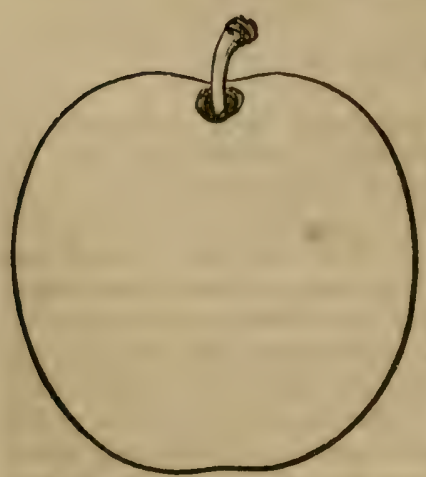

Fg. 109. Laverence's Favourite.

Lawrence's Favourite is a fruit of high merit, raised by Mr. L. U. Lawrence, of Ilud. son, N. Y., from a seed of the Green Gage. The exceeding congeniality to the plum of the soil of Hudson, which is almozi a stiff clay, is fully attested by the seemingly spontaneous pro. duction of such varieties as this, the Columbia and several others.

The general appearance of the fruit is like that of its parent, except that it is two or three times as large. It hangs well on the trec, and its remarkitble sizc, flavour and productivenese, 
will soon give it a place in every garden, and we think it de. serving our highest commendation. Specimen trees only 8 feet bigh, have borne abundantly with us this season.

Law rence's Favourite forms an upright tree of tirifty growth, with dark green leaves, (which are rather below the medium size, and upright growing short-jointed shoots. Young branch. es, downy.

Fruit large, heavy, roundish, a little flattened at either end. Skin dull yellowish-green, clouded with streaks of a darket shade beneath, and covered with a light bluish-green bloom. The upper part of the fruit, when fully ripe, is covered with a peculiar brozonsh net-work, and a few reddish dots. Stalk short, only half an inch long, slender, inserted in a narrow cavity. Flesh greenish, resembling that of the Green Gage, remarkably juicy, and melting, perhaps scarcely so rich as the latter, but with a very rich, sprightly, vinous favour, and one of the most delicious of plums. Stone five eighths of an inch long, fiat. tened; the flesh sometimes adheres a little, when not fully ripe, but then separates freely. Ripens at the middle of $\mathrm{A} \dot{u}$. gust.

23. Luconibe's Nonesucrr. Thomp. Lind. P. Mag.

An Fnglish plum raised by Lucombe, of the Exeter Nursery It is a large, yellowish green clingstone fruit, of good quality, but, unless fully ripe, not very rich in flavour. Branches smooth.

Fruit above medium size, roundish, shaped and colnured much like the Green Gage, but much more distinctly streaked with yellow and orange, and covered with a whitish bloom. Suture broad. Stalk straight, three fourths of an inch long, set in a wide hollow. Flesh pretty firm, greenish, rich, sweet mingled with acid, adheres to the stone. Bears well, and ripeng about the middle of August.

\section{Large Green Drying. § Thomp.}

\section{Knight's Large Drying. Ken.}

A new late variety, raised, we believe, by Mr. Knight, and introduced here from the garden of the Horticultural Society, of London. It has produced fruit for the first time this scason, scarcely giving us an opportun ty of judging, but Mr. Thoinp. son, the head of the fruit department, in that garden, describes it as of the first quality, bearing "fruit as large as that of the Washington, which when perfectly ripened, is excecdingly rich." The tree is vigorous, and the branches are suroth; the fruit large, round, greenish-yellow, the finsh yellowish, moderately 
juicy, rich and excellent, adheres to the stone. Ripens about the mildle of September, and is a moderate bearer.

\section{Niulberry.}

Raised by Isaac Denniston, of Albany, and is likely to prove a desirable sort. 'The leaves are remarkably luxuriant, broad and crumpled. Fruit large, oval, somewhat narrowest towards the stalk. Skin pale, whitish-yeliow, sprinkled with white dots, and dusted with a pale bloom. Stalk an inch long, rather slender, very slightly inserted. Flesh greenish-yellow, juicy, sweet and good; adheres slightly to the stone. The latter is long and pointed. First of September.

\section{Mirabelle. Thomp. Lind. O. Duh. \\ Mirabelle Petite. \\ Mirabelle Jaune.}

A very pretty little fruit, exceedingly orna. mental on the tree, the branches of which are thickly sprinkled with its abundant crops. The tree is small in all its parts, and although the fruit has a tolerable flavour, yet from its size and high perfume, it is chiefly valued for preserving.

Branches downy. Fruit quite small, obovate, with a wel marked suture. Stalk half an inch long, slightly inserted. Skin of a beautiful yellow, a little spotted with red at maturity, and covered with a white bloom.

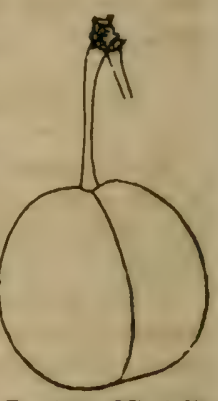
Flesh orange, sweet, and sprightly, becoming Fig. 110. Mirabelle dry when over-ripe, and separates from the stone. Ripens with the Green Gage.

\section{Orange. \\ Orange Gage, (of some.)*}

The Orange Plum is a new variety, which we have recent. Iy introduced, from the garden of Mr. Teller, of Rhinebeck, Dutchess co., N. Y. It is considerably disseminated about that locality, and undoubtedly oriminated there. It is only of second quality in flavour, but its extraordinary size, and showy appearance, as well as the abundance of its crops, will recom. mend it to all large planters of the plum. It is, perhaps, the largest of all plums, and has a pecuiliar bronze gold colour.

- There is a greas propensity for calling every plum of merit a Gage, in this part of the country. As this has no resemblance whatever to the originel type of this class, we drop that part of its nume. 
Branches stout and smooth. Fruit very large, oval, flattened at both ends. Skin bronze yellow, marked with roughish white dots, and cloured with

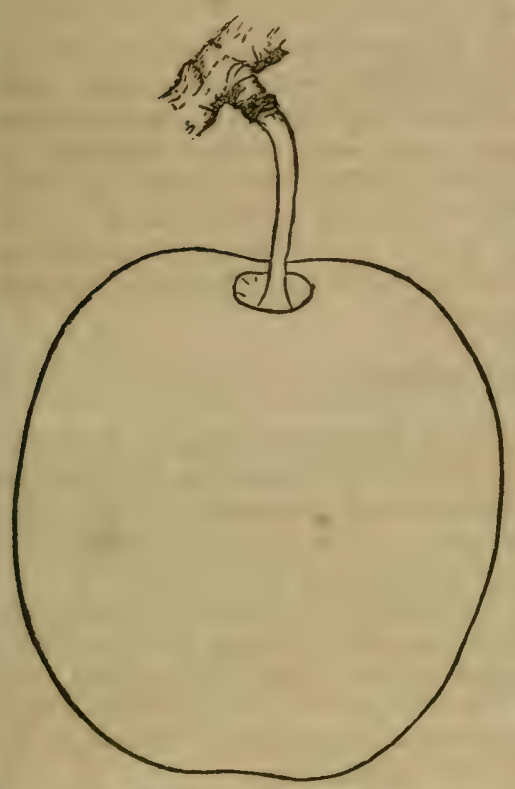

Fig. 111. Orange Plum. purplish red near the stalk. The latter is three-fourths of an inch long, rather rough, inserted in a narrow round cavity. Flesh deep yel. low, a little coarse grained, but with acid flavour when fully ripe. It ad. heres a little to the stone, which is much compressed and furrowed. Ripens the last of $\Lambda$ ugúst.

28. Saint Martin's QUfTSCHE. Thomp.

A very late variety of Prune, recently introdu. ced from Germany, and likely to take its place among the select sorts. Hardy and a good bearer.

Branches smooth. Fruit of medium size, ovate, or considerably broadest towards the stalk. Skin pale yellow, covered with a white bloom. Flesh yellowish, with a rich and excellent flavour, and separates readily from the stone. The tree is a good bearer, and the fruit hangs a long while on the tree, but we fear that to the northward of this it may not come to full maturity every season. Ripens the first of October, and will hang a month.

29. Sant Citherine: $\oint$ Thomp. Lind. O. Duh.

Among the fine old varieties of late plums, the St. Catherine is one of the most celebrated. In France it is raised in larure quantities, in some districts making the most de-

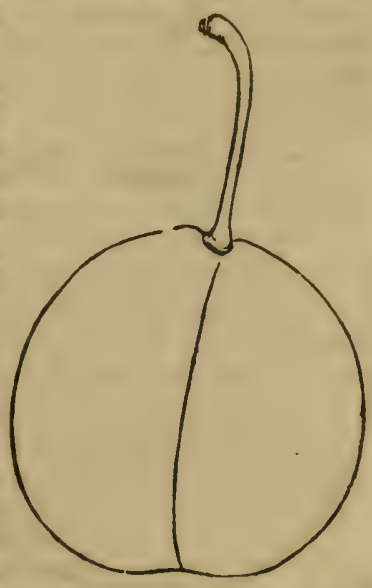

Fig. 112. St. Catherine. 
licate kind of prunes. It is also much esteemed for preserving,

- and is of excrllent quality for the dessert. It bears regularly: and abundantly in this part of the country, and deserves a place in every good garden.

Branches smoxth, upright, rather slender. Fruit of medium size, obovate, narrowing considerably towards the stalk, and having a strongly marked suture on one side. Stalk threefourths to an inch or more long, very slender, inserted in a slight cavity. Skin very pale yellow, oversprear with thin white bloom, and occasionally becoming a little reddish on the sunny side. Flesh yellow, juicy, rather firm, and adheres to the stone: in flavour it is sprightly, rich and perfumed. Ripera? the middle and last of September.

\section{Siamese.}

A curious growing varicty, as its name indicates, in pairs, attached on one side, and hanging by a common stalk. Nearly all the fruit on the tree exhibits this peculiarity, and grafts taken from it continue its habit. The original seedling tree stands in the garden of $\mathrm{Wm}$. Roe, Esq., of this place.

Branches long, s!ender, and smooth. Fruit mostly in pairs, distinct, but ciosely joined on one side, modium sized, obovate. Skin pale yellow, with a white bloom. Stalk rather long and slender, slightly inserted. Flesh yellow, juicy and sprightly, of second rate flavour, and adheres to the stone. Bears abun. dantly, and ripens about the 10 th of September.

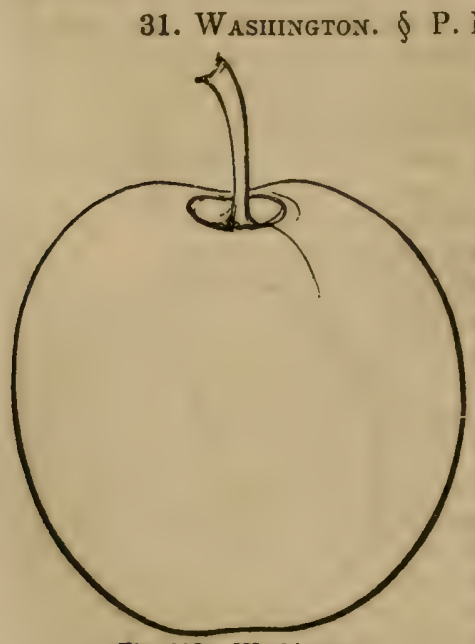

Fig. 113. Washington
Bolmar.

Bolmor's Washington.

New Washington. Franklin.

The Washington undoubt. edly stands higher in general estimation in this country, than any other plura. Alihough not equal to the Green Gage and two or three others, in high flavour, yet its great size, its beauty, and the vigour and hardiness of the tree, are qualities which have brought this noble fruit into inotice every where. 'The parent tree grew originally on De. lancey's farm, on the east side 
of the Buwery, New-York, but being grafted with anothet sort, escaped notice, until a sicker from it, planted by $\mathbf{M r}$. Bolmar,* a merchant in Chatham-strcet, came into bearing about the rear 1818, and attracted universal attention by the romarkable beauty and size of the fruit. In 18\%1, this sort was first sent to the Horticultural Society of London, by the late Dr. Hosack, and it now ranks as first in nearly all the European collections.

The Washington has remarkably large, broad, crumpled and glossy foliage, is a strong grower, and forms a handsome round head. Like several other varieties of plum, the fruit of this, especially in sandy soils, does not attain its full perfection until the tree has borne for several years. We have measured them very often six inches in circumference, and once froin $\mathrm{Mr}$. Bolmar's original tree, seven and a ruarter inches.

Wood light brown, downy. Fruit of the largest size, rounit. ish-oval, with an obscure suture, except near the stalk. Skin dull yellow, with faint marblings of green, but when well ripened, deep yellow, with a pale crimson blush or dots. Stalk scarcely three-fourths of an inch long, a little downy, set in a shallow, wide hollow. Flesh yellow, firm, very sweet and lus. cious, separating freely from the stone. Stone pointed at each end. Ripens from about the middle to the last of August.

32. White Inperatrice. Thoinp. Lind. P. Mag.

White Empress.

Imperatrice Blanche, o. Duh.

The White Imperatrice is but little known in this country. In the habit of the tree, appearance and flavour of the fruit, and season of maturity, it strongly resembles the St. Catherine, but is a freestone. It is not equal to the latter in flavour, though estecmed by some persons, neither does it hang well after ripening.

Branches smooth. Fruit of medium size, obovate, a little flattened at the ends, suture rather obscure. Skin bright yel. low, covered partially with a thin white bloom, and spotted with a little red. Stalk a little more than half an inch long, set in a narrow cavity. Flesh yellow, very juicy, crisp, sweet, and quite transparent in texture; separates freely from the stone, which is small and oblong. Ripe early in September.

* Which he purchased of a marliet woman 
33. White Magnun Bonunt. Thomp. Lind.

Yellow Magnum Bonum.

\section{Erg Plum.}

Yellow Egg.

White $\mathrm{Egg}$.

Wiuto Mugul.

Wen:wort?.

White Imperial.*

White Hollaru.

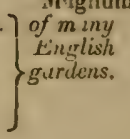

of $m$ iny

English

$\int$

The White Magnum Bonum, or Egg Plum, as it is almost universally known here, is a very popular fruit, chicfly on account of its large and splendid appearance, and a slight acidity, which renders it admirably fitted for making showy sweetmeats

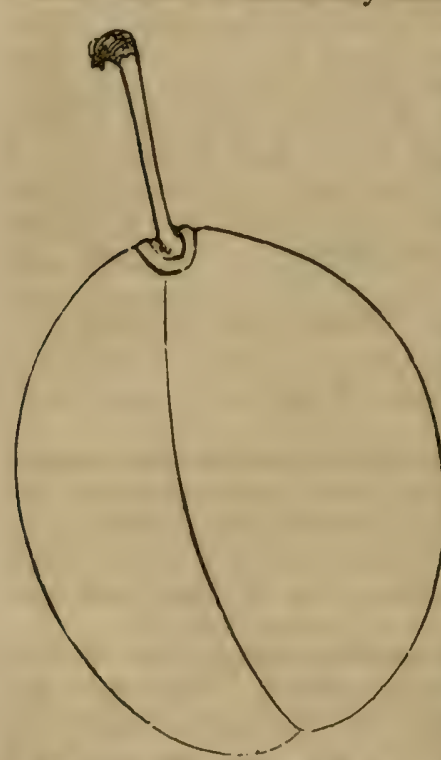

ol preserves. When it is raised in a fine warm situation, and is fully matured, it is pretty well flavoured, but ordinarily, it is considered coarse, and as be. longing to the kitchen, and not to the dessert.

Branches smooth, long. Fruil of the largest size, measuring six inches in its longest circumference, oval, narrowing a good deal to both ends. Suture ivell marked. Stalk about an inch long, stout, inserted, without cavity, in a folded border. Škin yellow, with numerous white dots, covered with thin white bloom-when fully ripe, of a deep gold colour. Flesh yellow, adhering closely to the stone, rather acid until very ripe, when it becomes swect, though of only second rate fla-

Fig. 114. Whute Magnum Bonum. vour. Stem long, and pointed at both unds. A pretty good bearer, though apt, in light soils, to drop fiom the tree before matured. Middle of Aurust.

* There is really no practical difference between the White, and the Yellow Magnum Bonum. The fruit is preciselv similar in appeurance and quali'y. Hougit the growth of the two trees may not fully agree. 


\section{White Damson. Thomp. Lind.}

Date Yollow Damson.

Whito Prune Damson.
Shailer's Whitc Damson. -White Vamascene.

A very pretty and pleasant little plum of the damson class. In England it is thought of very little value, except for preserving, but here, where it matures more perfectly, it becomes a very pleasant table fruit, and from its lateness, may be considered a very desirable variety. The long slender branches are loaded, so as to be pendant, with the weight of the abundant rich clusters of fruit.

Branches smooth, and of thrifty growth. Fruit small, about an inch long, oval. Skin pale yellow, with a white bloom, and sprinkled with reddish brown spots at maturity. Stalk rather more than half an inch long, downy, inserted without depres. sion. Flesh adheres slosely to the stone, yellow, and when fully ripe, of a rich, sprightly, sub-acid, agreeable favour. Ripens alinut the last of September, and will hang, shrivelling some. what, until severe frosts.

\section{White Perdrigon. Thomp. Nois.}

Perdrigon blanc. O. Nuh.

Minitre Claude.

Brignule?

A very sugary plum, which is largely cultivated in Provence, along with the Brignole, for drying, to form the colebrated Brignole prunes-so called from the little town of that naine. Thompson, indeed, makes this and the Brignole synonymous, but the French consider them distinet, the Brignole being larger, rather yellower, and dryer. It is probable that the difference is very slight.

Branches downy. Fruit middle sized, oval, narrowing towards the stalk. Strin pale greenish-yellow, with numerous small white dots, and a few red spots on the sunny sile-thinly coated with blonm. Stalk three-fourths of an inch long, slenuer. Flesh pale yellow, very sweet with a slight perfume, and ad. heres to the stone. Ripens last of A ugust.

\section{Yellow Gage, Prince's. $§$ P. Man.}

American Yellow Gage, (of some.)

White Gage, (of soms.)

The Yellow Gagge was raised, so long ago as the year 178:, oy the elder Mr. P'rince, of Flushing, L. I. It is very common on the Hudson river, but we do not find any description of it in Manning or Kenrick. We have noticed that it is sometimes confounder, at Boston, with the Imperial Gage, which is really 
quite distinct. Its great hardiness and productiveness, joinel to its rich sugary flavour, make it a favourite sort. There is a tree in the gariluns here, thirty years old, which still bears inost excellent crops annually.

Branches smnoh, short-jointed, with glossy leaves, and form. ing a large spreading head. Fruit a little above medium size, oval, rather broadest towards the stalk. Suture a mere line. Skin golden yellow, a little clouded, and covered with a copious white bloom. Stalk an inch long, inserted in a small round cavity. Flesh deep yellow, rich, sugary and melting, though sometimes rather dry; parts freely from the stone. Ripens rather early, about the first week in August.

The growth of this plum is not only very different from the Imperial Gage, but the fruit of the latter is readily distinguished by its abundant juiciness, its green. ish colour, and the superiour swrightliness of its flavour.

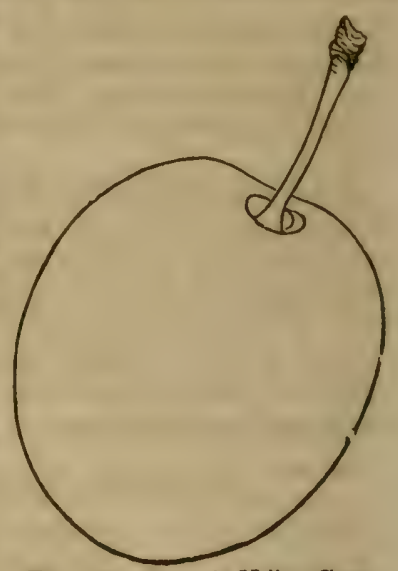

Fig. 115. Prince's Iellow Gage.

37. Yellow Gage, [of the English.] Thomp.

Little Queen Clande. Mill. Lind.

Petite Keine Claude, O. Duh.

Rein. Claude blanche.

$\longrightarrow$ perite éspéce.

Small Green Gage.

Gonne's Green Gage. of some

White Gage.

This plum, formerly known, we believe, as the Little Queen Claude, but which has now received the soubriquet of Yellow Gage, we suppose for good reasons, from the head of the fruit department, in the London Horticultural Society's garden, is an old French variety, described by Duhamel. It is of smaller size than the true Green Gage, much inferior in flavour, and does not appear to us much worthy of cultivation, when that plum, the Jefferson, and Lawrence's Favourite can be had.

Branches smooth and rather long. Fruit below medium size, round, with a distinct suture on one side. Stalk half an inch long, rather slender, inserted in a slight hollow. Skin pale yel. lowish.grem, speckled with a few reddish dots, and overspread with a grod deal of blonm. Flesh pale yellow, sweet, ana pleasant, separates freely from the stone. Ripens about the middle of August. 


\section{Elass II. Red, Blue, or Purple Plums.}

33. Abricotée Rouge. Thomp. O. Duh. Nols

The Abricotée Rouge, or Red Apricot plum, is a French va. riety, of rather protty colour, but indifferent in flavour, and not comparable to several of our native sorts.

Branches smooth. Fruit of medium size, oval, considerably flattened at each en:. Stalk nearly an inch long, set in a slighit eavity. Skin of a fine clear red in the shade, violet in the sun, covered with an abundant blue bloom. Flesh orange colour, sweet, but rather dry, and without much flavour; separates freely from the stone. Ripens the last of August.

\section{American Wheat.}

A singular little plum, of second quality, received by us from the late Robert Manning. Branches slender, smooth, leaves quite small and light coloured. Fruit quite small, of the shape of a small bullet, dropping from the tree when ripe. Skin palo blue, covered with a white bloom. Stalk slender, half an inch long. Flesh greenish, melting, juicy, and sweet, adheres to the stone. Last of August. Bears abundantly.

\section{Blue Gage. Lind. Mill. \\ Azure Hâtive. Thomp. \\ Black Perdrigon. \\ Little Blue Gage.}

An ordinary little round blue plum, the Azure Hative (f the French, of sweet and pleasant flavour, and very hardy, but the most indifferent of all the Gages. It bears most abundant crops every season, and we have found the seedlings to make gond stocks.

Branches slender and downy. Fruit quite small and rounci, about three quarters of an inch in diameter. Skin dark blue, covered with light blue bloom. Stalk three fourths of an inch long. Flesh greenish, juicy, a little acid, somewhat rich, and separates from the stone. Ripe the middle of August.

\section{Brevgort's Purple. Floy. Ken.}

New.York Purple. Floy.

Brevoort's Purple Bolmar.

Brevoort's Purple Washington.

Originated by Henry Brevoort, Esq., of New.York, from a 
stone of the Washington planted in 1819. It is a nandsome and most productive plum, uut appears to us to have been sver praised as regards its flavour, which is of second quality.

Branches long, smooth. Fruit large, oval, suture distincr at the base. Skin reddish purple, or reddish, covered with a violet bloom. Stalk three fourths of an inch long, set in a deep but narrow cavity. Flesh yellowish, soft, juicy, not very sweet, but with considerable vinous flavour, adheres closely to the stone. Ripe the first of September.

\section{Blue Perdrigon.}

\section{Violet Perdrigon. Blue Perdrigon. Perdrigon Violette. O. Duh. Brignole Violette.}

The Blue Perdrigon is a very old variety, having been intro. duced into England from Italy, as long ago as 1582 . It is a sweet and pleasant plum, and is largely employed with the White Perdrigon in making the Brignole prunes.

Branches downy. Fruit of medium size, oval, narrowing a little towards the stalk. Skin at first reddish, but becoming purple, sprinkled with many brown dots, and covered with a very thick whitish bloom. Stalk three fourths of an inch long, set in a small cavity. Flesh greenish.yellow, rather firm, sügary, rich and excellent, adhering to the stone. Last of August.

43. Blue Imperatrice. $\oint$ Thomp. P. Mag.

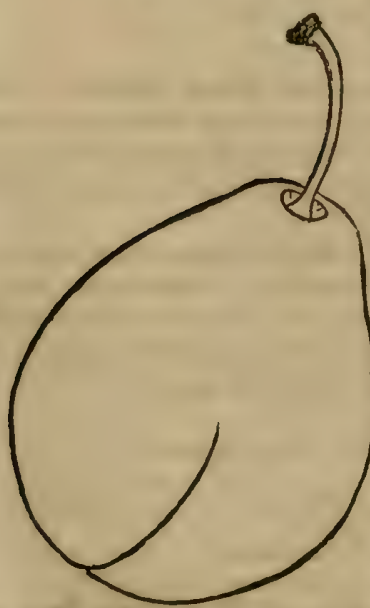

Fis. 116. Blue Imperatrice.
Impératriçe. Iind. Mill.

Véritable Impérairice.

Violette.

Impératrice Violette. O. Duh.

The true Blue Imperatrice is an admirable plum, one of the finest of the late plums, hanging for a long time on the tree, and may be kept in the fruit room a considerable period after being gathered. It is rich, sugary and excellent. The branches are long, smooth, and slender, and the smaller twigs start out at nearly right angles with the main branches.

Fruit of medium size, obovate, tapering most towards the stalk. Stalk nearly an inch long, set in a slight bollow. Skin deep purple 
covered with a thick blue bloom. Flesh greenish-yellow, pretty firm, rather dry, but quite rich and sugary, athering closely to the stone. Ripens in October, and will liang, in shelte:ed situ. ations, till the middle of Novemher.

The so.called Semina, or Blue Imperatrien of 3oston, has been considered, until lately, as identical with this pium. It is an acid, rather harsh fruit, only lit for preserving, and should not, therefore, be confoundea with the true Blue Imperatrice, which is sweet and excellent.

The growth of this spurious tree resembles that of the true Inperatrice, the fruit is oval, narrowing to the stalk, which, however, is scarcely more than half an inch long, very slender, and set without depression. Skin dark blue, with little blomm, flesh rather acid, and adheres to the stone. An abundant bearer, and hangs till late frosts.

This fruit, so well known about. Boston, seems to agree with the figure and description of the Imperatrice Violette of the old Duhamel, and we doubt, therefore, the identity of the English and French Plums of this name. Dulamel, even in the dryes and finer climate of France, only says, "assez douce pour une Prune tardive." This will apply to the Imporatrice or Semiana of Boston, but not to the Blue Imperatrice of the English.

\section{Cooper's Large. Coxe. Thomp.}

Cooper's Large Red.

Cooper's Large American.

La Délicieuse? Lind.

Coxe who first described this plum, says it was raised by $M_{\mathrm{c}}$ Joseph Cooper, of New. Jersey, from a stone of the Orleans. He considers it as a fine large plum, but exceedinerly liable to rut upon the tree, and we learn from Mr. Ives, of Salem, that he same complaint was made by former cultivators of this sort in his neighbourhood, where it is now nearly abandoner. It seems to be scarcely known now in this comntry-that is to say, in gardens* - as we have made diligent search for it, the last two yeats, without being able to obtain the fruit of the true sort.

Lindley describes a plum, La Déliceuse, as having bren brought from New Jersey about 1815, and which was sold by Mr. Kirke, for a guinea a plant. And Mr. Thompson gives this fruit as identical with Cooper's Large. We hope another season to be able to compare the two.

Thompson's description of the Cooper's Lare is as follows.

- Some nurserymen here, we regr't to say, do not scruple to fill large cataloguea with the names of varieties which have no corresponding existence in theis crounds. 
Branches smooth. Fruis, purple, oval, of medium size, sepr. rates from the stone, of second quality, ripens at the end of September, and a great bearer.

Coxe discribes it as ripening in August, and of the largest size.

The following is Lindley's description of La Déliceuse, which we rive in order to assist in identifying the two, if they prove really distinct. Branches long and smooth. Fruit oval, about two inches long, and one and three quarters in diameter. Suture rather broad, shallow, swelled a little more on one side than on the other. Stalk an inch long, slender, slightly inserted. Skin pale yellow on the shaded side, but in the sun deep purple full of brown specks. Flesh yellow, and separates from the stone. Iuice peculiarly rich and abundant. Ripe in October, with the Imperatrice.

\section{Columbia. \&}

Columbian Gage.

A noble plum, a contemporary of the Lawrence's Favourite, already described, and like that, raised by Mr. Lawrence, of Hudson, from a pit of the Green Gage. It is a superb looking, and a rich fiavoured variety-undoubtedly one of the finest of the large dark coloured plums. The tree is remarkable for its very stout blunt shoots, large roundish leaves, and the spreading horizontal form of its head. It is also highly productive. Branches and upper side of the leaves downy. Fruit of the largest size, six or seven inches in circumference, nearly globular, one half rather larger than the other. Skin brownish purple, dutted with numerous fawn-coloured specks, and covered with much blue bloom, through which appears a reddish brown tint on the

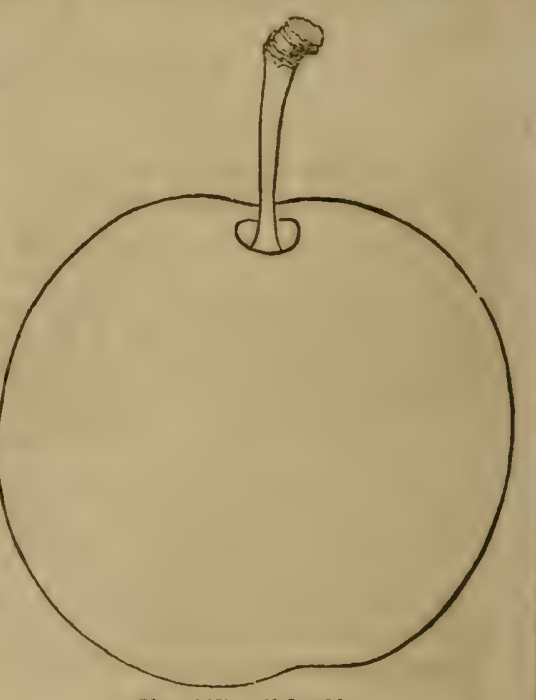

Nig. 117. Columbia. shaded side. Stalk about an inch long, rather stout, inserted in a narrow, small cavity. Flesh orange, not very juicy, but 
when at full maturity, very rich, sugary and cxcellent; it sepa. rates frecly from the stnne, except a little on the edgt:. 'The sinne is quite small and compressed. Last of Augrust.

\section{Corse's Aduiral.}

A rather large, light purple plum, which, like the two or three following ones, was raised by Henry Corse, Esq., an intelligent cultivator, of the neighbourhood of Montreal, Canada. 'They are all well adapted to a northern climate.

Branches quite downy. Fruit above medium size, oval, or a little ohovate, cousiderably enlarged on one side of the suture. Slin linht purple, covered with a pale lilac bloom, and dottrd with yellow specks. Stalk nearly an inch long, hairy, slightly inserted. Flesh greenish-yellow, juicy and sprightly, but se. cond rate in flavour, and adhering closely to the stone. A pro. lific tree. September.

\section{Corse's Field Marsital.}

Handsome in appearance. Skin lively purplish red. Fruit rather large, oval. Stalk rather slender, three fourths of an inch Inng, slightly inserted. Flesh greenish.yollow, juicy, but a little tart, arlheres closely to the stone, which is long, and pointed at both ends. Ripe middle and last of Aurust.

\section{Corse's Nota Bexr. Ken.}

This is the best of $\mathrm{Mr}$. Corse's varieti s that have been proved in the United States.

Branches smooth. Fruit of rather large sizn, round. Skin pale lilac or pale brown, ofien dull green on the shaded sile, wilh much light blue bloom. Stalk half an inch longe, set in a round hollow. Flesh greenish, rather firm, juicy, sweet and rich, and separates from the stone. The tree is a very grreat bearer, and is very hardy. First of September.

\section{C:uger's Scarlet. $\$$ \\ Cruger's. \\ Cruger's Seedling. \\ Cruger's Scarles Gage.}

An exceeding delicate and beautiful dessert fruit, raised from seed, by Lenry Cruger, Esq., of New-York, and first dis_eminated from the gardens here. Its milul and agreeable flavour is preferred by many who do rot like the more luscious plums. and its sure and abundant crops render it a favourite on Jight soils, where the curculio destroys many less hardy. Mr. Ives in. 


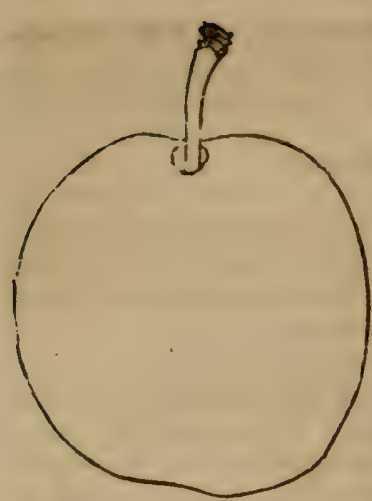

Fig. 118. Cruger's scarlet.

forms us, that with him, it is less liable to drop from the tree than any other sort.

Branches downy. Fruit rather larger tlan a Green Gagr, roundish. oval, with an obscure suture. Skin, when fully exposed, a lively red, but usually a bright lilac, covered with a thin bluish bloom; and speckled with numerous golden dots; in the shade it is pale fuwn-coloured on one side. Stalk half an inch long, set in a shallow depression. Flesh deep orange, not very juicy nor rich, but with a very agreeable, well after ripening. Last of August.

\section{Cherry. Thomp. Coxe.}

Farly Scarlet.

Alyrubo'an.
Virginian Cherry. of Equrpean

$\left.\begin{array}{l}\text { De Vi ginie. } \\ \text { D'Amerigue Ronge. }\end{array}\right\}$ gardens.

Prubs IIymbolant. O. Duh. Lind.

P'runiss Cerasifera. Pursh.

Miser l'lum, of Hoffy.

The Cherry Plum, or Early Scarint, is a very distinct specirs. It has been consilererl a native of this country, but we doubt this, and think, with Pursh, that it is only found here in the neighhourhoot of houses. The tree grows pretty rapisly, forms a small, bushy head, and is easily recomnized by the slenderness of its branches, and the smallness of its leaves. It bears the greatest profusion of snowy blossoms in the spring, which from the early date at which they appear, are rather liable to be cut off by frost.

There are several varieties produced from seed, but that most common herc, is round, about an incis in diameter, of a lively red, with very little bloom, and a very slender, short stem, set in a narrow cavity. On the trees they resemble cherries, rather than plums. The flesh is greenish, melting, soft, very juicy, with a pleasant, lively, sub-acid flavour-neither rich nor high flavoured, and adheres closely to the stone. The stone is oval, and pointed. It ripens about the middle of July, before most other plums, and this, and its pretty appearance at the dessert, are its chief merits. Branches smonth.

The cominon cherry plum, or Mrrobolan, of Europe, is 
rather larger, and shaped like a heart. In all other respects the saine.

Goldex Cherry Plum. Mr. Samuel Reeve, of Salem, NewJersey, has produced a seedling of the cherry plum, which is worthy of notice.* It is heart-shaped, yellow, speckled with scarlet in the sun, but of a glossy waxen yellow in the shade. The habit of the tree is exactly that of the common cherry plum, but as it is a very abundant bearer, and ripens early in July, Mr. R. has found it one of the most profitable plums for the market. It is worthy of more extensive trial.

\section{Coe's Late Red. $\S$ Thomp. Lind.}

\section{Saint Martin. $\quad$ of the \\ Saint Martin Rouge. French. \\ Prune de la St. Martiu. Nois}

This plum (which should properly be called the St. Martin's, though as it was also claimed to have been raised by an English nurseryman, it seems difficult to rid it of that title,) proves, with us, to be an exceedingly valuable, late variety. Indeed, it is sc late, that we fear, to the north of this, it would not come to maturify. It grows vigorously, bears regularly and heavily, and would prove a valuable market fruit. The flavour is excellent.

Branches downy. Fruit of me. dium size, nearly round, with a well marked suture running along one side. Skin light purplish-red, with a thin blue bloom. Stalk pretty stout, three-fourths of an inch long, set nearly even with the surface. Flesh yellowish, rather firm and crisp, juicy, with a rich vinous flavour, separating almost entirely from the stone. Cetober and November.

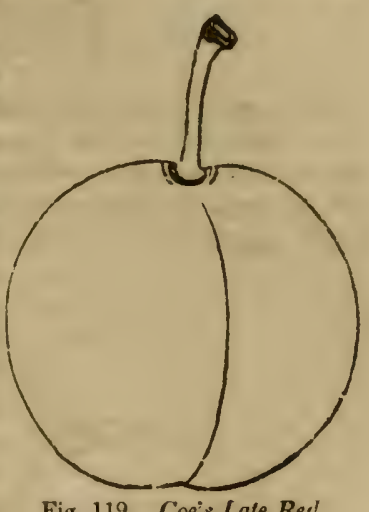

Fig. 119. Coe's Late Red.

52. Crifston. Thomp. Lind.

Matchless. Lang.

Diapreé Violetto. ? ac. to

Viulet Diaper. $\}$ Thomp.

A pleasant, early pium, but superseded now by better ones. Branches downy. "Fruit rather small, oval. Skin dark purple,

- Nescribed in Hofry's Orchardist's Companion, (Philadelphia,) as the sararer puva. 
with a blue bloom. Stalk quite short, set without depression. Flesh yellow, firm, sweet, and rather sprightly, separating from the stone. Last of July, and first of August.

\section{Denniston's Red.}

A strikingly handsome, new seedling, which has newly come into bcaring, in the celebrated plum orchard of the gentleman whose name it bears, at Albany.

Branches smooth, dark coloured. Fruit rather large, round. ish-oval, narrowed towards the stalk. Suture running half round. Skin of a beautiful light red, sprinkled with many small, fawn coloured dots, and dusted with a very light bloom. Stalk very long and slender, slightly inserted. Flesh amber colour, juicy, rich, and sprightly, with an excellent flavour. It separates from the stone, which is small, oval, and compressed. I,ast of August.

54. Domine Duzl. $\oint$ Floy. Thomp.

German Prune. Man. and of some

Dutch Prune. $\}$ American gardens.

Dutch Quetzen.

This good American prune was raised from a seed brought from IIolland, by the Rev. Mr. Dull, a Dutch minister, who afterwards resided at Kingston, N. Y. The parent tiee was the common Dutch prune, which this strongly resembles. The same gentleman's little parcel of plum stones from "fader.

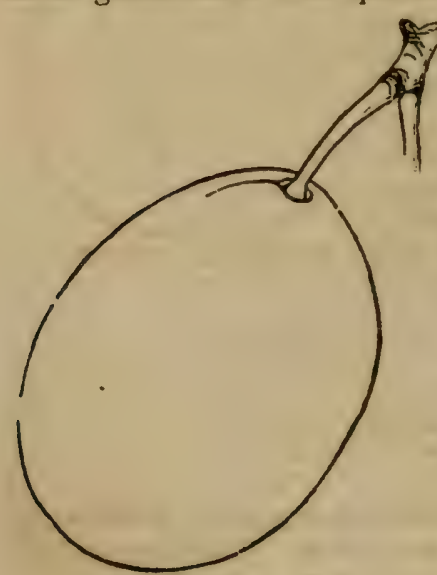

Kig. 120. Domine Dull. land," it will be remembered, gave origin to Bleecker's Gage, one of the finest of our yellow varieties.

Branches long and smooth. Fruit of medium size, longoval, with little or no suture. Skin very dark purple, nearly black, dusted with some blue bloom. Stalk nearly an inch long, inserted with very little cavity. Flesh yellow, quite juicy at first, but if allowed to hang on the tree becomes dry, rich and sweet; it adheres closely to the stone. A pro digious bearer, and a really good fruit. September. 


\section{Damson. Thomp.}

Common Damson.

Purple Damson.

Black Damson.

Early Damson, (of many.)

The common, oval, blue Damson is almost too well known to r.eed description, as every cottage garden in the country con. tuins this tree, and thousands of bushels are annually sold in $t^{\prime}$ 'e market for preserves. The tree is enormously proluctive, but in the hands of careless cultivators is liable to be rendered worthless by the knots, caused by an insect easily extirpated, if the diseased branches are regularly burned every winter or spring.

Branches slender, a little thorny and downy. Fruit small, oval, about an inch long. Skin purple, covered with thick blue bloom ; flesh melting and juicy, rather tart, separates partially from the stone. Septcmber.

As the Damson is frequently produced from seed, it varies somewhiat in character.

The Suropshire or Prune Danson is an English purple va. riety, rather obovate in figure, but little superiour to our common sort. The Sweet Danson resembles the cornmon Dam. son, and is but slightly acid.

The W WNTER Danson is a valuable market sort, from its extreme lateness. It is small, round, purple, covered with a very thick light blue bloom; flesh greenish, acid, with a slight astringency, but makes good preserves. It bears enormous crops, and will hang on the tree till the midcile of Novembers six weeks after the common Damson, uninjured by the carly frosts

\section{Duane's Purple. $\S$ P. Man. Ken.}

A superb looking purple fruit of the largest size, and of very fuir quality, -occasionally, in warm dry scasons, first rate. It was originally grown by James Duane, Esq., of Juancsburght, N. Y., and probably sprune from at serd of the Purple Magnum Bonum. We have seen this fruit, about Albany, confounded with the raricty just named. The tree is easily known by the grou appearanne of the wood, and large leaves, which are unusually woolly on the under surface. It is a highly attractive dessert iruit, ripening rather before the plum scison, and bearing well. 
Branches very downy. Fruit very larene, oval or oblong, considerably swol. ten on one side of the suture. Skin reddish-purpie in the sun, but a very pale red in the shade, sparingly dotted with yel. low specks, and covered with lilac bloom. Stalk three-fourths of an inch long, slender, set in a narrow cavity. Flesh amber coloured, juicy, sprightly, moderately sweet, adheres partially to the stone. Ri. pens with the Washington, (or a little before,) about the 10th of August.

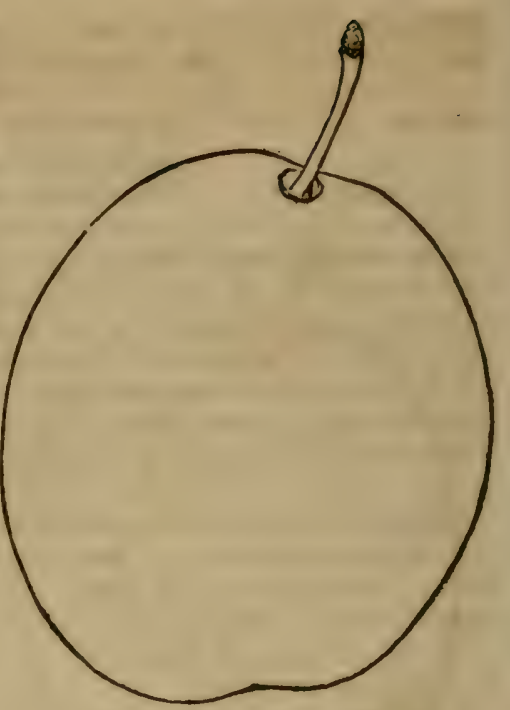

Fig. 121. Duane's Purple.

57. Diamond. Thomp. Man.

A very large plum, but exceedingly coarse in flavour, and of zo value, except for cooking. It grows thriftily and bears regularly and abundantly with us, and is very showy on the tree, but it is, otherwise, scarcelv third rate. It was raised from seed, by an Englishman, in Kent, named Diamond.

Branches long, downy. Fruit of the largest size, oval, shaped like an Eigg Plum or Magnum B snum. Skin black, covered with a blue bloom. Stalk three-fourths of an inch long, set in a narrow cavity. Flesh defp yellow, coarse-grained, and rather dry - a litile acid, and without flavour ; separates from the long pointed stone. ['The author of the American Orchardist says, "flavour superior!"]

58. Diaprée Rouge. $\oint$ Thomp. Poit. O. Duh.

$\left.\begin{array}{l}\text { Rorhe Corbon. } \\ \text { Mimms. } \\ \text { Imperial Diadem. }\end{array}\right\} \begin{aligned} & a c . \text { to } \\ & \text { T\%omp. }\end{aligned}$

The Diapreé Rouge, or Red Diaper, is a very large ana handsome French plum. Mr. Thompson considers it synnnymous with a fine Finglish variety, better known here as the Minms, or Imperial Diadem. As the Mimms plum has been fully tested by us, and proves to be a first rate fruit in all ro 
Epects, in th.is climate, we give the following description and outline diawu from the fruit, as produced by us.

A rather slow grower. branches almost smooth. Fruit large, obovate. Skin of a reddish-purple, with a few golden specks,

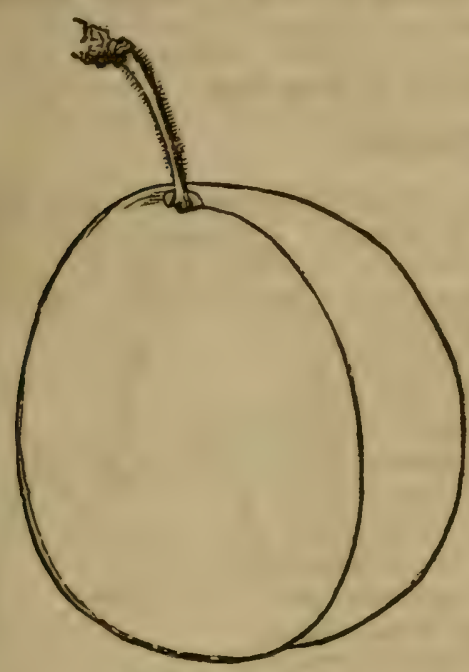

Fig. 122. Red Diaper. and a light blue bloon: easily rubbed off. Stalk three-fourths of an inch long, slender, hairy, slight. ly inserted. Flesh pale green, juicy, very melting, rich and delicious; separa. ting from the stone, which is quite small. Last of August.

The Diapree Rouge is described by Poiteau as having a thick, rather bitter skin, exactly the op. posite to that of the fruit we have described. It is probable, however, that our climate, more favourable for the plum, may produce it in greater per. fection:

59. Elfrey. Coxe. Man.

Elfry's Prune.

A native plum, first described by Coxe. It belongs to the class of prune plums, with dry, sweet flesh, and is much esteemed by many persons. The tree is thrifty, with rich glossy leaves, and bears to a fitult.

Branches smooth. Fruit rather below medium size, oval. Skin blue. Flesh greenish, very sweet, dry and firm, farting very freely from the stone-indeed, often splitting open when fully ripe.

60. Fotheringhani. Thomp. Lind. Mill.

\section{Sheen.}

\section{Grove House Purple.}

An old English plum of good quality. It is not unlikely that it originated at Sir William Temple's seat-Sheen, in Surrey, where, according to Lindley, it was grown before 1700, under the name of the Sheen plum.

Branches smooth. Fruit of medium size, obovate, with a 
distinct suture. Skin purple, where exposed, but in the shade redilish, sprinkled with small specks, and covered with a pale blue bloorn. Stalk an inch long. Flesh pale greenish-yellow, juicy, sprightly, and rich, separating from the stone. Ripens about the middle of August.

\section{Frost Gage. $\oint$ Pom. Man}

\section{Frost Plum.}

A most valuable late plum, scarcely yielding to any other late variety in the excellence of its favour. It appears to have originated in Fishkill, Dutchess co., N. Y., where it has, for many years past, been most extensively cultivated for market. Bufore fully ripe it abounds with sprightly, sub. acid juice, and is highly esteemed for preserving, and when mature is a sweet and luscious fruit for the dessert. It will hang on the tree till very late frosts. The tree is a tall upright grower, with smooth and rather slender shoots, and bears abundantly. The fruit is in perfection about the first of October, and from its lateness and good quality commands from two to five dollars a bushel, even when hundreds of bushels are sent to New-York market at once.*

Branches smooth. Fruit rather below

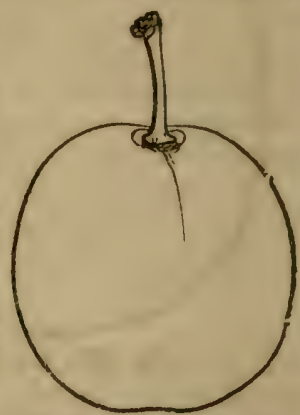

Fig. 123. Frost Gage. medium size, roundish-oval, with a distinct suture on one side. Skin deep purple, with a few brown specks, and a thin bloom. Stalk half to three-fourths of an inch in length, inserted with little or no depression. Flesh greenish-yellow, juicy, sweet, rich and melting, adhering to the stone.

62. Goliath. Thomp. Lind.

Caledonian, (of some.)

Saint Cloud.

Steers's Emperor.

Wilmot's late Orleans.

A large and handsome plum, not quite first rate, but well deserving cultivation. It is easily distinguished from the Necta rine plum, with which it has been confounded by its gray, very downy shoots. It bears fine crops.

* Eighteon hundrod dollars have beon received by a single farmer in ths ricinity, fur a single soason's erop of this plum. Haring some affinity to the Damson, it is, in some district, fiablo to the knots, but trifling care will soos banish this enemy. 
Fruit large, roundish oblong, enlarged on one side of the suture. Skin a fine deep red, approaching purple, a little paler in the shade, dusted with a thin blue bloom. Stalk three quar. ters of an inch long, deeply inserted in a well marked hollow. Flesh yellow, adheres considerably to the stone, rather juicv with a brisk, sprightly flavour. Last of August.

\section{Gwalsh. Thomp.}

A fruit little known out of New-Jersey, where, we believe, i is a native. We received it from Thomas Hancock, of Burling ton, N. J. It is large, showy and prolific, and about the quality of the Red Magnum Bonum.

Branches nearly smooth. Fruit large, regularly formed, obo. vate, with scarcely any suture. Skin rich, dark purple, cover. ed with blue ulnom. Stalk rather slender, not quite an inch long, slightly inserted. Flesh yellow, juicy, of sprightly fiavour, mixed with a slight acid, and adheres to the stone. Second quality. Middle of August.

\section{Holland, Pom. Man. Ken.}

Blue Holland.

Holland Prune.

A pleasant, late plum, of second quality, handed down from the old Dutch gardens of New.York, and perhaps, originally brought over by the first settlers of that city.

Branches downy, rather slender. Fruit round, slightly flat. tened. Skin blue or light reddish-purple, covered with a blue bloom. Stalk set in a small cavity. Flesh juicy, melting, sweet and pleasant, separating freely from the stone. It hangs a long while on the tree, to which the stalk adheres rather closely, ripening from the last of August to the middle of Sep. tember.

\section{Horse Plưr. Thomp. Floy.}

Large Early Damson. ? of Prince Sweet Damson.

A very common and inferiour fruit, which reproduces itself irom seed, and is almost naturalized in the gardens of the middle states. The seedlings make good stocks for the nursery.

Branches downy. Fruit of medium size, oval, with a deep suture on one side. Skin purple in the sun, red lish on the shaded side, with blue bloom. Flesh greenish-yellow, ratiex dry and acid, saparates from the stone. Laast of Angust. 


\section{Howeld's Early.}

This is a very desirable early fruit, ripening about the 20th of July, a few days before the Morocco. It takes its name frorn Mr. B. Howcll, of Newburgh, N. Y., who brought the paren tree when a sucker, from Virginia. It appears to us unliko any other described variety. The fruit is remarkably fragrant.

Wood slender, gray and downy. Leaves small, oval, downy. Fruit rather below medium size, oval, without any suture, a little angular. Stalk slender, three fourths of an inch long, set even with the surface. Skin light brown, often greenish-yellow on the shaded side, covered with a thin blue bloom. Flesh am. ber coloured, melting, juicy, with a sweet and perfumed flavour, separates from the stone, which is quite small and oval. Very productive.

\section{ICKivorth IIIPeratrice. $\oint$ Thomp.}

Knight's No. 6.

The Ickworth Imperatrice was raised by Mr. Knight, of Downton Castle, and is a hybrid between Blue Imperatrice and Coe's Golden Drop. It is one of the numerous recent and valu. able additions to the class of late plums, prolonging this formerly flecting fruit the whole autumn. It hangs a long while on the tree, and if gathered and wrapped in soft paper, will keep many weeks-much longer than any other variety, and is, perhaps, one of the best late dessert sorts.

Branches smooth. Fruit rather above medium size, obovate. Skin purple, peculiarly traced or einbroidered with streaks of golden fawn colour. Stalk moderately long and thick. Flesh greenish-yellow, sweet, juicy and rich, mostly adhering to the stone, which is rather small. Ripens early in October, and may be kept till Christmas, gradually becoming dryer and more sugary. It will, even if laid away in paper in a dry place, be. come an excellent prune, and it has been found in this state, and with an excellent flavour, the next summer.

\section{Italtan Damask. Lind.}

\section{Damas d'Italie. O. Duh. Thomp.}

Branches smooth. Fruit middle sized, nearly round, a little flattened at the base, and having a well marked suture extending from the stalk to the apex. Stalk half an inch long, slender, inserted in a small round cavity. Skin violet, becoming brown when fully ripe. Flesh yellowish-green, firm, and separates clean from the stone. Juice very sweet and high flavoured. Stone oval, rather thick. End of August. [Lindley's Guide.] 
To this we will add that the Morocco (with dorny shoots) is oiten inistaken for this plum in this country.

\section{Lombard. Ken.}

Bleocker's Scarlet.

Beekman's Sicarlet.

The Lombard is an exceedingly pretty plum, of pleasant fla. vour, and it has qualities that will always make it popular;great hardiness and productiveness, and the power of holding its fruit uninjured in those light sandy soils where most other sorts are punctured and fall by the curculin.

It was called the Lombard plum by the Massachusetts Horti. cultural Society, in compliment to Mr. Loinbard, of Springfield, Mass., who first brought it into notice in that state; and it is said to have been received by him from Judgre Platt, of Whitesborough, N. Y., who raised it from seed. But it was previously well known here by the name of Bleecler's Scarlet. Never having

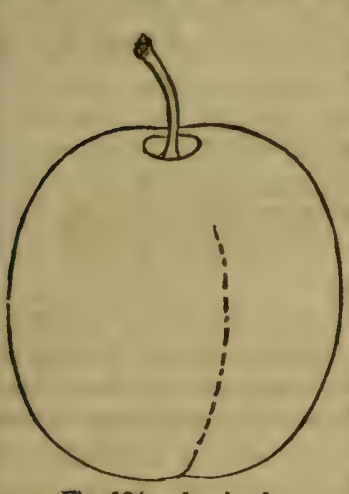

Fig. 124. Lombard. been described under that name, how. ever, we adopt the present title. The tree has strikingly crumpled leaves, thrifty; bright purple, glossy shoots, and grows with much vigour.

Branches smooth. Fruit of mediuın size, roundish-oval, slightly flattened at either end; suture obscure. Stalk quite slender, scarcely three-fourths of an inch long, set in a broad, abruptly narrowing cavity. Skin delicate violet red, paler in the shade, dotted with red, and dusted thinly with bloom. Flesh deep yellow, juicy, and pleasant, but not rich; arlhering to the stone. Middle and last of August.

\section{Long Scarlet}

\section{Scarlet Gage.}

Red Gage, (incorrectly, of some.)

A bright red, oblong fruit, very handsome upon the tree, which usually hangs heavy laden with its fruit. It is a native of this part of the Hudson, and has been disseninated by us. It is a little tart, and of second rate flavour, but it is highly valued for the bright red transparent jelly, that is made from the fruit, surpassing that of any other variety.

Shoots downy. Fruit of medium size, oblong-obovate, swol. len on one side of the suture and tapering to the stalk. S!kin bright red in the sun, pale yellowish-red on the shady side, 
covercd with a fine lilac bloom. Stalk three.fourths of an inch long, set in a narrow cavity. Flesh deep yellow, juicy, acid at first, but, if allowed to hang, it becomes rather rich and sweet. It adheres to the stone. Last of August.

\section{Orlfans. Lind. Thomp.

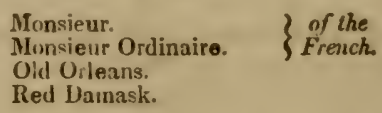

'The most popular Engrlish marliet plum, being hardy and uniformly productive. It is not generally cultivated here, being considered a second rate fruit, and is supplanted by ietter Ane. rican sorts. As a kitchen fruit, it is chiefly esteemed.

Branches gray, and very downy. Fruit middle sized, round, a little cnlarged on onc side of the distinct suture. Skin dark red, becoming purple in the sun. Stalk little more than half an inch long, set in a wide hollow. Flesh yellowish, sweet, mixed with acid, and separates freely from the stone. Ripens a l.ttle after the middle of August.

72. Orleans, Early. Thomp. Lind.

\section{New Early Orleans. \\ New Orleans. \\ Grimwoul's Early Orleans. \\ Hampton Court.}

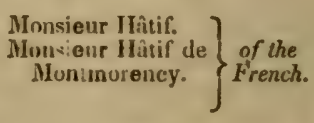

The Early Orleans is very near like the foregoing in all re. spects, except that it ripens ten days earlier-about the first of August here, with the Morocen-which makes it far more desirable. Branches downy. Fruit of the size and colour of the common Orleans, a little more oval, and with a more shallow suture. Stalk sometimes half an inch long and stout, some. times longer and more slender, set in a moderate hollow. Skin a little marbled. Flesh yellowish-green, of brisk flavour, rather richer than the old Orleans, and separates from the stone. A good bearer.

Wilmot's Netw Early Orleans, (Wilmot's Large Orleans, \&c.,) so strongly resembles the foregoing in appearance, time of ripening, etc., as to be scarcely worthy of a separate description

\section{Ordeans, Surtu's. $§$ Porn. Man.*}

Violet Perdrigon. $\quad$ incorrectly, of some Red Magnum Bonum. $\{$ American gardens.

Smith's Orleans, the largest and finest of this class of plums

- Descrihed. ty an orror in tisc l'omu!orical IInual, as a frestone. 
is a native variety raised from the old-Orleans about 20 years ago by Mr. Smith, of Gowanus, Long lsland. It is one o. the most vigorous of all plurn trees, making straight, glossy, red. disli-purple shocts, seven or eight feet long in the nursery, with dark green, crimped leaves. It bears regularly and well, in almost any soil, its fruit is large and handsome, and has that blending of sweet and acid in its flavour, which renders it, to our taste, one of the most agreeable of all plums. It is deservedly a favourite in American gardens.

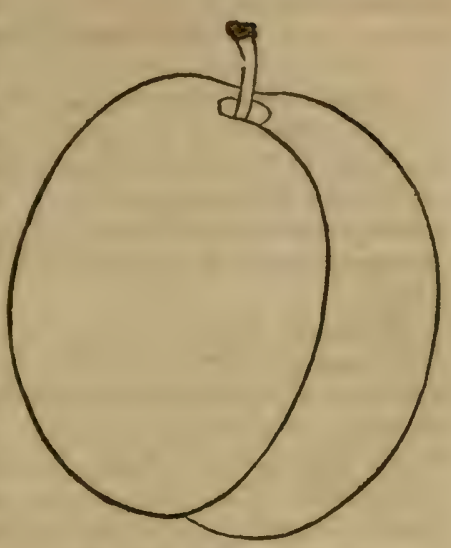

Fig. 125. Smuth's Orleans.

Bearing branches smooth, or nearly so. Fruit large, often of the largest size, oval, rather widest towards the stalk, a little irregular, with a strongly marked suture on one side. Stalk quite small and slender, little more than half an inch long, in. serted in a deep narrow cavity. Skin reddish purple, covered with a deep blue bloom. Flesh deep yellow, a litule firm, very juicy, with a brisk, rich vinous flavour, (not sweet and cloying,) and adheres to the stone. Ripens from the $20 \mathrm{th}$ to the last of August, and hangs for some time on the tree, becoming very dark in colour.

By an error, this variety was sent out from the gardens here for several years, as the Violet, or Blue Perdrigon, a smaller, and very different fruit, and bears this name still, in some col. lections.

\section{Isabella. Thomp.}

Tlis is an attractive looking English plum, of a fine red co lour, and of good flavour, though scarcely so beautiful as the coloured plate in the Pomological Magazine would lead one to suppose ; but well worthy of a place in a large collection.

Branches quite downy and gray, like those of the Orleans. Fruit medium size, oval, rather narrower towards the stalk. Skin dark dull red in the sun, paler in the shade, and thickly sprink. led with darker coloured dots. Stalk three-fourths of an inch long, a little hairy, set in a moderate hollow. Flesh yellow, rich, juicy, with a smart flavour, and adheres to the pointed stone. Last of August. 


\section{KIRKE's. Thomp. Lind}

Kirke's flum is a viriety which came to us from Fingland, where it was first brouglit into notice by Mr. Kirke, the nur. seryman at Brompion. Its cxcellent favour and proluctivesesa will bring it into favour here. The sort usually known in our nurseries under this name, is incorrect. In general appearance it resembles a good deal the Reine Claude Violette, or Purple Gage.

Branches smonth. Fruit of medium size, round, with very little suture. Skin dark purple, with a few golden dots, and coated with an unusually thick blue bloom, which adheres pretty closely. Stalk three-fourths of an inch long, inserted in a very slight depression. Flesh greenish-yellow, firm, and very rich in flavour. It separates freely from the stone, which is flat and broad. Ripens the last of A ugust and first of September.

76. Morocco. § Thomp. Lind.

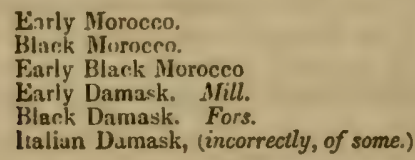

Eirly Moroceo.

Blarek Murocen.

Farly Black Morocco

Early Damask. Mill.

Black Damask. Fors.

Italiun Dumask, (incorrectly, of some.)

One of the very best of the early purple plums, ripening at the beginning of August, ten days before the Washington, and therefore worthy of a place, even in small gardens. It is a moderate bearer.

Branches downy. Fruit of medium size, roundish, with a shallow suture on one side; a little flattened at both ends. Skir dark purple, covered $"$ " $h$ a pals thin blonm. Stalk half an inch long, rather stout. Flesh greenish-yellow, adhering slightly to the stone, juicy, with a smart, rich flavour, becoming quite sweet at maturity.

77. Nectarine. Thomp. Lind.

Caledonian.

Howell's Large.

Jenkins' Imperial.
Pench Plum. Z incorrectly

Prune Pêche. $\}$ of some.

Louis Philippe.

A finc looking fruit, probably of English origin, and confound. ad by somn with the Peacrr Pluñ of the Freneh. Its size, and hantsome apparance, will alwalys sive it a place in the plum Jechurr', but it must be confisserl that it will hardly mink as a first rate d.sstrt fruit, being decitiedly inferiour to the (olumbia

- For the trire Peacri Prum, See Supplement. 
a plum of even largerdimensions. The young trees are readily known by their straight, large, blunt purplish shonts, neariy smooth, and not gray and downy, like those of the Goliath.

Fruit of the largest size, renularly formed, roundish. Stalk ahout half an inch long, rather stout, and set in a wisle shallow depression. Skin purple, dusted with a blue bloom. Elesh dull greenish yallow, becoming tinged with red at maturity, a little coarse grained, with a rich, brisk flavour, and arllering partially to the stone. A grood and regular bearer. Ripens about the 15 th of August.

Mr. Rivers has lately sent to this country trees of the PEACI Plum, which he says is the Prune Peche of Brittany, superiour to, and quite distinct from the Nectarine.

73. Précoce de Tours. $\S$ O. Duh. Thomp. Lind.

\author{
Farly Tours. \\ Early Violet. Vio!etre Hative. Lang. Lind. \\ Noire Hative. \\ Violet de 'Tours. \\ Perdrigon Violet. ? incorrectly \\ Blue Perdrigon. $\}$ of some.
}

The Early Tours plum is yet very little known in the United States, but deserves a more general trial, as it is esteemed abroad as an excellent very early plum, ripening the last of July, among the first of the season.

Branches downy. Fruit rather more than an inch in diameter, oval, with a shallow suture. Skin deep purple, covered

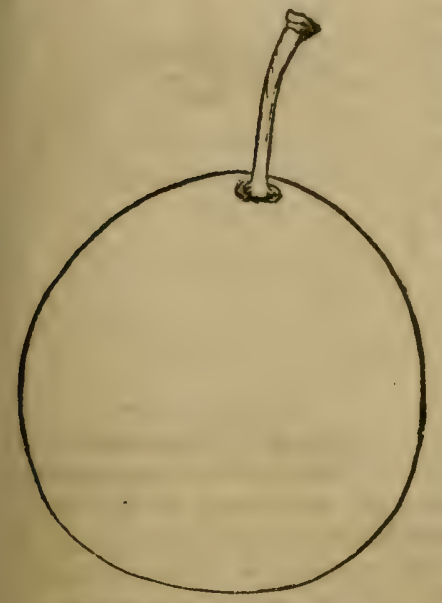

Fig. 126. Purple Fuvour'e. with a thick azure bloon. Stalk half an inch long, set in a narrow cavity. Flesh at first greenish, but becoming dull yellow at maturity; a litlle fibrous, but juicy, sweet, melting, and slightly perfumed; it adheres considerably to the stone.

\title{
79. Purrle Favourite. $\$$
}

This delicious fruit received its name from us some years ago. The tree from which the stock now in this country was derived, stood for many years (until it died of old age,) in the centre of the principal garden here, and was planted bv the 
father of the ambor. Its origin we were never able to learn and we have not been able during all nur pomological re. searehes and comparisons, to identify it with any other sort.

The Purple Favourite, when in perfection, is not surpassed by any other plum in luscious flavour. It is more juicy and melting than the Purple Gage-and has some affnity to the Diapré Rouge, or Mimms. It should have a place in every rarden, as it brars well, and is very hardy. In the nursery it has the dwarfish habit of the Green Gage, but nore slender shoots.

Branches nearly smooth, short-jointed. Fruit medium size, often larere, roundish.obovate. Suture none. Skin light brown in the shade, brownish-purple in the sun, dotted with numerous golden specks, and dusted with thin, light blue blonm. Stalk three fourths to one inch long, set in a very slight depression. Flesh pale, grcenish, very juicy, tender, melting, with a luscious sweetness. Parts freely trom the stone, which is very small and roundish. Begrins to ripen about the 20th of august, and will hang for a fortnight on the tree.

This is linown, incorrectly, as the Purple Garge, in some parts of the country.

\section{Purfle Gage $§$ Lind. Pom. Mag.}

Reine Claude Violette. Thomp. Nins. Die Violette Küning Claudie. Sickler. Violet Queen Claude.

'The Purple Gage holds the first place for high flavour among pusple plums abroad. Athough it is as well known in France under the title of the Reine Claude Violette, as in Eng. land under that of the Purple Gage, yet its native country is not precisely determined.

Branches smooth, much like those of the Green Gage. Fruit medium sized, shaped like the Green Gage, roundish, a little flattened. Suture shallow, but distinct. Stalk an inch long, Fig. 12\%. Purple (inge.
rathr thick, set in a narrow cavity. Skin a little thick, vinlet,

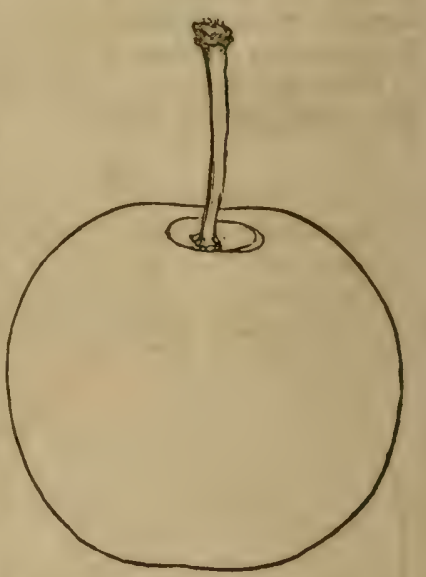
dotted with pale yellow and covered with light blue blonm. Flesh greenish yellow, rather firm, rich, sugary, and verv high flavoured, separates from the stone, which is oval and com. pressed. Ripens rather late, and will hang on the trec-shrivelling a little, but not cracking-all the month of September. 


\section{PoND's SeEdLing.}

\section{Pond's Purple. Ken.}

A productive plim of only second quality. It was brought into notice by Mr. Samuel Pond, a nurseryman near Boston, but the original tree grew in the garden of Lenry IIill, Lisq., in the city of Boston.

Branches downy. Fruit middle sized, roundish. Skin purple. Stalk short. Flesh yellowish, rather dry, separates froin the stone, sweet, mingled with acid, of tolerable flavour. Ripens carly in August, and hangs a long time.

\section{Peoly's Eatsly Blee.}

This is a native fruit, of medium quality, which we received from Mr. Manning. Branches very downy. Fruit middle sized, oblong, suture scarcely visible. Skin very dark blue, covered with light blue bloom. Stalk short, uneven. Flesh yellow, of pleasant flavour, adhering partially to the stone, which is not large, but rather bluntly terminated. Ripens about the 10th of August.

\section{Prune D'Agen. Nois.}

\section{D'Agen. Prune d'Ast. \\ liobe de Sergent.}

Agen Datte.

Si. Maurin.

Prune de Brignole, (of some.)

A French prune, of good quality, chiefly used for drying or preserving. Branches smooth, leaves narrow. Fruit of medium size, obovate, flattened on one side. Skin purple, with a blue bloom. Stalk short. Flesh greenish-yellow, sweet. It is a freestone, and makes an excellent prune. It ripens late in September, and bears prodigious crops.

\section{Prune, Manning's Lowg Blue. $\oint$}

\section{Large Long Blue. Man.}

\section{Manning's Long Blue.}

Manning's Long Blue Prune, we received from the late Mr. Manning, with the account that it was had by him without a name, from Landreth's Nursery. Philadelphia. It is undoubtedly a seedling of the common Quetsche, and is one of the best of this family of plums. lis large size, long keeping, and lateness, added to the fuct that it bear's most abundant crops, make it a good market fruit. 


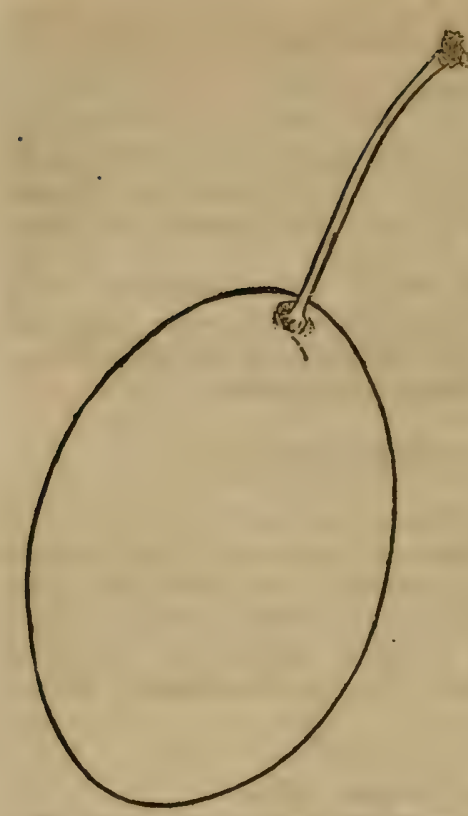

Branches smooth. Fruit quite large, long-oval, a littlo one-siderl, with an obscure suture. Stalk very long, and slender, set in a very trifling depression. Skin dark pur. ple, with a thick blue bloom. Flesi greenish-yellow, firm, rather juicy, with a sweet, sprightly, pleasant flavour. It separates pretty readily from the stone, which is long and pointed. First to last of September. Ripens gradu. ally, and bears carriage well.

\section{Queen Mother. Thos'p. Ray. Lind.}

Red Quven Mother. Pigen's Heart. Damas Viulet.

A neat little reddish plum, long known in European Fig. 123. Munning's Long Blue Prune. gardens. Branches smooth, rather feeble in growth. Fruit rather small, round, about an inch in diameter. Slin dark, purplish red in the sun, pale red. dish amber in the sharle, with many reddish dots. Stalk half an inch long. Flesh yellow, sweet and rich, separating freely from the stone, which is quite small. September.

86. Quetsche, or Gernan Prune. Thomp.

Common Quetsclie.

True Larue German Prunc.

T'urk:sh Quetsche.

Leipz:c.

Sweet Prune.

Damask.
Zwetsche.

Quetwche Grosse.

I'ru' e d'Al'emagne.

Quetsche d'Allemagne Grosse. Themp Damats Gro:

Covetche.

Imperatrice Violetie.

Imp ra rice Violette Grosse.
Damas Violet Gros.

So many plums are cultivated under the name of German Prune, that it is difficult to fix this fickle tille, a circumstance owing to the fact that the prune frequently comes the same, or nearly the sane, from seerl, and in prune growing districts this is a popular way of increasing them, while it, of enurse, gives rise to many shades of character. It is a valuable class of piums, of fair quality for the table. but most esteemed for dry 
ing and proserving-abundant bearers, and hangring long on the tree. The common German Prune is described as follows.

Branches sumoth. Fruit long-oval, near two inches long, peculiarly swollen on on? sids, and drawn out towards the stalh. Suture distinctly marked. S'in purple, with a thi,k bilı blonm. Stalk three-fourths of an inch long, slender, slishily inserted. Flesh firm, green, sweet and pleasant, separisles from the stone, which is flat, very long, and a little curved. Ripens about the 10th of Septernber.

This prune is, perhaps, the most universal and most valuzble fruit tree in Germany, Hungary, Saxony, and all central Europe. Preserved, it is used in winter as a substitute for butter, by the laboring peasantry; and dried, it is a source of large profit in cominerce. In this country, it is yet but little known, but from tha great hardiness and productiveness of the tree, it may be worth trial on a large scale.

The Austrian Quetsche, Thomp, (Quetsche de Brême, Bre men Prune, is a sub.variety, much like the formoing, purple, a freestone, of rather better flavour, and ripening somewhat later.

St. JAMes' Quetsche, is anther variety, with smooth branch. es, and oblong fruit of medium size; flesh purple, adheres to the stone, of very good havour. It yields great crops. September.

\section{Royale. O. Duh. Thomp. Nois.}

La Royale. Lind. Hooker.

The Royale, a French variety, is undoubtedly one of the richest plums. It is peculiarly crisp, with a very high flavour, and is remark. able for the exceedingly thick coat of bloom which covers the skin. The tree is a slow grower, forms a bushy, spreading head, and its very downy shoots have a gray or whitish appearance. It bears regularly, but moderately, and, though not fit for the orchard, it is a first rate garden fruit.

Fruit of medium size, often quite large; round, lessening a little to. wards the stalk. Suture distinct at the apex on one side only. Skin

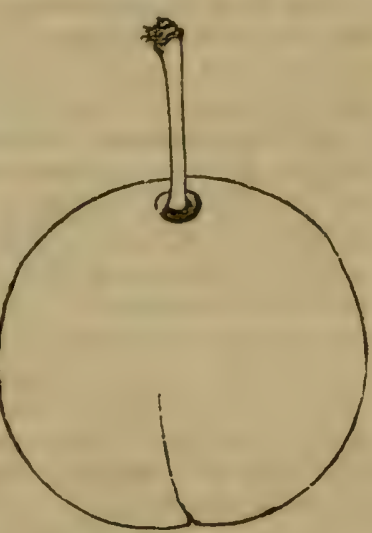

Fig. 1:9. Royale. redulish-purple, dotted with light brown specks, and covered with a thick pale bloom, which adheres closely. Stalk three. fuurths of an inch long, downy, set in a narrow cavity. Flesh 
dull yellow, rather firm, but melting, very juicy, with an ex. ceedingly rich, vinous flavour; it separates from the stone, which is small, roundish, pointed at both ends. Ripe the last of August, and will hang, dropping gradually, till the middle of September.

\section{Red Perdrigon. Lind. Fors.}

Perdirigon Rouge. Nois.

An agreeable late French plum, which hangs a good while on the tree, improving in flavour, till it becnmes very sweet and excellent. It appears to be a very hardy grower.

Branches downy. Fruit of medium size, mundish, slightly oval. Skin at first pale, but becoming fue deep red, dotted with fawn-coloured specks, and dusted with much lilac bloom. Stalk an inch long, rather stout, set in a small round cavity. Flesh bright yellow, a little crisp and firm, quite juicy and siveet, and parts freely from the stone. Last of August to the middle of September.

89. Red Magnual Bovun. Lind. Thomp. Mill.

Purple Egg̣.

Red Imparial.

Imperial.

Purple diagnum Bonum.

Flurence.

Imperial Violat.
Impériale Violette.

Impériale Rouge.

Dame Aubert Vivlette.

Impériale.

Prune d'ceuf.

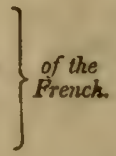

'The Red Magnum Bonum is a large, handsome, egg-shaped fruit, seen in abundance in cur markets, and chiefly valued for cooking and preserving, being rather harsh for the dessert. In fine dry seasons, it becomes of tolerable flavour.

Branches smooth. Fruit large, much like the White Magnum Bonum in form, oval, with a strong suture, on one side of which the fruit is more swollen. Skin rather pale in the shade, but deep red in the sun, sprinkled with many gray dots, and dusted vith but little pale bloom. Stalk an inch or more long, slender, set in a narrow cavity. Flesh greenish, rather firm and coarse, with a sub-acid flavour, separating from the stone, which is oval and pointed. Last of Aurust and first of September.

It is proper to state here, that this plum has been several tin:es reproduced from secd, on the North River, and with little difference of character, except that some are freestones and others clingstones.

Duane's purple is often confounded with the Red Magnum Bonum. It is a much better plum, and is easily distinguished, even when not in fruit, by its very gray, downy shoots. 
90. Royale de Tours. $\S$ O. Duh. Poit. Thomp.

Royal Tours.

This capital, early plum, from the neighbourhood of 'Tours, in France, is yet very scarce in this country, (two or three spurious sorts having been received by this name, ) but deserves to become generally known and cultivated. Its flavour is of the finest, and it commences ripening at the last of July, before most of the fine varieties.

Branches always quite downy. Fruit large, roundish, but marked with a large and deep suture extending quite half round, anil enlarged on one side. At the apex is a small white depressed point. Skin lively red in the shade, deep vio. let in the sun, with many minute golden dots, and coated with a thick blue bloom. Stalk half to three-fourths of an inch ling, stout, set in a narrow cavity. Flesh greenish, rather firm, with a rich, high flavoured, abundant juice. It adheres closely to the stone, which is large, oval, and flattened.

\section{Royale Hâtive. $§$ Thomp. Nois.}

\section{Early Royal.}

\section{Mirian.}

A new early plum of French origin, and the highest excellence. It is yet very scarce with us, having lately been re. ceived from the garden of the London Horticultural Socis:y. It strongly resembles, both in appearance and flavour, the Purple Gage, or Reine Claude Violette, but ripens a month earlier.

Branches very donony. Fruit of medium size, roundish, a little wiler towards the stalk. Skin light purple, dotted, (and faintly streakef,) with brownish-yellow, and covered with a blue bloom. Stalk half an inch long, stout, inserted with little or no depression. Flesh amber yellow, with an unusually rich, high flavour, and parts from the stone, (adhering slightly, till ripe.) Stone small, flattened, ovate. Begins to ripen about the 20th of July.

\section{RED GAGE. $§$ Pom. Man.}

An American plum, of delicious flavour, very hardy, and a prodigious bearer. It is a seedling raised from the Green Gage, by the elder $\mathrm{Wm}$. Prince, of the Flushing Nurseries, in $\mathbf{1 7 9 0 .}$ Ii grows very vigorously, and is distinguished, when young, by ts deep green, crimped foliage.

Branches dark reddish, smooth. Fruit about as large as tho 


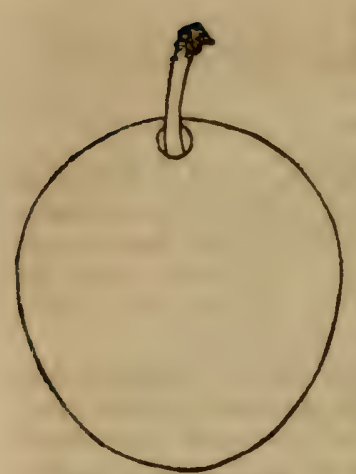

Green Gage, but more oval, regularly formed. Skin brownish or briek red: with little bloom. Stalk rather slen. der, set in a narrow cavity. Flest: greenish-amber, very juicy, meling, sugary, and luscious. It paris lirecly from the stone, which is small. Mid. dle of August.

['This is quite distinct from the LoNg Scarlet, (which see,) sometimes called Red Gage, or Scarlet Gage.]

Fig. 130. Red Gage.

\section{RIVER's EARLY.}

Two new seedlings raised by $\mathrm{Mr}$. Thomas Rivers, an English nurseryman of reputation. Their parent was the Précnce de Tours, but they are said to be earlier, hardier, and more prolific than that varjety.

"River's Early, No. 1," has downy shoots; No. 2, hiod smooth shining shoots. Both bear oval, purple fruit, of medium size; flesh yellow, sweet and excellent. They ripen the last of July.

94. Suisse. Thomp. Poit.

\section{Simiana.}

Monsieur Tardif.

Prune d'Altesso.

Prune Suisse.

Swiss Plum.

A handsome October plum, bearing some affinity to the $\mathbf{S t}$. Martin, or Coe's Late Red, and ripening about the same time, or a little earlier. It is very different from the oval plum, in. correctly known as the Semiana about Boston.*

Branches smooth. Fruit globular, rather large, with a broad shallow suture on one side, and terminating in a depressed point. Skin pale red in the shade, but lively violet red in the surn, dot. ted with numerous specks-a little marbled, and coater with a thick blue bloom. Stalk nearly an inch long, pretty stout, set in a wide hollow. Flesh greenish.yellow, crackling and meit. ing, with a brisk, rich flavour, in which there is a slight, but pleasant sharpness. It adheres to the stone, which is thick, with a rough edge. September, to the middle of October 
95. Sirarp's Emperor. Thomp.

Denyer's Victoria?

Quetn Victuria?

A brautiful new plum from Enerland, which will prors az eddition to our collection. It bears abundautly, and has a peculiarly tender stone.

Branches stroner, downy, and foliage, arere. Fruit quite large, roundish oval. Skin, whon exposed, of a fine br wht, lively red, paler in the shade, with a delicate bloom. Flesh decp ycllow, separates from the stone, of a pleasant, moderately' rich flavour. Middle and last of Septomber.

l)enyer's Victoria rescmbles this, but we require another trial before pronouncing them identical.

96. Thontas.

A new plim, of a lively, drop salmon colour, with a red cheek; a very attractive contribution to the dessert. thouen not of first rate flavour. It is a native variety, and the fruit was first exhibited by $\mathrm{Mr}$. W Wm. 'Thomas, of Boston. who has a fine tree in his garlen. It was thence named the Thomas Plum, by the Massachusetts Horticultural Society. It has sorne resem. blance to Sharp's Emperor.

Branches slightly downy. Fruit large, roundish-oval, a little irregular, and rather compressed in the dircetion of the suture. Stalk hairy, half an inch or more, long, stout, set in a small narrow cavity. Skin salmon colour, with numerous dots, and a soft red cheek. Flesh pale yellow, a little coarse grained, but with a milsl, pleasant flavour, separating frecly from the stone. The stone is peculiarly light coloured. Ripe the last of August, and bears adinirably.

\section{VIRGIN. Thomp.}

Lately received from England, where it has the reputation of bearing "some resemblance to the Reine Claure Violette, thoug! scarcely so rich." It has not yet been tested here.

Branches smooth. Fruit of medium size, roundish. Shm purple. Flesh yellow, of rich flavour, and separates from tho stone. Ripens the last of August and beginning of September.

Ornamental Varieties.

There are few varieties of plums, which are considered 
purcly ornamental. One, however, is a remarkable exception to this, as it is scarcely exceeded in beauty in the month of May by any other flowery shrub-we mean the Doubie Frow. ERING SLOE. It is a la rige shrub, only $\mathbf{1 0}$ or 12 feet high, with quite slender shoots and leaves, but it is thickly sprinkled, every spring, with the prettiest little double white blossoms about as large as a sixpence, but resembling the Lady Banlis' roses. It is one of the greatest favourites of the Chinese and Japanesethose flower-loving people.

The Common Exglish Sroe, or Blackthorn, (Prunus spinosa,) is rather an ornamental tree in shrubbery plantations. The branches are more thorny than those of the common damson, and the fruit is nearly round, quite black, but covered with a thick blue bloom. In the spring, this low tree is a perfect cloud of white blossoins.

'The Double blossonied Plun has large and handsome, double white towers. Except in strong soils, however, they a re apt to degenerate and become single, and are, indeed, always inferiour in ellect to the Double Sloe.

'The Cherry Plum we have already described. It is one of the fruit bearing sorts.

1. Selection of choice varielics for a small garden. Royal Hätive, Hudson Gage, Green Gage, Jefferson, Lawrence's Fa. rourite, Huling's Superb, Purple Favourite, Purple Gage, Coe's Golden Drop.

2. Platms that will bear uell in light soils, and generally withstand the curculio. Lombard, Cruge:'s, Blue Gagce, Roe's Iutumn Gage, Red Gage, Long Scarlet, Bleccker's Gage, Coe's Folden Drop, and all the Damsons.

3. Plums suitable for a cold northern climate. Smith's Urleans, Bleecker's Gage, Denniston's Superb, Corse's Nota Bene, Orleans, Cruger's Scarlet, WVashington, Duane's Purple.

4. Plums suit ible for a southern climate. Bingham, Imperial Garre, Washington, Larre Long Blue, Huling's Superb, Coe's Lrote Red; Joe's Golden Drop.

\section{SHAPTER XXI.}

\section{THE PEAR.}

Pyrus communis, I. Rosacea, of botanists.

Ponre, of the French; Birndar m. German; Peer, Dutcls: Perc Italian; and Pera, Spanish.

Tre Pear is, undeniabiy, the favourite fruit of modern tinzes, and modern cultivators. Indeed, we believe the Pear of modern 
times, thanks to the science and skill of horticulturists. is quite a different mors'l for the palate, from the par of iwro or three centuries ago. In its wild state it is one of the most austere of ali fruits, and a choke pear of our fields, really a great improve ment on the wild type, seizes ones throat with such an unnerciful gripe, as to leave behind it no soothing remembrances of nectar and ambrosia.

So long agu as the earliest time of the Romans, the pear was considerably cultivated. It was common in Syria, Egvpt, and Greece, and from the latter country, was transplanted into Italy. "Theophrastus speiths of the productiveness of old pear trees, and Virgil mentions snme pears which he received from Cato. Pliny in his 15 th book describes the varieties in cultivation in his time, as exceedingly numerous; and mentions a number which were named after the countries from which they were rec-ived. Of ail pears, he says, the Costumine is the most de. licute and agreeable. The Ealernian pear was esteemel for its juice; and the Tibernian, because it was preferred by the Emperor Tiberius. There were 'proud pears' which were so. called, because they ripened carly and would not keep, and 'winter pears,' pears for baking, as at the present day."* None of these old Roman varieties have been handed down to us, and we mirht believe some of them approached the buttery lusciousness of our modern pears, did not Pliny pithily add, most unfor. tunately for their reputation, "all pears whatsonver are but a heavy meat, unless they are well boiled or baked."

In fact the really delicious qualities of this fruit wore not developed until about the seventeenth century. And within the last sixty year's the pear, subjected to constant reproduction from seed by Van llons and his followers, and to hybridizing or erossing by Mr. Knight and nther English cultivators appears, at lenghth, to have reached almost the summit of perfection, in beauty, duration, and havour. Of l'rofessor Van Mons ant this labours of a whole life, almost devoted 10 pears, we have alrearly spoken in our first chapter. From amoner the 80,030 seedliners raised by hinself, and the many thousands reared by other zeal. ous cultivators abroad, especially in B lgium-the Eilen of the pear tree-there have been selected a large number of variftics of high excellence. In this country, we are continually adding to the number, as, in our newer soil, the pear, following the natural laws of successive reproduction, is coustantly appearing in new seedling forms. The high flavour of the Seckel pear, an American variety, as yet unsurpassed, in this reopect, hy any Eu:npean sort, proves the natural congeniality of 11 .. cli. mate of the nort Iern states to this fruit.

'The pear tree is not a native of North America, but was in.

- Arboretum Brittanicrem. $27 *$ 
troduced from the other continent. In Furope, Western Asia, and China, it grows will, in company with the apple, in herlges and wouly wastes. In its wild state, it is hardier an! longer lived lhan the apple, making a taller and more pyramilal head, and becomingr thicker in its trunk. There are trees on record abroal, of great size and arge for fruit trees. M. Bose mentions several which are known to be near 400 years old. 'There is a very extraordinary tree in Horne Lacy, Herefordshire, Englanil-a perry pear-from which were made more than once, 15) horshearls of perry in a single year. In 1805 it covered more thit half an acre of land, the branches bending down and taking root, and, in turn, producing others in the sane way. Louilon, in his recent work on trees, says that it is still in fine health, though reciuced in size.

One of the most remarkable pear trees in this country, is growing in Illinois, about ten miles north of Vincennes. It is not believed to be more than forty years old, having been planted by Mrs. Ockletree. The girth of its trunk one font above the irround, is ten feet, and at nine feet from the grouml, six and a half feet; ; and its branches extend over an area sixty-nine feet in diameter. In 1834 it yielded 184 bushels of poirs, in 1840 it yielded 140 bushels. It is enormously proiluctive always; the fruit is pretty large, ripening in early autu:nn, and is of tolerable flavour.* Another famous sp:cimen, perhaps the olilest in the country, is the Stuyvesant Pear tree, originally planted by the old governor of the Dutch colony of New-York, more than two hundred vears ago, and still standing, in fine vigour, on what was once his farm, but is now the upper part of the cify, quite thickly covered with houses. The fruit is a pleasant summer pear, somewhat like a Summer Bonchretien.

Usf.s. 'The great value of the pear is as a dessert fruit. Next to this, it is highly esteemed for baking, stewing, preserv. ingr and inarmalades. In France and Belgium the fruit is very generally dried in ovens, or much in the same way as we th the apple, when it is quite an important article of food.

Dessert pears should have a melting, soft texture, and a sugary, aromatic juice. Kitchen pears, for baking or stewing, should be large, with firm and crisp flesh, moderately juicy.

The juice of the pear, fermenter, is called Perry. This is made preciscly in the same way as cider, and it is richer, and more estecmed by many persons. In the midland coun. ties of England, and in various paris of France and Germany, what are called perry pears-very hardy productive sorts, hav ing an austere juice-are largely cultivated for this purpose In several places in our eastern states, we understand, perry is now annually made in considerable quantities. 'The fruil

* Rev. II. W. Beecher, in Hovey's Magazine. 
should be ground directly after being gathered, and requires rather nore isinclass-(say $1 \frac{1}{2} \mathrm{oz}$. to a barrel, ) to fine it, on racking, than cider. In suitable soil the yield of perry to the acre is usually about one third more than that of cider.

'ithe wood is heavy and fine grained, and makes, when stain. ed black, an excellent imitation of ebony. It is largely em. ployed by turners for making joiners' tools. The leaves will dye yellow.

Gathertag and keeping the fruit. The pear is a pecu. liar fruit in one respect, which should always be kept in mind; viz. that most varieties are much finer in flavour if picked from the tree, and ripened in the house, than if allowed to become fully matured on the tree. There are a few exceptions to this rule, but they are very few. And, on the other hand, we know a great many varieties wrich are only second or third rate, when ripened on the tree, but possess the highest and richest flavour if gathererl at the proper time, and allowed to mature in the house. This proper season is easily known, first, by the ripening of a few full grown, but worm-eaten specimens, which fall sonnest from the tree; and, secondly, by the change of colour, and the readiness of the stalk to part from its branch, on gently raising the fruit. The fruit should then bo gathered-or so much of the crop as appears sufficiently matured-and spread out on shelves in the fruit room* or upon the floor of the garret. Here it will gradually assume its full colour, and become de. liciously melting and luscious. Many sorts which, ripened in the sun and open air, arc rather dry, when ripened within door, most abundantly melting and juicy. They will also last for a considerably longer period, if ripened in this way-maturing grarlually, as wanted for use-and being thus beyond the risk of loss or injury by violent storms or high winds.

Winter dessert pears should be allowed to hang on the tree as long as possible, until the nights become frosty. They should then be wrapped separately in paper, packed in kegs, barrels, or smiall boxes, and placed in a cnol, dry room, free from frost. Some varieties, as the D'Aremberg, will ripen finely with no other care than placing them in barrels in the cellar, like apples. But most kinds of the finer winter dessert pears, should be brought into a warm apartment for a couple of weeks before their usual season of maturity. They should be kept covered, to prevent shrivelling. Many sorts that are comparatively tough if ripened in a cold apartment, become very melting, buttery and juicy, when allowed to mature in a roor kept at the temperature of 60 or 70 degrees.

* So important is the ripening of pears in the house that most amateurs of this fruit, find it to their advantage to lave a small room set apart, and fitted up with shelves in tier, to be used solely as a fruit room. 
Propagation. The finer sorts of pears are continued or in creased, by grafting and budding, and the stocks, on which to work, are either seedlings or suckers. Sucker stocks have usu ally such indifferent roots, they are so liabie to produce suckers, continually, themselves, and are so much less healthy than seed. lings, that they are now seldom used by good cultivators; though, if quite young and thrifty, they will often make good stocks.

Seedlings, however, are, by far, the best stocks for the pear, In all cases; and seedlings from strong growing, healthy pears, of common quality - such as grow about most farmer's gardens, are preferable, for stocks, to those raised from the best varie. ties-being more hardy and vigorous.

As it is, usually, found more difficult to raise a good supply of seedling pear stocks in this country, than of any other fruit tree, we will here remark that, it is absolutely necessary, to ensura success, that two points be observed. The first, is to clean and sow the seed as soon as may be, after the fruit is well matured; the second, to sow it only in deep rich soil. It should be previously trenched-if not naturally deep-at least twenty inches or iwo feet deep, and enriched with manure or compiost mixed with ashes. This will give an abundant supply of uutriment to the young seedlings, the first year-without which, they becone starved and parched, after a few inches growth, by our lot and dry summer, when they frequently fall a prey to the aphis and other insects at the root and top. A mellow, rich soil, whose depth ensures a supply of moisture, will give strong seedlings, which are always, at two years growth, fit to go into the nursery rows for budding. While a dry, thin soil, will seldom produce good stocks, even in half a dozen years.

The seeds should be sown precisely like those of the apple, in broad drills, and the treatment of the stncks, when planted in the rows for budding, is quite similar. Buddiug is almost uni. versally preferred by us, for propatating the pear, and this tree takes so readily, that very few failures can happen to an experienced hand. Abnut the first of August, in this latitude, is the proper season for performing this operation.

We may add here, that one ycar old pear seedlings, a re often winter-kiljed, when the autumn has not been such as to ripen the wood thoroughly. A few branches of evergreens, or some slight covering laid alone the rows, will prevent this. Or, they may be laid in by the heels, in a sheltered place.

The thorn makes very good stocks for the pear, except, that if grafted above ground, the tree is often apt to be broken off at the point of union, by high winds. This is obviated by grafting a little below the surface. Grafting on the thorn is a very use. ful practice for strong clayey soils, as, on such stocks the peas may be grown with success, when it would not otherwise thrive 
It also comes rather earlier into bearing. Grafting on the mounitin ash is thought to render the pear more hards. and it retaris the blosinming so much as to prevent their being in. jurer by spring frosts. The pear is sometimes budded on the apple, but it is then usually very short-lived.

For rendering the pear dioarf, the Quivce stock is almosi universally used, as the pear unites readily with it, becomes quite divaif in habit, and bears very early. Som? large grow. ing pears-as the Duchess of Angouleme-extremely liable to be blown off the tree, bear much better on the Quince strock, and others are considerably improved in flavour by it. The divarf par, lowever, it must be confessed, rather belongs to the small garden of the amateur, than to the orchardist, or him who desires to have regular large crops, and long-lived trees. The dwarf tree is usually short.lived, seldom enduring more than a dozen years in bearing-but it is a pretty, and eco. nomical way of growing a good many sorts, and getting fruit speedily, in a small garden.

The pear not being very abundantly supplied with fibrous mots, should never be transplanted, of large size, from the nursery. Sinall, thrifty plants, five or six feet high, are much to be preferred.

SolL, SITU' toN, AND CILTURE. The best soil for this fruit tree, is a strong loam of moderate depth, on a dry subsoil. The pear will, indeed, adapt itself to as great a variety of soils as auy fruit trec, but, in unfavourable soils, it is more liable to sulfer from disease, than any other. Soils that are damp during any considerable portion of the year, are entirely unfit for the pear tree; and soils that are over-rich and deep, like some of the western alluvials, force the tree into such over luxuriant growth, that its wood does not ripen well, and is liable to be killed by winter blight. The remedy, in this case, consists in planting the trees on slightly raised hillocks $\rightarrow$ say eight inches above the level of the surface, and using lime as a manure. Soils that are too light, on the other hand, may be improved by trenching, if the subsoil is heavier, or by top dressing with heavy muck and river mud, if it is not.

In a clinate rather cold for the pear, or on a cold soil, it is advantageous to plant on a southern slope, but in the middle states, in warm soils, we do not consider a decidedly southern expcsure so good as other, rather cooler ones.

The pear succeeds so well as an open standard, and requires so little care or pruning-less, indeed, in the latter respect, than any other fruit tree, that training is seldom thought of, except ir the gardens of the curious or skilful. The system of quenouille or disiaff training, an interesting mode of rendering trees very productive in a sinall space, we have already fully described in $\mathrm{p} . \mathbf{3 7}$, as well as root pruning for the same purpose in p. 32 
In orchard culture, the pear s usually planted about thirty feet listant rach way; in frui: gardens, where the hearls aro somewlat kept in by pruning, 20 feet is considered sufficient by many.

Pear trees in a bearing state, where the growth is no longer luxuriant, should have, every autumn, a moderate top dressing of manure, to keep them in goed condition. This, as it promotes stealy and regular growth, is far preferable to occasioual heavy manuring, which, as will presently be shown, has a tendency to induce the worst form of blight to which this tree is subject.

Diseases. As a drawback to the, otherwise, easy cultivation of this fine fruit, the pear tree is, unfortunately, liable to a very serious discase, called the pear tree blight, or fire blighl, appearing irregularly, and in all parts of the country; sometimes in succeeding seasons, and, again, only after a lapse of spveral years; attacking, sometimes, only the extremities of the limbs, and, at other times, destroying the whole tree; producing, occa. sionally, little damage to a few branches, but often, also destroy. ing, in a day or two, an entire large tree; this disease has been, at different times, the terror and despair of pear growers. Some parts of the country have been nearly free from it, while others have suffered so much as almost to deter persons from ex. tending the cultivation of this fine fruit. For nearly an hundred years, its existence has been remarked in this country, and, antil very lately, all notions of its character and origin have veen so vague, as to lcad to little practical assistance in removitig or remedying the evil.

Careful observation for several years past, and repeated com. parison of facts with accurate observers, in various parts of the country, have led us to the following conclusions:

$1 s t$, That what is popularly cilled the pear blight, is, in fact, two rlistinct disnases. $2 n d$, that one of these is caused by an insect, and the other by sudden freezing and thawing of the sap in unfivourable autumns. The first, we shall therefore call the insect blight, and the second, the frozen-sap blight.

1. 'The insect blight. The symptoms of the inser, bitight are as follows: In the month of June or July, when the rree is in full luxuriance or growth, shoots at the extrenities of the branches, and often extending down two seasons' growth, are ois. ${ }^{2}$ edel sudderily to turn brown. In two or three days the leaves become quite black and dry, and the wood so shrivelled and hard as to be cut with difficulty with a knife. If the branch is aliowed to remain, the discaso sometimes extends a short dis. tance further down the stem, but, usually, not much furthe: that the point where the insect harl mate his lodernent. "The ins:ent

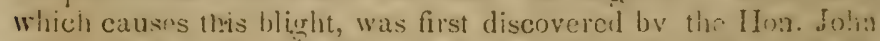
Lowell, of Buston, in 1816, and was described by Professor ['eck; ander the nams of Scolytus pyri. It is very minute, heing 
scarcely one-tenth of an inch long; and it escapes from the branch almost as soon as, by the withering of the leaves, we are aware of its attack; hence, it is so rarely seen by careless obscrvers. In the perfect state, it is a very small beetle, deep brown, with legs of a paler colour. Its thorax is short, convex, rough in front, and studded with erect bristles. The wing covers are marked with rows of punctured points, between which are also rows of bristles, and they appear cut off very obliquely behint.

'This insect deposits its egr some time in July or August, eithes behind, or below a bud. Wherher the egg hatches at once, wo are $n \mathrm{t}$ aware, but the following spring, the small grub or larva grows throw the sap wood or tender alburnum, beginning at the root of the bud, and burrows towards the centre of the stem. A round this centre or pith, it forms a circular passage, some. times devouring it altogrether. By thus perforating, sawing off, or girdling, internally, a considerable portion of the vessels which convey the ascending sap, at the very period when the rapid growth of the leaves calls for the largest supply of fluid from the roots, the growth and the vitality of the branch are checked, and finally extinnuished. The larva about this time, completes both its transforination, and its passage out, and, in the beetle form, emerges, with wings, into the air, to seek out new positions for laying i:s eggs and continuing its species. The small passage where it makes its exit, may now more easily be disco. vered, bolow (r) by the side of the bud, resembling a hole bored with a nendle or pin.

It is ' $v e l l$ to remark here, that the attack of this blight insect is not crinfined to the pear, but in some parts of the country we have ohserved it preying upon the apple and the quince in the same manner. In the latter tree, the shoots that were girdled, were shorter, and at the extremities of the branches only; not learlirs, therefore, to such serious consequences as in the pear.

The ravages of the insect blight, we are inclined to think, do not extend much below the point where the insect has deposited its $\mathrm{egg}$, a material point of difference from the frozen-sap blaght which often poisons the system of the whole tree, if allowed to rcurain, or if, originally, very extensive.

2he remerly for the insect blight is very distinct. It is that originally suggested by Mr. Lowell, which we and many others nave pursued wich entire success, when the other form of the discase was not also present. This remedy consists, at the very first indications of the existence of the enemy, in cutting off and hurning the diseased branch, a foot below the lowest mark of discoloration. The insect is usually to be found at the bottom of this blackened point, and it is very important that the branches be removed early, as the Scolytus is now about emerging from his burrow, and will speedily escape us, to multiply his mischief 
elsewhere. If there is much appearance of the insect blight, the tree should be examined every noon, so long as there are any indications of disease, and the amputated branches carried at once to the fire.

II. The FROzen SAP BLight. We give this term to the most formidable phase of this disease that affects the pear tree. Though it is, by ordinary observers, often confounded in its effects, with the insect blight, yet it has strongly characteristic marks, and is far more fatal in its effects.

The symptoms of the frozen-sap blight are the following. First; the appearance, at the season of winter or spring pruning, of a thick, clammy sap, of a sticky nature, which exudes from the wounds made by the knife; the ordinary cut showing a clean and smooth surface.

Second; the appearance, in the spring, on the bark of the trunk or branches, often a considerable distance from the extremities, of black, shrivelled, dead, patches of bark.

Third; in early summer months, the disease fully manifests itself by the extremities shrivelling, turning black, and decay. ing, as if suddenly killed. If these diseased parts are cut off, the inner bark and heart-wood will be found dark and discoloured some distance below where it is fresh and green outside. If the tree is slightly affireted only, it may pass oft with the loss of a few branches, but if it has been seriously tuinted, the disease, if not arrested, may, sooner or later, be carried through the whole system of the tree, which will gradually decline, or entirely perish.

To explain the nature of this disease, we must first promise that, in every tree, there are two currents of sap carried on, 1st, the upward current of sap, which rises through the outer wood, (or alburnum,) to be digested by the leaves ; 2nd, the downward current, which descends through the inner bark, (or libar,) furming a deposite of new wood on its passage down.*

Now let us suppose, anterior to a blight season, a very sudden and early winter, succeeding a damp and warm autumn. $\dagger$ 'The summer having been dry, the growth of trees was completed early; but this excess of dampness in autumn, forces the trees into a vigorous second growth, which continues ate. While the sap vessels are still filled with their fluids, a sharp and sud. den freezing takes place, or is, perhaps, repeated several times, followed, in the day time, by bright sun. The descending current of sap becomes thick and clammy, so as to descend with difficulty; it chokes up the sap-vessels, freezes and thaws

\footnotetext{
- Being distributed towards the centre of the stem by the meduliary ray which communica: efr m the inner bark to the pith.

+ Which always happen: previously io a sunmer when the blight. is very pre valent and will b:- remeinhsred, by all, as haviug heen especially the cane in the eutum, of 1813 , whicts procoded the extensive blight of the past season.
} 
agam, loses its vitality, and becomes dark and discolourer, and in somn cases, so poisonous, as to destroy the leaves of other plants, when applied to them. Here, along the inner bark, it lodyes, and remains in a thick, stıcky state, all winter. If it happens to flow down till it meets with any obstruction, and re. mains in any considerable quantity, it freezes again beneath the bark, ruptures and destroys the sap-vessels, and the bark and some of the wood beneath it shrivels and dies.

In the ensuing spring, the upward current of sap rises through its ordinary channel - the outer wood or alburnum - the leaves expand, and, tor some time, nearly all the upward current being taken up to form leaves and new shoots, the trec appears flourishing. Toward the beginning of summer, however, the leaves commence sending the downward current of sap to increase the woody matter of the stens. This current, it will be remembered, has to pass downward, through the inner bark or liber, along which, still remain portions of the poisoned sap, arrested in its course the previous autumn. This poison is diluted, and taken up, by the new downward current, distributed toward the pith, and along the new layers of alburnum, thus tainting all the neighbouring parts. Should any of the adjacent sap-vessels have been ruptured by frost, so that the poison thus becomes mixed with the still ascending current of sap, the branch above it immediately turns black and dies, precisely as if poison were introduced under the bark. And very fre. quently it is accompanied with precisely the odour of decaying frost-bitten vegetation.*

The foregoing is the worst form of the diseas ${ }^{\circ}$ and it takcs place when the poisoned sap, stagnated under the bark in spots, remains through the winter in a thick semi-fluid state, so as to be capable of being taken up in the descending current of the next summer. When, on the other hand, it collects in sufficient quantity to freeze again, burst the sap vessels, and afterwards dry out by the influence of the sun and wind, it leaves the patches of dead bark which we have already described. As part of the woody channels which convey the ascending sap probably remain entire and uninjured, the tree or branch will

- We do not know that thi-form of hlight is common in Curope, be: the fol. lnwing extrut trom th- celebrated work of Duhamel on fruut trees, published in

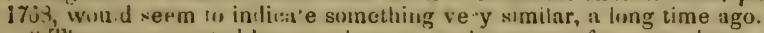

"Tlie sap currupted by putr.d willer. or the exie-s of manure bursts the rellu. far memhrities $i_{1}$ some places, extends itself helween the wond and the hath, whi.h it - ep:tra es, ant raries its pesismous as: iw intuence. to all the ncighbmuring parts, like a gan trene. When it attacks the smatl brameh.'s, they sh uid he cut off; if tt appears in the large branches or budy of the tree. al! the cankered pa:ts must be eat ont down to the somd womk and in womml covered with coms. po-ilius. If the evil be produred hy manure or alagnant water, (and it maly be poduces by other causes, the ald ear:t! mant he removert from ih roots, and fest soil pul in its plice and mea.1s taken to diaw off the water from the rowts

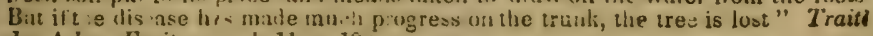
Les Arbres Fruitiers, v.1. 1!, p. 10 s. 
perhaps continue to grow the whole season and bear fruit, as if unthing had hapuened to it. drying down to the shrivelled spor of bar.: the next spring. 'The effect, in this cas', is precisely that of girdling only, and the branch or tree will die after a tims, but not suddenly.

From what we have said, it is easy to infer that it would not be dificult on the occurrener of such an autumn - when surdden congelation takes place in unripenerd wood-to prestict a blignt soason for the following summor. Such has several times been done, an l its fulfilment may he looked for, with certainty, in all trens that had not previously ripened their wood.*

So, also, it would and does naturally follow, that trees in a damp, ricn soil, are much more liahl - to the frozen sap blight, than those unon a dryer soil. In a soil over moist or too rich, the pear is always liable to make late second growths, and its wood will often be caught unripened by an early winter. For this reason, this form of blight is vastly more extensive and rle. structive in the deep, rich soils of the western states, than in the dryer and porer soils of the east. And this will always be the case in over rich soils, unless the trees are planted on raisel hil. locks, or their luxuriances checked by ront prunine.

Again, those varieties of the pear, which have the hahit of maturing their wool early, are very rarely affecterl with the frozen sap blicht. But late growing sorts, are always more or less liable to it, especially when the trees are young, and the excessive growth is not reduced by fruit-bearing. Every nurseryman knows that there are certain late growing sorts which are always more liable to this blight in the nursory. Among these we have particularly noticed the Passe Colmar and the Forelle, though when these sorts become bearing trees, they are

* Since the above was written, we have had th? pleasure of sceing a bighly intere ting att co hy tho kev. H. IV. Beedher, of Indiana, one of the most intelligeut observers in the cumrry. Mr. B esher mut only agrees in the main with us, but he fortifies our upition with a number of aclditimal fincts of great vaine. We shisl! e:slract sumo of lhis lestimony, which is vouched fur by MIr 13 , and for the publication of which the cultivators of pears owe him many thanks.

"Mr R. Reagan of Putnam county, Ind, has for more than twelvo years, susperted that this diseise uriginnted in the fall previous to the summer on whicb it d (Jires itself. During the last winter, Mr Reagan predicted the bligit, ns w II b. remembered by some of hi: arqualintances in Wayne Co., and in his peat urchard he maked the trees thit wusld snffer, and pointed to the spot whirh wuld be the seat of the disease, and his pugnomtirations were striclly verified. Out of his or.hard of 2.0 pear tree-, during the previous h ight of $18,2$. , ot: y finur escaped, and tliuse had been trans planted, and l.ad, therefore, mado litlle or nog owih.

Hi. Whi.e, a nurseryman, near Mnoresville, Ind, in an orrhard of orer 150 trees, hat not a cing'e rase of hliwht in the year 1814 , thongh all arumal him its ravazes w re telt. What were th: fic:ts in this ease? His orchard is planted on a mouml-like piece uf ground, is high, of a saudy, gravelly soil; earlier hy a week, than nursery suils iu this country; and in the summer of 1813, his trees grow through the summer, ripened and shed their leaves early in the fall, and curing the warm spell made no second growth." 
not morm lizuic tiran many others. The Seckel pear is cele brateu tor is gerseria freerlom from blight, which we attribute cutirely, to ths uavil of making short jointed shoots, ancl ripening its wona very early.

To distingusti vise bigit of the frozen sap from that causea by the attack or the jeyluzes pyri, is not difficult. The effects of the latter cease betuw the spot where the insect has perforated and eaten its Durrow in the brancls. 'The former spreads gradually down the bianch, which, when dissected, shnws the marks of the puisus in the discoloration of the inner bark and the pith, exterung down some distance below the external marks of injury. If the poison becomes larg ly diffused in the tree, it will sometımes die outright in a day or two; but if it is only slightly present, it will often entirely recover. The presence of black, dry, snrivelled spots of bark on the branches, or soft sappy spots, as well as the appearance of thick clainmy sap in winter or spring pruning, are the infallible signs of the frozen-sap blight.

The most successful remedies for this disastrous blight, it is very evident, are chiefly preventive ones. It is, of course, impossi. ble for us to avoid the occasional occurrence of rainy, warm autumns, which have a tendency to urge the trees into late second growth. The principal means of escaping the danger really lies in always studiously avoiding a damp soil for the fruit tree. Very level or hollow surfaces, where heavy early autumnal rains are apt to lie and saturate the ground, should also be shunned. And any summer top dressing or enriching, calculated to stimulate the tree into late growth, is pernicious. A rich, dry soil, is, on the whole, the best, because there the tree will make a grood growth in tirne to ripen fully its wood, and will not be likely to make second growth. A rich, moist soil, will, on the contrary, serve continually to stimulate the tree to new growth. It is in accordance with this, that inany persons have remarked, that those pear trees growing in common mealow land, were fiee from blight in seasons when those in the rich garden soils were continually suffering from it.

The tirst point then should be to secure a rich but dry, well drained soil. Cold aspects and soils should be avoided, as likely to retard the growth and ripening of the wood.

The second is to reject, in blighted districts, such varieties as have the habit of making wond late, and chonsing rather, these of early habit, which ripen the wood fully before autumn.

Severe summer pruning, should it be followed by an early winter, is :ikely to induce blight, and should therefore be avoid. ed. Indece, we think the pear should always be pruned in winter or early spring.*

- The only sev sre case of blight in the gardans here, during the summer of 184 , was in the head of a Gilogil pear-a very hardy sort, which had never be 
As a remedy for bligh. actually existing in a tree, we know of no other but that of firely cutting out the diseased liranches, at the earliest moment after it appears. The amputatiou should be contunued as far down as the least sign of discoloration, and consequent poisoning is perceptible, and it should not be neg. lected a single day after it manifests itself. A still better remedy, when we are led to suspect, during the winter, that it is likely to break out in the ensuing summer, is that of care. fully looking over the trees before the buds swell, and cutting out all branches that show the discoloured or soft sappy spots of bark that are the first symptoms of the disease.

Finally, as a proventive, when it is evident, from the nature of the scason and soil, that a late autumnal growth will take place. we recommend laying bare the roots of the trees for two or three weeks. Root pruning will always check any tendency to over-luxuriance in particular sorts, or in young bearing trees, and is therefore a valuable assistance when the disease is fear. ed. And the use of lime in strong soils, as a fertilizer, instead of manure, is worthy of extensive trial, because lime has a tendency to throw all fruit trees into the production of short. jointed fruit-spurs, instead of the luxuriant woody shoots in. duced by animal manure.

In gardens, where, from the natural dampness of the soil $n$ locality, it is nearly impossible to escape blight, we recommend that mode of dwarfing the growth of the trees-eonical standarls, or quenouilles, described in the section on pruning. 'This mode can scarcely fail to secure a good crop in any soil or climate where the pear tree will flourish.

After the blight, the other diseases which affect the pear tree are of little moment. They are chiefly the same as those tc which the applo is liable, the same insects occasionally uffecting both trees, and we therefore refer our readers to the section on the apple tree.

There is, howevor, a slug worm, which occasionally does great damage on the leaves of the pear tree, which it sometimes entirely destroys. This slug is the Selandria cerasi of Harris. It appears on the upper side of the leaves of the pear tree, fron the middle of June till the middle of July. It is nearly half an inch long when fully grown, olive coloured, tapering from the head to the tail, not much unlike in shape a miniature tarlpole. 'The best destructive for this insect is Mr. Haggerston's mixture of whale oil soap and water, ${ }^{*}$ thoroughly showered or sprinkled over the leaves. In the absence of this, we have found ashes or quicklime, sifted or sprinkled over the leaves, early in tho

fore sutfered. The previous midsummer it had bicn severely pruner, and head back. which threw it in:o late growth. The next season nearly the who's remaining part of the tree died with the frozen-say blight.

- See page 5 . 


\section{morning, to have an excellent effect in ridding the trees if this} vigilant enemy.

VARIETIES. T'he varieties of pear have so multiplied withiu the last thirty yea s, that they mi.y almost be considereu eud. less. Of the new varieties, Belgium has produced the greatest number of high quality. England and France many of excel. lence; and, lastly, quite a number of valuable sorts have ori. ginated in this country, to which some additions are mado annually. 'l'he latter, as a matter of course, are found even more generally adapted to our climate than any foreign sorts. But we believe the climate of the middle states is so nearly like that of Belgium, that the pear is grown here as a standard to qs great perfection as in any other country.

More than 700 kinds of pears, collected from all parts of the world, have been proved in the celebrated experimental garden of the Horticultural Society of London. Only a small proporion of these have been found of first rate quality, and a very arge number of them are of little or no value. 'The great tifficulty, even yet, seems to be, to decide which are the reallyvaluable sorts, worth universal cultivation. We shall not, per. haps, arrive at this point, in this country, for several yearsnot until all the most deserving sorts have had repeated trialsand the difficulty is always increased by the fact of the diforence of climate and soil. A variety may be of second quality in New-England, and of the first merit in Pennsylvania or Ohio. This, however, is true only to a very limited extent, as the fact that most sorts of the first character receive nearly the same praise in Belgium, England, and all parts of this country, clearly proves. High flavour, handsome appearance, productiveness, and uniformly good flavour in all seasons-these are the cri terions of the first class of pears.*

There is an idea prevalent, which has been greatly extended by Kenrick, in his American Orchardist, that all the finest old varietie; of pears are worthless and unfit for cultwanum, by reason of their dengeneration. It is but justice to say that this 130. tion owes its origin to $\mathbf{M r}$. Knight, but $\mathbf{M r}$. Kenrick living licar the sea-coast, in a climate, naturally rather unfavourable to the pesir, has fortified it by what he has observed in his own neigh. bourhood, forgetting that facts in the country at large, do not bear testimony to the doctrine. W' should be glad to show

- The mosi successful rultuvator of penrs in this country, whose collection comprises hundreds of varieties, lately assured us, that if he were asked io name all the sirts that he co:tidered of anvarying and unqu'stionable excellence in all re

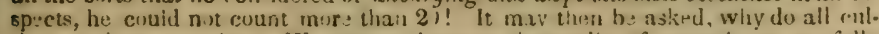
tivate so larre a variety. We answ r. beranve the quality of many is yet not fully decided; again, there is a great diff rione. in taste, as to the merits of a given sort; there are alou some sorts si profuctive, or hand:ams, \&ce.. that they are highly esteemed, thoury only second rate. In a work like the present. wa are also ibliged to describs many sorts of seenal ifuality, in order to assist in identi fying them, as diey are already in general cultivatima. 
him here, in the pear scason, a great many varieties, which he boldly dennunces as "rejected nutcasts," hearing as handsnme an! abuindant crnps as any kinds originated withm the last ten years. IVe shall recul to this sulject nore at lengh, hrere. after, and will only state now, that by propagation on unhralihy stocks, in a bad soil or climate. many sorts of pear liave becone so eufeebled, as to be nearly worthless, near the sea-coastwhere, indeed, only the hardier sorts will long cortinue fair and excellent. On the other hand, the same sort, (if the tree has not been brought already diseased from the sea-board.) will thrive and bear with all its natural vitrour in the interiour. And, finally, we have observed, that some of the newest Flemish p'ars, b:ing naturally of feeble habit, already show the same marks of decay or want of vigour as the oldest sorts.

In describing pear's, we shall, as usual, designate the size by comparison, as follows. Largr, as the Beurré Diel or Bartlett; morlium, as the Doyenné or Virgalieu; small, as the S.ckel. IVith regard to form, pyriform, as the Beurré Bose; obtuse pyriform, as the Bartlett; obovate, (egg-shaped reversed,) as the Inyenné or Virgalieu; turbinute, (top shaped,) as the Dearborn's Seedling; roundish, as the Gansel's Bergamot.

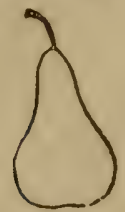

Pyriform.

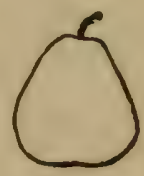

Oóovale.

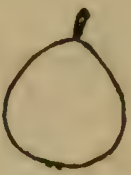

Turbinzle.

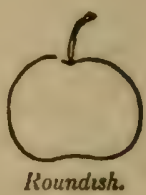

Koundesh.

Fig. 131. Forms of Pears.

With regard to the texture of the flesh; butlery, as the Doy. enné anll Barlett; crisp, as the Summr Bunchretien; juicy, as the Napoleun, and St. Germain ; as, in apples, the blossom end is called the eye, the remains of the blossom found there, the calyx, and the hollow in which it is placed, the basin.

\section{Class I. Summer Pcars.}

1. Amiré Johnnet. Thomp.

Enrly suzar, Pom Man.

Sugar Pear.

llarvest Pear.

St. Jean.

Joannefte.

St. Julun: Pear.

Archaluc d'ete?

This fruit, better known here, as the Early Sugar pear, is one of the very earliest, ripening at the beginning of July-in 
France, whence it originally comes, about St. John's daywhence the nam, bannet. It is a pleasant, juicy fruit, of second quality, and lasts but a f:w days in puffection. It opens tise pear season, with the little Muscut, to which it is superioner. Eruit below the millle size, regularly pyriform, tapering to the stalk, which is an inch and a half long, and thickest at the point of junction. Skin very smouth, at first light green, but becomeg bright lemon color at inaturity-very rarely with a faint blust. Calyx large, with reflexed segrnents, even with the surface. Flesh vhite, surary, delicate and juicy at first, but soon becomes mealy; seeds very pointed. Head of the tree open, with a few declining branches.

\section{Ambrosia. Lind. Thomp.}

Early Beurré.

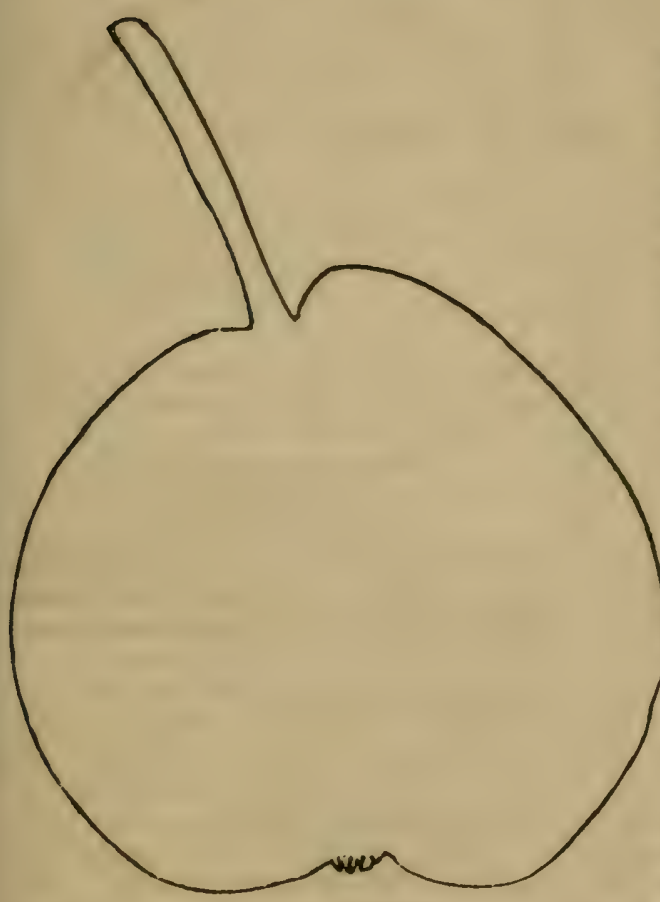

Fis. 131. Ambrosin.

The Ambro. sia is a French pear, which has been about thirty years in cul. tivation. It is a very sugary and pleasant early fruit, but it keeps only a few days after ripening. It has been very lately introduced into the United States.

It is very distinct from the Julienne, which is sometimes callod the Ear. ly Beurré in this country.

Fruit nearly of medium size, roundish - obo. vate. somewhai flattened. Skin smooth, greenish-vellow, thickly dotted with small gray specks, and a little russetterl. Stalk about an inch and a half long, sler der, and placed in a rather broad cavity. Caigx closerl, sel 
in a moderately deep basin. Flesh buftery and melting, with a sweet, rich, nerfumed flavour. Last of August and first n September.

\section{BLOODGOOD. § Man.}

\section{Early Beurré, of some.}

The Bloodgood is the highest flavoral of all early pears, and deserves a place even in the smallest garden. It was named from the circumstance of its. having been brought into notice about 1835 , by the late James Bloodgood, nurseryman, Flushing, L. I. 'The sort was brought to that nursery as a new varie. ty, without a name how. ever, by some person on Long Island, unknown to Mr. B., who was never able afterward to trace its history further. The tree is rather short jointel, with deep reddish brown wood, grows mo. derately fast, and bears early and regularly. 'The fruit, like that of all

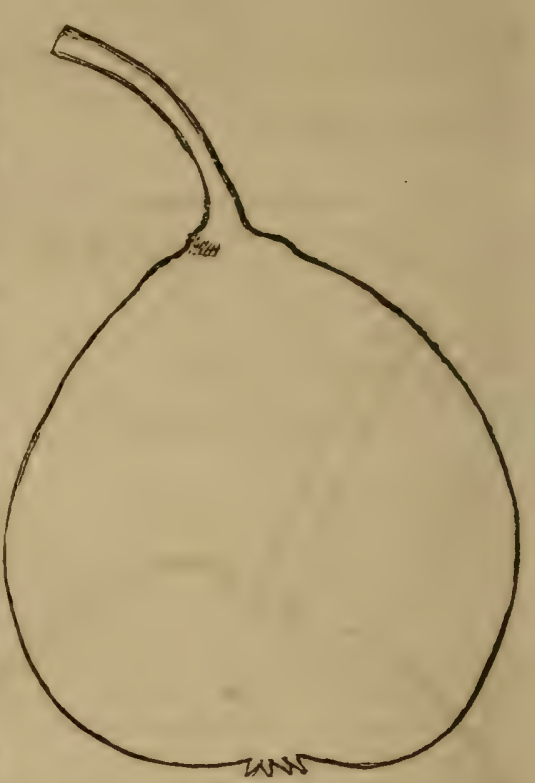

Fig. 132. Bloodgood.

carly pears, is better if ripened in the house. It surpasses every European variety of the same season, and together with the Dearboru's Seedling, another native sort, will supplant in all our gardens the Jargonelle, anc all inferiour early prars.

Fruit of medium size, turbinate, inclining to obovate, thicken. ing very abruplly into the stalk. Skin yellow, sprinliled with russet dots, and net-work marlings, giving it a russetty look on one side. Calyx strong, open, set almost without depres. sion. Stalk obliquely insertel, without drpression, short, dark brown, fleshy at its base. Flesh yellowish white, buttery and melting, with a rich, sugary, highly aromatic favour. The thin skin has a musky perfume. Core small. Ripe from the 2ivth of July to the 10th of August. 


\section{Beurré Haggerston. Man.}

No. 8 of Van Mons. Man.

This is one of Van Mons' Seedlings, sent with others to out American pomologist, the late Mr. Manning, with permission to bestow a uame. As it has noi fruited here with us, we annex Mr. Manning's description.

"Medium size, obiong, obtuse at the stem, which is one inch long; colour yellow; flesh juicy, sharp, agreeable and very abundant." It will ripen here about the middle of August.

\section{Berganot, Early. Thomp. Lind. P. Mag.}

A second rate, French sort. Frvii of medium size, roundish, rather flattened, and a little angular towards the eye. Skin pale yellowish-green, with a few streaks of duli red in the sun. Stalk rather thick, a little more than an inch long, set in a shal. luw cavity. Calyx moderately sunk. Flesh quite juicy, crisp, with a pleasant, sweet flavour. Ripe about the 20th of August

\section{Berganot, Summer. Thomp. Coxe.}

The Summer Bergamot is an old foreign variety, of small size, and second quality, quite supplanted now by such sorts as the Bloodgood, Dearborn's Seedling, \&c. The tree is of feeble growth.

Fruit quite small, round. Skin yellowish.yreen, becoming brownish in the sun, and full of small russet dots. Calyx set in a wide basin. Flesh juicy, and pretty rich in flavour, but quickly becomes mealy and dry. Last of July.

There is a Large Sunmer Bergainot, cultivated in this coun. try, quile distinct from the above. It resembles the Doyenné, but is broader and rounder, dryer and inferiour in flavour. Skin smooth, clear yellow, with very few dots. Stalk 11.2 inches long, curved, set in a narrow, deep cavity. Basin narrow, deep, smooth, with a small calyx. Flesh breaking and half buttery, not rich. September. The tree grows and bears finely.

\section{Berganot, Hampden's. Thomp.}

Summer Bergamot. I.ind. Mfill

Brrgainot d'Eté. O. Duk.

Bergamotte d'Angleterre.

Scotcl Bergamot.

Fingal's.

Ellanrioch.

Hampden's Bergamot is a strong growing, hardy tree, and a handsome, showy fruit, sometinies as attractive as the Bartlett, but of breaking texture, and not so high flavoured. 
Fruit large, rcundish, inclining to obovate. Skin at first green, becoming clear yellow at inaturity, with small dors, and sometimes with greenish spots in the shaile. Stalk scaicel: thren guarters of an inch loner, rather stout, curverl, and set in a small round cavity. Calyx small, closed, in a shallow basin. Flesh white, braking, a little coarse in texmere, but, if gatheresl early and riponed in the house, it uccones half buttery, sweet and agreeable. First of September.

\section{Belle de Bruxelles. Nois. Thomp.}

\section{Bellè d'Août.}

A large and handsome fiuit, of good quality, little known in this country, as two other sorts, Angleterre, and Flemish Beauty, have been wrongly imported under this nam?.

Fruit large, about four inches long, pyriform, tapering gradually to the stalk. Skin pale yellow, with a soft red cheek when fully exposed, otherwise entirely yellow. Stalk an inch and a half long, rather stout, obliquely inserted under a slight lip, fleshy at the lower end. Flesh white, juicy and melting, sweet, and slightly perfumed. Middle of $\mathrm{August}$

9. Bartlett, or Wilimims' Bonchretien. $\oint$ 'Thomp. Man.

Bartlett. of all American gardens.

Williams' Bonchretien. Thomp. Lind.

Poire Guiliaume, of the Fiench.

This noble pear is, justly, one of the most popular of all the summer varieties. Its size, bealuty and excellence, entitle it to this estimation, apart from the fuet that it hears very early, regularly and abundantly. It is an English variety, originated about 1770 , in Berlishire, and was afterwards propagated by a London grower by the name of Williams. IVhen first introduced to this country its name was lost, and having been culti. vated and disseminatud by Enoch Bartlett, Esq., of Dorchester, near Boston, it became so universally known as the Bartlett pear, that it is impossible to dispossess it now.* It suits our cli. mate admirably, ripening better here than in England, and has the unusual property of maturing perfectly in the house, even if it is picked before it is full grown. It has no competitor as a summer market fruit. The tree grows upright, with thrifty, yellowish brown shoots, and narrow, fulded leaves.

Fruit of large size, irrenularly pyramidal. Skin very thin and smooth, clear ycllow, (with a soft blush on the sunny side, in exposed specimens, ) rarely marked with faint russet. Stalk one

\footnotetext{
- The rirst iniported tree in MIr. Bartlett's grounds, was sent from Englan to 1799.
} 

Fruit middle sizen, obovate, regularly formed. Skin lign yellow, tinged with brown in the sun. Flesh white, buttery uweet, and of a tolerably pleasant flavour. August.

\section{Citron. Wilder. MSS.}

One of Krov. Edwards' seedlings, lately originated at New. IIaven. The trial of two seasons indicates that it is nearly, if not quire, first rate.

Fruit of medium size, roundish, and shaned somewhat like a Bergamot, to which it has some affinity. Skin dull grreen, regularly sprinkled with small russetty dots. Flesh greenish-white, a little coarse, melting and juicy, with a rich perfumed flavour Last of August.

\section{Dearborn's Seedling. $\S$ Man. Thomp.}

A very admirable, early pear, of first quality, raised in 1818 , by the Hon. H. A. S. Dearborn, of Boston. It bears most abundant crops in every soil, and is one of the most desirable early varieties, suc. ceeding the Bloodgood, and preceding the Bartlett. Young shoots long, dark brown. Fruit scarcely of medium size, turbinate, and very regularly formad. Skin very smooth, clear light yellow, with a few minute dots. Stalk slender, rather more than an inch long, set with very little depression. Calyx with delicate, spreading segments, set in a very shallow basin. Flesh white, very juicy and melting, sweet and sprightly in favour. Ripens about the middle of August.

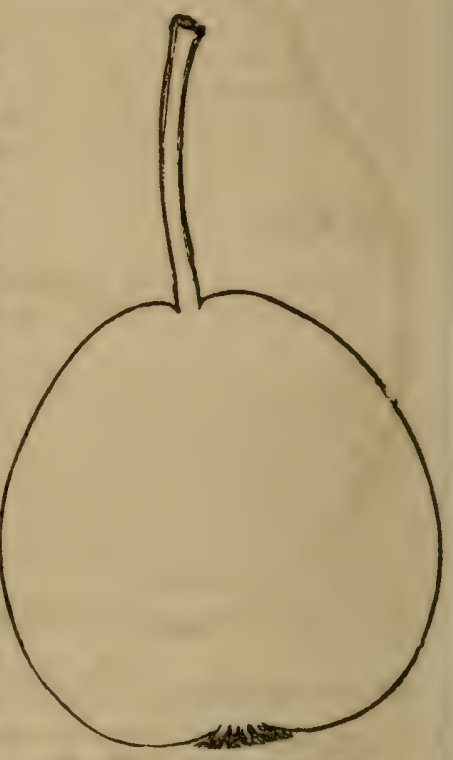

No. 135. Dearborn's Seedling.

13. Doyenné D'Eté. Nois. Bon. Jard.

Summer Doyenné.

The Doyenné d'Eté is shaped verv much like a small Whire Doyenné. The skin is smooth, shining, clear yellow, marked vith very small dots; and sometimes washed with faint red next 
the sun. Stalk short, thick, and feshy. Calyx small, closed, basin very slightly sunk. Flesh white, melting, very juicy, sweet, with a little acid, and of excellent flavour. It ripens at the last of July and beginning of August. The tree bears abun dartly, but is quite different from the Doyenne in its growth. M. Poiteall remarks that this pear has been cultivated for many yours at Nantes, though, till lately, little known in Paris.

\section{Green Chisel. Thomp. Fors. Lind. \\ Green Sugar. \} of some English \\ Sugar. $\{$ gardens.}

A pleasant old English pear, but not at all comparable with the new early sorts already described. The shoots grow quite erect, and the fruit is borne in clusters.

Fruit small, nearly round, tapering a little to the stalk. Skin quite green, with, occasionally, a dull brown cheek at full matu. rity. Stalk straight, three-fourths of an inch long, set almost without depression. Calyx open, crumpled, rather large. Flesh juicy, a little gritty in texture, with a sweet and pleasant favour. Ripe the middle of August. This is quite distinct from the Madeleine, an obovate pear, with which it is sometimes confounded.

\section{Hesser. Thomp.}

Hazel.

A Scotch pear, enormously productive, pretty, and of agreeable flavour, though it lasts only a few days in perfection. Tree with weeping branches.

Fruit rather below medium size, obovate. Skin yellowish. green, strongly marked with numerous dots, which give it a brownish, freckled appearance. Stalk an inch lnng, obliquely inserted. Calyx small, set in a shallow basin. Flesh whitish, iuicy, with a pleasant, sugary flavour. First of September.

16. Jargonelle, (of the English.) Thomp. Lind. P. Mag.

Epargne. O. Duh. Poit. Pom. Man.

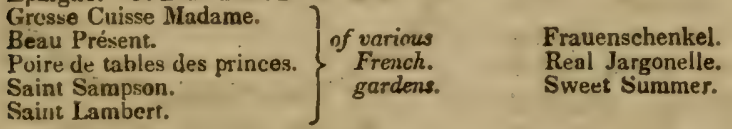

'This fruit, the true Jargonelle pear, ${ }^{*}$ was for a long tıme considered the finest of Summer pears, and Thompson yet says

- Although called by Thompaon the English Jargonelle, to distinguish it from the fruit more common under that name on the continent, thers is no doubt that 
"the best of its season." We think, that no man will hesitara however, to give the most decided preference to our native sorts, the Bloodgood, and Dearborn's Seedling. It is still, however, one of the most com. mon fruits in the New-York market, partly, because it bears abundant crops, and partly, because these superiour new sorts, have scarcely yet, had time to dis. place it. We con. sider it only a second rate fruit, and one that quickly decays at the core.

Fruit pretty large, long pyriform, tapering into the stalk. Skin greenish-yellow, smooth, with a little brownish colour on the sunny side. Stalk nearly two inches long, rather slender, curved, ob. liquely set. Calyx open, with quite long projecting segments, and sunk in a small and furrowed basin. The flesh is yellowish. white, rather coarse grained, juicy, with a sprightly, refresh. ing flavour. The tree is a strong grower, with a rather straggling, pendant habit. Ripens the last of. July and first of August.

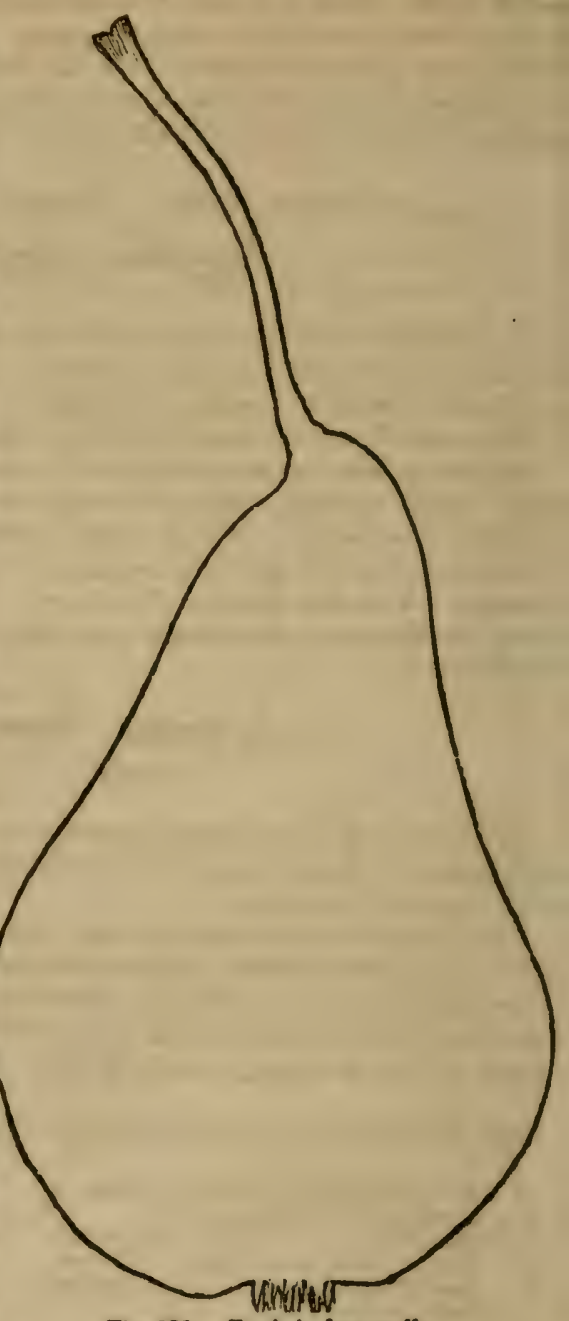

Fig. 135. Englush Jargonelle.

it was introduced orig inally from France. Antiquarinns derive its name trom Gergon, Italian, a cor uption of Gracum, whence Merlet supposes it to be the Numidianum Grecum of Pliny, and the Greculsm of Macrobius This, if cat oet, wonld prove is to bo a very apriens adort. 
The common Ciusse Madane of the French authors and gardens, is an inferiou: and smaller variety of Jargonelle, nu: worth cultivating. It has long, straight, raiher slender, hrown. ish red branct es, while the true Jargonelle has long stricgrting, dangling branches. The blossoms of the latter are also un usually large. [See also Windsor Pear.]

\section{Jargonelle, (of the French.) Thomp.}

Bellisime d'Eté. O. DıŁ. Nois.

Suuréme.

Bellissine Supréme. of French

$\left.\begin{array}{l}\text { Beilisime Jarronelie. } \\ \text { Vermuliiun d'Été. }\end{array}\right\}$ gurdens.
Red Muscadel. Lind. Mill

Sahine d'Elé.

Summer B.auty. Pom. Man.

Euglish Red Cheek. Lof minny Ame lied Che ek. \}ricun garden.s

This, which Mr. Thompson calls, by way of distinction, the French Jargonolle, because it is most comminly received under that name from France, is a hicher coloured and handsomer fruit thar the English Jargonelle, though much inferiour in quality, and, in fact, lasts nnly a day or two in perfection, and is often mealy and over-ripe, wnile the exteriour is fair and tempting. It has a bright red cheek, and a shoiter obovate form, blunt at the stalk.

The tree is of very strong, upright growth. Fruit of medium size, obovate in form. Skin shining, light green, becom. ing lemon colour, with a very rich, deep red chcek. Stalk

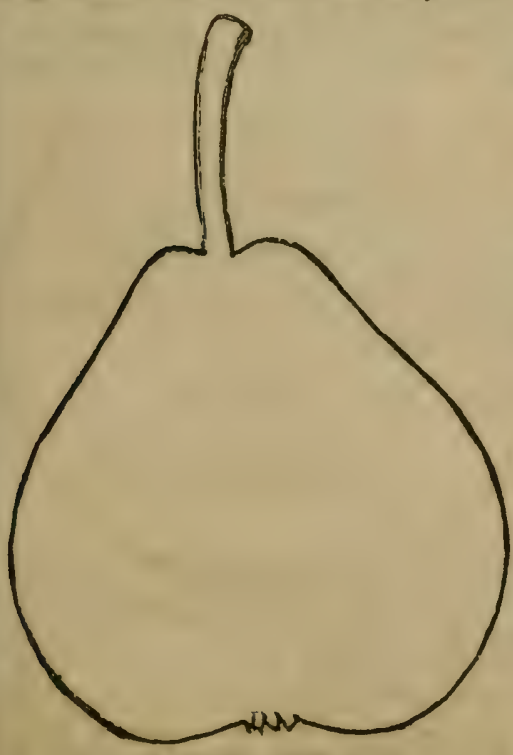

Fis 137. Julienna about an inch long, rather stiff and stout, and set in a blunt repression. Calyx in a shallow, slightly irreurular basin. Flesh white, coarse, breaking, sweet, and soon rots at the core. Ripens the last of July and first. of August.

\section{Juhinne. Coxe. Man.}

A handsome summes pear, which so much rc. sembles the Doyeune ot St. Michael, as to be called, by some, tl.e Sum. mer St. Michael. It is a beautiful and most pro. duc:ive fruit, and comes into bearing very early. It is often of excelleut 
favour, and of the first quality ; but, unfortunately, it is variable in these respects, and some sensons, it is comparatively taste less and insipid. In rich, warm, and dry suils, it is almost al. wars fine. It is a protitable market fruit, and will always sommand a prominent place in the orchard. Thes tree is of thrilty upright wrowth, with light ycllowish-brown shonts.

Fruit of medium size, but varying in different soils; obovate, recularly formed. Skin very smooth and fair, clear bright yellow, on all sides. Stalk light brown, speckled with yellow, a little more than an inch long, pretty stout, inserted in a very shallow depression. Calyx small, closed, set in a basin slightiy stink, but often a little plaited. Flesh white, rather firm at first, half buttery, sweet, and moderately juicy. Ripens all the month of August.

Coxe considered this synonymous with Archiduc d'Ete of Duharnel and Lindley-the Ognonet pear, a distinct and in feriour fruit, with a brownish cheek, and we therefore follow Mr. Man. ning in keeping it distinet. It inay yet prove synonymous with the Doyenne d'Ete of the French, which has not yet been fairly proved in this country. (See Doyenné d'Eté.)

\section{Lrimon. Van Mons. Man. in H. M.}

A fine, sprightly, Belgian pear, origrinated by Van Mons. The fruit resembles, in outward appearanes, the White Doyenne, but it is distinguished from that well known fruit, by its ripening a month earlicr. The young shoots are long, slender, reddish brown.

Fruit rather large, obovate. Skin sinooth, yellow, with a faint red cheek. Stalk an inch and a half lonir, rather stout, set in a moderately depressed, rounil cavity. Calyx set in a rather shallow, round basin. Flesh white, butterv, melting and juicy, with a sprightly, high flavour. Middle of August.

'This is evidently quite distinct from the Limon de Louvain, of the Jurdin Frutier, a winter pear.

20. Litrle Mijscat. Thomp. Lind. Mill.

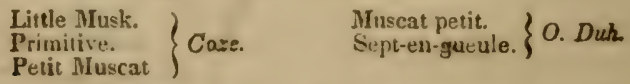

This very little, French pear, well known in many of our gardens, is allowed a place there, chiefly, because it is the earl. iest of all pears, ripening at the becrinning of July. The tree s of very handsonse, pyramidal wrowth, and bears the most enormous crops of pears, in clusters. The fruit, which is but little more than an inch in diameter, is shaped like a little rounded top, and is just passably good at its season. 
Fruit very small, turbinate. Skin vellow, with a dull rel cherk. Staik half, to one and a half inches long, set alunost without depression. Calyx open, set nearly level. Flesh breaking, sweet, with a slight musk flavour. Shoots dark browı

\section{Muscat Robert. Thomp. O. Duh. Lind. \\ Poire à la Reine. \\ I) Ambre. \\ St. Jean Jilusqueé Gros. \\ Nusk Robine. Lind. \\ Eiarly Quren. \\ Queou's Pear.}

A larger and hetter kind of Museat, which mirght be esteremed first rase, laal we not the Bloolyend to compare it wish. Shonta yelinwish-brown. Middle of July, and lasts ouly a few days.

Fruit small, about an inch and a half in dianeter, turbinate Skin clear greenish-yellow. Stalk nearly an inch long, set with a little unevenness, but un depression. Calyx large, open, scarcely sunk. Flesh white, tender, juicy and pleasant.

22. Madeleine, or Citron des Carmes. $\$$ Lind. P. Mag. 'Thomp

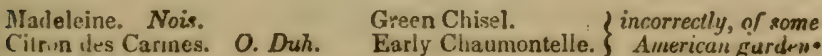

Magdelen.

'The Madeleine is one of the most refreshing and excellent of the early pears; indeed, as yet, much the best at the time of its ripen. ing-before the Blootrood. It takes its name from its being in perfection, in France, at the feast of St. Madeleine. Citron des Carmes comes from its being first cuitivated by the Carmelite monks. It is much the finest early French variety, and deserves a place in all collections. 'The tree is fruitful and vigorous, with long erect olivecoloured branches.

Fruit of medium size, obo. vate, but tapering gradually to the stalk. Stalk long and slender, often nearly two inches, set on the side of a small swelling. Skin smooth, pale yellowish-green, (very

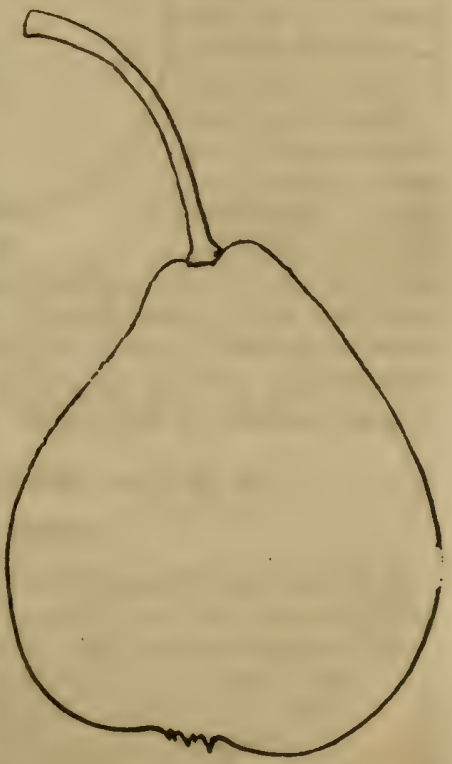

Fig. 133. Mudeierne. 
rarely, with a little brownish blush and russet specks around the stalk.) Calyx snall, in a very shallow, furrowed lsasin. Fiesh whise, juicy, melting, with a sweet and delicate flavour, slighty perfumed. Middle and last of July.

The Muscadine is a first rate pear, remarkable for its high musky aroma. It was first dissemi. nated by us, the original tree grow. ing on the firm of the late Dr. Fowler, in this county, by whoms it was named. Its history is unsertain, and it is be. lieved to be a nalive. lt bears very heavy crops, and if the fruit is picked, and ripened in the louse, it is not sur. passed in flavourby any pear of its time.

Fruit of medium size, roundish obo. vate, regularly formel. Skin pale ycllowish green, a

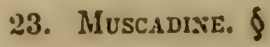

little mugh, thickly sprinkled with brown dots. Stalk ahout an inch long, set in a well formed, small cavity. Calyx with re. flexed serments, set in a shallow basin. Flesh white, buttery and meltintr, with an agrefable, rich, musky flavour. Aast of Augus", and first of September. Shoots stout, dark gray-brown.

\section{Passays du Portugal. $\&$ Thomp.}

\section{Summer Portugal.}

A delicate and pleasant pear, which comes early into hearmg, anit proiluces very large crops. Shoots upright, reddish-brnwn.

Fruit bclow mediunn size, roundish anıl much flattenerl. Skin pale yellow, with a chreck of fairest brown, becominer red in the sun. Stalk nearly an inch lonğ, inserted in a round, regular hollow. Calyx stili; basin moderately sunk. Flesh white, juicy, urezking, of tery delicate; agrecable flavour. Last of August. 
25. Rousselet Hâtif. U. Dul. Thomp.

Early Catherine. Care.

Kattern, of Boston.

Cyprus Pear.
Early Roussele\& Linut

Perdreau.

Puire de Chypre. Poit.

The Rousselet Hâtif, better known in our markets as the Early Catherine Pear, though not a first rate fruit, has good qualities as an early variety. It bears very heavy crops as soon as the tree is well grown, when its willowy limbs bend with the weight of the fruit. It is, therefore, profitable for the market. The fruit is thought better when ripened on the tree.

Fruit rather small, pyriform, the neck narrowing into the somewhat fleshy stalk, which is one, to one and a half inches long. Skin when fully ripe, yellow, with a brownish red cheek. Calyx small, placed in a shallow basin. Flesh tender, a little coarse grained, sweet, pleasant, and slightly perfumed. Ripens the last of July. Young shoots stout, olive coloured.

\section{Rousselet de Rheims. O. Duh. Thomp.}

Rousselet.

Petit Rousgelet. Nois.

Spice or Musk Pear.

This nice French pear, originally from Rheims, is sup. posed to have been the parent of our Seckel. There is a pretty strong resemblance in the colour, form, and flavour of the two fruits, but the Seckel is $m$ ich the most de. licious. 'T/ıe growth is quite different, and this pear has remarkably long and thrifty dark brown shoots. It is su. gary, and with a peculiarly aromatic, spicy flavour, and if it were only buttery, would be a first rate fruit.

Fruit below medium size, sbovate, inclining to pyriform. Skin yellowish-green on the shady side, but nearly cover. ed with brownish red, with russetty specks. Stalk rather nore than an inch long, cur. vel, and inserted without de-

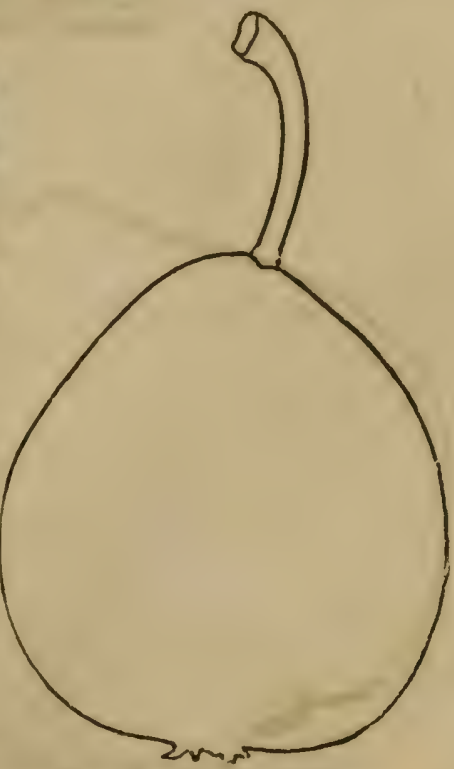

Fig. 140. Rousselet de Rheims. pression. Calyx spreading, set even with the fruit. Flesit 
breaking or half buttery, with a sweet, rich, aromatic flavour Ripe at the beginning of September.

\section{Sugar Top. Thomp. \\ July Pear. \\ Prince's Sugar. \\ Prince's Sugar Top.}

The Sugar Top is one of those indifferent pears, which, from their great productiveness and good appearance, make a figure in our markets, though not worthy of a place in a good garden. Great quantities of the Sugar Top pear may be seen in the NewYork markets in July.

Fruit of medium size, very regular, roundish-top-shaped. Skin smooth, and very bright, clear yellow over the whole surface. Stalk stout, obliquely inserted, with a thickening at the point of junction. Caly $x$ in a narrow basin. Flesh white, somewhat juicy and breaking, sweet, but with little flavour. Last of July.

29. Summer Franc Réal. \$ Thomp. Lind. P. Mag.

Frane Réal d'Eté. Diel.

Gros Miceb d' Fté.

Yondante. Ḱnoop.

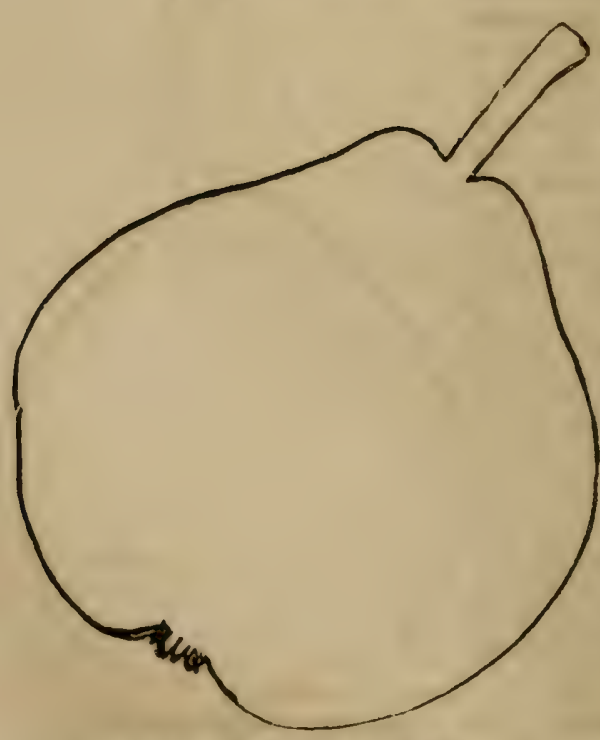

The Summer Franc Réal is one of the best summer pears, always melt. ing and delicious, it fills, along with Dearborn's Seed. ling, the space, in ripening, between those favourite sorts, the Bloodgood ana the Bartlett. Réa. is a Spanish gold coin, and we pre. sume, this fruit must have been named from its slerling me. rit, as it is not gold] colour. The tree is thrifty, hardy, and bears well, and is easily known by its Fig. 141. Summer Franc Rical. rounded, light co 
loured leaves. It is hardy, and bears admirably in all kinds nf snil:

Fruit of medium size, obovate, but largest in the midlde, ind tapering each way. Skin green at first, becoming pale yehow. ish-green, dotted ith small, brownish-green dots. Stalk short, thick, and rather uneven, inserted in a shallow cavity. Calyx small, closed, with long segmente, sct in a furrowed basin. Flesh white, fine grained, buttery and melting, with a rich, sugars. excellent favour. Core large. Ripe early in September

29. Stixseau, or Skinless. Thomp. Lind. Mill.

Poire Sans Peau. O. Duh.

Flour de Giuignes.

The Skinless is a very nice little pear, with a remarkably thin, smooth skin, and a delicate, perfumed flavour. It bears in clusters, and very regularly. It is not first rate, but is esteem. ed by many.

Fruit below medium size, long pyriform. Skin very smooth and thin, pale green, becoming light yellow, speckled with light red in the sun. Stalk long, slender, curved, inserted in a very trifling cavity. Calyx closed, set in a sinall basin. Flesh white, juicr, half melting, with a siveet and slightly perfumed flavour. First of $\mathrm{Au}$. gust.

This is quite distinct from the Early Rous. selet.

30. Suminer Rose.

Epine Rose. Duh. Nois. Poire de Rose.

Caillot Rusat d'Eté.

Epirse d'Eré Cualpur Rose. J'horny Kase. Mill.

Rosenbirue, of the Germans. Oinnon. 'Eté. $\left\{\begin{array}{l}\text { wrongly, of } \\ \text { some. }\end{array}\right.$

A handsome and peculiar summer pear, very popular, and well known on the other continent. It is quite nat, and remarkably like an apple in ap. pearance.

Fruit of medium size, round, flattened at

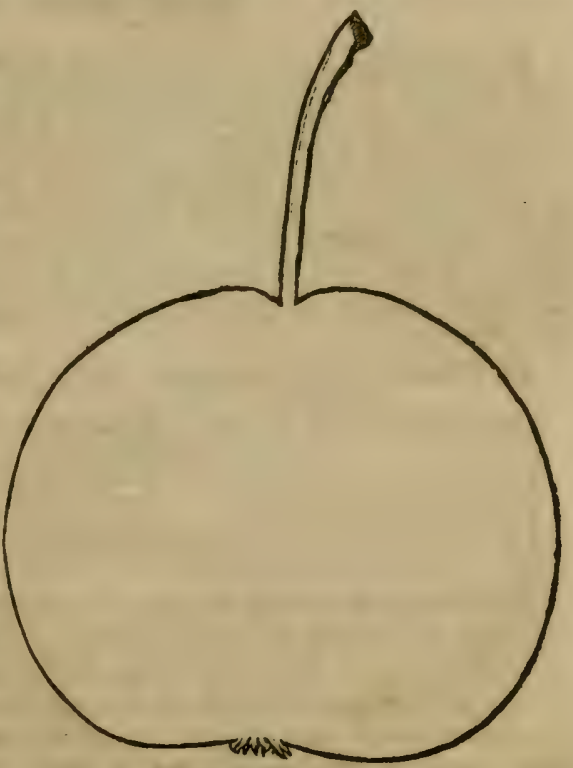

F.g. 112. Summer Rose. 
both ends. Slin faint yellow, blended and speckled with russe in "ic shade, with a red russet cheek, marked with brown dots. Sta i rather more than an inch long, slenter, curved, inserted in a very small hollow. Calyx open, small, set in a very shal. low basin. Flesh white, juicy, rich and sugary, hardly fisst rate. Last of August. Shoots upright, gray-olive.

\section{Sucrée de Hoyersiverda. Thomp.}

Sugar of Iloyersworda.

A pleasant German pear, of peculiar flavour, excellent when ripened in the house. It bears immense crops. Leaves very narrow.

Fruit of medium size, obovate-sometimes oblong, lengthening into the stalk, which is curved and obliquely inserted. Skin smonth and fair, pale yellowish-green, thickly sprinkled with greenish russet dots. Calyx very small, and placed in a very shatlow basin. Flesh white, quite juicy, with a sweet and piçuant flavour. It does not keep long. Last of August. Slivots long, olive brown.

32. Erine D'Eté. Thomp. Lind.

Summer Thorn.

Forulante Musqueb

Satin Vert.

A second rate, juicy, and pretty good fruit, which may be introducen in a large collection. It looks a little like a small Jargnnelle. A good bearer. Shoots yellowish-Lrown.

Fruit middle sizerl, pyriform. Skin smooth, greenish-yellow; a little darker on the sunny side. Stalk stout, about an inch long, set without depression. Calyx short, set in a small plaited basin. Flesh tendrer, melting, with a sweet, musky, prculiar fluvour. Last of August and first of September. Set with little or no cavity.

33. Sumiler Box Chrétien. Mill. Thomp. Lind. P. Mag.

Bun Chretien d'Eté. O. Duh. Summer Good Christian.

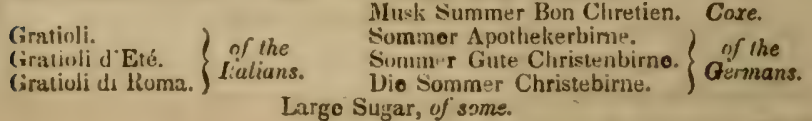

This is one of the oldest pears, having heen cultivated for tho is it wo centuries, all over Europe. It is common with us, but the stock is generally somewhat diseased. The tree has dron, ing shoots, and bears at the extremities of the branches Though a sweet and pleasant pear, it wants the favour of ous finer sorts, and does not deserve a place in a small garden. 
Fruit large, irregularly bell-shaped or pyriform, with swollen, nnnbbu sides. Skin yellow, with an orange-blush in finely ripened specimens, dotted with many green specks. Stalk long, irregular, curved, obliquely inserted in a knobby depression. Calyx small, in a narrow, uneven, shallow basin. Flesh yel. lowish, coarse grained, very juicy, and of a pleasant, simply sweet flavour. Very large blossoms and dangling leaven Last of August, or early in September.

\section{Summer St. Germain. 'Thomp. \\ Short's Saint Germain. \\ Saint Germain de Martin. \\ St. Germain d'Eté. N. Duh.}

A pleasant, juicy, summer pear, of second rate flavour, bear ing laige crops, and growing vigorously.

Fruit of medium size, obovate. Skin pale green all over the surface. Stalk an inch and a quarter long, obliquely inserted. Caly $\mathrm{x}$ in a basin scarcely sunken. Flesh juicy, tender, sweet. with a very slight acid, and very good.

35. Vallée Franche. Thomp. Duh.

\section{De Vallée. Nois. Poit. \\ Bonne de Keinzheim. \\ De Keinzheim.}

A second rate sweet, summer pear, productive, but by no means, in our opinion, of first quality. It ripens with the Bart. lett, and is immeasurably inferiour to it in this climate.

Fruit of medium size, obovate, or turbinate, and tapering to the stalk. Skin pale green, becoming pale yellowish-green, regularly sprinkled with numerous small, gray dots. Stalk about an inch long, set with little or no cavity. Calyx in a shallow basin. Flesh white, not fine grained, quite juicy, but not buttery, and of a simply sweet flavour. Last of August.

\section{Windsor. Lind. Thomp.}

Summer Bell:

Cuisse Madame, of some.

Konge.

The Windsor is an old European pear, very commonly known in some parts of this country, as the Summer Bell pear. Large quantities are grown for market. It is, however, only a third rate fruit. The tree is remarkable for its stout, perfectly up. right dark-brown shoots.

Fruit large, pyriform, or bell-shaped, widest above the middle, narrowing to the eye, and slender in form, tapering into the stalk Skin yellowish-green, dotted with small green specks, and tinged 
with a little dull orange next the sun. Stalk an inch and a hall long, slender. Calyx small, closed, set with little or no depres. sion. Flesh white, tender, or soft, a little cuarse-grained at the core, sweet, with a somewhat astringent juice. Last of August.

37. Williams' Early. $\oint$ Man.

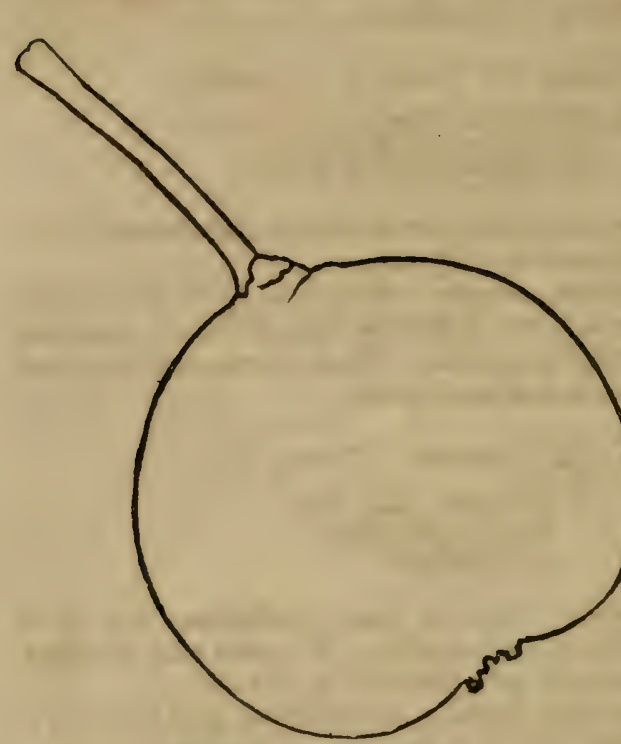

Fig. 143. Williams' Early.

A native fruit, which originated on the farm of Mr. A. D. Wil. liams, of Roxbu. ry, Mass. It is a very handsome, small pear, of ex. cellent quality, and a good bearer.

Fruit below me. dium size, round. ish-turbinate, regularly formed. Skin bright yel. low, thickly sprin. kled with rich scarlet dots on the sunny side. Stalk an inch and a hal! long, straight, a little fleshy where it joins the fruit. Calyx very short, open; basin shallow, and slightly plaited. Flesh white, a little coarse-grained at first, but, when ripe, very juicy, half buttery, rich, with a slightly musky flavour. First, to the middle of September. Young wood dark.

\section{Class II. Autumn Pears.}

\section{Alpha. Thomp.}

A Belgian seedling, received from Dr. Van Mons. It is a pleasant pear.

Fruit of medium size, obovate, a little inclining to oblong. Skin smooth, pale yellowish-green, dotted with reddish poirts, and having a thin, pale brown blush. Stalk little more than an inch long, inserted in a slight depression. Calyx stiff, open, set in a round basin of moderate size. Flesh white, fine grained, buttery and good. Middle of October. 


\section{Andrews. Man. Ken.}

\section{Amory.}

\section{Gibsun.}

The Andrews is a favorite native seedling, found in the neighbourhood of Dorchester, and first introduced to notice by a gentlcman of Boston, whose name it bears. It has, for the last 15 years, been one of the most popular fruits. It is of most excel. lent flavour, a certain and regular bearer, even while young, and the tree, which is very har. dy, never suffers from blight.

Fruit rather large, pyriform,one-sided. Skin smooth, and rather thick, pale yellowish - green, with a dull red cheek, and a few scattered dots.

Sitalk about an inch and a quarter long, curved, set in a

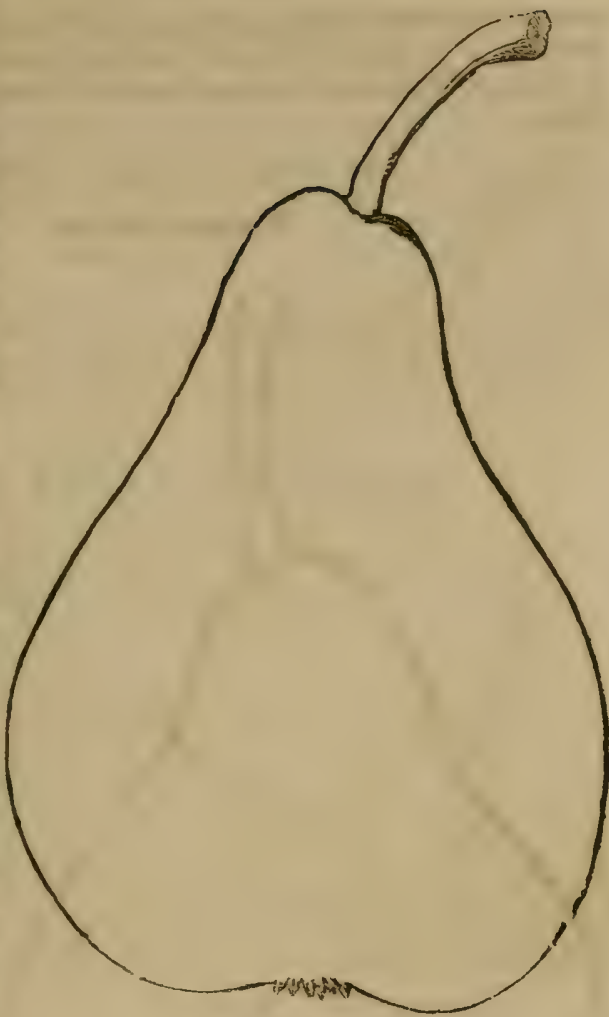

Fig. 144. Andreuns. very shallow, blunt depression, or often without depression. $\mathrm{Ca}$. lyx open, placed in a small basin. Flesh greenish-white, full of juice, melting, with a fine vinous flavour. Early in Septernber. Shoots diverging, light olive.

\section{Avanas. \& Bon. Jard.}

Poire Ananas. Nois.

This new and delicinus pear was introduced very recently ir, $m$ France, by Col. WVilder of Boston. It is a rich flavoured fruit, of the first quality, with an agreeavle pesfume, not how. 
ever resembling that of the pine-apple, as its name would leaa one to suppose.

Fruit of medium size, roundish-obovate. Skin greenish-yel. low, slighly market with russet, and recasionally with red on the sunny side. Stalk rather stout, thicker at the point of inser. tion. Calyx closed, and set in a shallow basin. Flesh white, melting, v ry juicy, with a rich and perfumed flavour. Ripen 3 the last of September and beginning of October. Young wood olive.

41. Axasas D'Eté. Thomp.

Ananas, (of Manning.)

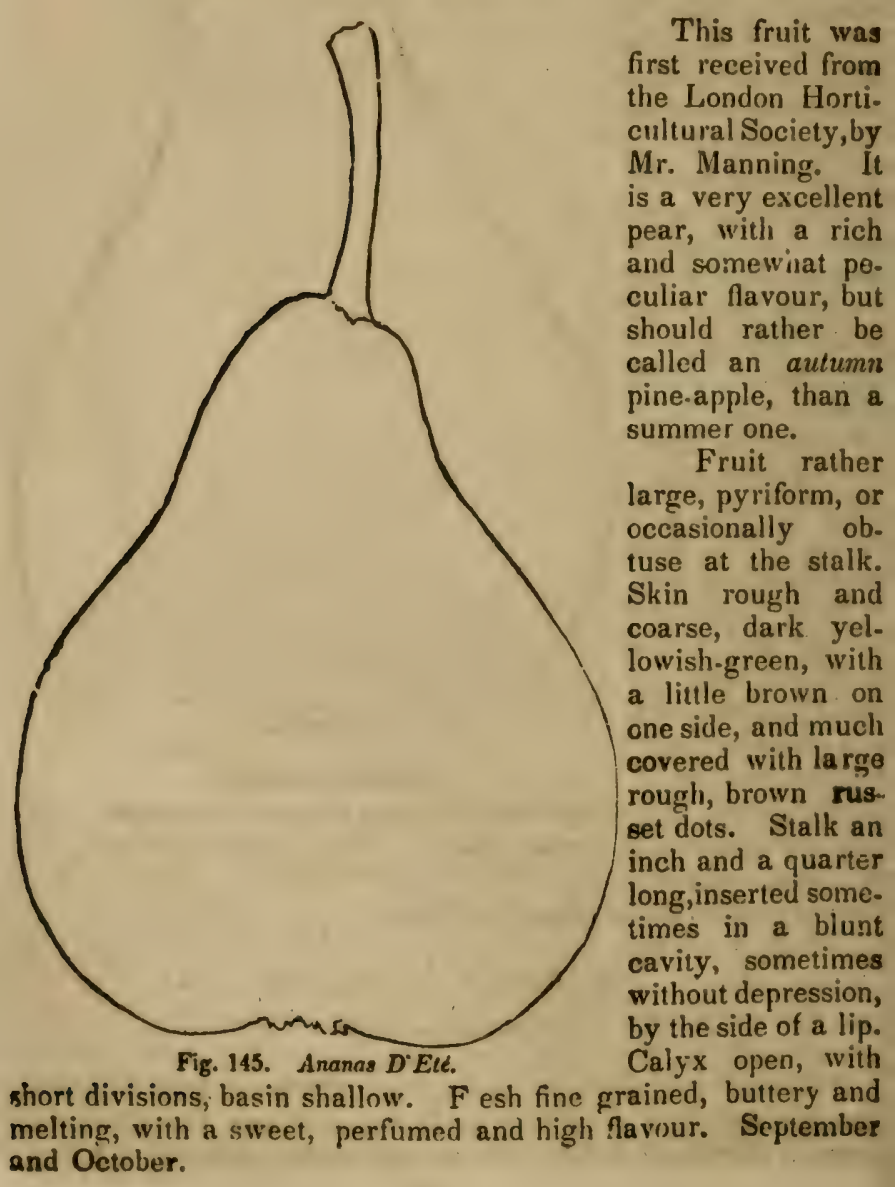




\section{Avglfitrore. Thomp.}

Engliah Benrré. Jind.

Beurré d'Angleierre. Nois.

A most productive pear, which has some affinity to Bınwn Beurré, but is inferiour to it in favour. It is a erual urchand fruit. but is not worthy of a place in a small garden. 'The tre forms a very ereet, pyramidal head. Youner worl olive. It is one of the most common fruits in the market of Paris.

Fruit of medium size, pyriform, very evenly shaped, tapering very regularly to its union with the stalk, which is slender, and rather more than an inch long. Skin rather thick asil hard, dull light green, thicklv sprekled with russet dots, and having a thin brownish russet cheek. Calyx set in a very smouth, scarcely sunk basin. Flesh white, buttery and melting, full of juice, and of pleasant, though not high flavour. Middle of S̈eptember.

\section{Asron Town. P. Mag. Thomp. Lind.}

A very hardy little pear, from the village of Aston, in Chester, England. It is of tolerable llavour, sometimes excel. lent, and the tree, when in bearing, is characterized by its long slender branches, which have a half-Iwisted, dangling appearance. It bears grcat crops, and is especially worthy of notice in an unfavourable soil, and cold climate.

Fruit rather small, forn roundish-turbinate. Skin a little rough, pale brownish-green, becom. ine yellowish when ripe, and thickly dotted with brown specks. Stalk an inch and a half long, rather straight and sleniler, inserted with little or no cavity. Calyx nearly closed, in a very

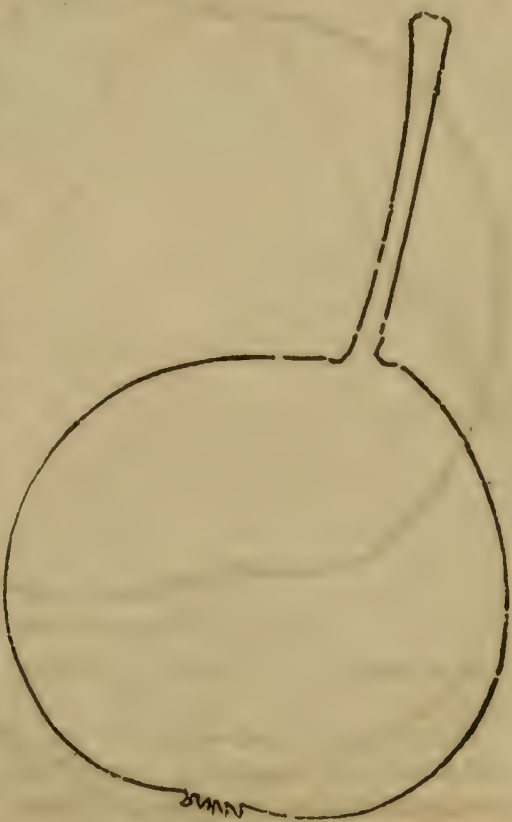

Fig. 146. Axton Toum. 
shallow basin. Flesh soft, buttery, moderate y sweet, periumed, and good. Midule and last of September.

\section{Altuorpe Crassane. Thomp. Lind}

This fine English pear is a seerlling raised by the late T. A Knnight, Esq., Presilent of the Lonion IIorticultural Sxicity. It was sent by him to the IInn. John Lowell, of Boston, in 18:32. It is very highly rated in England, and is recmmmended as a very hardy tree. The specimens as yet raised in this country have proved of excellent quality, but not quite equal to its repuration.

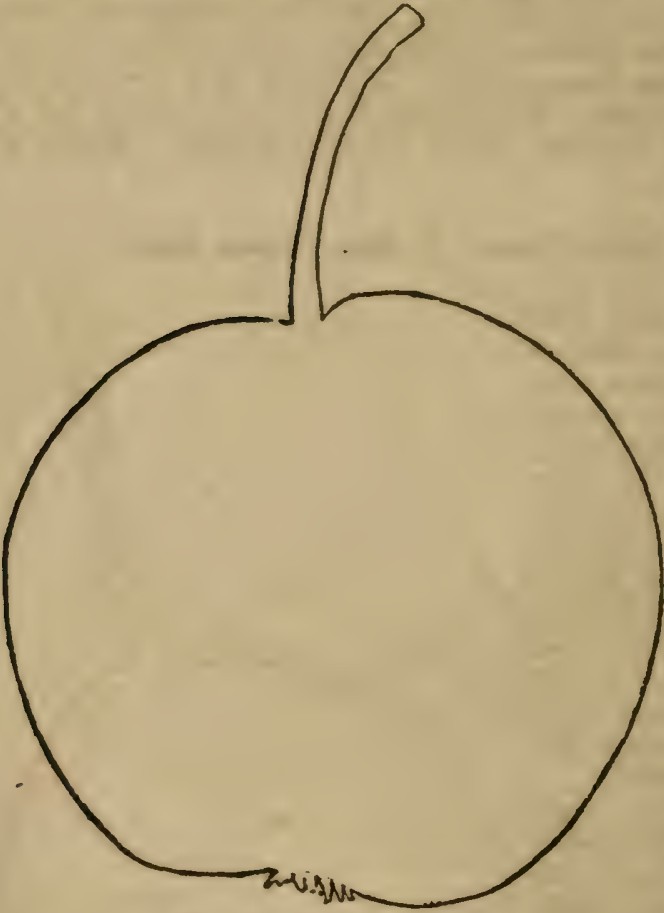

Fig. 147. Althorpe Crassane.

Fruit of merlium size: roundish-obo. vate, but nar. rowing rather more to the eye than the sialk. Skin pale green, dotted with small russetty points, and having a lit. tle tinge of brown on one side. Stalk about an inch and $a$ half long, slender, curyed, and slightly in. serted. Calyx with many divisions, set in a shallow basin; having a few plaits. Flesh white, buttery, and quite juicy, with a rather rich, slightly perfumed juice. October and No. vember.

Either there is a spurious sort strongly resembling this, or the Althorpe Crassane is somewhat variable in quality, as we have seen specimens quite indifferent. 


\section{Amande Double. Van Mons.}

Amanda's Double. Man. in Hov. Mag.

Une of Van Mons' seedlings, received by Mr. Manning, and we suppose named by Van Mons, in allusion to its having double kernels. It is a very handsome fruit. By misconcep. tion it has been called here Amanda's Double. Mr. Manning's description of it is as follows.

"Medium size, pyriform, stem short, fleshy at its junction with the fruit. Skin yeliow and bright red. Flesh coarse grained, sweet, tender and excellent. Ripe the middle of Sep. tember." Shoots stout, upright, dark olive.

A subsequent examination of this pear leads us to think it $\mathrm{drv}$ and inferiour in many seasons.

\section{Autumin Colitar. Thomp. Lind.}

A Flemish pear, of fair quality, and a good bearer.

Fruit of medium size, oblong or obtuse, pyriform, a lit:le uneven. Skin pale green, dotted with numerous russety specks. Stalk about an inch long, straight, planted in a small, uneven cavity. Calyx small, closed, set in a slight basin, a little furrowed. Flesh a little gritty at the core, buttery, with a rich and agreeable flavour. October.

\section{Belmont. Thomp.}

An English kitchen pear, considerably like the Althorpe Crassane, and of the same origin. It bears abundantly and constantly with us, and is remarkably fine for cooking and pre. serving, but is scarcely fit for the table.

Fruit roundish-obovate, medium, snmetimes of rather large size. Skin fair, yellowish-green, inarked with numerous dots, and a lintle brownish next the sun. Stalk quite long, (two it ch. es or more, slender and curved. Flesh rather coarse, juicy, and sweet. October.

49. Belle et Bonne. Thomp. Lind. P. Mag.

Schiine und Gute.

Grarieuse.

Belle de Brissels, (ircorrectly.)

The Belle et Bonne (beantiful and good,) pear is a variety fiom Bulgium, of large size, fine appearanes, and succharine llavour. It is a showy and good fruit, but whever reads Mr. Kenrick's description, and expects tn find it "a delicious Ber 


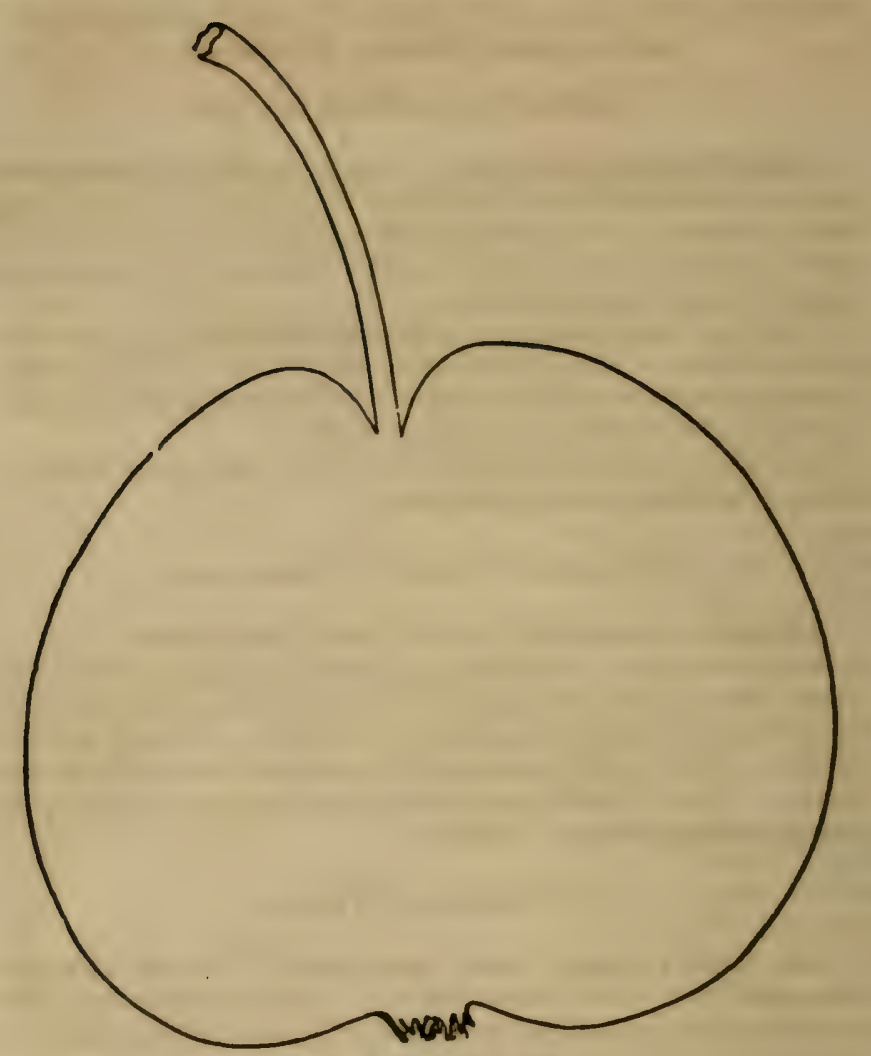

Fig. 148. Belle at Bonne.

famot of the best kind," will be disappointed. It is very far be. fow Gansel's Bergamot in richness. The tree is a strong grower.

Fruit large, roundish, a little greater in width than in height. Skin pale greenish-ycllow, with numerous russet green dots, especially near the eye. Stalk long, rather slender, deeply in. serted in a very narrow cavity. Calyx with crumpled divisions, set in a shallow, rather uneven basin. Flesh white, a little coarse grainer, tender, and when well ripened, buttery, with a very siveet and agreeable juice. Middle of September.

\section{Brovghas. Thomp.}

A new English variety, not yet proved here. It is said to bo very hardy and very productive. 
The fruit is described by Thompson as large, roundish-obo. vate. Skin yellow, a grond deal covered with russet. Flesh buttiry, quite melting, and of very excellent flavour. It ripens in November.

50. Bleeker's Mfeadow. Ken. Pom. Man.

\section{Large Seckel.}

A native fruit, said to have been found in a meadow in Penn. sylvania. It is a handsome, hardy fruit, and bears large crops, but it has been sadly over-praised as to quality. The truth is, it srems at first to grive promise of high flavour, but it rarely betomes mellow, but retains its crisp, hard state. We have raised many fine crops, but cannot recommend it much. In a very dry, warm soil, it is some. times excellent.

Fruit small, or of medium size, round. ish, very regular and smooth. Skin bright clear yellow, nccasion. ally sprinkled with crimson dots on the sunny side. Flesh very white, firm, with a peculiar musky or wasp. like aroma, and spicy taste, but mostly re. mains crisp and hard. Slalk straight and stiff. Basin shallow. Calyx

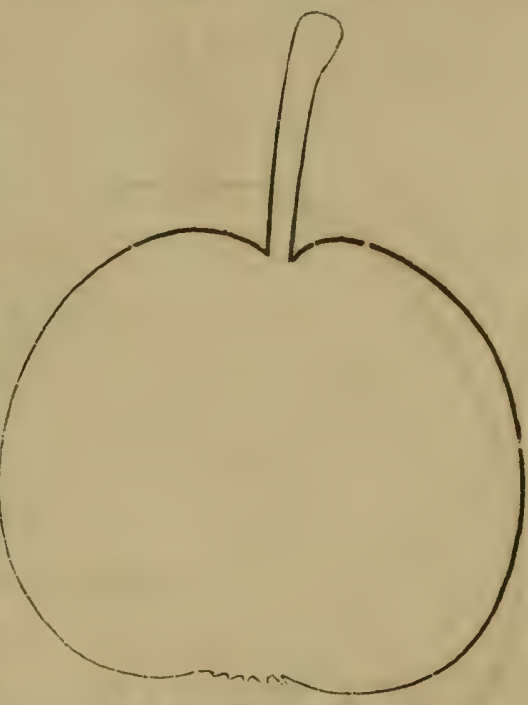

Fig. 149. Blecker's Sterdors. open and reflexed. ()ctober and November.

\section{Bovcquila. LHov. Mag.}

Beurré Bonequia. Ken.

A new Flemish pear, raised by Dr. Van Mons, and sent to Mr. Manning in 1836 .

Fruit rather large, nne-sideit. nval-turbinate. Skin pale yel. Inw, with a pale rol chne'., thickly sprinkled with redilish, and dark rtussty dots. Stalk an inch or more lnng. set a little oh. "quely, snd either fleshy at the point of junction, or set in a 
vel'y siight depression. Calyx large, basin scarcely sunk. Flesh yollowish-white, aboun ling with a very swect, rict juice, of excellent flavour. October. Rather liable to rot at the enre.

\section{Buffay. Man.}

\section{Buftum.}

'The Bufiam is a native of Rhode Islan', an! from its general resemblance to the Doyenue, it is, no doubt, a seedling of that

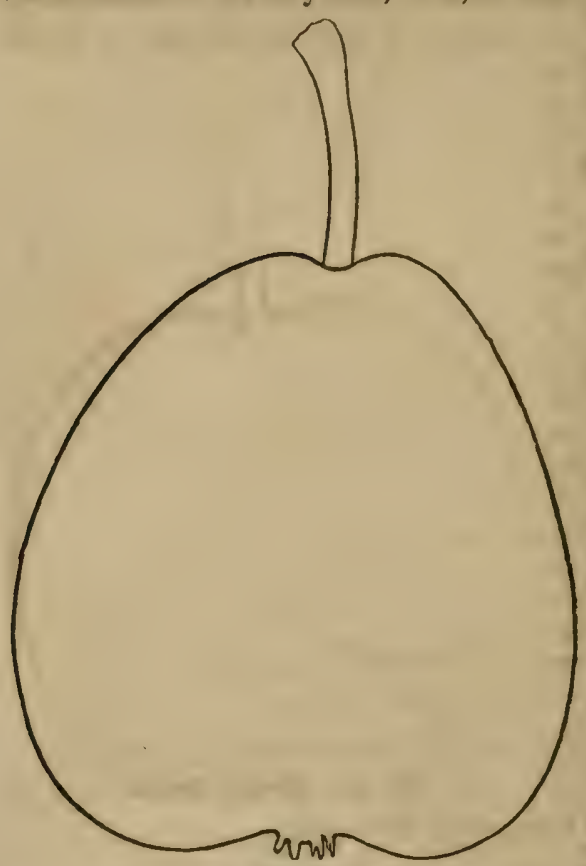
fine sort. It is an orcharil pear of the first quality, as it is a very sirong, upright grower, bears large, regular crops, and is a very handsome and saleable fruit. It is a little variable in quality. $\quad$ We have frequently eaten them so fine, as scarcely to be distinguished from the Doyenné, and again, when ra. ther insipid. It may be considered a beau. tiful and good, though not first rate varicty.

Fruit of medium size, oblong, obovate, a little sinaller on one side. Skin fair, deep yellow, (hrownish. green at first, ) finely sutfused over hali the Fig. 150. Buffurn.

fruit, with bright red, sprinkled with small lonwn dots, or a little russet. Stalk an inch long, inserted in a very slight cavity. Caly' $\mathbf{x}$ with small segrnents, and basin of moderate size. Flesh white, buttery, not so juicy as the Doyenne, but sweet, and of excellent flavour. The stroner upright redelish-brown shoots, and peculiar, brownish green apprarance of the pear, before ripening, distinguish this fruit. September. 
53. Beurré de Capiaumont. Thoinp.

Capiumont. Lind.

A Flemish pear, very fair, and handsomely formed, ana suct. a capital bearer, and so hardy in all soils and seasnns, that it is alrearly a very popular orchard and garden fruit. It is al. ways good, sometimes first rate, but when the tree is heavily laden, it is apt to be slightly astrin. gent. It grows freely; branches a little pendant, gravish yellow.

Fruit of medium size, long turbinate, very even, and tapering regularly into the stalk. Skin smooth, clear yellow, with a light cinnamon or cinnamon red cheek, and a fow small dots and streaks of russet. Calyx large, with spreading seginents, prominently placed, and not at all sunk. Stalk from three. fourths to an inch and a half long, curved. Flesh fine grained, buttery, melting, sweet, and when not astringent, of high flavour. September and Octnber.

This is quite distinct from the Frederick of

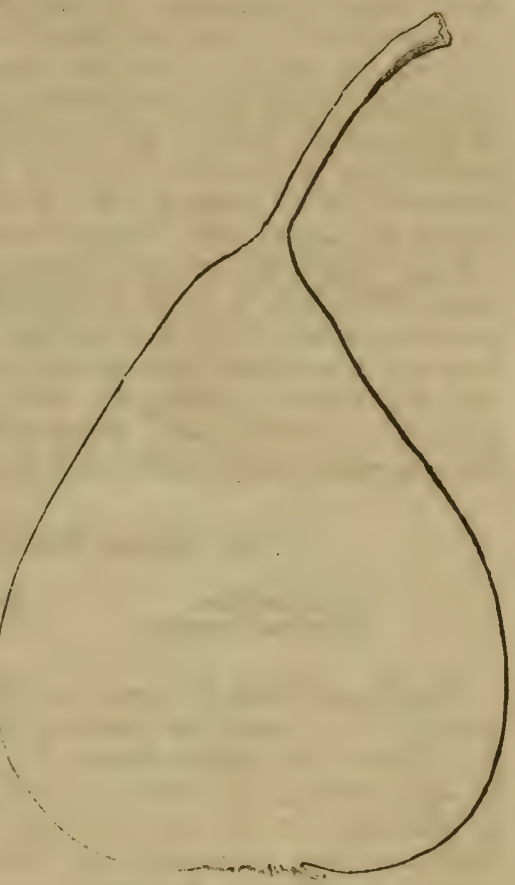

Fig. 151. Beurre de Capiunont.

IV urtemburgh, an irregular fruit, sometimes called by this name

54. Beurré, Brown. Thomp. Lind. Mill.

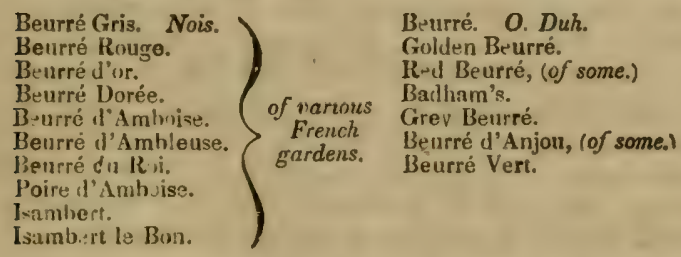

The Brown Beurré, almost too wcll known to need descrip 
tion, was for a long time, considered the prince of pears in France, its native countrv, and for those who are partial to the bigh vinous tlavour-a rich mingling of sweet and acill-it has, still, fitw enmpetituis. It is, however, quite variable in different soils, and its variety of appearance in diferent garilens, hits given rise to the many naines, gray, brown, red and golden, under which it is known. Kenrick calls it "an outcast," but our readers will pardon our dissent from this opinion, while we have the fact in tnind, of its general excellence in this region; and especially that of a noble tree, now in view from the library where we write, which is in luxuriant vigour, and gives us, an. nually, from five to eight bushels of superb fruit. 'The truth is, this pear is rather tender for New England, and requires a warm climate an 1 strong soil. Shoots diverging, dark brown.

Fruit large, oblong-obovate, tapering convexly quite to tho stalk. Skin slightly rough, yellowish-green, but nearly covered with thin russet, often a little redilish brown on one side. Stalk from one to one a half inches long, stout at its junction with the tree, anil thickening obliquely into the fruit. Calyx nearly closed in a shallow basin. Flesh greenish white, melting, buttery, extremely juicy, with a rich sub-acid flavour. September

\section{Beurré Bosc. Thomp.}

Cnlahasse Bosc.

Murianne Nuuvelle.
Bosc's Flaschenbirne.

Beurré d'Yelle, (of sume.)

The Beurre Bosc is a pear to which we give our unqualified praise. It is large, handsome, a regula bearer, always perfoct, and of the highest flavour. It bears singly, and not in clusters, looking as if thinned on the tree, whence it is always of fine sire. It was raised in 1807 by Van Mons, and named Calebasse Bose in honour of M. Bosc, a distinguished Belgian cultivator. Having also been received at the garden of the Horticultural Sociny of London under the name of Burre Bosc, Mr. 'Thompson thought it best to retain this name, as loss likely to lead to a confusion with the Calehasse, a distinct fruit. The tree grows vigorously; shoots long, brownish oive.

Fruit large, pyriform, a little uneven, tapering long and gradually into the stalk. Skin pretty smooth, dark yellow, a good deal covered with streaks and dots of cinnamon russet, and slightly touched with red on one side. Stalk one to two inches long, rather slender, curved. Calyx short, set in a very shallow basin. Flesh white, melting, very buttery, with a rich, delicious and slightly perfumed flavour. Ripens gradually from the last of September to the last of October. 


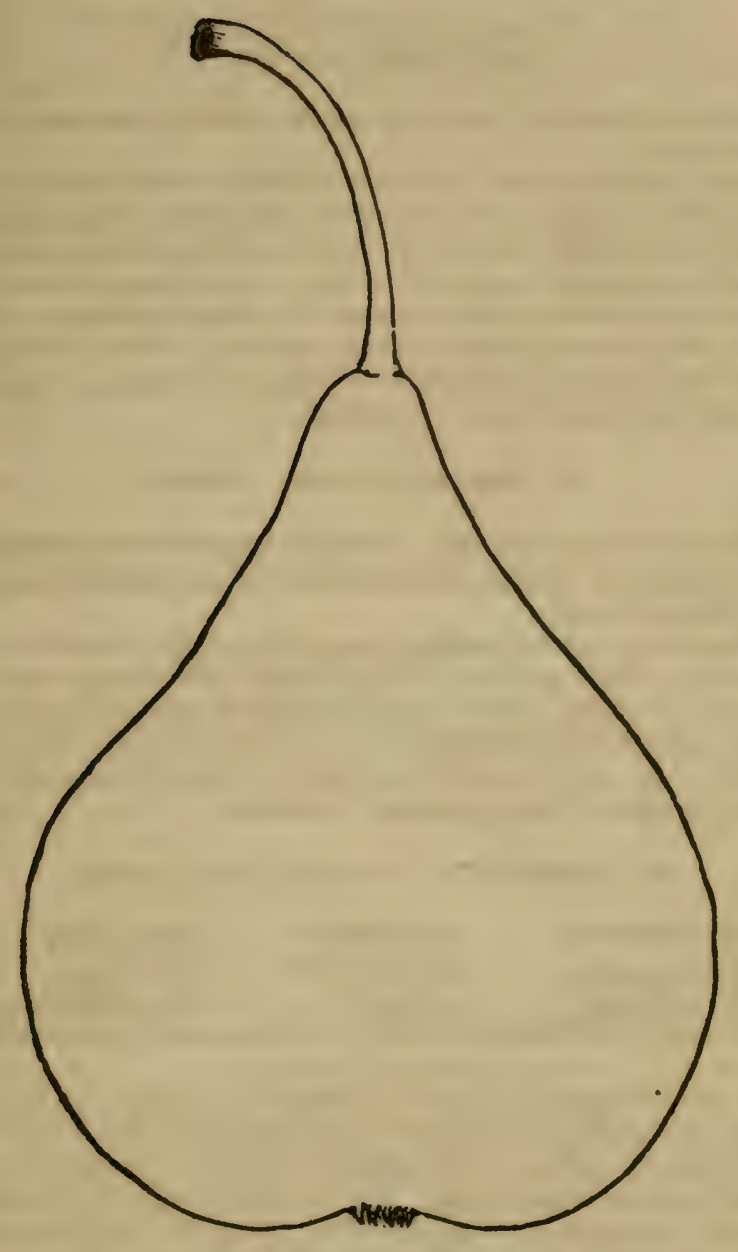

Fig. 152. Beurré Bosc. 


\section{Becrré d'Amalis. Thomp. Nois.}

Belrré d'Amanlis. Nous. Bon. Jurd

Beurré d'Amaulis. Ken. Man.

A Belgian pear, of excellent quality, nearly first rate. Very productive.

Fruit large, obovate, not very regular, a little sivollen on its sides. Slin rather thick, dull yellowish-green, with a pale red. dish brown cheek, overspread with numerous brown dots and russet streaks and patches. Stalk a little more than an inch long, set rather obliquely in a shallow, irregular cavity. Calyx open, with broad divisions, basin shallow. Flesh yellowish, sonnewhat coarse, but buttery, melting, abundant, rich, with slightly perfumed juice. September.

\section{Beurré D'ANJou. Thomp.}

This is a first rate pear, recently imported from France by Col. Wilder, of Boston, which appears to us quite distinct from the Brown Beurré.

Fruit rather above medium size, very regular, obovate. Skin greenish-ycllow, smoth, a little clouded with russet, especially around the calyx. Calyx small, open, in a round, smooth basin. Stalk rather short, straight, set in a slight cavity. Flesh yellowish-white, very fine grained, buttery, slightly sub-acid, with a rich, sprightly vinous flavour. October.

\section{Beurré Diel. § Thomp. Lind. P. Mag.}

Diel's Butterbime.

$1 \mathrm{H}$.

Ihvototieé Rorale.

Cir zsse Dorotheé.

Sys vanche vert d'hiver.
Beurré Royale.

Dorotieé Royalo.

Gros Dillen.

Dillen.

Des Trois Tours.
Beurré d'Yelle.

De Melon.

Mulin de Kons.

Beurré Magnifique.

Beurré Incumpirable.

A noble Belgian fruit, raised from seed, in 1895 , hy Dr. Vau Mons, and named in honour of his friend Dr. Augustus Fred. erick Adrien Diel, a distinguished German pomologist. Its sigour, productiveness and beauty, have made it already a general favouri ${ }^{*} \mathrm{c}$ with our planters. It is in every respect, a first rate fruit in fav urable situations, but on very young trees and in cold soils, it is apt to be rather coarse d astringent. The tree has long, very stout, twisting branches, and is un. commonlv vigornus. Young shoots dark grayish-brown.

Fruit laige, varying trom obovate to obtuse-pyriform. Skin ratner thisk, lemon yellow, becoming orange yellow, marked with large biown dots, and marblings of russet. Stalk an inch 


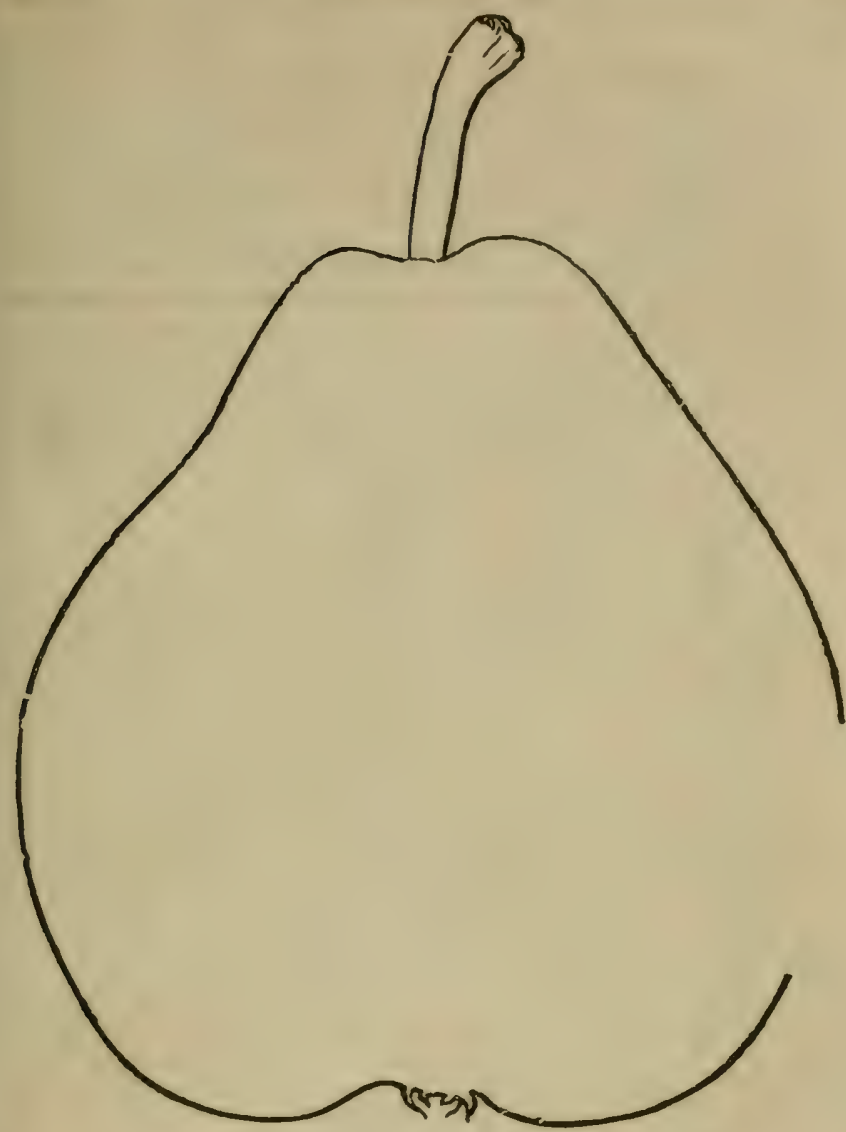

Fig. 153. Beutré Diel.

to an inch and three quarters long, stout, curved, set in a rather uneven cavity. Calyx nearly closed, and placed in a slightly furrowed basin. Flesh yellowish-white, a little coarse grained, especially at the core, but rich, sugary, half melting, and in good specimens, buttery and delicious. In eating, in this coun. try, from September to December, if picked and ripened in the house.

\section{Beurré KNox. Thomp. Lind.}

The Beurré Knox is a pleasant, second rate fruit, of large and handsome appearance, but a little liable to rot at the core. 
It is shaped a good deal like the Brown Beurre. A Flernist variety.

Fruit large, oblong, obovate, tapering to the stalk, which is about an inch long, curved, and set below a fleshy protuberance or lip-and without depression. Skin pale green, with thin russet on one side. Calyx open, and set in a small, narrow basin. Flesh tender and soft, juicy and sweet, but not high flavoured. Last of September.

This fruit is abundant near Boston. We received, by some error, a winter fruit, under this name, from the Horticultural Society of London.

\section{Beurré Kenrick. Man. in Hor. Mag.}

No. 1599 of Van Mons

\section{A Flem.} ish seedling, sent to this country and named by Manning.

"Medium size, flat at the blossom. end, tapering to the stalk, colourgreenish-yellow, with indistinct russet spots; stem one inch long; flesh good, juicy, sweet and buttery.Ripe in Septem. ber." Man.

61. BeurRÉ, GOLDEN OP BilboA. $\S$ Man.

Hooper's Bilboe.

The Gold. en Beurré

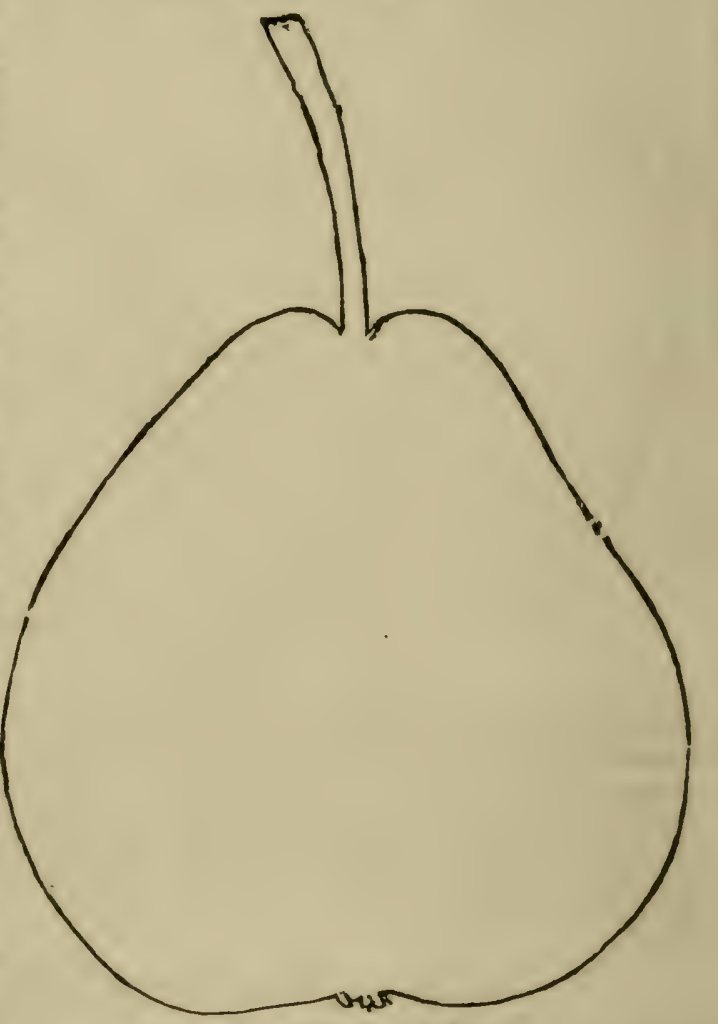
of Bilboa was

Fig. 154. Golden Beurrd of Bithoo.

as imported from Bilboa, Spain, about eighteen 
years ago, by Mr. Hooper, of Marblehead, Mass. Its European name is unknown, and it has become a popular fruit here under this title. It is of a fine golden colour, relieved by a little russet, and is certainly a beautiful early autumn pear of the first quality. It bears regularly and inost abundantly, and will become a favourite fruit in all parts of the country. In cold soils, it is particularly fine on quince stocks. Shoots stout, up. right, light yellowish.brown.

Fruit rather large, regular obovate. Skin very fair, smooth, and thin, golden yellow, evenly dotted with small brown dots, and a little marked with russet, especially around the stalk. Stalk about an inch and a half long, rather slender, set in a moderate depression. Calyx small, closed, placed in a slight basin. Flesh white, very buttery and melting, and fine grained, with a rich vinous flavour. First to the middle of September.

\section{Beurré Duval. Thomp.}

A new Belgian pear, raised by M. Duval. It is good, and bears abundantly. Fruit of medium size, obtuse pyriform. Skin pale green. Flesh white, buttery, melting and well fla voured. October and November.

\section{Beurré Preble. Man. in H. M.}

A large and excellent pear, named by $\mathrm{Mr}$. Manning in honour of Commodore Edward Preble, U. S. N., and raised from seed, by Elijah Cooke, of Raymond, Maine.

Fruit large, oblong-obovate. Skin greenish-yellow, mottled with russet and green spots. Stalk about an inch long, very stout, set in a moderate hollow. Flesh white, buttery, and melting, with a rich, high flavour. October and November.

\section{Beurré Colmar. Van Mons. Nois.}

Beurré Cơlnar d'Automne

A pleasant, juicy pear, ripening in October. It is one of Dr. Van Mons' seedlings, and is quite distinct from the Autumn Colmar.

Fruit of medium size, almost eliptical, or oval-obovate, regu. larly formed. Skin smoorh, pale green, becoming yellowish at maturity, with a blush next the sun, and thickly sprinkled with dots. Stalk an inch long. Calyx expanded, and set in a very shallow, narrow, irregular basin. Flesh very white, slightly crisp at first, but becoming very juicy and melting, with a slightly perfumed flavour. October. 


\section{Beurré de Beaunont § Thomp.}

A new and highly delicious pear, lately received from France, It appears, to us, to be worthy of extensive dissemination.

Fruit of medium size, roundish-obovate. Skin pale yellow. ish-green, thinly sprinkled with large dark green dots, and thin. ly washed with dull red on the sunny side. Stalk about an inch long, obliquely inserted, under a lip, or in a very slight cavity.

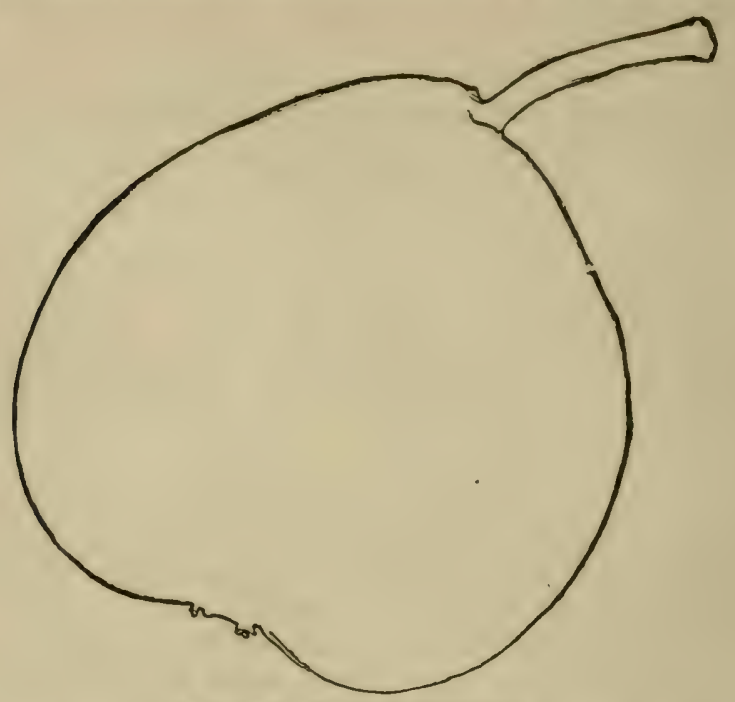

Fig. 155. Beurré de Beaumont.

Calyx small, with little or no division, and set in a shallow, smooth basin. Flesh white, buttery, melting, abounding with a rich, sprightly flavoursd juice. October.

\section{Beurré Van Mons. Thomp.}

The Beurré Van Mons is but just received in this country. It bears the reputation of a first rate fruit; it is of medium size, pyriform, skin yellowish, nearly covered with russet. Flesh buttery, melting and excellent. October.

\section{Beurré Romain. Thomp. N. Duh.}

A melting, juicy pear, of seconl quality. Fruit of medium size, regularly formed, obovate. Skin pale yellowish-green, dotted with numerous gray specks Stalk short, inserted with. 
out depression. Calyx prominently placed, even with the fruit, flesh white, juicv, melting, sweet and agreeable. September ta October. Bears abundantly.

\section{Beurré Van Maruni. Thomp.}

$A$ raiher large, and very good, juicy pear, one of the Flemish varieties. It comes early into bearing, and produces well.

Fruit large, oblong-pyriform, not very regular. Skin yellow, rarely with a little red. Stalk rather long and slender, inserted in a flattened cavity. Calyx large, set in an irregular shallow basin. Flesh white, melting, juicy, sweet and agreeable. First nf October.

\section{Beurré Spence? Thomp.}

It is probable that there may be a true Beurré Spence, since Ur. Van Mons claims to have raised one, and once pronounceci it the finest of all pears. But it is certain that neither the pomologists of England or America have yet been able to ob. tain it correct. Beurré Diel, Urbaniste, B. de Capiumont, and one or two others, of very inferiour quality, have been imported into this country for Beurré Spence. We have, however, received a tree from Mr. Rivers, the English nurseryman, which may prove correct. IIs says " this is the Beurré Spence of the Parisians. I ate it there in October, and thought it, simply, a good pear, scarcely deserving the high encomiums given by Vian Mons to Mr. Brāwuick."':k

70. Beurré Crapaud. Thomp.

A new foreign pear, resembling the Doyenné in flavour. Fruit of medium size, obovate. Skin pale greenish-yellow. Flesh buttery, fine-grained and excellent. Ripens in October.

\section{Beurré Picquery.}

The Beurré Picquery has lately been received from ìrance, where it has the character of a first rate fiuit, somewhat resembling the Urbaniste in general appearance; of rather larger size, melting, equally fine in flavour, ripening in October, and keeping a month or more. Shoots dark olive.

"In the mean time we annex Van Mons' original description. "Woa' shortjointed, leaves small, branches horizontal or declining. The fruit is of the shape and size of the Brown Beurré ; skin green, handsomely sprinkled and martied with reddish brown and reddish purple. Flesh tender, juicy, sugary and perfumei. It ripens about the last of September."-Revue des Revucs, 1330, p. 180. 
72. Bengantot, Autunn. Mill. Lind. Thomp.

\author{
Einglish Bergamot. \\ Yurk Bergainot. \\ Common Bergamot, (of England.) \\ English Autumn Bergamot.
}

The Autumn Bergamot is one of the oldest of pears, being sapposed by pomologists to have been in England since the time of Julius Cæesar. It is believed by Manger to be of 'Turkish origin, and originally to have been called Begarmoud,princely pear-from the Turkish, beg, or bey, and armoud, a pear. Since that time, the standard of excellence has risen much higher, and the title could, with more justice, be applied to the following variety than to this. The Autumn Bergamot bears well with us, and is of good flavour, but it is going out of cultivation, though the tree is thrifty, and bears well.

Fruit rather small, roundish and flattened. Skin roughish green, dotted with rough gray specks, and often with a faint or dull brown cheek. Stalk short, about half an inch long, stourt, inserted in a wide, round hollow. Calyx small, set in a shallow smooth basin. Flesh greenish-white, coarse-grained at the core, juicy, sugary and rich. September.

The BERganotTe D'Automne of the French, is a distinct fruit from this, usually more pyramidal; the skin smooth, light yel. lowish-green, with a brownish red cheek, and speckled with small, grayish dots. Stalk nearly an inch long, set in a slight cavity. Calyx very slightly depressed. Flesh breaking, juicy, and refreshing, but not high flavoured. A second rate fruit, though of fine appearance.

73. Berganot, Gansel's. § P. Mag. Thomp. Lind.

\section{Brocas Bergamot. Caxe. Ives' Bergamot. Staunton.}

Bonne Rouge.

Gurle's Beurré.

Diamant.

Gansel's Bergamot is a well known and delicious pear, raised sevcnty-seven years ago, from a seed of the Autumn Berga. mot, by the English Lieut. General Gansel, of Donneland Hall. Though a little coarse-grained, it is, in its perfection, searcely surpassed by any other pear in its peculiarly rich, sugary flavour, combined with great juiciness. It is stated, by some, to be an unfruitiul sort, and it is, in poor, or cold soils, only a thin bearer, but we know a very large tree near us, in a warm, rich soil, which frequently bears a dozen bushels of superu fruit. The mealy leaves, and spreading, dark gray shoots, distinguish this tree.

Fruit large, roundish.obovate, but much flattened. Skin roughish brown, becoming yellowish-brown at maturity, tinged sometimes with a russet red cheek, and sprinkled with spots of 


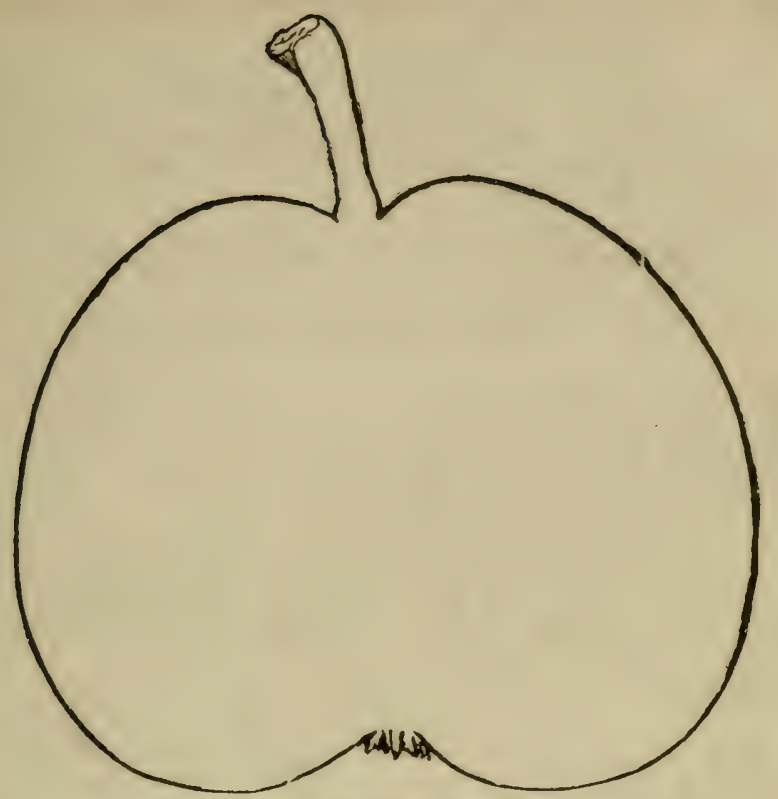

Fig. 156. Gansel's Berg imot.

russet. Stalk short, fleshy at both ends. Cavity moderate. Calyx short and small, placed in a smooth, moderate hollow. Flesh white, melting, very juicy, rich, sweet and aromatic. Ripens during all September.

\section{Bergamotte Suisse. O. Duh. Lind.}

Swiss Bergamot. Lind.

A very pretty, roundish, striped pear, which is a handsome addition to the dessert, and occasionally, when it ripens late, it is juicy, melting and excellent, but it is frequently of indifferent flavour. The tree is, with us, one of the strongest and most vigorous, and bears well. Branches striped.

Fruit of medium size, roundish, a little inclined to turbinate. Skin smooth, pale green, striped with yellow and pale red. Flesh melting, juicy, sweet and pleasant. October.

75. Bergamotre Cadete. O. Duh. Thomp.

Beurré Beauchamps. Poire de Cadet.

Beauchamps. Ognonet, (incorrectly, of some.)

A very good Bergamot from France, not, by any means 
equal, however, to Gansel's, but productive, and ripening for some time, in succession.

Fruit middle sized, roundish obovate. Slin smonth, pale green, rarely with a pale red cheek. Stalk an inch long, thick, set in an angular, shallow cavity. Calyx small, closed, basin nearly flat. Flesh buttery and juicy, sweet, and rather rich. October and Novemiver.

\section{Jezi* DE Montigny. Thomp. Lind. Poit.}

\section{Trouvé de Montigny.}

Beurré Romain? of some American gardens.

A p'easant, juicy fruit, with a musky flavour, but not firs! rate. The skin is remarkably smooth, and the pear is evenly fcrmrd. It is a goo' bearer. Fruit of medium size, very re. gulasly obovate. Skin pale yellowish-ereen, with numerous gray dots. Stalk stou, thickest at the point of insertion, an inch long, inserted in a small shallow cavity. Calyx small, firm, open, reflexed, in a very smooth basin, scarcely sunk. Flesh white, melting, juicy, half buttery, with a sweet, musky flavour. First of October.

77. BEZI DE LA MotTe. $\&$ O. Duh. Thomp.

Bein Armudi. Beurrś blane de Jersey.

This admirable old French pear is an es. pecial favourite of ours. Its flesh is solid and heavy, at the same time highly buttery, with a peculiaily pleasant flavour and aroma. The tree is exceed. ingly vigorous and productive,

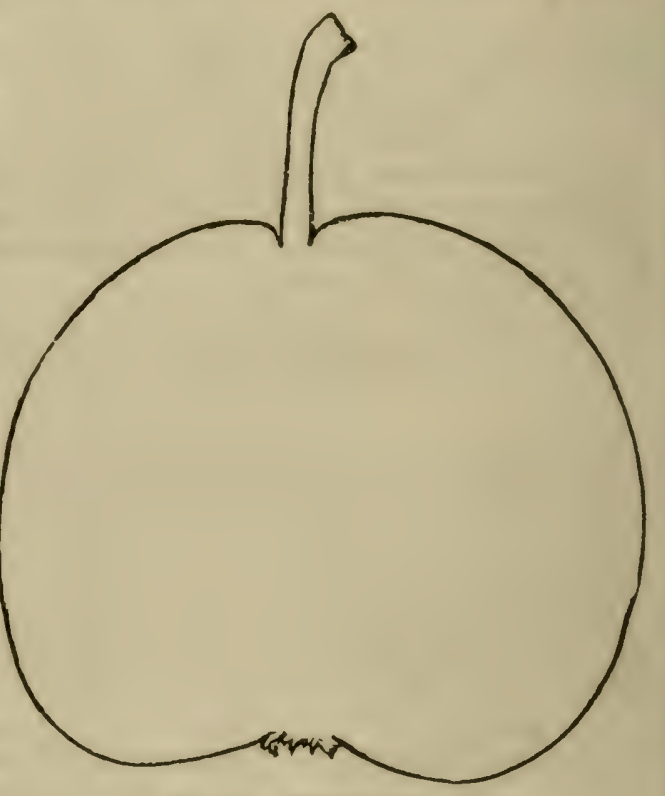

Fig. 157. Bexi de la Molle.

- Bezi signifies wilding, i. e. natural seedling found near Montigny, a lown in Frasice. 
and the grayish-olive shoots, like the fruit, have a peculiarly speckled appearance. Every garden should have a specimen of this fruit. It ripens gradually, and may be kept a good while.

Fruit of medium size, bergamot shaped, roundish, flattened at the eye. Skin pale yellowish-green, thickly sprinkled with conspicuous russet green dots. Stalk about an inch long, green, slightly curved, and inserted in a slight, flattened hollow. Calyx small, open, set in a shallow, rather abruptly sunken basin. Flesh white, very fine-grained, buttery, juicy, with a sweet, delicate, perfumed flavour. October.

78. Bishop's Thumb. Thomp. Lind.

A long, oddly-shaped, English pear, but jui. cy and excellent in flavour, indeed usually considered first rate. The tree bears abun. dant crops. Shoots grayish-olive.

Fruit rather large, oblong and narrow, and tapering irregularly, usually a little knobbed. Skin dark yellowish-green, dotted with russet, often nearly covered with russet specks, and having a russet red cheek. Stalk one to two inches long, slen. der, crooked, and set in a fleshy enlargement. Calyx with spreading divisions, and set in a flat basin. Flesh juicy, melting, with a good, rich vinous flavor. October

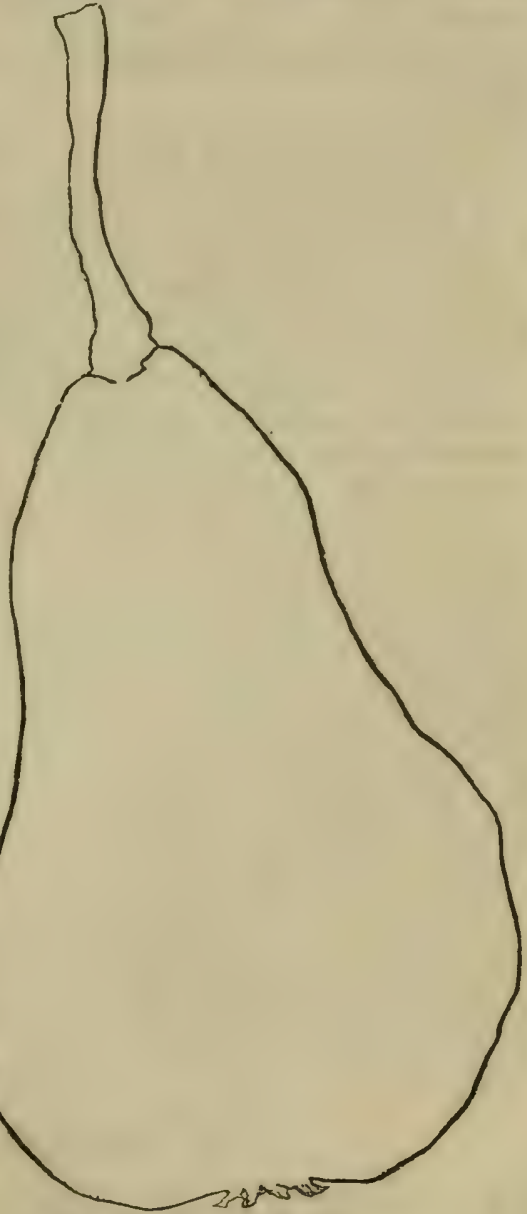

Fig. 159. Bishop's 'Thumb 


\section{Bon Chretien Fondante. Thomp. Liud.}

A recent Flemish pear, abounding with juice, and having a refreshing agreeable flavour. In good seasons, it is first of the quality, and it bears early and abundantly. Young shoots slender, diverging, olive gray.

Fruit pretty large, roundish-oblong, regularly formed. Skin pale green, sprinkled with small russet dots, and considerably covered with russet. Stalk three-fourths of an inch long, curved, inserted in a slight depression. Calyx small, set in a narrow hollow. Flesh yellowish-white, gritty round the core, exceedingly juicy, tender and melting, with a rich and pleasant slavour.

\section{Burnett. Ken.}

A pleasant, sweet pear, of large size, raised by Dr. Inel Burnett, of Southborough, Mass.

Fruit large, obtuse pyriform. Skin smonth, pale ycllow, with numerous greenish-gray dots, and sometimes a little russet. Stalk an inch and a half long, planted in a swollen base, or with a blunt depression. Calyx open, stiff, placed in a shallow basin. Flesh greenish-white, a little coarse grained, but juicy, sweet and good. First of October.

\section{Caвot. Man.}

Originated from the seed of the Brown Beurré, by J. S. Cabot, Esq., of Salem, Mass. It has a good deal of the flavour of its parent, and is an acreeable, sub-acid fruit. The tree grows upright and very strong, and produces amazing crops, but the fruit, with us, decays very quickly-though, we understand that, in older specinens, this is not the case. It merits a general trial. Col. M. P. Wilder, of Boston, informs us, that with him, it is of the first quality, nearly as good as Fondante d'A utomne.

Fruit pretty large, roundish-turbinate, narrowing rather abruptly to the stalk, which is bent obliquely, and inserted on one side, of a tapering summit. Slin roughish, bronze yellow, pretty well covered with cinnamon russet. Calyx small, open, set in a round, smooth basin. Flesh greenish-white, breaking, juicy, with a rich, sub-acid flavour. Middle and last of Sep. tember.

\section{Chelnjford.}

A native pear, from the neighbourhood of Boston of large 
and showy appearance and of second rate flavour, but much esteemed for stewing. It makes very strong wood, the young shoots yellowish-brown.

Fruit very large, irregular pyriform, with a wide crown. Skin deep yellow, at maturity, with a fine red cheek, sprinkled with distinct brownish-green dots. Stalk an inch and a half long, curved, planted in a crumpled shallow cavity. Calyx large, set in an irregular basin. Flesh white, juicy, rather .risp, with a saccharine flavour. Last of September.

\section{Compte de Lamy. $\S$ Thomp.}

Beurré Curté.

Dingler.

Marie Louise Nova.

$\left\{\begin{array}{c}a c . t o \\ \text { Thomp }\end{array}\right.$

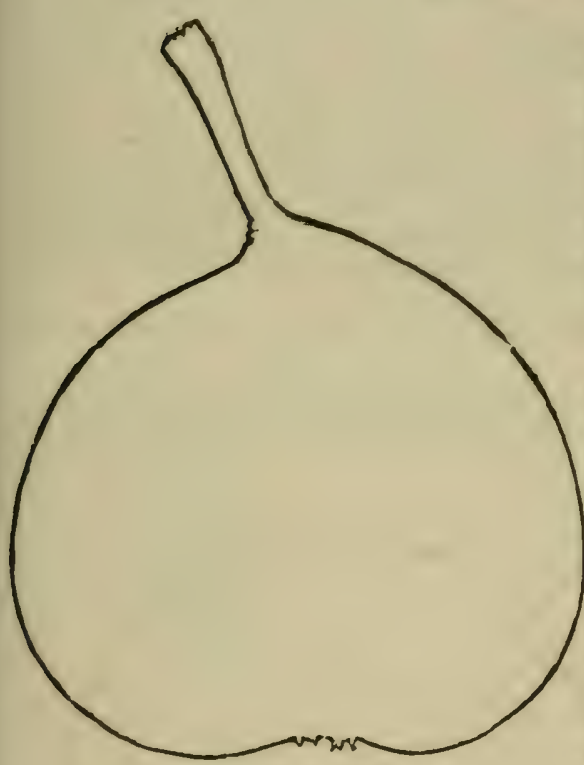

A rich, truly delicious, sugary pear, of the highest quality. It is one of the late new Flemish varieties, and is worthy of unusu. al attention. Young shoots pretty strong, upright, dark co. loured.

Fruit of medium size, roundish-obo. vate. Skin yellow, with a brownish red cheek, and sprinkled with small russetty dots. Stalk an inch long, straight, obliquely inserted under a lip, or planted in a slight cavity. Calyx small, set in a shallow, smooth baFig. 158. Compte de Lamy. sin. Flesh white, fine grained, buttery, melting, saccharine, and high flavoured. Last of September to middle of October.

This is quite distinct from the Marie Louise Nova of some Arnerican gardens, received from Van Mons. [See Marie Louise Nova.]

84. Comprette. 'Van Mons.

The Comprette is a very fine, new, Flerrish seedling, of $\mathrm{Dr}$. Van Mons', which has just begun to jear in this country, and 
was introduced by Col. Wilder, of Boston. It is, undcubtedly, a fruit of the first quality, and resembles in flavour tne Passe Colmar.

Fruit rather above medium size, obtuse-pyriform ; the short, stout stalk thickening into the termination. Skin yellowish green, thickly sprinkled with brown dots, and, occasionally. marked with a litile russet. Calyx pretty large, with few seg ments, set in a shallow bariu. Flesh white, buttery and meltIng, with a rich, perfiumed juicr. October to November.

\section{Conmodore. Man. in Hov. Mag.}

Van Mons, No. 1218.

A Belgian seedling, named by Mr. Manning, and promising to be of good quality, not quite first rate. Branches slender.

Fruit of medium size, very regular-obovate, tapering to the Stalk. Skin yellow, marked with a little red, some russet in patches, and a very few small dots. Sialk an inch or morr long, planted on the slightly flattened summit. Basin scarcely sunk, and having a small calyx. Flesh somewhat like that of the Doyenné-buttery, melting, with a sweet and excellent fla. vour. Last of October, to last of November.

86. Croft Castle. Thomp.

The Croft Castle is a recent English variety, peculiar in itg shape, and especially so in its flavour; the latter being greatly

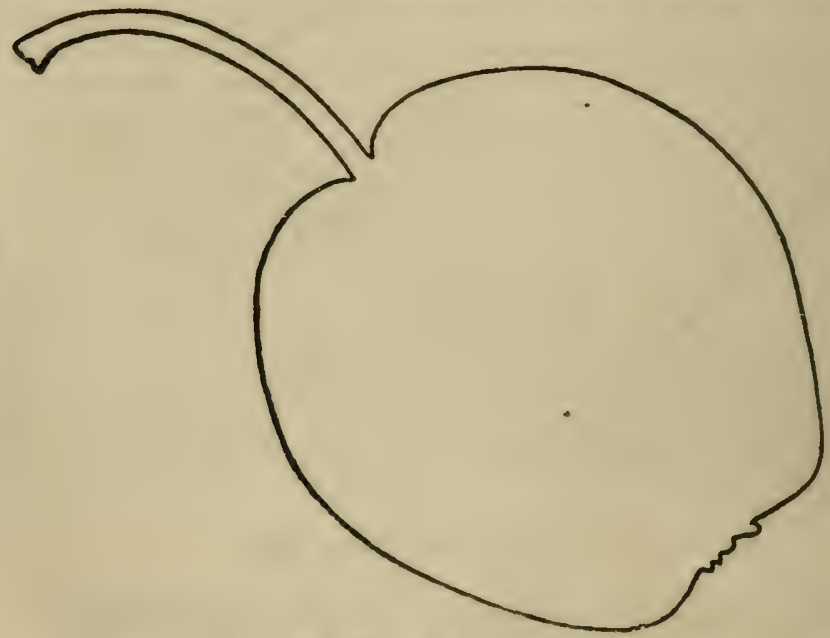

Rig. 159. Crnfl Custhe 
relished wy some persons, and not at all by others. It is very productive.

Fruit of medium size, oval, inclining to flattened ovatenarrowing most towards the rye. Skin pale greenish-yellow, marked with brown dots, and often a little russet. Stall: long and slender, curved, and planted almost even with the flattened summit. Calyx projecting a little beyond the level of the fruit, open, and stiff. Core large. Flesh juicy and a little crisp sweet, with a piquant perfume and flavour. October.

\section{COPIA.}

A Philadelphia seedling, named in thonour of the originator. Jacob Copia, Pine street, Philadelphia. It is a large and pretty good pear, resembling somewhat the Beurré Diel in flavour, but rather inferiour to it. Young shoots very stout, upright, olive brown.

Fruit large, broad-turbinate, tapering into the stalk, whicl is long, stout, and fleshy at the bottom, obliquely inserted. Ski yellow, with slight traces and specks of russet. Calyx large basin somewhat furrowed. Flesh rather coarse grained, but rich, juicy, and sugary. September and Octuber.

\section{Cushing. §Man.}

The Cushing is a native of Massachusetts, having originated on the grounds of Colonel Washington Cushing, of Hingham, about forty years ago. It is a very sprightly, delicious pear, and like many of our native varieties, it produces most abundant erops. Branches rather slender, diverging, grayishbrown.

Fruit medium size, often large, obovate, tapering rather obliquely to the stem. Skin smonth, light green-

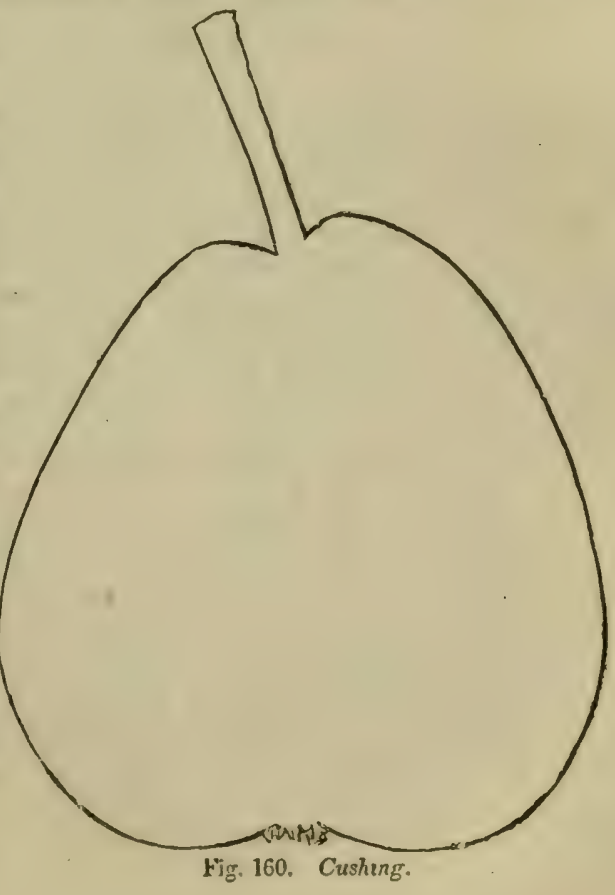


ish-yellow, sprinkled with small gray dots, and occasionally a dull red cheek. Stalk an inch long, planted in an abrupt cavity. Calyx rather small, set in a basin of mode. rate size. Flesh white, fine grained, buttery, melting, and abounding in a sweet, sprightly, perfumed juice, of fine flavour. A hardy and capital variety for all soils. Middle of September.

\section{Capsheaf. Man. Ken.}

This is believed, by the eastern cultivators, to be a native of Rhode Island. It is a very agreeable fruit, not first rate, but from its great hardiness, and steady habit of bearing, is well worthy of the attention of pear growers. Young shoots stout, upright, yellowish-brown.

Fruit of medium size, roundish-obovate. Skin deep yellow, nearly covered with cinnamon russet. Stalk an inch long, stout, inserted in a shallow hollow. Calyx small; basin slightly sunk. Flesh white, juicy, and melting, very sweet and pleasant, but lacking a high flavour. October.

90. Calebasse. Thomp. Lind.

Calebasse Double Extra.

Calebasse d'Hollande.

Beurré de Payence.

The Calebasse is a very grotesque-looking Belgian fruit, named from its likeness to a calabash, or gourd. It is a good deal esteemed, especially by curious amateurs, being a crisp, sweet, juicy pear, of second quality, and producing good crops.

Fruit of medium size, oblong, a little crooked, and irregular or knobby in its outline. Skin rough, dull yellow, becoming orange russet on the sunny side. Stalk about an inch and a half long, curved, and planted on the side of a knobby projection. Calyx very short, set in a small basin. Flesh juicy, crisp, a little coarse-grained, but sugary and pleasant. Middle of September.

This is the Calcbasse Bosc of the Jardin Fruitier, but incor rectly. [See Beurré Bosc.] The Calebasse Grosse, [Mon. strous Calabash, etc.] of Van Mons, is a prodigiously large, pyramidal fruit, 5 or 6 inches long, in the shape of a conical gourd. Skin smooth and shining, yellowish green, with a good deal of reddish gray in the sun. Stalk short and stout, about an inch long. Calyx rather small, but with large divisions. Flesh white, a little coarse, juicy, half melting, sugary and tolerably good. October. (The grafts sent out for this kind, by Van Mons, proved incorrect.) 


\section{Carucin. Van Mons.}

Capuchin.

This promises to be a very good pear. It is one of Var. Mons' Seedlings. Young shoots stout, diverging, dark coloured.

Fruit pretty large, oval, or sometimes obtuse-turbinate. Skin green, becoming pale yellow, a litule russeted towards the eyo and stalk, and distinctly dotted elsewhere, with also a red cheek. Staik nearly an inch long, placed in an obtuse hollow. Calyx small, deeply sunk in a narrow, irregular basin. Flesh green. ish, juicy, crisp, sugary and rich. October.

5

\section{Clara. Van Mons.}

Claire. Nois.

Another seedling, raised by the Belgian pomologist. The young shoots are stout, upright, dark brown. It is of medium size, oval-pyriform. Skin clear yellow, dotted with red, and having a blush on the sunny side. Stalk stout and straight. Calyx small, set in a small, round basin. Flesh white, melting, very juicy ard sweet, relieved by a slight acid; of good quality. In cold seasons it is liable to be too acid. September and October.

\section{Cumberland. Man. Ken.}

This is a native fruit, and the original tree is still growing in Cumberland, Rhode Island. It is inferiour to the Cushing, and though sometimes very handsome, and always productive, can scarcely be ranked higher than a second rate fruit, and occasionally it is quite indifferent. Branches rather slender.

Fruit rather large, obovate. Skin orange yellow, with a little russet, and a pale red cheek on the sunny side. Stalk about an inch and a half long, stout, rather obliquely planted, in a very slight depression. Calyx with expanded divisions, and placed nearly even with the surface. Flesh white, melting, buttery, and tolerably rich and juicy. September and October.

\section{Crassane. Thomp. Lind. \\ Bergamotte Crassane. \\ Cresane. \\ Beurré Plat.}

A celebrated, old French pear, which is said to take its name from ecrase, flattened or crushed, from its depressed, Bergamotlike shape. Its flavour is extolled by all the Eurnpean writers, but we have never been able to find it to equal its foreign cha. racter here, and cannot recommend it. Young shoots stout, diverging, grayish-olive. 
Fruit large, roundish, flattened. Skin greenish-yellow, embroidered thinly with russet. Stalk long, slender, curved, and planted in a slight, shallow cavity. Calyx small, set in a narrow rather deep basin. Flesh whitish, juicy, soft, sweet, and tole. ralily pleasant. October, and may be kept for a month longer.

\section{Charles of Austria. Thomp. Lind.}

Charles d'A utriche.

A large and handsome Belgian pear, which is likely to be. conse a favorite here. Raised by Van Mons. Young shoots stout, upright, yellow-olive.

Fruit large, roundish, a little uneven. Skin greenish-yel. low, a little russeted and thickly dotted with conspicuous brown specks, which give it a brownish appearance. Stalk an inch long, slightly inserted. Calyx set in a rather narrow hollow Flesh white, tender, quite juicy, sweet and agreeable. October

96. Colmar Epine. Van Mons. Man. in H. M.

An agreeable, juicy pear, sent to this country by Van Mons, and originated by him. Young shouts stout, upright, brown.

"Fruit large, roundish-oblong, tapering, gradually, to all obtuse point at the stem, which is one inch long; colour green ish-yellow; flesh white, sweet, very melting, juicy, high fla voured, and good." Middle of September.

\section{Chinton. Man. in H. M.}

Van Mons, No. 1233.

A second rate fruit. Mr. Manning says, "Large size, shaped like the Bezi de Montigny ; light yellow skin, flesh soft, tuttery and good, but not high flavoured." Middle of November.

'The wood is stout, and dark brown.

\section{Calfoun. Wilder Mss.}

New, and recently originated by Gov. Edwards, of New. Haven. It promises to be a fruit of the first quality.

Fruit of medium size, obovate, terminating obtusely at the insertion of the stalk. Skin usually smooth and handsome, pale yellow, occasionally with a pale red cheek. Flesh juicy, melting, with a rich, sub-acid, or vinous flavour. October to November.

99. Colmar Neill. Thomp.

This is a new variety, lately received from abroad, where it 
Mus a high reputation. It is a very handsome pear, very pro. ductive, and of most excellent flavour.

Fruit large, obovate. Skin pale yellow. Flesh white, but Les $y$, melting, of high flavour. Ripens at the middle of October

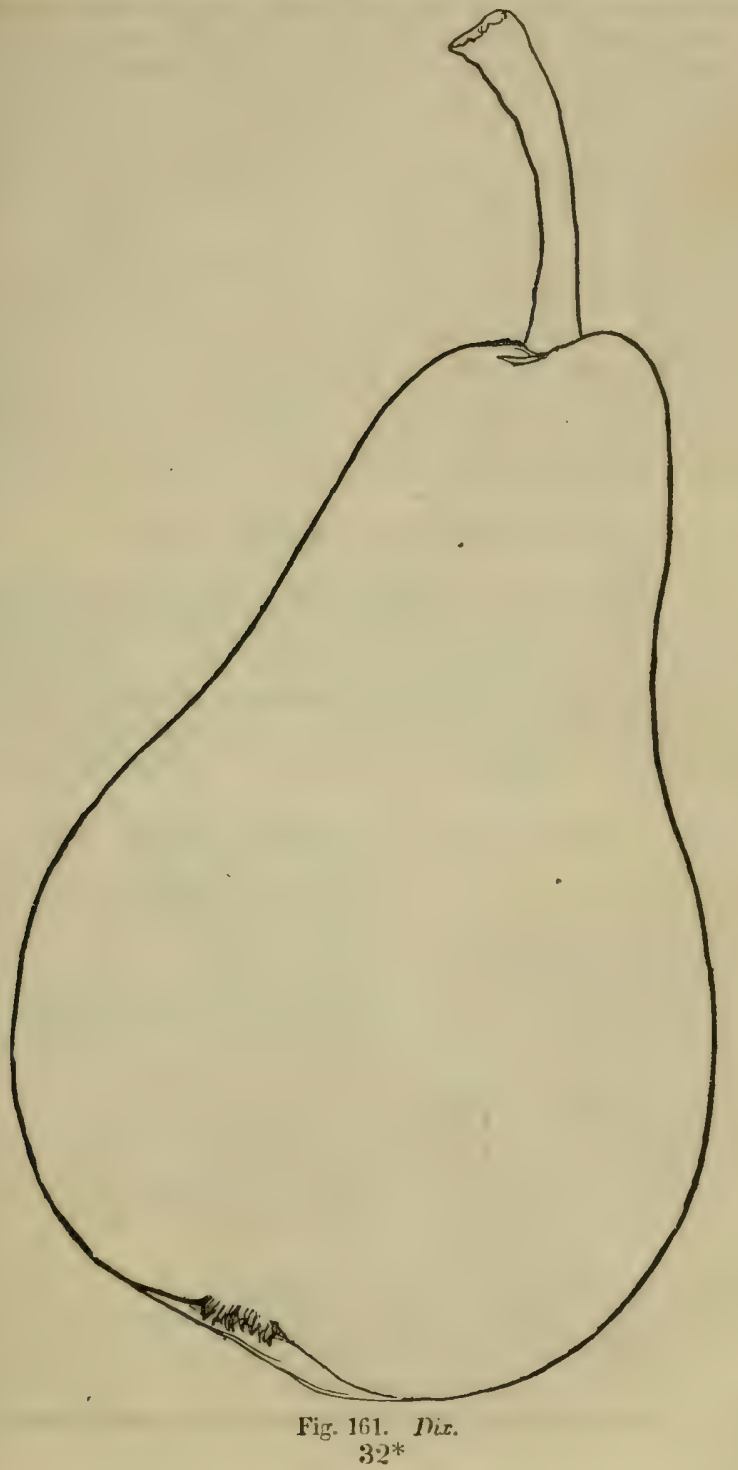




\section{Drx. $\S$ Man. Ken.}

The Dix is, unquestionably, a fruit of the highest excellence, and well deserves the attention of all planters. It is one of the hardiest of pear trees, and although the tree does not come into bearing intil it has attained considerable size, yet it pro. duces abundantly, and from its habit, will undoubtedly prove remarkably long-lived, and free from disease. The young branches are pale yellow, upright and slender. The original tree, about thirty-five years old, stands in the garden of Madam Dix, Boston. It bore for the first time in 1826 .

Fruit large, oblong, or long-pyriform. Skin roughish, fine deep yellow at maturity, marked with distinct russet dots, and sprinkled with russet around the stalk. Calyx small, for so large a fruit, basin narrow, and scarcely at all sunk. Stalk rather stout, short, thicker at each end, set rather obliquely, but with little or no depression. Flesh not very fine grained, but juicy, rich, sugary, melting and delicious, with a slight perfume. October and November.

\section{Dumortier. $\oint$ Thomp. Nois.}

A very excellent little Belgian pear, often remarkably high flavoured. Fruit nearly of medium size, obovate. Skin dull yellow marked with russet patches and dots. Stalk nearly two inches long, slender, planted without depression. Calyx open, set in a slight basin. Flesh greenish-white, juicy, melting and sweet. It keeps but a short time. September.

\section{Doyenné, White. $\S$ Thomp. Lind. P. Mag.}

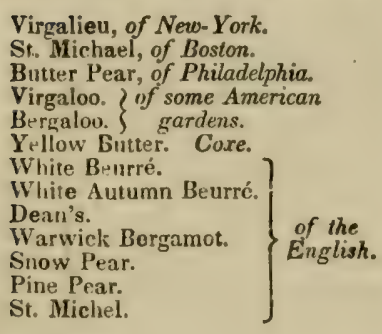

Virgalieu, of New-York.

St. Michael, of Boston.

Butter Pear, of Philadelphia.

Virgaloo. I of some American

Bergalou. $\}$ gardens.

Yullow Butter. Coxe.

ean's.

Pine Pear.

St. Michel.
White Autumn Beurré.

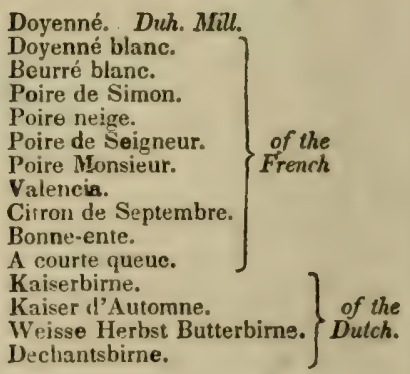

The White Doyenne is, unquestionably, ore of the most perfect of autumn pears. Its universal popularity is attested by the great number of names hy which it is known in various parts of the world. As the Virgalieu in New.York, Butter Pear in Philadelphia, and St. Michel's in Boston, it is most commonly 
known, but all these names, so likely to create zonfusion, should bo laid aside for the true one, White Doyenné. It is an old

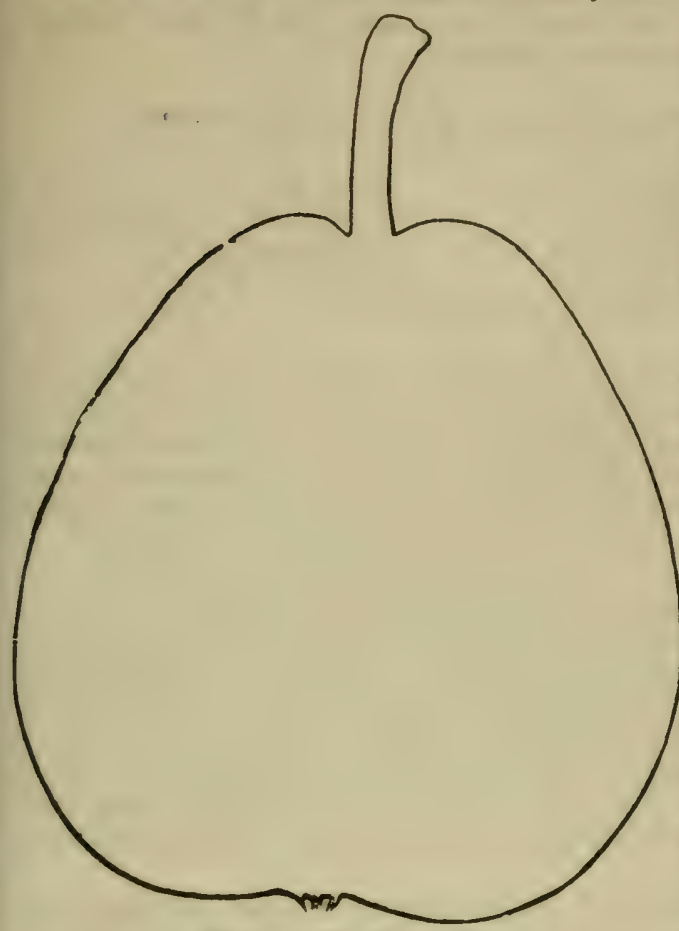

Fig. 162. White Doyenné.

French varie. ty, but with us, is in the most perfect health, and bears annually large crops of superb fruit. On the sea-coast, and in various old, or exhausted soils, it has lately become so liable to cracking as to be nearly worthless. In this case it is only necessary to renew the elements wanting-probably potash and lime -and, if the trees arc diseased, to plant healthy ones. The branches are strong, up. right, yellowish-gray or light brown.

Fruit of medium or large size, regularly formed, obovate. It varies considerably in different soils, and is often shorter or longer on the same tree. Skin smooth, clear, pale yellow, regu. larly sprinkled with small dots, and often with a fine red cheek. Stalk brown, from three-fourths to an inch and a fourth long, a little curved, and planted in a small, round cavity. Calyx always very small, closed, set in a shallow basin, smooth or delicately plaited. Flesh white, fine-grained, very buttery, melting, rich, high-flavored, and delicious. September, and, if picked early from the tree, will often ripen gradually till December.

* Virgalieu seems an American name, and is always liable to be confounded with the Virgouleuse, a very different fruit. The Doyenne, (pronounced dwoy-an. nay, literally dennship, is probably an allusion $v$. the Dean, ky whom it was first brouglit into notice. 
The Doyenne Panache, or Striped Dean, is a variety rather more narrownt, to the stalk, the skin prettily striped with yellow, green, and red, and dotted with brown. Flesh juicy, melting, but not high flavouxed. October.

103. Doyenne, Gray. § Thomp. Lind. P. Mag.

Gray Butter Pear.

Gray Deans.

Gray Doyenné.

Red Doyenné.

St. Nichel Doré"

Doyenné Galeux.

Doyenné Boussouck, (of some.)
Doyenné Gris, Duh.

Doyenné Rouge.

Doyenné Roux. Nois. Poit.

Doyenne d'Automne.

Red Beurré. \&incorrectly

Beurré liouge. $\}$ of sme.

The Gray Doyenne strongly resembles the White Doyenne in flavour and general appearance, except that its skin is covered all over with a fine, lively cinnamon russet. It is a beautiful pear, usually keeps a little longer, and is considered by many rather the finer of the two, but in the valley of the Hudson where both are remarkably fine, we do not perceive its superiority. It is much less known than the foregoing sort, and richly deserves more general attention. Shoots upright, grayish-brown.

Fruit of medium size, obovate, but usually a little rounder than the White Doyenné. Skin wholly covered with smonth cinna. mon russet, (rarely a little ruddy next the sun.) Stalk half, to three-fourths of an inch long, curved, set in a narrow, rather deef and abrupt cavity. Calyx small, closed, and placed in a smooth, shallow basin. Flesh white, fine grained, very buttery, melting, rich, and delicious. Middle of October, and will keep many weeks.

['The tree received in this country for Doyenne Boussouck, and Bossouck Nouvelle, have proved synonymous with this variety.]

\section{Dunmore. $\S$ Thomp.}

'The Dunmore is a large, and truly admirable pear, raised by Knight, which has been introduced into this country from the garden of the London Horticultural Society. It is a strong growing tree, bears exceedingly well, and is likely to become a very great favourite. Its blossoms resist even severe frosts.

Fruit large, oblong-obovate, rather swollen on one side. Skin greenish, dotted and speckled with smooth, brownish-red russet. Stalk stout, one to two inches long, fleshy at the base, planted obliquely on an obtuse end, or in a very fiat depression. Calyx rather small, open, sunk in a narrow basin. Flesh yellowish. white, buttery, exceedingly melting, with a rich, high-flavour, September. 
AUTUMN PEALE.

981

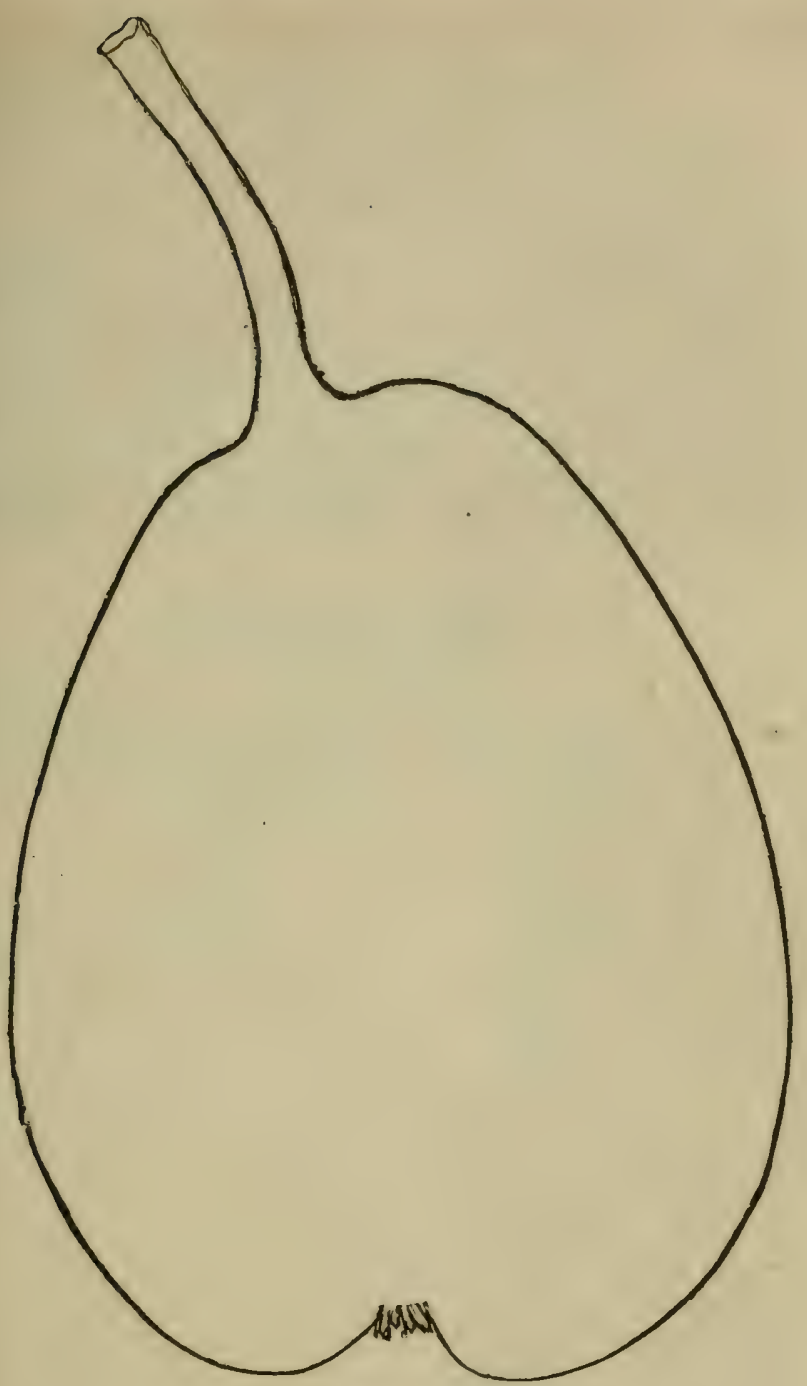

Fig. 163. Dinmore.

105. Duchess D'Angoûlene. $§$ Lind. Thump.

A magnificent large dessert pear, sometimes weighing a pound and a quarter, named in honour of the Ditches of An. 
gouleme, and said to be a natural scedling, found in a forest hedge, near Angers. When in perfection, it is a most delicious fruit, of the highest quality. We are compelled to adsi, how. ever, that the quality of the fruit is a little unceriain $c n$ young standard trees. On the quince, to which this sort seems we:l adapted, it is always fine. The tree is a strong grower, tue shoots upright, light yellowish.brown, and it is cieserving nial in all warm dry soils.

Fruit very large, oblong-obovate, with an uneven, somewhat knobby surface. Skin dull greenish-yellow, a good deal streaked and spotied with russet. Stalk one to two inches long, very stout, bent, deeply planted in an irregular cavity. Calyx set in a somewhat knobby basin. Flesh white, buttery, and very juicy, with a rich and very excellent flavour. October.

The quality of the fruit is often injured by the excessive luxuriance of the tree. This should, in such cases, be obviated by root pruning. (See p. 32.)

\section{Duchesse de Mars. Thomp.}

\section{Duchesso de Mars.}

The Dutchess of Mars lately received from France, and first introduced by $\mathrm{J}$. C Lee, Esq. of Salem, Mass., proves to be a rich, melting pear, in this climate, with a peculiar and good flavour.

Fruit nearly of medium size, obovate. Skin dull yellow, con. siderably covered with brown russet, and becoming ruddy on the sunny side. Stalk an inch long, inserted with little or no depression. Calyx smail, stiff, closed and placed in a slight basin. Flesh very melting and juicy, somewhat buttery, with a rich and perfumed flavour. October and November.

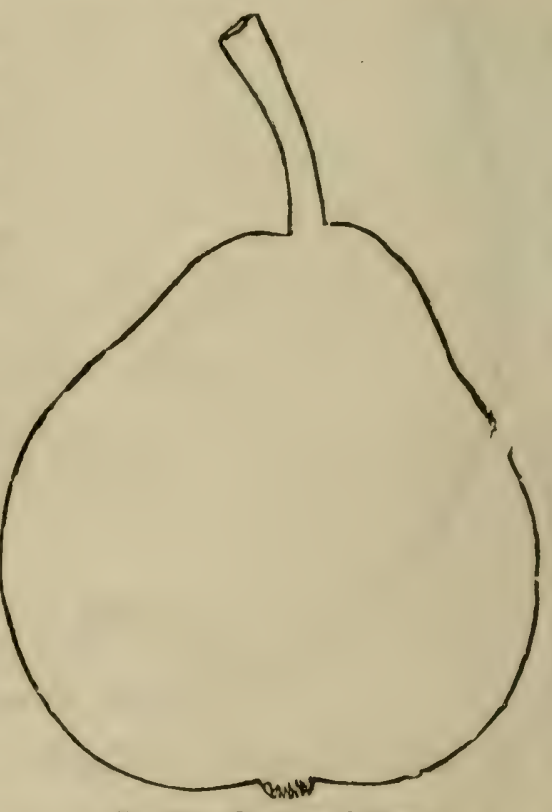

Fig. 164. Drachess of Mars. 


\title{
107. D'Amour.
}

\author{
Ah! Mon Diou. O. Duh. Lind. \\ Mon Vieu. \\ D'A bondance. \\ Poire d'Amour.
}

This little French pear, once considerably esteemed, is now little cultivated. We have sometimes tasted it of very rich fla. vour. It is a very fruitful tree.

Fruit small, obovate, inclining to turbinate, the end taperirg and swelling regularly into the stalk. Skin pale yellow, l,ut nearly covered with red, which is sprinkled with nume:ous darker dots next the sun. Stalk an inch long, curved, set. in a swollen base. Calyx small, nearly level, the shallow basin having a few plaits. Flesh white, very juicy, tender and melt. ing, with a sweet, rich flavour.

\section{De Louvain. Van Mons.}

Poire de Louvain. Nors. Lind.

A pear of the finest quality, raised by Van Mons in 1827 while his "Nursery of Fidelity" was at Louvain.

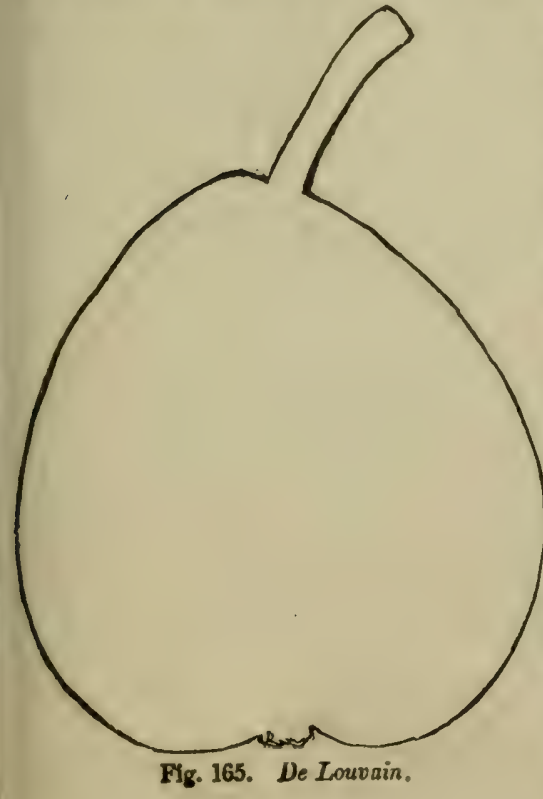

Fruit of medium size, obovate, inclining to pyriform, and tapering to the stalk. Skin rather uneven, clear light yellow, a little marked with russet, and dotted with brown points, which take a ruddy tinge next the sun. Stalk about an inch long, stout, insert. ed obliquely without depression, or by the side of a fleshy lip. Calyx placed in a very narrow, shallow basin. Flesh white, buttery and melting, with a rich, perfumed, and delicious fiavour. Ripers the last of Sep. tember, and keeps till November. 


\section{Duchesse D’Orleans.}

Latey received from France, where it has the reputation Deing a very handsome fruit, of the first quality, with precisely the flavour of the old, and much admired Gansel's Bergamot. Young wood light green.

Fruit large, long-pyriform. Skin golden yellow, dotted an streaked with a little russet. Flesh buttery, melting, rich, sugary and aromatic. Very productive, and ripens in October.

\section{Délices D'Hardenpont. Thomp.}

Délices d'Ardenpont. Lind.

A melting, buttery pear, one of the new Flemish varieties, and raised by the counsellor Hardenpont, of Mons. It has borne for several seasons in this country, and proves of rich and excellent flavour. The tree is moderately thrifty; shoots upright, yellowish-brown.

Fruit of medium size, obtuse-pyriform, with its widest part above the middle, and a little uneven in surface. Stalk an inch long, curved, and set rather obliquely in a narrow, shallow cavity. Skin pale yellow, dotted with numerous small gray dots in the shade, and somewhat russetted in the sun. Calyx very small, closed, and placed in a small, uneven basin. Flesh buttery, melting, with an abun. dant, slightly perfu. med, and rich juice. Middle of October.

111. Dundas. $\S$ Van Mons. Man. in H. M.

A very brilliant coloured fruit, remarka. bly handsome for the dessert, and of rich fla. vour. It is a recent Belgian variety, sent to this country by Van Mons, in 1831.

Fruit medium size,

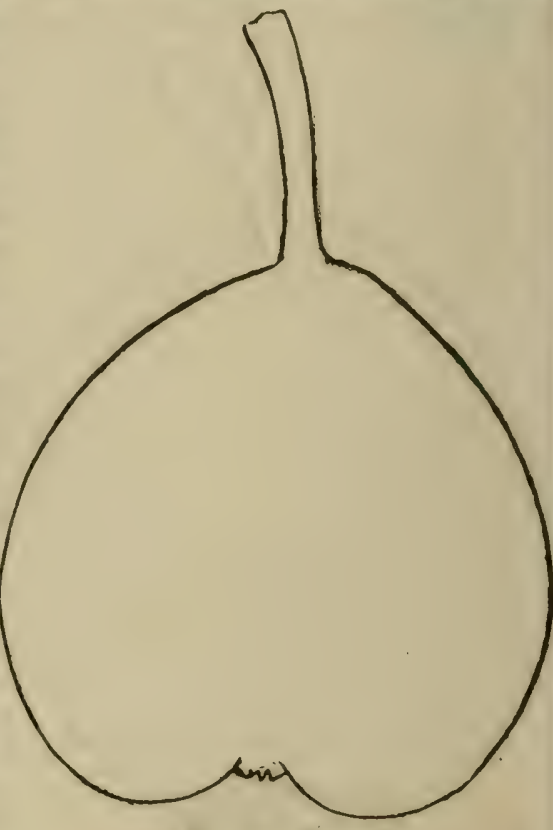

Fig. 166. Dundase. 
obovate, inclining to turbinate. Skin clear yelllow, sprinkled with greenish-black dots, and heightened by a very brilliant red cheek. Stalk dark brown, an inch long, stout, inserted without depression. Calyx small, placed at the bottom of a aeep round basin. Flesh yellowish.white, half buttery, melting, with a rich, perfumed juice. First of October, and keeps some time.

\section{Elizabeth, Edvards'. Wilder. Mss.}

Edwards' Elizabeth is a seedling pear of great beauty, and nearly, if not quite, of first rate quality, raised by ex-governor Edwards, of New-Haven, Conn.

Fruit of medium size, often large, obtuse-pyriform, angular, and oblique at the base, the stalk frequently planted in a lleshy protubcrance, like a fold. Skin smooth, pale lemon yellow, very fine, and of a peculiar waxen appearance. Flesh white, buttery, slightly sub-acid and good. October.

113. Elizabetir, Manning's. Man. in H. M.

Van Mons. No. 154.

Manning's Elizabeth, a seedling of Dr. Van Mons', named by Mr. Manning, is a very sweet and sprightly pear, with a peculiar flavour.

Fruit below madium size, obovate, shaped like the Julienne, or a small White Doyenné. Skin smooth, bright yellow, with a lively red cheek. Stalk. one inch long, set in a shallow, round cavity. Flesh white, juicy, and very melting, with a saccha. rine, but very sprightly, perfumed flavour. Last of August.

\section{Edwards' Henrietta.}

This is also one of Gov. Edwards' new Seedlings, raised at New-Haven. It bears most profusely, is a very agreeable fruit, and deserves a trial generally.

Fruit nearly of medium size, obovate, flattened at the base, sloping to an obtuse point at the stalk. Skin smooth, pale yellowish-green, with few dots. Stalk an inch and a half long, inserted in a very slight depression. Calyx closed, and set in a shallow, faintly plaited basin. Flesh melting, juicy, sub-acid and rich. Middle and last of August.

\section{Enfant Prodige. Van Mons.}

This is one of Dr. Van Mons' seedlings, which, from its name, Enfunt Prodige-wonderful child-must have been considered 
one of his most remarkable. 'The fruit is often remarkably ugly, and at times remarkably good. The tree bears abundantly with us, and the pears vary much, both in shape and quality-some. times indifferent, and at others first rate, with a rich sub-acid flavour, between a Brown Beurré pear, and a Banana.

Fruit of medium size, varying in form, obovate, always narrow at the stalk. Skin rough, and a little uneven, pale tawny yellow, a little russeted, and dotted with small specks, gray in the shade, and reddish gray on the sunny side. Stalk one and a half Inches long, a little curved, and set in a very slight depression, or under a slight lip. Calyx closed, crumpled, set in a slight. narrow, furrowed basin. Flesh melting, full of rich, sub-acid, vinous juice, of very agreeable flavour. October, and will keep a month. Shoots diverging, dark-olive.

\section{Eyewond. 'Thomp.}

A seedling of Mr. Knight's, not yet fairly proved in this country, but coming to us from Mr. Thompson, as of first rate quality, the tree vigorous, hardy, and a sure bearer.

Fruit of medium size, oblate or flattened; skin much covered with russet. Flesh buttery, rich and excellent.

\section{Flemish Beauty. $\oint$ Lind. Thomp.}

Belle de Flanders.

Bouche Nouvelle.

Bosch.

Bose Sire.
Impératrice de France.

Josephine. incorrecily,

Fondant Du Bois. 5 of some.

Boschpeer.

In good soils and open situations, the Flemish Beauty is certainly one of the most superb pears in this clinate. IVe have seen specimens, grown on the banks of the Hudson, the past summer, which measured 12 inches in circumference, and were of the finest quality. The tree is very luxuriant, and bears early and abundantly; the young shoots upright, dark brown. It should be remarked, however, that the fruit requires to be gathered sooner than most pears, even before it parts readily from the tree. If it is then ripened in the house, it is always fine, while, if allowed to mature on the tree, it usually becomes soft, flavourless, and decays soon.

Fruit large, obovate. Skin a little rough, the ground pale ye!low, but mostly covered with marblings and patches of light russet, becoming reddish brown at maturity, on the sunny side. Stalk rather short, from an inch, to an inch and a half long, and pretty deeply planted in a peculiarly narrow, round cavity. Calyx short, open, placed in a small, round basin. Flesh yel. lowish-white, not very fine grained, but juicy, melting, ver. saccharine and rich, with a slightly musky favour. Last a September 


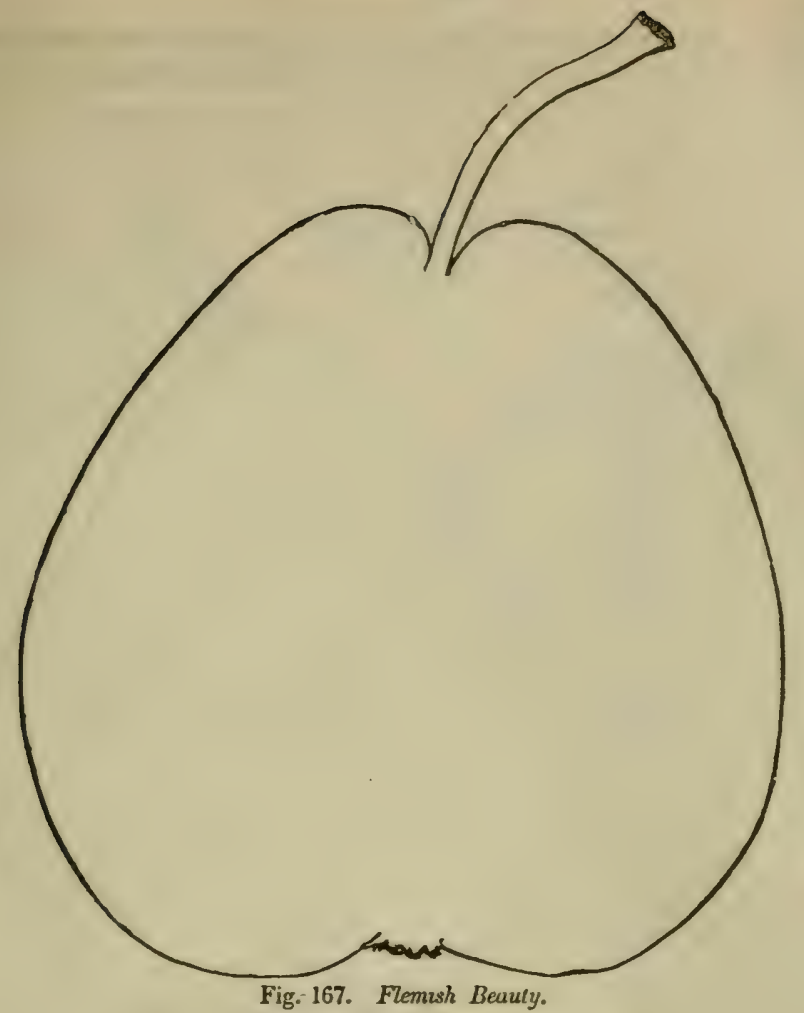

118. Fondante Van Mons. Thomp.

An excellent melting pear, raised by Dr. Van Mons, and first introduced by Mr. Manning. It bears abundantly.

Fruit nearly of medium size, roundish, a little derressed. Skin pale yellow. Stalk stout, an inch and a half long, planted in a rather deep cavity. Calyx set in a pretty deen hasin. Flesh white, juicy, melting, sweet, and of very agreeable na. vour. First of November.

\section{Fondante D'Autonne. \& Thomp.}

Belle Lucrative.* Lind. Man. und of most American gardens.

If we were asked which are the two highest flavoured pears

* This is the pear described by Lindley as Bel!e Lucrative. By some error. Mr. Thompeon, in the last edition of the Catalugue of the London Hurticutcura Suciety, bas made the two sort distinct. They are identically the Eame. 
known in this country, we should not hesitate to name the Seckil, and the Fondante d'Automne (Autumn melting.) It is a new Flemish pear, and no garden should be diestitute of it. The tree is of moderate growth, the young shoots long, yellow. ish.gray.

Fruit medium size, obovate, narrow, but blunt at the stalk. Skin pale yellow. ish-green, slightly russeted. Stalk little more than an inch long, stout, often fleshy, obliquely inserted in a slight, irregular cavity. Calyx very short, open, with few divisions, set in a basin of mo. derate depth. Flesh exceedingly juicy, melting, sugary, rich and delicious. Last of September.

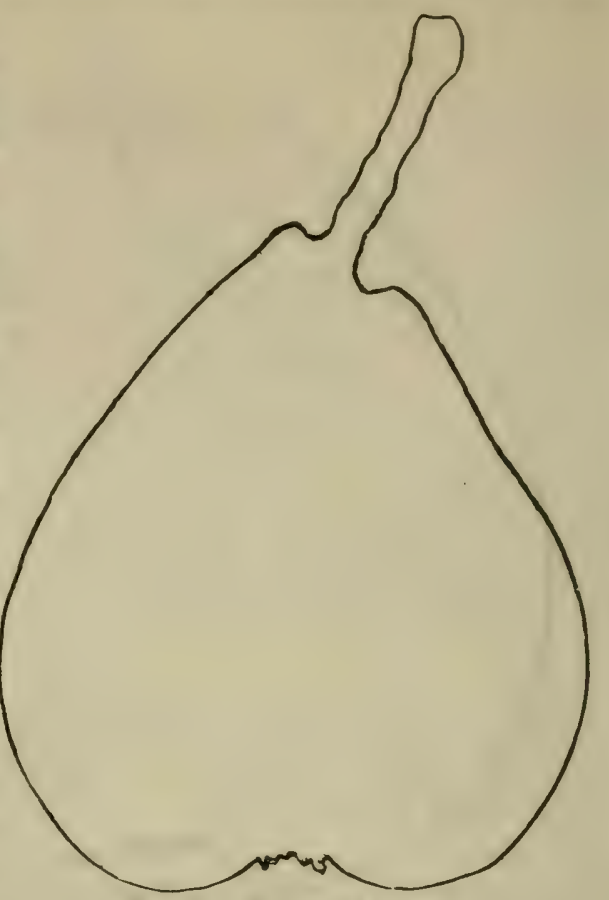

Fig. 163. Fondante d'Astomne.

\section{Forde de Délices. Thomp.}

A new Flemish pear, of excellent quality, received from the I.ondon Horticultural Suciety. Young shoots stout, upright, yellowish-green.

Fruit of medium size, obovate. Skin rough, yellowish, a good deal markel, or nearly covered with dull russet. Sialk an inch long, planted in a smooth, round cavity. Calyx wide, open, large, projecting. Flesh buttery, melting, somewhat dry, but sweet and good. Last of October.

\section{Figue de Naples. Thomp.}

Comtesse de Frénol. De Vigne P'elone.
Beurré Bronzée, incorrertly of some.

Fig Pear of Naples. Man.

A very goorl, late autumn pear, but inferiour to several thers. It grows vigorously and bears well. 
Fruit of rather large size, oblong-ousvate. Slin nea:'y covered with brown, and tinged with red next the sun. Flesh buttery, melting, and agrecable. Norember.

122. Forelde. Thomp. P. Mag. Lind.

Forellen-birne.

Poire Truite.

Trout pear.

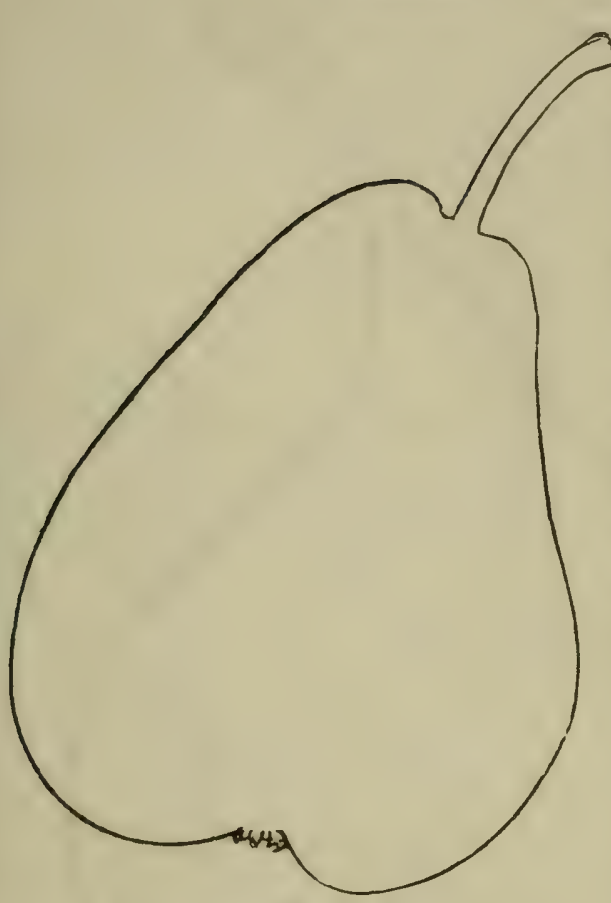

Fig. 169. Forelle.

This exquisite. ly beautiful Ger. man pear-called in that language Forellen-birnei. e. trout pear, from its finely speckled appear. ance, is one of the most attractive dessert fruits. It requires a warm soil and exposure, and well deserves to be trained as an espalier. It does not appear to have succeeded. well near lioston, but it fully sus. tains its high foreign cliaracter with us. Young shoots long, with few, and dark coloured branches.

Fruit oblong-obovate, inclining to pyriform. Skin smooth, nt first green, but, when fully ripe, lemon yellow, washed with rich deep red on the sunny side, where it is marlsed with large, marrined, crimson specks. Stalk about an inch long, rather slender, slightly curved, rather obliquely planted, in a shallow, uneven cavity. Calyx rather small, basin abruptly sunk. Flesh white, fine grained, buttery, melting, with rich, slightly b..bus juice. Beginning of November, and may be kept, with care, till Christmas. 


\section{Frédéric de WVurteniburf. $\S$ Van Mon. Nois.}

\section{Frederick of Wurtemburg."}

it is remarkable that this extremely handsome and very good dessert fruit, originated by Van Mons in 1812, should not to this day have found its way into the large collection of the London

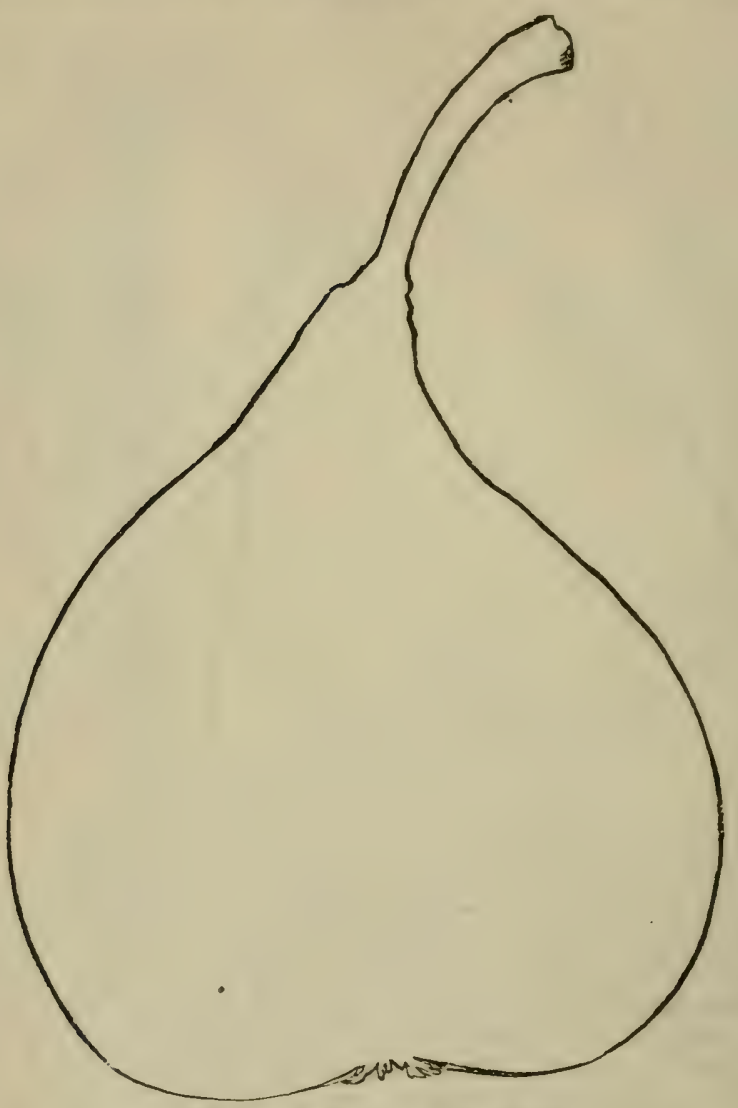

Fig. 170. Frederick of W'urlemherg.

Inrticultural Society. It is very distinct from the Beurre Ca. piumont, with which it is sometimes confounded in this coun.

* 'The Napoleon is sometimes incorrectly rereived under the name of "Wurcemberg," and the Glout Morceau as "Roi de Wurtumberg," both of which names have also been applied to this pear in America. 
try-the latter being very smooth, with a promirent calyx, whila this is rather uneven, with a somewhat sunken basin. The young wood is very stout and blunt, yellowish-brown, and the tree bears very young. (Part of the stock in this country scems stunted; it may be renovated by severe pruning back and grafting on thrifty stocks.) This is a pear that every annateur will cultivate.

Fruit large, one-sided, pyriform, rather uneven in its surface. Skin deep yellow at maturity, with a remarkably rich crimson cheek. Stalk quite stout, rather more than one inch long; curved, sometimes placed in a blunt hollow, but usually thickening into the fruit. Calyx open, large, set in a shallow, slightly furrowed basin. Flesh white, very juicy, melting and siveet; and when in perfection, buttery, and delicious. September.

\section{Fultow. Man. Ken.}

This American pear is a native of Maine, and is a seed. ling, from the farm of Mrs. Fulton, of 'Top. sham, in that state. It is very hardy, and bears every year abundant crops 0 . nice, small, grayrusset pears, which, if picked pretty ear. ly and ripened in the house, are of very excellent quality. Ripened on the tree they are worthless. Young shoots rather slender, and reddish. brown.

Fruit below medium size, roundish, flattened. Slin, at first, entirely grayrusset in colour, but

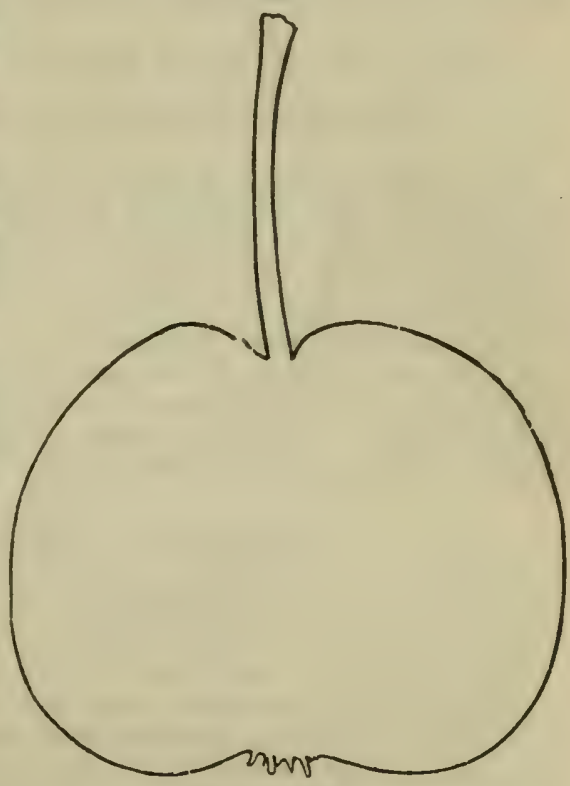

Fig. 171. Fulton.

at maturity, of a dark cinnamon russet. Stalk one to two inches long, slender, planted in a narrow cavity. Calyx with long segments, sunk in an uneven hollow. Flesh half buttery, moderately juicy, with a sprightly, agreeable flavour. Seeds comnressed, October and November. 


\section{Gendeshein. Thomp. Lind.}

A Flemish pear, which has but lately come into bearing, but promises well.

Fruit large, obtuse pyriform, a little irregular. Skin palo greenish-yellow, much dotted with gray, and marked with a little russet. Stalk an inch long, obliquely planted, in a slignt cavity, which is sometimes swollen. Calyx small, set in a narrow, irregular depression. Flesh rather gritty near the core. elsewhere buttery, rich and excellent. October and November.

\section{Green Pear of Yair. Thomp.}

\section{Green Yair.}

The green pear of Yair is an European fruit, which proves but little worthy of cultivation here. It bears abundantly.

Fruit of medium size, obovate; skin green; flesh very juicy, but not high flavoured or rich. September.

\section{Great Citron of Bomenia. Man. in I. M.}

Citronenbirne Bümische grosse, punctirte. Baum. Cat.

This pear was imported some years ago, by Mr. Manning, from the nursery of the brothers Baumann of Bolwyller, on the Rhine. It has not yet fruited with us, or any where, that we can learn, except in Mr. M.'s garden. We therefore give his notes, with the remark that its merits will soon be fully tested here. Young shoots very stout, dark gray.

"Fruit large, oblong, yellow, spotted and tinged with red on the side of the sun; stem one inch long; flesh sugary, juicy, and very fine." The specimen we tasted was a litile coarro grained. Ripens the last of September.

\section{Harvard. Man. Ken.}

\section{Boston Epame.}

Cambridge Sugar Pear.

The Harvard is one of the best and most profitable orchard pears, to plant in quantity for market purposes. It produces cnormous crops of fine looking fruit, which is of fair quality, and commands the best prices. The tree is remarkably hardy and vigorous, its upriglit shoots forming a fine hrad. It origi. nated at Cambridere, Mass, the seat of Harvard University.

Fruit rather large, oblong-pyriform. Skin russety olive-yel. low, with a brownish red cheek. Stalk rather stout, inserted rather obliquely on the narrow summit or in a small cavily. Calyx st in a narrow basin. Flesh white, tender, juicy and 
meltirg, of excellent flavour, but liable, if not pickec carly, to rot at the core. Beginning of September.

\section{Henry tife Fousth. $\oint$ Lind.}

\section{Henri Quatre. Thomp.}

Jacquin.

This little pear, perhaps not very attractive in appearance, being small, and of a dull colour, is one of our greatest favour. ites as a dessert fruit. It always bears well-often too abun. dantly-and the very melting fruit abounds with delicious, high

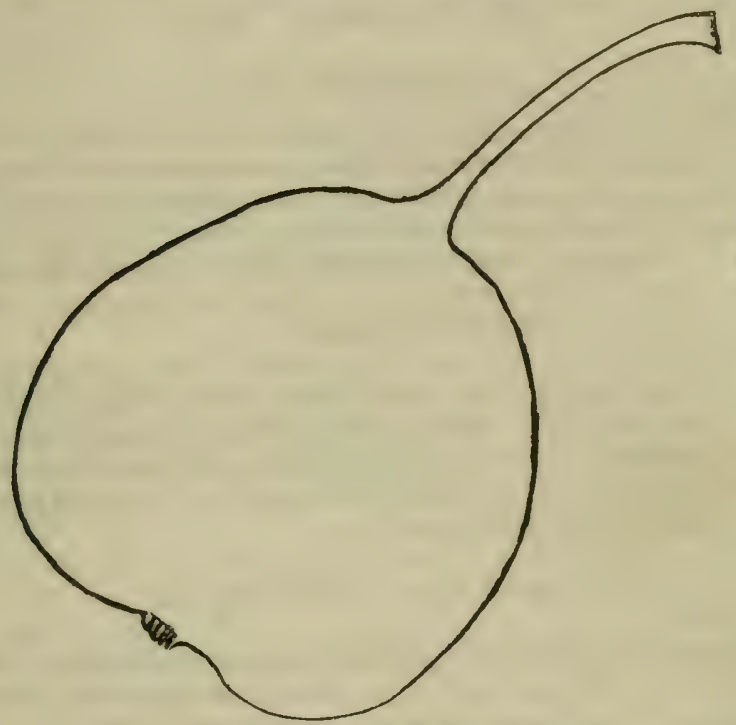

Fig. 172. Henry $\Gamma$.

flavoured juice. Every good collection of pears should com. prise it. The tree is hardy, and the branches, very thick of foliage, are a little pendant. Young shoots diverging, yellow. ish-brown.

Fruit below medium size, roundish-pyriform. Skin pale greenish-yellow, dotted with small gray specks. Stalk rather more than an inch long, slender, bent, and obliquely planted on a slightly flattened prominence, or under a swollen lip. Calyx small, placed in a shallow, abrupt basin. Flesh whitish, not very fine grained, but unusually juicy and meiting, with a rich, delicately perfumed flavour. It should always be ripened in hie house. Early in September. 


\section{Héricart. Van Mons.}

A second rate, Belgian pear, with a pleasant, perfumed juice, ripening early in Autumn.

Fruit of medium size, obovate, often rather oblong and irregu. lar. Skin pale green, slightly tinged with yellow, and doited with many greenish and russety specks. Stalk an inch or more long, rather slender, set in a small cavity. Calyx set in a shallow basin. Flesh white, fine grained, buttery, not rich, but with a delicate, peculiar aroma. The fruit ripens from the last of August, for a month or more.

\section{Heathcot. Man.}

\section{Gore's Heathcot. Kien.}

The heatheot, one of our most excellent native pears, will al. ways compete with the best foreign ones, especially for orchard culture. It is a hardy, thrifty tree, bears abundant crops of fair fruit, which is always of good quality. It was originated on the farm of Governor Gore, in Waltham, Mass., by Mr. Heatheot, then a tenant, and the original tree came into bearing in 1824 . Young shoots upright, reddish-brown.

Fruit of medium size, regularly obovate. Skin pale greenish. yellow, with very few dots, and a few russet streaks. Stalk an inch long, planted in a very small cavity. Calyx closed, and set in a rather narrow and shallow basin. Flesh white, buttery and melting, moderately juicy, with an agreeable, vinous flavour. Middle and last of September.

\section{Huld. Hov. Mag.}

A new pear, which originated in the town of Swanzey, Mass. It received a premium and high commendation at the annuas exhibition of the Massachusetts Horticultural Society, in 1843, when it was first presented.

Fruit of medium size, obovate. Skin yellowish-green, a good deal sprinkled with russet. Flesh white, a little coarse grained, but meiting, juicy, with a sweet, slightly perfumed flavour.

\section{Huguenot.}

A fruit of second quality, originated by Mr. Johosnot, of Salem. It bears abundantly, but is rather dry, and not worthy of general cultivat.on. Young shoots strong, upright, yellow. ish.brown.

Fruit of medium size, roundish. Skin smooth, pale yellow, sprinkled with large round spots of bright red. Stalk rather 
slender, curved, and inserted without depress on, on the slightly flattened end. Calyx small, set in a nicely rounded basir. Flesh white, fine grained, half breaking, sweet, but wanting in flavour and juice. October.

\section{Hacon's Inconparable. Lind. Thomp.}

Downham Seedling.

A capital English fruit, of modern origin, raised by $\mathbf{M r}$. Hacon, of Downham Market, Norfolk. It is a hardy, produc. tive tree, with rather depending branches, and the fruit is of the finest quality. Young shoots rather slender, diverging, olive-coloured.

Fruit rather large, roundish, inclining to turbinate. Skin slightly rough, pale, and dull yellowish-green, mixed with pale brown, sprinkled with numerous greenish russet dots, and russet streaks. Stalk an inch or more long, straight, inserted in a broad, shallow depression. Calyx with many small divisions, set in a wide, shallow basin. Flesh white, buttery, melting, with a rich vinous flavour. October and November.

\section{Johonnot. Man.}

This excellent native pear, which we received from the late Mr. Manning, originated in the garden of George S. Johonnot, Esq., of Salem, Mass., and bore first in 1823. The fruit is of medium size, of a roundish, and peculiar irregular form. Skin very thin, dull yellowish.brown, and obscurely marked with russet. Stalk short and thick, planted by the side of a swollen protuberance. The flesh is fine grained, melting, buttery and very goood. The tree is not very vigorous, but it bears good crops, and is in perfection from the middle of September to the middle of October.

\section{Jalousie, Duh. Nois. Thomp.}

A very unique looking, old French pear, with the richest reddish-russet skin, admired by the curious amateur, but not by the general cultivator. It makes a handsome appearance on the fable, but is only of second rate flavour, and soon rots at the core. Young shoots stout, olive.

Fruit rather large, varying in form from roundish to obovate, and more frequently pyriform. Skin rough, of the deepest russet, ruddy in the sun, and singularly marked with conspicu. ous, lighter coloured specks, which are slightly raised. Stalk an inch or an inch and a half long, planted in a very slight: cavity. Calyx small, set in a rather narrow basin. Flesh a 
little coarse grained, soft, sweet, and of pleasant flavour. Last of September.

\section{Jalousie de Fontenay Vendée. $\oint$ Man in H. M.}

This excellent French pear, was imported from Vilmorin, of Paris. It is greatly superiour in flavour to the old Jalouste, though having a little of its peculiar appearance. Young shoots upright, long, brownish-yellow.

Fruit of medium size, turbinate, or ob. tuse - pyriform. Skin dull yellow and green, considerably marked with russet patches and dots, and tinged with a red cheek. Stalk about an inch long, set obliquely, without depression on an - obtuse point. Calyx with closed and stiff segments, set in a shallow, round basin. Flesh white, buttery, melting, with a rich fiavoured juice. First of October.

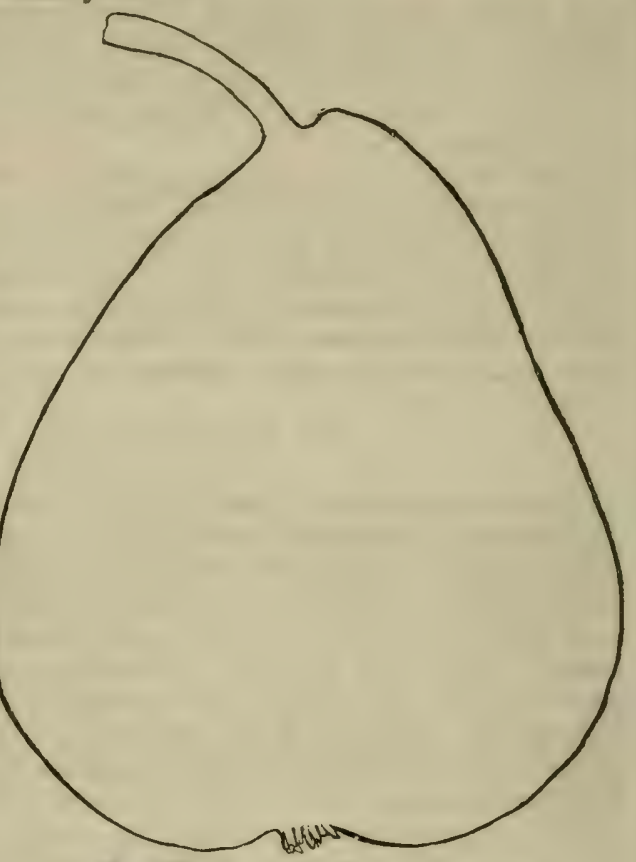

Fig. 173. Jalouse de Fontenay Vendke.

138. Kiṇg Edward's. Thomp. Jackman's Melting. Man.

King Edward's is a large, and very handsome fruit, which was received from England, with a high reputation, but which proves a very uncertuin fruit in this climate. Occasionally, it is of excellent flavour, but very often it is quite astringent and indifferent. The tree is very thrifty. Young shoots stout, upright, dark brown.

Fruit large, pyriform, tapering gradually to the stalk, which 
Is very short, and inserted without depression. Skin rather rough, yellow, a little mottled with patches of grcenish russet, and marked with a fine red cheek. Calyx sinall, somewhat projecting, basin very slight. Flesh jellowish, buttery, melting and good, when the season is favourable. October.

139. Lotise Bonne of Jersey. § Thomp.

Louise Bonne de Jersey.

Louise Bonne d'Avranches.

Beurré, or Bonne Louise d'Araudoré.

William the Fourth.

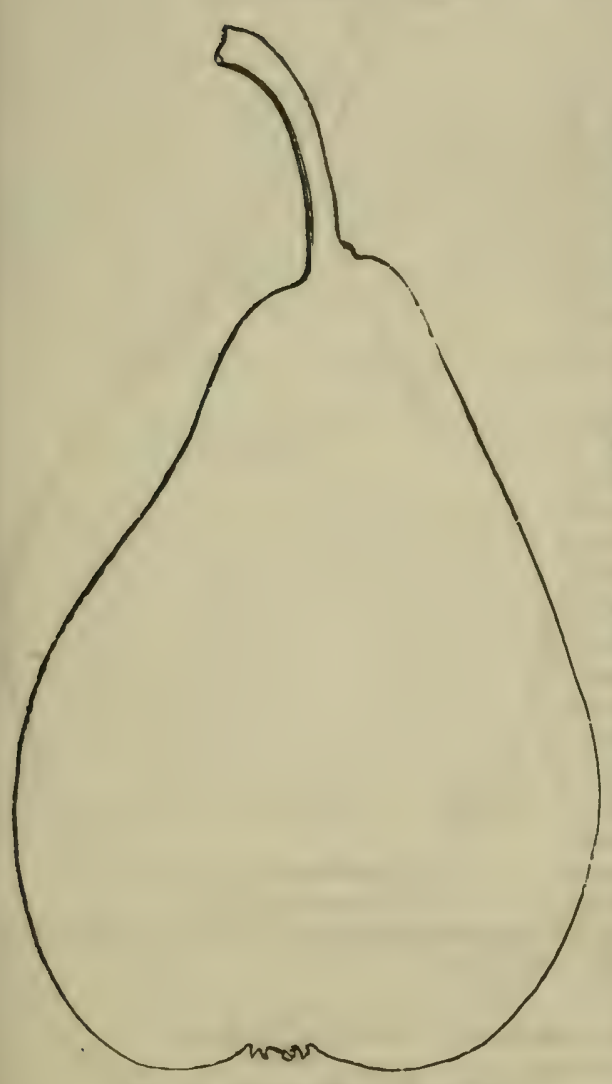

Louise Bonne, of Jersey, is one of the best new autumn pears, fair and glossy, exceedingly juicy, and well flavoured. It is claimed by English cultivators for the Isle of Jersey, and by the French for the neighbourhood of Avranches, and there is no doubt it originated in the latter place. The first fruit scen in England, was sent to the London Horticultural So. ciety's exhibition, from the gardens of

Gen. Gordon, of Jersey, in 1820. In this country it succeeds admila. bly, and will become a very popu. lar fruit, being har. $\mathrm{dy}$ and productive, the tree making fine upright shoots Fruit large, py-

Fig. 174. Lundise Ronne sf Jersey. riform, a little onesided. Skin smooth and glossy, pale green in the shade, but overspread with brownish red in the sun, ard dotted with nume. rous gray dots. Stalk about an inch long, curved, rather 
obliqueiy i iserted, without depression, or with a fleshy, enlarged base. Calyx open, in a rather shallow, uneven basin. Flesh greenish-white, very juicy and molting, with a rich and excel. ient flavour. September and October. [This is very distinct from the old Louise Bonne, a green winter fruit, of third quality.

\section{LoDge. Ken.}

The Lodge Pear is a native of Pennsylvania, and is understood to have originated near Philadelphia. It is a very agreeable sub. acid pear, and has so much of the Brown Beurré character, that we suspect it is a seed. ling of that fine old variety. Kenrick com. pares it to the Seckel, to which it has, no points of resemblance.

Fruit of medium size, pyriform, tapering to the stem, and one-sided. Skin greenish brown, the green becoming a little paler at maturity, and much covered with patches of dull russet. Stalk an inch and a fourth long, obliquely planted at the point of the fruit, which is a little swollen there. Flesh whitish, a little gritty at the core, which is large ; juicy, and melting, with a rather rich flavour, relieved by pleasant acid. September and October.

\section{Michaux. Man. in H. M.}

Compte de Michaux.

A fruit imported from the nursery of the Messrs. Baumann, of Bolwyller, France, by Mr. Manning. Young wood light green. It is of medium size, and nearly round. Ŝkin light yel. 
lowish green, with a faint blush on the sunny side. Calyx open, sligl.tly sunk. Stalk an inch and a half long, rather slen. der, inserted with little or no depression. Flesh white, half.but. tery, juicy, sweet, but second rate. September and October.

\section{Moor-Fowl EgG. Lind. Thomp. \\ Little Swan's Egg. \\ Knevett's New Swan's Egg.}

The Moor-fowl Egg is a Scotch pear, very hardy, and therefore, popular in that climate, which is cold and unfavourable for the pear. It is a third rate fruit, much like the Swan's Egg, and unworthy of cultivation in this country. [The Moorfowl's Egg, of some Boston gardens, is the Swan's Egg.]

Fruit rather small, roundish. Skin dull green, washed with brown on the exposed side, and dotted with minute russet dots. Stalk long and slender, planted in a slight hollow, or by the side of a fleshy lip. Basin narrow, slightly sunk. Calyx open. Flesh yellowish-white, soft, a little gritty, juicy and sweet. September and October.

\section{Marie Louise. $\oint$ P. Mag. Lind. Thomp.}

Forme de Marie Louise. Marie Chrétienne.
Princesse de Parme.

Braddick's Field Standard.

This truly delicious pear was originated from seed, by the Abbé Duquesne, of Belgium, in 1809, and its fruit was first sent to England by Van Mons, in 1816. It was introduced into this country, along with many other fine Flemish pears, about 15 years agro, and is every where held in the highest estimation, keeping for a long time in the house. The tree is hardy, but has an awkward, rather crooked, and declining habit, asd very narrow leaves. In the nursery it is best, therefore, to graft it standard high, when it soon makes a good head. The young shoots are olive-gray. It is a pear for every garden, bearing very regularly.

Fruit pretty large, oblong-pyriform, rather irregular or onesided in figure. Skin at first pale green, but at maturity, rich yellow, a gond deal sprinkled and mottled with light russet, on the exposed side. Stalk an inch and a half long, nbliquely planted, snmetimes under a slightly raised lip, sometimes in a very small, one-sided cavitv. Calyx small, set in a narrow somewhat plaited basin. Flesh white, exceedingly buttery and melting, with a rich, very saccharine, und vinous flavour Last of Sepiember and middle of October. 


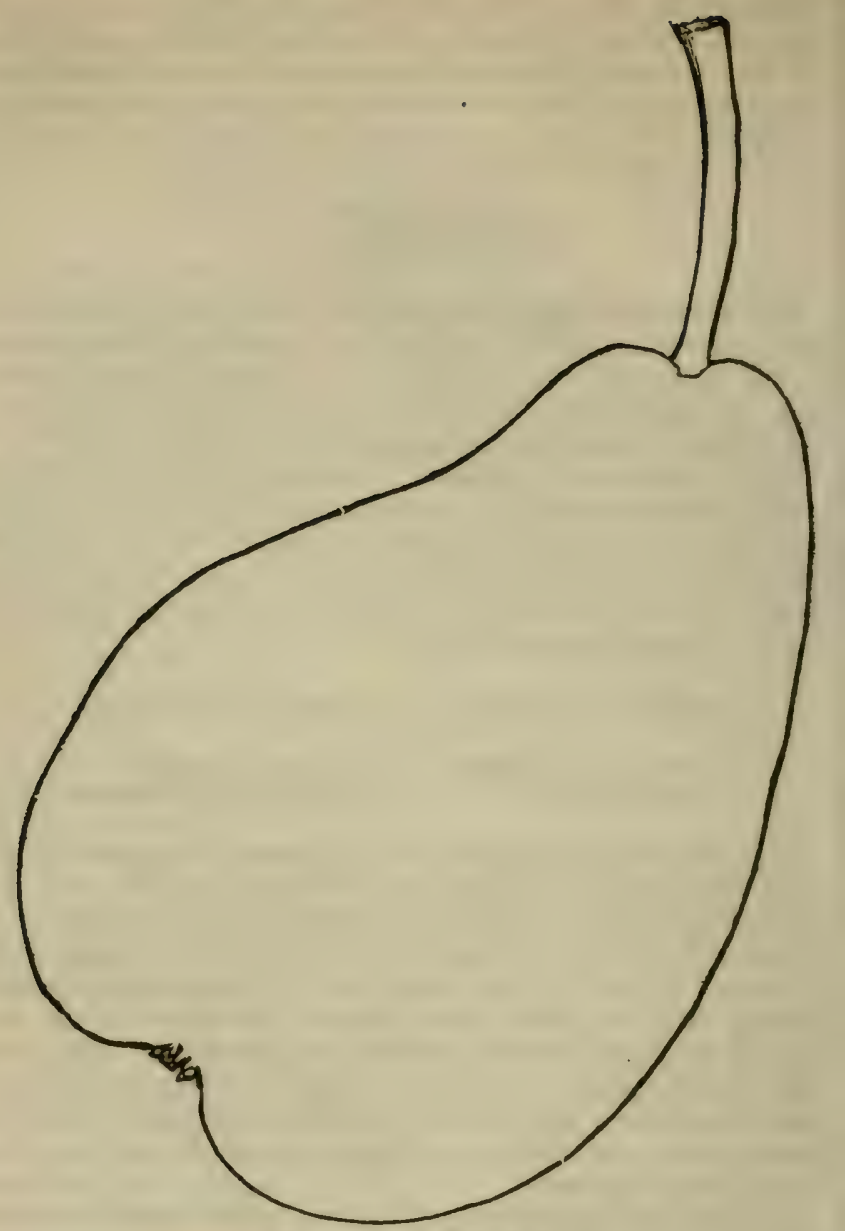

Fig. 176. Marie Louise.

144. Marie Louise Nova. Van Mons. Ken.

This variety, was sent by Van Mons to Mr. Manning. It will by no means bear a comparison with the Marie Louise, thougn in some seasons a very good fruit. Col. Wilder, of IBoston, considers it "hardly second rate," while the Salem cultivators "think it an execllent, juicy, rich pear, though sorne. times a little rough." It has borne two scasons with us, and is 
enormously productive, but, even with thinning the crop, it is an indifferent fruit. The wood is very strong, and dark coloured,

Fruit rather large, regular pyriform, tapering into the stalk. Skin smooth, yellow, with a brownish-red cheek. Sialk one to two inches long, rather slender and curved. Calyx set in a shallow depression. Flesh at first melting, juicy, and some. times rich, but quickly decays. Last of Sêptember.

\section{Niell. Thomp. Van Mons.}

Beurrè Niell. Man. zn H. M. Colmar Bosc.

Poire Niell. Lind.

Fondante du Bois, (incorrectly of some.)

A large and handsome Belgian variety, raised by Van Mons, fron seeds sown in 1815, and named in honor of Dr. Niell, of Edınburgh, a distinguished horticulturist, and man of science. The tree bears plentifully. Its quality is not yet fully ascertained, but specimens obtained here, promise well. Young wond stout, diverging, gray.

Fruit large, obovate, inclining to pyriform, rather shortened in figure on one side, and enlarged on the other-tapering to the stalk which is about an inch long, obliquely planted, with little or no cavity. Skin pale yellow, delicately marked with thin russet, finely dotted, and sornetimes marked with faint red. Flesh white, buttery, sweet, with a plentiful and agreeable juice. Last of September.

\section{Napoleon. Lind. P. Mag. Thomp:}

Medaille.

Sucree Dorée, (of some.)

Charles d'Autriche. incorrcctly

livi de Rume

The Napoleon is a pear of many fine qualities. As a tree it is very hardy, thrifty, and bears abundunt crops, even while very young; and its fruit is exceedingly juicy, melting, and agreeable in flavour. In poor soils, or in unfavourable ex. posures only, it is a little astringent. The leaves are broad, and the shoots are upright, and olive-coloured.

Lindley gives this as a seedling of Dr. Van Mons-but we believe, incorrectly, though Van Mons first sent it to England in 1816. It was raised from seed in 1808 , by M. Liart, gardener at Mons; exhibited by him before the Horticultural Society of Mons, which decreed him a medal for it, [whence the synonymo Médaille;] the original tree was then purcháed for 33 francs, by the Abbe Duquesne, who bestowed on it the name of Napoleon.

Fruit pretty large, obtuse pyriform, (but varying more than al. most any other pear in form.) Skin smooth, clear green a! 
first, but be. coming pale yellowishgreen at maturity, slightly brighter and darker on its expo. sed cheek. Stalk vary. ing from half an inch to an inch long, pretty stout, set in a slight depression or under a swollen lip. Ca. lyx set in a basin of mo. derate depth. Flesh white, melting, remarkably full of juice, which sweet, sprightly and excellent.

Shinuld be ripened in the house, when it will be fit

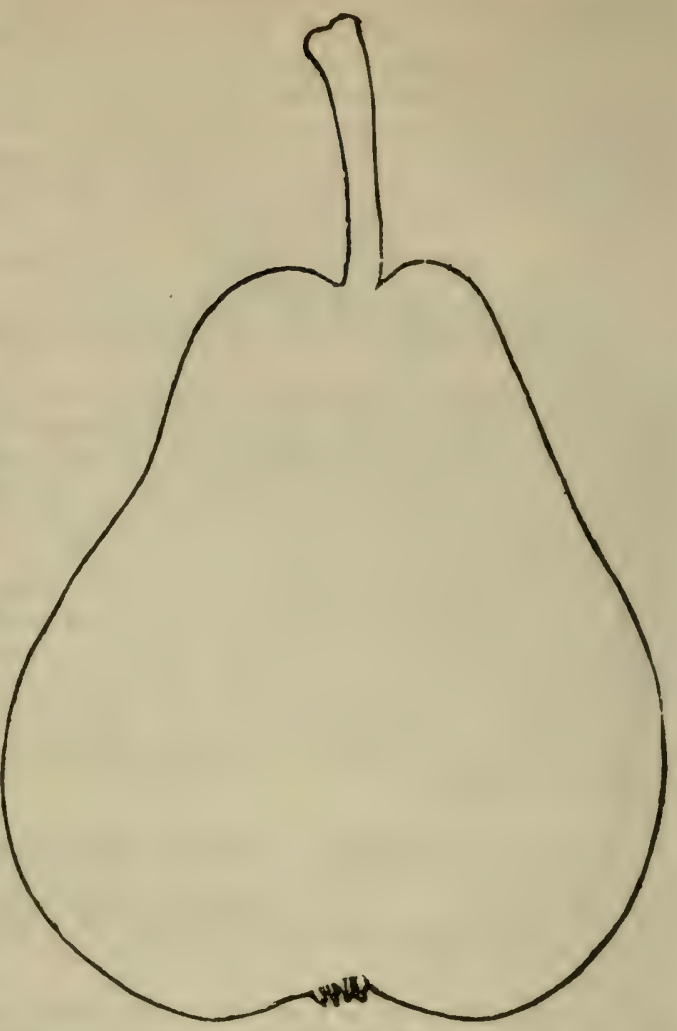

Fig. 17\%. Napoleon. for use in September, and may be kept for weeks.

\section{NaUmKeAg. Man.}

A second rate fruit, a native of Salem, Mass. In wood and leaf it resembles the Brown Beurré. Its appearance is ordinary, and it is often rather astringent. Fruit of medium size, roundish. Skin yellow russet, marked with brown russet in the sun. Stalk set in a very slight depression. Flesh juicy, meltir , but rather astringent in flavour. Bears abindantly. Ocinber.

148. Paradise D'Autonne. Thomp.

A newly imported pear, and the few specimens that we have 
seen here, so strongly resemble Beurré Bosc, as to lead us to suspect its identity. The following description is from a fruit of the present autumn.

Fruit large, pyriform, tapering into the stalk, which it joins by a fleshy base. Skin dull yellow, russeted, a gond deal like the Brown Beurré. Calyx quite small, open, stiff, set in a shallow basin. Stalk an inch and a half long, curved. Flesh white, fine grained, buttery, with a high, rich flavour. Last of September.

\section{Petré}

An American pear, of the highest excellence. The original tree is growing in that interesting place, the old Bartram Bo. tanic Garden, near Philadelphia. Cal. Carr, the proprietor, who has disseminated this tree, informs us that in 1735, a seed was received by the elder John Bartram, from Lord Petré, of London, as being the seed of a fine butter pear. Twenty-five

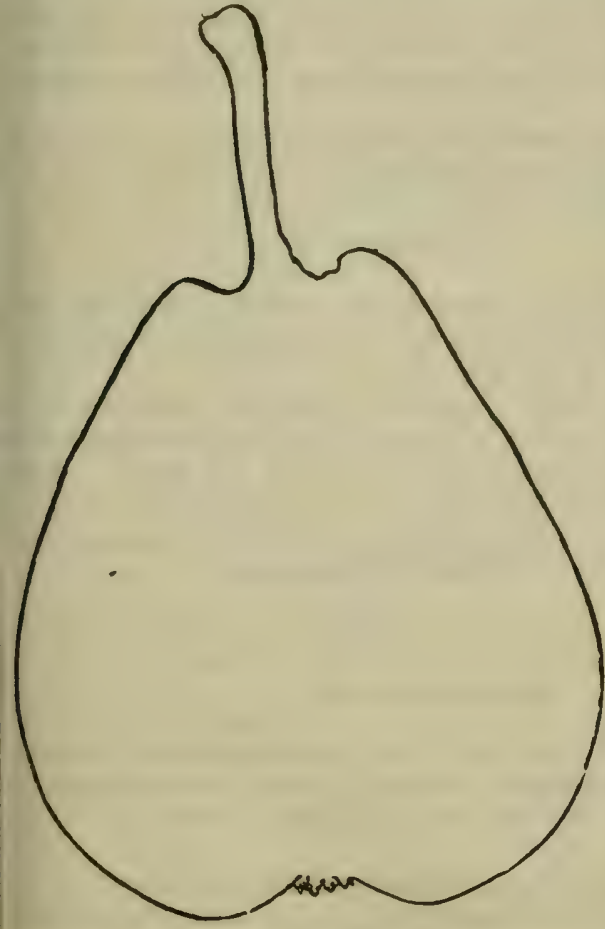

Fig. 178. Petré.

years after, ripe fruit was returned him from this seed. ling-called the Petre pear-which he pronounced su. periour to that of the original tree.

The tree is not a rapid grower, but produces very regular and abundant crops. The fruit has much of the quality of a fine Doyenné with a higher perfume. Young wood slender, yellowishbrown.

Fruit of mediurn size, or rather large, obovate. Skin very thin, pale yellow, (some. times marked with greenish russet, and sprinkled with russet about the eye.) Stalk stifl 
and strong, abou: an inch long, stout at the lower end, and se! in a peculiar, abruptly nattened cavity. Caylx small, set in a narrow, but sinooth basin. Flesh whitish, fine grilned, buttery, and very melting; witi a perfumed, slightly inusky, high tlavour. October, and if picked early, will keep a long iirne.

\section{Pitr's Prolific.}

Pirt's Surpasse Marie. Ken.

Surpass Mlaria Louise, (incorrectly of some Amencan gardens.)

An English market fruit, introduced by Mr. Kenrick. It was raised from the seed of the Marie Louise, but is greatly infe. riour to it. Its principal merit seems to us, to be its beauty and surprising fertility, its long, thrifty branches bcing literally load. ed with fruit. It is handsome, but in flavour it is third rate, quite poor, and soon decays.

Fruit of medium size, oblone-pyriform, (sometimes turbinate, ) usually shaped a little like a Jargonelle. Skin yellow, but nearly covered, in the sun, with brownish-red, and a little russetted. Stalk curved, fleshy at the base where it joins the firuit. Flesh juicy, soft, sweet, rather coarse, and of indifferent quality. September.

[The Surpasse Marie Louise of some European gardens, is the Compte de Lamy, a very fine pear.]

\section{Paquency.}

A new pear, introduced from France, by Col. M. P. Wilder, President of the Massachusetts Horticultural Society. It proves to be a fruit of the first quality.

Fruit of medium size, regularly pyriform. Skin green al first, becoming dull yellow at maturity, marked with patches of russet at both extremities, and dotted with the same. Stalk long, inserted without depression. Calyx stiff, open, set in a very shallow basin. Flesh white, buttery, with sweet, rich, and perfumed flavour. October to November.

\section{Pennsylvania.}

\section{Smith's Pennsylvania.}

The Pennsylvania is a seedling, originated by J. B. Smith, Esı., of Philadelphia, a well known amateur. It is a handsome and goor pear, of second quality. Young shoots diverging, reddish-brown.

Fruit of medium size, obovate, a good deal narrowed towards the stalk. Skin brown russet, nearly covering a dull yellow grou:rd, and becoming russet red on the sunny side. Stalk an 


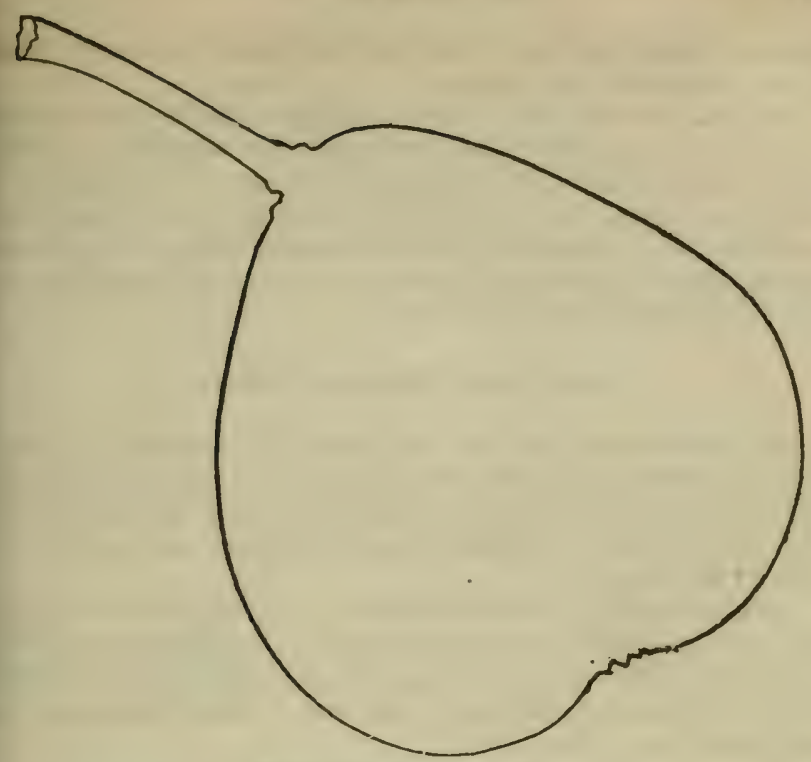

Fig. 179. Pennsylvania.

Inch and a half long, obliquely planted, without depression, but a fleshy base. Calyx small, basin very shallow. Flesh yel. lowish-white, not very fine grained, juicy, half melting, sweet and rich, with a highly perfumed. musky navour. Middle and last of September.

153. Princess of Orange. Lind. Thomp. P. Mag.

Princesse d'Orange.

P'rincesse Conquête.

The Princess of Urange is a pleasant, crisp, juicy pear, of second quality. Its long and upright shoots bear, with us, very regular crops of rich looking: ruddy pears.

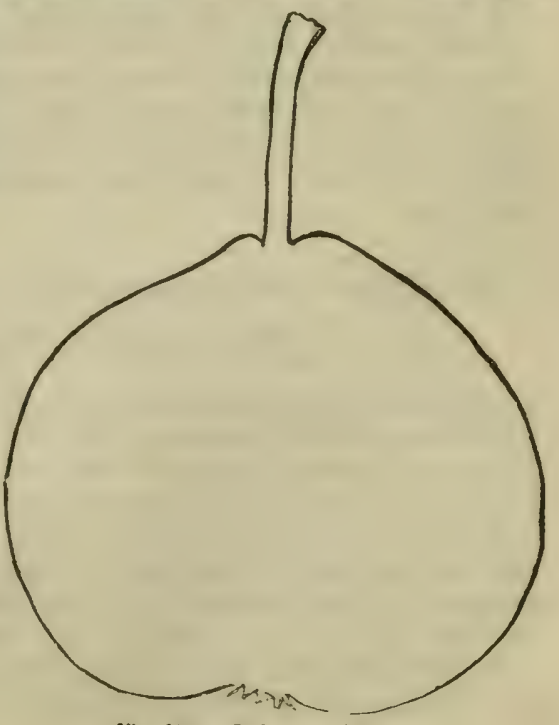

P'ig. 180. Princess of Orange. 
It is a Flemish variety, raised by the Count Coloma, in 1802. Young wood long, light olive.

Fruit of medium size, or a little less, roundish. Skin cinnamon russet in the shade, but nearly covered with bright reddish russet, mixed with a little orange, in the sun. Stalk an inch or more long, planted in a very slight cavity. Calyx small, in a shallow basin. Flesh pale yellowish-white, crisp, juicy, flavour vinous-sugary, relieved by acid, and when in perfection, excellent. October and November.

\section{Pope's Scarlet Major.}

We have discontinued the cultivation of this pear, as, though very handsome, it is quite inferiour. Fruit rather large, obo. vate, yellow, with a bright red cheek. Stalk long and thick. eye rather small. Flesh white, breaking, and rather dry, Last of August.

POPE'S QUAKER is another variety, a little better in quality, but not comparable to many other sorts of the same time. Fruit very fair, middle sized, oblong-pyriform, smooth yellow-russet, juicy, melting and pleasant. October. Both these pears are natives of Long Island, N. Y.

\section{Patlleau. Van Mons. Man. in H. M.}

A Belgian pear, of good quality, but rather coarse grained.

Fruit medium size, turbinate, inclining to pyriform. Skin rough, greenish-yellow, dotted with greenish gray dots, and marked with patches of russet. Stalk about an inch long, very stout, obliquely inserted with a fleshy base. Calyx in a basin slightly depressed. Flesh juicy, sweet and good. Early in Septernber.

\section{Queen of the Low Countries. Ken. Man. in H. M.}

\section{Reine des Pays Bas. Van Mons.}

This fine, large, and handsorne fruit, was transmitled by Dr. Van Mons to Mr. Manning, with the assurance that it was "the inost perfect of pears." Without, as yet, quite equalling this high character here, it proves worthy of extensive trial.

Fruit large, ofien very large, broad pyriform, tapering ab. ruptly to the stalk. Skin in the shade, dull ycllow, dotter and russetted around the eyc, and overspread with fine dark red on the side next the sun. Stalk an inch and a half long, curved, and planted without depression. Calyx very small, and with tew divisions, set in a pretty deep basin. Flesh white, buttery, 


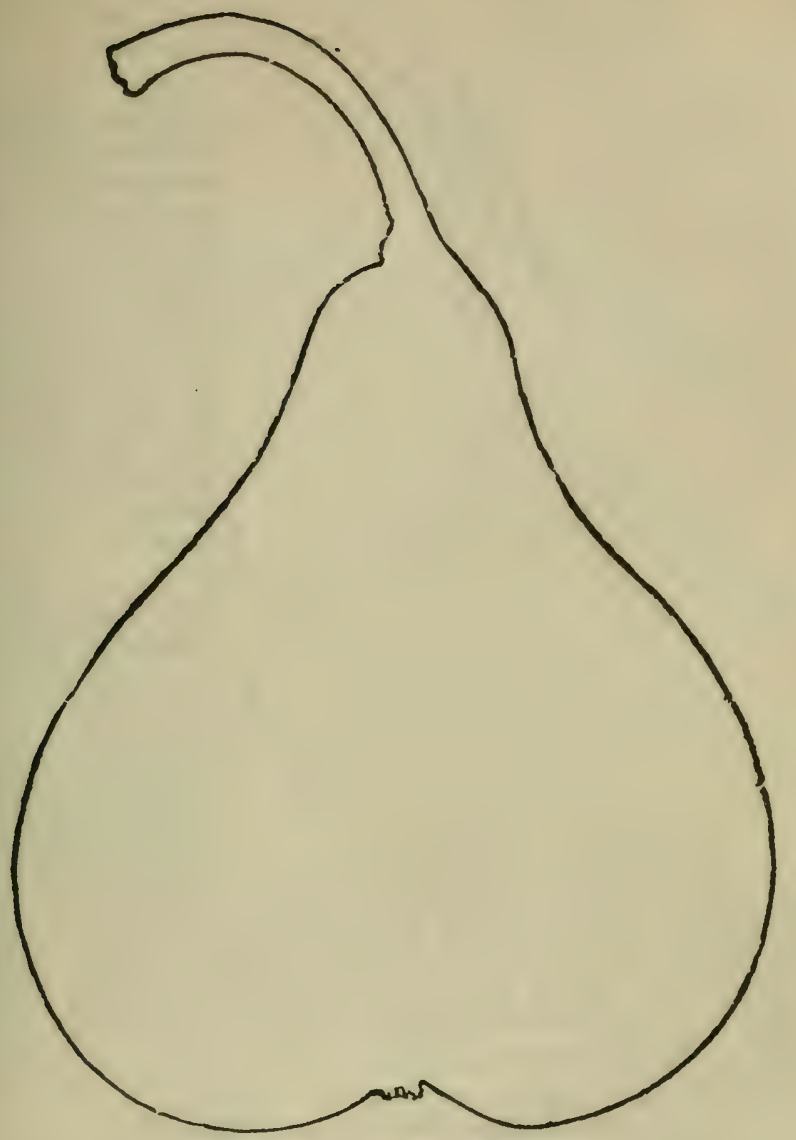

Fig. 181. Qucen of the Low Countries.

melting and very juicy, with a rich, sub-acid, vinous favour. Early in October.

\section{Quilletette. Van Mons.}

A new, and odd-looking, late autumn fruit, of the first qual. ity, recevied from Van Mons, and which deserves a genera. trial.

Fruit nearly of medium size, roundish, a little fiattened. Skin greenish, nearly covered with dull, iron-coloured russet. 


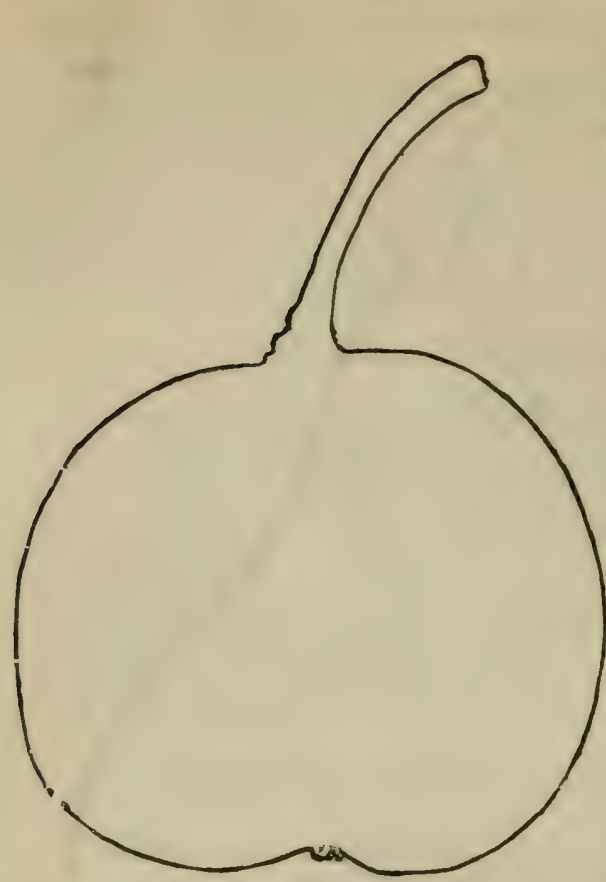

Fig. 182. Guillstetle.

Stalk about an inch and a half long, and set with. out depression, but with a peculiar fleshy swelling at its point of inser tion. Calyx extremely small, sometimes abortive, set in a narrow, rather deep basin. The flesh is white, buttery, and melting, rich, sweet, and perfumed. Novem ber.

158. Reine Caro LINE. 'Thomp.

Queen Caroline.

A pretty looking, European pear, ripening late in autumn, but

coasse, and only fit for cooking. Fruit of medium size, narrow-pyriform. Skin smooth yellowish-green, becoming yellow at maturity, with a rich, brownish-red cheek. Stalk an inch Ioner, curved, planted with little or no cavity. Flesh white, crisp, rather dry and indifferent in quality. November-and will keep for several weeks.

\section{Reine des Poires. Thomp.}

This French pear, with its fine name, unfortunately froves very poor and worthless. It is regularly formed, and handsome-quite distinct from the Cumberland, with which it is con. sidered synonymous, by some. It bears abundantly.

Fruit rather large, varying from turbinate to obtuse-pyriform, regularly shaped. Skin smooth greenish-yellow, with rarely a very little red on its cheek. Stalk an inch and a fourth long, slender, inserted with little or no depression. Calyx set in a shallow basin. Flesh dry and poor. September and October 
160. Rousseler de Meester. Van Mons. Man. in II. M.

\section{Ferdinand de Meester? Nois.}

This is a seed. ling of Dr. Van Mons', and is a very excellent pear in this climate, the flesh melting and sugary, though a little rough.

Fruit of medium size, roundish, somewhat flattened. Skin pale. yellow, marked with very light russet dots, and washed with pale red next the sun. Stalk an inch and a half long, rather slender, and planted somewhat obliquely in, or by the side of the swollen, abrupt end. Calyx large, open, placed in a very slight and irregular basin. Flesh

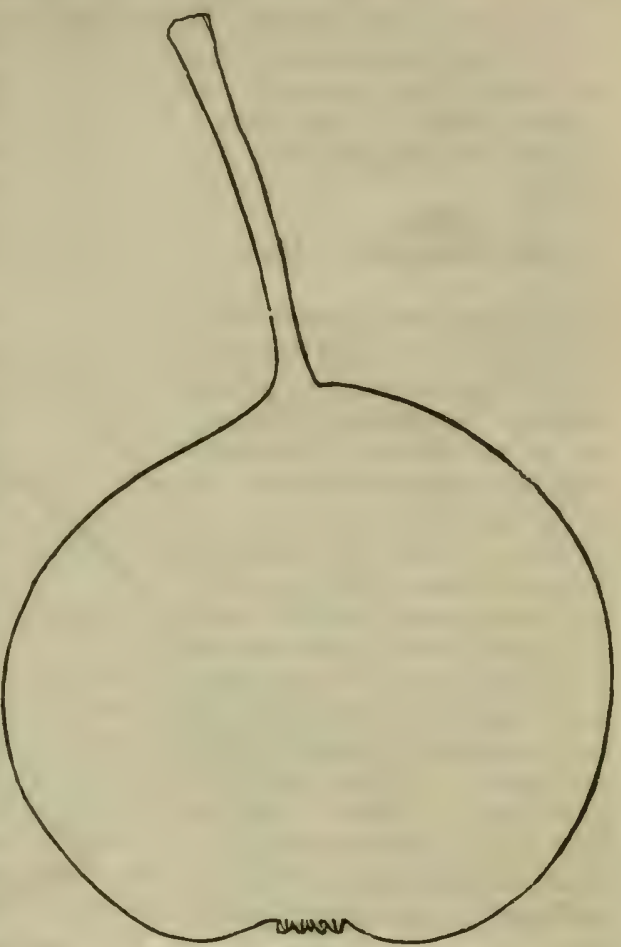

Fig. 183. Roussellet de Mtester. juicy, melting, sugary and rich, but a little rough, which does not prevent its being of excellent flavour. October.

Rousselet de Meester is the name by which this fruit was re. cerved, but we suspect that it is the Ferdinand de Meester, a larger and better pear than the former sort, which was named by Van Mons after his gardener. [See Annale's de la Sociele d'Horticullure. Paris. Vol. 15, p. 362.]

\section{RaYnond. Man.}

The Raymond is a native of Maine, and originated on the farm of Dr. I. Wight, in the town of this name. It has a good deal of the flavour of the IVhite Doyenne, and is a productive pear, often of the first quality, and if the tree were a little moro 
vigorous, would become a popular variety. Young shoots very slender, dark yellowish.brown.

Fruit of medium size, obovate, shaped like the Doyenne Skin yellow, marked with russet near the stalk, and tinged with a little red towards the sun. Stalk an inch or more long, inserted with little or no depression. Calyx round, firm, open, set in a shaliow basin. Flesh white, buttcry, melting, and very excellent.

\section{Rostiezer. Man.}

The Rostiezer is, we believe, a German pear, and was re. ceived from the nursery of the brothers Baumann, of Bolwil. ler, on the Rhine. It is likely to prove a capital variety. It bears abundantly.

Fruit of medium size, oblong-pyriform. Skin a dull yeliowish-green, with a red. dish-brown cheek, and whitish dots, light russet. Stalk very long, nearly two inches, irregular, slender, set with very little depression. Calyx open, but little sunk. Flesh juicy, a little coarse, but very melting, sweet and delicious, with a rich perfume. August and Septem. ber.

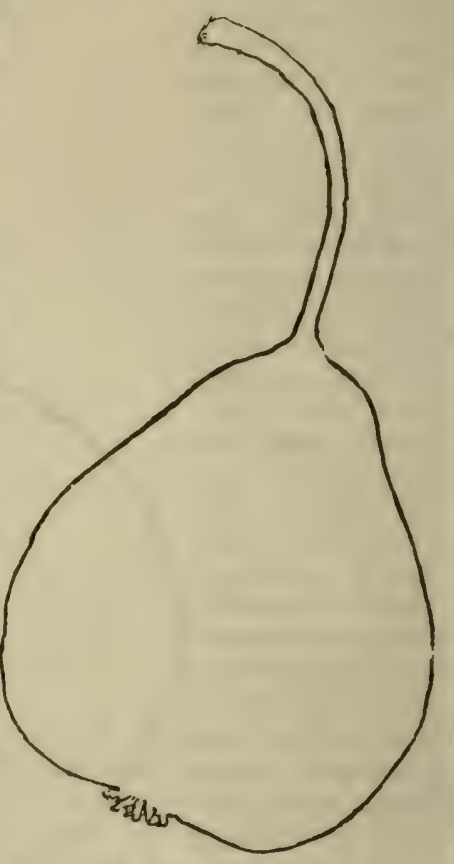

Big. 184. Rostiezer

\section{Sr. Ghislain. $\oint$ Thomp.}

A most excellent Belgian pear, recently originated by M. Dorlain, and introduced into the United States by S. (T. Perkins, Esq., of Boston. When in perfection, it is of the highest quality, but on some soils it is a little variable. 'The tree is remarkab!e for its uprightness, and the great beauty and vigour of its growth. Young shoots light brown.

Fruit of medium size, pyriform, tapering to the stalk, to which it joins by fleshy rings. Skin pale clear yellow, with a few gray specks. Stalk an inch and a half long, curved. Calyx rather small, open, set in a shallow basin. Core small. Elesh white, buttery and juicy, with a rich, sprightiy flavour. 


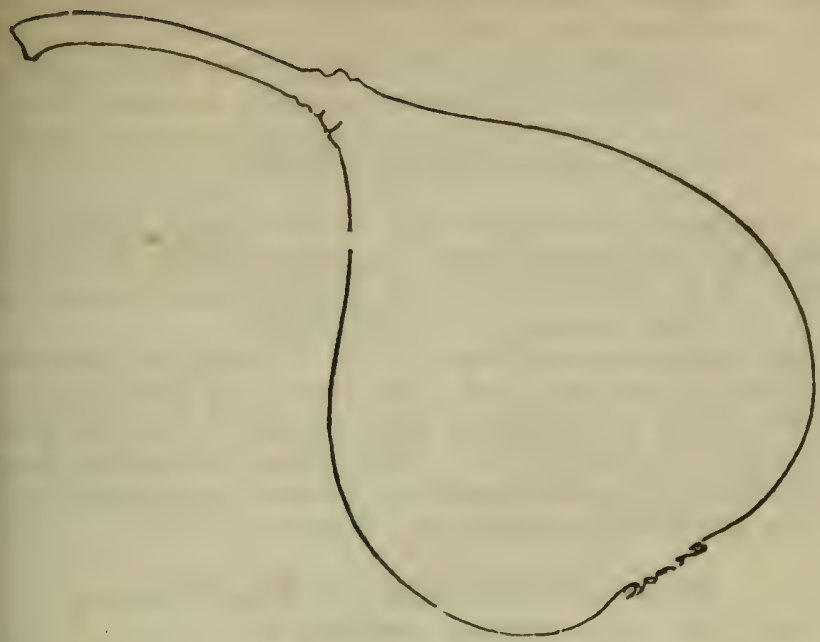

Fig. 185. St. Ghulain.

\section{Superfondante. Thomp.}

The Superfondante is a fine French pear, of the same clasy as the White Dovenué, which indeed, it strongly resanbles in appearance and flavour. Young shoots slender, yellowish-brown.

Fruit of medium size, obovate. Stalk an inch long, slightly inserted. Skin smonth, pale ycllow, marker with a few dots, and sonetimes marker with russet. Stalk an inch and a fourth loner, set in a slieght eavity. Calyx rather large, in a shallow basin. Flesh white, buttery, melting, and very grood. October

165. ST. ANDré. Man. in H. M.

Imported by Mr. Manning, from the Brothers Baumann, of Bolwyller. A first rate variety.

Fruit medium size, olsovate, shaped like IIcnry the $4 \mathrm{th}$. Skin light greenish-yellow, somewhat dotted with red. Stalk about an inch and a quarter long. Calyx sinall. Flesh white, fine grained, buttery, melting, and excellent. Early in Scp. tember.

166. Sulutvan. Man. in H. M.

Van Mons, No. 899.

A second rate seedling, sel to this country by Van Mons, and named by Mr. Manning. Young shoots slender, divarging, 
reddish-brown. Fruit of medium size, oblong-pyriform. Skin pale greenish.ycllow. Stalk an inch and a half long, stout, inserted at the tapering, pointed end. Flesh juicy, melting, sweet and pleasant. September.

\section{Styrian. Thomp.}

This very bright coloured and excellent pear, comes from England. We think it worthy of a creneral trial in the middle states. Like the Passe Colmar, it often produces a second crop of fruit, which, however, is seldom good.

Fruit rather large, pyriform, a little one-sided and irregular. Skin deep yellow, with a bright red cheek, and streaks of lisht russet. Stalk an inch and a half loner, curved, slender, fleshy where it tapers into the fruit. Calyx large, open, and set in an irrngular basin. Flesh yellowish, not very fine grained, crisp: with a rich, high flavoured juice. October.

\section{Stevens' Genesșee. $§$ Man. Thomp.}

Guernsey. Pom. Man.

Steplien's Genessee.

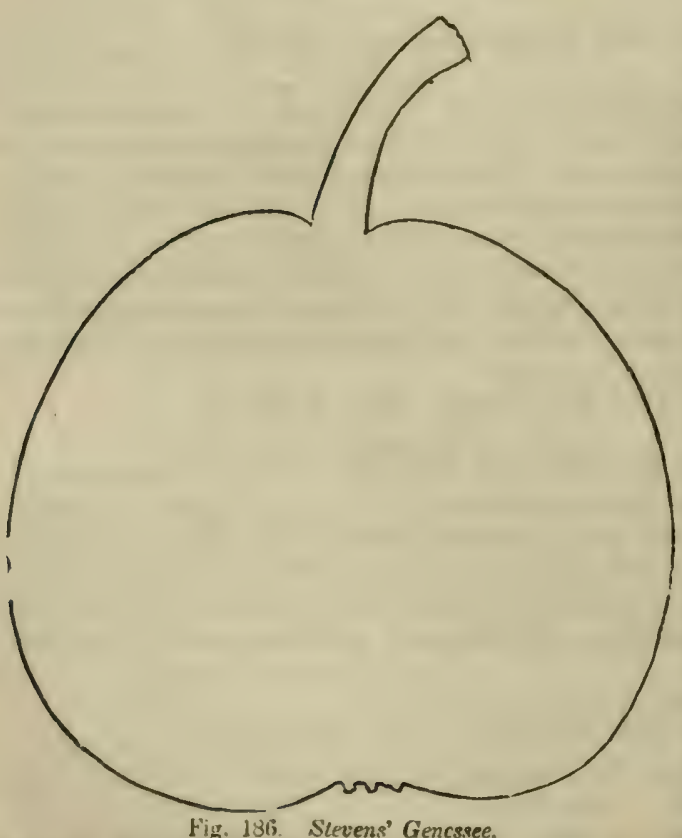

This admirable pear, combining in some degree, the excellence of the Doyenné and Berramot, is reputed to be a secdling of IVestern

New - York. It originated on the furm $M i$. F. Sto. vens, of Lima, Livings:ton Co., N. Y. Altho' placel among Autumn pears, it frequently ripens liere at the end of August, a. mong the late

Summer varietics. Young shoots diverging, dark.gray. 
Fruit larce, roundish-obovate, and of a yellow colour, resem. bling that of the Doyenne (or Virgalieu.) Stalk about an incli Inuer stout, thicker at the base, and set in a slight, Iather noe. siled depression. Calyx with short, stiff divisions, placed in a sinooth basin of only molerate depth. Flesh wh:c, hulf butterv, with a rich, aromatic flavour, somewhat like thai of (iansel's Bergamot. First of September.

\section{Sylvange. Nois. Thomp.}

Bergamotte Sylvange.

Green Sylvange. Lind.

A pleasant, juicy pear, which is much estecmed by srime persons, and alivays hears gnod crops with us. Young shouts stout, upright, dark olive.

Firuit roundish-obovate, shaped much like a bergamot. Skin rough, pale green, with a slightly rarker green cheek, a grond deal marked with dark dots. Stalk three fourlhs of an inch long, slender, slightly inserted. Calyx small, set in a rather uneven, shallow basin. Flesh greenish-white, juicy, tender and melting, with a rich, sweet, agreeable flavour. October, and keeps a long time.

\section{Sitexiss.}

A new native pear, from the castern states. It sonn grows mraly if left on the tree, but ripened in the house is remarkably juicy and sprightly. Fruit rather larese, obovate, and shaped somewhat like IIenry the 4th. Slin light yellowish. green. Stalk an inch long, slender. Calyx in a narrow, ruther derp basin. Filesh white, tenter and molting, with a juicy, and very sprightly flavour. Octolier.

\section{Sieulle. Thomp.}

Beurré sioulle.

A new fruit, very lately received from the Jondon Socintv's farden, and so far as it is yct proved, of very excellent cha. racter.

Fruit of modium size, roundish, flattened. Skin pale yellow, with a little red on the sunny side. Stalk an inch and a quarter longr, set in a shallow cavity. Calyx closerl, basin scarcely at all sumken. Flesh buttery, melting, rich, and very good. October

\section{2. 'Tirmeson's. § Thomp.}

This new, and very rich flavoured pear, received by us from the Horticultural Society of London, was named in honour of 
Mr. Rohert 'Thompson, the head of the fruit department, in the Suciety's garden, to whose pomological acumen, the horticultu. ral world is so larerely indebted. It is, appropriately, a frut of high merit, having the qualities of the Passe Colmar and Duyeuré somewhat combined, but with most of the richness of the former. It is very productive, and merits a place in every collection of pears. Young shoots diverging, yellowisholive.

Fruit of medium size, obovate, slightly irregular in surface. Skin pale lemon yellow, with a few small, russetty dots and strealis. Stalk pretty stout, an inch or more long, inserted in a blunt, uneven cavity. Caly'x open, stiff, often without divisions, basin slightly sunk. Flesh white, buttery, melting, with a ricli, sugary, slightly aromatic flavour. October and Novem. ber.

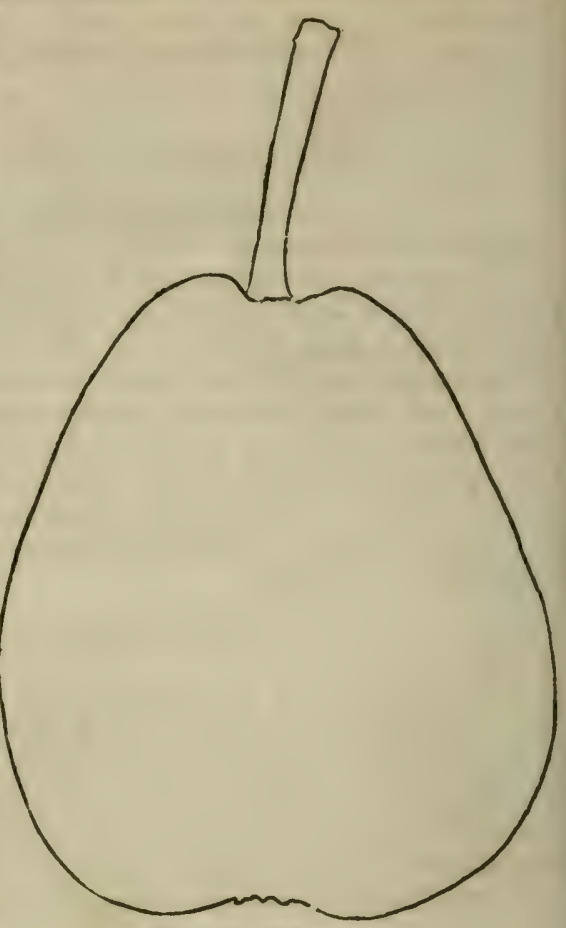

Fig. 187. Thompson's.

\section{Swan's EgG. Thomp. Lind.}

\section{Mour-fowl Egg, incorrectly of scme Boston gardens.}

The Swan's Figg is an old English pear, valued in Britain, for its great harliness and the large crops it bears as a stan. dard, where comparatively few pears succeed without being trained. In this country it is little esteemed, for no man, where so delicinus a fruit as the Seckel can be hail merely for the trouble of planting, will care to retain so ordinary a lind as the Swan's Eggr. Eranches long, upright or waving, dark colentred.

Fruit small, oval, inclining to obovate. Stalk an inch or more long, slender, inserted with very slight depression. Shin 
I: was sent to Europe by the late Dr. Hosack, in 1319, and the fruit was prnnounced by the London IJorticultural S. ceiety, ox. ceeding in flavour the richest of their autumn pears.

Fruit small, (exeept in rich soils,) regularly formed, obovate. Skin brownish-green at first, becoming dull yellowish. brown, with a lively russet red cheek. Stalk half to three-fourths of an inch long, slightly curved, and set in a trifling depression. C.a. lyx smali, and placed in a basin scarcely at all surk. Flesh whitish, buttery, very juicy and melting, with a peculiarly rich, spicy flavour and aroma. It ripens gradually in the house from the end of August to the last of October.

\section{Surpasse Virgalieu. $§$ Man.}

Surpasse Virgouleuse.

The precise origin of this very delicious fruit is not known. It was first sent out from the nursery of the late M. Andrew Parmen. tier, of Brooklyn, under this name, and is, perhaps an unrecognized fo. reign pear, so named by him in allusion to its surpassing the fa. vourite Virgalieu, (White Doyenné) of New-York. We consider it one of the finest of $\mathrm{Au}$. tumn pears, de. serving extensive dissemination. It bears regularly and well. Young shoots long, up. right, yellowish. brown.

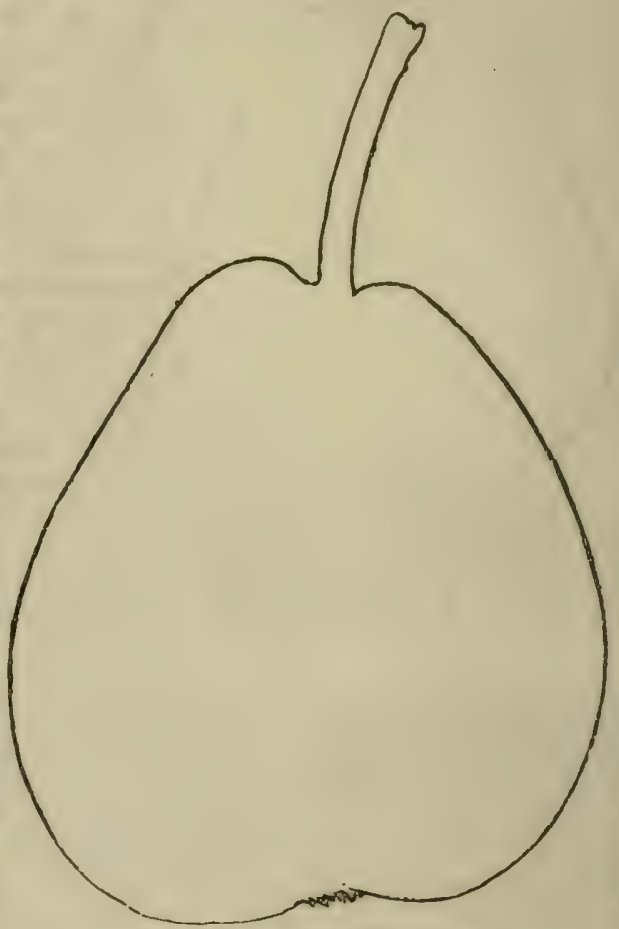

Fig. 189. Surpasse Virgalieu.

puhlie notice, and it received his name. Aferwards the property was aldind to the vast estate of the late Stephen Girard. 'I'lie original tree s'ill exists, (or ilul a few years ago, vigurous and fruitfu.. Sperimers of its pears wero, quite lately exhibited at the annual shows of the Pennsylvania Horticulural Suciezy. 
Fruit rather large, obovate, sometimes roundish-obovate. skin smooth, pale lemon yellow, with a very few minute dots, and rarely a little faint red on the sunny side. Stalk rather more than an inch long, not deeply planted in a cavity rather ligher on one side. Calyx rather small, and pretty firm, set in a slight, smooth basin. Flesh white, exceedingrly fine grained and buttery, abounding with delicious, high flavoured, aromatic juice, diflerent from that of the Doyenné. October.

\section{URBANiste. $\oint$ Thomp. Lind.}

'The Urbaniste is a fruit for which we confidently predict itie highest popularity in this country. In its delicious flavour it compares, perhaps, inore nearly with the favourite old Doyenné or Virgalieu, than any other fruit, and adds, when in perfection; a delicate perfume, peculiarly its own. Its handsome size ana appearance, and remarliably healthy habit, commend it for tirase di.utricts where, from negrlect or bad soil, the Doyenné does not flourish. The tree is a moderately vigorous grower, and the agh:

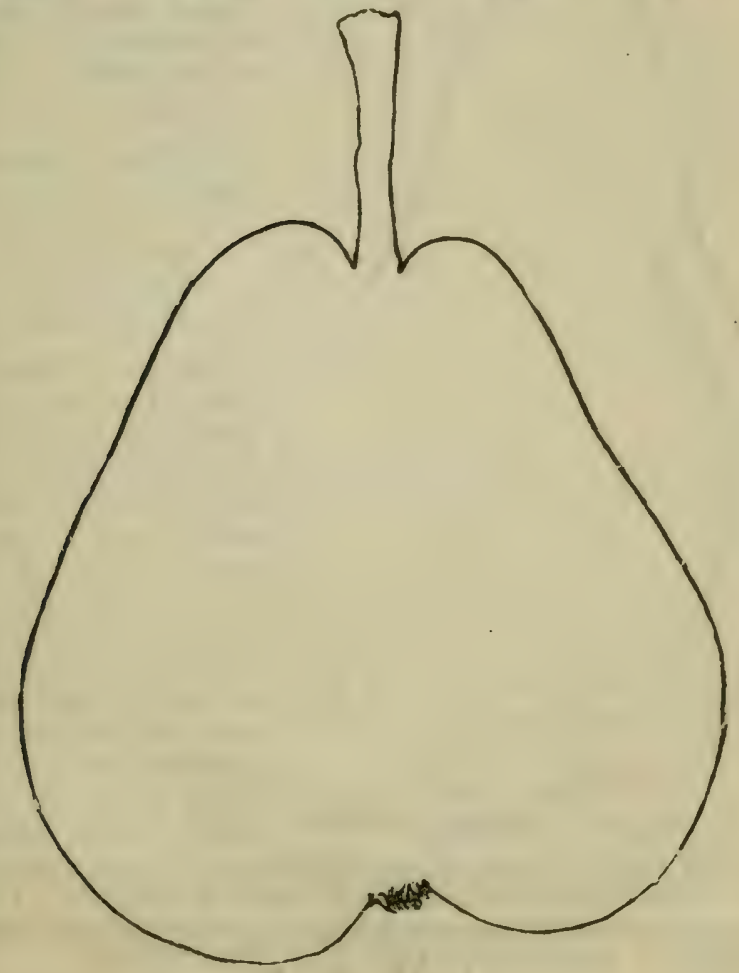

Fig. 190. Urbanis 
$t$ does not bewin to bear sn early as some of the new varieties i vields abuniant and regular crops, and gives every indication of a lomg-lived, hardy bariety. For the orchard or garden in he midlle states, therefore, we consider it indispensuble. With so many other fine sorts, we owe this to the Flmish, it having been originated by the Connt de Coloma, of Malines. It was first introllued into this country in 1823. Young shoots upright, short-jointed, grayish yellow.

Fruit of medium size, often large, pyramidal obovate. Skin smooth and fair, pale yellow, with gray dots, and a few russet streaks. Stalk about an inch long, rather stout, and inserted in a well marked or rather broad depression. Calyx small, closed and set in a narrow basin, which is abruptly and rather deeply

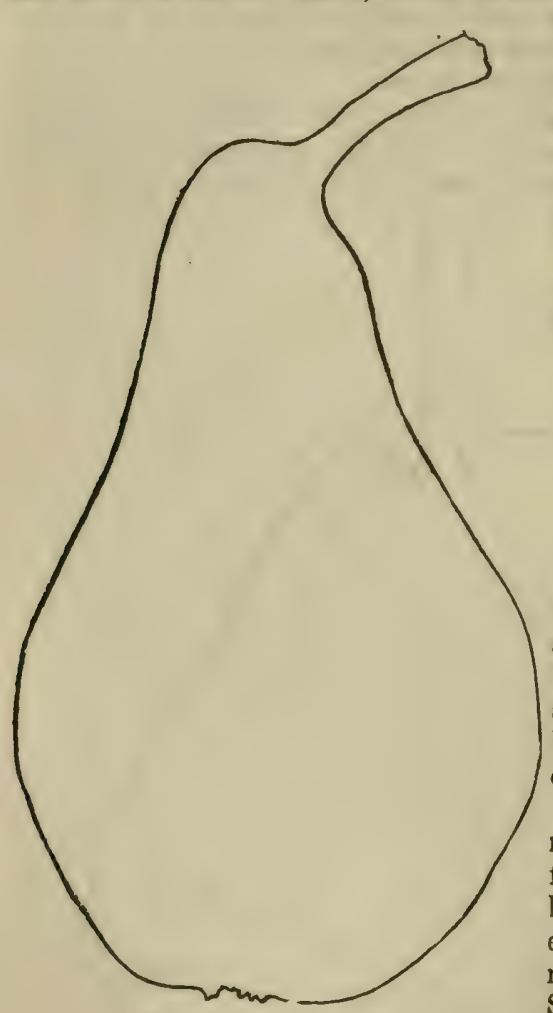
sunk. Flesh white, (yellowish at the core, ) buttery, very melting and rich, with a copious, delicious juice, delicately perfumed. Ripens from the last of September till the end of November, if kept in the house.

177. Verte Longue. Duh. Lind. Thomp.

Long Green.

Muuth Water.

New Autumn.

Muscat Fleuré.

The Long Green, or Verte Longue, is an agreeable, refreshing fruit, remarkably juicy and sprightly. It also bears most abundant crops.

Fruit long-pyriform, narrowing a good deal from the middle towards both ends. Skin green even at maturity, with numerous minute dots. Stalk about an inch Fig. 191. Terte Longue. long, straight, planted a little on one side, and without depression. Calyx small, set on the very narrow crown, which is scarcely hollowed. Flesh white, very juicy, with a sweet, slightly perfumed, vey excelient flavour, Last of September to middle of October. 
There is a small and inferiour variety, known also as the Mouth Water.

Verte Longue Panachée, (Verte longue, Suisse, Culotte de Suisse, ) or Striped Long Green, resembles the foregoing in all respects, except that the first is prettily striped with yellow and green It ripens at the same time.

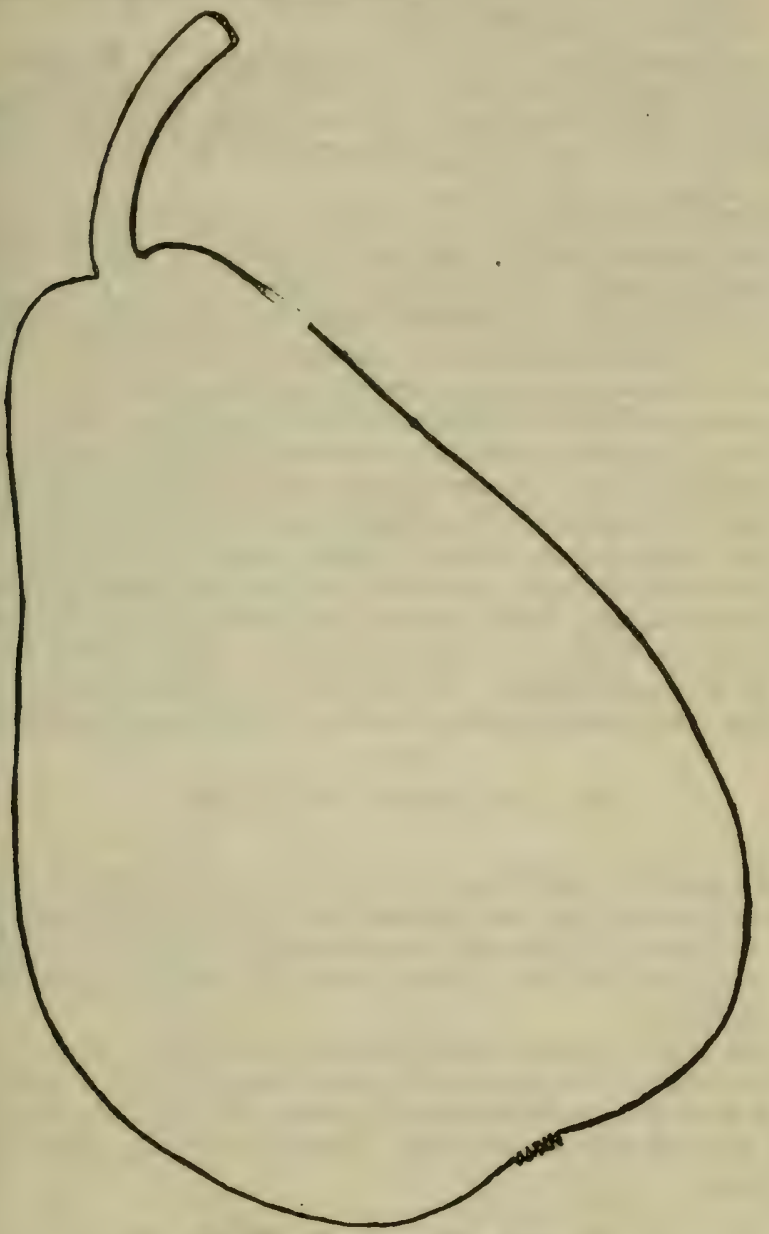

Fig. 192. Van Mons Leon le Clerc.

178. Vay Moss Léon Le Clerc. § Thomp. in Gard. Mag.

This new fruit, which has of late created so much sensation 
in the pomological world on both sides of the Atlantic, has borne for the first time in this country, the past seasoll, and is likrly to sustain its high character as one of the most delicious of Autumn Pears, "combining the properties of large size, handsome appearanee, and rich flavour." Our outline is from a specimen just produced by Col. Wilder, of Boston, whose standard pears are unrivalled in New-England.

Van Mons Léon le Clerc was originated hy M. Léon le Clerc, an amatcur cultivator, of Laval, in France, who, it. naming it, desired to couple his own name, with that of his friend, Dr. Van Mons-"le grand prêtre de Pomoua." Its shoots strong, upright, olive.

Dr. Van Mons himself, had previously raised a large winter pear of tolerable quality, but very inferiour to this, which he had named simply Léon le Clerc. [See Léon le Clerc in a succeeding page.] As this was known by many as Van Mnns's Leon le Clerc, it was naturally confounded with the Van Hons Léon le Clerc-the present variety, and when the latter came into notice, and was sold in England at a guinea a plant, liun. dreds of the inferiour kind were sold under the same name, and confounded with it. Incorrect grafts were also sent to this coun try, but the genuine kind has finally been obtained, and proved.

Fruit large, oblong-obovate. Skin yellowish, much mingled with brown over nearly the whole surface, and slightly russet. ed near the stalk. Stalk an inch and a half long, rather stout obliquely inserted, with little depression. Calyx small, open: set in a shallow basin. Flesh yellowish-white, buttery and melting, with a rich, sugary flavour. October and November

\section{Van Buren. Wilder, Mss.}

An American seedling, raised by Gov. Edwards of New. Haven, for which we are indebted to Col. Wilder of Boston. It is a most beautiful fruit, of second quality only for the table, but very excellent for baking and preserving, and kitchen use generally.

Fruit large, obovate, rather flattened at the eye. Skin clear yellow, with a rich orange-red blush next the sun, regularly dotted with conspicuous brownish specks, and slightly toucherl with greenish and russet spots. Flesh white, crisp, sweet and perfumed.

\section{Willian Edwards'. Wilder. Mss.}

This fruit is from the same source as the foregoing, and pro. mises to take its place among the buttery, autumn pears of the first quality. 
Fruit of medium sizc, obtuse-pyriform, terminating rather ab ruptly at the stalk. Skin yellow, and at maturity, profusely dottrd with red and russet points or dots on the sunny side. Flesh yellowish-white, buttery, melting, very sugary and rich. Sep tember.

\section{WiLbUr.}

The Wilbur is a native fruit, which originated in Somerset, Mass., and has recently been brought into notice. It will not rank above second quality. Shoots slender, yellowish. lirown.

Fruit of medium size, obovate. Skin dull grern and and rus. seted. Stalk three-fourths of an inch long, inserted with littlo or no depression. Caly $\mathbf{x}$ prominent, basin scarcely sunk. Flesh melting, juicy, sweet and pleasant, but glightly astrin. gent. September.

182. WuLKinson. \$ Man. Thomp.

This is a native pear, first brought into notice about 15 years ago. 'The original tree grows on the farm of $\mathrm{Mr}$. J. Wilkinson, Cumberland, Rhode Island. In the middle states it proves a most excellent late pear, coming between the autumn and winter sorts, worthy of seneral cul. tivation. The tree is very thrifty, har$\mathrm{dy}$, and a regular bearer. 'The shoots re long, upright, stout, greenish-yellow. The fruit is very fair. In the neighborhood of Boston, as a standard tree, it does not succeed so well.

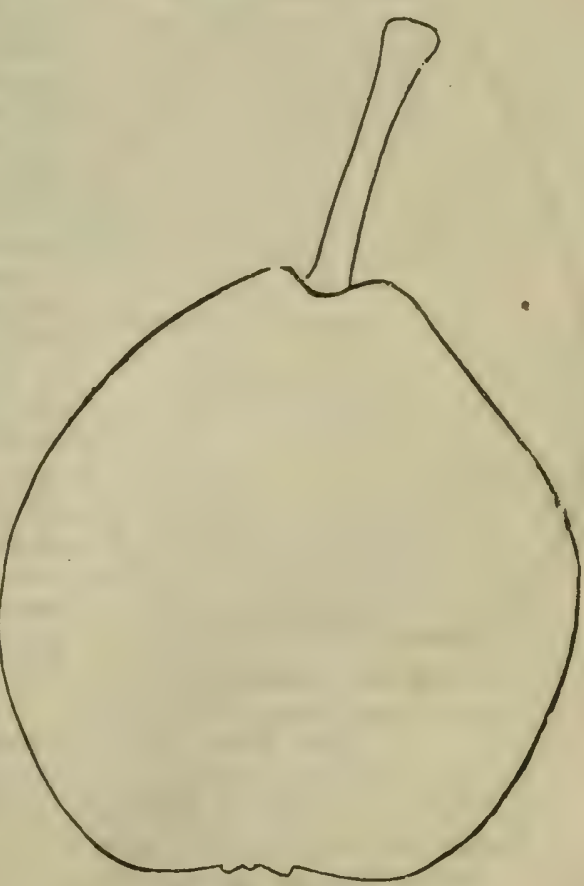

Fig. 193. Wilkinson 
Fruit of medium size, obovate, inclining to oval. Skin smoota and glossy, bright yellow, dotted with brown points. Stalk an inch and a quarter lone, rather stout, inserted with little or no depression. Calyx small, open, and firm, set in a shallow hasin. Flesh very white, juicy, melting, sweet and rich, with a slight perfume. October to December.

183. Wasmington. $\oint$ Man. Ken.

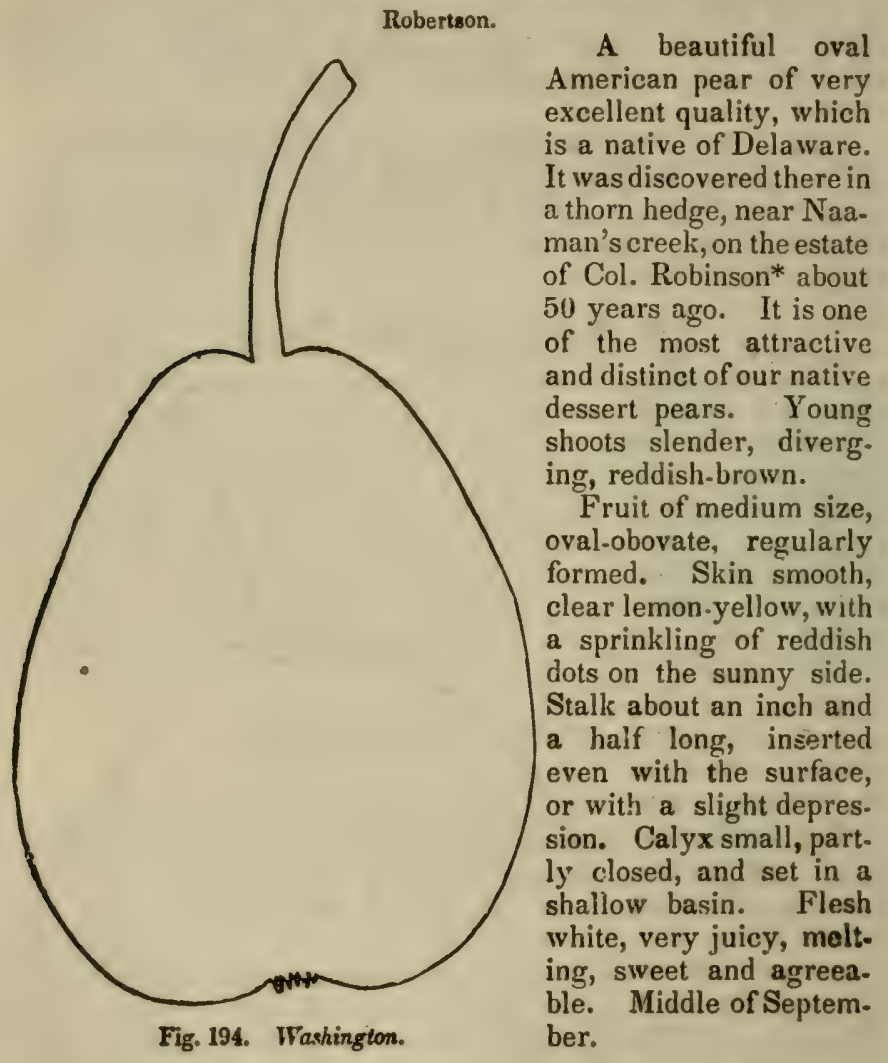

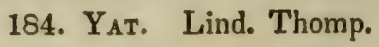

Yutte.

A Dutch pear, recently introduced, which is said to be a very

- The original tree in said to be still standing, and bears 14 to 16 bushels onnually 
excellent variety, and a very abundant bearer. The trees have slender, drooping branches.

Fruit rather small, turbinate, inclining to pyriform, and a little compressed on its sides. Skin dense brown russet, thickly sprinkled with round, gray specks. Stalk an inch long, sleniler, and planted obliquely, without depression. Calyx very small, set in a shallow basin. Flesh white, tencier, juicy, with a sugary, perfumed flavour. September.

\section{Class III. Winter Pears.}

\section{Angora.}

This pear, recently introduced into the United States, pur. ports to come from the town of Angora, near Constantinople. and it is said to be one of the largest and most delicious winter pears of the latter city. The fruit is said to weigh from two to five pounds, to be yellow, with a red cheek, to have a crisp flesh and sprightly flavour, and to keep till May. It will no doubt bear the coming season, and it is doubtful, taking into account the difference of climate in Turkey, whether it proves much more than a good cooking pear here.* Spurious sorts have been sold for the $\Lambda$ ngora-such as the Pound Pcal and Catillac; and we are inclined to believe that the latter sort is what has been received in this country as the true Angora.

186. Beurré D'Aremberg. $§$ Thomp. Lind.

$\begin{array}{ll}\text { Duc d'Aremberg. } & \text { D'Aremherg Parfait. } \\ \text { Deschamps. } & \text { L'Orpheline. } \\ \text { Colmar Deschamps. } & \text { Beurré des Orphelines. }\end{array}$

The Beurre d'Aremberg is certainly one of the first of winter dessert pears in our climate. It is a fine, large fruit, very high flavoured, bears most abundantly, and always keeps and matures, with perhaps less care than any other winter fruit in the house. Its flavour is of the rich vinous kind-sugar, mingled with acid-and, when in perfection, is not unlike that of the pine apple. This vinous flavour is not so agreeable to some persons as the sugary, and such will prefer the Winter Nelis and Glout Morceau to the present variety.

The Beurré d'Aremberg was raised, not long since, by the Abbé Deschamps, in the garder of the Hospice des Orphelines,

- We notice, since writing the ahove, that two sorts introduced into the Paris garilens from Constantinnple under this nume have borne, and buth are very in. diterent, one being the Catillac. 
at Enghein. Noisette, the French nurseryman, having intro. ducerl, about the sarme time, another fine pear from the garden of the Dulie of Aremberg, gave it the name of Beurre d'Arem. berg. This latter pear proved to be the Glout Morceau, and hence arose the confusion, which still, in some measure, exists between the English and French works respecting it-the Beurré d'Aremberg of many French calalogues, being the Gluut Morerau. The two sorts are, however, easily distinguisherl. The fruit of the d'Aremberg has a short, or thicker stalk, usually bent to one side; its flavour is vinous, instead of sucrary, and its wood is stronger, with more deeply serrated leaves. Branches clear yellowish-brown, dotted with pale specks.

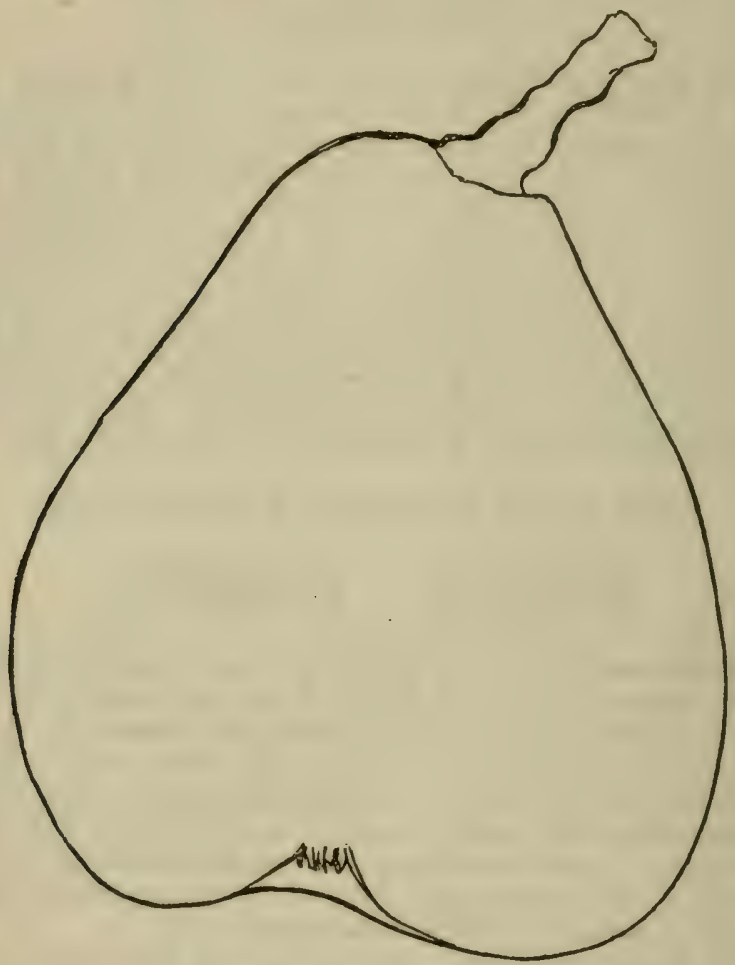

Fig. 195. Beurré d'Aremberg.

Fruit obovate, but narrowing a good deal to the stalk. Skın thick, rather uneven, pale greenish-yellow, becoming yellow at maturity, with many tracings and spots of light russet. Stalk short, half an inch, to an inch long, thick, and very fleshy 
especially where it joins the fruit, and usually planted very obliquely. Culyx short and small, set in a deep basin. Flesh while, liuttery, and melting, with an abundant, rich, delicious vinous juice. December.

\section{Beurré, Easter. P. Mag. Thomp.}

Bergnmotte de la Pentecôte.

Beurré de la Pentecûte.

Beurré d'Hiver de Bruxelles.

Doyenné d'Hiver.

Duyenné du Printemps.

Beurré Roupé.

Du Pâtre.
Beurré de Pâques

Pliilippe de Pâques.

Brzi Chaumontelle tres gros

Chaumontel tres gros.

Canting.

Seigneur d'Hiver.

The Easter Beurré is considered abroad, one of the very best late winter or spring pears. It seems to require a rather warmer climate than that of the eastern states, to arrive at full nerfection, and has disappointed the expectation of many culti.

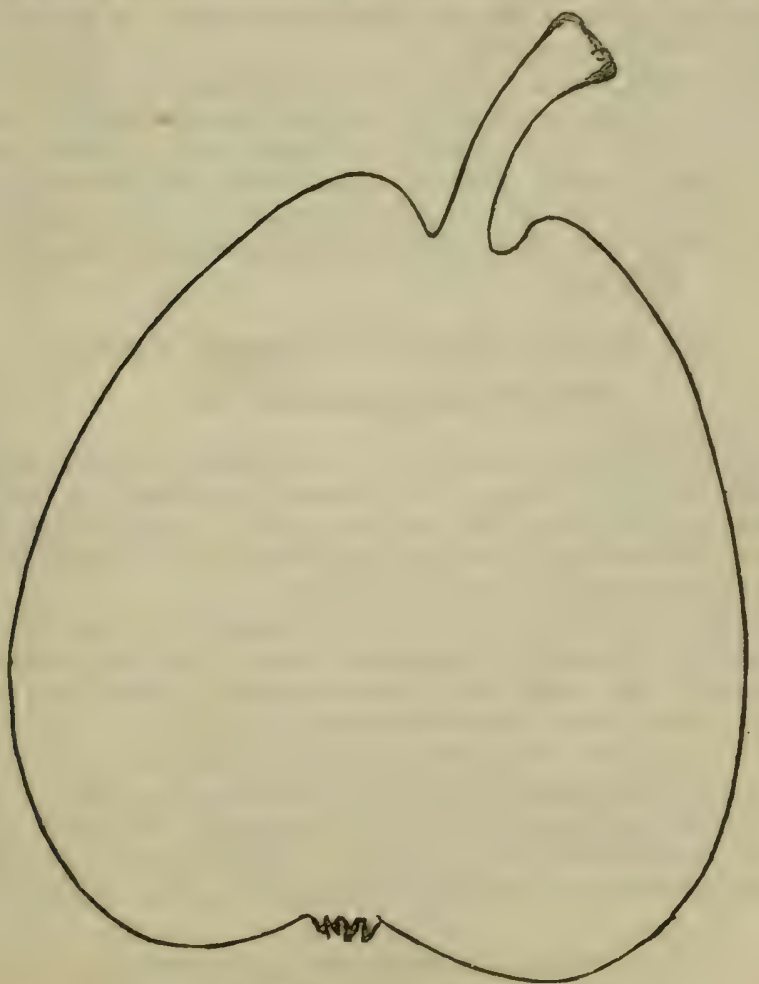

Fig. 196. Enster Bcurré 
vators. It hears well here, but is rather variable in quality In good seasons, if packed away in boxes and ripened off in a warm room, it is a delicinus, molting, buttery fruit. The tree grows upright, and thriftily, with reddish yellow shoots. It requires a warm exposure and a rich soil, to give fine fruil as an open standard tree.

Fruit large, roundish-obovate, often rather square in figure. Skin yellowish-green, sprinkled with many russetty dots, and some russet, which give it a brownish cheek in some specimens. Stalk rather short, stout, planted in an abruptly sunken, obtuse cavity. Calyx small, closed, but little sunk among the plaited folds of the angular basin. Flesh white, fine grained, very buttery, melting, and juicy, with a sweet and rich flavour.

\section{Beurré Gris d'Hiver Nouveau. Thomp.}

A new variety, which comes to us from France, with a high reputation, as one of the best of all late pears. It is just introfluced into this country.

Fruit large, almost round. Skin rather smooth, entirely russeter and having a slight red tinge on the sunny side. Stalk vory thick and short, inserted in a slight cavity. Calyx very small, basin slightly sunken. Flesh white, very melting and fure grained, with an abundant, sugary, slightly perfumed juice, rather richer, but somewhat resembling a fine Brown Beurrè. Eebruary.

189. Beurré, Mollett's Guernsey. Thomp.*

Mollet's Guernsey Chaumontelle. Ken.

Mollett's Guernsey Beurré is a new English variety, raised by Charles Mollet, Esq., of the Island of Guernsey ; it has the reputation of a fruit of the highest quality, "very melting and buttery, with a very rich Chaumontel flavour." Its adaptation to our climate remains yet to be proved.

Fruit of medium size, oval-pyriform-there being often a remarkable extension, or prolnnged neck of the frut where it unites with the stalk. Skin rather uneven, yellow and yel. lowish-green, nearly cuvered on one side with dark cinnamon brown russet, in stripes and tracings. Stalk an inch long, pretty stout, and planted in the fleshy extended neck of the fruit. Calyx large, with widely expanded divisions, and placed in a shallow basin. Flesh yellowish, exceerlingly molt. ing and butterv, with a rich vinous flavour. December.

- In Gardener's Clironicle, 1812, p. 37 and 85. 
190. Peurrí: Rance. $\oint$ Thomp.

Beurré Rance, Lind.

IIardenpont du Printemps.

Beurre Epine.
Beurre de Flondre.

Jusephine, incorrectly of some

Beurıé de Ranz.

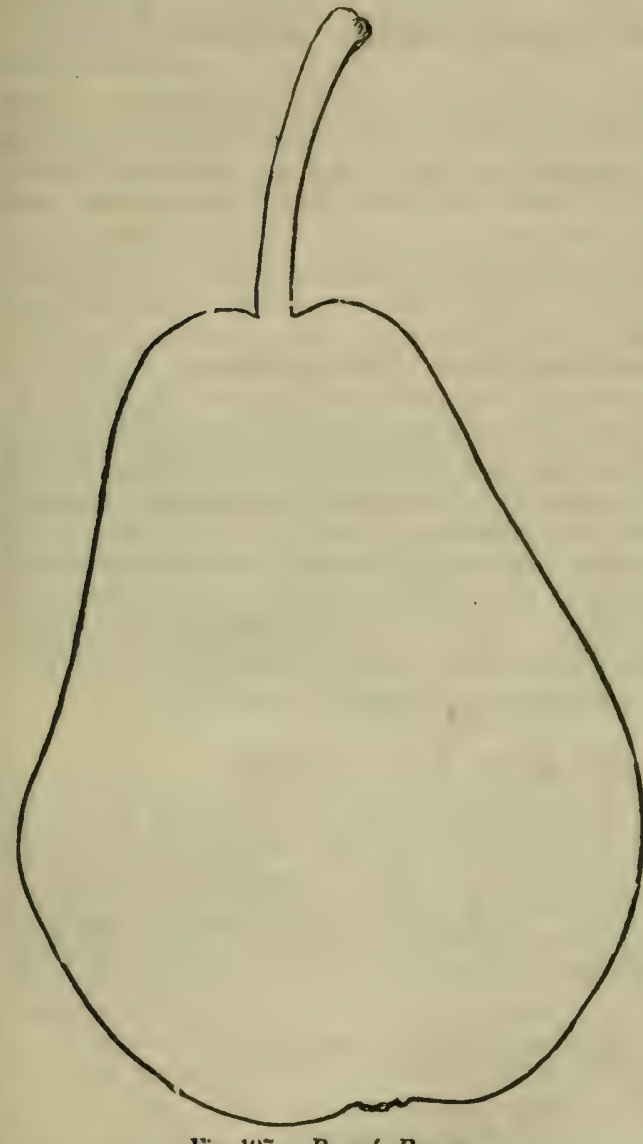

Fig. 197. Beurre Rance.

The Beuris Rance is corsidered by oll English cultiva. tors, the best very late pear yet generally known. With us it is in eating from March to May, ripening in succession if brought in separate parcels from a cool to a warm apartment. It is not a handsome fruit, always remaining green and rather rougl, but its long kerp. ing quality ren. der's it an indis. pensable variety in every good garden. In New England it does not mature well, and is frequent. ly second rate. It is excellent here, and still better farther south. The wood is brownish.yellow, straggling in growth, and rather pendulous when in bearing; and when the tree has attuined a mode. rate size it bears well.

Fruit of medlum size, obtuse pyriform. Skin dark green, even at maturity, rather thick, and dotted with numerous russet specks. Stalk rather slender, an inch and a half long, set in a slight, blunt depression, or often without any cavity. Calyx quite small, and set in a basin very little sunk. Flesh 
greenisn-whife, moltine, a little erritty at the core, full of sweet. rich juice, of excellent flavour.*

This is a Elemish pear, raised by M. Hardenpont, of Mons. Its name is, properly, Be'urré de Lunce, from the name of the rillage in which it was raised, near Mons.

\section{Beurré Bronzée. Thomp.}

This pear has as yet, only proved second rate with us. Fruit pretty large, roundish. Skin rough -ish, greenish, nearly covered with ilull russ 't, becming red next the sun. Stalk an inch or mure long, slightly inserted. Flesh white, crisp, juicy and sweet, of tolerable flavour.

[The Betirré Bronzée of some Boston gardens, is the Figue de Naples.]

\section{Bezi VAet. Thomp. Lind.}

The Bezi Vaet has been considerably cultivated in this coun. try, but is not generally considered more tian a grood second rate pear. The young shoots are upright, long, dark coloured.

Fruit of medium size, obovate, narrowing to the stalk. Skin rough-ish, pale green, becoming yellowish, with many russetty spots and a brownish cheet. Stalk an inch or more long, inserted in a slight cavity. Calyx set in a small basin. Flesh yellowish-white, melting, juicy, with a sweet, somewhat per. fumed flavour. November to January.

\section{Broon PakK. 'Thomp.}

This new prar, a seedling of $M r$. Knight's, comes to us with a high reputation, "partaking," says Mr. Thompson, "of the favour of the melon and pine apple." Fruit of mediurn size, roundish. Skin brown, flesh white, juicy, melting, perfumed and delicious. Shoots strong, diverging, dark brown. Decem. ber to January.

\section{Bezi d’Héri. Thomp.}

Bezi Roval.

Franzüsische Rumelbirne.

This is a very excellent, winter stewing pear, which beary

* $\Lambda$ we have stated hefore. a great deal depends on the mole of koeping wintor pears. 'Tliey are best whin packed in small hoxes or kiges, will layers of paper fretween them 'These buxes stould be kept in a coul, ilry cellar or garre?, free from frosi. till within two or three weelis of the swasun of their maturity, when they shmuld be brousht into a clo-et or room of warn temperature to rizen. In this way the fruit will attain a much higher flavour than it ripened in the ordinary way, and without shrivelling. 
most abundantly. It is of no value for the dessert, but would probably prove a good market fruit It derives its nanis from the forest of Héri in Bretagne.

Fruit of medium size, rounuish. Skin greenish-yellow, with a reddish blush. Stalk an inch and a half long, slender, curved. Caiyx open, slightly sunk. Flesh tender, juicy, free from grit, with an anise-like flavour. Fit for cooling from October to January.

\section{Bzack Worcester. § Thomp.}

Black Pear of Worcester. Lind. Man. Parkinson's Warden.

A very profitable market fruit, bearing always heavy crops of kitchen pears, which are esteemed for conking. The branches incline downwards with the weight of the fruit. Young shoots dark olive, diverging. Fruit large, obovate or oblong. Skin thick, rough green, nearly covered with dark russet. Stalk one half to an inch long, stout, planted with but little depression. Calyx small, and set in a moderate hollow. Flesh hard and coarse, but stews and bakes well. November to February.

\section{Berganot, Easter. Mill. Lind. Thomp.}

$\begin{array}{ll}\text { Bergamntte de Pâques. Duh. } & \text { Winter Bergamot. } \\ \text { Bergamutte d'Hiver. } & \text { Paddring'un. } \\ \text { Bergamutte de Bugi. } & \text { Ruyal 'Tairling. } \\ \begin{array}{ll}\text { Bergamotto de Toulouse. } & \text { T'erling. } \\ \text { Robert's Koeping. }\end{array}\end{array}$

The Easter Bergamot is a second rate, winter dessert pear, lut it is one that we consider woll worthy of cultivation. It bears, with us, very large crops of handsome pears, which are very tender, excellent stewing pears, all winter, and keep adinirably till late in the spring, whon thcy are agreeable for the table. It is much inferiour to the Easter Beurrế for eating, and It is readily distinguished trom that variety by the rounder form and lighter colour of its fruit, as well as the greenish hue of the young shoots. It is a thrifty old French variety.

Fruit mediuin to large, roundish-obovate, narrow at the stalk. Skin smooth, pale green, thickly speckled with conspicuous, light gray dots, and becoming pale yellowish at maturity. Stalk varying from, three-fourths to an inch and a half in length, set in a very slight depression. Calyx small, and placed in a very shallow basin. Flesh white, crisp, juicy and melting at maturity, with a sprightly fldvous. Febsuarv to May. 
197. Berganotte d'Hollandz. Thomp. Duh.

$\begin{array}{ll}\text { Hollanil Bergamnt. Lind. } & \text { Bergamotte de Fougére. } \\ \text { Beurre d'Allewy.un. } & \text { Amostille. } \\ \text { Bergamote d'Alleçnon. } & \text { Lurd Clioeney's. }\end{array}$

Ar excellent kitchen fruit, which will keep sound till May or June, and becomes then of good second rate quality for the table. Shoots stout, diverging, olive brown.

Fruit rather large, rouzdish. Skin green, much marbled and covered with thin brown russet, but becoming yellowish at maturity. Stalk an inch and a half long, slender, crocked, and planted in a rather shallow, one-sided cavity. Calyx small, with few or no divisions, and set in a wide, rather deep basin. Flesh white, crisp, with an abundant, sprightily, agreeable juice.

193. Bon Chrétien, Spanish. § Mill. Lind. Thomp.

Bon Clirétien d'Espagne.

Spina.

The Spanish Bon Chrétien is a kitchen fruit of excellent quality, the handsome appearance of which, joined to its occasional good flavour when raised on warm soils, renders th worthy of a place among dessert fruits.

Fruit large, pyriform, rather irregular and one-sided in figure. Skin at maturity, deep yellow, with a brilliant red chesk, and dotted with reddish-brown specks. Stalk an inch and a half long, bent, ard slender, inserted on the narrowed end, anil usually with very little depression. Calyx small, placed at the bottom of a rather deep, narrow, irregular basin. Flesh whice, crisp, or half breaking, and of moderately rich, good flavour.

\section{Bon Chrétien, Flemisir. Thomp.}

\section{Bon Chrétien Turc.}

The Flemish Bon Chrétien is an excellent cooking pear. The tree a most abundant bearer, and the fiuit fair. Young shoots diverging, gray.

Fruit of medium size, obovate. Skin pale green, and brown on the side exposed to the sun. Flesh crisp, juicy, and stews very tonder. November to March.

200. Columbia. $\S$

Columbian Virgalieu.

Columbia Virgaluuse.

This splendid American pear is one of the most excellent yualities, and will, we think, becorno more generally populas 
WINTER TEARS.

431

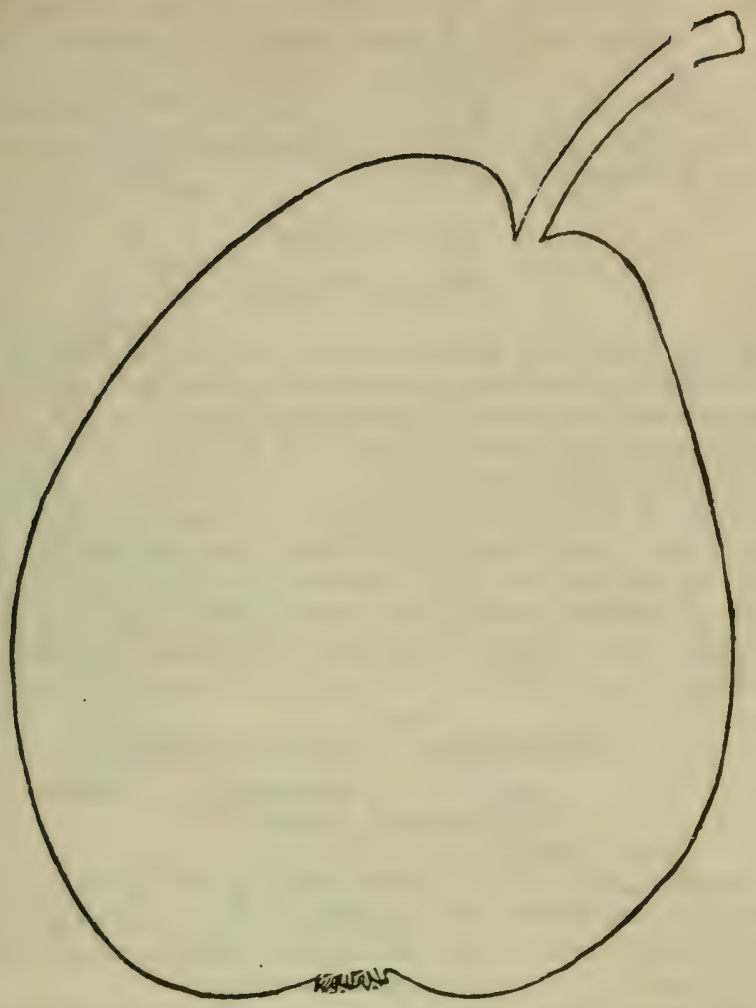

Fig. 198. Columbia.

than any other early winter fruit. It is large, handsome, very productive, and has a rich, sugary flavour, resembling, but often surpassing, that of the Beurre Biel. The original tree grows on the farm of $\mathrm{Mr}$. Casper, in Westchester co., 1:3 miles from New. York. Its productiveness may be judigerl of from he fact that a single graft, five years inserted l, has borne over four bushels in a single season, and its value as a market fruit, from the pears having readily brought six dollars per bushel in the New.York market. The tree grows upright, with stout brownish-yellow shoots. This fine pear was first brought into notice a few years since, by Blond good \& Co., of Flushing. Young wood stout, upright, yellowish-brown.

Fruit large, regularly formed, obovate, usually a little ob song, and always broadest in the middle. Skin smooth and fair pale green in autumn, but when ripe, of a fine golden yellow 
with occasionally a soft orange tinge on its cheek, and dottod with small gray dots. Stalk rather more than an inch long, slender, slightly curverl, placed towards one side of a narrow sepression. Calyx of medium size, partially open, set in a very shallow basin. Flesh white, not very fine grained, but melting, juicy, with a swect, rich and excellent, aromatic lla vour. November to January

\section{Comstock}

\section{Comstock Wilding.}

A very handsome, bright coloured pear, crisp, and of good second quality, and a very ornamental winter dessert fruit. It is a native of the town of Washington, Dutchess co., N. Y., and was brought into notice by Mr. J. R. Comstock, an orchardist there. Shoots long, upright, reddish-yellow.

Fruit of medium size, regularly formed, obovate. Skin smouth and glossy, bright yellow, with a crimson chepk. Stalik about an inch long, straight, inserted in a slight caviiy. Calyx set in a shallow basin. Flesh white, juicy, crisp, and if well ripened, with a sweet and sprightly flavour. November to January.

202. Catrllac. Mill. Duh. Thomp.

Cadillac.

Groote Mogul.

Grand Monarque. Katzenkopt.

The Catillac is an old French baking and stewing pear, of very large size and of good quality for these purposes, stewing tender, and of a fine light red colour. In rich soil the fruit is often remarkauly large and handsome.

Fruit very large, broadiy iurbinate, (flattened top-shaped.) Slin yellow, dotted with brown, and having sometimes a brownish-red cheek at maturity. Stalk stout, about an inch Iong, curved, and placed in a very narrow, small cavity. Calyx short and small, and set in a wide, rather deep plaited basin. Flesh hard and rough to the taste. November to March.

203. Cross. Hovey's Mag.

Winter Cross.

A new and delicious melting winter pear, which origrinated on the premises of $\mathrm{Mr}$. Cross, of Newburyport, Mass. 'The ori. ginal tree is not more than 19 years old, and is an abundant and constant bearer. It will, no doubt, prove a very harly va. ricty. It deserves a general trial in all parts of the country Branches rather slender, grayish-yellow. 
Fruit of medium size, roundish.

Škin smooth, at first pale, but ripening to a deep yellow, with a red cheek, and marked with numerous russet dots, and patches of russet around the eye. Stalk three. fourths of an inch long, very thick, planted in a slight depression. Calyx small, basin a good deal sunk. Flesh white, melting, jui$\mathrm{cy}$, and sweet, with a rich and perfu. med flavour. In eating from the last of November to the middle of Janua-

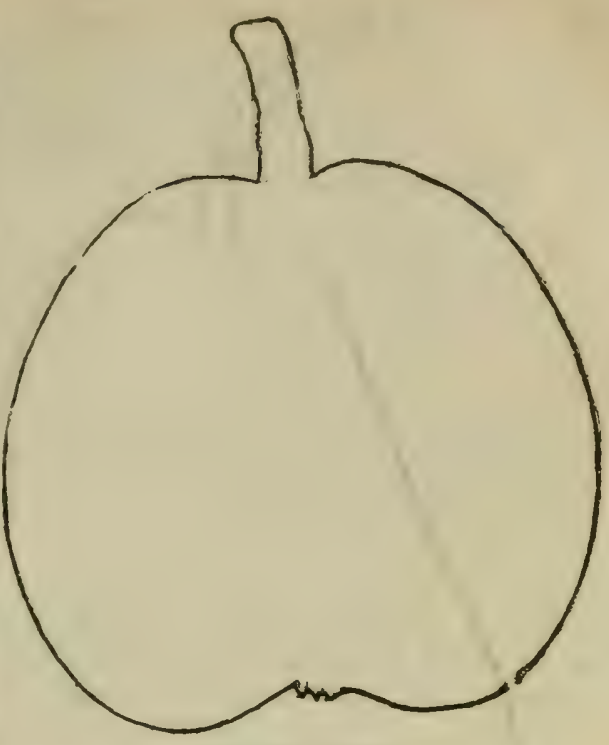

Fig. 199. Cross. rv, but chiefly in December.

204. Chaumontel. $§$ Lind. Thomp. Nois.

Bezi de Chaumontelle. Beurré d'Hiver. Roz.

o. Duh. Poils

Winter Beurré.

Osford Chaumontel.

This grand old French pear, which takes its name from the village of Chaumontelle, in France, is a very desiraole variety, where it can be cultivated to advantage-that is, in a warm rich soil; as it is seldom seen in perfection in a cold climate, or indif. ferent soil. When grown in favourable positions it is an exceedingly rich and excellent fruit, of very large and magnificent appearance. Young shoots long, slender, dark brown.

Fruit large, varying from obovate to oblong, but usually oblong and irregular, largest in the middle, and narrowing each way. Skin a little rough, yellowish in the shade, dotted with many brownish russet dots and brownish-red or rich deep red in the sun. Stalk about an inch long, inserted moderately deep, in an angular cavity. Calyx placed at the bottom of a deep, uneven, angular basin. Flesh buttery and melting, sugary, with a peculiar and agrecable perfume. November to February. 


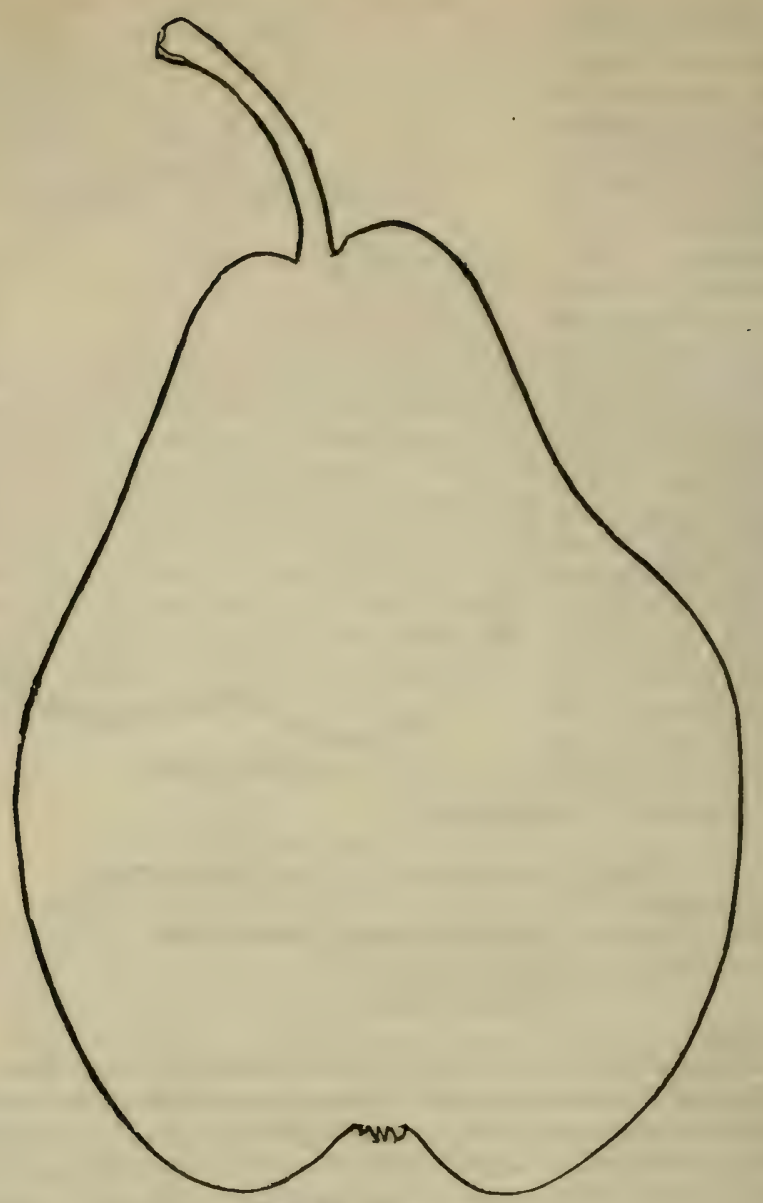

Fig. 200. Chaumontel.

205. Colmar. O. Duh. Lind. Mill.

D'Auch.

De Manne.

Colmar Doré.

Incompi.rable.

Winter Virgalieu, (of some.)

The place of this fine old variety, has of late heen mostry taken by the newer sorts-the Passe Colmar, Winter Nelis, $\& c .$, which are not only superiour in flavour, but much hardier trees. Still it is a good variety, and well deserves a place in col. lections-more especially in the middle states. The hark of this tree is remarkably rough. 
Fruit medium or large, obtuse-pvriform. Skin smooth, pale greenish-yellow, becoming light yellow at maturity, dotted with a few light gray specks. Stalk an inch or an inch and a balt toner tolerably stout, bent, and planted in a cavity often one-sided and uneven. Calyx rather small, set in a wide deep basin. Flesh melting, half buttery, juicy, sweet, rich and excellent. De. cember.

\section{Euhassery. O. Duh. Lind. Nois. \\ Ecluasserie. Thomp. Bezi d'Echassey \\ Bezi de Chasserie. Jagdbirne.}

A rich, melting, French pear, which has been a rool while in cultivation, but still holds its place as a first rate fruit. It is but little known in this country. The wood is rather weak, with crooked joints.

Fruit of medium size, roundish-oval. Skin smooth, pale green, yellowish at maturity, slightly dotted with gray. Stalk an inch and a half long, straight, somewhat uneven, planted in a narrow, irregular depression. Calyx open, with flat divisions, placed almost level. Flesh melting, buttery, with a sweet, perfumed and sugary flavour.

\section{Emerald. Thomp.}

A Belgian variety, recently introduced. It resembles very considerably in appearance the Glout Morceau, and is likely to prove a fine variety in the middle states. New-England may be too cold for it.

Fruit of medium size, obovate, rather square in figure, onesided, and somewhat knobby. Skin green, dotted with brcwn, and having a pale brown cheek. Stalk an inch and a half long, planted obliquely in a slight cavity. Calyx with short, stiff divisions, and set in a rather narrow, plaited, irregular basin. Core large. Flesh melting, buttery, sweet and excellent. December.

\section{Fondante du Bois. Thomp.}

This pear has not yet been proved in this country, the trees first received under this name having proved to be the Flemish Beauty. Mr. Thompson says it. "resembles the Passe Colmar, is alınost equal to it in quality, and keeps longer."

"Fruit of" medium size, obovate; skin greenish-yellow; flesh juicy, melting, of first quality. Ripens from December : February." 
209. Fortunée. Bon. Jard. Thomp.

La Fortunée de Parmentier.

La Furtunee de Paris.

Beurré Fortunée.

A new, round, russet pear, raised by M. Parmenties of Enghein. It carnc to us with the reputation of a fruit of the first quality, and as keeping till June and July. It has fruited the past season, and proves to be a small pear, of fair quality, juicy and sprightly, but rather astringent, and in eating until March or A pril. It deserves further trial.

Fruit below medium size, roundish, depressed. Skin covered with gray russet. Stalk short, with a fleshy base, tapering abruptly into the fruit. Calyx smail, in a round, smooth basin. Flesh white, juicy and sprightly, but not high flavoured. De. cember to April.

\section{Franc Réal d'Hiver. Thomp.}

Franc Erinal Lind. O. Duh.

Fin Or d'Hiver.

The Winter Franc Réal is a good cooking pear, which bears abundantly with us, and is esteemed for stewing-as its flesh becomes very tender, and takes a pretty, light purple colour. It bears well, and grows upright, with wavy loaves.

Fruit of medium size, roundish. Skin yeliow, speckled with russet brown, and having a brownish check. Stalk an inch long, set in a small cavity. Calyx small, set in a shallow basin. Flesh crisp and firm. In use from December to March.

\section{Gilogiz. Lind. Thomp. \\ Gile-o-gile. Poire à Gobert. Jilogil.}

A large, showy, globular Frencb pear, only fit for cooking. The French, we sce, by recent accounts, esteen it highly for preserving. It grows very strong and uprigut, and boars large crops.

Fruit large, roundish. Skin thickly covered with russet, with a reddish russet check. Stalk an inch and a half long, set in an uneven carity. Calyx large, set in a deep plaited hollow. Flesh very firm and crisp. November to February. 
149. Glout Morcenu. Thomp. Lind.

Gloux Morcenux.

Beurré d'Ilardenpont.

liardenpent d'Her.

Colmar d'lliver.

lieture d'lliver Nouvelle.

Linden d'Automne.

Leurré d'Aremberg, (wrongly.)
Goulu Murceau.

Roi de Wurtenberg.

Kronprinz Ferdinand.

Uen Uestreich.

Beurré de Cambron.

Got Luc de Cambron.

Hardenpunt's Winterbutterbirne

The Glout Morceau is universally admitted to be one of the most delicious of the recent Flemish winter pears; and as it is

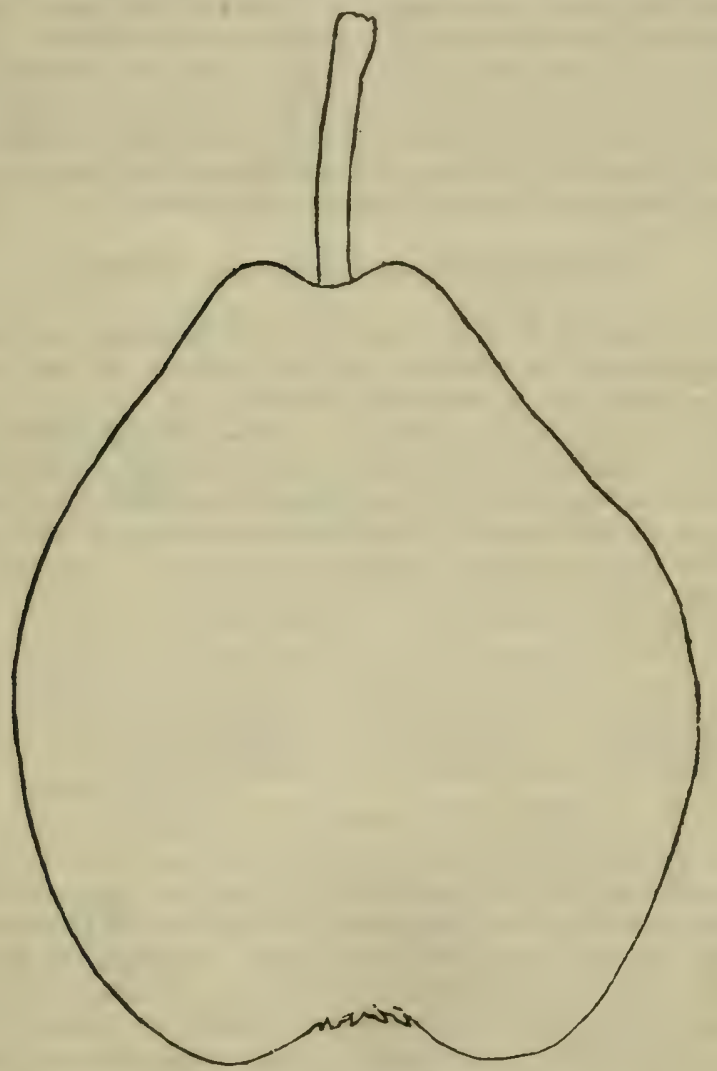

Fị. 201. Glout Morceau.

perfectly suited to our climate, bearing excellent crops, it should have a place in every good garlen. It has been confounded with the Beurré d'Aremberg, as has already been explained, $37^{*}$ 
but is readily distinguished from that pear, by its swceter, more sucrary flavour, more oval figure, and more slender stalk.* 'i'he' growth of the tree is also distinet, having darle olive slouts, spreatian and declining in habit, with wavy leaves.

The signification of Glout Morceau, is greerly morsel ; but Mr. Thompson suggests that this or the synonyme Goulu Morcrau is used (in the same sense as pris golitu, i. c. sugar pras,) to signify honied, or sugared pear, which is most appropriately applied to the present fruit.

Fruit rather large, varying in form, but usually obtuse-nval, and wider towards the stalk than Beurre d'Aremberg. Skin smooth, thin, pale greenish-yollow, marked with small green dots, and sometimes with thin patches of greenish-brown. Stalk rather slender and straight, an inch or more long, planted in a small, regular cavity. Calyx usually with open divisions, set. in a moderately deep basin. Flesh white, fine grained, and smooth in texture, buttery, very melting, with a rich, sugary flavour, with no admixture of acid. December.

\section{Groom's Princess Royal. 'Thomp.}

A new English fruit, not yet proved in America, but having the reputation of a variety of the first quality. It was raised by Mr. Groom, the famous tulip grower.

Fruit of medium size, roundish. Skin greenish-brown, with a tinge of brownish-red, and some russet tracings. Stalk short and thick, set in a very trifling depression. Calyx small, open, set in a shallow basin. Flesh buttery, melting, a little gritiy near the core, but sweet, and high flavoured. January and February.

\section{Janinete. Thomp.}

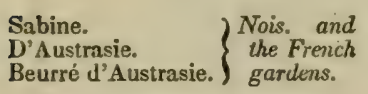

Josephine. Colmar Jaminette. Hardenpont du. printemps, (of some.)

The Jaminette (or Josephine, of American gardens,) is a very excellent winter pear, which grows strongly, produces abundant and regular crops, and is well worthy of general cultivation. It was raised from seed by M. Jaminette, of Metz.

Fruit of medium or large size, varying in form, but mostly obnvate, a good deal narrowed at the stalk. Skin clear green, paler at maturity, considerably marked with russetty brown, especially near the stalk, and sprinkled with numerous brown

* Mr. Hovey evidently figures the d'Aremberg for this peur in his Magazine, rol. ix. p 260 . 


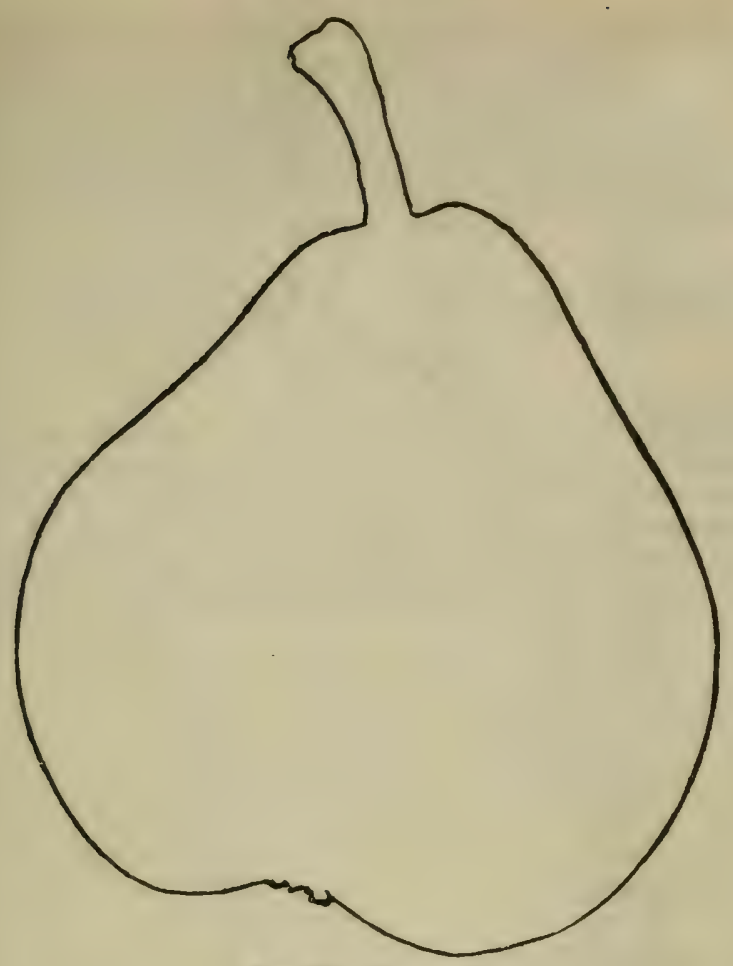

Fig. 202. Jaminette.

tots. Stalk scarcely an inch long, rather thick, and obliquely planted, without any depression. Calyx open and firm, set in a basin of moderate depth. Flesh white, a little gritty near the core, but very juicy and melting, with a sugary, aromatic flavour. November and December.

\section{Knight's Monarch. Thomp.}

This now and most delicious pear was originated from seed by the late Thomas Andrew Knight, Esq., of Downton Cast!e, England, to whom the horticultural world is so largely indebted. He called it the Monarch, because he considered it superiour to all others, and on account of its first perfecting its fruit in the first year of the rcign of William IV. It seems likely to stand equally high in this country, and will, we hope, soon become widely disseminated. The tree is a strong grower and bears abundantly.

By some unlucky error, Mr. Knight transmitted to this coun 
try, and disseminated partially in England, scveral years aga grafts of a worthless sort for this fine pear, which in no way re sembled it. 'The false sort was pretty large!y prorasatrianu distributed before the rror was discevered. The two are rea. dily distinguished by their wood. The true Monarch having yellowish or light olive shoots, the spurious, dark olive or violet.*

Fruit large, obovate. Slin yellowishbrown, tinged with red on the sunny. side, and thickly dotted with pale, gray specks. Stalk remarkably short and thick, set with little or no depression. Ca. lyx open, placed in a rather shallow basin. Flesh yellowishwhite, buttery, melting, and very rich, with a slightly musky, and

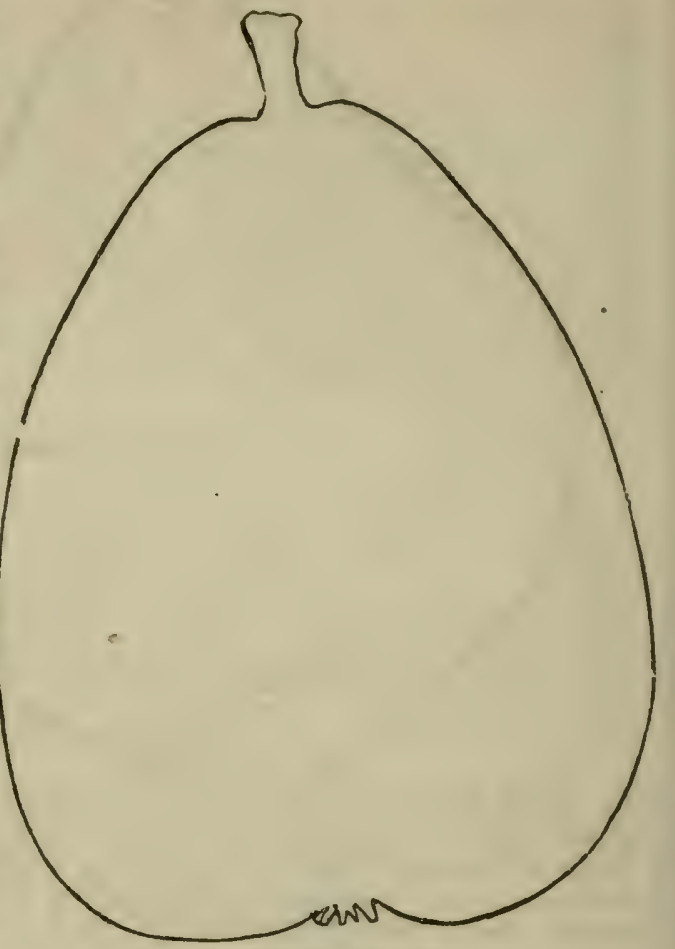
very delicious favour. In perfection in January.

\section{LÉon le Clerc. Thomp.}

Lón io Clere de Laval. Nois.

This is a good cooking pear, of large size, and very distinct from the celebrated "Van Mons Léon le Clerc," described in a foregoing page. In favourable seasons it is of tolerable quality for the table.

* Mr. Knight was deeply mortified at th.is accidental error. and is saill 10 have remarked, that he wonld gladly have sacrificed flo,non rather than it should havo occurred. Would that some nurserymen were as cunselentious! 
Fruit large, obovite, but swollen at the crown, and narrow. ing a goosl deal at the stalk. Skin yellow, smooth, a little grlossy, with russetty spots at either end, and some large dots. Calyx large, with long, straight, narrow divisions, and placed in a slight basin. Stalk an inch and a half long, pretty stout, swol. len at its point of insertion. Flesh white, juicy, crisp, and rather firm, with a tolerably pleasant flavour. December to April.

\section{Louise Bonne. O. Duh. Lind. Thomp.}

\section{Louise Bonne Réal.}

St. Germain blanc.

An old French, winter pear, which bears regularly with us, but is so inferiour to many more newly originated, that we do not think it worthy of cultivation.

Fruit large, pyriform, a little rounded towards the stalk. Skin smooth, pale green. Stalk short, seldom an inch long, straight, a little swollen where it is set on the fruit. Calyx

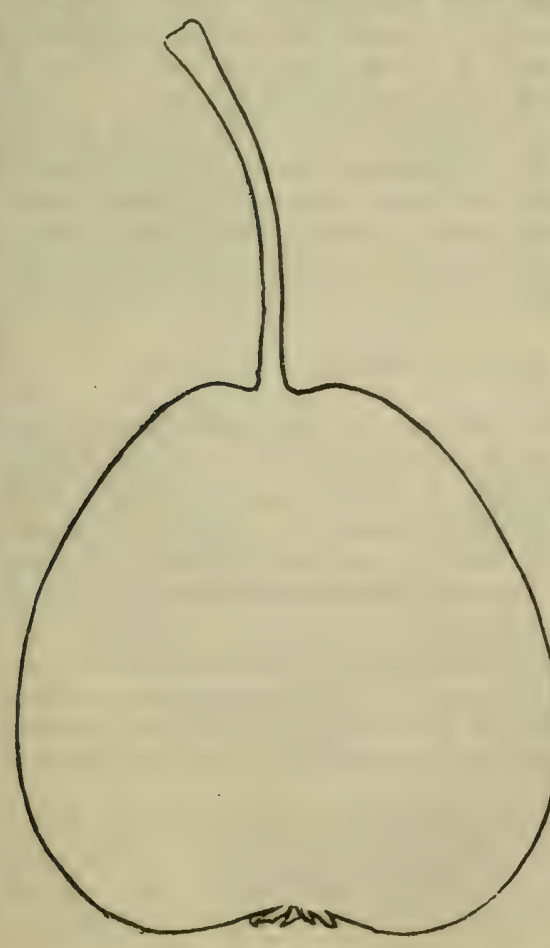

Fig. 204. Leuris. small, open, very slishtly sunk. Flesh white, rather coarse grained, melting, sweet, and pretiy good. December.

218. LEwIS. $\oint$ Man. Ken. Thomp.

This is an excellent winter pear, which originated some thirty years ago, on the farm of Mr. John Lewis, of Roxbury, and was first described and brought into notice by that veteran and zealous amateur of fruits, Samuel Down. er, Esq., of Dorchester, near Boston. It is a very profitable market fruit, bearing enormous crops; indeed, this is the chief fault of the tree, and the soil should there. fore be kept rich, or 
the pears will necessarily be small. The fruit has the good quality of arlhering closely to the tree, is not liable to be bluwn of or injured by early frosts, and should be allowed to remain on till late in the season. The tree grows vigorously, and has long, drooping branches, of dark olive colour.

Fruit scarcely of medium size, obovate. Skin thick, dark ereen in autumn, pale green at maturity, with numerous russetty specks. Stalk long and slender, inserted nearly even with the surface. Calyx large, with wide spread divisions, basin almost level. Flesh yellowish-white, rather coarse grained, melting, juicy and rich in flavour, with a slight spicy perfume. November to February.

\section{Locke. Hov. Mag.}

Locke's New Beurré.

This is a new native fruit, very lately originated from seed by Mr. James Lncke, an extensive orchardist in West Cam. bridge, Mass. From the few specimens we have tasted, we should judge it to be a fruit of excellent quality. The wood considerably resembles that of the St. Germain, from a seed of which Mr. Locke believes it to have been produced.

Fruit of medium size, roundish-obovate. Skin dull yellow. ish-green, slightly mottled with spots of darker green and bits of russet. Stalk an inch long, set without depression. Calyx small, closed, set in a shallow basin. Flesh greenish-white, very melting and juicy, with an excellent, sprightly, vinous flavour. November and December.

\section{LAWRENCE.}

The Lawrence pear is a new variety, which will, undoubtedly, take its place among those of the first quality. It is a seedling, which sprung up in Flushing, L. I., in the neighbourhuod of two other pear trees only, the St. Germain and the White Do. yenné, and bears some proofs, in its qualities, of being a natural cross between the two. Messrs. Wilcomb and King, nurserymen, of that place, first introduced this pear to notice; we learn from them that it produces regular and abundant crops, and the fruit is not inclined to rot or shrivel, commencing to ripen in October, and will keep till March. The tree is moderately vigorous, and has thorny, rather slender, light yellowish-brown shoots.

Fruit rather large, obovate, narrowing to an obtuse end, and a little irregular; pale, yellowish-green, marked with small patches of greenish-brown. Calyx set in a rather deep basin. Flesh 
yellowish-white, melting, juicy, with a very rich and sugary flavour.

\section{Moccas. Thomp.}

The Moccas is one of the many seedling fruits, originated by $\mathbf{M r}$. Knight. It has just begun to pro. duce fruit in this country, and proves to be a win. ter pear of high zuality.

Fruit of medium size, obovate. Skin pale green, or a little yellow. ish, with a brown cheek, and pretty thickly sprinkled with small brown russet dots and streaks. Stalk long, curved, and inserted without depression. Calyx short, partially closed, set in a narrow, rather shallow basin.

Flesh whitish-yellow, juicy, melting, with a rich

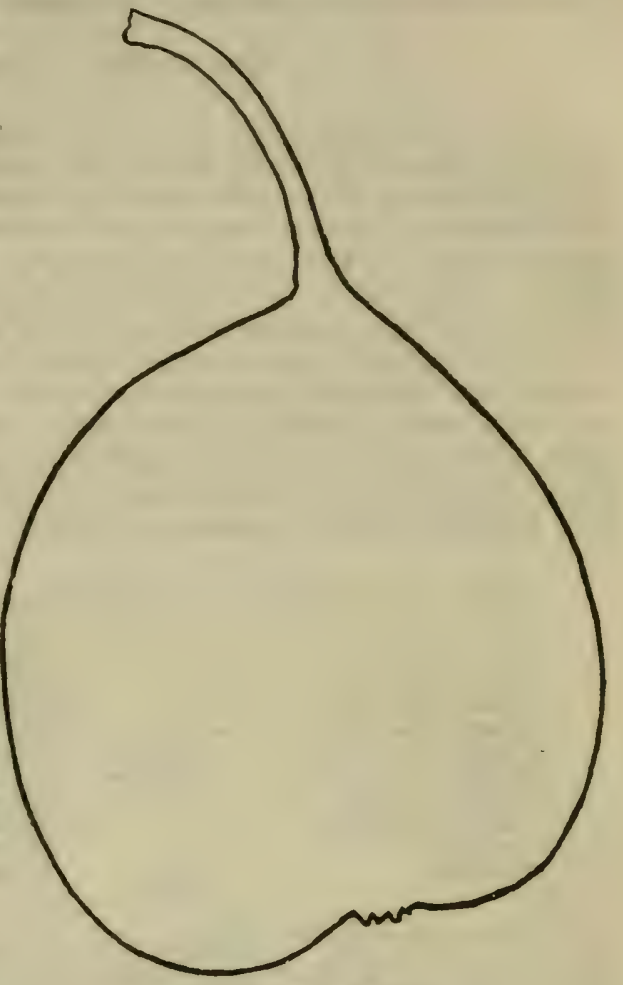

Fig. 204. Moccas.

end excellent flavour. December.

\section{Messire Jean. O. Duh. Mill. Thomp. \\ Monsieur Jean. Messire Jean Gris. Messire Jean Blanc. \\ Messire Jean Doré. Mr. John. \\ John.}

The Messire Jean is a rich, sugary, old French pear, but rather coarse grained and gritty, and therefore only of second juality, and not worthy of general culture. Shoots dark gray.

Fruit of medium size, turbinate, but narrowed a little to. wards the eye also. Skin somewhat rough, yellow, nearly 
cuvered with brown russet. Stalk an inch long, inserted in a small wide cavity. Calyx smaii, open, set in a shallow, plaited basin. Flesh gritty, white, crisp, juicy, and breaking, with a very sweet, rich flavour. In deep, warın, and favourable soils, it is sometimes highly excellent. November and December.

\section{Ne Plus Meuris. Thomp.}

This is a Belgian pear, one of Dr. Van Mons' seedlings, named in allusion to Pierre Meuris, his gardener at Brussels, when his garden there was about to be destroyed. It is an unprepossessing looking, uneven, dull russet fruit, but keeps admirably, and in February and March, is really of very fine flavour. The tree grows upright, has short-jointed, olive coloured shoots, and bears in thick bunches or clusters.

Fruit medium or rather small, roundish, usually very irregular, with swollen parts on the surface. Skin rough, dull yellowish-brown, partially covered with iron-coloured russet. Stalk quite short, set without depression, in a small cavity. Flesh yellowish-white, buttery, melting, with a sugary, and very agreeable flavour. January to March.

\section{Passe Coliar. \& Lind. Thomp. P. Mag.}

\begin{tabular}{|c|c|}
\hline $\begin{array}{l}\text { Passe Colmar Epineaux. } \\
\text { Colmar Gris. } \\
\text { Passe Colmar Gris. } \\
\text { Beurré Colmar Gris, dit précel. } \\
\text { Précel. } \\
\text { Fondante de Panisel. } \\
\text { Fondante de Mons. } \\
\text { Bourré d'Argenson. } \\
\text { Regintin. } \\
\text { Chapman's. }\end{array}$ & $\begin{array}{l}a c . \text { to } \\
\text { Thomp. }\end{array}$ \\
\hline
\end{tabular}

Colmar Hardenpont.

Présent de Malınes.

Marotie Sucrée Jaune.

Souverain.

Culmar Souveraine.

Gambier.

Cellite.

Colmar Preule

Colmur Dorée.

D'Ananas, (of some.)

The Passe Colmar is a Belgian pear of comparatively recent origin, raised by the counsellor Hardenpont. It is a fruit of the first quality; and has become one of the most popular winter pear in the middle states, on account of its excellent flavour, vigorous growth, and abundant bearing. It grows indeed al. most too thrifty, making long, bending shoots, and owing to this over-luxuriance, the fruit is often second rate on young trees. This should, therefore, be checked by occasional root-pruning, or cutting off the leading roots with a sharp spade. The young shoots are of a lively brownish-yellow, and the tree frequently bears a second crop of fruit on its after growth.* It is every way superiour to the old Colmar.

insure fine fruit of the Passe Colmar, prune or thin out half the fruit -.. in the month of March. 


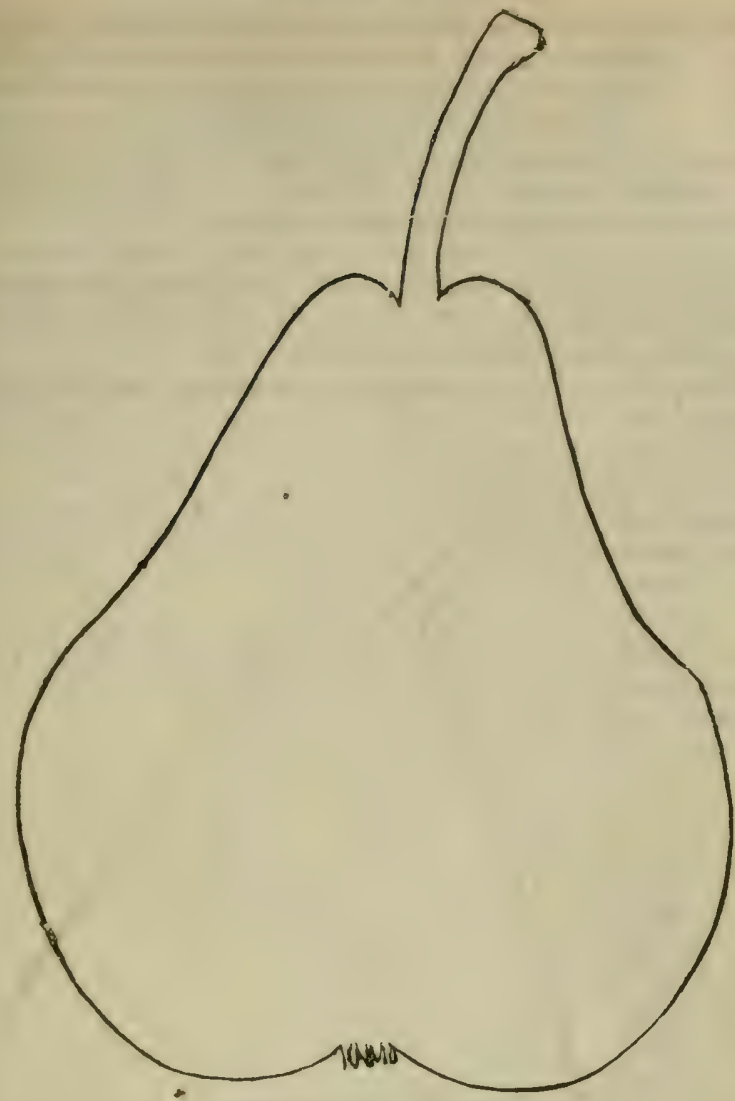

Fig. 205. Passe Colmar.

Fruit rather large, varying considerably, from obovate in obluse-pyriform, but most usually as in Fig. 23.5. Skin ratlur. thick, yellowish-green, becoming yellow at maturity, a gool deal sprinkled with light brown russet. Stalk an inch and a half long, inserted in an obtuse uneven cavity, or sometimes without depression. Calyx open, basin shallow. Flesh yel. lowish-white, buttery and juicy, with a rich, sweet, aromatio flavour.

\section{Pound. Coxe.}

Winter Bell.

Bretagne lo Cour.

ne round, or Winter Bell pear, valued only for cooking, is 
one of the most common fruits in the middle states. Indeed, this and the Black Pear of IVorcester, so common in New Eng land, are the only two kitchen pears extensively grown in this country. The pound jear is the larger of the two, often weigh. ing a couple of pounds each. It is also an abundant bearer, and a profitable orchurd crop. The trees are strong and healthy, with very stout, upright, dark coloured wood.

This is, no doubt, an old European pear, though it does not appear to be described in the books. A Belgian pear under the name of Bretagne le Cour, which has fruited with us for thret years past, appears to be identical with this.

Fruit large, pyriform, swollen at the srown, and narrowing gradually to a point at the insertion of the stalk. Skin yellowish - green, with a brown cheek, (yellow and red when long kept,) and sprinkled with numerous

brown russet dots. Stalk two inches or more long, stout, bent. Calyx crum. pled, set in a narrow, slight basin. Flesh firm and solid, stews red, and is excellent, baked or pre. served.

226. ST. GERMAIN. O. Duh. Lind. Thomp.

St. Germain Gris. Saint Germain Jaune.

Incunnue la Fare.

This is a woll known

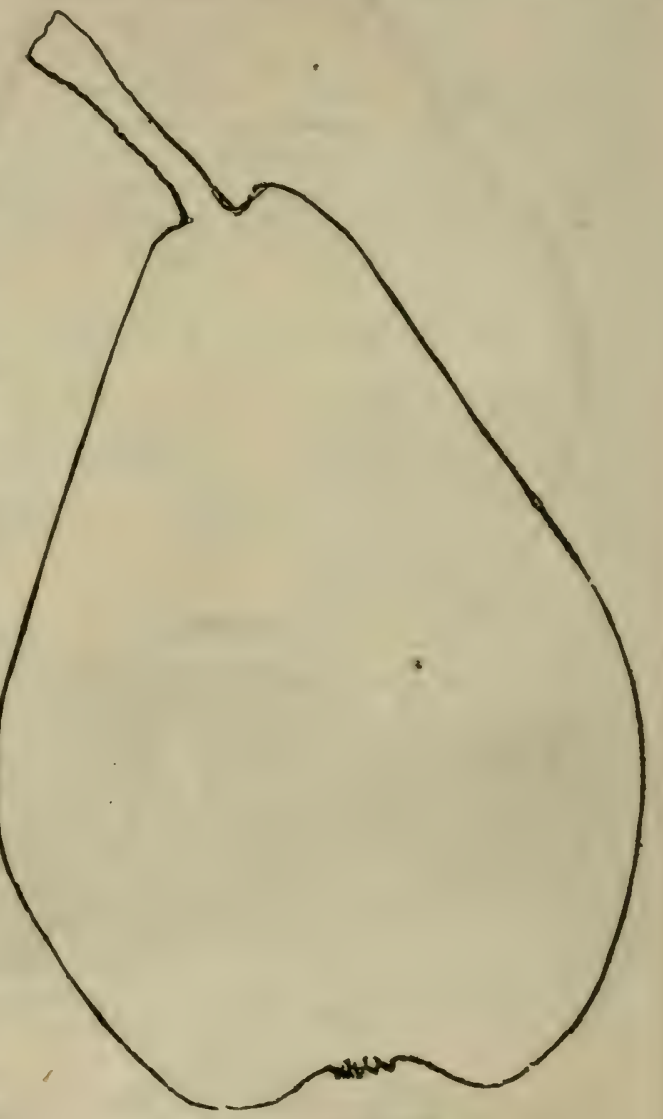

Fig. 206. St. Germain. 
and capital old French variety, and when in perfection, is scarcely surpassed by any other juicy pear. Unfortunately, how ever, it is not a very hardy tree, and is therefore worth litile, near the sea-coast. In the interiour, and in the warm, rich soils of the west, it is, on the contrary, highly deserving of general cultivation. The tree is rather a slow grower, with a dense head of foliage, - the leaves narrow, folded, and curved; the wood slender, and light olive coloured.

Fruit large, pyriform, tapering regularly from the crown to the stalk. Skin yellowish-green, marked with brownish specks on the sunny side, and tinged with a little brown when ripe. Stalk an inch long, strong, planted obliquely by the side of a small, fleshy swelling. Calyx open, set in a shallow basin. Flesh white, a little gritty, but full of refreshing juice, melting, siveet, and agreeable in flavour. November and December.

The Striped Germain, (St. Germain Panachée,) is a pretty variety of this fruit, differing only in being externally striped with yellow.

227. St. Germain, Prince's. $§$ Pom. Man. Thomp.

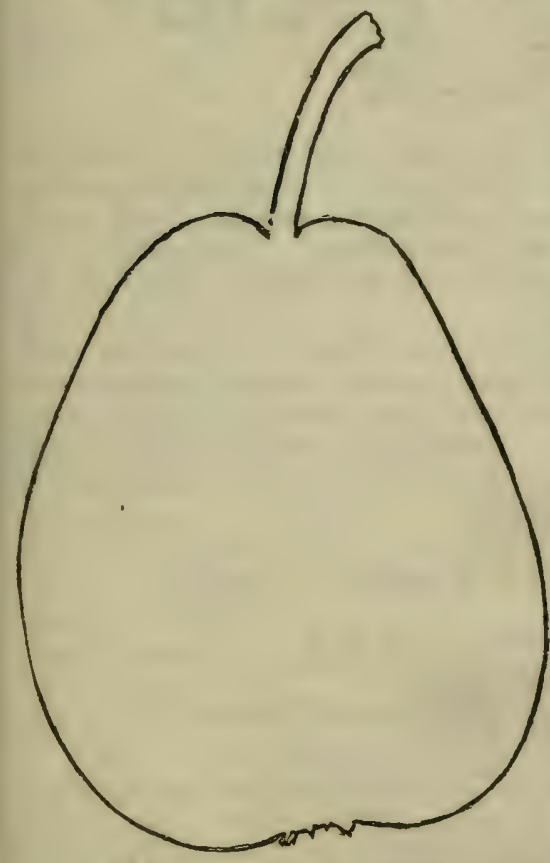

Fig. 207. Prince's St. Germain.

\section{Brown St, Germain.}

New St. Germain.

Prince's St. Germain is a seedling from the foregoing pear, raised at Prince's nurseries, at Flushing, about forty years ago. It is a most thrifty and hardy tree, with dark red. dish brown shoots. The fruit keeps as well as a russet apple, is uniformly good, and is certainly one of the best late pears when under good cultivation. It is much more es. teemed in the eastern states than the old St. Germain.

Fruit of melium size, obovate, inclining to oval. Skin nearly covered with brownish russet over a green。 ground, and becoming 
dull red next the sun. Stalk an inch or more long, a little curved, and placed in a slight, flattened depression. Calyx large, open, firm, and nearly without divisions, set in a smooth, ncarly flat basin. Flesh yellowis:-white, juicy, melting, with a sweet, somewhat vinous and vely agreeable flavour. November to March.

\section{Saint Geritain, Brande's.}

This is a new variety, received, we believe, from England. It has fruited in Sulem, Mas.., and proves of the first quality.

Fruit of rnedium size, oval, narrowing towards both ends. Skin vellowish-green. Stalk short, three-fourths of an inch long, thick, set obliquely on one side of the end of the fruit. Calyx small, stiff, placed on the narrow crown, mostly without a basin. Flesh melting, juicy, with a rich and cxcellent flavour. November and December.

229. St. Germain, ITvedale's. Mill. Lind. Thomp.

\begin{tabular}{|c|c|}
\hline $\begin{array}{l}\text { Uvedal's Warden. } \\
\text { Germain Baker. } \\
\text { Len: St. Germain. } \\
\text { Picliering Pear. } \\
\text { Chambers' }\end{array}$ & 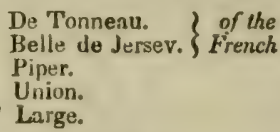 \\
\hline
\end{tabular}

Uvedale's St. Germain is a very large winter pear, only fit for cooking, for which it is very good. It is an English variety, which has been 100 years in cultivation, and frequently grown to the size of three pounds in that country. In this country it is not so much planted as others, being less hardy. It is very distinct from the pound pear.

Fruit very large, oblong-pyriform, obtuse at the end, and taper. ing to the eye. Skin yellowish green at maturity, with a brown cheek. Stalk an inch long, bent and planted in a rather derp, oblique, angular cavity. Calyx large, set in a deep hollow. Flesh white, hard and astringent, but bakes and stews well. In use from January to April.

\section{Vicar of WinkField. Thomp. -

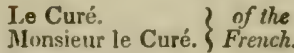 Dumas. Bourgermester, incorrectly of Boston. Clion. K' Kenrick.}

'This large and productive pear was discovered not long since, as a natural seedling in the woorls of Clion, France, by a French surate, whence it obtained in France, the familiar names of $L e$ Cure, or Monsicur le Cure. A short time after it became known 
dt Paris, it was imported into England by the Rev. Mr. Rham, of Winkfield, Berkshire, and cultivated and disséminated from thence, becomingknown in the neighbourhood of London as the Vicar of Winkfield. Now, although we think Mr. 'Thompson erred in adopting this English name instead of. continuing the French title, yet for the sake of having some uniform standard, we shall follow him, con. sidering, however, Le Curé as the genuine name.*
We should add that the same fruit was imported to Boston and here, a few years ago, under the erroneous name of Bourgermester, and considera. bly disseminated.

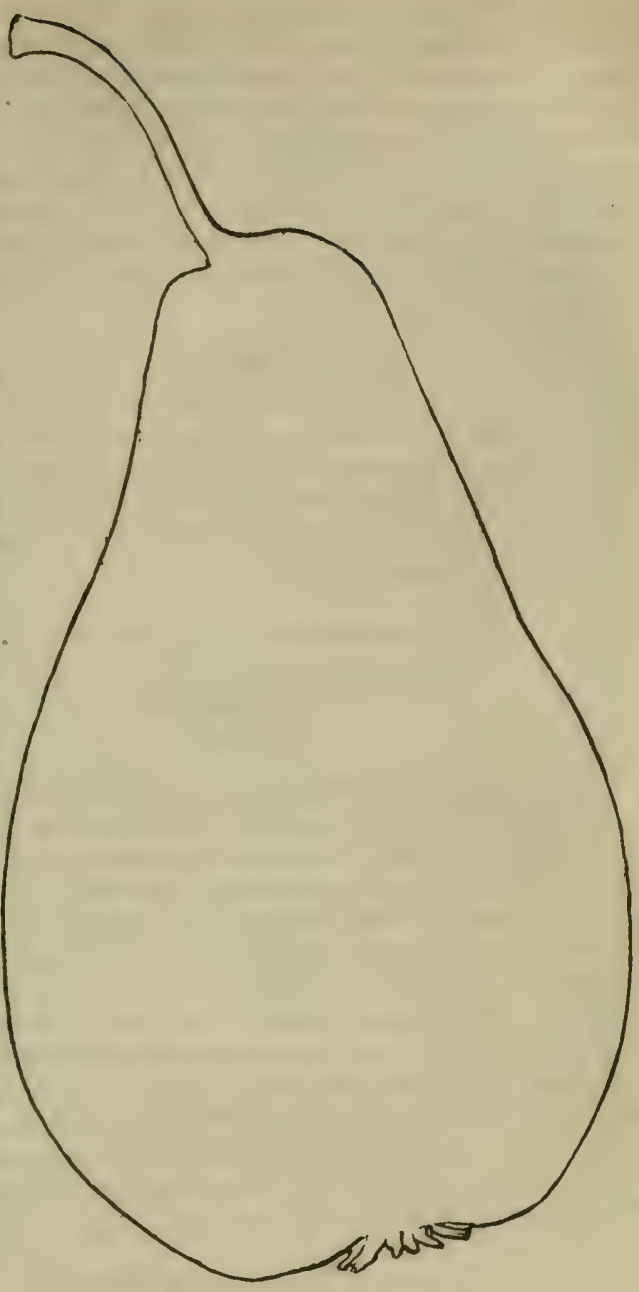

Fig. 208. Vicar of Winistuld.

With regard to its merits there is some difference of opinionsome persons considering it a fine fruit. It has borne very ad.

- The only reason that can be given for an English re-christening, is that the French (see Bon Jardinier, 1314, confuse this pear. Le Curé, with the St. Lozin an inferic ur firm fleshed pear, fit only fur stewing and cooking. 
mirably with us for some years past. It is always remarkably large, fair and handsome. We think it always a first rate baking pear. Occasionally we have tasted it fine as a table pear, but generally it is astringent, and only third rate for this purpose. If ripened off in a warm temperature however, it will generally prove a good, second rate eating pear. But its great productive ness, hardiness, and fine size, will always give it a prominent place in the orchard as a profitable, market, cooking pear. 'The tree grows thriftily, with drooping fruit branches. Shoots $\mathrm{d}_{\mathrm{l}}$ verging, dark olive.

Fruit large and long-pyriform, often six inches long, and a little me-sided. Skin fair and smooth, pale yellow, sometimes with a brownish cheek, and marked with small brown dots. Stalk an inch or an inch and a half long, slender, obliquely inserted withrut depression. Calyx large, open, set in a basin which is very slightly sunk. Flesh greenish-white, generally juicy, but sometimes buttery, with a good sprightly flavour. November to January.

\section{Virgouleuse. O. Duh. Poit. Thomp. \\ Poire-glace. \\ Chambrette. \\ Bujaleuf.}

An excellent old French variety, which, in consequence of it s indifferent crops, is scarcely cultivated in the middle states. In the warmer and richer western states, it is well worthy of a trial. The tree grows strongly. It takes its name from Virgoulé a sinall French village-the place of its origin. It is, however, a very different pear from the Virgaliou of New-York, which is the White Doyenné.

Fruit rather large, oval, obovate-handsomely rounded at both ends. Skin very smooth, yellowish-green at maturity, sprinkled with numerous gray or reduish dots. Stalk about an inch long, set in a very trifling depression. Calyx small, placed in a wide, shallow basin, somintimes scarcely at all sunk. Flesh white, buttery, melting, and of excellent flavour. November to Januayy.

\section{WintFị Nelis. $\oint$ Lind. Thomp. \\ Nélis d'Hiver. \\ Bonne de Malines. \\ Beurré de Malines. \\ La Bonne Malinoise. \\ Milanaise Cuvelier. \\ Etourneau.}

The Winter Nelis holds, in our estimation, nearly the same rank amoug winter pears, that the Seckel does among the au. tunınal varieties. We consider it unsurpassed in rich, delicious flavour, and indispensable „) every garden, however small. It 
is a, very hardy and thrifty tree, and bears regular crops of pear; which always ripen well. and in succession. Branches diverging, rather slender, light olive.

It is a Flemish pear, and was originated, above twenty years since, by M. Nelis, of Mechlin.

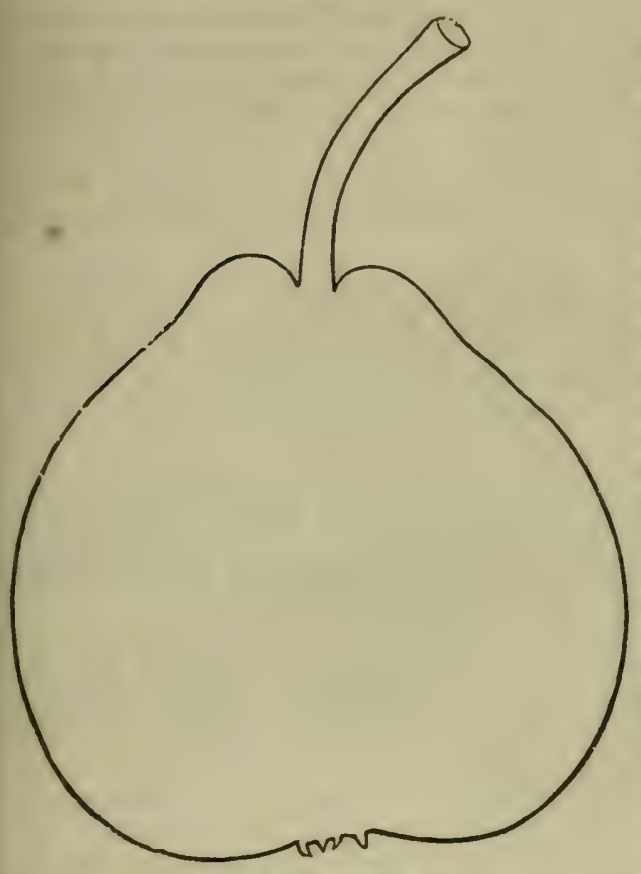

Fig. 208. Winter Nelis.

Fruit of me. dium size, of usually a little below it, roundish-obovate, nar. rowed.in near the stalk. Skin yellowish-green at maturity, dot. ted with gray russet, and a good deal cov. ered with russet patches and streaks, especially on the sunny side. Stalk an inch and a half long, bent, and planted in a narrow cavity. Calyx open, with stiff, short divisions, placed in a shallow basin. Flesh yellowish-white, fine grained, buttery and very melting, abounding with juice, of a rich, saccharine, aromatic flavour. In perfection in December, and keeps till the middle of January.

\section{Wilhelmine. Nois. Bon. Jard. Thomp.}

\section{Wilhelmina. Ken.}

New, and lately reccived from France, where it has the reputation of being a late winter fruit of the first quality.

Fruit of medium size, obovate, rather narrowed.in towards the stalk. Skin greennish-yellow, lotted with distinet gray specks, and washed with a little red towards the sun. Stalk an inch and a quarter long, inserted in a slight, rather blunt depression. Calyx large, open, set level with the surface, or a 
little projecting. Flesh yellowish-white, buttery and molting, with an abundant, sugary, perfumed juice. February and March.

Sclection of choice pears for a smail garden, to ripen in succession from Iuly to A pril. Maleleine, Bloodgrond, Dearborn's Seedling, Bartlett, or William's Bon Chrétien, Andrews, Sum. mer Frane Ré:al, White Doyenné, Seckel, Fondante d'Automne, Gray Doyenné, Urbaniste, Dunmore, Marie Louise, Van Mons Léon le Clere, Beurré Bosc, Dix, Columbian, Winter Nelis, Beurré d'Aremberg, Linight's Monarch, and (for deef: warm soil,) Beurré de Ranz.

Selection of very hardy and good pears for a cold climate. Fulton, Blooilgoud, Seclsel, Stevers' Genesee, Heathcot, Marie Lonise: Beurré Bosc, Dix, Hacon's Incomparable, Buflum, Beurré Capiumont, Andrews, Bartlett, Washington, White Doyenné, Beurré Diel, Winter Nelis, Beurré d'Aremberg, I'rince's St. Germain.

Almost all the varieties do well in the interiour; the old French sorts usually better than with us, and the following sorts are generally finer in a warmer climate, say that of Maryland, than here; Beurré de Ranz, Glout Morceau, Easter Beurré, Messire Jean, St. Germain, \&cc.

Perry pears. 'These are little attended to in this country; perry being made from the most common varieties. 'The best English perry pears are the following; Oldfield, Barland, Longland, and Teinton Squash.

\section{CIIAPTER XXII.}

\section{THE PEACH.}

Persica vulgaris, Dec.; Rosacea, of botanistg.

Pecher, of the French; Pfirschbuum, German; I'ersichtithow, Dutch; Persica, Ihalian; and El Melocoton, Spanish.

Trre peach tree is a native of Persia and rhina, and was brought from the former country to Italy by the Roinans in the time of the Emperor Claudius. It was considerably cultivated in Britain as early as the year 1550, and was introduced to this country by the early settlers somewhere about 1680. From Persia, its native country, its name in all languages-PersicoPecher-Peach, has evidently been derived. 
The peach is a rather small fruit tree, with narrow, smooth, serrated leaves, and pink blossoms. It is inore tender, and of shorter duration than most other of the fruits usually g "own in temperate climates. It is never raised in England, ani not generally in France, without the aid of walls. Even at Mon. treuil, near Paris, a village whose whole population is mainly cmployed in cultivating the peach for marke:, it is grown entircly upon white-washed walls. China and the United States are, therefore, the only temperate countries where the peach and the apple both attain their highest perfection in the open orchard. 'The peaches of Pekin are celebrated as being the finest in the world, and of double the usual size.*

It is a curious fact in the history of the prach, that with its delicious flavour were once coupled, in the East, certain notions of its poisonous qualities. This idea seems vaguely to have accompanied it into Europe, for Pliny mentions that it was sup. posed that the king of Persia had sent them into Egypt to poison the inhabitants, with whom he was then at war. As the peach and the almond are closely related, it has been conjectured by Mr. Knigit that the poisonous peaches referred to, were swollen almonds, which contain a considerable quantity of prussic acid. But it is also worth remarking that the peach tree seems to hold very much the same place in the ancient Chinese writings, that the tree of knowlerlge of the old scriptures, and the golden Hesperides apples of the heathens, do in the early history of the western nations. 'The traditions of a peach tree, the fruit of which when eaten conferred immortality, and which bore only once in a thousand years - and of a nother peach tree of knowledge, which existed in the most remote period on a mountain gua rded by an hundred demons, the fruit of which produced death, are said to he distinetly preserved in some of the early Chinese writings. Whatever may have been the nature of these extruordinary trees, it is certain that, as Lord Bacon says, "not a slip or sucker has been left behind." We must tharefore content ourselves with the delight which a fine peach of modern times affords to the palate and the eye.

We believe there is at the present time, no country in the world, where the peach is grown in such great quantities as in the United States. $\dagger$ North of a line drawn from the Mohawk river to Boston, comprising most of the eastern states, they do not indeed flourish well, requiring some artificial aid to produce

* 'l'he Horticultural world since our intercourse has been put upon a moro invorable fonting with tho "Celestial E:npire," are looking with great eagernoss to th: introtaction of miny valuable plants and trees, the chinese boing tho most curiuus and slzilful of inerely practical gardeners.

t It will amise our readers to read in Mc.Intoslt's work, "The Orchard," that "the Americaus ustally at the clumstones, waile they reserve the fre zstones for fecding tho pigs?" 
regular crops, but in all the Middle, Southern, and Western States, they grow and produce the heaviest crops in every garder, and orchard. Thousinds of acres in New-Jersey, Delaware and Maryland, are devoted to this crop for the supply of the markets of New.York and Philadelphia, and we have seen ir. scasons oi great abundance, whole sloop loads of fruit of second quality, or slightly decayed, thrown into the North river in a single morning. The market price usually varies from fifty cents to four dollars per bushel, according to the abundance of the crop, and to the earliness or lateness of the season at which they are offered; one hundred and fifty cents being considered a good retail price. Many growers in New. Jersey have or. chards of from 10,000 to 20,000 trees of different ages, and send to market in gond scasons as many bushels of fruit from the bearing trees. When the crop is not universally abundant, the profits are very large, if the contrary, they are often very little. But, as in some districts, especially in New-Jersey, peaches are frequently grown on land too light to produce good crops of many other kinds, the investment is a good one in almost all cases. Undoubtedly, however, the great peach growing district of the United States, will one day be the valleys of the Ohio and Mississippi. With an equally favourable climate, that portion of the country possesses a much finer soil, and the flavour of it peaches is unusually rich and delicious.

The very great facility with which the peach grows in thi: country, and the numerous crops it produces, almost without care, have led to a carelessness of cultivation which has greatly enfecbled the stock in the eastern half of the Union, and, as we shall presently show, has, in many places, produced a disease peculiar to this country. This renders it necestary to give some additional care and attention to the cultivation of the peach, and with very trifling care, this delicious fruit may be pro. duced in great a! Jundance for many successive years.

UsEs. Certainly no one expects us to write the praises of the peach as the most delicious of fruits. " "To gild refined gold," would be a task quite as necessary, and if any one doubts the precise rank which the peach should take among the different fruits of even that cornucopian month-September-and wishes to convinee us of the higher flavour of a Seckel or a Monarch pear, we will promise to stop his mouth and his argument with a sunny cheeked and melting "Gcorge the Fourth," or luscious "Rareripe!" No man who lives under a warm sun will hesi. tate about giving a due share of his garden to peaches, if he have no orehard, and even he, who lies north of the best lndian corn limits, ought to venture on a small line of espalier, for the sake of the peach. In pies and pastry, and for various linds of preserving, the peach is every where highly esteemed. At the south and west, where peaches are not easily carried to narket, a con. 
घंderable quantity of peach brandy is annually aistilled from them, but we believe, by no means so much as formerly. Hogs are fattened, in such districts, on the refuse of the orchard and distillery.

In Western New-York, and indeed in most parts of the coun. try where peaches are largely cultivated, the firuit is dried, and in this state, sent to market in very large quantities. The drying is performed, on a small scale, in spent ovens; on a large scale, in a small drying house heated by a stove, and fitted up with ventilated drawers. These drawers, the bottoms of which are formed of laths, or narrow strips sufficiently open to allow the air to circulate through them, are filled with peaches in halves. They are cut in two without being peeled, the stone taken out, and the two halves placed in a single layer with the skin dowr. ward. In a short time the heat of the drying house will com. plete the drying, and the drawers are then ready for a second filling. Farther south they are spread upon boards or frames, and dried in the sun merely; but usually, with the previous pre. paration, of dipping the peaches, (in baskets,) for a few minutes . $\mathrm{n}$ boiling water before halving them.

The leaf of the peach, bruised in water and distilled, gives the peach water, so much esteemed by many for flavouring articles of delicate cookery; and steeped in brandy or spirits, they communicate to it the flavour of Noyeau. Indeed a very good imitation of the celebrated Noyeau is made in this way, by using the best white brandy, which, after being thus flavoured, is sweet. ened with refined sugar mixed with a small quantity of milk, and afterwards decanted.

Propagation. 'The peach is the most easily propagated of all fruit trees. A stone planted in the autumn will vegetate in the ensuing spring, grow three or four feet high, and may be budded in August or September. Two years from this tirne, if left undisturbed, it will, usually, produce a small crop of fruit, and the next scason bear very abundantly, unless the growth is over-luxuriant.

In nursery culture, it is customary to bury the peach stones, in autumn, in some exposed spot, in thick layers, covered with earth. Here they are allowed to lie all winter. As early in the spring as the ground is in fine friable condition, the stones are taken out of the ground, cracked, and the kernels sown in mellow, prepared soil, in the nursery rows, where they are to grow. 'They should be covered about an inch deep. Early in the following September they will be fit for budding. This is performed with great eare on the peach, and grafting is there. fore seldom or never resorted to in this country. The buds should be inserted quite near the ground. The next season the stock shcuia be headed back in March, and the trees will, in good soil, grow to the height of a man's head in one year. This 
is, by far, the best size for transplanting the peach-one year old from the bud.

For northern latitudes, for cold soils, and for training, the plum stock is much preferable to tise peach for burding the fine varieties. In England the plum strek is universally comployed. The advantage gained thereby is, not only, greater hardihood, but a dwarfer and neater habit of growth, for their walls. In France, some of the best cultivators prefer the almond stock, and we have no doubt, as it would check the over productiveness of the peach, it would be desirable to employ it more generally in this climate. Still, healthy peach stocks afford the most natural foundation for the growth of standard, orchard trees. At the same time we must protest against the indiscriminate employment (as is customary with some nurserymen,) of peach stones from any and every source. IVith the present partially diseasel state of many orchards in this country, this is a prac. tice to be seriously condemned. And, more especially, as with a little care, it is always easy to procure stones from sections of country where the Yellows is not prevalent.

For rendering the pcach quite dwarf, the Mirabelle plum stock is often employed abroad.

Soil And situation. The very best soil for the peach is a rich, deep sandy loam; next to this, a strong, mellow loam; then a light, thin, sandy soil, and the poorest is a heavy, compact clay soil. We are very well aware that the extensive and profitable appropriation of thousands of acres of the lightest sandy snil in New-Jersey and Delaware, has led many to believe that this is the best soil for the peach. But such is not the fact, and the short duration of this tree in those districts, is unquestionably owing to the rapidity with which the soil is impoverished. We have, on the contrary, seen much larger, finer, and richer flavoured peaches, produced for a long tzme successively, on mellow loam, containing but little sand, than upon any other soil whatever.

It is a well founded practice not to plant peacli orchards suc. cessively upon the same site, but always to choose a new one. From sixteen to twenty-five feet apart may be stated as the limits of distance at which to plant this tree in orchards-more space ieing required in warm climates and rich soils than under tho contra:y circumstances. North of New.York it is better al. ways to make plantations in the spring, and it should be done pretty early in the scason. South of that limit it may usually be done with equal advantage in the autumn.

In districts of country where the fruit in the blossom is liable to be cut off by spring frosts, it is found of great advantage to make plantations on the uorth sides of hills, northern slopes or elevated grounds, in preference to warm valleys and southern aspects. In the colder exposures the vegetation and blossoming of the tree is retarded until after all danger of injury is past. 
Slluations near the banks of large rivers and inland lakes are equally arlmirable on this account, and in the garden where we write, on the banks of the Huason, the blossoms are not injured once in a dozen years, while on level grounds only five miles in the interiour, they are lestroyed every fourth or fifth season.

With regard to the culture of peach orchards, there is a secming disparity of opinion between growers at the north and south. Most of the cultivators at the south say, never plough or cultivate an orchard after it has borne the first crop. Plough. ing bruises the roots, enfeebles the tree, and lessens the crop. Enrich the ground by top-dressings, and leave it in a state of rest. The best northern growers say, always keep the land in good condition,-mellow and loose by cultivation, - and crop it very frequently with the lighter root and field crops. Both are correct, and it is not difficult to explain the seeming differ. ence of opinion.

The majority of the peach orchards south of Philadelphia, it will be recollected, grow upon a thin, light soil, previously rather impoverished. In such soils, it is necessarily the case, that the roots lie near the surface, and most of the food derived by them is from what is applied to the surface, or added to the soil. Ploughing therefore, in such soils, wounds and injures the roots, and cropping the ground takes from it the scanty food annually applied or already in the soil, which is not more than sufficient for the orchard alone. In a stronger and deeper soil, the roots of the peach tree penetrate farther, and are, mostly, out of the reach of serious injury by the plough. Instead of losing by being opened and cxposed to the air, the heavier soil gains grcatly in value by the very act of rendering it more friable, while at the same time it has naturally sufficient heart to bear judicious cropping with advantage, rather than injury, to the trees. The growth and luxuriance of an orchard in strong land, kept under tillage, is surprisingly greater than the same allowed to remain in sod. The difference in treatment therefore, should always adapt itself to the nature of the soil. In or. dinary cases, the duration of peach orchards in the light sandy soil is rarely more than three years in a bearing state. In a stronger soil, with oroper attention to the shortening system of pruning, it may be prolonged to twenty or more years.

Pruving. It has always been the prevailing doctrine in this country that the peach requires no pruning. It has been allowad to grow, to bear heavy crops, and to die, pretty much in its own way. 'This is very well for a tree in its native climate, and in a wild state, but it must be remembered that the peach comes from a warmer country than ours, and that our peaches of the present day are artificial varieties. They owe their origin to artificial mears, and require therefore, a system of culture to correspond. 
In short, we view this absence of all due care in the manago ment of the peach tree, after it comes inte bearing, as th:e princl. pal original cause of its present short duration, and the discase which preys upon it in many of the oliter parts of the country. We therefore earnestly desire the attention of peach growers tc our brief hints upon a regular system of pruning this valuable tree. Of course we speak now of common standard trees, in the orchard or garden.

A peach tree, left to itself after being planted, usually comes into bearing the third or fourth year, and has a well shaped, rounded head, full of small hearing branches, and well garnish. ed with leaves. It must be borne in mind that the fruit is only horne on the young shoots of the provious summer's growth. in a young tree these are properly distributed throumhout. But in a couple of seasons, the tree being left to itself, the growth being mostly produced at the ends of the principal branches, the young shoots in the interiour of the head of the tree, die out. The consequence is, that in a short time the interiour of the tree is filled with long lean branches, with only young shoots at their extremities. [See Fig. 209.] Any one can see that such a tree can be provided with but half the number of healthy strong shoots for bearing, that one would have if filled throughout with vigorous young wood. The sap flows tardily through the long and rigid branches, and not half leaves enough are provided to secure the proper growth of the fruit. Fig. 209. A peach tree, without pruning,
And, finally, all the fruit

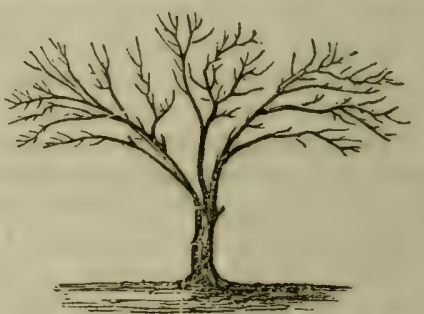
which the tree yields being allowed to remain at the ends of the branches, they often break under its weight.

Now, we propose to substitute for this, what is generally known as the shortening-in system of pruning. We affirm, hoth from its constant success abroad, and from our own experience aud observation in this country, that putting its $t$ wo discases out of the question, (which we will presently show how to avert,) the peach may be continued in full vigour and production in any good soil, for from ten to thirty years.

Let us take a healthy tree in the orchard or garden, in its first blossoming year. It is usually about 6 to 8 feet. high, its well.siaped head branching out about three feet* from the

- We think low heada much preferable to high ones on many arcosnts They shade the root, which insects are therefore much less linble to attack, and they are more within reach both for pruning and gnthering: 
ground. It has never yet been trimmed except to regrulate any deformity in its shape, and this is so much the better.

At the end of February or as early in the snring as may be, we cornmence pruning. This consists only of slentening-in. i. c. cutting off half the last year's growth over the whole outside of the head of the tree, and also upon the inner branches. As the usual average growth is from one to two feet, we shall necessarily take off from six to twelve inches. It need not be done with precise measurement; indeed, the strongest shoots should be shortened back most, in order to bring up the other's, and any long or projecting limbs that destroy the halance of the head should be cut hack to a uniform length. This brings the tree into a well rounded shape. By reducing the young wool one half, we at the same moinent reduce the coning crop one half in number. The remaining half, receiving all the sustenaisce of the tree, are of double the size. The young shoots which start out abundantly from every part of the tree, keep it well sup. plied with bearing wood for the next year, while the greater luxuriance and size of the foliage, as a necessarv consquence, produces larger and higher flavoured fruit.* Thus, while we have secured against the prevalent evil, an over crop-we have

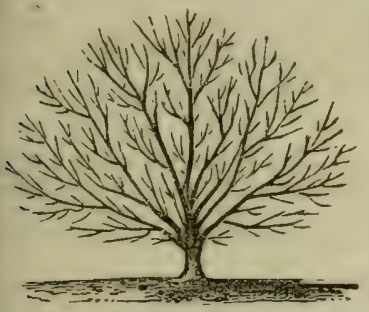

Fig. 210. A peuth tree, pruned by the shortening-in mode. also provided for the full nourishment of the present year's fruit, and induced a supply of fruit bearing shoots throughout the tree, for the next season.

This course of pruning is fol. lowed regularly, every year, for the whole life of the tree. It is done much more rapidly than one would suppose ; the pruned wonnds are too small to cause any grum to flow; and it is done at the close of winter, when labour is worth least to the cultivator.

The appearance of a tree pruned in this way, after many years of beuring, is a very striking contrast to that of the poor skeletons usually seen. It is in fact, a fine objjet, with a thick low bust.y hearl, filled with healthy young wood, [Fig. 210,] and in the summer with an abundance of dark gret 11 , boaltny foliage, and handsome fruit. Can any intelligent man hestate about adopting so simple a course of treatment to secure such valuable results? We recommend it with cntire confidence to

- It is well, in shortening-back, to cut off the shoot close al rove a wnod-luad rather than a blossom-bud. Few perons are? aware how much the size and beauty of the fruit depends on the size and vigour of the leaves. We hase seull wo peach trees of the same age side by sid-e, one unpruned, and the nther 10.12 . larly shortened-in, and both bearing abuut four bushels. That of the lutter was bowever, of double the size, and incomparably finer 
the practic: of every man in the country that cultivates a peach tree. After he has seen and tasted its good effects, we to not fear his laying it aside.*

Trainug the peach tree against walls or espaliers is but little practised in this country, except in the neighbourhood of Boston. Espalier training, on a small scale, is however, highly worthy of the attention of persons desiring this fiuit in the colder parts of the country, where it does not succeed well as a standard. Every where in New-England excellent crops may be pro. duced in this way. Full directions for training the peach with illustrations are given in page 38 .

INSEC IS AND DISEASES. For a considerable time after the peach was introrluced into America, it was grown every where south of the $40^{\circ}$ of latitude, we may say literally without cul tivation. It was only necessary to plant a stone in order to obtain, in a few years, and for a long time, an abundance of fruit. Very frequently these chance seedlings were of ex. cellent quality, and the finer grafted varieties were equally luxuriant. In our new western lands this is now true, excep. where tic disease is carried from the east. But in the older Atlantic states, two maladies have appeared within the last twenty years, which, because they are little understood, have rendered this fine fruit tree comparatively short lived, and of little value. 'These are the Peach borer, and the Yollows.

The: Peacil Bonen, or Peach-worm (Ageria exitiosa, Say.)

- While this is gning through the press our atten:ion is drawn to the following temarkiable exainiles of the gool effects of regular pruning, which we translate from the lading French Juinual of IIoriculiure. We ask the attention of our reat rs to these cases, especially after perusing our remarks on the lellows and its cauce.

"M. Duvilliers laid before the lioyal Society of IIorticulure, an account of some old peoch trees that he had lately seen at the Clatean de Villiers, neap Ferté-1lęs (Soine-ct-Oise.) These trees, eight in number, are growing upon a terrace wall. which they cover perfectly, and yold abundant erops. The gard:ner assured H. Wasillers that they had hasen witer his care during the thrty years that he ha.l b-ill a: the chateau. that h.'y were at large when he first saw iliem as at pri:eat, and that he supjowed the in we be at liast sixty yenrs old. We connot

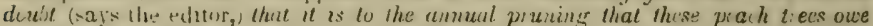

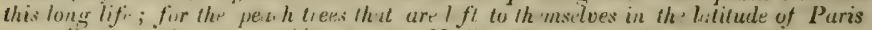

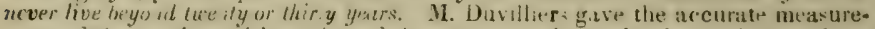
ment of the undse and b anches of these tres, and shatcd, what it is more inte resting to know, that aldusugh all their trunks are hollow, like those of old wil

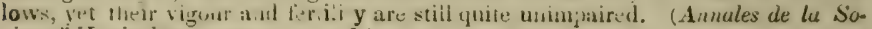
ciete diHorticultur, , iome $\mathrm{xxx}$. p. 53)

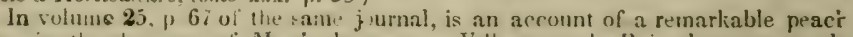
tree in the deme-ne of 31. jubbrt, near Ville-neuve-fe Roi, (departement de l'Yunne.) it is trabed again tone of the wings of the mansion, covers a large space with its basches, and lle circunterence of its trumk, taken at some dis.

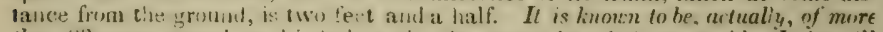

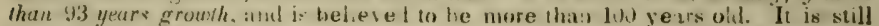

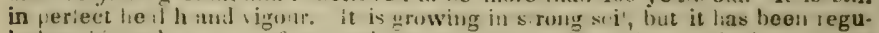

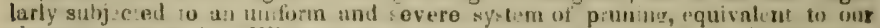

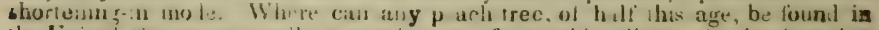
the Lisiel status-maturally a much mure fivourable elinabie for it, thas that of France? 
does great mischief to this tree by girdling and devouring the whole circle of bark just below the surface of the ground, when it soon languishes and dies.

'The insect in its perfuct state is a slenler, tark blue, four winced moth, somewhat like a wasp. It commences lepositing its egrs in the soft and tender bark at the base of the trunk, usually about the last of June, but at different times, from June to October. The egg hatches and becomes a small white borer or grub, which eventually grows to three-fourths of an inch loner, penetratr's and devours the bark and sap woorl, and, after passinin the winter in the tree, it enf lds itself in a coconon under or upru the bark, andemerges acrain in a perfict or winged form in June, and commences depositing its egrgs for another generation.

It is not difficult to rid our trees of this enemy. In fiact nothing is easier to him who is willing to devotr a fi womonts every season to each tree. The eges which produce the horer. it will be recollected, are depositer in the suft portion of bark just at the surface of the earth. Exprerince has conclusively proved that if a small quantity, say half a pecte of air-shatied lime, is heaped around the trunk of each tres at the end of 11 ity and suffered to remain till Octotser, the prach bores will unt attack it. It has been tried most suceessfully in larese orehards, where the protected trees have long remain sombl, while thrse unprotected have been sperdily destruyed by the horer. The remedy undoubiedly lies chiefly, in crivering the most vulner. able portion of the tree from the attack of the insect; and therefure persous have been more or less stecessful with ashes, chareoal, clay, mortar, and other protectives. But we recommend for this purpose air-slaked lime or ashes, * hecausin these more fully answer the purpose as protectives, and whon spread over the surface, as they should the every autumn, they form the best fertilizers for the peach tree.

This is the easiest and the most successful mode, and it should not be neglected a single scason. Many carcful and rigid cultivators prefer a regular examination of the trees every spring and autumn. On removing the rarth, for a few inches, the appearance of gum or castings quickly indicales where the borer has made his lodging. A feiv moments with the knife will then eradicate the insect for the season. This is a very effectual mode, but not, on the whole, so simple or sn good as the other, because the tree is always left exposed to attack, and to consequent injury, before the insect is dislodged.

The Yeloows. This most serious malady seerns to belong axclusively to this country, and to attack only the peach tree. Although it has been the greatest enemy of the prach planter for the last thirty years-rendering the life of the tree uncertain, and frequently spreading over and destroying the orchards $39^{*}$

* Bleached ashes. 
of whole districts ; still, little is known of its nature, and nothing with certainty of its cause. Many slight observers have con. founder it with the effects of the peach-borer, but all persons who have carefully examined it, know that the two are totally distinct. 'Trees may frequently be attacked by both the yellows and the borer, but hundreds die of the ycllows when the most minute inspretion of the roots and branches can discover no insect or visible cause. Still, we believe proper cultivation will entirely rid our gardens and orchards of this malady, and this belief is in part borne out by experiments under our own inspection. In order to combat it sucerssully it is necessary that the svmptoms should be clearly understood.

Symptoms. The Yellows appears to be a constitutional dis. casr, no external cause having yet been assigned for it. Its infallible symptoms are the following:

1. The production upon the brariches, of very slender wiry shoots, a fe'w inches long, and hearing starved, diminutive leaves. These shoots are not protiuded from the extremities, but from latent buds on the main portions of the stem and larger branches. The laves are very narrow and small, quite distinct from those of the natural size, and are either pale-yellow or destitute of colour.

2. The premature ripening of the fruit. 'This takes place from two to four weeks earlier than the proper scason. The first srason of the disease it grows nearly to its natural size; the following season it is not more than half or a fourth of that size; but it is always marked externally, (whatever may be the natural colour) with specks and large spots of purplish red. Inter1)ally, the flesh is more deeply coloured, especially around the stone than in the natural state.

Either of the foregoing symptoms (and sometimes the second appears a season in advance of the first,) are undeniable signs of the vellows, and they are not produced by the attacks of the worm or other malady. We may add to them the following additional remarks.

It is established beyond question, that the yellows is always propagated by budding or grafting from a diseased tree; that the stock, whether peach or almond, also takes the disease, and finally perishes; and that the seeds of the diseased trees produce young trees in which the yellows sooner or later break out. Tothis we inay add that the peach budded on the plum or apricot is also known to die with the ycllows.

'The most luxuriant and healthy varieties appear most liable to it. Slow growing sorts are rarely affected.

Very frequently, only a single branch, or one side of a tree, will be affected the first season. But the next year it invaria. bly spreads through its whole system. Frequently, tree; badly affected will die the next year. But usually it will last, growing 
more and more feeble every year, for several seasons. 'T'ine roots ou digging up the trec, do not appear in the least diseased.

The soil does not appear materially to increase or lessen the liability to the Yellows, though it first originated, and is most de structive in light, warm, sandy soils. T'rees standing in hard trodden places, as in, or by, a frequented side-walk, often outlive all others.

Lastly, it is the near $y$ universal opinion or all orchardists that the Yellows is a contagious disease, spreading gradually, but certainly, from tree to tree through whole orchards. It was conjectured by the late William Prince that this takes place when the trees were in blossom, the contagion being carried from tree to tree in the pollen by bees, and the wind. This view is a questionable one, and it is rendered more doubtful by the fact that experiments have been made by dusting the pollen of diseased trees upon the blossoms of healthy ones without communicating the Yellows.

We consider the contagious nature of this malady an unse tled point. Theoretically, we are disinclined to believe it, as we know nothing analagous to it in the vegetable kingdom. But on the other hand, it would appear to be practically true, and for all practical purposes we would base our advice upon the supposition that the disease is contagious. For it is on:y in those parts of the Atlantic states where every vestige of a tree showing the Yellows is immediately destroyed, that we have seen a return of the normal health and longevity of the tree.*

Cause of the Yellows. No writer has yet.ventured to assign a theory, supported by any facts, which would explain the cause of this malady. We therefore advance our opinion with some diffidence, but yet not without much confidence in its truth.

We believe the malady called the Yellows to be a constitutional taint existing in many American varieties of the peach, and pro. duced in the first place by bad cultivation, and the consequent

* The following extract from some remarks on the Yellows by that careful observer, Noyes Durling. Fsq., of New-Haven, Ct., we recommend as worthy the attention of those who ihink the disease contagions. They do not seem to indicate that the disease spreads from a given point of contagion, but breaks out in spots. It is clear, to our mind, that in this and hundreds of other similar cases the disease was inherent in the trees, they being the seedlings of diseased parents.

"Whien the discase cummences in a garden or orchard containing a considerable number of trees it dues not aliack all at onre. It breaks out in putches which are progressively enlarred, till evenually all the irees become victims to the malady. Thus in an orchard of two and a half acres, all the trees were hea!hy in 1827. The mext year two trees on the wsist side of the orchard, within a rod of each ullar, tock the lelluws. In 1329 , six trees on the cast sile of the orchard were ataclie.l; five of them standing withn a circle of four rods diameter.

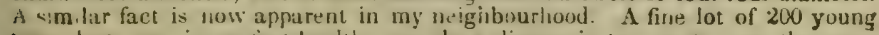
tree-, last year in periect liralth, now sluw dis:ase in tw, spots near the opposite ends of the lot, taving exactly six diseased trees in ea th patch contiguous to each other; while all the other trees are free from any other marks of disease." 
exhaustion arising from successive over-crops. Afterwarls it has been ustablished and perpetuated by sowing the seeds of the enfeebled tree either io o'btain varistios or f. $1^{\circ}$ stculs.

Let us look for a moment into the history of the peach eslture in the United States. For alınost an hundred yea:s after this tree was introduced into this country it was largely cultivated, especially in Virginia, Maryland, and New.Jersey, as we have already stated, in perfect freedoin from such disease, and with the least possible care. The great natural fertility of the soil was unexhausted, and the land occupied by orcharils iwas seldom or never cropped. Most of the soil of these states, however, though at first naturally rick. was light and sandy, and in course of time became comparatively exhausted. 'The peach tree, always productive to an excess in this climate, in the impoverished soil was no longer able to recruit its energies by an. nual growth, and gradually became more and more enfeebled and short-lived. About 1800 , or a few years before, attention was attracted in the neighborhood of Philadelphia to the sudden decay and death of the orchards without sudden cause. From Philadelphia and Delaware the disease gradually extended to New-Jersey, where, in 1814 , it was so prevalent as to destroy a considerable part of all the orchards. A bout three or four years later it appeared on the banks of the Hudson, (or from 1812 to 1815, gradually, and slowly, extending northwarl and westwart, to the remainder of the state. Its progress to Connecticut was taking place at the same time, a few trees here and there showing the disease until it became well known, (though not yet generally prevalent,) throughout most of the warmer parts of New-England.

It should be here remarked that, though the disease had been considerably noticed in the Maryland and Middle States, previously, yet it was by no means general until about the close of the last war. At this time wheat and other grain crops bore very high prices, and the failing fertility of the peach orchard soils of those states was suddenly still more lowered by a heavy system of cropping between the trees, without returning any thing to the soil. Still the peach was planted, produced a few heavy crops, and declined, from sheer feebleness and want of sustenance. As it wis the custom with many orchardists to raise their own seedling trees, and as alınost all nurserymen gathered the stones indiscriminately for stocks, it is evident that the con. stitutional debility of the parent tree would naturally be inherit. ed to a greater or less degree by the seedlings. Still the system of allowing the tree to exhaust itself by heavy and repeated crops in a light soil was adhered to, and generation after generation of seedlings, each more enfeebled than the former, at last produced a completely sickly and feeble stock of peach trees in ihose districts. 
The great abundance of this fruit caused it to find its way, more or less into all the markets on the sea-coast. The stonis of the enfeebled southern trees were thus carried north, and, being es. teemed by many better than those of home growth, were every where more or less planted. They brought with thern the cn. feebled and tainted constitution derive f from the parent stock. They reproduced almost al ways the same disease in the new soil and thus, little by little, the Yollows spread from its original neighborhood, below Philadelphia, to the whole northern and eastern sections of the Union. At this moment it is slowly, but gradually moving west; though the rich and deep soils of the western alluvial hottoms will, perhaps, for a considerable time, even without care, overpower the original taint of the trees and stones receiver fiom the east.

Let us now look a little more closely into the nature of this enfeebled state of the peach tree, which we cal! the Yellows.

Every good gardener well knows that if he desires to raise a healthy and vigornus seedling plant, he must solect the seed from a parent plant that is itself decidedly healthy. Lindley justly and concisely remarks, "all seeris ivill not equally pro. duce vigorous seedlines; but the bealthiness of the new plant will correspond with that of the seed from which it sprang. For this reason it is not sufficient to sow a seed to oblain a given plant; but in all cases when any importanc? is attached to the result, the plumpest and healthiest seeds should be solected, if the greatest vigor is roquired in the seedling, and fireble or less perfectly formed sceds, when it is desirable to check natural luxuriance."**

A rain, Dr. Van Mnns, whose experience in raising serdling fruit tree's was more extensive than that of any oth $\cdot r$ man,.de. clares it as his opinion that the more frcquently a tree is repro. duecd continuously from seed, the more feeble and short-lived is the seedling produced.

Still more, we all know that certain peculiarities of constitution, or habit, ean be propagated by rrafting, by slips. and "ven by seeds. Thus the viriegaled follage, which is a disease of some sorts, is propragat $d$ for ever by budding, and the disposition to mildew of some kind: of peaches, is continued almost always in the seedlings. That the peach trec is peculiarly constant in any constitutional variation, the Necturine is a well known prouf. That fruit tree is only an accidental variety of the peach, and yet it is continually reproduced with a smooth skin from seed.

Is it $\mathrm{n} \cdot \mathrm{t}$ evident, from these premises, that the constant sonving of the seeds of an enfecbled slock of peaches would naturally prociuce $a$ sickly and discased race of trees. 'I he seedlings 
will at first, often appear healthy, when the parent had been only partially diseased, but the malady will sooner or late show itsclt, and especially when the tree is allowed to produce an over-crop.

That poor soil, and over-bearing, will produce great debility in any fruit tree, is too evident to need much illustration. Even the apple, that hardiest orchard tree, requires a whole year to recover from the exhaustion of its powers caused by a full crop. The great natural luxuriance of the peach enables it to lay in new fruit buds while the branches are still loaded with fruit, and thus, except in strong soil, if left to itself it is soon enfeebled.*

There are some facts, in our every day observation, which may be adduced in proof of this theory. In the first place, the varieties of this tree always most subject to this disease are the yellow peaches; and they, it is well known, also prorluce the heaviest crops. More than nine-tenths of the victims, when the disease first appeared, were the yellow fleshed peaches. On the oth-1 hand, the white fleshed kinds (those white and red ex. ternally) are much more rarely attacked; in some parts of the country never. They are generally less vigourous, and bear more inoderate crops. And it is well worth remarkine that cer. tain fine old sorts, the ends of the branches of which have a peculiar, mildewed appearaner, (such as the old Red Rareripe, the Early Aune, \&c., ) which scems to check the growth without im. palring the health, are rarely, if ever attacked by the Yellows. Slow growing, and moderately productive sorts, like the Nut. mer peaches, are alinost entirely exempt. We know an orchard in the adjoining county, where every trec has gradually died with the Vellows, except one tree which stood in the centre. It is the Red Nutmer, and is still in full vigour. It is certainly true that these soris iften decay and suddenly die, but we be. lieve chiefly from the neglect which allows thein to fall a prey to the Peach Borer. Indeed the frequency with which the Borer has been confounded with the Yellows by ignorant obscrvers, renders it much more difficult to arrive at any corret conclu. sions respeting the contugious nature of the latter disease.

It may be said, in objection to these vinws, that a disease which. is only an enfeeblement of the constitution of a tree, would not be sufficient to alter so much its whole nature and duration as the Yellows has done that of the peach. The answer to this is, that the dubility profued in a single generation of trees, proba. b) would not have led to such effects, or to any settled form of constitutional disease. But it must be borne in mind that the same barl management is to a great extent sooing on to this day, the whule country over. Every year, in the month of August.

\footnotetext{
" Th: mikerably enfeebled state of sume kinds of ptars on the sea-const, arising grom usisilathe clims e und the continual propuga:lun ty graftiug from tho same iebilitated stock, is ouly a fair paralle! to the Yellows in the peach tree.
} 
the season of early peaches, thousands of bushels of frutt, show . ing the infallible symptoms of the Yellows-a spotted skin, \&c.. are exposed at d sold in the markets of New.York, Philadelphia and Boston. Every year more or less of the stones of these peaches are planted, to produce, in their turn, a generation of diseased irees, and every successive generation is even mord feeble and sickly than the last! Even in the north, so feeble has the stock become in many places, that an excessive crop oi fine fruit is but too frequently followed by the Yellows. In this total absence of proper care in the selection both of the seed and the trees, followed by equal negligence of good cultivation, is it surprising that the peach has become a tree comparatively difficult to preserve, and proverbially short-lived!

Abroad, it is well known that the peach is always subjected to a regular system of pruning, and is never allowed to produce an over-crop. It is not a litile singular, both that the Yellows should never have originated there, and that, not:vithstanding the great number of American varieties of this fruit that have been repeatedly sent to England and are now growing there, the disease has never extended itself, or been communicated to other trees, or even been recognized by English or French horticulturists. We must confess these facts appear to us strong proofs in favour of our opinion as to the nature and origin of the malady.

Remedy for the Yellows. It may seem to many persons a difficult task to rid ourselves of so wide spread a malady as this, yet we are confident that a little perseverance and care will certainly accomplish it. In the present uncertainty with regard to its contagious nature, it is much the wisest course to reject "the benefit of the doubt," and act upon the principle that it is so. We know at the present moment several gardens, where the trees are maintained in good health by immediately rooting out and destroying every tree as soon as it shows marked symptoms oi the malady.

1. We would therefore commence by exterminating, root and branch, every tree which has the Yellows. And another tree should not be planted in the same spot without a lapse of several years, or a thorough removal of the soil.

2. The utmost care should be taken to select seeds for planting from perfectly healthy trees. Nurserymen to secure this should gather them from the latest ripening varieties, or procure them from districts of the country where the disease is not known.

3. So far we have aimed only at procuring a healthy stock of rees. The most important matter remains to be stated-hero to preserve them in a healthy state.

The answer to this is emphatically as follows; pursue steaaity, from the first bearing year, the shorlening.in system of pruning, already expiained. This will at once secure your trees against 
the pussibility of over-bearing, and its consequences, and nrain tains them in vigour and productiveness for a long time.* It will, in short, effectually prevent the Y'ellows where it does not already exist in the tree. To whoever will follow these pre. cautions, pursue this mode of cultivation, and adopt at the same time the remedy for the Borer, alres.dy surgested, we will confidently insure healthy, vigourous, long-lived trees, and the finest fruit. Will any reasonable man say that so fine a fruit as the peach does not fully merit them?

Whether the system of shortening-in, and careful culture, wil, prevent the breaking out of the Yelluws when constitutionally latent in the tree we will not yet underake to say. A fi'w more experiments will prove this. In slignt cases of the discase we believe that it may. Of one thing, huwever, we are certain; it has hitherto failed entirely to reclaim trees in which the malarly had once broken out. Neither do we know of any well attested case of its cure, after this stnge, by any means what. ever.t Suclı cases have indeed been reported to us, and pub lished in the journals, but, where investigated, they have proved to have been trees suffering by the effects of the borcs only.

A planter of peach trees must, even with care, expect to ser. a few cases of Yellows occasionally appear. The malady in too widely extended to be immediately vanquished. Occasion. ally, trees having the constitutional taint will sliow themselves where least suspected, but when the peach is once properly cultivated, these will every day become more rare until the ori. ginal health and longevity of this fruit tree is again established.

The Curl is the name commonly given to a malady which often attacks the leaves of the peach tree. It usually appears in the months of May or June. 'I'he leaves curl up, become thickened and swollen, with hollows on the under, and reddish swellings on the upper side, and finally, after two or three weeks, fall off. They are then succeeded lyy a new, and healthy crop of foliage. This malady is eaused by the punctures of very minute aphides, or plant lice, (Aphis l'ersice?), which attack the under side of the leaves. Although it does not appear materially to injure either the tree (or the crop,) yet it greatly dis. figures it for a time. In orchards, perhaps few persons will

* The following remarks, directly in point, are from Loudon's last work. "The effect of shortening the shouts of the peach is not merely to throw more * p into the fruit, but to adil vigour to the tree generally, by increasing the puwea of the roots relatively to the branches. The peach being a short-lived tree, $u$ kas been justly remarked by Mr. 'Thompson, were it allowed to expend all its acrumulated sap every year, th would soon exhaust uself and die of old age." Suburban Horticulturist.

+ All the specific applications to the ront of such substances as salt, ley, brine, altpetre, urine, \&c., recommended for this disease, are founded on the ir good effects when applied agninst the borer. They have not bee found of ary value for the Yellows. 
trouble themselves to destroy the insect, but in garduns, it is much better 10 do so. A mixture of whale oil soap or strong noff soap and water, with some tobacco stems boiled in it, and the whole applied to the branches from below, with a syringe or garden engine, will soon rid the tree of the iusects for one or more years. It should be done when the leaves are a third grown, and will seldom need repeating the same scason.

VARIFTIES. The variety of fine peaches cultivated abroan is about fifty ; and half this number embraces all that are highly esteemed, and generally cultivated in Europe. Innumerable scerilings have been produced in this country, and some of them are of the highest excellence. One or two of our nurserymen's cataIngues enumerate over an hundred kinds, chiefly of native origin. Half of these are second rate sorts, or merely lncal varie. tie's of no superiour merit, and other's are new names for old sorts or seedling; newly produced, and differing in no essential respects from old varieties. It is very desirable to reduce the collection of praches to reasonable limits, because, as this fruit neithes offers the same variety of flavour, or the extent of sfason as the apple and pear, a moderate number of the choicest kinds, ripening from the earliest to the latest is in every respect better than a great variety, many of which must necessarily be scrond rate.

It is worthy of remark that most of our American varieties, of the first quality, have proved second rate in England. 'This is owing to the comparative want of sun and heat in their climate. Indeed our finest late peaches will not ripen at all except under griass, and the early varieties are much later than with us. On the other hand many of the hest European sorts are finer here than in England, and we have lately endeavoured to introduce. all of the foreign sorts of high quality, both with the view of improving our collection, and because we believe they are gene. rally purer and healthier in constitution than many of our own native kinds.

In the description of peaches and nectarines the form, and outlines, of many kinds are so nearly similar that we are obliged to resort to other characteristics to distinguish the varieties. 'The two most natural classes into which the kinds of this fruit are livided, are free-stones, and cling-stones, (melters and pavies, uf the English;) the flesh of the former parting freely frorn the stone, that of the latter adhering.

Next to this the strongest natural distinction is found in the leaves of the peach. At the base of the leaves of certain kinds are always found small glands, either round and regular, ur ob. Inng and irregular, while the leaves of certain other kinds rave no grlands, but are more deeply eut or scrrated on the margin. These peculiarities of the foliage are consiant, and they und us greatly in recognizing a variety by forming three di stinct 40 

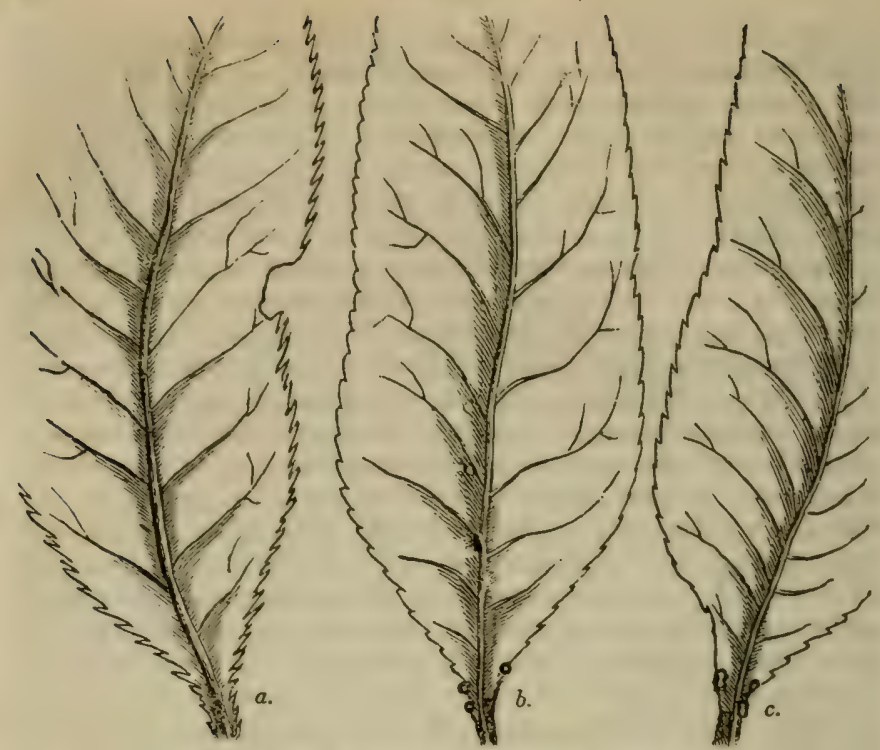

Fig. 211. Characters in the leaves of peaches.

classes, viz. 1. Leaves serrated and without glands, Fig. $211, a$. 2. Leaves with small round, or glohose glands; $b$. 3. Leaves with large irregular, reniform glands; $c$.

This distinction of leaves is valuable, because it not only as. sists us when we have the fruit before us, but it may be referred to, for the sake of verifying an opinion, at any time during the season of foliage.

There is also another class of characteristics to be found in the blossoms which is constant and valuable; though not so much so as that of the leaves, because it can only be referred to for a few days in the spring. The blossoms afford two well marked sub-divisions; 1st, Large flowers, always red in the centre, and pale at the margin; $2 d$, small flowers, tinged with dark at the margin.*

The most des:able peaches for market growers in this country are very carly, and very late kinds. These command double the price in market of kinds ripening at the middle season. For New. England, and the north, only the earliest kinds are desirable, as the late ones seldorn mature well.

We shall divide peaches into three classes. 1. Freestone Peaches with pale Resh. 2. Freestone peaches with deep yellow flesh. 3. Clingstone Peaches.

- Lindipy makes a third division, embracing a few sorts with blossoms of an anternediate size. But it is of no practical value, as any doubt as to whicb of the two divisions any blossum helungs is immediately set at res: by the calour of the blossom. 
Elass I. Freestone Peaches, with pale Resh.

\section{Acton Scotr. Lind. Thomp.}

The Acton Scott, an English peach, raisnd by Mr. Kright, is one of his cross bred seedlings, between the Noblesse and the Red Nutmeg. It is an excellent early fruit, and will thrive and ripen well at the north.

Leaves with globose glands. Fruit of medium size, rather narrow and depressed at the top, with a shallow suture. Skin rather woolly, pale yellowish-white, with a marbled, bright red cheek. Flesh pale quite to the stone, melting, sugary and rich, with sometimes a slight bitter flavour. Middle of August. Flowers large.

\section{Astor. Floy.}

An American peach, which originated in New.York, twenty. five years ago. It is good, but hardly first rate.

Leaves with glohose glands. Fruit large; rather flattened, or broad, and slightly sunk at the top, suture well marked. Skin pale yellowish-white, with a deep red cheek. Stone strall. Flesh melting, very juicy, sweet, and of excellent fa. vour. Ripens the last week in August. Flowers large.

\section{Bellegarde. $\S$ O. Duh. Lind. Thomp.}

\begin{tabular}{|c|c|c|}
\hline & \multicolumn{2}{|c|}{ Galande. isois and the } \\
\hline Viclette Hâtivo. & of many & Brentford Mignonne. \\
\hline Violette Hitive Grosse. & English & Ronald's Mignomne. \\
\hline French Royal George. & gardens. & Larye Viulet. \\
\hline Early Roval George. ? incorrect & of $\mathrm{son}$ & \\
\hline Magdalen. & gar & \\
\hline
\end{tabular}

This very excelent French peach is the one most highly esteemnd by the Montreuil growers, who supply the Paris markets, and it is equally valued by the English. It is also one of the handsomest and most delicious fruits here.

Leaves with globnse glands. Fruit large, round and regular, the suture shallow, the top slightly hollowed, and having a little projecting point. Skin pale yellowish-green, with a rich red cheek, often streaked with darker purple. Flesh slightly mark ed with red at the stone, a little firm: out very melting. juicy, rich and high flavoured. Stone rather large. End of August, and first of September. Flowers small. 


\section{Brevoort. $\oint$}

\section{Brevoort's Morris.}

\section{Brevourt's Seedling Melter. Floy.}

One of the richest and most delicious of Amorican peaches, and one of the favourite sorts for garden cultivation. It was raised some years ago by Henry Brevoort, Esq., of New.York. Mr. Floy describes this, in his edition of Lindley, as a sinall fruit. It is almost always large on the Hudson river, and bears regular, moderate crops.

Leaves with uniform glands. Fruit medium or large, round and rather broad, with a distinct suture, deep at the iop. Skin pale yellowish-white, often a little dingy, with a bright red cheek. Flesh rather firm, slightly red at the stone, rich, sugary and high flavoured. First of September. Flowers sinall.

\section{Belle de Vitry. Duh. Lind. Thomp.}

Admirable Tardive.

Bellis. Mill.

This is not the Belle de Vitry of most of our gardens, which is the Enrly Admirable. It is quite distinct also, fiom the Late Admirable; but is the Polle de Vitry, described by Duhamel, and is a very firm fleshed and excellent French variety, little known in this country.

Leaves serrated, without glands. Fruit midlle size, rather broad, with a deep suture, the top depressed. Skin pale yellowish-white, tinged and marbled with bright and dull red. Flesh rather firm, red at the stone, melting, juicy and rich. Ripens here the last of September. Flowers small.

\section{Barrington. P. Mag. Thomp. Lind.}

Buckingham Mignonne.

Culunel Ausleys.

A handsome, very fine, and very hardy English peach. The tree is vigorous and healthy. The fruit ripens at the medium season, about a week after the Royal George.

Leaves with globose glands. Fruit large, roundish, inclining to ovate, and rather pointed at the top, with a moderate suture on one side. Skin pale yellowish-white, with a deep red, mar. bled elseek. Flesh but slightly tinged with red at the stone melting, juicy, very rich, and of the first quality. Stone ruir sed, dark brown. Beginning of September. Flowers la rge. 


\section{Chistos.}

A native variety, of second rate flavor $x$.

Leaves with globose glands. Fruit of medium size, round. ish, a little depressed at the top, but nearly without suture. Skin pale yellowish.white, with a red cheek marked by broken stripes of dull red. Flesh scarcely stained at the stone, juicy and good. Last of August. Flowers large.

\section{Cole's Earlj Rev}

A now American peach, which is a very fruitful and excellent variety, for market culture.

Leaves with globose glands. Frait of medium size, round. ish, with but little suture. Skin pale in the shade, but nearly a!l covered with red, becoming dark red on the s'inny side. Flesh melting, juicy, rich, and very sprightly. Beginning, to the middle of August. Flowers small.

\section{Cooledge's Favourite. $\oint$ Man. Ken.}

\section{Cooledge's Early Red Rareripe}

This most popular early New England peach, was rais? from seed by Mr. J. Cooledge, of Watertown, Mass. It is unusually productive, and a very bright coloured, handsome peach, of excellent quality; and its hardiness renders it valu able at the north.

Leaves with globose glands. Fruit large, roundish (the suture prominent at the top only), but rather the largest on one side. Skin clear smooth white, with a fine crimson mottled cheek. Flesh very melting and juicy, with a rich, sweet, and high flavour. Middle of August. Flowers small.

\section{Chancerlor. Mill. Lind. Thomp.}

Chancellière, var. O. Duh.

Noisette.

Late Chancellor.
Stewart's Late Galande Edgar's Late Melting.

The Chancellor is a celebrated French peach, long cultivated and highly esteemed abroad. It is said $t=$ have been originated by M. de Seguier, of Paris, then Chancellor of France.

Leaves with reniform glands. Fruit large, oval, with a well marked suture. Skin pale yellowish-white, with a dark crimsor. cheek. Flesh very deep red next the stone, melting, and pos. sessing a rich, vinous flavour. Stune oblong. Middle of Sep tember. Flowers small. 


\section{Double Montagne. $\oint$ Lind. Thomp.}

Double Mountain

Montagne.

Montauban.

A ingh flavoured and beautiful peach, much resembling the Noblesse. It is of French origin, and is a favourite variety with the English gardeners. We think it one of the finest peaches in this climate.

Leaves serrated, without glands. Fruit of medium size, roundish, but somewhat narrower at the top. Skin pale greenish-white, with a soft-red cheek, which is marbled with darker red at maturity. Flesh white to the stone, very delicate and melting, with a plentiful and high flavoured juice. Stone ovate and rugged. Middle of August. Flowers large.

\section{Druid Hill. $\oint$}

This new freestone peach, of splendid size, high flavour, and very late maturity, we think will prove one of the greatest acquisitions to our gardens. It was originated about six years ago, by Lloyd N. Rogers, Esq., of Druid Hill, near Baltimore, and we named the variety after his country seat, where may be four d one of the largest collections, not only of peaches, but all other fine fruits, in Maryland. We know no other late freestune variety which equals it in flavour and size. 'The tree is unusually vigorous, the shoots and leaves very large, and it beurs abundantly. The very late season of its maturity renders it valuable, as most of the luscious sorts are then gone.

Leaves with globose glands. Fruit very large, roundish, the cavity at the stalk rather narrow, the suture very slight, and the swollen point distinct, but scarcely prominent. Skin pale greenish-white, clouded with red on the sunny side. Flesh greenish-white, purple at the stone, very juicy, and melting, with an exceedingly rich, high virous flavour. Stone long and rather compressed, much furrowed. Ripens from the 20th of Septer

\section{Early Anne. Lind. Thomp.}

Anne. Lang. Forsyth.

Green Nutmeg.

The Early Anne is an old and familiar English sort. It is .he first peach of any value that ripens, the Red and White Nutmegs heing tos small, and of indifferent flavour; and the Early Anne, itself, is so inferior to the Early Tillotson (which 
ripens at the sime time), that it will soon searecly be collinated, except by amuteurs. 'The tree is of slender growth.

Leaves scrited, without glands. Fruit ruther small, round. Shin white, with a faint tinge of red next the sun. Flesh white to the stone, soft, melting, swcet, and of pleasint flavour. Last of July, and first of Augrust. Flowers large, nearly white.

\section{Ehrly Trllotson. $\oint$}

The Early Tillotson is considered by many persons one of the best of the very carly freestone peaches. It is a variety from central New York, first introduesd to notice by our fricnd, J. J. Thomas, of Macidon, Wayne county. It is considernd a native of that part of the State.

It ripens early in August, about the same time as the Early Iork, Royul Goorge, and the other very choice carly kinds, and only a few days after the Karly Anne. It is much higher fiavouied than any peach that ripens, except the true Farly Yori, and as a garden variety, is entitled to considerable favour. The tree grows slowly when young, but frecly and more vigorously afterwards, and is a great and constant bearer. It is very hiridy, but a good deal inclined to mildew at the end of the shoots-a drawback to its culture.

Leaves deeply serratcd, without glands. Fruit of medium size, round. Skin nearly covered with red, the ground-colour -pale yellowish-white, being thickly dottel with red, and the exposed cheek being a dark red. Flesh whitish, but red at the stone, to which, though a freestone, it partially adheres: melting, juicy, with a rich, highly excollent flavour. It ripens the middle of August. Flowers small.

\section{Early York.}

\section{Large Early York.}

The Finly Fork has long been the most popular of ealy peaches in this country. It is at least a week carlier than the (true) Royal George, more melting and juicy, though not quite so rich, and deserves a place in every garden. In unfavourable soil, the ends of the branches are a little liable to mildew; but the tree is very hardy and productive. There are one or two newer senllings raised from this, and bearing the sane name, in New Jirs: y, which are rather more thrifiy for the orchard, but do mot priss ss the high flavour of the old siad. 'They are easily known from it by the absence of crlands in the leaves and by the lutge fowers of the true sort. It is quite distinct from the Red Rareripo, which is large, broader, decply marke. with is suture, later in ripening and richer flavoured." 
Leaves serrated, without glands. Fruit of medium sizes coundish, inclining a little to ovate, with a slight suture unly. Skin very thin, pale red thickly doited over a palc gromul, in the shadi, but quite dark red in the sun. Flesh greenish white, remarkably tender and melting, full of rich, sprightly juice. Ripens about the 18th of August. Flowers large.

\section{Early Newington Freestone. $\oint$}

$\left.\begin{array}{l}\text { Newington Peach, } \\ \text { Early Newington, }\end{array}\right\}$ of many $A$ m. garlens.

This is a large, and excredingly high-flavoured, early peach; mdeed, we: consider it without a superior, at its season. It ic quite distinct from the other Newingtons, which are clings, and rather late, while this is early, and generally parts from the stone, though it frequently happens that some of the fruit on the same tree adheres partially, or wholly to the stone; and this peculiarity (common, so far as we know, to but one other hind) is one of its constant characteristics. It has been cultivated here and disseminated, fir the last twenty year's, and we sup. pose it to be an American variety. The tree is only a moderate bearer. Leaves with globose glards. Fruit rather large, round, with a distinct suture, and one-half the fruit always the larger. Skin pale yellowish white, dotted and streaked with rerl, the cheek a rich red. Flesh white, but red at the stone, to which many particles adhere. If not fully ripe, it has the habit of a cling. Flesh juicy, melting, with a rich vinous Aavour. Ripens directly after the Early York, about the 24th of August. Flowers small.

\section{Early Siveet Water. $\oint$ Floy. Thomp.}

Sweet Water.

Lar je American Nutmeg

A very early, and very agreeable white peach, among the best of its season, as it ripens early in August, not long after the Early Anne, and ten days or more befure the Early York. It is an American peach, raised from a stone of the Early Anne. It is so much larger and superior to the Early Anne, or any of the Nutmeg peaches, that it has almost driven them out of our gardens. The tree is thrifty and productive, with pale shoots, and nearly white blossoms.

Leaves with globose glands. Fruit of medium size, sometimes large, roundish, with a slight suture. Skin pale white, very seldom with a faint blush when fully exposed. Flesh white, slightly stained at the stone, melting, juicy, sweet, 
and of very agreeable flavour. Ripe about the 8th of August. Stone small. Flowers large.

\section{Emperor of Russia. Floy. Thomp.}

\section{Cut-Leaved.}

New Cut-Leaved.

Serrated. Unique.

A very rich and fine-flavoured peach, raised by Mr. Floy, in 1812. Its growth is slow, and its shoots are inclined to become mildewed. It is rather a shy bearer here, but is an adnirable sort in the Western States. The leaves are very deeply cut, or serrated on the edges.

Leaves serrated, without glands. Fruit large, roundish, and broad, with one-half more swollen than the other. Skin downy, dull yellowish-white, with a dark red cheek. Flesh yellowish. white, rather firm, rich and high flavoured. Last of August. Flowers small.

\section{Early Admirable. Lind. Thomp.}

Admirable.

L'Adinirable.

Belle de Vitry, (Bon Jardinier.)

A very excellent French peach, wrongly known by many in this country as the Belle de Vitry, which is a distinct variety. We find it early, and very prolific.

Leaves with globose glands. Fruit not quite round. Skin pale yellowish-white, with a lively red cheek. Flesh red next the stone, melting and juicy, with a good, rich, sweet flavour. Middle of August. Flowers' large.

\section{Favourite. Coxe.}

Favourite Red.

A capital orchard fruit, of large size, hardy and a most abundant bearer. It is a very good native peach, though not of high flavour.

Leaves with obscure globose glands, often with none. Fruit large, oblong or oval. Skin white, rather downy, much covered with red, which becomes a very dark red, when fully exposed in the sun. Flesh red at the stone, a little firm, but juicy, with a good vinous, but not rich flavour. Second week in Septem. ber. Flowers small. 


\section{Fox's Seevling.}

A good and productive late peach, a native of New. Jersey. Leaves with globose glands. Fruit round, a little compressod, cavity at the stalk narrow. Skin white with a red cheek. Flesh melting, juicy, sweet and good. Middle of September Flowers small.

\section{George the Fourth. $\S$ Floy. Lind. Thomp.}

This is certainly the most popular peach for garden culture in the United States. It is large, bears regular and abunciant crops, is of the highest flavour, and the tree is unusually hardy and vigorous, succeeding well in all parts of the country. No garden should be without it. The original tree stood, not long since, in the garden of Mr. Gill, Broad street, New.York.

Leaves large, with globose glands, often obscure. Fruit large, round, deeply divided by a broad suture, and one-half a little larger than the other. Skin pale, yellowish white, finely dotted with bright red, and deepening into a rich dark red cheek on one side. Flesh pale, marked with red at the stone (which is small), melting, very juicy, with a remarkably rich luscious flavour. Ripens the last of August. Flowers small.

\section{Grosse Mignonne. $\S$ O. Duh. Lind. Thomp.}

Royal Kensington.

Grimwood's Royal Georye.

New Royal George.

Large French Mignonne.

French Mignonne.

Swiss Mignonne.

Purple Avant.

Early Purple Avant.

Early May.

Early Vineyard.

Neil's Early Purple.

Johnson's Early Purple.

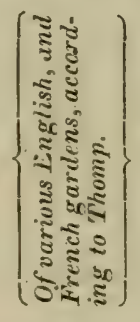

Vineuse de Fromentin

Mignonne.

Velou'és de Merlet.

Vineuse.

Pourprés de Normandie.

Belle Beauté.

Belle Bausse.

La Royal (of some.)

Pourprée Hâtive (of some.)

Ronald's Seedling Galande.

Royal Suvereign.

Superb Royal.

The Grosse Mignonne is certainly the "world renowned " of peaches. In France, its native country, in England, in America, in short everywhere, it is esteemed as one of the most delicious of varieties. It is a grood and regular bearer, a large and handsome fiuit, is a favourite for those who have to grow peaches under glass, and ripens the best crops even in a rather unfavourable climate, like that of Boston. The great number of names hy which it is known abrond (and we have not quoted all) proves the universality of its cultivation.

Leaves with globose glands. Fruit large roundish, always *omewhat depressed and marked with a hollow suture at the 
top. Skin pale greenish yellow, mottled with red, and having a purplish red cheek. Flesh yellowish white, marked with red at the stone, melting, juicy, with a very rich, high, visous tha vour. Stcne small, and very rough. Middle of August, betore the Royal George. Flowers large.

\section{Haines' Early Red.}

An early peach, newly originated in INew-Jersey, of very fine flavour, and so hardy and productive as to be a popular orchard fruit.

Leaves with globose glands, fruit of medium size, round, depressed at the top, with a well-marked suture extending round the fruit, one half larger than the other. Skin pale white marked with red, and nearly covered with deep red. Flesh greenish white, very juicy, meling, sweet and well flavoured. Middle of August. Flowers small.

\section{Kenrick's Heath. Ken.}

Freestone Heath.

A large, showy, oblong peach, often growing to the largest size, and a very hardy tree, but the quality of the fruit is only second rate. 'This sort, which is a native of New-England, is vigorous, and bears large crops. It is quite distinct from the celebrated Heath Cling.

Leaves with reniform glands. Fruit very large, oblong, with a slight suture, and a small swollen point at the top. Skin pale greenish white, with a purplish red cheek. Flesh greenish white, deep red at the stone, a little coarse, melting, quite juicy, with a pleasant sub-acid flavour. Middle of September. Flowers small.

\section{Late Admirabe. $\S$ Lind. Thomp.}

Royale. O. Duh
La Royale.
Pèche Royale.
Bourdine.
Boudin.
Narbonne.

Têton de Verus. French Bourdine Judd's Melting. Mutteux's.

Pourprée Tardive. Z incorrectly Late Purple. $\quad\}$ of some.

"The Late Admirable," says Mr. Thompson, "is one of the very best of late peaches, and ought to be in every collection," an opinion in which we fully concur. It is one of those delicious sorts that, originating a long time ago in France, have received the approval of the best cultivators everywhere. It is hardy and productive in this climate. 
Leaves with globose glands. Fruit very large, roundish, in. ciining to oval, with a bold suture dividing the fruit pretty deeply all round, and a small, acute, swollen point at the top. Skin pale yellowish green, with a pale red cheek, marbled with darker red. Flesh greenish white, but red at the stone, very juicy, melting, and of delicate, exquisite flavour. Middie of September. Flowers small.

\section{La Grange. $\oint$}

The La Grange is a new white freestone peach, of very late maturity, large size, and fine flavour. It was originated from seed five or six years ago, in the garden of Mr. John Hulse, Burlington, New-Jersey.

Its late period of maturity, its colour, its productiveness, and size, have already given it quite a reputation among the extensive growers of New-Jersey, and it is undoubtedly a most valuable fiuit, not only for the rable but for preserving at the most desirable period for this purpose-late in the season. Its flavour is remarkably rich and delicious, equalling, in this re. spect, almost any peach of its season of maturity. It was first brought into notice and disseminated by Mr. Thomas Hancock.

Leaves with reniform glands. Fruit large, oblong, shaped somewhat like the Heath Cling. Skin greenish white, with occasionally sume red on the sunny side. Flesh pale, juicy, melting, very rich, sweet, high flavoured and delicious. Last of September, and beginning of Oetober. Flowers small.

\section{MorRis's RED RARERIPE.}

Morris Red.

$\left.\begin{array}{l}\text { Red Rareripe. } \\ \text { Large Red Rareripe. }\end{array}\right\}$ of some

This very popular and well-known American peach, has the reputation of having originally been disseminated from the garden of Robert Morris, Esq., of Philadelphia. It is everywhere justly esteemed for its acknowledged good flavour, beauty, and productiveness. Mr. Kenrick, and some other American writ. ers, have erred in supposing it synonymous with the Grosse Mignonne, which is quite difierent, both in the colour of its skin and fiesh as well as in its flavour and blossoms.

Leaves with small glohose glands. Fruit large, roundish, a little depressed at the top, with a moderately well-maıked suture. Skin fune pale greenish white, a little dotted, and with a lively, ricis red cheek. Flesh pale, greenish white, quite red at the stone, very melting and juicy, with a sweet and rich flavour Last of August. Flower's small. 


\section{Morris's White Rareripe. $\zeta$}

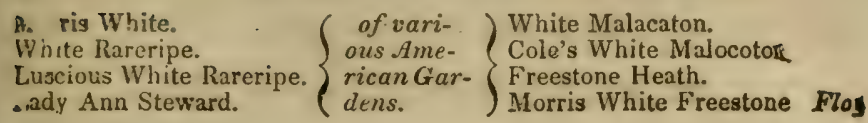

Morris's White Rareripe, a native, is the most popular and well. known white peach, and is everywhere cultivated in this coun. try, either under this, or some of the other names quoted above. It is a rich fruit in a warm climate, but is not quite so high lavoured at the north or east. The tree is vigorous and sealthy, and bears fair crops.

Ledves with renıform glands. Fiuit rather large, oval; su. urre oniy of moderate depth, swollen point small. Skin rather úwny, gleenish white on all sides, at first, but white with a creamy tint when fully ripe; and when fully exposed, sometimes with a slightly purple cheek. Flesh white to the stone, a little firm, melting, juicy, sweet and rich. Middle of September. Flowers small.

\section{Morrisania Pound. Thomp.}

Hoffman's Pound. Floy. Morrison's Pound.

vory large and late variety, originated many years ago,

Martin Hoffiman, Esq., but first disseminated from the garden of Governor Morris, of Morrisania, near New York. It is a good fruit, but its place has been taken, of late, by other more popular sorts.

Leaves with globose glands. Fruit very large and heavy, nearly round. Skin, dull greenish white, with a brownish red cheek. Flesh, pale yellow, firm, juicy, sugary, and rich in fiavour. Ripens the middle and last of September. Flowers sinall.

31. Madeleine de Courson. Thomp. Lelieur. Lind.

Red Magdalen (of Ailler).

True Red Magdalen

French Magdalen.
Madeleine Rouge, O. Duh

Rouge Paysanne.

The Red Magdalen, of Courson, is a favourite old French peach, very little known in this country; the Red Magdalen of many of our gardens being either a spurious sort, or the Royal George. It is an excellent, productive peach, hardy, and worthy of more general cultivation.

Leaves serrated, without glands. Fruit of medium size, or rather below it, round, flattened, with a deep suture on one side. 
Skin pale yellowish white, with a lively red cheek. Flesk white, slightly red at the stone, juicy, and melting, with a rich vinous flavour. Middle and last of August. Flowers large

\section{Malta. $\S$ Lind. Thomp. P. Mag.}

Póche Malte. O. Duh. Malte de Normandie.
Italian.

Belle de Paris.

A most delicious, old European peach, of unsurpassable fla. vour. The tree is not a great bearer, but it is hardy and long lived, and richly deserves a place in every garden. There is a spurious sort sold under this name in the United States, which is easily known by its globose glands. The fruit of the Malta keeps well after being gathered.

Leaves serrated, without glands. Fruit of rather large size, roundish, flattened, with a broad, shallow suture, on one side. Skin pale, dull green, marked on the sunny side with broken spots, and blotches of dull purple. Flesh greenish, with a little dark red at the stone, very juicy and melting, with a pecu. liarly rich, vinous, piquant, and delicious flavour. Last of $\mathrm{Au}$. rust. Flowers large.

The trees of the true Malta are remarkably free from tho velows, in this country, aftording another proof of our theory regarding this disease, as they bear only moderate and regu. ar crops.

33. Nutueg, Red. Mill. Lind. Thomp.

Avant Rouge. O. Duh.

Avant Pèche de Troyes.

Red Avant.
Brown Nutmeg.

Early Red Nutmeg.

The Red Nutmeg is a very small and inferior peach, which tas long been cultivated, solely on account of its ear!iness. It is now seldom seen in our gardens, being abandoned for better sorts. Is is desirable, however, in a compiete collection. Both this and the following are European varieties. The tree grows slowly, and is of dwarf habit.

Leaves small, with reniform glands. Fruit small, roundish, with a distinct suture, terminating in a smali, round, swollen noint at the top. Skin pale yellow, with a bright, rich red cheek. Flesh yellowish white, red at the stone, with a sweet and rather pleasant flavour. Middle and last of July. Flow ers large. 
31. Nutneg, White. Mill. Lind. Thomp.

\author{
Avant Blanche. O. Duh. \\ White Avant. \\ Early White Nutmeg.
}

The Wlite Nutmeg resembles the foregoing in its genera. habit, being dwarfish, and of slender growth. It is the small. est of peaches, the flavour is inferior, and it is only esteemed by curious amateurs as ripening a fiew days earlier than any other variety.

Leaves serrated, without glands. Fruit very small, rather oval, with a deep suture extending a little more than half round. Skin white, or rarely with a pale blush. Flesh white to the stone, with a sweet and slightly musky, pleasant tlavour. Ripens about the 10th or 15th of July. Flowcrs largere

\title{
35. Noblesse. $\$$ Lang. T.ind Thomp.
}

\section{Vanguard. \\ Mellish's Favourite \\ Luid Montague's Noblesse}

An English peach of the highest reputation, and which, in this country, is esteemed wherever known, as one of the largest, most delicious, and most valuable varieties. 'The tree is hardy and productive, and every cultivator should possess it. In England it is one of the favourite kinds for forcing and wall culture, yielding regular and abundant crops of beitutiful, pale fruit.

Leaves serrated, without glands. Fruit large, roundish oblong, a littie narrowed at the top, and tcrminated by an acute swollen point. Skin slightly downy, pale green throughout, marked on the cheek with delicate red, clouded with durker red. Flesh pale greenish white to the stone, melting, very juicy, with a very high and luscious flavour. Last of August. Eowers large.

\section{Nivette. O. Duh. Lind. Thomp. \\ Nivette Veloutée. \\ Veloutée Tardive. \\ Dorst:tshire.}

The Nivette is an excellent French variety, much resemoling ine late Admirable.

Leaves with globose glands. Fruit largei, roundsh, inclining to oval, suture shallow, and the top slighttly depressed. Skin pale green, with a lively red cheek. F'lesh pale green, but deep red at the stone, juicy, melting, and very rich. Beginning, and middle of September. Flowers small. 


\section{Oldmixon Freestone. Pim. Man.}

\section{Oldmixon Clearstone. Coxe}

A large American peach, of late maturity and rich flavour. It was, we believe, raised either from a stone of the Catherine Cling or the Oldmixon Cling, the latter having been brought to this country many years ago, by Sir John Oldinixon. It bears good crops, and is a valuable variety.

Leaves with globose glands. Fruit large, roundish, or slightly oval, one side swollen, and the suture visible only at tine top; cavity but slightly sunk at the stalk. Skin palc yellowish white, marbled with red, the cheek a deep red. Flesh white. but quite red at the stone, tender, with an excellent, rich, sugary and vinous flavour. Beginning of September. Flow. ers small.

\section{President. P. Mag. Lind. Thomp.}

One of the best of our peaches, and a capital variety. originated, several years ago, on Long Island.

Leaves with globose glands. Fruit large, roundish oval, the suture shallow. Skin very downy, pale yellowish green, with a duil red cheek. Flesh white, but deep red at the stone, very juicy, nelting, rich and high flavoured. Stone very rough. Middle of September. Flowers small.

39. Pourpré Hative. O. Duh. Thomp. Lind.

Pourprée Hâtive à Grandes Fleurs.

Early Purple.

This is the Eurly Purple of Duhamel; what is often in. correctly called the Larly Purple, being the Grosse Mignonne.

Leaves with reniform giands. Fruit of medium size, globular and depressed, and having a deep suture running across the top. Skin pale, light yellow, with a inottled purplish red check. Flesh pale, but red at the stone, melting, very juicy, with a high vinous flavour. Stone broad and rough. Middle of $\mathrm{Au}$. gust. Flowers large.

The Pourpree Hative Veritable, of the French (Early Purple, True Early Purple, of some), with globose glands and large flowers, Thompson says, is "probably nothing different froin the Grosse Mignonne." 


\section{Royal Georee. $\$$ P. Mag. Lind. Thomp.}

Early Royal George.

Millet's Mignonne.

Lockyer's Mignonne.

Griffin's Mignonne.

Superb.
Red. Magdalen.

Madeleine Rouge à Petite Fleurs.

French Chancellor. )

Early Bourdine.

Double Swalsh.
Incorrectly, of some

Few of the early peaches surpass in flavour and beauty the Royal George. It is one of the finest European varieties, and attains the highest flavour with us. The points of its shoots are a little inclined to mildew, which is entirely, in our climate, prevented by the shortening-in pruning. It is a regular and morierate bearer, and is one of the varieties indispensable to every good garden, ripening directly after the Larly York.

The peach generally cultivated as the Royal George, Early Royal George, or Red Magdalen, in almost all parts of the United States, from Salem, Mass., to Baltimore (and described by Manning), is not the true Royal George, but the Bellegarde, or Smooth-leaved Royal George, which is rather later, not so rich, and has globose glands.

Leaves serrated, without glands. Fruit above the middle size, or rather large, globular, broad and depressed, the suture d.e $p$ and broad at the top, and extending round two-thirds of the $\mathrm{f}$ uit. Skin pale, or white, thickly sprinkled with reil dots, and th check of a broad rich, decp red slightly marbled. Flesh whitish, but very red at the sinc, meling, juicy, very rich; an of the highest flivour. From the 20th the last of August. Flowers small.

\section{Red Rareripe.}

Isrce Red Rareripe, of some. Early lied Rareripe.

This remarkably fine early peach, is a very ponplar one with us, and has been cultivated for many years in this State. It strongly resembles the Royal Genrge, and we believe it an American seedling from that variety, which is however distinct, and superior in flavour.

It must be observed that this is totally different both from the Eariy York, and Morris' Red Rareripe, with which it is often cunfounded by some nurserymen. The fruit is larger, broader, and a week later than the first; and its serrated leaven, and different fluvour, separate it widely from the latter. Ends of the branches sometimes slightly mildewed.

Leavis serrated, without glands. Fruit rather large, globular, but broad, depressed, and marked with a deep broad suture, extending nearly round the whole fruit. Slkin white, mottled, 
and marked with numcrous red dots, and the cheek of a rich dark red. Flesh whitish, but red at the stone, meling, juicy, sery rich and high flavoured. Middle and list of August. Flowers small.

\section{Rareripe, Late Red. $\$$}

\section{Prince's Red Rareripe.}

This noble American fruit, the late Red Rareripe, is unques. tionably one of the very finest of all peacher, even surpassing often the Late Admirable. Its large size, and great excellence, its late maturity, and its productiveness and vigour, all unite to recommend it to universal favour. We cannot praise it too highly. The rather grayish appearance of the fruit serves to distinguish it, at first sight, from all others.

Leaves with globose glands. Fruit large and heavy, roundish oval, suture depressed only at the top, where the swollen point is distinctly sunken. Skin downy, pale grayish yellow, thickly marbled and covered with reddish spots, the cheek dull teep red, distinctly mottled with fawn-coloured specks. Flesh white, but deep red at the stone; very juicy, melting, and of an unusually rich, luscious, high flavour, not surpassed by any other peach. First to the 10th of September. Flowers small

\section{Royal Charlotte. $\oint$ Thomp.}

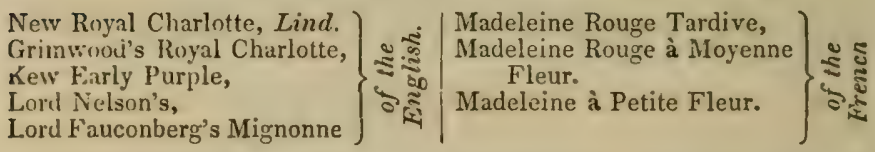

A very excellent peach, and a favorite variety with all European gardeners. Its leaves are more coarsely and decply serrated than those of other varieties.

Leaves serrated, without glands. Fruit rather large, inclining to ovate, being rather broader at the base than at the top; the suture of moderate size. Skin pale greenish white, with a deep red marbled cheek. Flesh white, but pale red at the stone, melting, juicy, rich and excellent. Beginning of Septem. ber. Flowers small.

\section{SNow. $\oint$}

The Snow peach is a remarhably fair and beautiful fruit, of American origin, which has but lately made its appearance in our gardens. The fruit and blossoms are white, and the foliage 
and wood of a light green. It is a very nardy, productive, and desirable variety.

Leaves with reniform glands. Fruit large, globular; suture fainitly marked except at the top. Skin, thin, clear beautiful white, on all sides. Flesh, white to the stone, juicy, and melt. ing, with a sweet, rich, and sprightly flavour. Beginning of September. Flowers small.

45. Van Zandt's Superb. Pom. Man.

Waxen Rareripe:

A very bright-coloured and handsome peach, originated some years ago by Mr. Van Zandt, of Flushing, Long Island. It is one of the most beautiful dessert peaches, though only of medium size, and possesses very agreeable flavour.

Leaves with globose glands. Fruit of medium size, roundish, the suture slight, but one-half the fruit larger than the other. Skin white, with a beautifully sprinkled red cheek, on a yellowish white ground, the union of the two softened by delicate dotting of bright carmine red. Flesh whitish, but tinted with red at the stone, melting, juicy, sweet, and of good flavour. Stone deeply furrowed. First of September. Flowers small.

\section{Scott's Early Red.}

Scott's Early Red is a new variety, of very excellent flavour, and a prolific bearer, which we have lately received frum New Jersey.

Leaves with obscure globose glands. Fruit of medium size, roundish, a little depressed, the suture distinctly marked, but not deep. Skin, pale greenish white, but much covered with red, which is mottled with fiwn-coloured dots. Flesh whitish very juicy, with a rich and luscious flavour. Middle of August. Flowers small.

\section{Strawberry}

Rose.

The strawberry peach we received from Mr. Thomas Han. cock, of Burlington, proprietor of one of the most respectable and extensive nurseries in New Jersey. It is esteemed one of the very finest early varieties for orchard culture in that State. It is quite distinct from the Early York.

Leaves with reniform glands. Fruit of medium size, oval, .he cavity at the stem deeply sunk, the suture extending half round. Skin marbled with deep red over almost the whole 
surface. Flesh whitish, melting, juicy, rich, and of very delk rious flavour. Middle of August. Hlowers small.

\section{Wasmington. Floy.}

\section{Washington Red Freestone Ken.}

The Washington is a handsome and very delicious peach, of American origin. It was named, and first introduced to notice, by Mr. Michael Floy, nurseryman, New York, about forty years acro. The fruit ripens late; the tree is vigorous hardy, and productive, and it is altogether a valuable variety.

Leaves with globose glands. Fruit large, broad, depressed with a broad deep suture extending nearly round it. Skin very thin, yellowish white, with a deep crimson cheek. Flesh pale yellowish white, very tender, juicy, and melting, with a sweet, rich, and luscious flavour. It often adheres slightly to the stone, which is quite small. Middle of September. Flowers small.

\section{Walter's Early. Ken.}

Walter's Early is esteemed as one of the most popular, early varieties for orchards in New Jersey, where it originated. It is remarkably well adapted to the light sandy soil of that State, bearing abundant crops of excellent fruit. At the north it is ruch inferior to the White Imperial, and the Royal George.

Leaves with globose glands. Fruit large, roundish. Slin white, with a rich red cheek. Flesh whitish, a little touched with red at the stone, melting, juicy, sweet, and of very agr reable flavour. Ripens about the 20th of August.

\section{White IMperial. $\oint$}

The White Imperial is a new early fruit, of most estirsable quality. We consider it quite a valuable variety for every garden north of New York, as its flavour is very excellent, it is extremely hardy and vigorous, it bears good and regular crops. without the bad habit of overbearing, and its fruit is uniformly excellent in all seasons.

This fine peach originated (it is believed, from the Noblesse) in the garden of David Thomas, of Cayuga county, N. Y., su long known for his skill and science as an amateur horticul. turist. It was first made known to us by his son, J. J. Thomas, of Macedon, N. Y. Leaves with globose glands. Fruit rather arge, broad, depressed, hollowed at the summit; with a wide, deep cavity at the stem; the suture moderately deep, and the frut enlarged on one of its sides. Skin yellowish white, with 
only a slight tinge of red next the sun. Flesh nearly white very melting and juicy, of a very delicate texire, and the flavour sweet and delicious. Ripens amor.g the earliest, a few days after the Early York, about the 25th of August. Flowers small.

31. White-Blossomed Incomparable. P. Man. Thomn.

White-Blossom.

Willow Peach.

This is a native fruit of se ond quality, much inferior, botk, in flavour and appearance, to the Snow peach. Its seeds very frequentiy produce the same variety. 'The flowers are white, the leaves are of a light green, and the wood pale yellow, Leaves with reniform glands. Fruit large, oval. Skin fair, white throughout. Flesh white to the stone, melting, juicy, sweet and pleasant. Beginning of September. Flowers large, white.

Class II. Free-Stone Peaches with deep yellow flesh.*

52. AвRicotée. Thomp. O. Duh.

Yellow Admirable. Admirable Jaune, O. Duh. Nois. Apricot Peach. D'Abricot.

Grosse Jaune Tardive. "Orange. (Orange Peach., Ken.) .

The Apricot-Peach (or Yellow Admirable, as it is more frequently called), is an old French variety, but little cultivated in this country, though deserving of attention in the Middle States. It ripens very late, and is thought to have a slight apricot flavour. It grows with moderate vigour, and bears abundantly.

Leaves with reniform glands. Fruit large, roundish oval, with a small suture running on one side only. Skin cleat yellow all over, or faintly touched with red next the sun. Flesh yellow, but a little red at the stone, firm, rather dry, with a sweet and agreeable flavour. Stone small. Ripens at the beginning of October. Flowers large.

* Nearly all this class are of American origin, and the Yellow Alberqe of Europe is the original type. They are not so rich as Class 1, and require our hot suminers to bring out their flavour. In a cold climate, the rcid is always prevalent. Hence they are inferior in Ergland, and at the northern limits of the peach in this country. 


\section{Bergen's Yellow. $\$$}

Bergen's Yellow is a native, we believe, of $[$ ong Island. It is very large, and of very delicious flavour. It 's darker coloured, more depressed in form, rather finer flavoured, and ripens some day's later than the Yellow Rare-Ripe, which it much resembles. It is a moderate, but good bearer. It is earlier, and much superior to the Malacoton, and its glands distinguish it, also, from that variety.

Leaves with reniform glands. Fruit large (often measuring nine irches in circumference), globular, depressed, and broad ; the suture well marked, and extending more than half round Skin deep orange, dotted with some red, and with a very broad, dark red cheek. Flesh deep yellow, melting, juicy, and of rich and luscious flavour. Ripens at the beginning of Sep. tember. Flower small.

\section{Baltinore Beauty.}

A very good, and remarkably handsome peach, of nativo origin, ripening very early, which we received from Lloyd N. Rogers, Esq., of Baltimore.

Leaves with globose glands. Fruit rather small, roundish oval. Skin deep orange, with a rich brilliant red cheek. Flesh yellow, but red at the stone, sweet and very good-a little mealy if over-ripe. Ripens early in August. Flowers large.

\section{Crawford's Early Melocoton. $\oint$}

Early Crawford. Ken

Crawford's Early.

This is the most splendid and excellent of all early, yellow. fleshed peaches, and is scarcely surpassed by any other variety in size and beauty of appearance. As a market fruit, it is perhaps the most popular of the day, and it is deserving of the high favour in which it is held by all growers of the peach. It was originated a few years ago, by William Crawford, Esq., of Middletown, New Jersey. The tree is vigorous and very fruitful.

Leaves with globose glands. Fruit very large, ublong, the swollen point at the top prominent-the suture shallow. Skin ycllow, with a fine red cheek. Flesh yellow, melting, sweet, rich and very excellent. It ripens lere the last week in $\mathrm{Au}$. gust. Flowers small. 


\section{Crawford's Late Melocoton. $\oint$}

Crawford's superb Malacatune.

Crawford's Late Melocoton, from the same source as the foregoing, is one of the most magnificent American peaches. We think it unsurpassed by any other yellow-fleshed variety; and deserving of universal cultivation in this country. As a splendid and productive market fruit, it is unrivalled, and its size, beauty and excellence, will give it a place in every garden.

Leaves with globose glands. Fruit very large, roundish, with a shallow but distinct suture. Skin yellow, with a fine dark red cheek. Flesh deep yellow, but red at the stone, juicy and melting, with a very rich and excellent vinous flavour. Ripens from the 20th to the last of September. Flowers small.

\section{Columbia. Coxe.}

The Columbia is a singular and peculiar peach. It was raised by $\mathrm{Mr}$. Coxe, the author of the first American work on iruit trees, from a seed brought from Georgia. It is a very excellent fruit, which every amateur will desire to have in his garden. The tree is not a very rapid grower and bears only moderate crops, being, of course, all the less subject to speedy decay. The young wood is purple.

Leaves with reniform glands. Fruit large, globular, broad and much depressed, the suture distinct, extending half way round. Skin rough and rather thick, dull dingy red, sprinkled with spots and streaks of darker red. Flesh bright yellow, of the texture, as Coxe remarls, of a very ripe pine apple, rich, juicy, and of very excellent flavor. Ripens from the beginning to the middle of September.

\section{Poole's Large Yellow. Ken.}

Poole's late Yellow Freestone.

A very large and handsome peach, of the Melocoton family, which is worthy of general orchard cultivation. It lately originated near Philadelphia and bears the finest crops.

Leaves with reniform glands. Fruit large, roundish, with a suture extending from the base to the top. Skin deep yellow with a dark red cheek. Flesh yellow, but red at the stone, rich, juicy, and of exce!lent flavor. Ripens last of September. 


\section{Red Cheer Melocoto:.* Pom. Man.}

Malagatune.

Malaratune.

Hayr's Melocoton.

Yellow Malocoton.
Yellow Malagatune.

Red Cheek Malocoton. C'oxe.

The Melocoton (or Malagatune, as it is commonly called) is almost too well known to need description. Almost every orchard and garden in the country contains it, and hundreds of housarids of bushels of the fruit are raised and sent to market in this country, every year. It is a beautiful and fine fruit, in favorable seasons, though in unfavorable ones the acid frequently predominates somewhat in its flavor. It is an American seedling, and is constantly reproducing itself under new forms, most of the varieties in this section having, directly or indirectly, been raised from it ; the finest and most popular at the present time, being Crawford's Early, and Late, Melorotons, Doth greatly superior, in every respect, to the original Meln. coton.

Leaves with globose glands. Fruit large, roundish oval, with a swollen point at the top. Skin yellow with a deep red cheek. Flesh deep yellow, red at the stone, juicy, melting, with a good, rich vinous flavour. First of September. Flnwers small.

59. Smock Freestone. Ken.

St. George.

A variety which ripens late, and is much esteemed for or chard culture. It was originated not long since, by Mr. Smock, of Midd!etown, New Jersey, the centre of extensive peach cul. tivation.

Leaves with reniform glands. . Fruit large, oval, narrowed towards the stalk, and rather compressed on the sides. Skin light orange yellow, mottled with red, or often with a dark red cheek, when fully exposed. Flesh bright yellow, but red al the stone, moderately juicy and rich. Ripens last of Septen ber and first of October.

60. Yellow Alberge. Thomp.

Alberge Jaune, O. Duh Péche Jaune. Gold Fleshed Yellow Rareripe, of many .Am. Gardens.

Purple Alberge. Iind Red Alberge Golden Mignonne

\section{- Melocoton is the Spanish for peach.}


The Yellow Alberge is an old French variety, and one of the earliest of the yellow fleshed peaches. It is, no doubt, the original sort from which our Melocotons and Yellow Rareripes huve sprung in this country. It has only a second rate llavour, except in rich warm soils, and is not comparable to the Yellow Rareripe in size or quality.

Lea"es with globose glands. Fruit of medium size, roundish, with a well marked furrow running half round. Skin yellow, with a deep purplish red cheek. Flesh yellow, but deep red at the stone, soft, juicy, sweet, with a pleasant vinous flavour. Middle of August. Flowers small.

The Rosanna (Lind. Thomp.), Alberge Jaune of many French gardens, and Yellow Alberge of some gardens here, differs from the above only in having reniform glands, and ripen. ing ten or twelve days later. Flavour second rate.

\section{Yellow Rareripe. $\oint$}

Large Yellow Rareripe.

Marie Antoinette

One of the finest very early yellow fleshed peiches. It is an American seedling, produced about a dozen years ago, and well deserves the extensive cultivation it receives, both in the orchard and garden.

Leaves with globose glands. Fruit large, roundish, the suture slightly depressed, extending more than half round; the swollen point at the top small.

Skin deep orange yellow, somewhat dotted with red, the cheek rich red, shaded off in streaks. Flesh deep yellow, but red at the stone, juicy, melting, with a rich and excellent vinous flavour. Ripens from the 25th to the 30th of August. Stone small. Flowers small.

There is an inferior and older sort, very commonly known as the Yellow Rareripe and Yellow Malagatune, the fruit of which is scarcely of medium size, dull yellow, with very little red, and of a flavour very inferior to the true kind just described.

\section{ulass III. Clingstone Peaches (or Pavies).}

02. Brood Clingstone. Floy.

Claret Clingstone. Blood Cling.

'The Blood Clingstone is a very large and peculiar fruit, of 42 
no value for eating, but osteemed by many for pickling and preserving - the flesh very red, like that of a beet. 'This is an American seedling, raised many years ago, from the French Blnod Clingstone,-Sanguinole a Chair Adherente. It is a much larger fruit than the original sort, which has large fluwers, otherwise they are the same in a!l respects.

Leaves with reniform glands. Fruit often very large, roundish oval, with a distinct suture. Skin very downy, of a dark, dull, clouded, purplish red. Flesh deep red, throughout, firın and juicy-not fit for eating. September to October. Flowers small.

'There is a Frencir Blood Freestone (Sanguinole, Sanguine, Cardinale, or Betrave, Duh. Thomp.) of the same nature, an.: used for the same purpose as this, but smaller in size, and not equal to it for cooking. Leaves without glands.

\section{Catherine. Lang. Lind. P. Mag. Thorp.}

The Catherine cling is a very fine, old English variety, of excellent quality, but not, we think, equal to the Large White Clingstone, a native seedling, so much esteemed in the Middle States.

Mr. Manning, and, after him, Mr. Kenrick, have remarked that "the Catherine, the old Newington, and old Mixon Clingstone, cannot be distinguished from each other." This is an arror, probably from not having seen together, the genuine borts, as they are quite distinct fruits, and the glands of the leaves-that unerring characteristic-different in each variety.

Leaves with reniform glands. Fruit large, roundish oval, more swollen on one side than the other, and terminated by a small swollen point at the top. Skin pale yellowish green, much sprinkled with red dots, the exposed cheek of a bright lively red, streaked with darker red. Flesh firm, yellowish white, but dark red at the stone, to which it adheres very closely. juicy, rich and excellent. Middle and last of Septembes. Flowers small.

\section{Heath. $\delta$ Coxe.}

Heath Clingstone

Fine Heath.

Red Heath.

The most superb and most delicious of all late Clingstones. It seldom ripens in New England, but here, and to the southward, it is one of the most valuable kinds, of very large size, and the very finest flavour.

Coxe informs us that this is a seedling pioduced in Maryland from a stone brought by Mr. Daniel Heath from the Mediterra. nean; and it is frequently still propagated from the stone, with- 
out variation, in that State. The tree is vigorous, long lived and moderately productive; with the shortening-in norde of pruning, the fruit is always large and fine, otherwise often poor. This tree is well deserving of a place on the espalier rail a? wall, at the north.

Leaves nearly smooth on the edges, with reniform glands. Fruit very large, oblong, narrowing to both ends, and terminating at the top with a large swollen point: the suture distinct on one side. Skin downy, cream-coloured white, with a faint blush or tinge of red in the sun, or a brownish cheek. Flesh greenish white, very tender and melting, exceedingly juicy, with the richest, highest, and most luscious flavour, surpassed by no other variety. It adheres very closely to the stone. It ripens in October, and frequently keeps for a month after being gathered. Flowers small.

Bayne's New Heath, is a recent seedling, very similar in all respects, originated lately by Dr. Bayne, of Alexandria, D. C. it is considered rather finer by some.

\section{Incomparable. Lind. Thorp.}

Pavie Admirable. Bon. Jard. Ken。 Late Admirable Cling.

Larger than the Catherine, which it resembles. It is inferior to it and several others in flavour, and is only worthy of culti. vation for market.

Leaves with reniform glands. Fruit large, roundish, one side enlarged. Skin pale yellowish white, light red on the exposed side. Flesh yellowish white, red at the stone, juicy, melting, and of agreeable flavour. Last of September. Flowers small.

\section{Large White Clingstone. $\S$}

New York White Clingstone. Floy. Williamson's New York.

Selby's Cling.

The Large White Clingstone is by fal the most popular of this class of peaches in this State, and in New England. We think it superior to the Catherine, and old Newington, and only sur. passed in flavour by the old Mixon cling, and the Heath cling.

This variety was raised about forty years ago by David Williamson, a nurseryman, in New York, and vas first de. scribed by Floy as the Nezo York Clingstone. But as it is uni. versally known now by the present title, we have placed the ori. ginal names as synonymes. The light coleur, and oxcelien quality of this fruit, render it the greatest favourite for preserving 
in brandy o: sugar. The tree is remarkably hardy and long lived ; rarely, if ever, being attacked by the yellows. It beara regular and good crops.

Leaves with globose glands. Fruit large, round; the st.ture slight, and the swollen point at the top small. Slin whito (inclining to yellow only when over ripe), dotted with red on the sunny side, or with a light red cheek when fully exposed. Flesh whitish, tender, very melting, full of juice, which is very sweet, luscious, and high flavoured. Beginning and middle of September. Flowers small.

\section{Lemon Clungstone. $§$ Floy. Thomp.}

Kennedy's Carolina. Pom. Man. Long Yellow Pine Apple. Coxe. . Kennedy's Lemon Clingstone.

Largest Lemon.

Pine Apple Clingstone.

Yellow Pine Apple.

The Lemon Clingstone is one of the largest, and most beautiful of all the yellow fleshed clings, and though of course inferior in flavour to the white fleshed, is deserving of its universal popularity. It is originally a native of South Carolina, and was brought from thence by a Mr. Kennedy, of New York, before the war of the Revolution. 'There are now many seedlings reproduced from it, but none superior to the original. This is a very productive, hardy tree.

Leaves long, with reniform glands. Fruit large, oblong; narrowed at the top, and having a large, projecting, swollen point, much like that of a lemon. Skin fine yellow, with a dark brownish red cheek. Flesh firm, yellow, slightly sed at the stone, adhering firmly, with a rich, sprightly, vinous, sul). acid flavour. Middle and last of September. Hlower's small.

68. Late Yellow Alberge. Pom. Man.

October Yellow.

Alriers Yellow.

Algiers Winter.

A very late Clingstone Peach, entirely yellow, scarcely good for eating, but esteemed by some for preserving. It was originally introduced from the south of France, and has been considerabiy cultivated here but we have abandoned it. Thio Heath cling is in evry way greatly its superior.

Leaves with reniform glands. Fruit of medium size, round. ish oval, with a small distinct suture. Skin downy, green till the last of September, but at maturity being yellow. Flesh yellow to the stone, very firm, rather juicy, sweet. October Flowers large. 


\section{Oldnixon Clingstone. $§$ Coxe}

Oldmixon Cling.

Green Catherine, of the Americans Thomp.

The Cldmixon Clingstone is certainly one of the highest fla. bured of all peaches known in th.is country, where it is raised in perfection, and should have a place in every good garden Indeed we consider this, the large White Cling, and the Heath Cling, as being the sorts among the most desirable of this class of peaches for small collections.

'This fruit is quite distinct from the Catherine cling of Europe, or the old Newington, as a single glance at its leaf glands will show, to say nothing of its superior flavour. It can scarcely be the "Green Catherine of the Americans" of the London Horticultural Society's Catalogue, as that is said to be a poor fruit IVe are not familiar with it. Coxe says the Oldmixon cling was introrluced by Sir John Oldmixon, from Europe. It is more probable that he introduced the stone only.

Leaves with globose glands. Fruit large, roundish oval, the suture distinct only at the top, on one side of which the fruit is slightly enlarged. Skin yellowish white, dotted with red, or with a red cheek, varying from pale to lively red. Flesh pale white, very melting and juicy, with an exceedingly rich, lus. cious, high flavour. First of September. Flowers small.

\section{Orange Clingstone.}

The Orange Cling is a very large, handsome, and excellent fruit, somewhat resembling the Lemon Cling in colour, but glob. ular in form, rather richer in flavour, and quite a distinet sort

Leaves large, serrated, without glands. Fruit large, round, the suture distinctly marked, and extending nearly round the fruit-swo!len point at the top, none. Skin deep orange, with a rich dark red cheek. Flesh dark yellow, rather firm, juicy with a rich vinous flavour. September. Flowers small.

72. Old Newington. Lang. Lind. Thomp.

Newington. Parkinson. (1629.) Large Newington. Coxe

A celebrated English clingstone which has been in cultiva. tion more than 200 years, and still is perhaps the best in the English climate. Although excellent, it is not so generally es. teemed here as the Large White Cling, and Oldmixon Cling. stone.

Leaves serrated, without glands. Fruit large, roundisin, the suture slight. Skin pale yellowish white, with it fine red check, 
marked with streaks of darker red. Flesh pale yellowish whte, deep red at the stone, to which it always adheres very firmly; melting, juicy, and rich. Ripens about the 15th of September. Flowers large.

73. Pavie de Ponpone. Bon. Jard. Lelieur. Thomp.

$\begin{array}{ll}\text { Monstrous Pomponne. ? Lind. } & \begin{array}{l}\text { Pavie Ronge de } \\ \text { Pompone. O. Duh }\end{array} \\ \begin{array}{l}\text { Mavie de Pomponne Grosse. } \\ \text { Pavie Monstrueux. }\end{array} & \begin{array}{l}\text { Pavie Camu. } \\ \text { Gros Mélecoton. } \\ \text { Gros Persique Ro'dge }\end{array}\end{array}$

A very large and magnificent old French cingstone, not se woll known in this country as it deserves. 'The fruit is very solid in flesh, and much sweeter here than in France. The tree is of very strong growth.

Leaves with reniform glands. Fruit very large, roundish oval, with a well marked suture extending to the top, and terminating there in an obtuse swollen point. Skin yellowish white, a gnod deal covered with the broar, very deep red colour of its chee's. Flesh firm, yllowish white, deep red at the stone, to which it adheres very firmly, and which is rather small; juicy. flavour sweet and grood. First of October. Flowers large.

\section{Situ's Newington. Lind. Thomp.}

\section{Early Nervington. Of the \\ Smith's Early Newington. $\}$ English \\ Early Newington. Coxe.}

'This is one of the best Early Clingstone Peaches. It is of English origin, and is little cultivated in this country. The Early Newington of our gardens as generally known (see Early Newington Freestone), is earlier and a very much finer variety, with reniform glands, being a partial clingstone, but most fiequently parting from the flesh, has quite supplanted it.

Leaves serrated, without glands. Fruit middle sized, rather oval, narrower at the top, and one half a little enlarged. Skin pale straw colour, with a lively red cheek streaked with purple. Elesh firm, pale yellow, but light red at the stone, to which it adheres closely; juicy, and of very good quality. Last of August. Flowers large.

What Mr. 'L'hompson calls "Newington of the Americans" is a seed'ing cling with globose glauds, and of second quality: iuite distinct from sur Early Newington Freestone. 


\section{Tippecanoe.}

Hero of Tippecanoe.

A new, very large, and handsome clingstone, origınaud by Mr. George Thomas, of Philadelphia, and first exhibited thefora tne Horticultural Society there in 1840. Its lateness alle beauty rerder it a valuable kind.

Leaves with reniform glands, the shoots dark purplish red. Fruit very large, nearly round, a little compressed on the sides. Skin yellow, with a fine red cheek. Flesh yellow, juicy, with a gond vinous flavour. It ripens from the 20th to the last of September. Flowers small.

\section{Wasington Clingstone. $\oint$}

An American variety, remarkably juicy and sweet. Although 'Thompson finds it third rate in England, it is here scarcely surpassed. 'To use the expressive words of nne of our friends in Maryland, a good judge of fruit, "there is nothing hetter than this peach out of paradise." It is weither handsome nor prepossessing externally.

Leaves with reniform glands. Fruit of medium size, round. ish. Skin yellowish green, marked with grey specks, and with a slight tinge of red on the sunny side. Flesh very juicy, tender, and melting, with a very sweet and luscious flavour. Last of September. Flowers small.

\section{Curious, or Ornamental Varieties.}

\section{Double Blossoned. Thomp.}

Double Flowering Peach.

Ruse Fluwering
Pécher à Fleurs Doubles. Bon. Jard. Pécher à Fleurs Semi-Doubles. O. Duh

The Double Blossomed Peach is, when in full bloom, one of the gayest and most beautiful of fruit trees, and blooming with its lovely companion, the Double Flowering Cherry, finds a place in all our pleasure grounds and ornamental plantations. its flowers are three times the size of those of the cornmon peach, of a lively rose colour, nearly full double, and so thickly disposed on the branches as to be very striking and showy They are produced at the usual season o1 a fow days later 
This sort is rendered more dwarf for shrubberies, by budding it upon the ilirabelle, or the Cherry Plum stock.

The leaves have reniform glands. The fruit, which is sparingly produced, is roundish oval, pale greenish yellow, faintly tinged wih red, freestone, and of indifferent flavour.

78. Flat Peach of China. Lind. Thomp.

Chinese Peach.

Java Peach.

Peen To.

A very singular variety, from China, where the gardeners aflect all manner of vegetable curiosities. The fruit is of small size, about two inches in diameter, and so much flattened at the ends that only the skin and the flat stone remains, the fleshy part bsing crowded on either side. The tree is of rather dwarfish habit, and holds its leaves very late. 'The fruit is of very good flavour, and is well worthy of a place in the gardens of the curious.*

Leaves with reniform glands. Fruit small, so much flittened as to form a deep hollow at both ends, having at the top a sin. gular broad, roug!, five-an sled eye. Skin pa!e yellowish green, mottled with red on one side. Flesh pale yellow, with a circle of red round the stone (from which it separates), sweet, juicy, with a slight noyeau tizvour. Bexinning of September. Flowers large.

\section{Weerixg Peacir.}

\section{- Reid's Weeping Peach.}

A peculiar varlety, with pendant, weeping branches, and : habit much like that of the weeping ash. It was lately origi. nated by Mr. William Reid, the skilful nurseryınan at Murray Hill, near New York. To display itself to advantage, it should be grafted six or eight feet high, on the clean stem of a peach or plum stock. Reniform glands. Flowers large.

Selection of clioice peaches for a small garden, to furnish in succession. Freestones; Early York, White Imperial, Early Newington Freestone, Royal George, Grosse Mirnonne, George IV., Crawford's Early, Bergen's Yellow, Noblesse, Brevoort, Ma!ta, Late Red Rareripe, Druid Hill. Clingslones; Large white Clingstone, Oldmixon, and Heath.

* This variety $\mathrm{l}$ as been saveral times imported to this country and lost on the way. Should any one of our amateurs now possess it, we shall be much gratified to receive buds of it. 
Selection of hardy and excellent sorts, for a northern latitude. George the Fourth, Yellow Rareripe, Early York, Morris Red Rareripe, Grosse Mignonne, Noblesse, White Imperial, Craw. furd's Early, Favourite, Bullegarde Brevoort, Cuoledge's Fa. vourite, Morris', IWhite Rareripe, La rge White Ciingstone.

The bess vuricties for forcing, are the Grosse Mignonne, No. blesse, Bellegarde, Royal George White Irr.perial, Royai Charlotte, and Barrington.

\section{CHAPTER XXIII.}

\section{- THE NECTAPINE,}

\section{Persica vulgaris (v.) Lavis. Dec. Rosacea of botanists.}

Tue Nectarine is only a variety of the peach with a smooth skin (Peche lisse, or Brugnon of the Frnnch). In its growth, habit, and general appearance, it is impossible to distinguish it from the peach tree. 'The fruit, however, is rather smaller, perfectly smooth, without down, and is one of the most waxlike and exquisite of all productions for the dessert. In flavour, it is perhaps scarcely so rich as the finest peach, but it has more piquancy, partaking of the royeau or peach leat flavour.

The Nectarine is known in Northern India, where it is called moondla aroo (smooth peach). It aprears to be only a dislinet, accidental variety of the peach, and this is reidered quite certain, since there are several well litown examples on record of both peaches and nectarines naving been produced on the same branch*-thus showing a disposiion to return to the natural form. Nectarines, however, usually produce nectarines again on sowing the seeds, - but they also occasionally froduce peaches. The Boston Nectarine originated from a peach stone.

The Nectarine appears a little more shy of bearing in this country, than the peach, but this arises almost always from the destruction of the crop of fruit by the curculio, the destroyer of all smoth-skinned stone fruit in sandy soils. It is quite hardy here whe ever the peach will thrive, though it will not generally bear large and fine iauit unless the branches aro shortened-in annually, as we nave fully directed for the peach tree.

- Sce London Gardener's Magazine, Vol. 1, p. 471 ; Vol 14, p. 53. 
With this easy system of pruning, good crops are reanily ntrained, wherever the curculio je not very prevaleut.

Where this insect abounds, we nust recomment the stcarly anmual applieation of salt, spread over the surface of the gromol, - te surface being first made hard and firm. This should be tone when the punctured fruit commences to drop. (See the :'lum for further remarks on this insect.) And we would, as a proventive to the attacks of the insect, recommenr rags, dipped u coal tar, ${ }^{*}$ to be hung in the branches for two or three weeks after the fruit is formed. The coal tar should be renewed oc. casionally, as soon as it loses its powerful smell.

The culture of the nectarine is, in all respects, preciscly similar to that of the peach, and its habits are also completely che same. It is longer lived, and hardier, whon budded on the plum, but still the nurserymen here usually work it on the peach stock.

\section{Class I. Freestone Nectarines. (Pềches lisses, Fr.)}

[The same characters are used as in describing peaches, for which the seader is referred to that part].

\section{Bostox. §'Thomp.}

\section{Lewis'
Perkins' Seedling. KKen.}

This American seedling is the largest and most beautifti of all nectarines. It was raised from a peach stone by Mr. T. Lewis of Boston. The origina! tree was, when full of fruit, destroyed by boys, but the sort had been preserved by that most skilful cultivator, S. G. Perkins, Esq., and soon in his nands attracted attention by the uncommon beauty of its fruit. In 1821, this gentleman transmitted trees of this variety to the London Horticultural Socicty, of which he is a corresponding member, together with a very accurate drawing of the fruit glown by him, measuring eight and a half inches round, and "so beautiful, that its correctness was doubted abroad," until Mr. Kuight showed specimens grown there in 1823. The fruit, though not of high flavour, is excellent, the tree very harly and productive, and one of the best for general standard culture. Mr. Perkins' seculing, raised from the original Lewis tree, is quite identical, and we adopt the name of "Boston " nrctarine, as the standard one. Three trees of this sort corering fifty. five feet of wall at his place at Bruoline, are now very beau. tifui objects. [See Broomfield Nectarine.]

- To be had very cheap at the city gas works 
Leaves with globose glands. Fruit large and handsome, roundish oval. Skin, bright yellow, with very deep red cheek, shaded off by a slight mottling of red. Flesh yellow to the stone (which is small and pointed), sweet, though not rich, with a pleasant and peculiar flavour. First of September. Flowers small.

\section{Duc du Tellier's. §Lind. Thomp.}
Du Tilliers.
Duke de Tilley:
Du de Tello.
Du Tilly's.

A very excellent Nectarine, considerahly resembling the Elruge, but a much greater bearer.

Leaves with reniform glands. Fruit rather large, roundish oblong, being slightly narrowed at the top, and broad at the base or stalk. Skin pale green, with a marbled purplish-red cheek. Flesh greenish white, pale red at the stone, melting, juicy, sweet and good. Last of August. Flowers small.

\section{Downton. Thomp.}

The Downton is a seedling, raised by Mr. Knight. It is in quality, appearance, and season, an intermediate variety, between the Violette Hâtive and the Elruge, ripening a few days earlier than the latter.

Leaves with reniform glands. Fruit large, roundish oval. Skin pale green, with a deep violet-red cheek. Flesh pale green, slightly red at the stone, melting, rich, and very good. Kipens about the 25th of August. Flowers small.

\section{Elruge. § Thomp.}

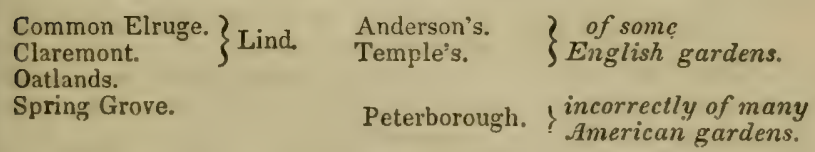

The Elruge is everywhere esteemed as one of the very finest Nectarines. It is an. English variety which has been a good while cultivated, and, with the Violet Hâtive, is considered indispensable in every collection. In this country, when the young wood is annually shortened-in, it bears good crops on standard trees, which ripen finely.

Without this precaution, like almost all other nectarines, the fuit is small, poor, and ripens imperfectly.

Leaves with reniform glands. Fruit of medium size, round. 
ish-()val, the suture slight, except at the top, where it is dis tinctly marked. Skin with a pale green ground, but when fully exposed, it is nearly covered with deep violet, or blooa red, dotted with minute brownish specks. Flesh pale green to the stone, or slightly stained there with pale red; melting, very juicy, with a rich high flavour. Stone oval, rough, of a pale colour. Last of August and beginning of September. Flowers srnall.

\section{Fairchild's Lind. Thomp.}

Fairchild's Early.

A very small, indifferent sort, only valued for its earliness, and scarcely worth cultivating when compared with the following.

Leaves with reniform glands. Fruit small, about an inch and a fourth in diameter, round, slightly flattened at the top. Skin yellowish green, with a bright red cheek. Flesh yellow to the stone, rather dry, with a sweet, but rather indifferent flavour. Beginning of August. Flower small.

\section{Hunx's Tawny. $\S$ Thomp.}

\section{Hunt's Large Tawny.
Hunt's Early Tawny. Lind.}

This is the best, very early Nectarine. It is a very distinct sort, with serrated leaves, and was originated in England abou thirty years ago. It is worthy of general cultivation, as it is not only early, but hardy, and an abundant bearer.

Leaves serrated, without glands. Fruit nearly of medium size, roundish-ovate, being considerably narrowed at the top, where there is a prominent swollen point; and the fruit is slightly enlarged on one side of the suture. Skin pale orange, with a dark red cheek, mottled with numerous russety specks. Flesh deep orange, juicy, melting, rich, and very good. It ripens from the 5 th to the 15th of August. Flowers small.

('The accidental variation of this sort, described as Hunt's Large Tavny, does not seem to have been permanently difie. rent from this.)

\section{Hapdwicke Seedling. Thomp.}

Hardwicke's Seedling.

A comparatively new variety, of high reputation, which we have lately received from $M r$. T. Rivers. It was raised at Hardwicke Honse, in Suffolk, England, and has the reputation 
st being " nne of the best and hardicst of nectarines, and a very axcellert bearer."

Leaves with reniform glands. Fruit very large, roundish, inclining to oval,and resembling the Elruge. Skin pale green, with a deep, violet-red cheek. Flesh pale green, slightly marke with red at the stonc, juicy, melting, rich, and high uav ured. End of August.

8. Murrey. Ray. Thomp.

Murry. Lind.

Black Murry.

Th- Murrey is an old English Nectarine, which, though of good quality, is rather a poor bearer, and is little known or cultivatcd in this country.

Leaves with reniform glands. Fruit of medium size, roundish-ovate, slightly swollen on one side of the suture. Skin pale green, with a dark rea cheek. Flesh greenish white, melting, sweet, and of good flavour. Stone almost smooth. Ripens about the 20th of August. Flowers small.

\section{New White. Thomp.}

Neat's White: Lina.

Flanders.

Cowdray White.

Emerton's New White.

Large White.

The N-w White is the finest light skinned variety, and is a beautiful, hardy, and excellent nectarine, bearing abundant crops, und is well worthy to be generally planted. It is an English seedling, raised by the Rev. Mr. Neate, near London, fifty years ago: from the seed of the following valiety.

Leaves with reniform glands. Fruit rather large, nearly round, skin white, with occasionally a slight tinge of red when exposed. Flesh white, tender, very juicy, with a rich, vinous flavour. The stone is small. Ripens early in September. Flowers large.

\section{Old White. Lind. Thomp.}

This nectarine is supposed to have been introduced from Asia into England, about sixty years ago. It is much like the foregoing in flavour, perhaps a litile richer, but it is less hardy and productive.

Leaves with reniform glands. Fruit rather large, roundish 
oval. Skin white, slightly tinged with red. Flesh white, tetder, juicy and rich. Early in September. Flowers !arge.

\section{Pitmaston's Orange. Lind. Thomp.}

\section{William's Orange.}

William's Seedling.

The Pitma.ston Orange, which is considered the best yellmo fleshed nectarine, was raised in 1816, by John Williams of Pitmastun, near Worcester, England. It is yet but little known, but will prove one of the best sorts for general cultiva. tion in this country. The tree is vigorous.

Leaves with globose glands. Fruit large, roundisl: ovate, the base (towards the stalk) being broad, and the top narrow, and ending in an acute swollen point. Skin rich orange yellow, with a dark, brownish red cheek, streaked at the uniors of the two colours. Flesh deep yellow, but red at the stone; melting, juicy, rich, sweet, and of excellent flavour. 'The stone is rather ssicll. Ripens middle and last of August. Flowers lange.

12. Peterborough. Mill. Lind. Thomp.

Late Green.

Vermash (of some).

This is the latest nectarine known. It is rather small, and of inferior quality, and scarcely deserves cultivation except to make complete a large collection. It was brought by Lord Peterborough, from Genoa to England.

We doubt if this fruit is now in this country. All the specimens that we have seen under this name, from different districts, being only the Elruge imperfectly grown.

Leaves with Ieniform glands. Fruit rather small, roundish. skin mostly green, or slightly tinged with dingy red on the sunny side. Flesh greenish white to the stone, somewhat juicy and of tolerable flavour. It ripens early in October. Flower small.

\section{Violette Hâtive Lind. Thomp.}

Early Violet.
Violet $\boldsymbol{P}$. Mag
Early Brugnon.
Brugnon Red at the Stone.
Hampton Court.
I.arge Scarlet.
New Scarlet.
Aromatia.

Early Violet. Violet P. Mag

Early Brugnon.

Brugnon Red at the Stone. I arge Scarlet. New Scar
PetiteViolet Hative. $O$ Itanh Brugnon Hâtif. Violette Angervillières. Viulctte Musinuée. Lord Selsey's Elruge. Violet Red at the Stone. Violet Musk. 
The Violette Hitive, or Early Violet Nectarine, everywhere takes the highest rank arnong nectarines. It is of delicious flavour, fine appearance, hardy, and productive. Listernally, the fruit is easily confounded with that of the Elruge, but it is readily distinguished by its dark coloured stone, and the deep red flesh surrounding it. The fruit is usually rather darker coloured. It is of French origin, and has been long cultivated.

Leaves with reniform glands. Fruit rather large, roundish, narrowed slightly at the top, where it is also marked with a shallow suture. Skin pale yellowish-green, in the shade, but, when exposed, nearly covered with dark purplish red, mottled with pale brown dots. Flesh whitish, but much rayed with red at the stone. The latter is roundish, the furrows not deep, and the surface reddish brown. The flesh is melting, juicy, rich, and very high flavoured. It ripens about the last of August. Flowers rather small.

The Violetre Grosse (Thomp.) resembles the foregoing in leaves and flowers, and general appearance. The fruit is, however, larger, but not so richly flavoured.

\section{Class II.-Clingstone Necturines, (Brugnons, Fr.)}

\section{Broomfield.}

Lewis, (incorrectly, of some.)

A handsome clingstone nectarine, of second quanty. It is an accidental seediling, which sprung up in the garden of IIenry Broomfield, Esq., of Harvard, Mass., and was first named and disseminated by S. G. Perkins, Esq. of Boston, thirty-five years ago.

Leaves with obscure, reniform glands. Fruit large, roundish. Skin rather dull yellow, with a dull or rather dingy red cheek. Flesh yellow, and adheres closely to the stone, juicy, rather pleasant, tut not high flavoured. First to the midule of September. Flowers small.

\section{Golden, Lang. Mill. Thomp.}

Orange.

Fine Gold Fleshed.

A very handsome looking nectarine, but of decidedly indit. ferent quality, when eompared with many others. Its waxen appearance, when fully ripe, is very beautiful. It is an old English variety.

I.eal es with reniform glands. Fruit of medium size, round. 
ish, ovate. Skin of a fine bright, waxen yellow colour, with a small scarlet check. Filesh orange-yellow, firm, juicy, sweet, and inlerably erood. It ripens alout the 10th of Septemiver. Flowers small.

Prince's (iolden Nectarive is of much larger size. It ripens about a wrek later, but is also only of second quality. Leares with reniform glands. Flowers large.

\section{Newington. Lang. Mill. Thomp.}

Scarlet Newington. Lind.

Scarlet.

Old Newington.

Smith's Newington.

French Newington.

Sion Hi!l.
Anderson's.

Anderson's Round

Rough Roman.

Brugnon de Newington

D'Angleterre.

A very good clingstone nectarine, of English origin. It should be allowed to hang on the tree till it begins to shrivel, when the flavour is much improved.

Leaves strated, without glands. Fruit rather large, roundish. Skin pale greenish-yellow, nearly covered with red, marbled with dark red. Flesh firm, pale, but deep red next the stone, juicy, sweet and rich, with an excellent vinous flavour. Ripens about the 10th of September. Flowers large

17. Newington, Early. $\S$ Lind. 'Thomp.

Early Black Newington.

New Dark Newington.

New Early Newington.

Black
Lucombe's Black.

Lucombe's Seedling. Early Black.

The Early Newington is one of the best of clingstone nectarines. It is not only a richer flavoured fruit than the old Newington, but it is larger, dark coloured, and earlier.

Leaves serrated, without glands. Fruit large, roundish, ovate, a little enlarged on one side of the suture, and terminating with an acute swollen point at the top. Skin pale green in its ground, but nearly covered with bright red, much mar. bled and mottled with very dark red, and coated with a thin bloom. Flesh greenish white, but deep red at the stone, juicy, sugary, rich and very cxcellent. Beginning of September. Flowers large.

\section{Red Ronan. $\oint$ Forsyth. Lind. Thomp.}

Old Roman.

\section{Roman.}

Jrugnon Violette Musquéc.

Brugnon Musquée.

O. Dus. 
The Red Roman is a very old European variety, havin peen en mmerated by Parkinson, in 1629. It is still esteemrit both in Europe and this country, as nne of the richest and best of clingstone nectarines. The tree healt'ny and productive.

The Newington is frequently sold for the Red Roman in this country, and the true Roman is comparatively scarce.

Iseaves with reniform glands. Fruit large, roundish, a little flattened at the top. Skin greenish yellow, with a brownish, muddy, red cheek, which is somewhat rough, and marked with brown russetty specks. Flesh firm, greenish yellow, and deep red at the stone, juicy, with a rich, high vinous flavour. Ripening early in September. Flowers large.

Selection of choice hardy Neclarines, for a small. Garlen.Early Violet, Elruge, Hardwicke Seedling, Llunt's 'Tawny, Boston, Roman, New White.

\section{CHAPTER XXIV.}

\section{THE QUINCE.}

Cydonia vulgaris, Dec.; Rosacere, of Botanists.

Coignassier, of the French; Quittenbun, German; Irivepeer, Dutch; Cotogno, Italian; and Membrillo, Spanish.

Tre Quince is a well-known, hardy, deciduous tree, of small size, crooked branches, and spreading, bushy head. It is indi. genous to Germany and the south of Europe; and it appears first to have attracted notice in the city of Cydon, in Crete o: Cantia-whence its botanical nume, Cydonia. 'The fruit is of a fine golden yellow, and more nearly resembles that of the orange than any other. It was even more highly cstecmed by the Greeks and Romans, for preserving, than by us. "Quinces," says Columella, "not only yicld ploasure, but health.".

The Quince seldom grows higher than fifteen feet, and is tisially rather a shrub than a tree. Its large white and pale pink blossoms, which appear rather later than those of other fruit trees, are quite omamental; and the tree, properly grown, is very ornamental when laden in October and Novem. ber with its ripe golden fruit.

Uses.-The Quince is, in all its varietics, unfit for cating raw. It is, however, much esteemed when cooked. For preserving, it is everywhere valued. and an excellent marmalade is also made from it. Stewed, it is very frequently used, to 
communicate additional fiavour and piquancy to apple-tarts, pics, of othel pastry. In Lingland, whe is fiequently made from the fruit, by adding sugiar and water, as it other fruit wines; and it is a popular notion there, that it has a most beneficial offect upon asthmatic paticnts. Iried Quinces are pxecllent.

In this country, large plantations are sometimes made of the Quince; and as it is, in good soil, a plentiful beares, it is consiblered one of the most valuable mariset fruits. The Apple quince is ti:e most productive and saleable; but as the Pear ynince ripens, and can be sent to market much later, it fir. quently is the most prolitable.

Plubstiation. - The Quince is easily propagater from seed, laycrs, of cuttings. From secds the quance is somewhan liable to vary in its seedlings, sometimes proving the appleshaped and sometimes the pear-shaped variety. Cuttings, planted in a sharled situation, early in the spring, root very easily, and this is perhaps the simplest and best way of continuing a good variety. 'The better sorts are also frequently budded on common seedling quince stocks, or on the common thorn.

Quince stocks are extensively used in engrafing or budding the l'ear, when it is wished to render that tree dwarf in its habit.

Sinl and Culturf.- The Quince grows naturally in rather moist soil, by the side of rivulets and streans of watcr. Hence it is a common idea that it should always be planted in some dinup neglected part of the grarden, where it usually receives little cure, and the fruit is cften knotty and inferior.

This practice is a very erroneous one. No tree is more benefiter by manuring than the quince. In a rich, mellow, deep soil, even if quite dry, it grows with thrice its usual vigour, and bears abundaut crops of large and fair fruit. It should, therefore, be planted in deep and good soil, kept in constant cultivation, and it should have a top-dressing of manure, cvery season, when fair and abundant crops are desired. As to pruning, or other care, it requires very little indeed-an occasional thinning out of crowding or decayed branches, being quite sufficient. 'Thinning the fruit, when there is an overcrop, improves the size of the remainder. 'Ten feet apart is a suitable distance at which to plant this tree.

'I'he Quince, like the apple, is occasionally sulject to the attacks of the. borer, and a few other insects, which a little care will prevent or destroy. For their habits we refer the reader to the apple.

VARIETIES.- Several varieties of the common Quince aro enumerated in many catalogues, but there are in reality only three distinct forms of this fruit worth enumerating, viz: 


\section{ApPle-Shaped Quince. Thomp.}

Orange Quince,

Cydonia v. Maliformis, Hort. Brit.

Coignassier Maliforme, of the Erench.

This is the most popular variety in this country. It bears large roundish fruit, shaped much like the apple, which stews quite tender, and is of very excellent flavour. It also bears most abundant crops. Leaves oval.

There are several inferiour varieties of the apple quince. The true one bear's fruit of the size of the largest apple, fair and smooth, and a fine golden colour.

\section{Pear-Shaped Quince. Thomp.}

Oblong Quince.

Coignassier pyriforme, of the French.

Cydonier sub. v. pyriform, Hort. Brit.

The pear-shaped quince is dryer and of firmer texture than the foregoing. It is rather tough when stewed or cooked, the flesh is less lively in colour, and it is therefore much less esteem. ed than the apple-shaped variety. The fruit is of medium size, oblong, tapering to the stalk, and shaped much like a pear 'The skin is yellow. 'The leaves are oblong-ovate. It ripens about a fortnight later, and may be preserved in a raw state considerably longer.

\section{Portugal Quiven. 'I'hoinp.}

Cydonia Lusitanica. Hort. Bril.

Coignassier de Portugal, of the French.

The Portugal quince is rather superior to all others in quality, as it is less harsh, stews much better, and is altogether of milder flavour, though not fit for eating raw. For marmalade and baking it is much esteemed, as its flesh turns a fine purple or deep crimson when cooked.

The leaf of the Portugal Quince is larger and broader than that of the common quince, and the growth of the tree is stronger. It is therefore preferred by many gardeners for stocks on which to work the pear.

'The fruit is of the largest size, oblong. The skin is in colour not so deep an orange as that of the other sorts.

The Portugal Quince is unfortunately a shy bearer: whicn is the reason why it has never been sn generally cultivated as the Apple Quince. 
Ornamental Varietics.-There are two or three ninamental varieties of the quince, which are natives of Chnna and Japan, and are now among the most common and attractive of ous garden shrubs. They are the following:-

\section{JAPAN QUINCE.}

Cydonia Japonica. Dec. Pyrus Japonica. Thunberg.

The Japan Quince is a ?ow thorny shrub, with small dark green leaves. It is the most brilliant object in the shrubbery, during the month of April, the branches being clothed with numerous clusters of blossoms, shaped like those of the quince, but rather larger, and of the brightest scarlet. The fruit which occasionally succeeds these flow rs, is dark green, very hard, and having a peculiar and not un pleasant smell. It is entirely useless.

The White, or Blush Japan Quince (C. jap. $\{$. albo), resembles the foregoing, except that the flowers are white and pale pink, resembling those of the co mmon apple-tree.

\section{Chinese Quince.}

Cydonia Sinensis. Dec.

We have had this pretty shrub in our garden for several years, where it flowers abundantly, but has, as yet, produced no fruit. The leaves are oval, somewhat like those of the common quince, but with a shining surface. The flowers are rosy red, rather small, with a delicate violet odour, and have a very pretty effect in the month of May, though much less showy than those of the Japan Quince. The fruit is described as large. eggshaped, with a green skin and a hard dry flesh, not of any value for eating. The leaves assume a beautiful shade of red in autumn.

\section{CHAPTER XXV.}

\section{THE RASPBERRY AND BLACKBERRY}

\section{The Raspberry}

Rubus Idaus, 4 : Rosacec, of botanists.

Framboisier, of the French; Himbeerestrauch, German; Framboos
Dutch; Rova ilco, Italian; and Frambueso, Spanish.

THe Raspberry is 3 . low deciduous shrub, which in several forms is common in the woods of both Europe and America: 
The large fiuited varieties most estecmed in our gardens hava ali originated from the long cultivated Rubus idreus, or Mount Ida bramble, which appears first to have been introduced into the gardens of the South of Europe from Mount Ida. It is now quite naturalized in some parts of this country. Besides this; we have in the woods the common black raspberry, or thimble. berry (Rubus occidentalis, L.), and the red raspberry (Rubus strigosus, Michx.), with very good fruit.

The name raspberry (Raspo, Italian) is probably from the rasping roughness of prickly wood. The term raspis is still used in Scotland.

UsES.-The raspberry is held in general estimation, not only as one of the most refreshing and agreeable sub-acid fruits fur the dessert, but it is employed by almost every family in making preserves, jams, ices, sauces, tarts and jellies; and on a larger scale by confectioners for making syrups, by distillers for making raspberry brandy, raspberry vinegar, \&c. Raspberry wine, made in the same way as that of currant, is considered the most fragrant and delicious of all home-made wines.

Succeeding the strawberry at the beginning of summer, when. there is comparatively little else, this is one of the most invalu. able fruits, and, with the strawberry, generally commands tha attention of those who have scarcely room for fruit trees. It is, next to the strawberry, one of the most wholesome berries, and not being liable to undergo the acetous fermentation in the stomach, it is considered beneficial in cases of gout or rheu. matism.

Propagation.-The raspberry is universally propagated by suckers, or offsets, springing up from the main roots. Seeds arn only planted when new varieties are desired. The seedlings come into bearing at two or three years of age.

Soll AND CUlture.-The best soil is a rich deep loam, rather moist than dry, but the raspberry will thrive well in any soil that is rich and deep, provided it is fully exposed to the sun and air.

In making a plantation of raspberries, choose, therefore, an upen sunny quarter of the garden, where the soil is good and deep. Plant the suckers or canes in rows, from three to four feet apart, according to the vigour of the sort. Two or three suckers are generally planted together, to form a group or stoot, and these stools may be three feet apart in the rows.

'The plantation being made, its treatment consists chiefly in a single pruning, every year, given early in the spring. 'To perform this, examine the stools in April, and leaving the strongest shoots or suckers, say about six or eight to each stooi, cut away all the old wood, and all the other suckers (except such as are wanted for new plantations). The remaining shoots should have about a foot of their ends cut off, as this part of the 
wood is fecble and worthless. With a light top-dressing of manure, the ground should then be lug over, and little other care will be requisite during the season.

When very neat culture and the largest fruit are desired, more space is left between the rows, and after being pruned, the canes are tied to long lines of rods or rails, like an espalier, by which means they are more fully exposed to the sun and light, and the ground between the rows is kept cropped with small vegetables.

A fine late crop of raspberries is readily obtained by cutting down the canes uver the whole stool, in the spring, to within a fiew inches of the ground. They will then shout up new wood, which comes into bearing in August or September.

We liave found a light application of salt given with the topdressing of manure in the spring, to have a most beneficial effect of the vigour of the plants, and the size of the fruit.

A plantation of raspberries will be in perfection at the third year, and after it has borne about five or six years, it must be broken up, and a new one formed, on another plot of ground.

In New England, and the northern part of this State, the Antwerp rasplerries are often liable to be killed to the ground by severe winters. In such situations, it is customary to prune them in fail, after which the cancs are bent down, and covered lightly with earth or branches of evergrcens till spring. Here, and to the south of this place, this is not necessary.

VAnIETHE. - The fincst raspberrics in general cultivation for the dessert, are the Red and the White Antwerp. The Franconia is a fine, large, and productive varicty, greatly estecmed at the North and East, as being hardier and later in ripening than the Antwerp. The Fastolff is a new and uncommonly fine fruit, which deserves a place in every garden. The common American Red is most cstcemed for flavouring liqueurs or making brandy, and the Ancrican Black is preferred by most oersons for cooking. The Ever-bearing and the Ohio Everbearing, are valuable for prolonging the season of this fruit till iate frosts.

\section{Antwerp, Red. $\oint$ P. Mag. Thomp.}

New Red Antwerp

True Red Antwerp

Howland's Red Antwerp.
Burley.

Knevet's Antwerp.

Framboisier à Gros Fruit.

The Red Antwerp is the standard varicty for size, flavour, and productiveness, wherever it is known. It is a Dutch sort, originally from Antwerp city. It bears early and ahun. dantly with us, and is one of the most profitable native fruits.

Fruit very large, conical, dull red; flavour rich and sweet. 
Canes mollcrately strong, yellowish green, becoming pale brows early in autumn, covered below with dark brown bristles; bu the upper portions, especially, of the bearing wood, nearly smooth. Leaves large, plaited. The fruit commences ripen. ing about the 10th of July, rather before the other varieties.

The Conmon Red ANTwerp, as known in this country, is a very inferior variety of the foregoing, with fruit half the size, less conical, and canes stronger, and more inclined to branch. It is not worth cultivating when compared with the foregoing, though it is almost universally known as the Red Antwerp, in this country-whence we are often obliged to call the foregoing the true, or New Red Antwerp, to prevent its being confounded with the common and inferior sort.

\section{ANTwerp, Yellow. $\oint$ Thomp. Lind.}

White Antwerp.

Double Bearing Yellow.

The Yellow Antwerp is the largest and finest light colourec raspberry, and indispensable to every good garden.

Fruit large, nearly conical, pale yellow, sweet and excellent. Canes strong and vigorous, light yellow, with many bristles or spines. On successive suckers, which shoot up, this raspberry will frequently continue somewhat in bearing for months.

\section{American Red.}

Common Red.

English Bed (of some).

Rubus Strigosus (Michx)。*

The Common Red Raspberry is a native of this, and all the middle states. It ripens nearly a week earier than the Antwerps, bears well, and though inferior in fiavour and size to these sorts, is esteemed by many persons, particularly for flavouring liqueurs.

Fruit of medium size, roundish, light red, pleasant sub-acia in flavour. Shoots very vigorous, long, upright, and branch. ing, grows from six to ten feet high. Light shi-aing brown, with purplish spines. Leaves narrow, light green.

\section{American Black.}

Common Black-cap.

Black Raspberry.

Thimble-berry.

Rubus Occidentalis

- This strongly resembles the Common Red Raspherry of Eumpe, $\boldsymbol{R}$. ldous. but according to Torrey and Gray is quite distinct. 
This raspberry, common in almost every field, with Iong ramoling purple shoots, and flattened small black berrits, is everywhere known. It is frequently cultivated in gardens, where, if kept well pruned, its fruit is much larger and finer Its rich acid flavour renders it, perhajis, the finest sort for kitchen use-tarts, puddings, \&c. It ripens later than other raspberries.

The AMERICAN White resembles the foregoing in all respects, except in the colour of its fruit, which is pale yellow or white.

\section{Barnet. P. Mag. Thomp. \\ Cornwall's Prolific. \\ Cornwall's Seedling. \\ Lord Exmouth's. \\ Large Red.}

The Barnet is a very large and fine English variety, conside. rably resembling the Red Antwerp; it ripens early, and is very productive, though not so good a market fruit, as it does not bear carriage well:

Fruit very large, roundish-conical, bright transparent pur. plish red, flavour very rich and agreeable. Canes long, yollow ish green, branching much more, especially towards the ground, than the Red Antwerp, and covered with numerous slender bristles, which are stronger towards the top of the cane. They become dull brown early in autumn. Bearing shoots nearly snooth.

\section{Brentford Cane. Thomp.}

A pretty good fruit, though much inferior to the best. Fruit of medium size, oval-conical, dark dull res Canes strong, branching, with purplish prickles.

\section{Cretan Red. Pom. Man.}

A rather late variety, of good quality, introduced some years ago from the Mediterranean. Its lateness renders it valuable, but it is inferior to the Franconia, which has taken its place in most of our gardens. It is a hardy sort.

Fruit of medium size, globular, inclining to conical, deep purplish-red, sub-acid and good. Canes upright, branclics dark grey, with few bristles. I,eaves rather narrow; dark green, but very light coloured on the lower side. It ripens about two weeks after the Whi:e Antwerp, and scntinues several weeks in perfection. 


\section{Uueble Bearing. Thomp.}

Perpetual Bearing.

Siberian.

Late Cane.

A very good variety of the Antwerp Rasplerry, chiefly es. eenied for its habit of bearing late in the season. A tiner crop, which will continue ripening till late frosts, is obtained by cut.ng down the shoots to within a few inches of the ground, every spring. It is a hardy variety.

There is a TwICE BEARING Raspberry known in some gardens in this country, which is an inferior small fruit, and bears sparingly.

\section{Franconia. $\oint$}

The hardiest large Raspberry, very productive, and very excellent. It was imported from Vilmorin, of Paris, under this name (which does not appear in any foreign catalogues) by $\mathrm{S}$. G. Perkins, Esq., of Boston, some years ago. It flourishes admirably in New England, and north of us, where the climate is tno cold for the Antwerps, and is by far the finest variety for a northern latitude. Its crops are abundant, the fruit is frm, and bears carriage to market well, and it ripens about a weck or ten days later than the Red Antwerp. It is perhaps the finest sort for preserving.

Fruit very large, obtuse conical, dark purplish red, of a rish acid flavour, more tart and brisk than that of the Red Antwerp. Canes strong, spreading, branching, yellowish brown, with scattered, rather stout, bristles. Leaves rather narrow, ard long, deep green.

\section{Fastolff. $\oint$}

The Fastolff Raspberry is a new English variety of the highest reputation. It derives its name from having originated near the ruins of an old castle, so called, in Great Yarmouth. It fruited with us last season, and fully sustained its character as the finest Red Raspberry known. The fruit is of the richest favour, and is borne for a long time in succession. It ripens about the same time as the Red Antwerp.

Fruit very large, obtuse, or roundish conical, bright purplish red, very rich and high flavoured. Canes strong, rather erect, uranching, light yellowish brown, with pretty strong tristles. 


\section{Knever's Giant.}

A capitai English Raspberry, a variety of Red Antwerp, ripening among the very earlicst. Its canes are very strong, and quite hardy. The fruit is very large, conical, deep reu, and of excellent flavour

\section{Notringhan Scarlet.}

An excellent variety, introduced from England by Cnl. wilder, of Boston. He considers it the richest in flavour of any of the older varieties. The fruit is red, obtuse-conical, of medium size.

\section{OHio, Everbearing.}

Ohio Raspberry. Ken.

This is a native of Ohio, and was first made known to Eastern cultivators by Mr. Longworth, of Cincinnati, though, we believe, it had been cultivated for some time previous, at a Quaker settlement, in Ohio. It is precisely like the American Black Raspberry, or Black-cap, in all respects, except that it has the valuable property of bearing abundant crops of fine fruit, till late in the season. We have seen a quart gathered from a single plant, on the Ist day of Novemher. It deserves a place in every larfo garden.

\section{ViCTORIA.}

A new English seedling of high reputation, wot yet fairly tested here. It is said to resemble and fully equal the Red Antwerp, in size and flavour, and to be everbearing in its habits -producing an abundant crop from July to December, on numerous side branches which it throws out, from eighteen inches to two feet long. Its value will soon be fully proved in this country.

\section{The Blackberky.}

There are several species of the Bramble indigenous to this ecurtry, which produce eatable fruit, but the two best for the iable, or for cooking, are the Low Blackberry, a trailing shrub, and the High Blackberry, a bush about four or five feet high.

The fruit is larger than that of the Raspberry, with fewer and larger grains, and a brisker flavour. It ripens about the last of July, or early in August, after the for ner is past and is 
much used by all classes in this country. The sorts are selàom cuitivated in gariens, as the fruit is produced in such great abundance in a wild sta!e ; but there is no doubt that varieties of much larger size, and greatly superiour flavour, inight be produced by sowing the seeds in rich garden soil, especially if repeated for two or three successive generations.

\section{t. Low Blackberry.}

Trailing Blackberry.

Dewberry.

Rubus Canadensis. Lin.

A low, trailing, prickly shrub, producing large white blos soms in May, and very large roundish-oblong black fruit in midsummer. Leaflets from three to five in number. The fruit, when in good soil, and fully exposed to the sun, is high flavoured, sweet, and excellent.

\section{High BlackberRy.}

Bush Blackb rrry.

Rubus Villosus. Tor, and Gray.

This is an erect growing blackberry, the stems tall, and mose or less branching. In its foliage it resembles the foregoing, but its flowers, which are white, are smaller. 'The fruit is also sinaller, rounder, not so dark coloured (being reddish-black), and though good, is seldom so juicy or high flavoured.

There is a variety; cultivated abroad, with white fruit.

Ornamental Varieties. The Dourle-White-Blossomed, and Dovble Pink-blossoned Branibes, are beautiful climbing shrubs, of remarkably luxuriant growth, which may be trained for a great length in a season, and are admirably adapted fos covering walls and unsightly buildings. 'The flowers are like small double roses, and are produced in numerous clusters in June, having a very pretty effect. North of New York these climbers are rather tender in severe winters.

The Rose Flowering Bramble (Rubus odoratus) is a very pretty native shrub, with large broad leaves, and pleasing rose. coloured flowers, and groups well with other shrubs in orna. mental plantations. 


\section{CHAPTER XXV.}

\section{THE ETRATWBERRY.}

Fragaria (of species) L. Rosacea, of botanists. Frasier, of the French; Erdiseerpflanze, German, Aadbezie, Dutch Pianta di Fragola, Italian; and Fresa, Spanish.

The Strawberry is the most delicious and the most wholesome of all berries, and the most universally cultivated in all gardens of northern climates. It is a native of the temperate latitudes of both hemispheres, - of Europe, Asia, North and South Ame. rica; though the species found in different parts of the world are of distinct habit, and have each given rise, through cuiti. vation, to different classes of fruit-searlet strawberries, pine strawberries, wood strawberries, hautbois, \&c.

'The name of this fruit is popularly understood to have arisen from the common and ancient practice of laying straw between the plants to keep the fruit clean. In the olden times the vari. ety of strawberry was very limited, and the garden was chiefly supplied with material for new plantations from the woods. Old Tusser, in his "Five Hundred Points of Good Husbandry," points out where the best plants of his time were to be had, ard turns them over, with an abrupt, farmer-like contempt of littlo matters, to feminine hands :-

"Wife, into the garden, and set me a plot

With strawberry roots, of the best to be got;

Such growing abroad, among thorns in the wood,

Well chosen and picked, prove excellent good."

The strawberry belongs properly to cold climates, and though well lnnown, is of comparatively little value in the south of Europe. Old Roman and Greek poets have not therefore sung its praises; but after that line of a northern bard,

"A dish of ripe strawberries, smothered in cream,"

which we consider a perfect pastoral idyl (as the German school would say), in itself, nothing remains to be wished for. We have heard of individuals who really did not, by nature, relish strawberries, but we confess that we have always had the same doubts of their existence as we have of that of the unicorn. 
Ripe, blushing, strawberries, eaten from the plant, or serred with sugar and cream, are certainly Arcadian dainties with a true pasadisiacal flavour, and fortunately, they are so easily grown that the poorest owner of a few feet of ground may have them in abundance.

To the confectioner this fruit is also invaluable, commuricating its flavour to ices, and forming several delicate preserves. In Paris a cooling drink, bavaroise $\dot{a}$ la grecque, is made of the juice of strawberries and lemons, with the addition of sugar and water.

'The strawberry is perhaps the most wholesome of all fruits, being very easy of digestion, and never growing acid by fermentation as most other fruits do. The often quoted instance of the great Linnæus curing himself of the gout by partaking freely of strawberries-a proof of its great wholesomeness-is a letter of credit which this tempting fruit has long enjoyed, for the consolation of those who are looking for a bitter concealed under every sweet.

Propagation atid SuIL. The strawberry propagates itselt very rapidly by runners* which are always taken to form new plantations or beds. These are taken off the parent plants early in August, and either planted at once where they are to grow, or put out in nursery beds, or rows, to get well established for the next spring planting. When the parent plants have become degenerated, or partially, or wholly barren, we should a void taking the runners from such, and choose only those which grow frum the most fruitful ones. In order to be sure of the latter point it is only necessary to mark the best bearing plants by small sticks pushed into the bed by the side of each when the fruit is in perfection. Some varieties, as the Prolific Hautbois, the English wood, and the Large Early Scarlet, are not liable to this deterioration, and therefore it is not necessary to select the runners carefully; but others, as the Pine strawberries, and some of the Scarlets, are very liable to it, and if the runners are taken and planted promiscuously, the beds, so made, will be nearly barren.

The best soil for the strawberry is a deep, rich, loam. Deep it must be, if large berries and plentiful crops are desired; and the wisest course, therefore, where the soil is naturally thin, lies in trenching and manuring the plot of ground thoroughly, before putting out the plants. But even if this is not necessary it should be dug deeply, and well enriched with strong manure Deforehand.

The best exposure for strawberries is an open one, fully ex. posed to the sun and light.

* Excepting the Bush Alpines, which have no runners, and are propa gated by division of the roots. 
Cultune in Rcws. The fincst strawberries are always ob trined when the plants are kept in rows, at such a distance aprart os to give sufficicnt space for the roots, and abundance of light and air for the leaves.

In planting a plot of strawberries in rows, the rows should te two fuet apart, and the plants, of the large growing kints, two feet from each other in the rows; of the smaller growing linds, from one foot to eighteen inches is sufficient. The runners must be kept down by cutting them off at least three times a year, and the ground must be maintained in good order by constant dressing. During the inrst year a row of any small vegetables may be sown in the spaces between the rows. Every autumn, if the plants are not luxuriant, a light coat of manure should be dug in between the rows; but if they are very thrifty it must be omitted, as it would cause them to run too much to leaf.

A lighi top-dressing of leaves, or any good compost, applied late in the fall, though not necessary, greatly promotes the vigor of the plants, and secures the more tender kinds against the effects of an unusually cold winter. Before the fruit ripens, the ground between the rows should be covered with straw, or light new-mown grass, to keep it clean.

A plantation of this kind in rows, will be found to bear the largest and finesi fruit, which, being so fully exposed to the sun, will always be sweeter and higher flavoured than that grown in crowded beds. A plantation in rows is generally in full perfection the third year, and must always be renewed after the fourth year.

Culture in alteinate strips. A still more easy and eco. nomical mode is that of growing the strawberry in alternate strips.

Early in April, or in August, being provided with a good stock of strong young plants, select a suitable piece of good deep soil. Dig in a heavy cuat of stable manure, pulverizing well and raking the top soil. Strike out the rows, three feet apart. with a line. The plants should now be planted along cach line about a foot apart in the row. 'They will soon send out rumers, and these runners should be allowed to take possession of every alternate strip of three feet-the other strip being kept bare by co.ıtinually destroying all runners upon it, the whole patch being kept free of all weeds. 'The occupied strip or bed of runners will now give a heavy crop of strawberrics, and the open strip of three feet will serve as an alley from which to gather the fruit. After the crop is over, dig and pre. pare this alley or strip for the occupancy of the new runners fur the next season's crop. 'The runners from the old strip wil? now speedily cover the new sprace aliotted to them, and will perhaps require a partial thinning out to have them evenly disiributed. As soon as this is the case, say about the middle of 
August. dig under the whole of the old plants with a light cos.: of manure. The surface may be then sown with turnips or spir. age, which will come off before the next season of fruits.

In this way the strips or beds, occupied by the plants, are re. yersed every season, and the same plot of ground may thus bs continued in a productive state for many years.

Both of the above modes are so superior to the common one of growing then more closely in beds, that we shall not give any directions respecting the latter.

It may be remarked that the Alpine and European Wood strawberries will do well, and bear longer in a rather shaded situation. The Bush-Alpine, an excellent sort, having no runners, makes one of the neatest borders for quarters or beds in the kitchen garden, and produces considerable fruit till the season of late frosts. If the May crop of blossoms is taken off, they will give an abundant crop in September, and they are, therefore, very desirable in all gardens.

To accelerate the ripening of early kinds in the open garden it is only necessary to plant rows or beds on the south side of a wall or tight fence. A still simpler mode, by which their maturity may be hastened teh days, is that of throwing up a ridge of soil three feet high, running east and west, and planting it in rows on the south side. ('The north side may also he planted with later sorts, which will be somewhat retarded in ripening.) The best early sorts for this purpose are Duke of Kent, and Large Early Srarlet.

Staminate and Pistillate Plants.-A great number of experi. ments have been made, and a great deal has been written latejy, in this country, regarding the most certain mode of producing large crops of this fruit. On one hand it is certain that, with the ordinary modes of cultivation, many fine kinds of strawberries have disappointed their cultivators by becoming barren; on the other, it is cqually certain, that, by the mode of cultivation practised at Cincinnati, large crops may be obtained every year.

The Cincimati culavators divide all Strawberies into two chasse s, cl aricterized l,y tlie ir biossoms. The first of these they citl steminate (or mals), from the stamens be ing chichy deve. lencel; the secrord are called pistillate (or fimale), from the pistils being elit fly dev loped.

The firsi class, to which belong vorious sorts, as licen's Secdling, Pritish Quern, cte., visually in this climate bear uncertain croins, from the ficet that only a part of the blossoms develop the pistils sufficiently to swell into perfect fruit.

'The se end class, to which brlong various other sorts, such as Hovey's Serlling, Black Prince, re., producing cnly pistil-bratring flewers, co not set fruit at all when grown quite apart by thensclve:; but when grown neas a proper number of staminate clants. so ats to be duly fertilized by them, they brar much larger 
erops, of much more perfect berrics, than s:an be produecd ir this elimnte in any other way.

'This is no longer a navilor of ther ory, for the market of Cincin. nati, in which are sold six tlousind bushe ls of strawberies amually, is supplicel more abundantly and re gularly than perhaps any other in the world, by this very mode of culture.

In planting strawberry beds, it is important, therefore, to the cultivator, to know which are the slaminate, and which the pistil. late, varicies-as they are found to be permanent in these characters. We have, accordingly, designated these tritis in the descriptions of the varietics which follow.

Unon the relative proportion of staminale to pistillate plants, cultivators are not absolutely agrecd. Where, however, such hardy sorts as the Large Liurly Searlet, or the Duke of Fent, are chosicn for staminates, it is sufficicnt to plant onc-fourth as many of these as of pistillates, to insure a full crop of the latter. When staminate sorts, like licon's Serdling, or like less hardy kinds, are chosen, then the proportion should be one-third to two-thirds of pistillates.

Thus, in planting in the alternate strip mode, let every twolve fect of each strip be planted with Hovey's Secdling, (pistzllate,) and the succecding four fect with Large Early Scarlet. $\Lambda$ very little trouble, bestowed when the rumers are extending across the open spaces, will preserve the proportion good from year to year. The appearance of a plat, planted in this way, will be as follows: S rrpresenting staminate, and P pistillate, varieties.

In planting in beds, the sime cours" may be arlopted,

g $8 \mathrm{~S}$

S S S

B $B$ S

I $P$ P

I) $\mathrm{P} P$

$p \stackrel{p}{p}$

$P$ P $P$

$P P P$

$P$ P P

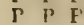

P P P

1. $\mathrm{P}$ J

P P P

P. P P

P P P

S S S

is $i s$ S

3 is $S$

5 is $\mathrm{S}$ or, what is perhaps better, every third or fourth bed may be entirly staminite, and the rest pistilliate sorts (the beds in this case being supposed to be side by side).

Nothing is easier than to distinguish the two clinses of strawberries when in blossom. In one, the staminate, the long yellow anthers $(a)$, bearing the fine dust or pollen, are abundint; in the other, the pistillute, only the cluster of pistils $(b)$, looking like a very minute green strawberry, is visible-(that is to the common observer, for the winting organs are morely rudimen. tary, and not developed).

\section{Fig. 211. Strawberry Blossoms.}

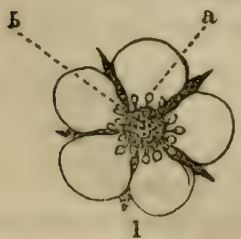

Perfert blossom.

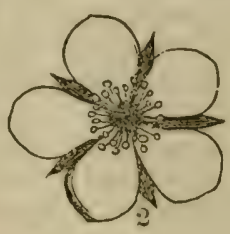

Staminate hlossom.

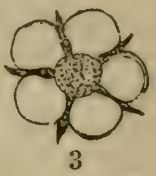

Pistillate hlossum 
Besides these, there is really a third class, quite distinct, the b!ossoms of which are regularly hermaphrodite, or perfect, in themsclves, and which always bear excellent crops-though not nernaps so large as some of the most prolific of the pistillates de

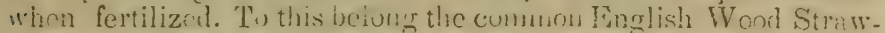
berries and the Alpines. Henen, these al I inhabitants of the Fardens have, from their uniform productiveness, long becn fitrourites with many who have not understood the character and habits of the larger staminate and pistillate varieties. Fig. 211 (d) shows the blnssom of this clnss of strawberries.

VARIETIES.-The varieties of this fruit are very numerous, indeed quite unnecessarily so for all useful purposes. 'They have chiefly been originated abroad within the last thirty years. The different species from which the varieties have been raised, have given a character to certain classes of Strawberries, pritty distinctly marked. Thus, from our own Wild Strawberry, or Virginia Scarlet, as it is called abroad, have origin. ated the Scarlet Strawberries; from the Pine or Suriuam Strawberry has been raised the class called Pines. From the common Wood Strawberry of Europe, another class, comprising the Woods and Alpines. Beside, there are the Hautbois, from a sort, a native of Bonemia, the Chili Strawberries, from Suuth America, the Green Strawberries, and the Black Strawberries.

Of these the Pines and the Scarlets are the largest and highest flayoured. The Wood and Alpine Strawberries are valuable for bearing a long time, and parting freely from the hull or talk, in picking. 


\section{Class I. Scarlet Stravberries.}

[Flowers strall. Leaves rather lone, of thin texture, and rather light Lolour, with sharp serratures; the fruit of a bri sht colour, and a sub-acid flavour: the seed sunk in ridged intervals. Fruit mediurn or large, acid or sub-acid.]

\section{Bishop's Orange. $\oint$}

\section{Bishop's New.}

Orange Hudson Bay:

A handsome and very highly flavoured variety, which has proved of the finest quality, in the Middle States, when planted in rich, deep, sandy soils. The fruit is a beautiful light scarlet, somewhat approaching orange-of large size, very regularly shaped, conical, borne in large clusters lying on the surface. Flesh firm. Flavour very high. Leaves hairy. Ripens rather late, and bears well. Pistillate.

\section{Black Roseberry. 'Thomp.}

A good, nearly round Strawberry, of rather large size, and a fine, dark, purplish-red colour. Flesh firm, with an excel. lent, rather peculiar flavour. Only a moderate bearer.

\section{DundeE. $\oint$}

A capital Scotch variety, extensively cultivated here, and esteemed for its great productiveness and hardiness. Leaves very long, foot-stalks and fruit also, supported on long stalks in large clusters. Fruit pretty large, roundish oval, regularly formed, and of a fine light scarlet. Flesh firm, and of a rich acid flavour. It ripens pretty late-at the sume season as the Hudson Bay. Pistillate.

\section{Duke of Kent's Scarlet. Thomp.}

Austrian Scarlet, Lind. Globe Scarlet.

Nova Scotia Scarlet. Early Prolific Scarlet, \&c.

We have long cultivated this, which is the eariest of all Strawberries. The fruit is rather small, and the sort is alto. gether inferior to the large Early Scarlet which ripens a week later. Still, it is valuable where the earliest fruit is desired Fruit small, varying from roublish, to long conical; bright scarlet: flavour sharp and goo!!. Ripens here in the middle or last of May. Flowers staminate. 
6. Grove End Scarlet. Thomp.

Atkinson Scarlet.

A very handsome English Strawberry, but not esteemed here so much as the Large Early Scarlet. Leaves very widely serrated. Fruit of medium size, very bright vermillion scarlet, glcbular and flattened. Seeds slightly imbedded. Flesh of a pleasant, mild flavour. Ripens pretty early. Staminate.

\section{Hudsox's BAy. Thomp.}

Hudson.

Late Scarlet.

American Scarlet. (of some.)
York River Scarlet. etc.

The Hudson Strawberry is perhaps more celebrated than uly other for the markets of Philadelphia and New-York. Its great firmness enables it to bear carriage well, and its deep acid flavour, and the late season at which it ripens, have made it the most popular sort for preserving.

Fruit pretty large, ovate (with a neck), of a rich dark shining red. Seeds deeply imbediled. Flesh very firm, of a high, but brisk acid flavour. It should therefore be allowed to hang late Ripens late, after most of the scarlets are gone. Hudson's Bay.

\section{Large Early Scarlet. Pom. Man.}

'T'he finest of all very early Strawberries, a regular, very abundant, and excellent bearer and indispensable in every garden. The flowers generally perfect It is an American variety, the leaves rather broad, and the flowers larger than the most of its class. It is so superiour to the Old Scarlet as to entirely supersede it wherever known.

Fruit pretty large, roundish ovate, regularly formed, handsome light scarlet, seeds deeply imbedded. Flesh tender, of a rich, excellent flavour. Ripens before all the other sorts, but Duke of Kent's Scarlet. Staminate.

8. Methyen's Scarlet. Thomp.

Methven Castle.

Southampton Scarlet.

Warren's Seedling.

An immense fruit, but hollow and of rather coarse flavour. The leaves are very broad. Fruit of the largest size, roundish or cnckscumb shaped, rather dull scarlet. Flesh soft, and of in. different flavour. Seeds not deeply imbedded. Ripens at me. dium season. 
[A bout Philadelphia, this sort was, a year or two ago, pretty generally cultivated as the Keen's Seedling, a very different: fruit.]

\section{MELON.}

A very good Scotch variety of dwarfish habit, and quite pro. ductive. Fruit of medium size, roundish, of very darkish co. lour; flavour rich and good.

10. Old Scarlet. Thomp.

\section{Original Scarlet.} Virginia Scarlet.
Scarlet.

Early Scarlet.

This is the common wild strawberry of this country, and is the type of the class. It is here quite supplanted by the Isarge Early Scarlet. Fruit roundish-conical, bright scarlet, and deeply imbedded; flesh of good flavour. It ripens f.retty early, three or four days after the Large Early Scarlet. Staminate.

\section{ROSEBERRY.}

Aberdeen.

Roseberry.

Scotch Scarlet.

A very good Strawberry not so much liked here as the Dun. dee, another Scotch variety. The fruit-stalks are short. It ripens gradually in succession, with a very short neck, dark red. Flesh firm, of tolerably good flavour. Ripens at a me. dium season. Flowers pistillate.

\section{Class II. Pine Strawberries.*}

[Flowers large, leaves rather broad, dark green with obtuse serratures, seeds prominent, on a smooth surface, fruit large, rich and swcet.]

\section{Black Prince. Thorp.}

\section{Black Imperial.}

The Black Prince which we imported a few years since from England, proves a variety of rare merit for this climate. The plants are very hardy, the fruit very handsome and large, always very dark polished red-almost black, roundish, or ovate

- Wo include in this class the small class of BLACK STRAwBERrres of the English authors, which we do not think need really be considereci äis. linct from the Pines, to which they have so much affinity. 
depressed; seeds slightly imbedded; flesh firm, very rich and high flavoured. It always bears heavily almost without care with us. It is highly worthy of a reneral trial in different sce tions of the country. Flowers pistillate.

\section{Brewer's EMTERor.}

A recent English variety. It may be compared in size and fluwour to Keen's Seedling, with the great superiority for our clinıate, of being quite hardy and an abundant bearer. Fruit large, ovate, dark red, excellert. Medium seasun. Staminate.

\section{Downton. Thomp.}

\section{Knight's Seedling.}

A very rich flavoured late Strawherry, but so uncertain ir. its crops as to have been nearly abandoned by our cultivators, The leaves are small and light green, but with the coarse ser. ratures of this class. Fruit-stallis very long and urright. Fruit pretty large, with a neck, ovate, or cockscomb.sinaped, tiark purplish scarlet; grains not deeply imbedded; flesh firm, very rich and aromatic. I'lowers staminate.

\section{Elton. 'Thomp.}

\section{Eaton Seedling. Lind.}

A very delicious, and very late Strawberry, ripening some time after most varieties hain passed by. The plants are ra. ther tender, and moth of Philadclphia. require a slight covering of straw in winter, which the anatcurs will not crudge so excell nt it sort. Leaves rather smaller and paler than in most of this class.

I'ruit la rere, nvate, most frequently cockscomb-shaped; glossy, light rerl at first, hut when fully ripe, dark rod; liesh rather firn, with a very rich favour. Flowe rs stininate. 
16. Hovey's Seedilig. $§$ Ifov. Mag.

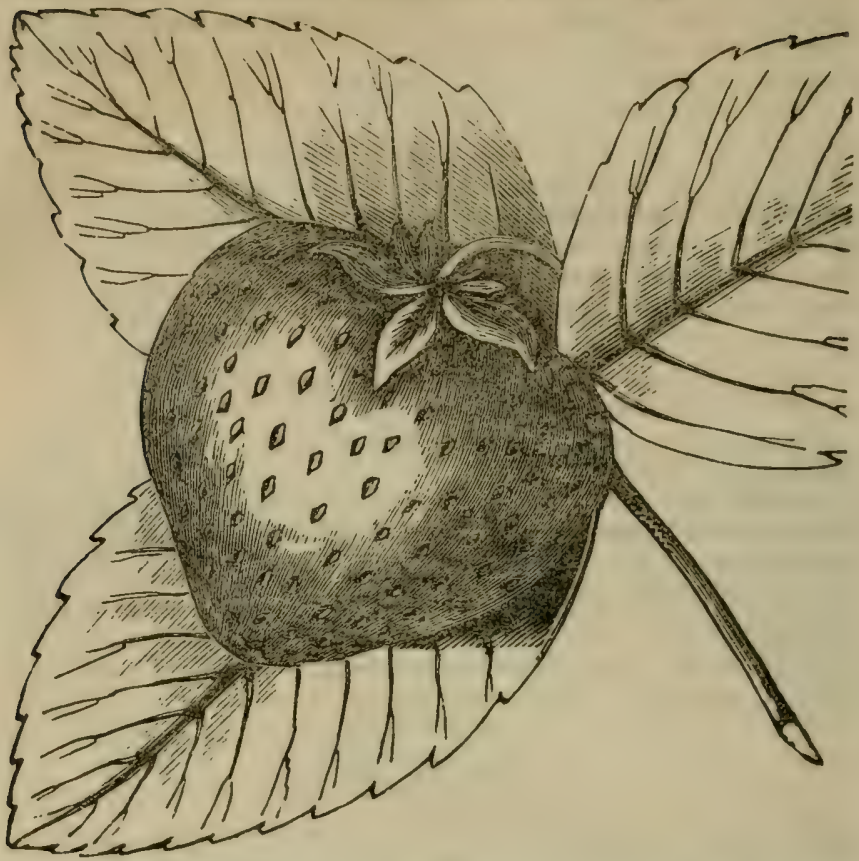

This splendid Strawberry was raised, in 1834, by Messrs. Ilovey, seedsmen, of Boston, and is undoubtedly, for this climate, one of the finest of all varieties. The vines are unusually vigor:ous and hardy, producing very large crops, and the fruit is al. ways of the largest size and finely fluvoured. It is well known at the present moment throughout all the states, and has everywhere proved superior, for all general purposes, to any ethes large-fruited kind. The leaves are large, rather light green, and the fruit-stalk long and erect.

Fruit very large, roundish oval, or slightly conical, deep shining scarlet, seels slightly imbedded; flesh firm, with a rich, agreeable flavour. It ripens about the medium season, or a few days after it. Flowers pistillate. 


\section{KeEn's Seeding. Thomp.}

Keen's Black Pine.

Murphy's Child.

This Strawberry, raised in 1821 by Keen of Isleworth, the celebrated English strawberry grower, has, ever since its intro. duction, enjoyed in England the highest reputation for produc. tiveness and excellence. In this country, though of the finest quality, it proves too tender for general cultivation, and has been supplanted by Ross' Phœnix, a perfectly hardy sort, fully equal to it, and by Hovey's Seedling.

Fruit very large, roundish, usually a little depressed, ofter: cocksccmb-shaped, dark purplish scarlet, surface polished, seeds slightly imbedded, fiesh firm, with a rich, high flavour. It ripens pretty early. Spurious sorts, especially the Methven Scarlet, are often sold for this, in this country. Staminate.

\section{Myatt's British Queen. Thomp.}

The largest, finest, and most productive, of all the new Straw. berries that have lately been originated in England. The fruit is borne on tall footstalks, and the lcaves are large. Its quali. ties for general culture are not generally tested yet in different parts of the country; but unfortunately it proves rather tender in our winters.

Fruit of monstrous size, roundish, occasionally of cockscomb slape, rich scarlet colour, favour rich and excellent. It ripens pretty early. Flowers staminate.

\section{Myatt's Pine. Thomp.}

A very large and splendid fruit of the richest flavour, but difficult of cultivation and usually a shy bearer. It requires a deep rich soil, rather light and warm, and the bed to be renewed every year. The fruit is large, round, bright scarlet. supported high, on stout footstallis. Flowers staminate.

\section{Mratt's Depteord Pine. $\oint$}

The Deptford Pine is a superb new English variety which we have just proved in this country. It is quite hardy, far more so than the British Queen, and will therefore be much more es. teemed here. The leaves are very downy underneath; the fruit is of cxtraordinary size, wedge shaped, of a clear bright scarlet. Flesh firm and solid, with a rich and excellent flavour. Ripens at the medium season, and is likely to prove hardy and productive. Flowers staminate. 


\section{Myatt's Ej.iza.}

One of the new sorts, second-rate in flavour, but aardy, though the plants make few rumners. Fruit large, handsome and juicy.

\section{Prince Albert.}

A very large and showy new English variety and a good bearer, but of second rate flavour as compared with Ross' Phoenix or Hovey's Seedling. Fruit of the largest size, ovate, rich scarlet; flesh rather solt and not high flavoured. Staminate.

23. Old Pine, or Carolina. Thomp.

\begin{tabular}{l|l} 
Pine Apple. & $\begin{array}{l}\text { Old Carolina. } \\
\text { Blood Pine. } \\
\text { Carolina. } \\
\text { Old Scarlet Pine. }\end{array}$
\end{tabular}

The Old Pine or Carolina Strawberry is believed to have been carried from Carolina to Fngland many years ago, and nearly all this class of strawterries have been directly or indi. rectly raised from it. It is one of the very richest flavoured sorts. Still, it is only rarely seen in our gardens, as it requirea a rich soil and a considerable degree of care to get crops of truit.

Fruit large, conical, with a longish neck, sometimes cocks. comb shaped; uniform bright scarlet, grains slightly imbelded; flesh solid, juicy, and very rich in Havour. Ripens at the mid. dle of the season. Flowers staminate. 


\section{Ross' Puanix.}

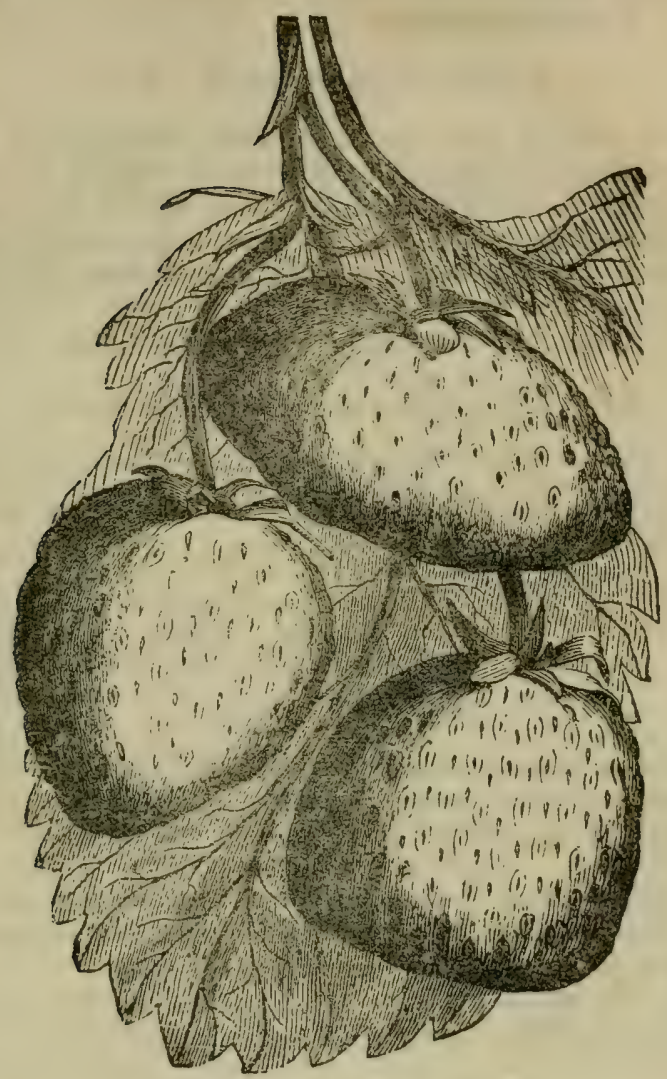

Ross' Phœnix appcars to be suited only to particular soils. In such, it certainly has all the good qualities of the celebrated Keen's Seedling, and is more hardy than that excellent old variety. It appears to demand a decp loamy soil; in such we have secn this sort succeed with the commonest culture, and produce fine crops of truly splendid fruit in gardens where the finest English Pines generally failed with much greater care. It is a native seedling, raised in 1837 , by $\mathrm{Mr}$. Alexander Ross, of Hudson, N. Y., from the Keen's Seedling, which it most resembles, but the leaves are broader, very dark green, with rery coarse serratures, and lie close to the ground. The clusters of fruit are very large.

Fruit very large, generally cockscomb-shaped, or compressed, $45^{*}$ 
of a very dark purplish red, with a polished surface. Flest. firm, of the richest pine flavour. Ripens about the mediun season. Flowers staminate.

\section{Swainstone's Seedling. Thomp.}

A new variety, from England, which fruited well in this country last season, and gives promise of being a very hardy and valuable sort. It ripens quite early, but the fruit matures a long time in succession, and sometimes a second crop is borne in autumn. It grows very strongly, the foliage and flowers are large, and the foctstalks long. Fruit large, ovate, of a heautiful light, glossy scarlet, flavour very delicious. Staminata

\section{Class III. Alpane and Wood Strawberries.}

[Flowers rather small. Leaves quite small, light green, and quite thin. Fruit small, roundish in the Wood Strawberries, and conical in the Alpines, parting more readily from the stalk than other sorts, wich very tender sweet flesh.]

\section{RED Woov. Thomp.}

English Red Wood.

Common Rouge.

Des Bois à Fruit Rouge.

This is the wild strawberry of Europe (F. vesca), long more commonly cultivated in ou: gardens than any other sort, and still, perhaps, the easiest of cultivation, and one of the most desirable kinds. It always bears abundantly, and though the fruit is small, yet it is produced for a much longer time than that of the other classes of strawberries, and is very sweet and delicate in flavour. Flowers always perfect.

Fruit red, small, roundish-ovate. Seeds set even with the surface of the fruit. It ripens at medium season.

\section{WiIte Wood. Thomp.}

This is precisely simlar in all respects to the foregoing, ex sept in its colour, which is white. It ripens at the same time.

\section{Red AlpiNe. Thomp.}

Red Monthly Strawberry.

Des Alpes à Fruit Rouge.

Des Alpes de Tous les Mois à Fruit Rouge, \&c.

The common Red Alpine or monthly bearing strawberry, a native of the Alps, and succeeds well with very trifling care in this country. The $\mathbf{A}$. pines always continue bearing from Iune till November; but a very fine autumnal orop is secured, 
by cutting off all the spring blossnms. The plant resembles the Red IVool, and the fruit is stmilar in Havou: and colour, but long-conical in form. Flowers always perfect.

\section{Vhite Alpine. 'Thomp.}

White Monthly,

Des Alpes à liruit Blanc.

Des Alpes de tous les mois à Fruit Blanc, \&c.

Preciscly similar to the Red $\Lambda$ lpine, except in colour. Fruit sonical, white.

\section{Red Bush Alpine. $\oint$ Thomp.}

Monthly, without Runners.

Buisson.

Commun sans Filets.

Des Alpes sans Filets

The Bush Aipines are remarkable among strawberries for their total destitution of runners. Hence they always grow in neat compact bunches, and are preferred by many per. sons for edging beds in the kitchen garden. The fruit is conical, and the whole plant, otherwise, is quite similar to common Alpines. We think it nne of the nost desirable sorts, and it bears abundantly through the whole season. The Bush Alpines were first introduced into the United States by the late Andrew Parmentier, of Brooklyn. 'To propagate them the roots are divided. Flowers always perfect.

\section{White Busa Alrine.§ Thomp.}

White Monthly, without Runners. Buisson des Alpes Blanc, \&c.

This differs firom the foregoing only in the colour of the fruit, which is conical and white.

\section{Class IV. Hauibois Strawberries.*}

[Leaves lartre, and crumpled, pale green, supported on tall staiks. Frui. stalk tall and erect. Fruit, pole at first, dull purplish at maturit ${ }^{\circ}$.]

\section{Prolific, on Conical. $\oint$ Thomp.}

Musk Hantbois. Double Bearing French Musk Hautbois. Caperon Royal Caperon Hermaphrodite. 
This is a captal variety, and the only one of this class that we consider worth cultivating here. Its strong habit, and very large, usually perfect, flowers, borne high above the leaves, distinguish it. The fruit is very large and fine, lark coloured, with a peculiarly rich, slightly musky flavour. It bears most aburdant crops. Fruit large, conical, light purple in the shade dark blackish purple in the sun, seeds prominent, flesh rathes firm, sweet and excellent. It ripens tolerably early, and some. lines gives a second crop. Staminate.

'I'he Comion Ilatrois, Giobe, Large Flat, \&c., aro scarcely worthy of cultivation here.

\section{Class V. Chili Strawberries.}

[Orixinally from Chili. The leaves are very hairy, the leaflets thick and obtusely serrated; the fruit of monstrous size, pale colsur, and rather insipid flarnur. They are too tender to withstand our cold winters well.]

\section{True Chis. Thomp. \\ Pataronian. \\ Greenwell's new Gianc. \\ Greenwell's French.}

Fruit very large, bluntly conical or ovate, dull rerl, seeds dark brown, projectung, flesh very firm, hollow-cored, of a rather indifferent, sweet flavour. Ripens late.

\section{Wilmot's Strerb. Thomp.}

An Finglish soedling, raised from the forecromg-very showy in size but indif rene fruit and a por bearer. Fruit roundish, sometimes cockscomb shaped, surface pale scarlet, polished, seeds projecting, flesh holluw and of only tolerable flavour. Medium season.

\section{Yellow Chili. Thomp.}

Fruit very large, irregular in form, yellow with a brown cheek, seeds slightly imbedted. Flesh, very firm, rather rich.

\section{Class VI. Green Strawberres.}

[I.ttle valued or cultivated, being nuore curic us than good. They rnsem ble, in cencral appeararce, the Wood strawberries. Lewes light green, much plaited. Flesh solid. There are severul surts growa by the French, bu: the fullowing is the only one of any value, and it is a shy bearer.] 
36. Green Stratherry. Thomp.

Green Pine. Fraisier Vert.

Green Wood. ' Powdered Pine.

Green Alpine.

Fruit small, roundish or depressed, whitish green, and at ma, turity, tinged with reddish brown on the sunny side. Flesk solid, greenish, very juicy, with a peculiar, rich, pineappla flavour. Ripens late.

Selection of the finest strawberries for a small garden. Large Early Scarlet, Hovey's seedling, Black Prince, Hudson's Bay, British Queen, Prolific Hautbois, Red, and White Alpine.

Selection of sure bearing and very hardy kinds for itiv North. Large Eurly Scarlet, Hovey's seedling, Black Prince, Prolific Hautbois, Red Wood, White Wood.

Varieties requiring care, but very fine in deep rich soils. Bishnp's Orange, Elton, Myatt's Pine, Uld Pine, Downton.

\section{CIIAPTER XXVII.}

Cucumis MIeln, L. Cucurbitacer, of botanists.

Melon, of the French: Melona. German; Meleon, Dutch; Melone, Italian; and Melon, Spanish.

The Melon (or musk melon) is the richest and most luscious of all herbaccous fruits. The plant which bears this fruit is a trailing annual, supposed to be a native of Persia, but which has been so long in cultivation in all warm climates that it is quite doubtful which is its native country.

The climate of the Middle and Southern States is remarkably favourable for it-indeed far more so than that of Englind, France, or any of the temp rate portions of Europe. Cons quently melons are raised as ficld crops by market garleners, and in the month of August the finest citrons or areen-fleshed melons nuy be seen in the markets of New York and Philarlelphia in immense quantities, so abundant in inost seasons as frequently to be sold at half a dollar per basket, containing nearly a bushel of the fruit. The warm dry soils of Long Island and New Jersey are peculiarly favourable to the growth of melons, and even at low prices the product is so large that this crop is one of the most profitable.

Cularure.- - The culture of the melon is very casv in all, exerpt the most northern portions of the United States. Warly in May, a piece of rich, light soil is selected, well manured and thorouglily dug, or prepared by deep ploughing and ha:rowing. 
IIills are then marked sut, six fect apart each way. These hulls are frepared by digrging a foct deep, and two feet across, which are filled half full of gool, well-rotted manure. Upon the latter are thrown three or four inches of soil, and both ina nure and soil are then well mixed together. More soil, well pulverized, is now thrown over the top, so as to complete the hill, making it three inches higher than the surface. Upon this, plant eight or ten grains of seed, covering them about half an incli deep.

When the plants have made two rough leaves, thin them so as to leave but two or three to each hill. Draw the carth nicely around the base of the plants with the hoe. And to pre. vent the attack of the striped cucumber bug (Galereuca villata), the great enemy of the milon and cucumber plants, sprinkle the soil just beneaih the plants, as soon as they come up, with guano. The pungent smell of this manure renders it an effect. ual protection both against this insect and the cucumber flea beetie, a little black, jumping insect, that also rapidly devours its leaves in some districts; while it also gives the young planis a fine start in the early part of the season.

As soon as the runners show the first blossom buils, stop them, by pinching out the bud at the extremities. 'This will cause an increased production of lateral shoots, and add to the size of the fruit. Nothing more is necessary but to keep the surface free from weeds, and to stir the soil lightly with the hoe, in field culture. In gardens, thinning the fruit, and placing bits of slate, or blackened shingles under each fruit, improve its size and flavour.

To retain a fine sort of melon in perfection, it should be grown at some distance from any other sort, or even from any of the cucumber family, otherwise the seeds of the next gene. ration of fruit will be spoiled by the mixture of the pollen.

Varieties. - More than seventy varieties are cnumerated in the catalogue of the London Horticultural Socity's garden, but many of these do not succeed without extra care in this coun. try, which their quality is not found to repay. Indeed what is popularly known as the Citron mclon, one of the finest of the green fleshed class, is the greatest favourite with all American gardeners. It is high flavoured, uniformly good, very productive, and in all respects adapted to the clinate.

Melons may be divided into three classes-the Grecn-Fleshed, as the citron, and nutmer; Yellow-Flesherl, as the cantelopes; and Persiun Melons, with very thin shins and the most melting honey-like flesh, of delicious flavour. The Green-Fleshed melons are of very rich flavour and roundish form; the Yellow. Fleshed are large, usually oval, and of second rate flavour: 
the Persian melon, the finest of all, but yet scarce with us, re. quiring much care in cultivation, and a fine warm season.*

\section{Class I. Green-Fleshed Melons.}

1. Crtron. This is much the finest melon for general cul ture. Fruit rather small, roundish, flattened at the end, regularly ribbed and thickly netted; skin deep green, becoming pale greenish yellow at maturity ; rind moderately thick, flesh green, firm, rich, and high flavored. Ripens pretty early and bears a long time.

2. Nutueg. An old variety, often seen impure, but when in perfection, very melting and excellent. Fruit as large again as the foregoing, roundish oval; skin very thickly netted, pale green, slightly but distinctly ribbed; rind rather thin, flesh fale green, very melting, sweet and good, with a high mushy flavour. Medium season.

3. Frankin's Green-Fleshed. Very excellent and pro. ductive. Fruit rather large, roundish; skin very slightly netted, greenish yellow when ripe; flesh green, exceedingly ten. der and rich.

4. Improved Green-Flesh. A new English variety, of exquisite flavour. Fruit pretty large, roundish, not ribhed, slight

* We hone te see the Persian melon more common in our gardeng, as its ex puisite flavour richly repays the additional care it requires It almost always requires in this country the aid of a slight hot-bed to forward the plants in the spring, and needs the use of the glass frequently even in summer, during cold nights and stormy weather. The foliowing treatment will produce fine Persian melons in this latitude, and south of this probably less care will 've necessary.

About the middle of April prepare a slight hot-bed, not more than three feet wide, and as lone as may be desired. Cover the manure of the hotbed with a mixture of two thirds fresh loamy soil taken from just below the turf o I the road sides, and one third sand, mixing with the whole guann, or p geon dung - the best manure for the melon, at the rate of half a peck of guano, or a peck of pigeon dung, to a bed ten feet long. Give the plants as much air as possible, consistent with vigorous growth, till the last of June-taking off the sashes altogether during a few hours in the middle of very warm bright days. All this time the soil must be kept pretty moist by frequent watering, which should be applied on the surface of the ground, and not over the leaves of the plants.

After the first of July, if the summer is a warm one, the glase may be taken off almost entirely during the day-or only restored to it in cold stormy weather, or in sudden changes of temperature. If the season should not be as warm and fine as ordinary, the beds may be kept partially covered to ripen the fruit. It should be borne in mind that the Persian melon requires a very dry, warm atmosphere, and a very moist soil. In Peraja the beds are irrigated by small streams of water running between them, and when it is practicabie this should be put in practice here, or otherwise cho soil should be reguiarly watered. It is best to lay some light branches : re: the surface, on which to train the vines. 
ly netted; skin thin, pale yellow at maturily; flesh thick, green, and of very delicious flavour.

5. BeEchwood. One of the very best of this class. Fruit of medium size, oval, netted, skin greenish yellow; flesh palo green, rich and very sugary. Ripens early.

\section{Class II. Yellow, or Orange-Fleshed Melons.}

6. Early Canteloup. Early and productive-its chief merits. Fruit small, nearly round, skin thin, smooth, ribbed nearly white; flesh orange colour, of sweet and pleasant flavour. The first melon ripe.

7. Netted Canteloup. The best flavoured of this class, often quite rich. Fruit rather small, round, skin pale green, closely covered with net-work; flesh dark reddish-orange, fla. vour sugary and rich.

8. Black Rock (or Rock Canteloup). A very large melon, frequently weighing 8 or 10 pounds, and of pretty good llavour. Fruit round, but flattened at botin ends, covered with knobs or carbuncles; skin dark green, thick; flesh salmon coloured, sweet, but not rich. Ripens rather late.

\section{Class III. Persian Melons.}

9. Keising. One of the very finest and most delicate fla voured of melons. Fruit rather large, egg-shaped, skin pale lemon colour, regularly netted all over; flesh nearly white, high flavoured, and "texture like that of a ripe Beurré pear."

10. Green Hoosainee. One of the best for this climate, and bears well. Fruit egg-shaped, of medium size, skin light green, netted; flesh pale greenish white, tender and abounding with sugary, highly perfumed juice. Seeds large.

12. Sweet Ispanian. The most delicious of all melons. Fruit large oval; skin nearly smooth, deep sulphur colour; flesh greenish white, unusually thick, crisp and of the richest and most sugary flavour. Ripens rather late.

13. LaRGE Genmek. Early, good bearer, and very excellent. Fruit of large size, roundish, flattened at the ends, and ribbed, skin green, closely netted; flesh greenish, firm, juicy, rich and high flavoured.

Besides the foregoing there are Winter Melons from the South of Europe, very commonly cultivated in Spain, which, if suspended in a dry room, may be kept till winter. The Green 
Vatiencta and the Danipsha are the three principal sorts; they are oval, skin netted, flesh white, sugary and good.

\section{CHAPTER XXVIII.}

\section{THE WATER-MELON.}

Cucurbita citrullus, L. Cucurbitacea, of botanists.

Pasteur, of the French; Wasser Melone, German; Cocomero, Italian

$\Gamma$ HE water-melon is a very popular and generally cultivated iruit in this country. The vine is a training annual of the most vigorous growth, and the fruit is very large, smooth, and green, with a red or vellow core. Though far inferior to the melon in richness, its abundant, cooling juice renders it very grateful and refreshing in our hot midsummer days. Immense fields of the water-melon are raised in New Jersey and Long Island, and their culture is very easy throughout all the middle and southern states.

The cultivation of the water-melon is precisely similar to that of the melon, except that the hills must be eight fect apart. The finest crops we have ever seen, were grown upon old pieces of rich meadow land, the sod well turncd under with the plough at the last of A pril, and the melons planted at once.

'The following are its best varieties.

1. IMPERIAL. A remarkably fine flavoured and very pro. ductive sort, from the Mediterranean. Fruit of medium size, nearly round. Skin pale green and white, marbled, rind re. markably thin, flesh solid to the centre, light red, crisp, rich, and high flavoured. Seeds quite small, light brown.

2. CA ROLINA. The large common variety. Fruit very large, oblong, skin dark green and white marbled, rind thick, fiesh deep red, hollow at the centre, sweet and good, seeds large black.

There is also a sub-variety with pale yellow flesh and white scerls.

3. Sraxisu. A rich and very excollent water-melon. Fruit large, oblong; skin very dark blackish-green, slightly mirbled, rind moderately thick, flesh red, solid, rich, and very sweet.

The Citron Water-Melon is a small, round, pale green, inarbled sort, ripening late, and csteemed by many for pre. serving. 


\section{CHAPTER XXIX.}

\section{The Orange Famili.}

\section{Citrus, L. Aurantiacea, of botanists.}

Tue Orange family includes the common orange, (Citru. aurantium;) the Lemon, (C. limonum ;) the Lime, (C. linclla ;: the Shaddock, (C. decumana;) and the Citron, (C. Medica;) all diferent species, with the same general habit.

The Orange, a native of Asia, is the most attractive and beautiful of fruit trees, with its rich, dark evergreen foliage, and its grolden fruit; and it may well therefore enioy the repu. tation of being the golden apple of the Hesperides. When to these charms we add the delicious fragrance of the blossoms, sur. passing that of any other fruit tree, it must be conceded that, though the orange must yicld in flavour to some other fruits, yet, on the whole, nothing surpasses an orange grove, or orchard, in its combination of attractions-rich verdure, the delicious aroma of its flowers, and the great beauty of its fruit.

'The south of Europe, China, and the West Indies, furnish the largest supplies of this fruit. But it has, for a considerable time, been cultivated pretty largely in Florida, and the orange rroves of St. Augustine yield large and profitable crops. Inileed, the cultivation may be extended over a considerable portion of that part of the Union bordering on the Gulf of Mexico; and the southern part of Louisiana, and part of Texas, are highly favorable to orange plantations. The bitter orange has become quite naturalized in parts of Florida, the so-called wild orange seedlings furnishing a stock much more hardy than thos a produced by sowing the imported seeds. By continually sowing the seed of these wild oranges, they will furnish stocks suited to almost all the Southern States, which will in time render the better kinds grafted upon them, comparatively hardy.

North of the latitude, where, in this country, the orange can be grown in groves, or orchards, it may still be profitably cul. tivated with partial protection. The injury the trees suffer from severe winters, arises not from their freezing-for they will bear, without injury, severe frost-but from the rupture of sap-vessels by the sudden thawing. A mere shed, or covering of boards, will guard against all this mischief. Accordingly, towards the south of Europe, where the climate is pretty severe, the orange is grown in rows against stone walls, or banks, in terraced gardens, or trained loosely against a sheltered tre!lis; and at the approach of winter they are covered with a slight moveable shed or frame of boards. In mild weather, the sliding 
doors are opened and air is admitted freely--if very severe, a few pots of charcoil are placed within the enclosure. This covering remains over them four or five months, and in this way the orange may be grown as far north as Baltimore.

Soll and Culture. - The best soil for the orange is a deep rich loam. In propagating them, sow, early in the spring, the seeds of the naturalized or wild bitter orange of Florida, which gives much the hardiest stock. They may be budded in the nursery row the same season, or the next, and for this purpose the earliest time at which the operation can be performed (the wood of the buds being sufficiently firm), the greater the suc:cess. Whip, or splice grafting, may also be resorted to early in the spring. Only the hardiest sorts should be chosen for orchards or groves, the more delicate ones can be grown easily with slight covering in winter. Fifty fect is the maximum height of the orange in its native country, but it rarely forms in Florida inore than a compact low tree of twenty feet. It is better therefore to plant them so near as partially to shade the surface of the ground.

INSECTS.--The orange plantations of Florida have suffered very severely within a few years, from the attacks of the scale insect (Coccus Hispcridum), which, in some cases, has spread over whole plantations and gradually destroyed all the trees. It is the same stuall, oval brownish insect so common in our greenhouses, which adheres closely to the bark and under side of the leaves. All efforts to subdue it in Florida have been nearly unavailing.

A specific, however, against this insect has lately been discovered in England. It is the use of the conmon Chamomile. It is stated that merely hancing up bunches of fresh chamomile herb in the branches, destroys the scaled insect, and that cultivating the plants at the roots of the trees is an effectual preventive to the attacks of this insect. Where the bark and leaves are much infested, we reconmmend the stem and branches to be well washed with an infusion of fresh chamomile in water, and the foliage to be well syringed with the same. Repeating this once or twice, will probably effectually rid the trees of the scaled insect.

Another very excellont remedy for this and all other insects that infest the orange, is the gas liquor, of the gas works, largely diluted with water, and showered over the leaves with a syringe or engine. As this liquor varies in strer.gth and is sometimes very strongly impregnated with ammonia, it is difficult to give a rule for its dilution. The safest way is to mix some, and apply it at first to the leaves of tender plants; if too strong, it will injure them ; if properly diluted it promotes vegretation, and estroys all insects.

VARIETIES.-From among the great number of names thas 
figure in the Europiean catalogues, we select a few of those really deserving attention in each class of this fruit.

\section{TIE ORANEE.}

The Orange (Oranger, French; Pomeranze, German; Arancio, Italian; and Naranja, Spanish) is on the whole the finest tree of the genus. Its dark green leaves have winged foot-stalks, its fruit is round with an orange coloured skin. It is one of the longest lived fruit trees, as an instance of which we may quote the celebrated tree at Versailles, called "the Granil Bourbon," which was sown in 1421 , and is at the prosent time in existence, one of the largest and finest trees in France.

The fruit of the orange is universally esteemed in its ripe state. 'The bitter orange is used for marmalades; the green fruits, even when as small as peas, are preserved, and used in various ways in corfectionary; the rind and pulp are used in cooking; and the orange flowers distilled, give the orange flower water, so highly esteemed as a perfume and in cook ry.

Besides the COMnon sweEt orange, the most esteemed sorts are the MALTESE, and the BLOOD-RED, both of execllent flavour with red pulp. The MANDARIN Orange is a small, flattened fruit, with a thin rind scparating very easily from the pulp, frequently parting from it of itself, and leaving a partially holInw space. It cones from China, and is called there the Manda. rin, or noble orange, from its excellent quality. 'The flesh is dark orange coloured, juicy and very rich.

The ST. MrchaEL's orange is a small fruit, the skin pale yellow, the rind thin, the pulp often seedless, juicy, and lusci. ously sweet. It is considered the most delicious ol all oranges. and the tree is a most abundant bearer.

The Seville, or bitter orange, is the hardiest of all the varieties, enduring very hard frosts without injury. It has the largest and most fragrant flowers: the pulp, however, is bitter and sharp, and is valued chiefly for marmalades. The Double Bigarde is a French variety, of this species, with fine double blossoms.

The Berganor orange has small flowers, an! pear-shaped fruit. 'The laves, fl wers, and fruit, being peuliarly fragrant, it is highly esteemed by the perfumer, and yields the bergamot essences. "The rind, first dried and then moist nod, is pressed in moulds into small boxes for holding swectuseas, to which it communicates a bergamot lavour."

Besides th' above, the Fingered, Suect-sinnerl, Pear-shaped and Ribbed oranges, are the most striking sorts, all chiefly cul. :ivated by curicus amateurs. 


\section{II -LEMIONS.}

Tyz Isenon (Limoner, of the French and German; Limone, Italian; Limon, Spanish) has longer, paler leaves than the orange, the footstallis of which are uaked or wingless; the fowers tinged with red externally, and the fruit is oblong; pule yellow, with a swollen point, and usually an acid pulp. Its principal use is in making lemonade, punch, and other cooling acid drinks.

Besides the common. Lemon, there is an Italian variety, called tire Sweer Lenow, the pulp of which is sweet and good.

\section{III.—THE LIME.}

THE Lime (Limetlier, of the French) differs from the Lemon by its snaller, entirely white flowers, and small, roundish, pale yullow fruit, with a slight protuberance at the end. 'The acid, thou sh sharp, is scarc ly so rich and high as that of the lemon, and is used for the.same purposes. The green fruit is more esteemed than any other for preserving. The Italians cultivate a curiously marked variety called Pomo d'Adamo, in which Adain is said to have left the marks of his teeth.

\section{IV.-THE CITRON.}

TuE Citron (Cidratier of the French; Citronier, German; Cedrato, Italian) is one of the finest growing trees of this family, with large, oblong, wingless leaves, and flowers tinged with purple externally. 'The fruit, shaped like that of the I: mon, is much larcer, of a yellow colour, warted and furrowed externally. The rind is very fragrant, and very thick, the pulp is subacit, and is used in the same way as that of the lemon. It is chicfly valued lowever for the rich sweetmeat or preserve, called catron, made from the rind.

The Madras citron is considered the largest and best variety.

\section{V.-THE SHADDOCK.}

Tre Shaddock (Pampelmous, French; Arancio massimn, Ita. lian) may be considered a monstrous orange, with a compara. tive! tastrless pulp. It is a native of China and Japan, and has its name from I.r. Shaddock, who first carried it to the West $46^{*}$ 
Indies. The !eaves are winged, like those of the orange, the flowers white, and the fruit globular. Its size is very large. as it often weighs six or eight pounds. 'The pulp is sweetish, or sub. acid, and the juice is rather refreshing. It is, however, more showy than useful, and certainly makes a magnificent appear. ance in a collection of tropical fruits *

\section{CHAPTER XXX.}

TIIE OLIVE.

Olea Europea, L. ; Oleince, of botanists.

Olivier, of the French; Oehlbaum, German; Ui:vo, Italian; Olivo, Spanish.

The Olive, which, as Loudon justly remarks, furnishes, in its invaluable oil, the cream and butter of Spain and Italy, will undoubtedly one day be largely culiivated in our Southern States. Already snall plantations of it have been formed by a few spirited gentlemen in Georgia and Mississippi, and its adaptation to the Southern parts of the Union near the sea-coast, tested. The apathy of Southern planters generally, respecting all products but cotton and rice, is the only reason for the tardy manner in which this and other valuable trees are introduced into cultivation there.

The uses and value of the olive-oil are still comparatively anknown in this country. In the South of Europe it is more raluable than bread, as, to say nothing of its wholesomeness, it enters into every kind of cookery, and renders so large a quantity of vegetable food fit for use. A few olive trees will serve for the support of an entire family, who would starve on what could otherwise be raised on the same surface of soil; and dry crevices of rocks, and aimost otherwise barren soils in the deserts, when planted with this tree, become flouristing and valuable places of habitation.

The olive is a native of the temperate sea-coast ridges of Asia aud Africa; but it has, time out of mind, been cultivated in the South of Europe. It is a low evergreen tree, scarcely twenty feet high, its head spreading, and clothed with stiff, nar. row, bluish green leaves. Its dark green or black fruit is oval,

* To those of our readers who desire to pursue this branch of the subject, we recommend that silsulid wor's, the Iristoire Naturlle des Orangers, of Risso and Poitesu, wilh superb culuured plates of every variety. Paris, folio, 1818. 
the hard fleshy pulp enclosing a stone. In a pickled state the fruit is highly esteemed. 'The pickles are made by steeping the unripe olives in ley water, after which they are washed and bottled in salt and water, to which is often adderl fennel. or some kind of spice. The oil is made by crushing the fruit to a paste, pressing it through a coarse hempen bag, into hot water, from the surface of which the oil is skimmed off. 'The best oil is made from the pulp alone: when the stone also is crushed, it is inferiour.

Propagation and Culture.-A very common mode of pro. parating the olive in Italy, is by means of the uovoli (little eggs). "These are knots or tumours, which form in considerable numbers on the bark of the trunk, and are easily detached by girdling them with a pen-knife, the mother plant suffering no injury. 'They are planted in the soil like bulbs, an inch or so deep, when they take root and form new trees. It is also pro. pagated by cuttings and sceds. The seedlings form the strongest and thriftiest trees; they are frequently soine months in vegetating, and should therefore be buried an inch deep in the soil as soon as ripe.

The wild American olive (Olea Americana, L.) or Devil-wood, a tree that grows more or less abundantly as far North as Virginia, will undoubtedly prove a good stock, on which to engraft the European olive. It is of a hardier habit, and though worthless itself, may become valuable in this way.

The olive-tree commences bearing five or six years after being plarted. Its ordinary crop is fifteen or twenty pounds of oil per annum, and the regularity of its crop, as well as the great age to which it lives, renders an olive plantation one of the most valuable in the world. IVith respect to its longevity, we may remark, that there is a celebrated plantation near Terni, in Italy, more than five miles in extent, which, there is every rea. son for believing, has existed since the time of Pliny.

The olive is not a very tender tree. It will thrive farther north than the orange. The very best sitcs for it are limestone ridges, and dry, crumbling, limestone, rocky regions al ways pro. duce the finest oil. 'The tree, however, thrives most luxuriantly in deep, rich, clayey loans, which should be rendered more suitable by using air-slacked lime as manure. It requires comparatively little pruning or care, when a plantation is once fairly established.

VARIETIES. - There are numberless varicties cnumerated in the French catalogues, but only a few of them are worth the attention of any but the curicus collertor. The cominon European olive is, on the whole, much the best for general cultivation, yieldin's the most certain and abundant crops.

The sub-variety most cultivated in France is the Loxg. LIVAed Olive (Olea, e. longifolia), with larger ond longey 
leaves; the fruit nearly of the same size as that of the com mon olive.

The favorite sort in Spain is the Broad-Leaved Olrve (Olca e. latifolia). Its fruit is nearly double the size of the common slive, and yiclds an abundance of oil, but the latter is so strong in flavour as to be more relished by the Spaniards than by stranger's.

The Olivier a Fruit arrond (Olea sphericas N. Duh.) is a hardy French varicty, which, in a moist rich soil, yields most abundant crops of fine oil.

The Olivier pleuleula (Olea eranimorpha, N. Duh.), or weep. intr olim, is one of the largest and finest trees. Its branches are pendant, its fruit excellent, and the oil pure and abundant. It is a very hardy sort, and grows best in damp valleys.

'The Olivier Picholive (Olea oblonga, N. Duh.) yields the fruit most esteemed for pickling. It grows quite readily in any tolerable soil, and is one of the hardiest varieties.

There are two varieties of the olive, which are said to have becn found not long since in the Crimea, lat. $45^{\circ}$ and $46^{\circ}$, which bear abundant crops of fine fruit, and the trees endure a temperature in winter, of zero of Fahrenheit. These sorts have not yet been introduced into this country, and though it is a desideratum to obtain them and test them at the South, yet it is not unlikely that, in common with many trees similarly reported, they may prove little different from the common olive.

\section{CHAPTER XXXI.}

\section{TIIE POMEGRANATE.}

Punica granatum, L. ; Granatacea, of botanists.

Grenadier, of the French; Granatenbaum, German; Melagrano, Italian; Granado, Spanish

'Trus unique fruit, the most singularly beautiful one that ever appears at the dessert, is a native of China and the South of Europe. It grows and bears very readily in this country, as far North as Maryland and the Ohio river, though the fruit does not always mature well north of Carolina, except in shel. tered places. It is even hardy enough to stand the winter here, and will bear very good fruit, if trained as an espalier, and protected in winter.

The fruit is as large as an apple. Its skin is hard and leathery, of a yellowish orange colour, with a rich red cheek. It is crowned in a peculiar manner with the large caly $x$, which 
remains and increases in size after the flower has fallen. There is a pretty bit of mythological history told by Rapin, the French poet, respecting this fruit. Bacchus once beguiled a lovely Scythian girl, whose head had been previously turned by the diviners having prophesied that she would some day wear a crown, and who therefore lent a willing ear to his suit. 'The fickle god, however, not long after abindoned her, when she soon died of gricf. Touched at last, he metamerphosed her into a pomegranate tree, and placed on the summit of its fruit, the crown (calyx), which he had denied to his mistress while living.

The fruit of the common pomegranate is acid, but the cultivated variety bears fruit of very agrecable sweet flavour. The interior of the fruit consists of seeds enveloped in pulp much like those of the gooseberry, int arranged in compartinents, and of the size and colour of red currants. Medicinally it is cooling and much esteemed, like the orange, in fevers and inflammatory disorders.

'The tree is of low growth, from twe!ve to twenty feet, with numerous slender twiggy branches, and is very ornmental in garden seenery, either when clad with its fine scarlet flowers or decked with fruit, which hangs and grows all summer, and does not ripen till pretty late in the seasun. It is well worthy of a choice sheltered place at the north, on a wall or espalier rail, where it can be slightly protected with mats or straw in winter; and it deserves to be much more popular than it now is in every southern garden. If raised in large quantities there, it would become a valuable fruit for sending to the northern cities, as it is now constantly sent from the south of Europe to Paris and London. Hedges are very often made of it near Genoa and Nice.

Propagation and Culture.-This tree is readily propa. gated by cuttings, layers, suckers or seeds. When by seeds, they should be sown directly after they ripen, otherwise they seldom vegetate. Any good rich garden soil answers well for the Pomegranate-and, as it produces little excess of wood, it needs little more in the way of pruning, than an occasional thinning out of any old or decaying branches.

Varieties. - There are several varieties. The finest, viz.:

1. 'The Sweet-Fruited Pomegranate (Grenadier à Fruit. Doux), with sweet and juicy pulp.

2. The Sub-Acid Fruited Pomegranate; the most com. mon variety cultivated in gardens.

3. 'The Wild, or Acid-Fruited Pomegranate, with a sharp acid flavour; which makes an excellent syrup.

Besides these, there are several double-flowering varicties of the Pomegranate which are very beautiful, but bear nn fruic 
They are also rather more tender than the rruit-bearing ones. The finest are the Double Red Pomegranate, with large and very splendid scarlet blossoms, and the Double White Pome. granate, with flowers nearly white. There are also the rarer varietics, the Yellow-Flowered and the Variegated Flower. ED Poinegranate-seldoin seen here exer.pt in charce green-house collections. 


\section{A P P E NDIX.}

FEMARES ON THE DURATION OF VARIETIES OF FRUIT TREES.

Ir was, for a long time, the popular notion that when a good variety of fruit was once originater from seed, it might be continued by grafting and budding, for ever,-or, at least, as some old parchment deeds pithily gave tenure of land-" as long as grass grows, and water runs."

About fourteen years ago, however, Thomas Andrew Knight, the distinguished President of the Horticultural Society of London, pubiished an Essay in its Transactions, tending entirely to overthrow this opinion, and to establish the ductrine that all varieties are of very limited duration.

The theory advanced by Mr. Knight is as follows: All tho constitutional vigor or properties possessed by any variety of fruit are shared at the same time by all the plants that can be made from the buds of that variety, whether by grafting budding, or other modes of propagating. In simpler terms, all the plants or trees of any particular kind of pear or apple being only parts of one original tree, itself of linited duration, it follows, as the parent tree dies, all the others must soon after die alse. "No trees, of any variety," to use his own words, "can be made to produce blossom or fruit till the original tree of that variety has attained the age of puberty ;* and, under ordinary modes of propagation, by grafts and buds, all become subject, at no very distant period, to the debilities and diseases of old age."

It is remarkable that such a theory as this should have been offered by $\mathrm{Mr}$. Knight, to whose careful investigations the science of modern horticulture is so deeply indebted-as, however common it is to see the apparent local decline of certain sorts of fruit, yet it is a familiar fact that many sorts have also been continued a far greater length of time than the life of any

* This part of the doctrine has of late been most distinctly refuted, and any one may repeat the experiment. Seedling fruit trees, it is well-known. are usually several years before they produce fruit. Rut if a graf. is shserted on a bearing tree, and after it makes one seison fair grcivth, this grafted shoot is bent directly down and tied there, with its point to the stock below, it will, the rext season-the sap being checked-produce flower-buds, and begin to bear, long before the parent tree 
one parent tree. Still the doctrine has found supporter's abroad and at least one huarty advocate in this country.

Mr. Kenrick, in his new American Orchardist, adopts this doc. trine, and in spealing of P'ears, says: "I shall, in the following pares, designate some of these in the class of old varieties, once the finest of all old pears, whose duration we had hoperl, but in vain, to perpetuate. For, except in certain sections of the city, and some very few and highly favoured situations in the country around, they (the old sort?) have becom' tither so uncertain in their bearing-so barren-so unproductive-or so miscaibly blighted-so mortaily diseased-that they are no longer to be trusted; they are no longer what they nnce were with us, and what many of them are still described to be by most forcign writers."

Mr. Kenrick accordingly arranges in separate classes the Old and New Pears; and while he praises the latter, he can hardly find epithets sufïiciently severe to bestow on the former poor unfortunates. Of the Doyeune he says: "This most emincnt of all Pears has now become an outcast, imolerable evin to sight ;" of the Brown Beurré, "once the brest of all Pear's-now become an outcast." The St. Germain "las lung sirce become an abandoned variety," \&c., \&c.

Many persons have, therefore, supposing that these delicious varietie's had really and quietly given up the ghost, marle no more inquiries after them, and only ordered from the nurscries the new varietics. And this, not always, as they have conf ssed to us, without some lintrering feeling of regret at thus abandoning old and tried friends for new comers--which, it must be added, not unfrequently failed to equal the good qualitic sof their predecessors.

But, while this doctrine of Knight's has found ready sup. porters, we are bound to add that it has also met with sturdy opposition. At the head of the opposite party we may raik the most distinguished vegetable physiologist of the age, Professor De Candolle, of Geneva. Varieties, says De Candolle, will endure and remain permanent, so long as man chooses to take care of thern, as is evident from the continued existence, to this day, of sorts, the most ancient of those which have been described in books. By negligence, or through successive bad seasons, they may become diseased, but careful culture will restore them, and retain them, to all appearance, for ever.

Our own opinion coincides, in the main, with that of De Candolle. While we admit that, in the common mole of propagation, varieties are constantly liable to decay or become comparatively worthless, we believe that this is owing not to natu. ral Imits set upon the duration of a variety; that it does not depend on the longevity of the parent tree; but upon the care 
with which the sort is propagated, and the nature of the climate or soil where the tree is grown.

It is a well established fact, that a scedling tree, if allowed to grow on its own root, is always much longer lived, and often more vigorous than the same variety, when grafted upon another st:ck; and experience has also proved that in propor. tior to the likeness or close relation between the stock and the graft is the long life of the grafted tree. Thus a variety of pear grafted on a hcalthy pear scedling, la sts almost as long as upon its own roots. Upon a thorn stock it does not endure so long. Upon a mountain ash rather less. Upon a quince stock still less; until the average life of the pear tree when grafted on the quince, is reduced from fifty years-its ordinary duration on the pear stuck-to about a dozen years. This is well known to every practical gardener, and it arises from the want of affinity between the quince stock and the pear graft. The latter is rendered divarf in its habits, bears very early, and perishes equally soon.

Next to this, the apparent decay of a variety is often caused by grafting upon unhealthy stocks. For although grafts of very vigorous habit have frequently the power of renovating in scine measure, or for a time, the health of the stock, yet the tree, when it arrives at a bearing state, will, sooner or later, suffer from the diseased or feeble nature of the stock.

Carelessness in selecting scions for engrafting, is another fertile source of degeneracy in varieties. Every good cultivator is aware that if grafts are cut from the ends of old bearing branches, exhausted by overbearing, the same feebleness of habit will, in a great degree, be shared by the young graft. And on the contrary, if the thrifty straight shoots that are thrown out by the upright extremities, or the strong limb-sprouts are selected for grafting, they ensure vigorous growth, and healthy habit in the graft.

Finally, unfavourable soil and climate are powerful agents in deteriorating varieties of fruit-trees. Certain sorts that have originaled in a cold climate, are often short-lived and unproductive when taken to warmer ones, and the reverse. This arises from a want of constitutional fitness for a climate different from its natural one. For this reason the Spitzenburgh apple soon degenerates, if planted in the colder parts of New England, and almost all northern sorts, if transplanted to Georgia. But this only proves that it is impossible to pass certain natural limits of fitness for climate, and not that the existence of the variety itself is in any way affected by these local failures.

Any or all of these causes are sufficient to explain the apparent decay of some varieties of fruit, and especially of Dears, over which some cultivators, of late, have uttered so 
many lamentations, scarcely less pathetic than those of Jere. miah.

Having stated the theories on this subject, and given an outline of our explanation, let us glance for a moment at the actual state of the so-called decayed varieties, and see whether they are really either extinct, or on the verge of annihilation.

Mr. Knight's own observations in England led him to cors. sider the English Golden Pippin and the Nonpareil, thcir two most celebrated varieties of apple, as the strongest examples of varieties just gone to decay, or, in fact, the natural life of which had virtually expired twenty years before. A few years longer he thought it might linger on in the warmer parts of England, as he supposed varieties to fall most speedily into decay in the north, or in a cold climate.

Lindley, however, his contemporary, and second to no one in practical knowledge of the subject, writing of the Golden Pippin, ${ }^{*}$ very frankly states his dissent as follows. "This apple is considered by some of our mudern writers on Pomology, to be in a state of decay, its fruit of inferior quality, and its existence near its termination. I cannot for a moment agree with suck. an opinion, because we have facts annually before our eyes completely at variance with such an assertion. In Covent Garden, and indeed in any other large market in the southern or midland counties of England, will be found specimens of fruit as perfect, and as fine, as have been figured or described by any writer, either in this or any other country whatever. Instead of the trees being in a state of "lapid decay" they may be found of unusually large size, perfectly healthy, and their crops abundant; the fruit, perfect in form, beautiful in colour, and excellent in quality." And the like remarks are made of the Nonpareil.

Certain French writers, about this time, gladly seized Knight's theory as an explanation of the miserable state into which several fine old sorts of pears had fallen, about Paris, owing to bad culture and propagation. 'They sealed the death. warrant, in like manner, of the Brown Beurré, Doyenrié, Chau. montel, and many others, and consigned them to oblivion in terms which Mr. Kenrick has already abundantly quoted.

Notwithstanding this, and that ten or fifteen years have since elapsed, it is worthy of notice that the repudiated apples and pears still hold their place among all the best cullivators in both England and France. Nearly half the pear-trees annu. ally introduced into this country from France, are the Doyenne and Beurré. And the "extinct varieties" secm yet to lid de. fiance to theorists and bad cultivators.

But half the ground is not yet covered. How does the theory

- Guxde to the Orenara, by Gearge Lindles. 
work in America? is the most natural inquiry. In this cour. try, we have soil varying from the poorest sand to the richust alluvial, climate varying from frigid to almost torrid-a range wide enough to include all fruit trees between the apple and the orange.

$1 i^{2}$ answer that the facts here, judged in the whole, are de cidelly against the theory of the extinction of varieties. IThie here, as abroad, unfavourable soil, climate, or culture, have produced their natural results of a fueble and diseaserl state of certain sorts of fruit, these are only the cxceptions to tlic general vigour and health of the finest old sorts in the cuutitry at large. The oldest known variety of pear is the Autumin Bergamot-believed by Pomologists to be identically the same fruit cultivated by the Romans in the time of Julius Cesarthat is to say, the varicty is nearly two thousand years old. It grows with a: much vigour, and bears as regular and ahunclant crops of fair fine fluit in our own garden, as any sort we culti. vate. Whole orchards of the Doyenne (or Virgalieu) are in the finest and most productive state of bearing in the interior of this State, and numberless instances in the western states-and any one may see, in Suptember, grown in the apparently coll and clayey soil near the town of Hudson, on the North River, specimens of this "outeast," weighing three fourths of a pound, and of a golden fairness and beauty of appearance and lusciousness of flavour worthy of the garden of the Hesperides.certainly we are confident never surpassed in the lustiest youth of the variety in France. The same is true of all the other sorts when propagated in a healthy manner, and grown in the suitible soil and climate. Wherever the snil is not exthulustra of the proper elements the fruit is beautiful and gnol. The largest and finest crops of pears regularly produced in our own gardens, are by a Brown Beurré tree, only too luxuriant and vigorous. Of the Golden Pippin apple, we can pnint out trees in the valley of the Hudson, productive of the fairest anci finest fru: , and the St. Germain Pears grown by a neighbour here, without the least extra care, are so excellent, that he may fai:' $y$ set them against any one of the newer varieties of $W$ intc $\cdot r$ fruit.

Cn the other hand, we candidly admit that these has becn for sonce time a failure of many sorts of pear and spple in certain aris of the country. All along the scit-const where the snil 2.3 lighl, and has been exhausted, by long cultivation, of lime, potash, and phosphates, the inorganic elements absolutely nccessary to the production of fine pears, many varieties that once flourished well, are now feeble, and the fruit is often blichted.*

'The apparent decline in these districts is owing to the lightzess

- Tha symptnms of the declire ar decay in the pear aro chieriy these 
of the snil, which in this climate, under our not sun (as we have already rematied), lity's the fundation of more than half the diseases of truit-trecs-because, after a few years, the necessary slistenance is exhausted by the roots of a bearing tree, and avery one knows how rarely it is re-supplied in inis country. We can from our cwn observation on the eflects of soil, take a map and mark out the sandy district on the whole sea-boarl, where certain sorts of pears no longer bear good fruit; whilt? within a few miles, on stroug deep loams, the fruit is fair and beautiful-the trees healthy and luxuriant.

Nothing is more convincing, on this point, than to compare the egour and productiveness of the old pears, at the prescnt mo - rent, in the new soils of Rochester and Syracuse, abounding, not i serely with vegetable matter, but with the necessary inorganic curt. with the same sorts grown along the sea-board, in light soils, inere the latter clements are no longer present in sufficient s Junarnce. In the former localities, it is as common to see trecs $\mathrm{c}:$ the nid varieties bearing from ten to twenty bushels of unblerashed rrut annually, as it is in the latter to see them bearing ouly croos of blighted pears.

Recent cxperiments have proved, that it is not sufficient to bring healihy tress oi the old varicties from the interior to the sea-board to insure, in the latter localities, fair and excellent crops. But, or the other hera.t, the complete renovation of blighted trees, in light and exhausec soils, by the plentiful use of wood-ashes, bone dust, lime, ant racksmith cinders, along with common manuse, shows us distinitiv that it is not the age of these varietipg of iruit which causes liteir arparent decline, but a want of that fooid absolutely necessary to the production of healthy fruit.

liat there is anorher nieresting point in this investigation. Do the newly originatea sor's really maintain in the unfavour. able districts the appearance n: perfect health? Are the new pears uniformly healthy where the nld ones are always fieble?

Urcion btedly this quistion must be answered in the negative. Some o: the latest Flemish pears atu ady exhibit symptoms of decay or urd health in these districts. Even Mr. Kenrick, with

The tree, apprarently healthy in the sprina, blossoms, and sets a crop of fruit. Towarde w...jusumer its leaves are disfirured with dark or black spots, and except a fow at the endis, fall from the branches. The fruit is covered with black snerks, often ceases growing when at half it s size, and in the worst cases the skin becomes hard, cracks, and the fruii is entirely worthless. This rusty and diseased state of the skin, is caused by the attack of a minute species of funci (Uredc, Puccinia, etc.), which fasten upon, or are generated in regetable surfaces in a languid state of health 
ull his enthusiasm for the new sorts, is cbligeil to make the

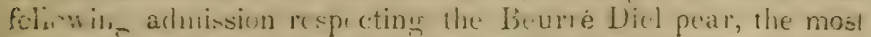

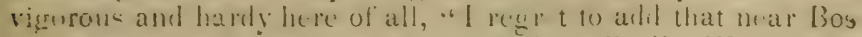
ton this noble fruit is liable to crack hadly." We prediet tha $n$ sny of the Flemish pears orizinated by Tan Hons will berome feeble, and the fruit liable to crack, in the neightnur. lonerl of Bestun, in a much less time than did the old varietics.

Ant this leals us to remark here, that the hardness of any ruruty d pends greatly upon the circumstances of its origin. Wheris a new variety springs up accilentally from a healihy steel in a semi-natural manner, like the Sectiel, the Dix, and other native sorts, it will usually prove the harditst. It is. as it were, an elfin of nature to produce a new individual out of the materials, in a prow ssive state, "hich garde 11 culture has af. forked. Criss-bred seerllings-one parent being of a harly nature, and both healthy-such as Ki.inht's own seedlings, the Monareh an I Dunm re Pears-are next in hardiness. Litstly, we rank varieties reared by Yan Mons' methorl-that of con. inually repeated reprociuctions. This, as Van Mons distinctly states, is an enfeebling process-without any compensating clemont of vigour. Hence it follows as a matter of course, that sterllings of the fifth or sixth generation, as are some of his varietits, must in the ir orierin be of fuble habit. Van Mons himseif was fully aware of this, and therefure resorted to "grafting ly copulation" - in fact, root grafting-well knowing that on common stocks these new varicties would in light soils soon become feeble and decayed. It is necdless for lis to adt that hence we consider the Belgrian mode of producing new varieties greatly inferior to the English one-sirce it gives us varieties often impaired in health in their very origin.

If any further proff of this is desired, we think it is easily foun iy comparing the robust vigour and longevity of many native pear lrees, to be found in the United States-some of them 83 or 100 years old, and still producing large crops of fruit-with the doli. cate trees of several new varieties now in our gardens, from Europe. These varieties are delicate not only with respect to their constitutional vigour, but they are also more susceptille to injury from the severity of our winter's cold and summer's sun.

There are great advantages, undoubtedly, for soils naturally unfivourable, and for small gardens, in grafting the pear upon quince stocks; yet, as it diminishes the vigour of the tree, it is not impossible that continued propagation from dwarf trees may some. what lesson the vital powers and the longevity of a given varieiy.

The decay of varieties of the Apricot, or Peach, much shorter lived trees by nature, we seldom or never hear of. Vari. eties of both are now in cultivation, and in the most perfect vigour of 208 vears' duration. This, probably, is owing to the 
more natural treatment these trees receives generally. Va. rictics of the vine are said never to degetrerate. and this is perhaps owing to their having very rarely been propagated by gratting.*

We are not without remedy for varieties that have partially decayed in a certain district. If the trees have once been pro. ductive of excellent fruit, and are still in a sound condition, though enfeebled, a thorough renewal of their powers will angin restore them to hcalth. To effect this, the soil about the roots should be replaced by new, enriched by manure or peatcompost, and mixcd with the mineral substances named in the preceding pange. The bark of the trunk and large branches sirould be well scraped, aud, as well as all the limbs, thoroughly washed with soft soap. The hear should be moderately pruned. And firally the tree should be suffered to bear no fruit for the two following srasons. After this it will generally bear excellent fruit for several years again. $\dagger$

In making plantations of fine old varieties, in districts where the stock has become fceble, something may be gained by procur. ing grafis or trees from nore fuvourable localitics, where the fruit is still as fuir as ever-and care should be exercised in selceting only the healthicst grafts or trees. Nurserymen in unfivourable districts should (ndeavour to propagate only from trees of henlthy character, and if those in their own variety are discascd, they slould spare ro pains to bring into their nurserics and propagate only such as they fecl confident are healthy and sound. On them, next to the soil, depends very considerably the vigour or debility of the stock of any given variety in the country around them.

In Mr. Knight's original essay on the decay of varietics, he clearly stated a circumstance that most strongly proves what we have here endeavoured to show-viz: that the local decline

* We do not deny that in any given soil there is a period at which a variety of tree or plant exhibits most vignur, and after having grown there awlile it ceases to have its former luxuriance. The same is true of wheat or pot toes, and accurdingly, farmers are in the habit of "changing their seed." The nutriment for a given variety is after a time exhausted from the soil, and uiless it is arain supplied the tree must decline. In light soils this silecdily happesis. In strong, clavey or rocky soils, the naturil decomposition of which affords a continual strire of lime, potash, etc., the necessiry suppiy of inorganic food is maintained, and the variety continues healthy and prodictive.

$T$ It is not uncoms:on to hear it said that the Newtown pippin-that finest of all apples-is de reaterating rapidly. The solution of this is easy. More than any other apple does this one need lime, and high culture. In proof, we may state that never have there been finer Newtown pippins raised, or in sn larre quintities, as at the present moment on the Hudson River One geufleman's urchards supply hundreds, we may say thousands of barrels to the London markets of the fairest, largest, and highest flavoured iruit we huve hrid the ple sure of seeing or tasting. It any one will turn to page 62 , he will speedily see why this variety has not fallen into decay at Pel. tam farm. 
of a variety is mainly owing to neglect, and to grafting on bad slock. We allude to the fact repeatedly verified, that healthy young shoots taken from the roots of an old variety in apparent decline, produce trees which are vigorous and healthy. "The decay," says he, "of the powers of life in the roots of seedling trees is exceeding slow, comparatively with that in the branches. Scicns (ur shoots) obtained from the roots of pear trees two hun. dred years old, afford grafts which grow with great vigour, and which are often covered with thorus like young seedling stocks; whilst cither grafts taken at the same time from the extremities of the branches of such trees present a totally different charac. ter, and a very slow and unhealthy growth. I do not conceive that such shoots possess all the powers of a young seedling, but they certuinly possess no inconsiderable portion of such powers."

This is nuthing more, in fact, than going back to the roots, the portion of the tree least exhausted, for the renewal of the health of a varicty when the branches of the tree have been exhausted by overbearing, etc. It is a simple and easy mode of in. crensiner the ricnur of a sort of delicate habit, to take scions from young root suckers for grafting anew. 'This can of course only be done with trees that grow on their own roots-or have not been grafted. And we suggest it as worth the attention of those interested in gardening to graft feeble sorts on pieces of roots, with a view to establishing them finally on their own roots; or to raise them from layers, a more simple mode of at. taining the object.

Mr. Knight's idea that old varieties first decay in the north, while they yet remain comparatively good in warmer and more southern districts, is by no means borne out by the existing facts in America. On the contrary the decline here, as we have al. ready stated, is almost entirely along the sea-board, and to the southward. In the interior, and to the north, the same sorts are universally fair and excellent, except in cases where a diseased stock has been obtainect from the sea-board, and has not recovered its health by removal. The whole middle and western sections of the country abound, more or less, with the finest pears, of sorts that are in a state of decline on Long Island, in portions of New Jersey, or near Boston. But the influence of the soil, so far as our own observations extend, is, after a certain time, always the same. In this light soil the pear and the npple soon become feeble, because the sustenance afforded by it is, after a time, insufficient to keep the tree in a continual healthy, bearing state. The moisture afforded by it is not great enough to answer the demand made upon the leaves by our hot summer sun. Unless this is remedied by skilful culture, theze fruits must, more speedily fail in health in such districts 
while in more fitoura le ones they will remin as sound ano inealthy as crer.

From these remarls, it will be pererived how important it is in all exhaustcd soils to surfly the necessary food to vario ties that have " in out" from the want of it, ind how unwise ve bu lic ve it to be to reject such incomparable fruits as the No w town pippin, and the Doyenne pear, bccause in certa n local districts, frum causes easily explained, they have become feeble and discased.

Note.-To prevent mice or rabbits from girdling trees.Great injury is done to young orchards in some districts by the meadow mouse. This little animal always works under cover, and therefore does its mischief in winter when the sunw lies deeply upon the ground. A common and effectual mode of deterring it is that of treading down the snow firmly about the stem directly after every fall of snow. But this is a very troublesome affair.

The following mixture will be found to be an effectual preven. tion. Take one spadeful of hot slaked lime, one do. of clean cows-dung, half do. of soot, one handful of flowers of sulphur, mix the whole together with the addition of sufficient water to bring it to the consistency of thick paint. At the approxch of winter paint the trunks of the trees sufficiently ligh to be beyond the reach of these vermin. Experience has proved that it does no injury to the tree. A dry day should be chosen for its application.

English nurserymen are in the halit of protecting nurseries of small trees from the attacks of ralbils, simply by distributing through the squares of the nursery coarse matches made by dip. ping bunches of rags, or bits of tow, in melicd sulphur, and fast. ening these in split stakes a couple of feet high. 'The litter aro stuck into the ground, among the trees, at from 12 to 20 fiel apart, and are said completely to answer the purpose.

NoTE. - Wash for the trunks and branches of fruit lrees.The best wash for the siems and branches of fruit trees is made by dissolving two pounds of potash in two gallons of water. This is applied with a birush at any season, but, per. haps, with most effect in the spring. One, or, at most, two applications will rid the stem of trees of the bark louse, and render it smooth and glossy. It is far more efficacious than whitewash, as a preservative against tue attacks of insects, while it promotes the growth of the tree, and adds to the natural lively colour of the bark.

The wash of soft soap is also a very good one for many purposes. 'Though not equal for general purposes to the potash wash, it is better for old trunks with thick and rigid bark, 
as a portion of it remains upon the surface of the bark for some time, and with the action of every rain is dissolveu, and thus penetrates into all the crevices where insects may bo lodged, destroying them, and softening the bark itself.

Note.-Key to French slandard names of Fruit.-To meat the "arts of some of our farming friends, in various parts of the country, who are zealous collectors of fruit, but at the same time are more familiar with plough-handles than with the sound of Monsicur Crapand's polite vernacular, we have prepared the following little key to the pronunciation of such French names as are necessarily retained among the standard varieties.

So long as these sorts must retain their foreign names, it is very desirable that they should be correctly pronounced. To rive to these French terms what appears to nerely English readers the proper sound is often as far as possible from the true pro. nunciation. A skilful Hibernian gardener puzzled l:is em. pioyer, a friend of ours, during the whole month of $S$ ptember with some prars that he persisted in calling the "Lucy Bony," until after a careful comparison of notes, the latter found he meant the Louise Bonne.

We have, therfore, in the following, eschewed all lotters with signs, and given, as nearly as types alone will permit us: the exact pronunciation of the French names.

KEY TO FRE NC II NANES .

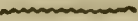

\section{APPLES}

Court Pendu Plat.-Coor Pahn du Plah.

Drap d'Or-Drah dor.

Fenouillet Gris-Fen-nool-yai Gree.

Male Carle.-Mal Carl.

Pomme de Neige.-Pum de Naije.

Reinette Blanche d'Espagne. - Ren-ett-Blansh d'Esp.gs.

Reinette Triomphante.-Ren-ett Tre-ome-fant.

\section{APRICOT.}

Albergier-m-Al-bare-je-ai. Briançon.-Bre-ahn-sohn. 
CHERIIES.

Bolle du Choisv. - Bel de Shwoi-sev.

Belle Magnifi j.,e,-Bel Man-gne-feek.

Bigarreau. - Be-gar-ru.

Bigarreau Rouze-Be-gar-ro Rooje.

Bigarreau Couleur de Chair.-Be-gar-ro Coo-lur de Shair

Birarrea' Gros Cœuret.-Be-gar-ro Gro Keur-ai.

Bigrarreau Tardif de Hildesheim.-Be-gar-ro Tar-deef de Hildeshesn.

Gros Biqarreau Rouge. - Gro Be-gar-ro Rooje

Griotte d'Espagne.-Gre-ote Des-pan.

GRAPES.

Chasselas Musqué.-Shah-slah Meus-kay

Chasselas de Frintainebleau. - Shah-slah de Fone-tane-bla

C'iotat, -Se-o-tah

Lenoir,-Lun-war.

\section{NECTARINES.}

Brugnon Violet Musqué.-Brune-yon Ve-o-lay Meus-kay.

Brugnon Musłué.-Brune-yon Meus-kay.

D'Angleterre.-Dahn-glet-are.

Duc du Tellier. -Deuk du Tel-yay.

Abricotée.-Ab-re-co-tay.

\section{PEACFES.}

h 3 lie de Vitry.-Bell de Ve-tree.

Gr. sse Mignonue.-Groce Mene-yon.

Madeleine de Courson.-Mad-lane de Coor-son.

Pavie de Pompune.-Pah-vee de Pom-pone.

Pourprée Háilve.-Poor-pray Hat-eve.

Sanguinole à Chair adhirente.-Sahn-gwe-nole ah Shair Ad-hay-ront

PEARS.

Amiré Joannet.-Am-e-ray Jo-ahn-nay.

Ananas.-An-ali-ah.

Ananas d' Eté.-An-an-ah Da-tay.

Angleterre.-Ahn-glet-are.

Beurré - Bur-ray.

Belle de Bruxelles -Bel-de Broos-ell.

Belle et Bonne-Bel-a-Bun.

Belle Lucrative-Bel-lu-crah-teve.

Beuri é de Capiumont.-Bur-ray de Cap-u-mohn.

Beurré d'Amalis.-Bur-ray Dah-mah-lee.

Beurré Gris d'Hijer Nouveau.-Bur-ray Gree Dee-vair Noo-ro.

Beurré Diel - Bur-ray De-ell.

D̉eurrè Bronzée. - Bur-ray Brone-zay.

Bezi d'Heri.-Ba-zee Daree.

Bezi Vact.-Bazee Vah-ai.

Beurré Crapaud.-Bur-ray Crah-po.

Bezi de Montirny.-Bay-zee de Mon-teen-gnee.

Bon Chretien Fondante-Bone Cray-t:-an Fone-donte.

Boucquia.-Boct-kiah.

Calebasse Grosse.-Cal-bass Groce.

Lapucin.-Cap.u-san.

Chaunonte L très Gros.-Sho-mone-tell tray Gro. 
Compte de Lamy.-Conte de Lah-me.

Colmar Epine.-Cole-mar A-pees.

Crassanne,-Cras-sahn.

Cuisse Madame-Kuees Mah-dam.

D'Amour. - Dam-onr.

De Louvain.-Dul-oo-van

Délices d'Hardenpont-Day-lece Dar-dahn pone.

Doyenné d'Eté -Dwoy-on-nay Day-tay.

Doyenné Panaché-Dwoy-on-nay Pan-ah-Shag.

Dumortier.-Du-mor-te-ay.

Duchesse d'Angoulême.-Du-shess Dong-goo-lame.

Duchesse d'Orléans.-Du-shess Dor-lay-on.

Enfant Prodige-On-font Pro-deeje.

Epine d'Eté. - A-peen day-tay.

Figue de Naples. - Feeg de Nah-pl.

Fondante d'Automne.-Fone-donte do-tonn.

Forme de Dólices - Form de Day-lece.

Forelle.-Fo-rel.

Fondante du Bois.-Fone-dont du Bwoi.

Fortunée.-For-tu-nay.

Franc Réll d'Hiver-Fronk Ray-ahl Dee-varr.

Glout Morceau.-Gloo Mor-so.

Héricart.-Hay-re-car.

Jalousie,-Jail-oo-zee.

Jalousie de Fontenay Vendée.-Jal-oo-zee de Fone-ten-ai Vor-day.

Léun le Clerc.-Lay-on le Clair.

Limon.-Lee-mohn.

Louise Bonne.-Loo-eze Bun.

Madeleine, or Citron des Carmes.-Mad-lane, or Cee-trone day Carp.

Marie Louise,-Mah-re Loo-eze.

Michaux. - Me-sho.

Passans de Portugal.-Pah-sahn de Por-tu-gal.

Pailleau - Pahl-yo.

Paradise d'Automne - Par-ah-deze do-tonn.

Passe Colmar.-Pass Col-mar.

Quilletette.-Keel-tet.

Reine Caroline.-Rane Car-o-lene

Reine des Poires.-Rane day Pwore.

Rousselet Hâtif.--Roos-lay Hat-eef.

Sanspeau. - Sahn-po

Sieulle.-Se-ull.

Sucréa de Hoyerswarda.-Seu-cray de Hoyersworda.

Surpasse Virgalieu.-Seur-pass Vere-gal-yu.

St. Germain,-San Jare-man.

Sylvange.-Seel-vonje.

Vallée Franche -Vol-lay Fronsh.

Verte Longue, - Vairt Longh.

Verte Longue Panachée.-Vairt Longh Pan-ah-shвy.

Virgouleuse.-Vere-goo-leuz.

Wilhelmine,-Wil-el-meen.

PLUMe.

Abricotée Rouge.-Ab-re-co-tay Rooje.

Diaprée Rouge.-De-ah-pray Rooje.

Drap d'Or.-Drah-dor.

Jaune Hâtive. -Jaun Hat-eve.

Mirabelle.-Me-rah-bell.

Précoce de Tours.-Pray-cose de Toor.

Prune Suisse.-Prune Su-ece.

Rovale Hative, - Rwoy-al Hat-evs. 


\section{INDEX TO TIIE DIFFERENT FILITS.}

[The standard names are in Roman letters. The synonymous names id ralic.]

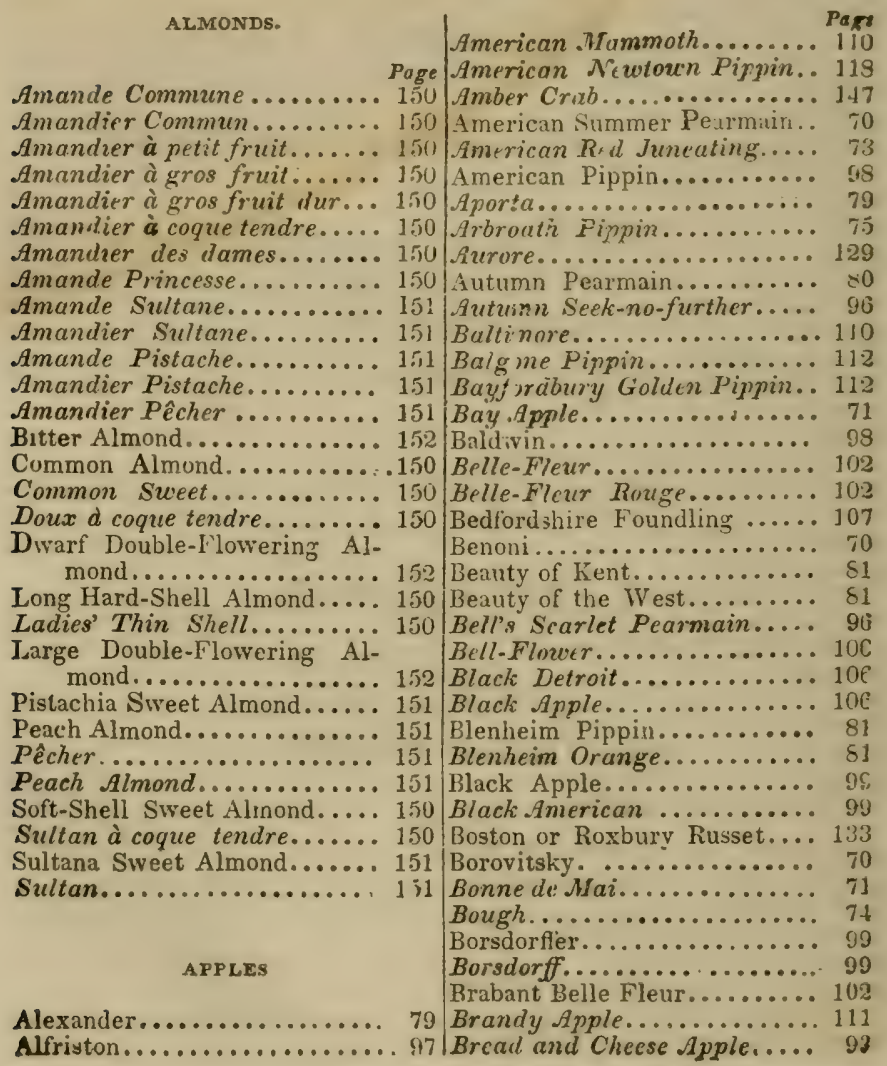




\begin{tabular}{|c|c|}
\hline 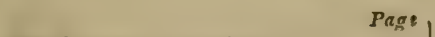 & \\
\hline urlington Greerir:g. & Duchess of Oldenburgh .. \\
\hline$\ldots 1013$ & Due d Arsel... \\
\hline$\ldots \ldots \ldots 121$ & Dutch Codlin.. \\
\hline ...... 103 & atch Mignonn \\
\hline Hiver..... 10.2 & $\ldots \ldots \ldots$ \\
\hline Tiver. ...... i03 & arly Sum \\
\hline e Ruuge .......... 1us & $n \ldots \ldots$ \\
\hline ........ & $\ldots \ldots \ldots \ldots$ \\
\hline$\ldots \ldots$ & e...... \\
\hline$\ldots \ldots$ & . \\
\hline .... & $\cdots$ \\
\hline 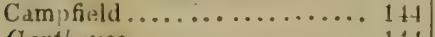 & $\cdots \cdots$ \\
\hline & . \\
\hline & 13 \\
\hline$\cdots$ & 19 \\
\hline ....... & ppin. \\
\hline .......... & $e r \ldots$. \\
\hline$\ldots \ldots \ldots \ldots 122$ & $e \ldots \ldots$ \\
\hline .......... & $\ldots \ldots$ \\
\hline$\ldots \ldots$ & ….... \\
\hline ...... & • \\
\hline 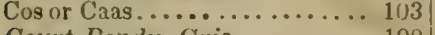 & 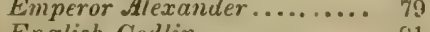 \\
\hline 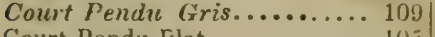 & $\cdots$ \\
\hline$\cdots$ & \\
\hline re & \\
\hline re. & \\
\hline$\cdots$ & \\
\hline$\cdots$ & 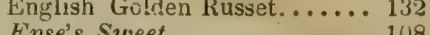 \\
\hline$\cdots$ & 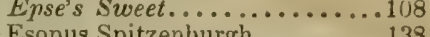 \\
\hline$\ldots \ldots$ & .. 1 \\
\hline ..... & $\ldots \ldots$ \\
\hline :........ & $\ldots \ldots \ldots$ \\
\hline pin... & - \\
\hline & $\ldots \ldots \ldots$ \\
\hline$\ldots .$. & -.......... \\
\hline$n \ldots .$. & - 109 \\
\hline .. 1 & 10 \\
\hline$\ldots \ldots \ldots$ & $(39$ \\
\hline$\ldots \ldots \ldots$ & \\
\hline$\ldots \ldots$ & 83 \\
\hline -.. & $F$ \\
\hline$\cdots \cdots$ & h...... \\
\hline ... & $F_{0}$ \\
\hline . & $\ldots .$. \\
\hline$\ldots \ldots \ldots$ & - 14 \\
\hline ........... 1 & - ings \\
\hline$\ldots \ldots \ldots$ & - $12 \mathrm{~L}$ \\
\hline -.. & . 109 \\
\hline 130 & $\mathrm{~F}$ \\
\hline & - \\
\hline & \\
\hline & \\
\hline 107 & \\
\hline 82 & 1 \\
\hline ton Golden Pippin..... 82 & \\
\hline 113 & Gloria Mundi. . 110 \\
\hline$\ldots \ldots \ldots \ldots \ldots 71$ & ande............ \\
\hline . 109 & $k \ldots$ \\
\hline & \\
\hline
\end{tabular}


Page.

Golden Ball............. 111

iolden Harvey............... 111

Golden Pippin............ 112

Golden Reinette............ 129

Golden Russet........... 131

Golden Sweet..............

Gray Apple..............

Green Bell-flower ...........

Green . Newtown Pippin.....

Green Winter Pippin.......

Grise ..................

Gross Reinette d'Angleterre..

Gregson Apple.............

Gravenstein...............

Grave Slije...............

Grand Sachem.............

Grindstone.................

Grosser Casselar Reinette...

Grune Reinette............

Hartford Sweeting...........

Harrison ................ 145

Hagloe Crab,............. 145

Hawthorden ..............

Hampshire Yellow...........

Hay's Winter.............. 143

Herefordshire Golden Pippin. 112

Herefordshire Pearmain....... 1:21

Hewe's Virginia Crab........ 145

Herefordshire Red Streak.... 146

Hinchman................ 121

Hoary Morning.............. 113

Hollow-cored Pippin......... 101

Holland Pippin............. 86

Hubbardston Nonsuch......... 113

Hunt's Nonpareil........... 120

Hutching's Seedling......... 76

Irish Peach Apple........... 74

Irish Russet............... 134

Ironstone................. 109

Jonathan................ 113

Jnnuarea............. 129

Jersey Greening............ 128

Jersey Sweeting............ 87

July Pippin............... 72

Juneating.............. 78

Kentish Fill-basket .......... 114

Keswick Codlin..............

Kenrick's Autumn ............

Kerry Pippin .................

King Philip................. 113

Kirke's Lord Nelson........... 114

Kirke's Lemon Pippin........ 115

Kirl's Golden Reinette....... 129

Kilham Hill.................

King of the Pippins.............

King George the Third.......

King ....................

Knightwick Pippin ...........

Knight's Golden Pippin......

87

87

88.

Nonpareil ............... 120

Northern Spy.............. 120

Norfolk Pippin............. 123

Norfolk Beaufin..........., $12 \mathrm{C}$

Nonsuch .................. 91

88 Nonsuch................. 91

99 Ohio Favorite............. 101

99 Old Golden Pippin.......... 112

105 Old English Codlin.......... 91

82 Oldaker's.New............ 97 
Page

Dld Nonpareil........... 120

Orange Sweeting.......... 84

Ortley Apple.............. 142

Ortley Pippin............ 142

Triginal Nonparei. ........ 12.

slin

Osgond's Favorite.............

Owen's Golden Beau'y......

Ox.Apple................ 110

Oxford Peach..............

Paternoster Apfel.......... 107

Parmin Royal............. 121

Parmain d' Elc............ 81)

Pepind $d^{\prime} O r \ldots . . . . . . . . . .6112$

Petersburgh Pippin......... 118

Pennock's Red Winter ........ 123

Pennock.................. 125

Pearson's Plate............. 126

Peck's Pleasant........... 126

Pennington's Seedling ........ 1:27

Peach Pund Sweet.............

Pecker.................. 8

Petit Api Rouge.......... 115

Philip Rick............. 113

Phillip's Reinette.......... 105

Pie Apple..................

Pine Apple Russet............

Pomme d'Api Rouge.........

Pompey................ 141

Pomme Grise........... 124

Ponme de Caractire........ 10s

Pomme Royals.............

Pomme Rose .............. 115

Pound Royal.............. 124

Pumme Regelans............ 102

Pomme de Berlin........... 105

Pomme d'Anis.............. 110

Pomme d' Or............. 112

Pomme de Charles.......... 116

Pomme Finale.............. 116

Pomme de Laak........... 107

Pound................... 127

Pomme de Caen.............. 129

Portugal................. 129

Pornme de Neige........... 91

Porter.................. 92

Princesse Noble Zoete...... 105

Priestley .................. 126

Priestley's American......... 126

Prince's Harvest, or Early

F'rench Reinette...........

Pumpkin Russet..............

Pumplkin Sweet.............

Yutnam Russet............. 132

Queen's...................

Rumbo.....................

Rambour Franc..............

Rambour d' Elé............

Ramsdell's Red Pumpkin, Sweet... ......... 13't

Ramsdell's Sweeting........ 137

Read's Baker............. 12C

Red Belle-Fleur ............ 102

Red Wiater C Llville.......... 103

Red Calville............. 103

Red I etroit................ Inf

Red Doctor............ 107

Red or Black Gilliflower...... 134

Red Pumpkin Swect........ 137

Rəd Streak............... 146

Red Quarrenden............ 71

Red Juneating.............., 73

Red As'ruchan .............. 75

Red Ingestrie............. 95

Reinette blanche d'Espagne... 130

Reinette Triomphante....... 130

Reinette $d^{\prime}$ Angleterre ....... 112

Reinette du Canada Blanche. 129

Reinette Grosse du Canada.. 129

Reinette du Canada a Cortes. 129

Reinette d'Aix............ 1:99

Reinette Dorée............ 107

Reinette d'Hollande........ 86

Reinette Batarde............ 99

Reinette de Misnie........... 99

Rhode Islınd Greenin

Ribston Pippin............ 131

Rival Golden Pippin........ 105

Round C'atshead............. 103

Royal Pearmain........... 121

Royale d'Angleterre......... 121

Roxbury Russeting........... 133

Rode Wyn Appel........... 77

Royal Pearmain............ 80

Roman Stem............ 131

Romanite................ 93

Ross Nonpareil ............ 95

Russian................. 105

Russet Golden Pippin...... 112

Russian Emperor........... 79

Saint Julian............. 108

Sam Rawlings............ 113

Sam Young............... 134

Sack Apple.............. 71

Sapson ................... 77

Sam's Crab................ 90

Sanguineus............... 91

Scudamore's Crab.......... 146

Scarlet Perfume............ 71

93 Scarlet Pearmain ........... 96

93 Scarlet Nonpareil............ 120

Seek-no-further............ 93

Seek-no-further ........... $90^{\circ}$

Sheep Nose............. 131

194 Siberian Bitter Sweet........ 146

94 Siberian Crab............ 144 


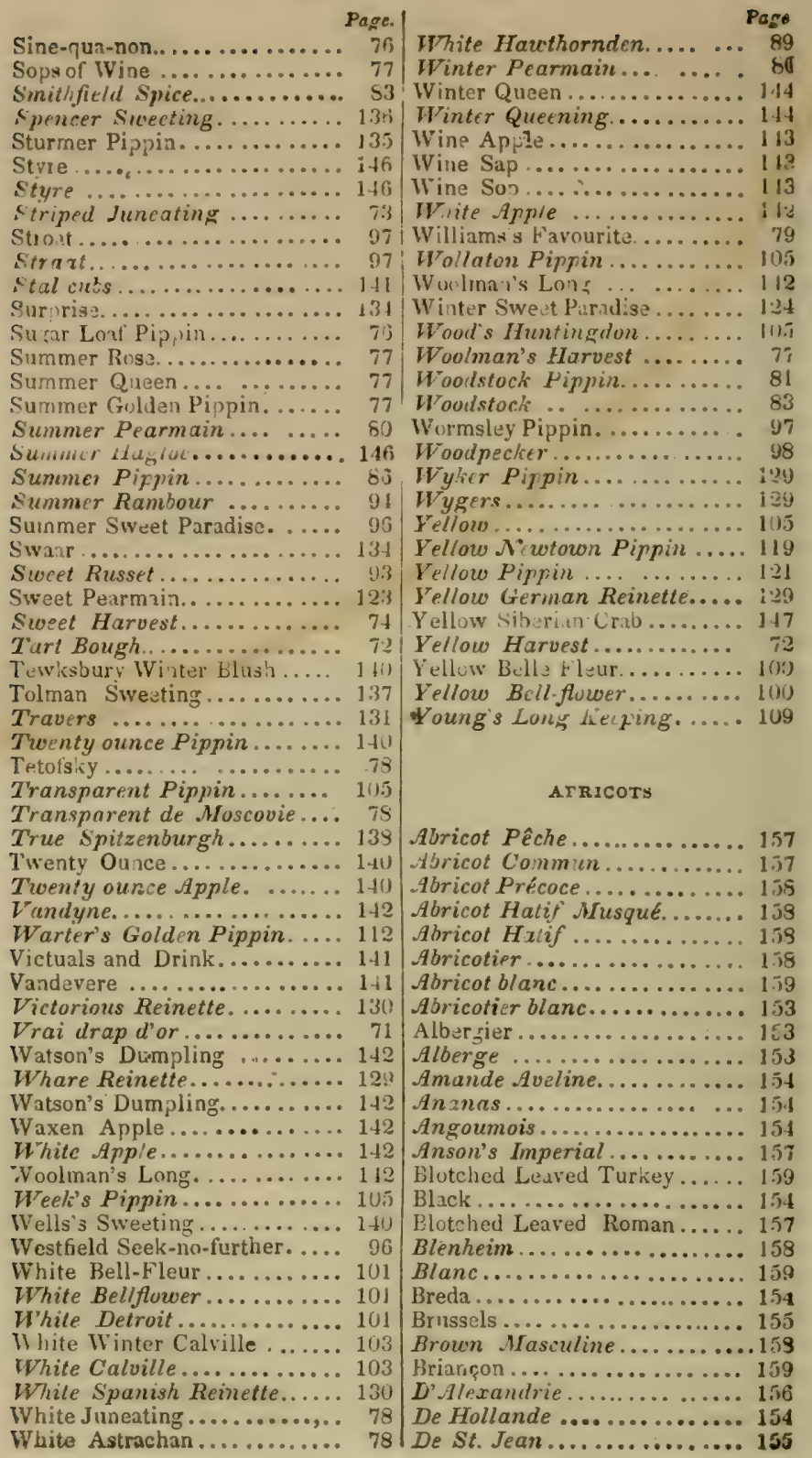


Page

De St. Jean Rouge ........ 1.i. De Nancy ................ 15i5

De Niancy ................ 157

De Nancy.............. 159

Dowhle fluwering Aprict....... litu

I) Pape................. 15.

Dunmore ............... 15.5

Dunmore's Breda........... 15.5

Du Luxembourg. ............ 157

Euriy Orange............. 1.56

Ear.y Masculine........... 1.58

Fariy White Hasculine.... 159

Frictier Miscateller........ 158

Germine................ 157

Gros Préruce............ 15.j

Gros d'Alexandrie........... 155

Gros Frïhe... ........... 155

Grosse Germine............ 157

Hasselnussmandel........... 154

Hemskirke................ 1.55

Hunt's Moorpark............ 1.55

Large Early ............... 155

Large Turkey............. 15?

Moorpark ............... 155

Muscin-MI usch............ 15th

Noir ..................... 154

Olduk sr's Moorpark.......... 15.)

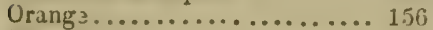

Persizue................. 1.54

Persian.................. 156

Pexch.................... 157

Pêche................... 157

Pêche Grosse............. 157

Pfirsche ................ 157

Purple ...................154

Red Masculine............. 158

Ruyal................... 158

Roman ............... 157

Royal Peach............. 157

Royal Persian...............156

Royal Orange............. 1.56

Royal George............. 156

Shipley's............... 15s

Shipley's Large............ 158

Sullow's Moorpark.......... 155

Temple's ................. 155

Transparent.............. 1.57

Turkey................... 1.50

Violet.................. 154

ITalton Moorpark......... 15.)

White Masculine............ 159

White Apricot............ 1.59

White Algzers.............. 159

Irurtemburg.............. 157

\section{BERBERRIES,}

Black Sweet Magellan $48^{*}$
Peor

Common Red............ 160

Mahonia............... 1

Nepal.................... 161

Seedless................ 161

Stoneless............... in1 $^{\prime}$

Vinetier Sans Voyeau....... 101

\section{CHERRIES.}

À Courle Queue de Provence. 195 Allen's Sucet Alontmorency.. 1:3 Allerheiligen Kirsche........ 2190 American Amber............ 167 Amber Gean............... 168 Ambrer Heart............ 173 Amber à petit fruit.......... 178 American Heart........... 178 Amber or Imperial......... 179 Ambrée de C'hoisy........... 100 Amürée à Gros Fruit.......... 190 Ansell's Fine Black......... 169 Anglaise Tardive.......... 191 Apple Cherry............. 187 Arden's Early White Heart.. 173 Armstrong's Bigarreau...... 181 Arch Duke............... 199 Baumann's May............ 168 Belle de Rocmont........... 182 Belle de Rocmont............ 1s3 Belle de Choisy............. 190 Benham's Fine Early Duke.. 191 Belle Magnifi ${ }^{\prime}$ e.......... 193 Belle et IIIagnifique......... 193 Bigarreau de MIai......... 169 Birarreau................. 17y Bigarreau Royal........... 179 Bigarreau Gros............ 179 Bigarreau Tardif.......... 179 Bigarreau Blanc........... 180 Bigarreau Rouge............ 181 Bigarreau d' Hollande........ 181 Bigarreau Couleur de Chair.... 152 Bigarreau à Gros fruit Blanc. 152 Bigarreau ds Rocmont....... 182 Bigarreau Lauemann....... 133 Bigarreau Gros Cæuret ....... 183 Bigarreau Gros Monstrutux.. 183 Bigarreau à Gros Fruit Rouge 183 Biyarreau Tardif de Hildesheim 1S4 Bigarreau Mfarbrée de Hildesheim.................. 184

Bigarreau Blanc Tardif de Hildesheim.............. 184

Bigarreau Noir............. 185

Bigarreau Gros Noir......... 188

Bigarreautier à Feuı!les de Tabac................ 189

Bigarreautier à Grandes Feu. illes ................. 183 


\begin{tabular}{|c|c|}
\hline$P$ & \\
\hline Bloodgood's Amber.......... 167 & Cluster. \\
\hline Bloodgood's Honey. & Common $I$ \\
\hline loolgood's $\mathcal{N}$ ew Honey..... 107 & me.... \\
\hline lack tieart................ 169 & Couronne. \\
\hline C Caroon.............. 169 & Coroun ... \\
\hline$\ldots 169$ & 15 \\
\hline 70 & $1:$ \\
\hline$\ldots 170$ & ze à Trochct......... 1 \\
\hline .......... 170 & Red......... \\
\hline .. 170 & Commune.. \\
\hline$\ldots \ldots \ldots 171$ & Common Red.......... \\
\hline Honey..............171 & Common So \\
\hline rleans............. 17: & ... $1: 8$ \\
\hline$t \ldots \ldots \ldots \ldots 17 t$ & $\ldots \ldots \ldots$ \\
\hline$\ldots \ldots \ldots \ldots 15 . j$ & y........... \\
\hline $\operatorname{voy} \ldots . .1$ & 3 \\
\hline$t \ldots \ldots \ldots$ & ack.... \\
\hline$\cdots \cdots$ & ...... \\
\hline ........ 1 & $\cdots$ \\
\hline ‥ 1 & \\
\hline ;. 18 & s. \\
\hline 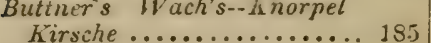 & $\begin{array}{l}\text { Downer.. } \\
\text { Downer's }\end{array}$ \\
\hline norpel & $\begin{array}{l}\text { Downer's Late Red........... } 173 \\
\text { Downing's Red Cheek....... } 196\end{array}$ \\
\hline .. 185 & $s \ldots \ldots \ldots \ldots$ 195 \\
\hline 's Early Duke. & Cherry \\
\hline Oetuber Murillo..... 193 & ntish... \\
\hline Osto3er Zusker & Heart. \\
\hline L............... 193 & 97 \\
\hline$\ldots 194$ & 10 \\
\hline$h \in \ldots \ldots \ldots \ldots \ldots 194$ & $E$ \\
\hline …....... 194 & E0 \\
\hline$\ldots 179$ & Ea \\
\hline re.... & $\ldots \ldots$ \\
\hline .. 19 & ... \\
\hline ... 19 & 95 \\
\hline Tendre..... 1 & $\ldots \ldots \ldots$ \\
\hline$\cdots \cdots$ & ............ \\
\hline e.. 194 & $E$ \\
\hline ugal ..... & $a d .$. \\
\hline et $\ldots . . .$. & hsel........ \\
\hline .. 194 & $E$ \\
\hline aे Fruit Rond 1! & $r y+\ldots$ \\
\hline à Fruit Rond.. & reau..... \\
\hline Fruit Pré & $\cdots \cdots$ \\
\hline Queue.. & 195 \\
\hline ... & $\cdots$ \\
\hline Doubles & k Tartarian... 170 \\
\hline Toussa & 's Black Heart.......... 170 \\
\hline ....... & 's Black........ \\
\hline .. 200 & arische........ 170 \\
\hline St. Martin.... & s White Tartarian.... 178 \\
\hline e Virginie.... & ite Transparent.. 178 \\
\hline$\cdots$ & Kleine Runde Zwerg \\
\hline & $\therefore 195$ \\
\hline & ........ 174 \\
\hline & - 168 \\
\hline Ch & $\because 190$ \\
\hline & \\
\hline
\end{tabular}


Page. ,

Fage

Grafion................ 179

Lauermann's Herz Rirsche. . $18 \AA$

Gruvie Princesse............. 179

Grosse Bigarrean Couleur de Cinair.................. 192

Gius Bigarreau Blane...... 19:3

Gros Ceuret............. 183

Gros Bigarreau Rouge....... 15:3

Gridley................. 157

Grosse Schwarze Knoorpel... 1ss

Griotte de Purtugal......... 199

Griotte Grosse . Vuir......... 191

Griotte d' Espagne........... I9t

Griotte Piécuse............ 191

Grosse Corise Ronge Pale.... 101

Griottier Rouge Pâle....... 11!4

Griotte de Villennes......... 101

$G$-iottier à Bouquet.......... 191

Grinttier Suin Précoce...... 19.5

Gros (robet.............. 19.5

Griotte Ordincire du Nord... 197

G linicr à Frust Noir........ litg

Guigne Grosse Nuir.......... Iniy

Grigne Rouge Hátive....... 174

Grigne Noir Tardive....... 1ss

G'ignier à Feuilles de Tabac. 19.

$G$ lignier à Rameaux Pendans 2.ji)

Hurrison Heart........... 1S1)

Hásive................. 19.)

Herefordshire Black........ 17:2

H:refordshire Heart........ 171

Hildesheimerganz Spale Knor-

pel Kirsche.............. 184

Hildesheimer Spate Herz

Kirsche............... 184

Hildesheim Bigarreau....... 194

Honey................. 17.5

Hollandische Grosse........ 179

Holland Bigarreau........... 1s!

Hulman's Duke............. 192

Hyde's Red Heart........... 17.5

Italian Heart............. 179

Jelirey's Duke............ 190

Jeffrey's Royal............ I90

Jeffrey's Royal Caroon....... 19i)

hentish................ 19.5

Kentish............... 196

Xentish.................. 196

Kentish Red.............. 1:16

Kentish Red.............. 197

K'nevett's Late Bigarreau.... I57

Knight's Early Black......... 175

Kentish................ 197

Large Wild Blach.......... 17:2

Large Huney............ 17.5

Large Wnite Bigarreau...... 18

Zarge Heart-Shapdd Bigarreau................. 142

Eusuermann's Kirsche....... 183

Lauermanits Grosse Kirsche. 183 Large Heart-Shaped Bigie?. reau.................. Ls? Lar se Red Bi Lady Southampton's Yelluw... 1s? Lady Southampton's Duie... 18', Lady Southampton's Golden Drop ................ 197

Large Black Bigurreau...... 188 Late Arch Duke............ 189 Late Duke................. 189 Late Duke................ 191 Large Mayduke............ 191 Late Kentish............. 197 Late May Duke............. 192 Large Alorello............ 197 Late Morello.............. 197 Large Double-Flowering...... 199 Lion's Heart............... 176 Mamning's Early Black Heart.. 169 Mazzard ............... 171 Manning's Mottled........... 176 Mxccarty $\ldots \ldots \ldots \ldots \ldots \ldots \ldots$ 187 Midison Bigarrean.......... 197 Manning's Late Bluck......... 198 MI enning's Late Black Heart. 189 May Duke................ 191 AI xy Cherry.............. 195 Martin's Weichsel........... 2.10 M. rry Cherry............. 171 M:risier ù petit fruit........ 171 M risier à petit fruit noir.... 171 Merisitr à fruit blanc...... 17.5 Mferisier à Aleurs Doubles..... 199 Bifillett's Late Heart Duke.... 191 Milan................... 1977 Mottled Bigarrau......... 176 MIorris' Early Duke......... 191 Montmorency............ 195 Montmorency à gros fruit.... 195 Montmorency............. 146 Afintmorency à longue queue. $1: 16$ Muntmoreacy.............. 1!6 Morello................... 197 .Monat's Amarelle.......... 2in) MLusrat de Prague........... 196

Napuleon Bigarreau......... 1 183

Nain Précoce............. 195

New Lar re Black Bigarreau... 155

Ochsen Herz Kirche........ 176

Ox Heart................ 176

Petite Cerise Rouge Précose. $1 ! 15$

Pie Cherry................ 193

Pie Cherry............... 197

Plumstoxe Morello.......... 1:19

Porlugal Du:C............ i

Purtigal butie............. 1:11

Prinzessin Kirscize......... 179

Prince's Duke............. 194 


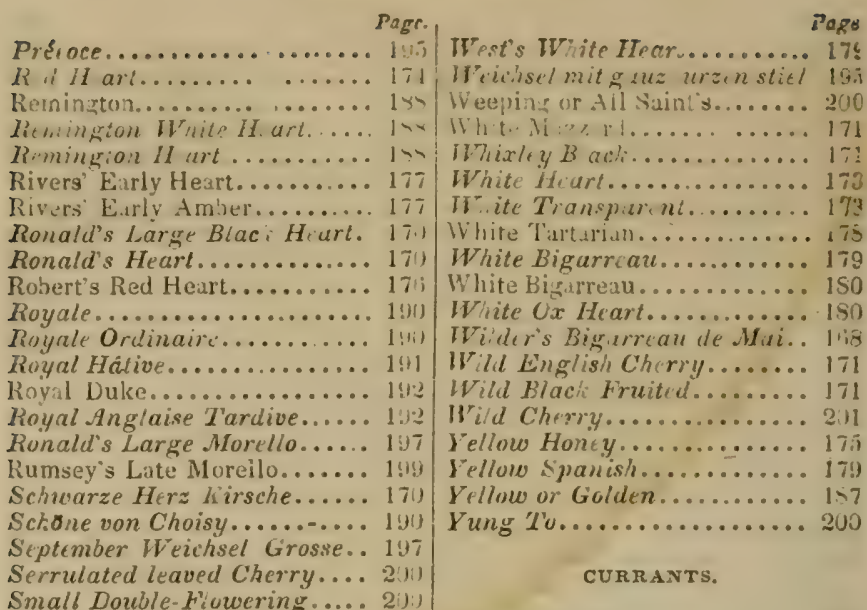

B?ach English.......... 2าน Elack Naples............. 21, C'assis.................... 21.! Champarne.............2. 2.4 Common blick............. 2us Gros.illiırRouge à vros Fruit 203 Gruseil'ier à fruit couleur de Chair................. 2i)t Knisht's Lar se Red.......... 214 Knight's Early Red......... 2.14 Knight's Siveet Red.......... 2.14 Large Red Dutch............ 20. 13 Large Bunched Red.......... 2.13 Long Bunched Red......... 213 May's Victoria.............. 24 Missouri Currant.......... 20.j Morgan's Red.............. 2i)3 Morgan's WWhitt............ 203 New Red Dutch............2:3 New White Dutch............. 2i)3 Pheasant's Eye.......... 2)t Red Dutch.............. 2!)3 Red Grape................ 2i13 Reeve's White............. 2!13 179 lRed Flowering Currant....... 2. 2j 150 Striped Fruited............ 201 176 Grosse Weiss und Rothge18! streijte Johannisbecre...... 201 196 White Dutch............. 203 21)l Uhite Crystal............. 2013 2)1 I1hit:ieghorn........... 2U3

Werder's Early Black....... Werdersche Frïhe Schwarze Herz Kirsche............ 169

\section{.}

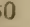
. . (1) 踏

\section{,} . . (n) 


\section{Page.}

Bauswater...............? . .

Black Naples.............. . 2.j9

Blärk Ischia...............210

Blue Ischia............. 2lu

Black Genoa..............210

Biordeaux.................211

Brutsswick.................2,

Broun Hamburg........... 2u: I

Brown Turkey...............210

Brown Italian............ 210

Brown Ninles..............2 210

Brown Ischia.............2i

Chestnut................210

Chestnut-coloured Ischia......

Clementine...............

Concourelle Blanche........

Early Forcing.............

Figue Blanche.............

Ford's Seedling.............

Green Ischia. . . . . . . . . . .

Hanover.................

Italian...................

Large Blue.................

Large White Genoa..........

Lee's Perpetual..............

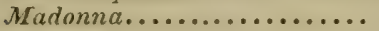

Malta....................

Marseilles................

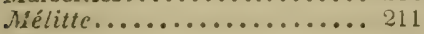

Murrey...................2,

Nerii................ 212

Pocock...................2 212

Pregussata.............. 21:2

Red...................2 2

Smali Brown............. 211

Small Brown Ischia..........

Violette..................

Vjolette de Bordeaux.........

White Marseilles.............

White $\mathcal{N}^{\prime}$ iples............ 21:?

Hrite standard .......... 2l:?

Wnite Ischia ............ 2L:2

Early Green IIaisy

Fage

Edwards's Jolly Tar......... 216

Farruw's Roaring Lion ........ 215

Glenton Green ........... $21 n^{n}$

Gorton's Viper ............. 210

Green Walnut ............ 217

iiartshorn's Lancashire Lad ... 216

Houley's Lady of the Mancr .. 217

Hepburn Green Prolific...... 217

Hill's Gulden Gourd........ 216

Keen's Seedling........... 216

Leigh's Rilleman. ........... 216

Massey's Heart of Oak ....... 217

Mellin's Cruwn Bob......... 216

Pitmaston Green Gage.........

Saunder's Cheshire Lass ...... 217

Taylor's Bright Venus....... 217

Wainman's Green Ocean...... 217

210 White Honey............. 217

209 Woodward's Whitesmith ..... 217

Yellow Champagne.........2 216

Yellow Ball.............. 216

Alexander's ................. 253

211 Amber Muscadine.......... 212

211 Amiens................... 24?

21:2 American Muscadine........ 2.59

Ansell's large Oval Black.... 238

Auverne.................. 236

Auvernal............... 23!

Auvernas Rouge. .......... 236

August Traube.............. 2:39

Black Cluster.............. 236

Blac's Morillon ............ 236

Berry's Greenwood.......... 216| Black Burgundy........... 236

Buardman's Britisi Crowa .... 215 Black Frontignan ........... 236

Buerdsill's Duckwing ........ 21li Black Frontignac.......... 239

Capper's Top-Sawyer ....... 21., Black Constantia .......... 230

Capper's Bunker's Hill ....... 2hij Blue Frontignan ............ 237

Capper's Bonny Lass ........ 217 Black Hambur. .......... 237

Champasne.............. 21; Blue Trollinger.......... 237

Cleworth's IVhite Lion....... 217 Black Prince............ 237

Colier's Jolly Angler........ 21 if Black Spanish............ 237

Cook's White Earle......... 217 Black V'alentia........... 237

Crouspton Sheba Queen ...... 21 i Blach Portugal .......... ¿.57

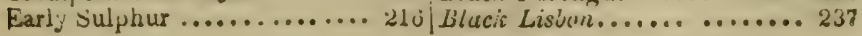




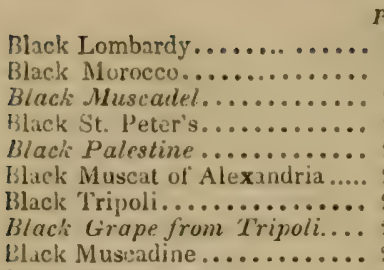

Black Chusselas...............

Black Sweetwater............

Blanc de Bonneuil ...........

Blacksmiti's Winte Cluster...

Bland ....................

Bland's Virkinia.............

Bland's Madeira ............

Bland's Pale Red........... 25:

Bourdales des Hautes Pyrenécs

Boston.....................

Brown Hamburgh ......... 237

Burgunder............... 236

Burgunder............... 239

Bull or Bullett............ 2.s

Cambridge Botanic Garden .. 237

C.nnon Hall Muscat......... 214

Cape Grape.............. 253

Catawba................ 2.54

Cutawba Tohay........... 2.54

Chasselas noir............. 239

Chassel as Mus jué............

Chadsworth Tokay ............

Chasselas Dork...............

Chasselas Blanc ........... 24.

C'inasselas de Funtainebleau..

Chasselas Précuce ...........

rhasselas Royal .............

Chasselas Panaché...........

Chasselas Rouge.............

Ciotut ....................

Clijton's Constantia.........

Clarenee...................

Cumberland Lodge...........

U.Arbois....................

De St. Jean...................

Diana.....................

Dutch Hamburg..............

Dutch Sweetwuter...........

Early Black................

Eurly black July ............

Eurly White Malvasia..........

Early Chasselas.............

Enrly White Teneriffe.......

Early Wiite Muscadine.....

Early Sweetwater...........

Elsinbur................

Eisenburough..............

Esperione.....................

Farineux Noir.
241

211

$2+2$

$24: 2$

21.1

$2+4$

2413
247

2411

2.3 .3

2.315

211)

2122

2339

25.5

237

241

2:3i.

$236 !$

211

211

214

214

2.5

2.).

210

210
Pags

Fleish Truube............. 237

Hox Grape............... 2.

Hrane Pineau.............236

Frankendule............. 237

Frankenthaler .............237

Frankenthules Gros $\mathcal{N}$ iir .... 237

Fromenté............... 2u

Frontiac of Ale andria....... 243

Genmine Tolkay............ 2ts

Gibraltar...................237

Golden Chasstlas........... 2.12

irrove End Sweetwater ...... 2-11

Gray Tolay.............. 244

Grosser Riessling .......... 24j

Grizzly Frontignan ......... 2 if

Grizzly Frontignac ......... 240

Grauir Muscuiller......... 2 ij

Hampton Court Vine........2237

Hardy Blue ITindsor........ 240

Hudler..................237

Isabella................. 25j

Jacos's Traube............ 2313

Jews................. 247

Jerusalem Muscat.......... 21

July Girape............... 23y

يleieer Kissling........... 245

Kunisht's Varie futed Chasselas.. 2.17

Kummel Traübe........... 24t6

Languedoc...............237

Le éceur.................. 23s

Le Minuier................ 240

Le Cuor...................211

Le M:licr ..............211

Lenoir...................... 2.th

Lombardy................217

Longworth's Oitio........... 2.57

Lunel .................. : 13

Madeleine...................30

Madeleine Noir............ 239

Malmsey Muscadine......... 240

Wadtira Wine Grape....... 2ł3

Muleira ............... 2$\rceil 3$

Malaga ............... 213

Malasa ....................

Maurillan Panaché....... 246

Maurillan noir Panaché .... 246

. Melier Blanc ............. 911

Miller's Burrundy........... 240

Miller Grape............. 210

Hissuuri................. 256

Hissouri seedling......... 2.56

Morillon Noir ............ 236

Mohrendutte................ 237

Money's ...................238

Murillon Hàtif............. 239

Morillon Taconne.......... 240

. Morone Farinaccio......... 240 


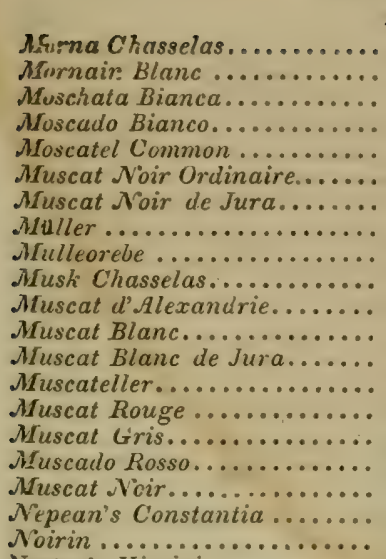

Norton's Virginia........... Nurton's Seedling........... Ohio

Cldaker's West's St. Peter's. Parsley-leaved.............. Parsley-leaved IÍscadine.... Passe longue Afusqué......... Pilsse Musqué Petit Riessling.............

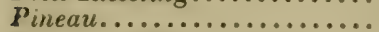
Pitmaston White Cluster........ P'ocock's Damascus.......... Poonah ..................

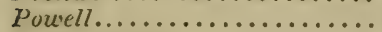
Pond' Seedling.............. Purple Frontignan......... Purple Constantia........... Purple Hamburg............ Pulverulenta............... Raisin de Bourgugne........ Raisin des Carmes........... Raisin de Cuba............. Raisin d'Espagne............ Raisin Précoce................ Raisin d'sutriche.............. Raisin de Champagne......... Raisin de Frontignan........ Raisin Suisse.............. Raisin d'Alep.............. Red Frontiguan............. Red Grape of Taurida....... Red Chasselas............... Red Muscadine................ Red Scupperriong.......... Red Muncy.............. Red Hamburgh............ Red Muscat of Alexandria... Heu Frontignan of Jerusalem.
Page.

241

241

244

244

244

236

236

240

241)

241

243

244

214

244

246

236

244

240

256

2.56

.257

235

2.10

240

243

243

245

$231 \mathrm{j}$

241

237

235

2.53

2.55

230

23r

237

240

230

238

233

2is

239

240

2.12

2.14

24ij

246

247

2.53

238
Reissting .............. 245

Red Frontignan.......... 246

Red Constantia............. 2-1ti

Rhenish Red..............24?

Roanoke................ 2.58

Rother.................. 236

Royal Muscadine............ 24:

kössting ...............24 24

Rudesheimerberg............ 24d

Salisbury Violtt........... 237

Saint Peter's.............. $23 s$

Sauvignien noir........... 240

Schwarzer Frihzeitt ger... . . 239

Scotch White Cluster........ 242

Schloss Johannisberg........ 245

Schuylkill Muscadell........ 253

Schuylhill Muscadine....... 2.)3

Scuppernurig............ 2.js

Schwarzer.............. 236

Segar-Box Grape......... 2.57

Shurtleft's Seedling......... 257

Sir William Rowley's Black . 236

Sir A. Pytche's Black ...... 237

Small Black Cluster........ 236

Smart's Ellsingburg......... 255

Spring Mill Cunstantia...... 2.53

Steward's Black Prince..... 237

Stilluard's siweetwater...... $2 \$ 4$

Striped Muscadine......... 240

Sumpter................. 256

Switzerland Grape......... 246

Syrian..................24?

Tasker's Grupe........... 253

'Tottenham Pari Muscat..... 213

Tokai Blanc.............. 244

To Kalon.................. 2.54

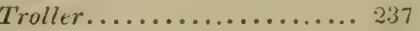

Trollinger............... 237

True Burgundy........... :230

T'urner's Black............ 240

Valentine's............... 237

Variegated Chasseias....... 246

Variegated Chasselas....... 247

Verde lho................ 2t3

Verdilhio.................2 24

Verdal................ 2tá

Victoria................... 2,5i

Warner's Black Hamburyh.. 237

Water Zoet Noir........... 23G

Water Zoete Blastc......... 24t

Wantage.................217

Warren................. 258

Welscher................237

IV tissholziger Trollinger.... 2.37

West's st. Peter's...........239

White Parsley-Leuved...... 210

White Mfeiver............ 2-11

White Chasselas........... 242 
Page.

White Muscat of Alexandria.. :2 i3

White Muscat............. 213

While . Muscut of Lunel..... 21 :;

White Frontignan.......... 2.14

White Constantia......... 214

White Frontniac........... 2.14

IVhite Sweet. Water......... 24t

White Muscadine.......... 244

White Tolkay.............. 2-14

White Hornburgh.......... 2 45

Wrhite Lisbon............ 245

White Portugal........... 245

White Raisin............2.245

While Nice............. 245

While Rissling........... 245

Winne..................

Wilmot's New Blick Hamburgh

Weisser Muscat ller.........

Weisse Muscaten Traube....

Zebilio.

MELONS.

Beechwood ................

Black Rock ..................

Citron......................

Dampsha.................

Early Canteloup..............

Franklin's Green Fleshed .....

Green Hoosainee.............

Green Valencia..............

Imploved Green Flesh .... ....

Keising ................

Large Germek . ............

Netted Canteluup ...........

Nutmeg..................

Rock Canteloup.............

Sweet Ispahan ..............

\section{MULBERRIES.}

Black or English............ Johnson.................. Red.....................

\section{NECTARINES,}

Anderson's................ 50s

Anderson's (of some)........ 5. 3 .Anderson's Round.......... 5us Arrmatic.................5.6i Black................... 5us

Block Murry........... 5 j.5

Boston................... 502

Bronmfield............... 507

Brugnon Hàtif............. 506

Bruswon, Red at the Stane..
Faga

Brugnon de Newington..... 50.

Brugnon Musqué........... 5ine

Brmenon Violette Musquée... 5is

Claremont................ 5us

Common Elruge........... 51)3

Cowdray White........... 50.5

$D$ Angleterre............. 5!s

Downton................ 5 5

Duc du Tellier's............ 5013

Duc de Tilly.............. 503

Duc de Tello............. 50)3

Du Tilley's..............503

Early Violet.............. 506

Early Brugnon.............. 5u6

Early Newington........... 508

Early Black Newington..... 5018

Early B.ack.............. 5us

Elruge................. 503

Emerton's New Wiate...... 5!)J

Fairchild's...............504

Fairchild's Early........... 504

Fine Gold-Fleshed.......... 507

Flanders................ 505

French Newingtun......... 50s

540 Golden.................. 507

Hardwicke Scedling......... 504

Hampton Court............. 506

Hunt's Tawny............. 504

Hunt's Large Tawny........ 504

Hunt's Early Tawny....... 50. 50

Large White............... 505

Late Green................ 506

Large Scarlet.............. 50t

Lewis................... 502

Lord Selsey's Elruge....... 506

Lucombe's Black............ 510s

Lucombe's Seedling.......... 509

Murrey.................. 5us

Mfurry.................. 5u5

New White............. 51

Neat's IVhite.............. 5us

New Scarlet................ 506

Newington................5us

Nicw Dark Niwington...... 51

New Early Newington..... 50s

Oalland's................ 5u3

Old White................5 5u5

O/d Newingtun........... 5118

Old Romun................ 508

Grange.................. 507

Perkins' Seedling........... 5u.

Peterborough (of some)...... 5il 3

Peterborough.............. 5:16

Petite Violette Hâtive....... Jun

Pitmaston Orange.......... 506

lied Roman................ 5us

Ruman.................58

Kough Roman............ 5us

Scarlet Newington ........ 508

Scarlet.................. 5as 
Page.

Snon Hill............... Silos

Shilh's Newingtun......... sus

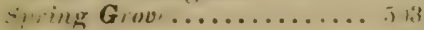

Tcmple's................ Tit 13

Vermash. (of some)......... sof)

Viclette Hâive............ 5nt

Violet................. $5(\pi)$

Irolette Angurvillieres....... 5i)ti

Violette Musquée........... sui

l'iolet, red at the stone...... silli

liulet Must: ............. jui

Urilliams' Orangr..............1i

Williams' Secdling......... Juti

NUTS.

Chestnut...............

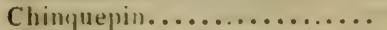

Cosfurd Filluert..............

European Walnut...........

Filbert.................

Friz\%led filbert.............

Hickory Nut..............

Northhamptonshire Prolific

Filbert.................

Red Filbert................

White $\mathrm{H}$ ilbert.

\section{OLIVES.}

Broad-Teaved Olive......... 549

Curnmon Olive.............5.17

Long-L.eaved................ 547

Olivier à fruit arrondi....... 519

Olivier Pleureur............. 5.15

Olivier Pichuline........... 5ts

IVild Ainerican..............

\section{ORANGE FAMILY.}

\section{Oranges.}

Rergamot................. 544

Blood Red................. 544

Common Sweet............. 544

Fingered................ 54.4 $B$

Maltese................. 5 54-1 $B$

Mandarin................. 5.44

Pear-Shaped.............. 544

Ribbed.................. 5.4

Seville.................. 514

St. Michael's.............. 514

Sweet-Skinned............ 514

2. Lemons.

Common................. 515

Sweet.................... 545

3. Limes.

Common
POMEER AN ITER.

Double Red............... B.

Double White............. 5.

Grenalier à Fruil Doux.... 5.

Sweet iruited............. 5.49

sub-acid Fruiterl............ 549

Varierated Flowered......... 550

Yellow l.lowered.............5.50)

Wild, or Acid-fruited........ 5.14

PEACHES.

Abriso é............... 4\&9

Acton scott............... 4it

Acimirable Turdior......... 47-3

Llmirable.................. 477

Admirable Jaune........... 459

Alberge Jaune............ 4!n2

.leviers Yrlione........... 4!16

Aluiers Winter............. $4 ! 6$

Inne................... 47.

tyricut Piach............. $4 \$ 9$

Astur................... 471

Avant Rouge.............. 4s:3

Avant Pêche de Troyes...... 452

Avrnt Blanche........... 453

Barrington.............. 472

Baltimore Beauty........... 490

Bellerarde...............4 471

Belle de Vitry............ 472

Bellis.................... 472

Belle Beauté.............. 478

Belle de Vitry............. 477

Belle Bausse............4 479

Belle de Paris.............. 4 52

Bergen's Yellow............ $4 ! 40$

Betterave................ \$4!

Blood Clingstone........... 443

Blood Cling................ 493

Blood Freestone............ 454

Bourdine................ 479

Boudin..................475

Brevoort.................4 472

Brevoort's Morris........... 472

Brevourt's Secilling Melter... 472

Brown Nulmeg............. 492

Buclingham Mignonne...... 472

Cardinale............... $4 y 4$

Catherine............... 44.

Chancellor............... 473

Chancellière............... 473

Chinese Peach............ 500

Clinton................ 473

Claret Clingstone.......... $4 \$ 3$

Cole's Early lied........... 473

Cooled re's l'avourite....... 473

Cooldalge's Eurly Red Rarerije 173

Coloncl Anstey's.......... 47a 


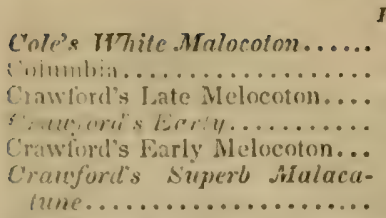

Cul-Lavid................

1) Jhrirnt................

Double Miontagne............

Double Mouniain............

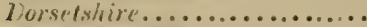

Domble swalsh.............

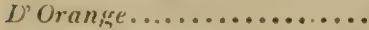

Double klossomed............

Double Flowering...........

Druid Hill.................

Eurly Amne.................

Euty Tillotson............

Early York.................

Eurly Newington kreestone...

Early Ni wiugton..........

Farly sweet-Water..........

Early Arimirable............

Early Purple Suant........

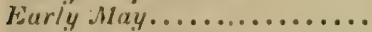

Early Vineyari...........

Early Red Nutmog..........

Early White Nulmeg.......

Early Purple.............

Early Royal George.........

Early Buurline............

Early Red Rareripe.........

Early Craufurd...........

Early Newington Cling....

Ellgar's Late dielling.......

Einperor of Russia...........

Favourite.................

Favourite Red.............

Flat Peacn of China..........

Fox's Seedling.............

French Mignonne..........

French Royal George........

French Magilalen..........

Freestone Heath...........

French Bourdine...........

Free-stone Heath...........

Galande..................

Georve the Fourth ..........

Guld Fleshed ..............

Golden Mignonne..........

Green . liutmeg.............

Grosse Mignonne............

Grimwood's Royal George...

Grimevod's New Royal Gicorge'

Griffin's Mignonne.........

Grimwood's Royal Charlotte.

Grosse Jaune 'Tardive........

Green C'atherine ............

Gros Malecaton.
Page.

4) 1

$4 ! 1 !$

(4)

$4 ! 10$

Hoffinian of Tippecande.........

Hlug

Incompurible ............... 4yî

Italian ................. $48 \mathrm{E} 2$

Java................. 506

Johnson's Early Purple......478

Judt's Afel'sing........... 479

Kenrick's Heath............ 479

licw Early Pirple......... 486

Lemnedy's Carolina........4 $4 ! 16$

Lennedy's Lemon Cling ..... 496

Lale Chancellor............473

Large Early York.......... 475

Large American Nutmeg..... 476

L'Arimirable ........... 477

Large French Mignonne.... 478

La Poyale................ 47s

La Fioyale............... 479

Late Admirable .......... 479

Late Purnle............... 479

475 La Grange............... 450

475| Large Red Rareripe........ 455

482 Large Red Rareripe......... 4\$ 4\$

483 Lady Ann Steward.........481

454 Late Red Rareripe............ 4S6

. 48.) Lady Gallatin............. 492

48.5 Large Yellow Rareripe...... 4!13

45.5 Late Admirable C'ling ....... 49é

490 Large White Clingstone....... $49 \%$

- 495 Late Yellow Alberige........ 490

473 Largest Lemun............. 496

477 Large Newington........... $4 \$ 7$

477 Lemon Clingstone........... 496

477 Lord Siontague's Noblesse.... 4\$3

5u() Lockyer's Mignonne..... . . . 435

478 Lord. Nelson's............. 486

475 Lord Fauconberg's Mignonne. 456

471 Long Yellow Pine Apple.... 496

451 Luscious White Rarerije.... 451

479 Madeleine de Courson......... 191

479 . Madcline Rouge............ 4s1

451 Mlalia................. 482

471 .alte de Normandie........ 452

475 Madeleine Rouge à Petites

.19.2 Fileur: .............. 455

492. Madeleine Rouge Tardive.... 486

474 Madelcine Rough à Moyennes

47s Flcurs................4 486

478 . Madeleine à Petites Fleurs... 486

478 . Malagatune .............. 492

485 Malacatune.............. 452

486 . Marie Antoinette.......... 493

489 . Mellish's Favorite.......... 45 3

497 . Nignonne .............. 478

498 Millet's .Mfignmne......... 485 
Page

M sntague............... 474

Montauban .............. 474

Motteux's................. 479

MInris's Red Rareripe....... 480

AInrris Red................

Morris White Rareripe........

Morris White............

Morris White Freestone......

Morrisania Pound............

Morrison's Pound...........

Munstrous Pavie...........

Munstrous Fompone........

Varbonne................

Newington Peach..........

New Cut-leaved .............

Neill's Early Purple........

New Royal Charlotte........

New York White Clingstone.

Newington...............

Nivette.................

Nivette Veloutée............

Noisette ................

Noblesse..................

. Noir de Montreuil...........

October Yellow..............

Oldmixon Freestone..........

Oldmixon Clearstone.........

Oldmixon Clingstone .........

Old Newington ............

Orange Clingstone...........

Orange Peach..............

Pavie Admirable...........

Pavie de Pomponne ..........

Pavie de Componne Grosse...

Pavie Rouge de Compone.....

Pavie C'aum................

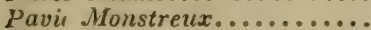

Péche Royale..............

PÉche Malte..............

Pêche Jaune..............

Pếche à Fleurs Doubles.......

Pêche à Fleurs Semi-Doubles.

Peen-To.................

Pine-Apple Clingstone.......

Pourprée de $\mathcal{N}$ ormandie......

Puurprée Hâtive............

Pourprée Tardive............

Pourprée Hûtive à Grandes

Fleurs.................

Pourprée Hâtive ............

Poole's Large Yellow..........

Pooles Late Yellow Freestone.

President .................

Prince's Red Rareripe.......

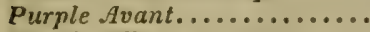

Purple Alberge............

Red Rareripe..............

Red Rareripe...............

Red MIagilalen...........
Red Nutmeg. $\mathrm{rag}^{\circ}$

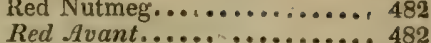

Red Cheek Melocoton........ 492

Red Magdalen........... 485

Red Cheek Malocoton........442

Red Alberge.............. 492

Red Heath............... 494

Reid's IVeeping Peach....... 500

Royal Ktnsington.........4 478

Ronald's Seedling Galande.. 478

Royal Sovereign.......... 478

Royale................ 479

Rouge Paysanne.......... 481

Royal George............. 485

Royal Charlotte.......... 486

Rose...................4 487

Rosanna ............... 493

Sanguinole à Chair Adherente. 494

Scott's Early Red.......... 487

Serrated.................4 477

Selby's Cling ............. 495

Smock Freestone............ 492

Smith's Newington........... 498

Smith's Early Newington.... 499

Smooth-leaved Royal George. 471

Snow....................486 48

Stewart's Late Galande...... 473

Strawherry .............. 487

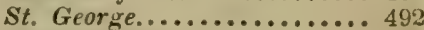

Superb Royal............ 478

Superb..................485

Sweet Water...............4 476

Swiss Mignonne...........448

Têton de Venus...........479

Tippecanoe.............. 499

True Red Magdalen......... 481

Unique................ 477

Vanguard................. 483

Van Zandt's Superb.........4487

Veloutée Tardive........... 483

I'eloutée de Merlet.........4 478

Violette Hâtive ........... 471

Vineuse............... 478

Vineuse de Fromentin.......478

Waxen Rareripe.......... 487

Washington ............. 498

Washington Red Freestone... 488

Walter's Early............. 489

Washington Clingstone....... 499

Weeping Peach ........... 500

White Rareripe........... 481

White Malacaton .......... 481

White Nutmeg. ............ 493

White Avant............. 493

White Imperial............ 498

White Blussomed Incomparable 459

White Blossom........... 489

Willow Peach.............. $48 \varepsilon$

Williamson's . New York...... 495 


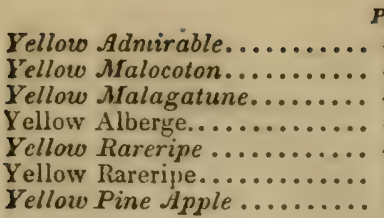

PEARS.

Ah! Mon Dieu

Alpha......................

Althorpe Crassaune......... Amiré Joannet. . . . . . . . . . Ambrosia.................

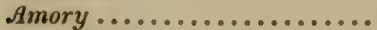
Amande Double............ Amanda's Double ........... Amoselle................... Andrews ................ Ananas ................ Ananas d'Eté..............

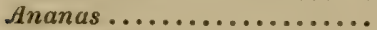
Angleterre ............... Angora . . ............... Archiduc d'Eté............. Aston Town................ Autumn Colmar............. Autumn Bergamot.......... 366 Bartlett, or Williams' Bonchré-

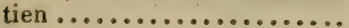

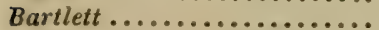
Badham Belle de Bruxelles...........

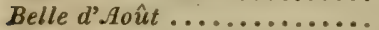

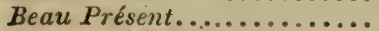
Bellissime d'Eté............ Bellissime Supréme......... Bellissime Jargonelle........

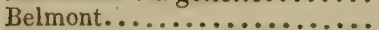
Belle et Bonne.............. Beurré Haggerston.......... Beurré d'Angleterre.......... Beurré Boucquia........... Beurré de Capiumont.........

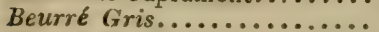

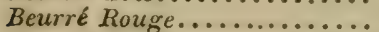
Beurré d' $0_{r} \ldots \ldots \ldots \ldots \ldots \ldots$

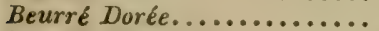
Beurré d'Amboise........... Beurré du Roi.............

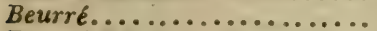
Beurré d'Anjou............ Beurré d'Anjou ...........

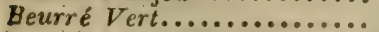

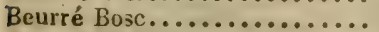

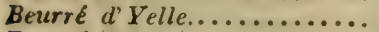

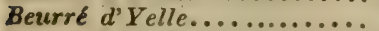
Beurré d'Amalis.
Page.

$4 \varsigma 3$ 492 $49 \cdot 2$ 492 $4 y 2$ 493 $4 y 6$

383 345 352 330 331 353 353 430 349 349 3.50 350 351 423 330 351 3.) 3 334 334 357 334 334 337 339 $33 y$ 339 353 353 333 351 $35 \dot{3}$ 357 357 360
Bcurré d'Amaulis.......... $3 \dot{3}$ C

Beurré d'Amanlis.......... 360

Beurré Diel. . . . . . . . . . . 350

Beurré Royale ........... \$00

Beurré Magnifique.......... 360

Beurré Incompurable....... 360

Beurré Knox............ 36

Beurré Kenrick............ 36ं2

Beurré Duval.............. 363

Beurré Preble............ 36

Beurré Colmar ............ 363

Beurré Colmar d'Automne... 3 rj3

Beurré de beaumont....... 3ij-1

Beurré Van Mons.......... 3ijs

Beurré Romain............ 3r 4

Beurré de Ranz........... 4:7

Beurré Rance.......... 427

Beurré Epine............. 427

Beurré de Flandre......... 427

Beurré Bronzée.......... 4.29

Beurré d'Alençon......... 430

Beurré d'Hiver........... 433

Beurré Furtunée.........4 436

Beurré d'Hardengront....... 437

Beurrés d'Hiver Nouvelle... . 437

Bunrré d.Aremberg........ 437

Beurré de Clambron ........4.4:37

Beurré d'Austrasit.......... 438

Beurré Colman Gris, dit Pré-

cel.................... 444

Beurré d'Argenson........ 444

Beurré de Mlalines ..........4 450

Beurré Van Marum.......... 365

Beurré Spence............ 3ti5

Beurré Crapaud........... 365

Beurré Piçuery........... 365

Beurré Beauchamps......... 367

Beurré Romain............ 3tis

Beurré Blanc de Jersey ...... 363

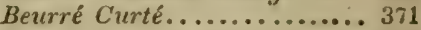

Beurré de Payence......... 374

Beurré Plat.............. 375

Bergaluo .................. 378

Bewrré Blanc............ 378

Beurré Rouge............. 350

Beurré Bronzée............ 383

Beurré or Bonne Louise-d'Araudoré.............. 397

Beurré Niell.............. 401

Beurré Sieulle............ 413

Beurré d'Aremberir .......... 423

Beurré des Orphelins........ 423

Beurré de la Pentecôte....... 4:55

Beurré d'Hiver de Bruxelles.. 4:25

Beurré Roupé............ 425

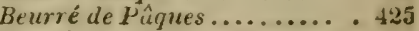

Beurré Gris d'Hiver Numveau.. 426

Belle de Brussels.......... 353

Bezi Vaet............... 4ta 
Bezi d'Héri $\quad$ Page

Bezi Royale.............. tzs

Burganotte de Pîques...... 42!

Beryamotte d'Hiver........ 429

Bergamotte de Bugi....... 429

B rgamotle de Toulouse ..... 424

Bel gamotte d'Hollande ......4430

Bergamotte de FouEgre..... . 430

Berganot d'Eté............ 333

Bergamotte $l^{\prime}$. In gleterre.... 333

Bergamotte Suisse.......... 367

Bergarcotte Cadette ......... 367

Bergamotte Crassane....... 375

Ber samotte Sylvange.......4 413

Bergamotte de la Pentecôte.. 425

Bezi de Chaumontelle....... 433

Bezi d'Echassey............ 435

Bezi de Chasserie.......... 435

Belle de Jersey. . . . . . . . . 448

Beauchamps............. 3f7

Bezi de Monisgny.......... 365

Bezi de la Mutte........... 368

Bein Armudi............. 36s

Belle de Flanders............ 356

Belle Lucrative........... 357

Be zi Chaumontelle très gros.. 42.5

Bishop's Thumb............ 369

Bloodgood ................ 332

Bleeker's Meadow ... . . . . . . . 35. 35

Black Worcester............ 429

Black Pear of Worcester.... 429

Bon Chrétien d'Ete.......... 346

Bonne de Keingheim ....... 347

Boucquia............... 355

Bosc's Flaschenbirne........ 359

Bonne Rouge............. 366

Bon. Chrétien Fondante......

Bonne-ente ...............

Bouche Nouvelle.............

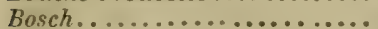

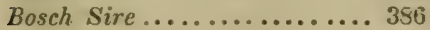

Bosch Peer............... 356

Boston Epargne........... 392

Bon Chrétien d'Espagne..... 430

Bon Chrétien Turc........ 430

Boulgermester ............ 448

Bonne de Malines.......... 450

Brougham ................ 35 4

Brown Beurré ............. 357

Fr.seas Bergamot .......... 3ri6

Braddork's Field Stanc ard.. 39 ?

Broom Park................ 428

Bretagne le Cour.......... 445

Brown St. Germain........ 447

Brande's St Germain.........44 44

Hitfirm ................. 356

Buffum............. 3.ji

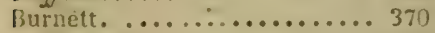

Butter Frur............. 37
Pago.

Bujalenf ... .......... $45 C$

Caillot Rosat d'Eté........ 345

Capiumont.............. 35)

Caluhasse Buse.......... 35

Cabut ................... 37r,

Capsheaf................ 374

Calebasse ............... 374

Calebisse Double Extra..... 374

Calebasse d'Hollande........ 374

Calebasse Grusse........... 374

Capucin.............. 375

Capuchin.............. 375

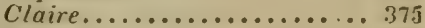

Calhoun................. 376

Cambridge Sugar Pear... . . 3132

Canning .............. 4.. 4. 25

Catillac................ 432

Cadilloc................. 432

Cellite.................444

Chelmsford.............. 370

Charles of Austria.......... 376

Charles d'Autriche......... 376

Charles $d$ Autriche..........4 401

Chaumontel très yros........ 425

Chaumontel ..............4 433

Chapman's................ 414

Chambers' Large........... 448

Chambrette............. 450

Citron ................. 336

Citron des Carmes......... 341

Citron de Septembre......... 37s

Citrunenbirne Bömische Grosse

Punctirte............. 3s?

Clara..................... 375

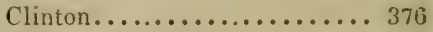

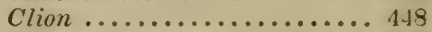

Common Bergamot.......... 366

Compte de Lamy............. 371

Comprette ............. 371

Commodore ............... 372

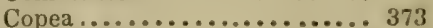

Colmar Epine............. 376

Colmar Neili............. 376

Comtesse de Frêsnol......... 3ss

Compte de Michaux.......... 3! $\$ 8$

Colmar Bosc.............. 401

Colmar Deschamps......... 423

Columbia.................4 430

Columbian Virgalieu....... 430

Columbian Virgalouse...... 430

Comstock............... 432

Cumstock Wilding.......... 432

Colnar .................. 431

Colmar Doré.............. 431

Colmar $d^{\prime}$ Hiver............ $1.3^{\prime \prime}$

Colmar Jaminette...........448

Colmar Gris.............. 444

Colmar Harlenpont........ 444

Colmar Souveraine........ $\$ 44$ 


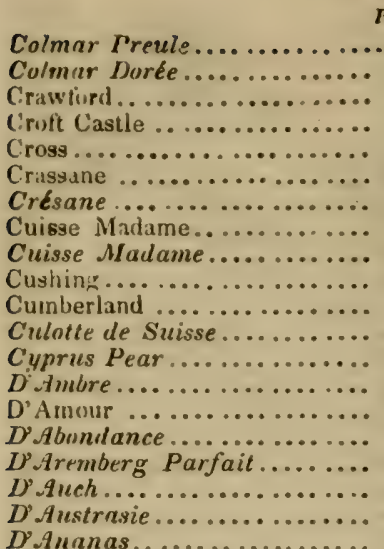

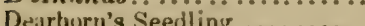

De l'ullée...................

De Keinzheim ............... 317

Des Truis T'uurs........... 3ij

De Melon.................. 3n

rean's.....................37s

Dechnntsbirne............. 378

De Louvain ............... 353

Délices d'Hardenpont......... 384

I) Slices d'Ardenpont........ 35t

De I Tigne Pelone............ 335

Deschamps.............. 423

De Mfaune.............. 434

De Tunneau.............. 445

Die Sommer Christebirne.... 346

Diel's Butterbirne........... 360

Thel ...................... 360

Jillen .................. 360

Diamant............... 366

Dingler.................... 371

Dix......................... 378

Dorothee Royaíe........... 360

Doyenné d'Eté..............

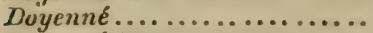

Doyenné Blanc.............

Doyenné Panaché............ 330

Doyenné Galeux........... 350

Doyenné Boussouck.......... 380

Doyenné Gris.............. 380

Doyenné Rouge.............

Doyenné Roux............. 33i)

Downham Seedling......... 395

Doyenné $d^{3}$ Hiver. .......... 425

Doyenne du Printemps....... 425

Doyenné d'Automne......... 350

Dumnrtier................ 378

Dunmore..................

Duchesse d'Angoulême........

puchess of $M$ Iars ............
Duchesse de Mars............ 382

Duchesse d'Orleans. .......... 394

bundas ..................... 384

Duc a Aremberg. ........... 423

Du Pûtre.................425

Dumas.................... 448

Early Sugar.............. 330

Early Beurré.............. 331

Early Beurré.............. 332

Early Bergamot............. 33 i

Early Queen................ 3.11

Early Chaumontelle......... 341

Early Catherine............ 343

Early Rousselet. ........... $3+3$

Easter Beurré............ 425

Easter Berramot............ 429

Echassery................ 435

Echasserie............... 435

Edivard's Elizabeth. ......... 355

Edwards' Henrietta.......... 38j

F.dwards' William........... 420

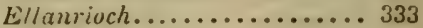

Emerald................. 435

Enylish Red-Cheek......... 339

English Beurré............ 351

English Bergamot.......... 366

English Autumn Bergamot... 366

Enfant Prodige............ 355

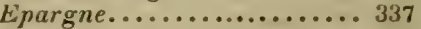

Fpine Rose............... 345

Epine d' Eté Couleur Rose... 345

Epine $d$ s'Éé............. 345

Evine d' Eté............... 346

Etourneau.............. 450

Eyewood............................ 45

Ferdinand de Meester....... 409

Fingal's.................. 333

Figue de Naples............ 358

Fig Pear of Naples......... 358

Fin Or d'Hiver............. 436

Fleur de Guignes........... 315

Flemish Beauty............. 356

Flemish Bon Chrétien....... 430

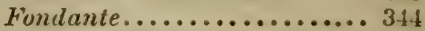

Fondante du Bois........... 356

Fondante Musquée......... 316

Fondante Van Mons.......... 387

Fondante d'Automne........ 387

Fondante du bois........... 401

Fondante du Bois........... 435

Fondante de Panisel........ 444

Fondante de Mons........... 444

Forme de Délices............ 3Ss

Forelle.................. 3s9

Forellen-birne.............. 359

Fornie de Marie Louise. ...... $39 y$

Fortunce.................. 436

Frauenschenkel.............. 337

382 Frédéric de Wurtemburg....... 390 
Page.

Frederick of Wurtemburg.... 390

Franzbsische Rumelbirne...... 4:35

Franc Ré.l d' Hiver.......... 43:j

Franc Kŕal d' Eté ......... 34t

Franc Réal............... 436

Fulton................. 341

Grnsel's Bergamot........... 3its

Garde d' Ecosse........... 43i;

Gambier................. 444

Gendesheim.............. 392

Germain Baker........... 448

Gibson................. 3.4!

tiil-o-gile..............4 43ij

Gil-0.gil............... 43ij

Glout Morceau............. 43:

Gloux .Morceaux.......... 437

Golden Beurré............ 3.57

Golden Beurré of Bilboa..... 3 $x^{j} 2$

Gore's Heathcot............ 344

Goulu Morceau........... 437

Got Luc de Cambron........ 437

Green Chisel............ 337

Green Chisel............ 3ł1

Green Sugar........................ 337

Grosse Cuisse Madame..... 337

Gros Aficet $d$ ' Ėté........... 344

Grativli................. 346

Gratioli d' Eté............ 346

Gratioli di Ruma.......... 346

Gracieuse....................53

Grey Beurré............. 357

Grosse Dorothée........... 360

Grosse Dillen............. 3ijo

Gray Doyenné.............. 350

Gray Butter Pear.......... $\$ s 0$

Gray Deans.............. 380

Green Pear of Yair.......... 392

Green Yair.............. 392

Great Citron of Bohemia...... 392

Green sylvange........... 413

Grand Monarque......... 432

Groote Mogul............. 432

Groom's Princess Royal....... 438

Gurle's Beurré............... 366

Guernsey............... 412

Harvest Pear.............. 330

Hamipden's Bergamot.......... 333

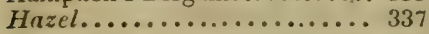

Harvard.............. 392

Havon's Incomparable........ 395

Hardenpont du Printemps. .. 4:27

Hardenpont d' Hiver........ 437

Hardenpont's Winter Butter.

birne...................

Hardenpont du Printemps.... $43{ }^{\circ}$

Hessel... ............... 337

Henry the Fourth.......... 393

Henri Quatre............. 3 3

Héricart...................
Page

Heathcot.................394

Hooper's Bilboa............ 364

Holland Bergamot........ 436

Hull...........................

Huguenot................. 394

Impératrice de France....... 380

Incomparable............. 434

Inconnue la Fare............ $4 \mathrm{Hb}$

Isambert................ 357

lsambert le Bom........... 357

lves' Bergamot.............. 366

Jargonelle, English.......... 337

Jargonelle, French........... 339

Jacquin ................. $3 ! 3$

Jalousie................. 345

Jalousie de f'ontenay Vendée. 396

Jackman's Melling ......... 396

Jagdbirne................. 4... 4.5

Jaininette .............. 438

jilogil ............... $43 t$

Joannetle .....................36

Johonnot ................ 395

Josephine ............... 386

Jusepline ............... 427

Josephine.............. 438

John ................... 443

July Pear................. 344

Julienne................... 3:3y

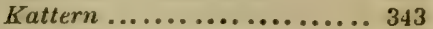

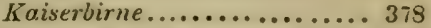

Kaiser d'Autonne.......... 378

Katzenkopt ............ 4i2

King Edward's............. 346

Knevett's New Swan's Egg .. 349

Kuight's Monarch.......... 439

Konge.................... 347

Kronprinz Ferdinand ...... 437

Kronprinz Vun Oestreich.... 437

Large Summer Bergamot...... 333

Large Sugar............. 346

Large Seckel.............. 355

La Fortunée de Parmentier... 436

La Fortunée de Paris ........ 436

Lawrence................ 442

La Bonne Malinoise........ 450

Lẻnn le Clerc.............. 440

Léon le Clerc de Laval........ 440

Lewis................... 441

Lent St, Germain......... 448

Le Curé.................. 14t

Limon................... 3is

Little Muscat.............. 340

Little Musk................ 340

Little swan's Egg. ......... 399

Linden d'Autumne ........ 437

Lodge................. 3us

Long Green.................... 4:s

L'Orpheline.............423

Lord Cheney's............ 430 
Page.

Toncise Bonne de Jersey...... 3!17

soluise Bunue of Jersey....... 3!17

Lonise Bunne d'.4vanches.... 3!'7

couise bonne............... 441

Louise Bunne Réal..........441

acke.................. 442

Wucke's . liew Beurré ........ 44:

Hadeleine, or Citrun des Carmes 341

Madeleine................ 341

Magdalen ...................311

Manning's Elizabeth ......... 35.

.Marianne Nouvelle.......... 358

Marie Louise Niva ........ 371

Marie Luuise the Second.... 371

Marie Luuise................ 3ay

Marie Chrétienne........... 3!y

Marie Louise Nova.......... 40.)

Marotte sucré Junne........ 44. 4

Mrlon de hopis............... 3tiv

Mérlaille................ 4til

Messire Jean...............4.13

Messire Jean Gris.......... 443

Mressire Jean Blanc......... 443

Messire Jean Dorḱe......... 443

Michaux................. 3!s

Milanaise Cuvelier.......... 4511

Mon Dieu.................. 3s

Moor-Fowl Egg ............ 399

.Hunr-Fowl Eigg ........... 414

Mouth Water...............4 418

Mollett's Guernsey Beurré..... 426

Mollett's Guernsey Chaumon-

telle.

Moccas ..................

Munsieur Jean ................

Monsieur le Curé............

Mr. John................443

Mruscat Petit............... 340

Muscat Robert................ 341

MIuscat F'leuré..............418

Musk Rubine.............. 341

Muscadine............... 342

Mrusk Summer Gool Christian 346

Napoleon.....................401

Naumkeag...............4402

New Yurk Red Cheek........4 415

.New Autumn..............4 418

Ne Plus Meuris............ 444

Niew St. Germain.......... 447

Nélis d'Hiver ............. 450

Niell....................4 401

. No. 8 of I'an Mons.......... 333

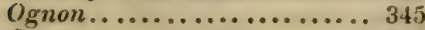

Oynonet.................367

Oxford Chaumontel......... 433

Passans du Portural......... 342

Parquency................ 404

Pailleau................. 406

Paradise d'Automne..........
Parkinson's Warden ...... 429

Paddinglon ............... 429

Passe Culmar.............. 14

Passe Culinar Enineaux.... 4.4

Passe C'olmar Giris............ 11!

Perdreau................. 343

Petit Muscat............... 340

Petit Rousselet............ $3 \$ 3$

Petre.................... 403

Pennsylvania .............. 404

Philijpe de Pâques............ 425

Pine Pear................. 378

Pitt's Prolific............... 40.

Pitt's surpasse Afarie ...... 4114

Pickering Pear.............. 448

Piper....................448

Poire Guillaume............. 334

Puire des Tables des Princes.. 337

Poire à la Reine............. 341

Peire de Chypre ........... 3.43

Poire Sans Peau............ 345

Pwire de Ruse.............. 345

Pvire Ananas .............. 349

Poire d' Ambuise............ 357

Poire de Cadet............. 367

Puire de Simun.............. 378

Poire Neige............... 378

Poire de Seigneur.......... 378

Poire Monsieur............ 378

Poire d'Amuur.............. 383

Puire de Louvain........... 353

Poire Truite.............. 359

Poire-Glace.............. 450

Poire Niell............... 401

Poire à Gobert............ 436

Pope's Scarlet Major ......... 406

Pope's Quaker.............. 406

Pound.................. 445

Primitive................ 340

Prince's Sugar............. 344

Prince's Sugar-Top........ 344

Princesse de Parme......... 39y

Princess of Orange........... 405

Princesse d'Orange......... 405

Princesse Conquête........ 405

Précel ................. 44

Présent de Malines......... 444

Prince's St. Germain......... 447

Queen's Pear.............. 341

Queen of the Low Countries.... 406

Queen Caroline............ 408

Quilletette................ 407

Raymond...............4409

Real Jargonelle............ 337

Red Muscadel.............. 339

Red Cheek ............... 333

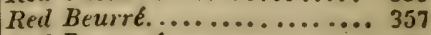

Red Duyenné .............. 350

Red Beur rk.............. 380 


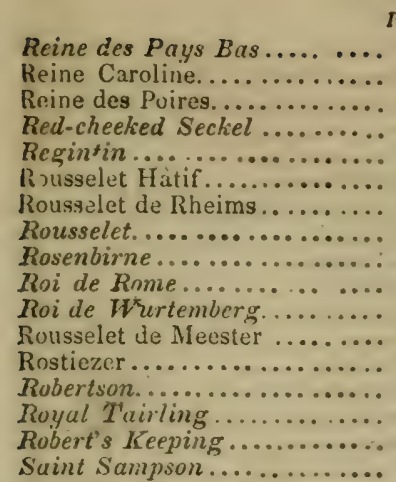

Saint Lambert.............. Saline d' Eté.................. Sanspeau or Skinless........... Satin Vert............... Saint Germain de Martin.... Sabine.................... Saint Germain Jaune........ Scotch Bergamot.............. Schöne und Gute............ Sept-en-gueule............... Seckel .................... Seckle...................... Seigneur d' Hiver............. Short's Saint Germain....... Shenks..................... Sieulle.................... Skinless................... Sickel .................... Smith's Pennsylvania........ Snow Pear................. Sommer Apothekerbirne ....... Sommer Gute Christenbirne.. Souverain

Spanish Bon Chrétien ......... Spice or Musk Pear .......... Spina.................... St. Jean ................. St. John's Pear.............. St. Jeair Mrusquée Gros...... St. Germain d' Eté........... St. Michael.................. St. Michel................... St. Michel Doré ............ St. Ghislain ................ St. André .................. St. Germetin Blanc.......... St. Germain ................ St. Germain Gris ............ St. Germain $P$ xnachée....... Styrian..................... Stevens' Genessce..............
Sieghen's G'Enessec.........
Page. 405 405 415 444 343 343 345 401 437 409 411) 422
$4 \cdot 24$ 499 337 337 339 345. 346 438 446 333 353 340 415 415 425 347 413 413 345 415 404
378 346 340 444 430 343 430 330 330 347 375 378 110 411 441 440 446 447 412 412
Pags

Striped Germain ........... 447

Striperl Long Green ......... 419 Staunton.................. 3ris Sugar Pear............... 33 C Sugar Top................. 344 Summer Bergamot........... 335 summer Bergamot.......... 333 Summer DoyennE............ 336 Supréme................. 339 Summer Beauty............ 339 Summer Portugal.......... 342 Summer Franc Real. ........ 344 Summer liose............. 34.5 Sucrée de Hoyerswerda. ...... $346^{\circ}$ singar of Ioyersworda...... $34 \dot{0}$ Summer Thorn............. 346 Summer Jjon Chrétien........ 346 Summer Giood Christian...... 346 Summer St. Germain........ 347 Summer Bell.............. $3 \$ 7$ Sucrée Dorée............. 4is Superfondante.............. 411 Sullivan................. 411 Surpasse Marie Louise...... 404 Surpasse Virgalieu............ 416 Surpasse Virgoneleuse....... 416 Swiss Bergamot............ 367 Sweet Summer............. 337 Swan's Egg............... 414 Sylvanche Vert d' Hiver...... 360 Sylvange................... 413 Syckle.................. 415 Terling................... 429 Thorny Rose.............. 345 Thompson's............... 413 Trouve de Montigny......... 3fis Trout Pear................ 389 Union...................448 Urbaniste.. ............. 417 Uvedale's St. Germain........ 448 Uvedule's Warden........... 448 Vallée Francine............. 347 Van Mrons, No. 1218....... 372 Valencia.................. 378 Van Mons, No.154......... 385 Van Mons, No. 859........411 Van Buren...............420 Van Mons Léon le Clerc...... 419 Van Mons, No. 1238........ 376 Vermillion de Eté........... 339 Verte Longue............... 418 Verte Longue Suisse........ 419 Verte Longue Panachée....... 419 Virgalicu................ s7s Virgaloo.................. s78 Vicar of Winkfield........... 448 Virgouleusc................4 450 Warvick Bergamot.......... 378 Washington.............. 422 


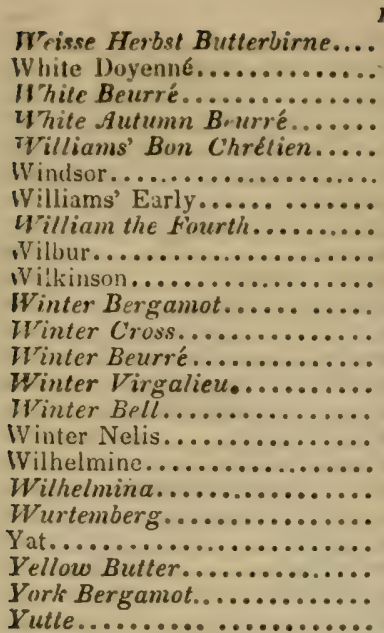

pLuMs.

Abricotee de Tours.......... 272 Abricolée.................... Abricot Vert.............. Abricotée Rouge............ Agen Datte................ Amber Primordian........... American Yellow Gage...... American Wheat............ A pricot.................. Apricot Plum of Tours...... Autumn Gage................ Austrian Quetsche........... Azure Hâtive............... Beeliman's Scarlet........... Beach Plum.............. Bingham................. Bleecker's Gage............... Blue Gage.................. Black Perdrigon............ Blue Perdrigon............. Blue Imperatrice.............. Blue Perdrigon.............. Black Damson.............. Blue Holland............... Bleecker's Scarlet............ Black Morocco.............. Black Damask..............

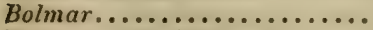
Bolmor's Washington........ 3ruyn Gage............... badford Gage............. rugnon Gage..............
Page. 375 378 334 347 348 397 433 434 445 $4.51)$ 422 378 3656 422 272 270 239 309 279 297 289 272 272 271 311 289 303 263 272 273 289 259 290 290 307 297 301 303 306 306 284 284 276 276 276
Brigncle................. 258

Brevoort's Purple............ 2s:

Brevourt's Purple Bolmar.... 289 Brevourt's Purple Washington 259 Brignwle Violette........... 290 Bucl's Favorite........... 272 Bury Seedling............ 273

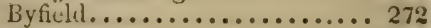

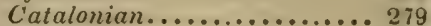

Caledonian.............. 300

Caledonian............... 306

Cherry..................294

Chestun................. 245

Chickasaw Plum........... $2 \nmid 3$

Coe's Golden Drop............ 273

Coe's Imperial............. 273

Cooper's Large............... 291

Cooper's Large Red.......... 291

Cooper's Large American.... 291 Columbia.................. 293 Columbian Gage........... 292 Corse's Admiral............. 293 Corse's Field Marshal......... 293 Corse's Nota Bene. . . . . . . . . 293

Coe's Late Red................. 295

Common Damson.......... 297

Common Quetsche........... 310

Cornmon English Sloe....... 316

Covetche.................. 310

Cruger's Scarlet............ 295

Cruger's..................203

Cruger's Seedling........... 293

Cruger's Scarlet Gage...... 293

Duna's Yellow Gage.......... 275

Damas Vert.............. 276

Dauphine............... 276

D'Avoine................. 279

Dame Aubert ..............286

Dame Aubert Blanche......... 286

Dane Aubert Jaune......... 286

D'Amérique Rouge......... 294

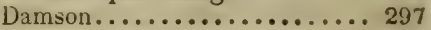

Damas d'Italie............ 302

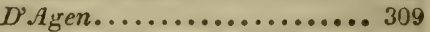

Damas I'iolet............. 310

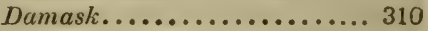

Damas Gros................ 310

Drmas Violet Gros.......... 310

Dame Aubert Violette........ 312

De Virginie.............. 294

Denniston Red............ 2y8

Denniston's Albany Beauty.... 275

Denniston's Superb.......... 275

Denyer's Victoria.......... 315

Diamond................. 298

Diaprée Violette.......... 295

Diaprée Rouge............. 298

Die Violette KöniginnClaudie. 308

Downton Imperatrice......... 275 


\begin{abstract}
Domine Dull.
Double-Flowering Sloe........

Double-Blossomed Plum......

Drap d'Or...

Dutch Prune.

Dutch Quetzen.............

Duane's Purple French........

Dwanf Texas Plum...........

Early Yellow...............

Early Scarlet...............

Early Damson..............
\end{abstract}

Early Orleans...............

Early AIorocco..............

Early Black Moro:co.........

Early Damask..............

Early Tours...............

Early Violet...............

Early Royal...............

Egg Plum..................

Elirey.....................

Elfry's Prune................

Emerald Drop................

Fair's Golden Drop...........

Flushing Gage.............

Florence...................

Fotheringham..............

Firanklin..................

Frost Gage.................

Frost Plum................

German Gage..............

German Prune..............

German Prune...............

Ghiston's Early..............

Golden Gage................

Gunne's Green Gage.........

Golden Cherry Plum..........

Goliath....................

Green Gage.................

Grosse Reine Claude.........

Grosse Reine................

Grosse Luisante.............

Grove House Purple.........

Grimwood's Early Orleans...

Gwalsh.....................

Hampton Court..............

Holland...................

Holland Prune.............

Horse Plum ...............

Howell's Early............. 30 ?

Howell's Large.............. 306

Hudson Gaye............. 277

Hnling's Superb............ 277

Ickworth Imperatrice........ 302

Imperial Ottoman........... 278

Inperial Gage............. 278

Imperatrice Blanche........... 285

Imperiale Blanche...........2 256

Imperatrice................ 290

Inspératrice Violette..........

Page.
Imperatrice

Imperatrice Violctte Gro..... 31

Imperial Violet............ 31 .

Impériale Violette........... 312

Impériale Ruuge............ 312

Imperiale................. 312

isleworth Green Gage...... 276

Isabella.................. 305

Italian Damask............. 31)6

Italian Damask............. $3^{1 / 2}$

Jaune Hâtive............. 279

Jaune de Catalogue......... 279

Jefferson................. 279

Jenkins's Imperial........... 306

Keyser's Plum............. 277

Kirke's.................. 306

K'nights' Large Drying...... 2S1

Knight s No.6........... 302

Lawrence's Favorite.. ...... 250

Lawrence's Gage............. 280

Large Green Drying.......... 281

Large Early Damson........ 301

Large Long Blue........... 309

Late Yellow Damson......... 287

La Délicieuse............... 291

La Royale................. 311

Leipzic................ 310

Little Queen Claude......... 288

Little blue Gage........... 2.89

Lombard................... 303

Long Scarlet.............. 303

Louis Philippe.............. 306

Lucombe's Nonsuch.......... 281

Magnum Bonum........... 286

MFaitre Claude............. 287

Bifatchless................ 295

Manning's Long Blue Prune... 309

Manning's Long Blue....... 309

Mirabelle Double........... 274

Mirabelle Grusise........... :774

Mirabelle.................

Mirabelle Petite............ 282

Mirabelle Jaune............ 25ล

Miser Plum............... 254

fifimms.................. 249

Mririam.................. 313

Mronsieur................. 304

Monsieur Ordinaire......... 304

Monsieut Hâtif............ 3C4

Lonsieur Hâtif de MIontmorency.................. 304

Mronsicur Tardiff........... 314

Morocco.................. 306

Mulberry.................252

Miyrobulan ............... 294

Myrobolan ................. 294

New Golden Drop........... 273

Nero Washington ............. 284 


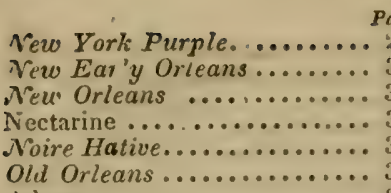

1) $r$ leans....................

Crange..................

Orange Gage..............

Petite Reine Claude...........

Peach Plum............... 306

Peach Plum.............. 306

Peoly's Early Blue ........... 3.J9

Perdrigon Rouge.......... 312

Perdrigon Blanc.......... 287

Perdrigon Violette......... 290

Perdrigon Violet........... 3u7

Pigeon's Heart............. 310

Pond's Seedling..............

Pond's Purple..............

Prince's Imperial............

Prune de St. Barnabé... ....

Drince's Yellow Gage.........

Prune Pêche...............

Précoce de Tours............

Prune de la St. Martin......

Prune d'Agen..............

Prune d'Ast. . . . . . . . . . . .

Prune de Brignole...........

Prune d'Allemagne..........

Prune d'UEf................

Prune d'Altesse..............

Prune Suisse...............

Purple Damson.............

Purple Favorite.............

Purple Gage................

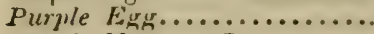

Purple Masnum Bonum......

Quetsche or German Prune....

Quetsche Grosse.............

Quetsche d'Allemagne Grosse.

Queen Victoria.............

Queen Mother...............

Reine Claude..............

Reine Claule Irioletie........

Reine C'laude Blanche.......

Reine Claude petite espèce...

Red Gage...................

Red Gage...............

Red Damask...............

Red AL anum Bonum. ......

Red Queen Nother...........

Red Perdrigon..............

Red Magnum Bonum.......... .

Red Imperial..............

Eivers' Early................

'Roe's Autumn Gage..........

Roche Curbon..............,

Rabe de Sergent..............
Page. Page

Royale ................. 311

Royale de Tours............ 313

Royal Tours................. 313

Royale Hâtive.............. 313

Saint Martin's Quetsche....... 283

Saint Catherine........... 283

Saint Martin .............. 295

Saint Martin Rouge ........ 295

St. Mraurin.............. 30

St. James Quetsche.......... 311

Saint Cloud............... 30n

Schuyler's Gage........... 276

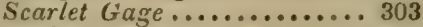

Semiana................. 291

Shailer's White Damson ..... 287

Shropshire, or Prune Damson.. 297

Sheen.................295

Sharp's Emperor............. 315

Siamese................ 284

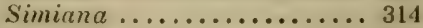

Small Green Gage.......... 258

Smith's Orleans............. 304

Steer's Emperor............ 300

Sucrin Vert.............. 276

Superior Green Gage....... 278

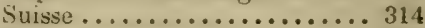

Sweet Damson............ 297

Sweet Damson ............ 301

Sweet Prune............. 310

Swiss Plum............... 314

Thomas................. 315

True Large German Prune... 310

Turkish Quetsche........... 310

Vert Bonne.............. 27to

Véritable lmpératrice....... 2(y)

Violet Perdrigon........... 290

Violette................ 290

Violet Diaper............. 295

Violet Perdrigon........... 304

Fiolette Hâlive........... 307

Fiolet de Tours...............307

Violet Queen Claude........ 308

Virginian Cherry......... 294

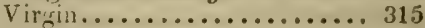

Washington .............. 2S4

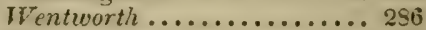

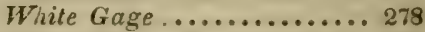

White Primordian..........279

White Imperatrice.......... 285

White Empress............ 255

White Magnum Bonum........ 286

White Iogul ............. 286

White Imperial............ 280

White Holland.............. 250

White Egg ................. 256

White Damson............. 287

Whitc Prune Dantson ...... 287

White Danuascene.......... 257

Whita Pardrigon........... 28' 
Page.

White Gage............... 257

Wilmot's Green Gage ........ 270

Wilmot's. New Green Gage... 276

Wilmot's Late Green Gage. . 2713

Winter Damson ............ 297

W'ilmot's Late Orleans....... 300

Wilmot's New Orleans........ 304

Wild Red or Yellow Plum .... 2ti3

Yellow Apricot............ 272

Yellow Perdrigon ........... 274

Yellow Magnum Bonum.....2 286

Felluw Egg................ 286

Yellow Gage .............. 2ss

Zwetsche .................310

\section{QUINCES.}

Apple-Shaped.............. 511 Chinese. ................ 51? Japan.................. 512 Oblong................... 511 Orange.................... 511 Pear-Shaped.............. 511 Portugal................. 511

IABPBERRIES AND BLACKBERRIES.

\section{RASPBERRIES.}

American Red............. 515

American Black............. 515

American White........... 516

Barnet................. 515

Black Raspberry............ 515

Brentford Cane ............. 516

Burley.................. 514

Common Red Antwerp........ 515

Commun Red.............. 515

Common Black-Cap........ 515

Cornwall's Prolific........... 516

Cornwall's Seedling..........

Cretan Red................ 516

Double Bearing............ 517

Double-Bearing Yellow....... 515

English Red, of some....... 515

Ever-Bearing Ohio ........... 518

Fastolff................. 517

Franconia............... 517

Framboisier à Gros Fruit..... 514

Howland's Red Antwerp..... 514

Knevet's Antwerp .......... 514

Knevet's Giant.............. 518

Large Red............... 516,

Late Cane............... 517

Lord Exmouth's............ 510

Nicto Red Antwerp.......... 514

Nottingham Scarlet.......... 51s

Okio Raspberry ............ 518
Page

Perpetual Bearing.......... 511

Red Antwerp.............. 514

Siberian................. sin

Thimble Berry ............ 515

True Red Antwerp......... 514

Trwice Bearing.............. 517

Victoria................. 519

White fintwerp ........... 515

Yellow Antwerp .......... 515

\section{BLACKBERRIES,}

Bush Blackberry........... 519

Dewuerry................ 519

Double White-blossomed Bram-

ble................. 519

Double Pink-blossomed Bramble 519

High Blackberry ........... 519

Low Blackberry............ 519

Rose Fluwering Bramble ...... 519

Trailing Blackberry......... 519

\section{STRAWBERRIES:}

Aberdeen................ 528

American Scarlet........... 527

Atrinson Scarlet........... 527

Austrian Scarlet............ 526

Bishop's Orange............ 5:6

Bishop's New............... 526

Black Prince................ 528

Black Imperial............ 528

Blood Pine............... 532

Black Roseberry ............. 526

Brewer's Emperor........... 529

Buisson ................. 535

Carolina................. 532

Caperon Royal............. 535

Caperon Hermaphrodite ..... 535

Common Hautbois........... 536

Commun Sans Filets ........ 535

Commun Rouge............. 534

Des Alpes à Firuit Rouge..... 534

Des Alpes à Fruit Blanc..... 535

Downton.................. 52 ?

Double-Bearing ............ 535

Dundee ................... 520

Duke of Kent's Scarlet....... 526

Early Scarlet.............. 528

Early Prolific Scarlet........ 526

Elton................... 529

Elton Seedling............. 529

English Red Wood......... 534

Fraisier Vert............. 53

French Musk Hautbois....... 535

Globe Scarlet.............. 526

Green Strawberry.......... 537

Green Alpille.............. 537

Green Pine............... 53 


\section{rage.}

Green Irind............. 537

Grrentevelrs . ime Giant...... 5.36

Gruve.Finl sirarlet........... 527

Jlusey"s Serelling. ........... E.3n)

Humbsin's Bay ............. 527

Hudsun................. 5:77

Keen's seedling............ 5531

Kipen's Blark Pine......... 5 531

Knight's Seedling......... 5:99

J.arife Farly Searlet......... 5:7

1.ate Scurlet ............ 5:27

Mulon ................ 52

Mh.lhsen scarlet............ $5: 27$

Mrthern C'astle............ 5127

Mtunl/s/y, withoul Runners... 53;)

Murphyis Child........... 5i31

. Husk Humtbois............. 53.

M!att's Brısish Queen.......5.531

Myalt's l'ille............... 5.31

Miatt's Deptford Pine....... 531

Mliatt's Eliza.............. 532

- Vina Scortia Scurlet........ 5. 5ti

Oli scarlet................ 52s

Old Pine................ 532

Orange Hudson's Bay ........ 520;

Uriganul Scarlet.............

Patagonian .............. 536

Pine Apple............... 536

Prince Allert . . . . . . . . . . . . 532

P'rolitic, or Conical Hautbois... 535

Red Bush Alpine.......... 535

Red Alpine................534

Red Monthly............ 534

Red IVond............... 534

Ruseberry ............... 528

Russ Phuenix ............. 538

Scarlet................. 52s

Scotich Searlet ........... 5.28

Southaminom sicarlet........ 527

Swainstmue Seedling ......... 531

True Chili............... 536

Virminia Scurlet.......... 523

Hirren's Seedling......... 5:77

Wilmot's superb........... 5i.31)

White Alpine............. 535

White Bush Alpine......... 535

Ithite Monthly........... 535

Ithite .Monthly, without Run-

ners................. 535

White Ivond................ 534

Felluw Chuli.............. 536

525 York River Scarlet........ 52? 


\section{GENERAL IN CEX.}

Almund, its history and uses, page 149 ; ciltivition, 140; varieties of 150 ; ornamental varieties, 152 .

American Blight, 66 .

Amelintation of Fruits, 1.

Apple, history of, 56 ; its uses, 56 ; criterion of qualities in, 55 ; propaga. tun, and soil and situation for, 54; grafting the trees, 17; pruning, 6.2 ; cultivation of orchards of the, 61 ; the bearing year of, to aiter, 61 ; insects injurious to, and mod ss of destroying, 62 ; gathering and keeping, 67.

Apple Borer, to destroy, 63 .

A pricot, its history, uses, and cultivation, 15 ; diseases of, 1.53 ; varietiea of, 153 ; ornamental sorts, 159 ; selection of choice varieties, 160

Aspect of fruit trees, $4 \mathrm{~S}$; effects of hills and valleys, 50

Bark Louse, to destroy, 66, 560 .

Bending the limbs, to induce fruitfulness, 34 .

Berberry, its uses, culture, and varieties, 160.

Birds, destroyers of insects, 55 .

B!ackberry, its culture and varieties, 518.

Black Gum, on the plum tree, 269.

Black Walnut, 261.

Butternut, 261.

Budding, the theory and practice of, 19 ; its advantages, best season for, 20 ; shield and American shield budding, 21 ; after treatment of the buds, 22 ; reversed shield, and annular, 23.

Canker Worm, its habits, 64 ; best modes to destroy, 65 .

Caterpillar, to destroy, 63.

Chestnut, its varieties, 262

Cherry, history and uses, 162; planted for avenues in Germany, 163; soil and situation for, 163; propagation (f, 164; classification of, and its varieties, 165; ornamental varieties, 199 ; selections of chuice sorts, 201.

Chamomile, used to destroy inseets, 54 ; for the scale insect $\mathrm{Cn}$ the orange tree, 543.

Cider, to make, 68 .

Citron, the, 545.

Coal-Tar, to prevent mice from girdling trees, 560 .

Codling Moth, 66.

Composition for wounds in fruit trees, 32.

Crab, the type of the apple, wild species of, 37.

Cranberry, its habits and culture, 205. 
Cross-Breeding, to obtain new varieties, 9.

Curl, in Peach trees, 468.

Curculio, its habits, 266 ; to destroy, 268.

Currant, its history, 201 ; uses and culture, 202; varieties, 203; crnamen. tal sorts, 205 .

Cultings, propagation 2 y 2 i

Duration of varieties of Fruit-Trees, 55!

De Candolle, his remarks on the decay of varieties, 552

Eyes, or Buds, propagation by, 27.

Fig, its history, 207; propagation and culture, 208; to hasten the ripenir. of the fruit, and its varieties, 209

Filbert, its varieties and culture, 261.

Fire-Blight, its nature, 322.

French Standard Names, key to the pronunciation of, 561 .

Frozen-Sap Blight, in the pear tree, 324.

Fruitfulness, induced by root-pruning, 32 ; by bending the limbs, and oy disbarking, 34 ; by salts of lime, 35 .

Grafting, its influence on varieties, $5,5.53$; its uses, 12 ; proper time for $12^{2}$; its limits, 14 ; splice and tongue-grafting, 15 ; cleft-grafting. 17 ; saddle-grafting, 18.

Graft, its influence on the stock, 26.

Grafting-clay, 19.

Grafting-wax, 19 .

Grape, its history, 21S ; soil and propagation, 210; culture of the foreign, 220 ; vinery culture, $22:$; insects and diseases of, 235 ; foreign varieties, 236 ; culture of the native, 217 ; vineyard culture of, 250 ; selection of choice sorts, 259.

Grape-Beetles, 252.

Gooseberry, its habits and uses, 213; propagation and culture, 214; vario. ties, 215 ; selection of choice, 217.

Hickory Nut, 261.

Hybridising plants, 9 ; its limits, 10.

Inoculating, or Budding Fruit-Trees, 19.

Insect Blight, in the pear, 322.

Insects, general remarks on, 51 ; to destroy by hand-picking, to kill in the grub state, 52 ; to kill in the winged state, 53 ; surts injurious to the apple, 62 ; to the grape, 235 and $25 \%$; to the plum, 266 ; to the pear, 322 ; to the peach, 460 ; to the melon, 535 ; to the orange, 543.

Knight, his mode of raising new varieties, 9 ; his theory on the decay of varieties, 551.

Knots, on the plum, 269.

Layers, propagation by, 28.

Laying-in-by-the-heels, 47.

Lemons and Limes, 545.

Longworth, on vineyard culture, 250.

Manure for fruit-trees, 45

Madeira Nut, 260.

Melon, its history and culture, 537 ; insects affecting, 538 ; culture of the Persian, 539 ; varieties, 538. 
Mice, to deter from girdling trees, 560

Mildew, on the grape, 252

Moths, to destroy, 54.

Mulberry, its habits and varieties, 259.

Mulching newly-planted trees, 45.

Nectarine. its habits and culture, 501 ; insects affecting, 502 ; varieties 562 ; selection of choice sorts, 509.

Nuts, description of, 260 ; Eliropean Walnut, Butternut, ₹61; Filberts of various sorts, 261; Chestnuts, 262.

Orange, its history and uses, 512 ; soil and culture, 543 ; scale insect on, 543 ; its varieties, 543.

Olive, its history and uses, 546 ; propagation, culture, and finest varieties, 547.

Pean.h, its history, 452 ; its uses, 45.4 ; propagation, 455 ; soil and situation for, 456 ; pruning, 457 ; insects and diseases of, 460 ; the Yellows in, its cause and remedy, 461 ; destruction in the leaves of, 470 ; varietres, 471 ; crnamental varieties, 499 ; selection of chcice sorts of, 500 ; tongue-grafting the, 15.

Peach Borer, 460 .

Pear, its history, 316 ; uses; and remarkable trees of, 313 ; gathering and keeping, 319 ; propagation, 320 ; soil and culture, 321 ; diseases of, blight, etc., 322 ; forms and character of varieties, 330 : selection of choice sorts, 452.

Planting deep, ill effects of, 45.

Plum, its histury and uses, 262 ; propagation and culture, 264; best soll for, $26 j$; insects and diseases of, curculio, etc., 266 ; varieties, 271 ; ornamental varieties, 315 ; selection of choice sorts, 316 .

Pomegranate, its history and uses, 549 ; propagation and culture 5.19 ; varieties, 550 .

Potash Wash for the Stems of Fruit-Trees, 560.

Position of Fruit-Trees, 48.

Preparing the Soil for Fruit-Trees, 43.

Propagation, by grafting, 12 ; by budding, 19 ; by cuttings, 26 ; by eyeg, 27 ; by layers, 23 ; by suckers, 29.

Prunes, to make, 263.

Pruning, to promote growth, 29 ; theory of, 30 ; to induce fruitfulness, 32 ; root pruning, 32 ; transplanted trees, 46 ; shortening-in, 453

Qenouille training, 36.

Quince, its history and uses, 509 ; propagation, culture, and varietien, 510; ortumental varieties, 512 .

Rabbits, to prevent their girdling trees, 560 .

Raspberry, its habits, 512 ; uses, culture, and varieties, 513.

Renewal Training of the Vine, 221

Ringing and Disbarking, 34.

Rivers' Remarks on Root-Pruning, 33.

Rnot-grafting, Dr. Van Mons' remarks on, 17.

Root-pruning, its advantages, 32.

Rust and cracking of the fruit of the Pear-tree, $55 \%$.

Salt, to destroy insects generally, 53; to destroy the curculio, 269.

Scions, to select, 13. 
Seedlings, to raise, 5 .

Sea Air, its effects on fruit-trees, 555

Shaddock, 545.

Shellac, composition for wounds in trees, 32.

Shortening-in, mode of pruning the peach, 458.

Stnells. their power of driving away insects, 53

Suil, the best for fruit-trees, 48 ; gravelly loam, sandy loan, clayen toase, 49 ; its effect on the health of the trees, 49.

Soft-Soap, for the stems of fruit-trees, 560.

Species of Fruit-Trees, 3; reproduce themselves, 3.

Slug-worm on the Pear, 325.

Spurring-in, training the vine, 221.

Stools, for propagation, 29.

Stocks, for grafting on, 13 ; their iufluence on the graft, 21 ; bad ones catee the decay of varieties, 553 .

Strawberry, its history and uses, 520 ; propagation and anil for, 521 ; culture in rows and in strips, 522 ; fertile and barran plants or, 523 ; varieties, 525 ; selections of choice sorts, 536 .

Taking-up trees, 42.

Thorn, the, as stocks for the pear, 320

Tobacco-water, for insects, 54.

Toads, destroyers of insects, 56.

Transplanting, directions for, 41 ; preparing places for 43

Training, general remarks on, 35 ; its objects, 36 ; cunical standards, and quenouille training, 36 ; fan training, 38 ; hor $1 z n t_{3} 1$, 10 ; renewa of grapes, 221.

Vallies, eflects of, 50.

Varieties, to produce new, 3 ; their tendency to change. 4 : do not produce the same, 4 ; influence of grafting on, 4 ; Van $M$ nns' mode of raising new, 5 ; raising new, by crop breeding, 9 ; pronagation of, 12 ; remarks on the duration of, 551 ; Knight's theory nin the decay of, 5.52; effects of climate on, 555; to restore decayed, 559.

Vine, grafting the, 18 ; culture of, 221.

Vinery, rheap mode of building, 222; for fire heat, 220; diary of sclture in the, 228.

Vineyard Culture, 250.

Wash for the Stems of Fruit-Trees, 560.

Water Melon, its uses, culture, and varieties, 541.

Whale oil soap, to destroy insects, 54.

Yejluws, a disease of the Peach, 402 ; its symptoms, 462 ; ite caure 192 ; remedy for, $4 \dot{j} 7$.

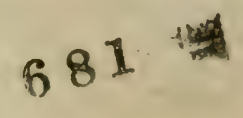







\section{K. $0=x^{\circ}$ s.}




\section{LIBRARY OF CONGRESS |||||||||||||||||||||||||||||||||||||||||

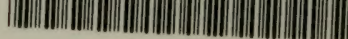 \\ 000091?1216}

